\title{
Canadian Rheumatology Association Meeting JW Marriott Parq Vancouver Vancouver, British Columbia, Canada February 21-24, 2018
}

\begin{abstract}
The 73rd Annual Meeting of The Canadian Rheumatology Association (CRA) was held at the JW Marriott Parq Vancouver, Vancouver, British Columbia, Canada, February 21-24, 2018. The program consisted of presentations covering original research, symposia, awards, and lectures. Highlights of the meeting include the following 2018 award winners: Distinguished Rheumatologist, Alan Rosenberg; Distinguished Investigator, John Hanly; Teacher-Educator, Anna Oswald; Young Investigator, Évelyne Vinet; Best Abstract on Research by a Rheumatology Resident, May Choi; Best Abstract on Systemic Lupus Erythematosus Research by a Trainee - Ian Watson Award, Lily Wang; Best Abstract on Clinical or Epidemiology Research by a Trainee - Phil Rosen Award, Sophie Wojcik; Best Abstract on Basic Science Research by a Trainee, Jessica Salituri; Best Abstract on Quality Care Initiatives in Rheumatology, Arielle Mendel; Best Abstract by a Post-Graduate Research Trainee, Victoria Stefanelli; Best Abstract by a Medical Student, Tedi Qendro; Best Abstract by an Undergraduate Student, Sujay Nagaraj; Best Abstract on Research by Young Faculty, Kimberly Legault; Best Abstract on Pediatric Research by Young Faculty, Roberta Berard and Dax Rumsey. Lectures and other events included Keynote Address by John Hanly, Distinguished Investigator Awardee: Lupus and the Nervous System; Keynote Lecture by Linda Li: Arthritis Care in the Digital Age: The Patient CAN See you Now; State of the Art Lecture: Immunotherapy: Immune Modulation to Cure RA by Ranjeny Thomas; and the Great Debate: Be it Resolved that Precision Diagnostic Tools such as Biomarkers, Advanced Immunology, and Artificial Intelligence will Reduce the Need for Rheumatologists in the Future. Arguing for: Susa Benseler and Walter Maksymowych, and against: Trudy Taylor and Johannes Roth. Topics including rheumatoid arthritis, systemic lupus erythematosus, systemic sclerosis, Sjögren syndrome, psoriatic arthritis, spondyloarthritis, vasculitis, osteoarthritis, fibromyalgia, and their respective diagnoses, treatments, and outcomes are reflected in the abstracts, which we are pleased to publish in this issue of The Journal.
\end{abstract}




\section{Podium}

14-3-3eta Synergistically Interacts with Anti-CCP2 Status and Simple Disease Activity Index (SDAI) Levels to Predict Rapid Radiographic Progression over the Following Year in Patients with Inflammatory Polyarthritis

Gilles Boire (Université de Sherbrooke, Sherbrooke); Nathalie Carrier (Centre Hospitalier Universitaire de Sherbrooke, Sherbrooke); Artur Fernandes (Université de Sherbrooke, Sherbrooke); Patrick Liang (Université de Sherbrooke, Sherbrooke); Ariel Masetto (Université de Sherbrooke, Sherbrooke); Norma Biln (Augurex Life Sciences Corp, Vancouver); Yuan Gui (Augurex Life Sciences Corp., Vancouver); Jane Savill (Augurex Life Sciences Corp., Vancouver); Sara Michienzi (Augurex Life Sciences Corp., Vancouver); Walter Maksymowych (University of Alberta, Edmonton); Anthony Marotta (Augurex Life Sciences Corp., Vancouver)

Objectives: Our objective is to examine whether HIGH 14-3-3-eta levels add significantly to anti-CCP2 positivity and elevated Simple Disease Activity Index (SDAI) levels measured at the same visit to predict rapid radiographic progression (RRP) over the following year. We previously reported that baseline 14-3-3-eta levels $\geq 0.50 \mathrm{ng} / \mathrm{ml}$ (HIGH 14-3-3-eta) were predictive of radiographic progression over 5 years, as were the serial 14-3-3-eta changes in serum levels over the course of disease.

Methods: The Sharp/van der Heijde (SvH) and SDAI scores, serum 14-3-3-eta and anti-CCP2 were all measured at initial and yearly follow up visits in patients with recent onset polyarthritis treated to remission. HIGH 14-3-3-eta was defined as $\geq 0.50 \mathrm{ng} / \mathrm{ml}$; anti-CCP2 positivity as $\geq 5 \mathrm{U} / \mathrm{ml}$ (EuroImmun); elevated SDAI as $>11$; RRP as an increase of $\geq 5$ Units in the Erosion component of the $\mathrm{SvH}$ score. Generalized Estimated Equations (GEE) were performed to assess the association with RRP of elevated SDAI, positive anti-CCP2 and HIGH 14-3-3-eta, alone and in combination.

Results: Mean age was 58.5 years, $61.2 \%$ female; median duration at inclusion 3.4 months; median (IQR) follow-up 17.6 (9.0-33.5) months. Out of 1529 complete evaluations in 533 patients, 511 (33.4\%) were HIGH 14-3-3-eta, 590 (38.6\%) anti-CCP2 positive and $722(47.2 \%)$ elevated SDAI. In univariate analyses, HIGH 14-3-3-eta, anti-CCP2, and elevated SDAI were each associated with significantly increased Relative Risks (RR) of RRP: $1.82(1.36-2.43) \mathrm{p}<0.0001 ; 2.33(1.65-3.30) \mathrm{p}<0.0001$; and 1.80 $(1.38-2.34) \mathrm{p}<0.0001$, respectively. In GEE, relative to being negative for all three, being positive for all of HIGH 14-3-3-eta, anti-CCP2, and elevated SDAI was associated with an increased RR of 4.67 (2.77-7.86), RRP then occurred following $25.5 \%$ of such visits. Being positive for only 2 variables identified subsets of patients at intermediate RR (2.61 to 4.42) depending on the combination, while only 1 positive variable was associated with lower RR (0.93 - 1.98) depending on the variable. The contribution of 14-3-3-eta was highest in patients with either anti-CCP2 positive or elevated SDAI alone.

Conclusion: In this cohort of patients with polyarthritis treated to remission, the presence of HIGH 14-3-3-eta amplified the risk for RRP conferred by anti-CCP2 and/or active disease. The highest risk of RRP over the following year was present in anti-CCP2 positive patients who also had active disease and HIGH 14-3-3-eta levels. Adding 14-3-3-eta measurement to anti-CCP2 and clinical measures over the course of RA may inform therapeutic strategies tailored to halt rapid joint damage progression in the most susceptible patients.

Suboptimal Immunization Coverage Among Rheumatology Patients in Routine Clinical Care

Tedi Qendro (McGill University, Montreal); Maria de la Torre (CEMIC, Buenos Aires); Pantellis Panopalis (McGill University, Montreal); Elizabeth Hazel (McGill, Montreal); Ines Colmegna (McGill University Hospital Centre, McGill University, Montreal); Marie Hudson (McGill University, Jewish General Hospital, Lady Davis Institute for Medical Research, Montreal)
Objectives: Vaccine-preventable infections pose an increased risk of disease and complications in patients with systemic autoimmune rheumatic diseases (SARD). As such, while recommendations highlight the importance of vaccination in this at-risk population, immunization coverage remains largely unknown. We assessed vaccination rates and predictors of vaccination among rheumatology patients in routine clinical care.

Methods: Consecutive patients presenting to a tertiary rheumatology clinic at the McGill University Health Center between May and September 2015 were asked to fill a survey on vaccination. Patients self-identified as having rheumatoid arthritis (RA) (RA, juvenile idiopathic arthritis), SARD (e.g., vasculitis, lupus, systemic sclerosis, myositis), spondyloarthropathies ( $\mathrm{SpA}$ ) (psoriatic arthritis, ankylosing spondylitis), or other diseases (Other). Multivariate logistical regression analyses were performed to evaluate patient and physician factors associated with vaccination rates (influenza, pneumococcus, hepatitis B virus [HBV], and herpes zoster [HZ]).

Results: 352 subjects were included in the analysis (RA:135, SARD:113, SpA:48, Other:56). Vaccination rates were reported as: (1) influenza: RA 48.1\%, SARD 42.0\%, SpA 33.3\%, Other 48.1\%; (2) pneumococcus: RA $41.5 \%$, SARD $37.8 \%$, SpA $31.6 \%$, Other $16.3 \%$; (3) HBV: RA $34.0 \%$, SARD 55.6\%, SpA 71.4\%, Other $36.8 \%$; and (4) HZ: RA 3.2\%, SARD $9.5 \%$, SpA $17.6 \%$, Other 7.0\%. In multivariate analyses, physician recommendation was the strongest independent predictor of vaccination across all vaccine types (influenza: odds ratio (OR) 12.6, 95\% confidence interval $(\mathrm{CI})$ 5.40-29.6; pneumococcus: OR 424, $95 \%$ CI 99.7-1801; HBV: OR 12.8, $95 \%$ CI 5.27-31.1). Patient-reported awareness of the benefits of vaccination was also a significant predictor of vaccination (influenza: OR $5.15,95 \% \mathrm{CI}$ 1.26-21.1; HBV: OR 11.5, 95\% CI 2.49-52.8). The effect of age varied by vaccine (influenza: OR 1.04, 95\% CI 1.02-1.06; HBV: OR 0.96, $95 \%$ CI 0.94-0.99). There were no other significant associations with vaccination, including disease group, disease duration, comorbidities (cancer, diabetes, renal disease), treatment type (disease-modifying anti-rheumatic drugs and/or biologics), and access to a primary care physician.

Conclusion: Despite national guidelines and recommendations for vaccination in this at-risk population, immunization coverage against influenza, pneumococcus, $\mathrm{HBV}$, and $\mathrm{HZ}$ is far from optimal among ambulatory rheumatology patients. An important role for both patient and physician education is highlighted from our study as both patient awareness of the benefits of vaccination, and physician recommendation were strongly predictive of vaccine uptake. These results will help inform strategies aimed at optimizing vaccination rates in this at-risk population. Best Abstract by a Medical Student.

\section{3}

\section{Citrullinated Provisional Matrix Proteins Influence Fibroblas} Activation

Victoria Stefanelli (Georgia Institute of Technology, Atlanta); Dwight Chambers (Georgia Institute of Technology, Atlanta); Vincent Yeh (University of Virginia, Charlottesville); Shilpa Choudhury (Georgia Institute of Technology, Atlanta); Kelly Pesson (Georgia Institute of Technology, Atlanta); Matthew Torres (Georgia Institute of Technology, Atlanta); Thomas Barker (University of Virginia, Charlottesville)

Objectives: Considering that both citrullination and activated fibroblasts are hallmarks of several chronic inflammatory conditions, including malignant cancers, lung fibrosis, and rheumatoid arthritis (RA)-and that especially in the case of RA, these are both correlated with a severe disease course-our objective is to better understand how citrullinated provisional matrix proteins may influence the initiation and maintenance of activated fibroblast phenotypes. Methods: Fibronectin, the primary adhesive matrix protein for fibroblasts within inflammatory environments, was citrullinated using a mixture of PAD2 and PAD4 enzymes. Cell phenotypes, primarily including adhesion, spreading, migration, and mechanosensing capacity, were evaluated through a combination of fluorescence microscopy, time-lapse microscopy, and atomic force microscopy (AFM), respectively. To begin elucidating the mechanism underlying observed phenotypic differences, mass spectroscopy (MS) was performed on citrullinated fibronectin (Cit-Fn) to identify specific

Personal non-commercial use only. The Journal of Rheumatology Copyright @ 2018. All rights reserved. 
regions of modification, and a Systematic Analysis of PTM Hotspots (SAPH-ire) was performed to evaluate which of these regions possesses the most probable biological significance. We explored the impact of these modifications on specific integrin subtypes by studying the ability of $\mathrm{CHO}$ cells transfected with single integrin subtypes to interact with Cit-Fn. Integrin preference and focal adhesion formation, along with associated downstream signaling, were analyzed using a combination of confocal microscopy and magnetic bead force-inducible co-immunoprecipitation assays.

Results: Phenotypic assays determined that healthy fibroblasts possess a decreased ability to attach and spread on Cit-Fn. Despite this deficit in attachment, however, AFM results show that fibroblasts on Cit-Fn are on average stiffer, indicating that mechanotransduction pathways are activated which allow the cells to interpret their environments as being stiff and to react in kind. One such resulting reaction is an enhanced migratory capacity as determined by improved defect closure in wound healing assays. Underlying these altered phenotypes are at least 20 unique sites of fibronectin citrullination, as identified by MS, three of which reside in the integrin binding domain and are predicted by SAPH-ire to have the largest biological impact. $\mathrm{CHO}$ adhesion assays, in part, confirm this prediction demonstrating that alpha-v-beta3 integrin binding on Cit-Fn is detrimentally impacted. In consequence, alpha-5-beta1 integrins appear to compensate by both increasing in abundance and recruiting additional integrin subunits and downstream signaling molecules, notably phospho-FAK and vinculin.

Conclusion: By itself, citrullination of fibronectin fundamentally alters how fibroblasts interact with the matrix, ultimately resulting in enhanced migratory capacity. An integrin switch, whereby alpha-5-beta1 dominates over alpha-v-beta3 in fibroblast attachment and subsequently enacts mechanotransduction signaling pathways, underlies this altered behavior. Best Abstract by a Post-Graduate Research Trainee.

4

Evaluating the Effectiveness of a Faculty-Resident Co-Learning Quality Improvement Curriculum

Shirley Lake (University of Toronto, Toronto); Brian Wong (University of Toronto, Toronto); Alex Kiss (University of Toronto, Toronto); Natasha Gakhal (Women's College Hospital, Toronto)

Objectives: One of the greatest challenges in quality improvement (QI) training is the lack of faculty experts who can teach and supervise QI projects. We implemented a faculty-resident co-learning QI curriculum, where faculty and residents experienced the curriculum together, which we hypothesized would allow faculty to role model the importance of QI. The purpose of our study was to evaluate the impact of the co-learning approach on resident knowledge of QI.

Methods: From July 2013 through June 2017, the authors conducted a yearly co-learning QI curriculum for the Division of Rheumatology at the University of Toronto. Faculty and resident participants learned together using didactic and interactive formats. Project work occurred between sessions, and at the end of the year, all programs presented their projects at a multidivisional QI half-day. We compared our model to 4 other Ontario rheumatology programs where a range of QI educational experiences exist (didactic rounds, reading materials, case based discussions, interactive role plays, and discussion of QI projects). We evaluated our program by collecting descriptive data from workshop evaluations. We conducted a yearend objective structured clinical exam (OSCE) in 2016 and 2017, where all Ontario rheumatology residents were presented a QI problem and had to identify an aim, a set of measures and potential QI interventions. They were evaluated using the Quality Improvement Knowledge Assessment Tool (QIKAT), a validated tool of QI assessment. The results of the OSCE station was examined using a non-parametric Wilcoxon rank sum test.

Results: Twenty-eight rheumatology residents had gone through the co-learning curriculum. Ninety-two percent ( 49 of 53 workshop evaluations) felt the course was excellent or outstanding and the majority felt it improved their QI knowledge and skills. Nineteen University of Toronto rheumatology residents and 29 other rheumatology residents completed the QI OSCE station. Residents who completed the co-learning QI curriculum scored higher on the QI station than other residents (Median 8/10 vs. 7/10, p = $0.0015)$. PGY5 residents who completed the co-learning project scored higher than PGY4 residents at $\mathrm{U}$ of Toronto who did not complete the project (Median $9 / 10$ vs. $7 / 10, p=0.0024$ ), and higher compared to other residents (Median $9 / 10$ vs. $7 / 10, \mathrm{p}<0.0001$ ).

Conclusion: We improved resident learning by better preparing faculty to do and teach QI using the co-learning framework. This was a small sample size and will be the basis for future work. Collaboration amongst schools to share QI resources and expertise may help improve QI skills for all rheumatology trainees.

5

Impact of Maternal Systemic Autoimmune Rheumatic Diseases (SARDs) on Neonatal Outcomes: A Population-level Analysis

Stephanie Keeling (University of Alberta, Division of Rheumatology, Edmonton); Anamaria Savu (University of Alberta, Edmonton); Padmaja Kaul (University of Alberta, Edmonton)

Objectives: We examined the association between SARDs and neonatal outcomes in a contemporary pregnancy cohort in the province of Alberta, Canada

Methods: The patient population consisted of women giving birth between January 1, 2005 to December 31, $2014(n=312,081)$. For women with multiple gestations during the period, one birth event was randomly selected. Women with SARDs included any of the following: systemic lupus erythematosus (SLE), systemic sclerosis, myositis and sjogren's syndrome, diagnoses based on the presence of International Classification of Disease version 9/10 codes in outpatient or inpatient records. Baseline characteristics, comorbidities, medication use (available for all births after January 1, 2009), and neonatal outcomes among women with and without SARDs were compared.

Results: Compared to women with no SARDs $(\mathrm{n}=311,755)$, women with SARDs $(\mathrm{n}=326,0.1 \%$ ) were slightly older (SARDs 31.3 vs No SARDs 29.3 years $(\mathrm{p}<0.01)$ ) but did not differ in terms of rural residence, ethnicity, median household income or nulliparity. Of the 326 women with SARDs, $271(83.1 \%)$ had SLE, $53(16.3 \%)$ had systemic sclerosis, $26(8 \%)$ had dermatomyositis, 17 (5.2\%) had polymyositis and 29 (8.9\%) had sjogren's syndrome. Rates of pre-term delivery, emergent caesarean section, induction, hypertensive disorders/eclampsia and mortality were higher among women with SARDs than those with no SARDs. Offspring of women with SARDs had lower birth weights, were more likely to have small for gestational age babies (SGA), and had longer stays in neonatal ICU. Among women with SARDs, prescription rates in the 270 days prior to delivery were highest for anti-malarials $(25.1 \%)$ followed by steroids $(16.3 \%)$. Regarding the impact of medication use during pregnancy, the unadjusted odds ratio was significant for steroids on preterm delivery (OR 3.84 (95\% CI 1.66, 8.89, p < 0.05 ) and hypertensive disorders of pregnancy (OR 3.67 (95\% CI 1.51, 8.93), p < $0.05)$; for nonsteroidal anti-inflammatories, the unadjusted odds ratio was significant for preterm delivery (OR 4.96 (95\% CI 1.55, 15.85, $\mathrm{p}<0.05)$ Antimalarials did not have an impact on pregnancy related outcomes in the unadjusted analysis. After multivariable adjustment, both NSAID use (OR (95\% CI): $5.24(1.57,17.52), \mathrm{p}<0.01)$ and steroid use (OR (95\% CI): 3.15 $(1.31,7.59), \mathrm{p}<0.01)$ were significantly associated with a higher risk of preterm delivery.

Conclusion: Women with SARDs are at an increased risk of adverse outcomes during pregnancy. The association between corticosteroid and NSAID use and preterm delivery requires further investigation. Our findings suggest the need for closer monitoring and coordinated care with obstetrics and perinatology in these high risk women.

6

Effectiveness of the Outreach Model for Rheumatology Specialty Clinics to On-Reserve First Nations in Alberta: System-level and Individual Measures of Performance and Outcomes

Sujay Nagaraj (University of Calgary, Calgary); Claire Barber (University 
of Calgary, Calgary); Margaret Kargard (Siksika Health Services, Siksika); Tyler White (Siksika Health Services, Siksika); Cheryl Barnabe (University of Calgary, Calgary)

Objectives: A model of care consisting of rheumatology specialty services embedded in the primary care context was instituted to reduce barriers to care and improve treatment outcomes for First Nations persons with Inflammatory Arthritis (IA) in one community. We assessed the system-level performance of the model as well as its effectiveness on disease activity measures and patient-reported outcomes over 7 years.

Methods: Patients were enrolled in a longitudinal cohort at the Siksika Nation in Alberta (2011-2017). Clinical characteristics, disease activity measures, and treatment recommendations were systematically recorded over follow-up. System-level performance was evaluated according to established measures developed by the Arthritis Alliance of Canada. Mixed-model regression analysis was performed to determine monthly rates of change for disease activity measures, adjusted for baseline demographics and disease activity measures.

Results: 59 participants (78\% female, mean age 47 (SD 13)), predominantly with RA ( $n=36$ ), were followed for a mean of 29 (SD 23) months with a mean of 6 (SD 5) visits per participant. At the system-level, the 50th and 90th percentile wait times were 69 and 695 days, respectively. Only 33\% of patients were seen in the benchmark waiting time of 4 weeks but $83 \%$ of patients were followed up in each measurement year. Nearly all (96\%) of patients received a DMARD in each measurement year and $90 \%$ were prescribed a DMARD within 2 weeks of diagnosis. At the baseline visit, $72 \%$ of participants with RA were in DAS28 moderate or high disease activity state. Disease-modifying agents were escalated for moderate or high disease activity at $65 \%$ of visits for RA with reasons for not escalating including pending investigations, being too soon to assess response to a new therapy, patient choice, contraindication to therapy, absence of joint swelling, or intra-articular injections provided. Swollen and tender joint counts significantly improved during follow-up (SJC28 $-0.20,95 \%$ CI -0.29 to -0.10 ; TJC28 $-0.20,95 \%$ CI -0.34 to -0.01 , both $\mathrm{p}<0.001)$. Pain $(0.01,95 \%$ CI -0.05 to $0.05, \mathrm{p}=0.97)$, physician global evaluation score $(-0.05,95 \% \mathrm{CI}$ -0.11 to $0.06, \mathrm{p}=0.08)$, and HAQ $(-0.01,95 \% \mathrm{CI}-0.01$ to $0.01, \mathrm{p}=0.45)$ did not significantly improve over time. Patient global evaluation score continued to worsen over time $(0.08,95 \%$ CI 0.03 to $0.13, \mathrm{p}=0.002)$.

Conclusion: The program met several system-level performance measure targets however patients still experienced long wait times. Despite improvement in swollen and tender joint counts and adherence to current treatment paradigms, patient-reported outcomes did not significantly improve during follow-up. Further innovation is required to meet relevant outcomes. Best Abstract by an Undergraduate Student.

\section{7}

Preventing Rheumatoid Arthritis (Pre-RA): Preferences of Potential Recipients, Patients, and Health Care Professionals for Preventative Treatment

Mark Harrison (University of British Columbia/Arthritis Research Canada, Vancouver); Nick Bansback (University of British Columbia/Arthritis Research Canada, Vancouver); Luke Spooner (University of British Columbia, Vancouver); Cheryl Koehn (Arthritis Consumer Expert, Vancouver); Marie Hudson (McGill University, Jewish General Hospital, Lady Davis Institute for Medical Research, Montreal)

Objectives: To (1) determine preferences of patients with rheumatoid arthritis, first-degree relatives (FDRs) of patients (as a proxy for pre-symptomatic, at risk people), and health care professionals (HCPs) for preventative treatment, and (2) predict the likely uptake of preventative treatments.

Methods: A discrete choice experiment with 5 key attributes of treatment (reduction in risk of RA, how treatment is taken, chance of side effects, certainty in estimates, their $\mathrm{HCPs}$ /patients opinion) was administered using a web survey to Canadian patients and FDRs of patients, and HCPs. This survey asked participants to first choose between sets of 2 hypothetical preventative RA treatments, then choose between their preferred treatment and 'no treatment for now'. DCE data was analyzed using conditional logit regression model to estimate the significance and relative importance of attributes in influencing preferences and a mixed logit model to explore preference heterogeneity.

Results: 78 patients, 30 FDRs and $47 \mathrm{HCPs}$ from nine Canadian provinces started and completed all tasks in the survey. HCPs were primarily rheumatologists $(81 \%)$ or rheumatology nurses (9\%). Patients (86\%) and FDRs (73\%) were predominantly female; patients were older (6\% aged 18-39) than FDRs (23\% aged 18-39). Preferences for levels within attributes were in the expected direction. The preference of the patient was the most influential attribute for HCPs (patient prefers vs. does not prefer: beta0.734, p < 0.001), followed by reduction in the risk of developing RA (risk reduced from 60 in 100 to 24 in 100 vs. 44 in 100) beta0.509, p < 0.001). The opinion of HCPs was also important to patients and FDRs (patients: beta0.461; FDRs: beta0.379), along with risk reduction (patients: beta0.413; FDRs: beta0.430). FDRs had stronger preferences for how treatment was taken (oral vs infusion: beta0.355, $\mathrm{p}=0.010$ ) than patients (beta0.257, $\mathrm{p}=0.010$ ) or HCPs (beta0.257, $\mathrm{p}=0.010$ ). Predicted uptake suggested that $19 \%$ of FDRs would not be willing to take any currently available treatment as preventative treatment, while $4 \%$ of HCPs wouldn't be willing to recommend a preventative treatment. HCPs were predicted to prefer non-biologic DMARDS (e.g. oral methotrexate 35\%; hydroxychloroquine 19\%) than biologic DMARDs (e.g. abatacept 5\%); FDRs preferred non-biologic DMARDS (oral methotrexate $24 \%$; hydroxychloroquine $32 \%$ ) and other options like statins (65\%).

Conclusion: Uptake of preventative strategies will be most influenced by discussions of preferred options between providers and recipients, potential risks and benefits, and convenience. This evidence will help policy makers identify the most likely preventative treatment strategies to be acceptable to at-risk people. Supported by a CIORA grant.

8

A Pragmatic Pilot Randomized Controlled Trial of the OA Go Away, a Symptom and Exercise Tracking Self-Management Behavioural Intervention for Promoting Adherence to Physical Activity Among Individuals with $\mathrm{OA}$ of the Hip or Knee

Gail Paterson (The Arthritis Society, Ottawa); Isabelle Gaboury (Université de Sherbrooke, Sherbrooke); Peter Tugwell (University of Ottawa, Ottawa); Karine Toupin-April (Children's Hospital of Eastern Ontario Research Institute, Ottawa)

Objectives: Despite the well-established benefits of exercise and physical activity, the majority of Canadians with OA of the hip or knee do not meet Health Canada guidelines for physical activity. Behavioural interventions are needed to help patients make the transition from supervised structured health care provider care to long term independent self-management. The objectives of this study were to assess the feasibility of conducting a full $\mathrm{RCT}$ to test the effectiveness of the OA Go Away self-management behavioural intervention and to determine its acceptability. The OA Go Away is an internal reinforcement adherence measure that includes a monthly personalized self-report symptom tracker, goals and action plan and a weekly exercise $\log$.

Methods: This study was a 3-month pragmatic pilot-test of the OA Go Away. 40 participants with OA of the hip or knee were randomized into either the treatment group who used the OA Go Away for 3 months, or the control group who received standard care at The Arthritis Society. Participants completed outcome measures at baseline and at 3 months. Outcomes were compared between study groups at the end of the trial using Mann-Whitney and 2-tailed Fisher's exact tests to assess adherence to prescribed exercise, level of physical activity, functional goal attainment, pain, stiffness, physical function, and quality of life. Treatment group participants completed a measure to determine the usefulness and acceptability of the OA Go Away. Results: 36 participants (16 in the treatment group and 20 in the control group) completed the protocol. At the end of the trial, participants in the treatment group were more physically active $(\mathrm{p}=0.02)$, had less pain $(\mathrm{p}=$ $0.03)$, better function $(\mathrm{p}=0.048)$ and more vitality $(\mathrm{p}=0.01)$. Adherence,

Personal non-commercial use only. The Journal of Rheumatology Copyright @ 2018 . All rights reserved. 
goal attainment and other quality of life scores, were greater in the treatment group but were not statistically significant. The majority of participants in the treatment group felt that the OA Go Away was acceptable in terms of time commitment, useful and motivational. Some of the participants proposed that an electronic version of the OA Go Away would facilitate visualization of changes in status, exercise progress and achievement of goals.

Conclusion: This pilot RCT justifies a formal randomized controlled trial of the OA Go Away and shows promising results concerning its acceptability and effectiveness. Its real potential may be best realized if translated into an electronic tool.

9

Disparities between Ultrasound and MRI Assessment of Erosions: Are they Dependent on Size and Location?

Hanyan Zou (McMaster University, Hamilton); Myriam Allen (Université Laval, Hamilton); George Ioannidis (McMaster University, Hamilton); Saara Totterman (QMetrics Technologies, Rochester); Karen Beattie (McMaster University, Hamilton); Maggie Larche (McMaster University, St Joseph's Healthcare Hamilton, Hamilton)

Objectives: Compared to Magnetic Resonance Imaging (MRI), studies have reported a high specificity of ultrasound (US) detection of bone erosions joints in patients with RA, but only low/moderate sensitivity. However, the nature of the US probe does not allow for erosions to be seen in regions directly adjacent to other bones. When examining sensitivity and specificity, this is an important factor to consider. Thus, we evaluated US assessment of erosions in the metatarsophalangeal joints (MTPJ) by characterizing the location of erosions on MTPJ bones observed by MRI.

Methods: Patients newly diagnosed with RA (ACR criteria) were recruited. Using US, the presence of erosions on 2nd-5th MTPJ was recorded. The same foot was imaged using MRI. Metatarsal heads and phalangeal bases were sectioned into dorsal, plantar, medial and lateral regions. Blinded to US results, a radiologist recorded the locations and grades (OMERACT criteria, grades 0-10) of MRI erosions on each bone of the 2nd-5th MTPJ. Dorsal and plantar surfaces and lateral 5th MTPJ were hypothesized to be within the field-of-view of US. We described the location and size of erosions seen on MRI, and compared these with erosions seen on US.

Results: This study included 39 patients $[\mathrm{n}=33$ female, mean (SD) age $=$ 51.6 (10.3) years]. MRI found erosion grade $\geq 1$ in 123 MTPJ bones (26 on the phalanx base, 97 on MTP head), totaling 101 unique joints (of 156 total) affected by erosion. The majority of erosions were grade $1[\mathrm{n}=107(87 \%)]$. Erosions were most common on the plantar surface $[n=110(89.4 \%)]$, with few on dorsal, medial or lateral surfaces of the bones $(n=5,21$ and 38 respectively). Despite MRI identifying 90 joints with erosions in regions hypothesized to be within the US field-of-view ( $57.7 \%$ of 156 joints), these erosions were rarely visualized on US $(n=5)$. Of 14 MTPJ with MRI erosion grade $\geq 2$ in either bone, US visualized erosions in 4 joints. US found erosion in 2 joints that were not seen on MRI.

Conclusion: Most MRI erosions in the MTPJs were on the plantar surface which can hypothetically be visualized by US. However, the lack of plantar erosions seen on US suggests i) US may have a smaller-than-expected field-of-view, ii) the cortical bone is not clearly broken or iii) the size of the cortical break may be too small to see. Erosions visualized on US were often grade $\geq 2$ on MRI. However, this early RA cohort had few such erosions.

10

Diagnostic Ascertainment of Axial Spondyloarthritis in Patients Presenting with Undiagnosed Back Pain: What is the Impact of MRI in Rheumatological Practice?

Walter Maksymowych (University of Alberta, Edmonton); Raj Carmona (McMaster University, Hamilton); James Yeung (Vancouver); Jonathan Chan (University of British Columbia, Vancouver); Liam Martin (University of Calgary, Calgary); Ariel Masetto (Université de Sherbrooke, Sherbrooke); Sibel Aydin (University of Ottawa, Ottawa); Dianne Mosher (University of Calgary, Calgary); Stephanie Keeling (University of Alberta, Division of
Rheumatology, Edmonton); Olga Ziouzina (University of Calgary, Calgary); Joel Paschke (Canadian Research Education (CaRE) Arthritis, Edmonton); Amanda Carapellucci (Canadian Research Education (CaRE) Arthritis, Edmonton); Robert Lambert (University of Alberta, Edmonton)

Objectives: In current rheumatology practice, the circumstances that prompt clinicians to order MRI in patients with suspected axSpA are unclear and the degree to which MRI changes diagnostic ascertainment of axSpA in patients presenting with undiagnosed back pain has not been formally studied. We aimed to determine whether any particular patient characteristic(s) is associated with rheumatologist ordering of MRI and to assess the impact of MRI evaluation on diagnostic ascertainment of axial $\mathrm{SpA}$ in patients presenting with undiagnosed back pain.

Methods: The multicenter Screening for Axial Spondyloarthritis in Psoriasis, Iritis, and Colitis (SASPIC) Study is aimed at early detection of axial SpA. Consecutive patients $\leq 45$ years of age with undiagnosed back pain with any one of psoriasis, acute anterior uveitis (AAU), or colitis undergo routine clinical evaluation by a rheumatologist for axial $\mathrm{SpA}$ and MRI evaluation is ordered per rheumatologist decision. The rheumatologist determines the presence or absence of axial SpA and the degree of confidence in the diagnosis at 3 consecutive stages: 1 . After the clinical evaluation; 2. After the results of labs (B27, CRP) and radiography; 3. After the results of MRI evaluation. Differences in patient characteristics between those who did or did not have MRI examination were assessed by chi-square and t-tests. We also assessed the degree of diagnostic reclassification after each step.

Results: 225 patients $(52.0 \%$ male, mean age 34.7 years, mean age at symptom onset 27.5 years, B27+35.1\%) were referred with AAU $(30.7 \%)$, psoriasis (20.4\%), Crohn's colitis (34.2\%), ulcerative colitis (19.1\%). A diagnosis of axSpA was made in $66.7 \%$ of patients after stage 1 clinical evaluation and in $55.8 \%$ in stage 2 after review of the labs and radiography. Radiographic sacroiliitis according to modified New York criteria (mNY) was reported in $30.7 \%$ according to local site reads. MRI evaluation was conducted in 129 patients and was ordered significantly more frequently when radiography was mNY- $(76.7 \%$ vs $57.8 \%, p=0.004)$ and in those without Crohn's colitis $(71.3 \%$ vs $51.8 \%, \mathrm{p}=0.004)$. After review of the MRI scan at stage 3, diagnostic categorization of axSpA decreased from $55.8 \%$ to $46.6 \%, 21(17.8 \%)$ patients being recategorized from SpA to non-SpA and 4 (3.4\%) from non-SpA to SpA. Confidence in diagnostic categorization was increased after MRI.

Conclusion: In a setting of undiagnosed back pain and higher risk for axial SpA, use of MRI is primarily driven by negative radiography. MRI was primarily helpful in ruling out SpA and reducing false positives.

11

Physician Global Assessments for Disease Activity in Rheumatoid Arthritis Are All Over the Map! Results from a CRA Survey

Matthew Turk (Schulich School of Medicine \& Dentistry, UWO, London); Janet Pope (Western University, Department of Medicine, Division of Rheumatology, London)

Objectives: A physician (MD) global assessment is part of routine care in monitoring patients with rheumatoid arthritis (RA) and in clinical trials and it is part of some composite measures (CDAI, SDAI). However, the variability between physicians with respect to their global ratings is not known. We wanted to determine the variability of MD global assessments in RA and if results varied between rheumatologists based on their age, sex, practice setting, experience (number of patients seen per year), and years in practice.

Methods: Our research goal was to determine the variability of MD globals and which factors are associated with discrepancies. We surveyed rheumatologists who were members of the Canadian Rheumatology Association using RA patient scenarios where each was rated for disease activity from 0 - 10. Cases were developed to span the spectrum of disease activity. Means, t tests, correlations and Fleiss' Kappa statistics were used to analyze the responses

Results: A total of 145 responded to the survey ( $40 \%$ response). MD globals 
were not significantly different between physicians in any demographic category. The range of answers for the same scenario was as high as 7.6 out of a possible 10, indicating vast discrepancies between physicians. Some scenarios outlined changes in individual patients, however physicians surveyed were often in disagreement as to how much the patient recovered or worsened but the direction was the same (i.e. if better all agreed). The average range for their answers was 7 out of a possible 10 with a mean standard deviation of each answer was 1.7. The average Q1-Q3 interquartile range was 3 and overall Fleiss Kappa was 1.16x10-3 (indicating poor to slight agreement for many case scenarios). Mean kappa for new questions was $1.16 \times 10-3$, whereas for change scenarios it was $2.513 \times 10-3$ (only slightly numerically better for changes in diseases activity).

Conclusion: This research emphasizes the need to establish evaluation criteria in RA for disease. Perhaps a catalogue of patient scenarios that range from 0 to 10 could be developed, standardized and agreed upon to decrease the wide variability of ranking by rheumatologists.

12

Pregnancy Outcomes in Patients with Childhood-onset Systemic Lupus Erythematosus

Lily Wang (University of Toronto Faculty of Medicine, Toronto); Carl Laskin (TRIO Fertility, Toronto); Karen Spitzer (TRIO Fertility, Toronto); Earl Silverman (Division of Rheumatology, SickKids Hospital; Divisions of Translational Medicine and Genetics, SickKids Research Institute; Faculty of Medicine, University of Toronto, Toronto); Michelle Hladunewich (Sunnybrook Health Sciences Centre, Toronto); Linda Hiraki (Division of Rheumatology, SickKids Hospital; Division of Genetics, SickKids Research Institute; Faculty of Medicine, University of Toronto, Toronto); Deborah Levy (Division of Rheumatology, SickKids Hospital; Faculty of Medicine, University of Toronto, Toronto); Amanda Steiman (Mount Sinai Hospital, Toronto)

Objectives: The literature suggests that patients with childhood-onset systemic lupus erythematosus (cSLE) have more severe disease than those with adult-onset, and that SLE can impact pregnancy outcomes. Hence cSLE patients with severe disease may be at risk for poor pregnancy outcomes. The purpose of this study is to describe pregnancy outcomes in cSLE patients.

Methods: A retrospective chart review was conducted of patients with cSLE (disease onset $\leq 18$ years), followed at the Lupus Clinic at the Hospital for Sick Children (HSC) in Toronto. Of those, we identified patients who had at least one pregnancy, through cross-referencing OHIP numbers of patients seen at the HSC Lupus Clinic and by one of the two specialists in pregnancy in the rheumatic diseases in Toronto, between January 1, 2007, and April 1, 2017. We assumed most cSLE patients would see one of these physicians in pregnancy. Pregnancy and SLE data were abstracted using a standardized form. Variables included age at diagnosis, autoantibody profile, including antiphospholipid antibodies (APLAs) and/or antiphospholipid syndrome, renal involvement, and SLE treatment. Obstetric information included pregnancy outcome and complications. Proportions of patients with features and outcomes were calculated using Microsoft Excel software.

Results: There were 56 pregnancies in 24 patients from the cSLE cohort of 646 female patients, most of whom were of post-pubertal age between 2007 and 2017, who fulfilled an average of 6 ACR and 7 SLICC criteria for lupus. The mean age at diagnosis of SLE was $14(10-17)$ years and mean age at pregnancy was $29(21-40)$ years. Of 56 pregnancies, 16 were term $(28.6 \%)$, 16 preterm (28.6\%), 12 miscarried (21.4\%), 9 therapeutically terminated $(16.7 \%)$, and 3 stillborn $(5.5 \%) .5 / 24(21 \%)$ patients had preeclampsia. Fifty percent (12/24) had SLE renal involvement; the majority (10) had proliferative (class III/IV) nephritis. Fifty percent (12/24) had APLAs. Of these, $2 / 12(17 \%)$ experienced thrombosis prior to pregnancy.

Conclusion: There were strikingly few pregnancies documented. The pregnancies were complicated, and had worse outcomes than those previously described for adult-onset cohorts. The patients had high rates of nephritis and APLAs, consistent with previous reports of cSLE. These findings could reflect uniquely poor pregnancy outcomes in cSLE, or a skewed sample of patients with more severe disease who, consequently, continued to be followed in tertiary care. We will attempt to expand this cohort by contacting all female HSC cSLE patients to document additional pregnancies and explore predictors for outcomes in this population. Best Abstract on SLE Research by a Trainee - Ian Watson Award.

\section{3}

Quantification of Leukocytes' Secretome to Guide Diagnosis and Treatment Options in Patients with Suspected Chronic Auto-Inflammatory Syndromes

Anne-Laure Nézondet-Chetaille (Department of Rheumatology, CHU de Québec-Université Laval, Québec); Marie-Pier Longchamps (CHU de Québec-Université Laval Research Center, Québec); Nathalie Pagé (CHU de Québec-Université Laval Research Center, Québec); Anne-Sophie Julien (Clinical Research Platform, CHU de Québec-Université Laval Research Center, Québec); Nathalie Amiable (CHU de Québec-Université Laval Research Center, Quebec ); Alexandra Albert (Department of Rheumatology, CHU de Québec-Université Laval, Quebec); Laetitia Michou (Department of Rheumatology, CHU de Québec-Université Laval, Quebec); Louis Bessette (Department of Rheumatology, CHU de Québec-Université Laval, Québec); Paul Fortin (Department of Rheumatology, CHU de QuébecUniversité Laval, Québec ); Philippe Tessier (CHU de Québec-Université Laval Research Center, Québec); Martin Pelletier (CHU de QuébecUniversité Laval Research Center, Québec)

Objectives: Auto-inflammatory syndromes are inherited conditions characterized by recurrent inflammation (fever, abdominal pain, dermatitis, arthritis). Diagnosis and treatments are challenging as detection rate of mutations in patients with high suspicions for auto-inflammatory syndromes is low, symptoms are reminiscent of other autoimmune diseases, especially Systemic Autoimmune Rheumatic Diseases (SARD), and the cytokines abnormally secreted are unknown. As a consequence, patients can be misdiagnosed, leading to inappropriate treatment, severe complications and substantial socio-economic costs, as AIS often severely affect young children, impeding their education and subsequent employability. Since autoinflammatory syndromes are characterized by abnormal secretion of cytokines, our objectives were to quantify the cytokines in the plasma and the cytokines secreted (secretome) by in vitro-activated peripheral blood mononuclear cells (PBMCs) to distinguish suspected auto-inflammatory syndrome patients from SARD patients and healthy individuals, and provide personalized biotherapy options.

Methods: Plasma and PBMCs were obtained from healthy controls, suspected auto-inflammatory patients, and definite rheumatoid arthritis (RA) and systemic lupus erythematosus (SLE) patients from the CHU de Québec SARD Biobank Repository Database (SBRD). PBMCs were stimulated with inflammatory and immune stimuli, and nine cytokines (IL-1-alpha, IL-1beta, IL-1RA, IL-18, IFN-gamma, IL-12, TNF, IL-6 and CXCL10/IP-10) were analyzed by multiplex assays in the plasma and cell supernatants.

Results: The cytokines found in the plasma of suspected auto-inflammatory patients were not different between both healthy donors and definite SARD patients. In contrast, PBMCs had a distinct profile of cytokine secretion. Stimulation of PBMCs with IL-15 or anti-immunoglobulins led to differential secretion of members of IL-1 cytokine family, IL-12 and IFN-gamma in definite SARD, but not auto-inflammatory patients. Moreover, suspected auto-inflammatory patients can be distinguished from healthy donors and definite SARD patients by quantification of IL-12, IL-1-alpha and IL-18 levels in leukocytes' supernatant following distinct inflammasome activation. Stimulation with inflammasome activators or pro-inflammatory cytokines led to selective secretion of IL-1-alpha, IL-1beta, IL-1RA, IL-18, IFN-gamma or IL-12 in suspected auto-inflammatory patients that can be targeted by biotherapies.

Conclusion: This study demonstrates that analysis of leukocytes' secretome is reliably more sensitive than plasma to reveal cytokine signatures and to predict treatment options in patients with suspected chronic auto-inflammatory syndromes. 
Risk of Major Congenital Malformations Associated with Exposure to Biologics Before or During Pregnancy: A Population-based Cohort Study

Nicole Tsao (University of British Columbia Faculty of Pharmaceutical Sciences; Arthritis Research Canada, Vancouver); Gillian Hanley (University of British Columbia, Vancouver); Mohsen Sadatsafavi (University of British Columbia, Vancouver); Larry Lynd (University of British Columbia, Vancouver); Carlo Marra (University of Otago, Dunedin); Mary De Vera (University of British Columbia Faculty of Pharmaceutical Sciences; Arthritis Research Canada, Vancouver)

Objectives: As most biologics cross the placenta during human pregnancies, there remains concern whether in-utero exposure may be associated with increased risk of major congenital malformations (MCM). A recent metaanalysis found a non-statistically significant trend towards increased risk of MCM, however most of the published studies have not adjusted for differences in disease activity between exposure groups. Our objective was to evaluate the association between biologics exposure and risk of MCM by applying high dimensional propensity scores (HDPS) in population-based administrative data.

Methods: We conducted a population-based, retrospective cohort study using British Columbia administrative data including all physician visits, hospital admissions, and dispensed medications, linked to a Provincial Perinatal Registry. Our cohort included women with autoimmune disease who had $\geq 1$ pregnancies, and infants born to these women, between $01 / 01 / 2002$ and $12 / 31 / 2012$. Pregnancies were defined as exposed if women had at least one biologic prescription 90 days before pregnancy or up to 13 weeks gestation (first trimester), and unexposed if there were no prescriptions for biologics during the same period. Each exposed pregnancy was matched with 5 unexposed pregnancies using HDPS. The outcome of interest was defined as the occurrence of $\geq 1 \mathrm{MCMs}$ identified at birth. Logistic regression models were used to evaluate the association between exposure to biologics and risk of MCMs in the HDPS-matched cohort. Sensitivity analysis was conducted using multivariable logistic regression models with deciles of HDPS.

Results: The cohort included 131 pregnancies (120 women) exposed to biologics 90 days before pregnancy or during the first trimester, and 599 HDPS-matched pregnancies (578 women) that were not exposed to biologics during that time. In the exposed group, $94 \%$ had prescriptions for infliximab, etanercept, or adalimumab. In the exposed and unexposed groups, respectively, 10/131 (8\%) and 40/599 (7\%) of newborns had $\geq 1$ MCMs at birth In the unmatched cohort, the odds ratio for those exposed to biologics was 1.46 (95\% confidence interval $[\mathrm{CI}] 0.78-2.72)$ compared to unexposed, whereas in the matched cohort it was 1.16 (95\%CI 0.56-2.37), suggesting no association between biologics exposure and MCM. Sensitivity analyses did not materially change our results.

Conclusion: These population-based data suggest that use of biologics before pregnancy or during the first trimester may not be associated with MCM in infants born to women with autoimmune inflammatory diseases. Given the effectiveness of biologics in controlling disease activity, findings emphasize the importance of balancing benefits and risks of treatments for patients who may be pregnant or considering pregnancy. 


\section{Posters}

1

Depression Increases the Risk of Developing Rheumatoid Arthritis: A Population-based Cohort Study

Isabelle Vallerand (University of Calgary, Calgary); Ryan Lewinson (University of Calgary, Calgary); Mark Lowerison (University of Calgary, Calgary); Alexandra Frolkis (University of Calgary, Calgary); Gilaad Kaplan (University of Calgary, Calgary); Andrew Bulloch (University of Calgary, Calgary); Scott Patten (University of Calgary, Calgary); Cheryl Barnabe (University of Calgary, Calgary)

Objectives: The underlying risk factors for development of Rheumatoid Arthritis (RA) remain poorly understood; however, prospective studies have demonstrated that individuals with elevated Tumor Necrosis Factor alpha (TNF-alpha), a pro-inflammatory cytokine, are at increased risk of subsequently developing RA. It remains unknown whether elevated TNF-alpha by means of a different disease process can subsequently increase the risk of developing RA. Recently, major depressive disorder (MDD) has been identified to have a direct effect on cytokines, including increased serum concentrations of TNF-alpha relative to healthy controls independent of underlying inflammatory disease. Based on this, it is possible that exposure to MDD may increase the risk of subsequently developing RA. In this study using a large population-based cohort, it was hypothesized that patients with MDD are at increased risk of subsequently developing RA compared to the general population without MDD.

Methods: A retrospective cohort study was conducted using The Health Improvement Network (THIN) Database between the years 1986-2012 for up to 26 years of follow-up. The MDD cohort comprised patients with a diagnostic (Read code) for MDD and the remainder of patients formed the referent cohort. Both cohorts were followed until patients developed RA or were censored. Cox proportional hazards models were used to determine the risk of developing RA among patients with MDD, as reported using Hazard Ratios (HRs) and 95\% confidence intervals $(\mathrm{CIs})($ alpha $=0.05)$. A backward elimination procedure was used to determine the presence of effect modification (using a Wald test) or confounding by age or sex.

Results: A cohort of 403,932 patients with MDD and a referent cohort of $5,339,399$ patients without MDD were identified in THIN. A total of 2,192 (0.54\%) patients with MDD developed RA, and 24,021 (0.45\%) patients without MDD developed RA over this period. Cox proportional hazards models identified a significant interaction by age $(\mathrm{p}<0.0001)$, whereby younger patients with MDD (age < 40) had a $42 \%$ increased risk of developing RA (HR $=1.42,95 \%$ CI: $1.31-1.53)$ after controlling for sex. In older patients (age > 40), the risk of developing RA among those with MDD was lower but demonstrated a $14 \%$ increased risk $(\mathrm{HR}=1.14,95 \% \mathrm{CI}$ : 1.08-1.21)

Conclusion: MDD was found to be a risk factor for the development of RA. This risk was highest among younger patients with MDD, and the risk was only somewhat increased among older patients with MDD. These results provide support to the hypothesis that MDD may be associated with the development of RA.

Case Series of 9 Patients with Immune Related Adverse Events Associated with Checkpoint Inhibitors

Daniel Ennis (Division of Rheumatology, University of British Columbia, Vancouver); Shahin Jamal (Division of Rheumatology, University of British Columbia, Vancouver); Fergus To (University of British Columbia, Vancouver)

Objectives: The advent of immune checkpoint inhibitors (ICI) has made a significant impact on the treatment of various advanced malignancies. There is little published on the rheumatologic complications of these treatments. We describe 9 new cases of rheumatologic IRAEs associated with ICI treatment.

Methods: We report patients evaluated in a general Rheumatology outpatient clinic from 2014 to 2017. Cases were defined as those with new rheumatologic symptoms in the context of treatment with an ICI for various cancers. Alternative explanations for the presenting syndrome were excluded clinically. Clinical data was extracted by retrospective chart review.

Results: This case series includes 9 patients ( 4 female, 5 male) with a median age at IRAE onset of 62.7 years (range 33-79). Multiple cancer types were represented including melanoma $(\mathrm{n}=7)$, Hodgkin's lymphoma $(\mathrm{n}=$ $1)$, and squamous cell lung cancer $(n=1) .5 / 9$ patients received Nivolumab, 5/9 received Pembrolizumab, and 2/9 received Ipilimumab. ICI exposure was associated with various rheumatologic IRAEs including PMR-like syndrome $(n=4)$, symmetric polyarthritis $(n=6)$, psoriatic arthritis $(n=1)$, oligoarthritis $(n=1)$. Other IRAEs were also noted including vitiligo $(n=$ $1)$, pulmonary capillaritis $(n=1)$, ulcerative colitis flare $(n=1)$, inflammatory seborrheic keratosis and psoriasis $(n=1)$. The median time of onset of the IRAE from the first exposure to an ICI was 6 months (range 0-21 months). In 6/6 cases who received ongoing ICI therapy, rheumatologic symptoms worsened with each dose. In the remaining 3 cases, no further ICI was indicated. Laboratory investigations demonstrated elevated CRPs in 6 cases (median 70.1; range 3.7-290.1), RF positivity in 2 cases, weak positive ANAs in 3 cases (1:80), and a single case where a pre-existing anti-CCP antibody was identified. Steroids were used in 8 cases at a median dose of $37.5 \mathrm{mg}$ (range $10-50 \mathrm{mg}$ ) by mouth daily for an average duration of 6 months (range 1-7 months). While only 3 patients experienced rapid improvement, 3 experienced gradual improvement, and one only achieved partial resolution of symptoms. Tumor response was observed in 9/9 patients.

Conclusion: This case series of IRAEs associated with ICI treatment suggest that symmetric polyarthritis and PMR-like syndromes are the most common rheumatologic IRAEs, although the spectrum is broad. IRAEs seem to develop around 6 months after first exposure, worsen with ongoing doses of ICI administration, and respond to treatment with corticosteroids (relatively high doses and prolonged courses). Those with IRAEs tend to have good tumor response. More experience is required around use of DMARDs and biologics in these patients.

3

A Retrospective Study of Clinical Factors Influencing the Development of Overlapping Disease Features in Pediatric Patients with Chronic Recurrent Multifocal Osteomyelitis (CRMO) and Spondyloarthropathies (SpA)

Lillian Lim (Dept. Rheumatology, The Hospital for Sick Children, Toronto); Jyoti Panwar (The Hospital for Sick Children, Toronto); Jennifer Stimec (The Hospital for Sick Children, Toronto); Shirley Tse (Hospital for Sick Children, Toronto); Brian Feldman (The Hospital for Sick Children, Toronto); Ronald Laxer (Dept. Rheumatology, The Hospital for Sick Children and University of Toronto, Toronto)

Objectives: This study was done to test whether gender, HLA-B27 positivity, or age at onset of disease, influence whether and when pediatric chronic recurrent multifocal osteomyelitis (CRMO) and spondyloarthropathy (SpA) patients develop overlapping features. Understanding of the clinical factors influencing overlap may have implications for earlier diagnosis, risk stratification, and treatment options.

Methods: This was a retrospective inception cohort study of SickKids hospital charts. Eligible pediatric patients diagnosed with either CRMO or SpA between January 2000 and December 2016 were collected using the SickKids Rheumatology patient database. Using a secure web application (REDCap), we collected and compared the clinical, laboratory, and radiographic data of 40 randomly sampled patients from each of the CRMO and SpA groups. The MRIs were re-read by a radiologist. A patient was considered to have an overlap diagnosis if they had radiologic and clinical features of both CRMO and SpA.

Results: Our results showed that 7 patients (17.5\%) and 8 patients (20.0\%) from the CRMO and SpA groups, respectively, developed overlap. The median time to overlap was 36.0 months and 31.5 months, respectively. A survival (time-to-event) analysis, using the Kaplan-Meier approach, was used to show the proportion of patients without overlap features, since time of symptom onset. Of the entire sample population, 28\% was HLA-B27 positive. Multivariable regression models using the Cox Proportional

Personal non-commercial use only. The Journal of Rheumatology Copyright @ 2018. All rights reserved. 
Hazards regression technique showed that the overall likelihood ratio test was not statistically significant $(\mathrm{p}=0.59)$, with hazard ratios of $1.11,0.91$, and 1.17 for each of male gender, HLA-27 positivity, and age at symptom onset, respectively.

Conclusion: Patient gender, HLA-B27 positivity, and age of symptom onset did not appear to increase the risk of developing overlap features of CRMO and $\mathrm{SpA}$ in our sample population. Nonetheless, overlap of clinical and radiologic features was found in approximately $20 \%$ of our patients. CRMO and SpA may represent two diseases on the same spectrum, though prospective studies with longer follow up are needed. Our study was limited by the small sample size and retrospective design, as it is unknown whether overlap may occur in the time exceeding clinical follow-up. Study of their clinical overlap is important to allow a better understanding of how to recognize and treat these rare diseases.

4

Evaluation of the Early Inflammatory Arthritis Detection Tool as an Aid in Triaging Patients with Possible Inflammatory Arthritis

Raquel Sweezie (Sunnybrook Health Sciences Centre, Toronto); Vandana Ahluwalia (William Osler Health System, Ontario Rheumatology Association, Brampton); Claire Bombardier (Ontario Best Practices Research Institute, University of Toronto, Toronto General Research Institute, Toronto); Sydney Brooks (The Arthritis Society, Toronto); Angela Cesta (University Health Network, Toronto); Mary Bell (University of Toronto, Toronto)

Objectives: Excessive delays to DMARD treatment have been documented for patients with inflammatory arthritis (IA). Many of these delays occur either prior to or during referral to rheumatology. Evidence suggests that self-assessment with the Early Inflammatory Arthritis Detection Tool (EIADT) may assist both primary care providers and rheumatologists in appropriately triaging patients with IA. We evaluated positive and negative predictive values (PPV and NPV), sensitivity and specificity of the EIADT for identifying patients with IA, as determined by a rheumatologist.

Methods: Patients with possible IA were identified from rheumatologists' wait lists through a paper triage process. Patients were included if they were adults and newly referred by a general practitioner or nurse practitioner within the previous month. Differential diagnoses by the rheumatologists were documented by chart review within a 6-month window following completion of the 12-question EIADT. Responses to each question were scored 1 point for "Yes" and 0 points for "No". Patients scoring $\geq 6$ were classified as IA and those scoring $<6$ were classified as non-IA. The rheumatologists were blinded to the patients' responses and scores. The proportion of patients given a differential diagnosis of IA by the rheumatologist and classified as IA by the EIADT was determined.

Results: Seven rheumatologists agreed to participate in the study. Of 390 patients identified from the rheumatologists' wait lists as having possible IA, $149(38 \%)$ completed the EIADT (female: 65\%; mean age (SD): $52.8(13.7)$ ), and 120 received a differential diagnosis from a rheumatologist. Of the patients classified as IA with the EIADT $(n=86), 50$ received a differential diagnosis of IA from their rheumatologist (PPV $=0.58)$. Of those identified as non-IA with the EIADT $(n=34), 15$ received a differential diagnosis from their rheumatologist that did not include IA (NPV $=0.44)$. Sensitivity of the EIADT was moderate (0.72) and specificity was low (0.29).

Conclusion: The moderate sensitivity of the EIADT suggests that this tool has potential for providing primary care providers and rheumatologists with additional information that may assist in determining the urgency of a given case where IA is a possibility. As indicated by the low predictive values, this tool is not intended as a stand-alone method for identifying patients with IA More work needs to be done to determine whether it can impact the triage of patients with possible IA in primary care.

5

Methotrexate Exposure and Risk of Strongyloidiasis: A Systematic Review

Ceri Richards (University of Manitoba, Winnipeg); Hal Loewen (University of Manitoba, Winnipeg); Addisu Melkie (Addis Ababa University, Addis Ababa); Michele Meltzer (Jefferson University, Philadelphia); Rosie Scuccimarri (McGill University, Montreal); Yewondwossen Mengistu (Addis Ababa University, Addis Ababa); Zenebe Melaku (Addis Ababa University, Addis Ababa); Ines Colmegna (McGill University Hospital Centre, McGill University, Montreal); Carol Hitchon (University of Manitoba, Winnipeg)

Objectives: Rheumatoid arthritis (RA) patients receiving immunosuppressants or corticosteroids have increased infection rates. Strongyloides, a global endemic intestinal parasite, can cause significant or fatal disease, usually in immunocompromised patients. The risk of serious Strongyloides infection occurring with methotrexate doses recommended for treating rheumatic disease is unknown, but must be considered when developing recommendations for treating RA for regions with high rates of Strongyloides carriage.

Methods: We performed a systematic literature review. EMBASE, Pubmed and Web of Science databases were searched using the terms Strongyloide (exploded subject heading) OR Strongyloide* (keyword) and Methotrexate (subject heading) OR Methotrexate (Keyword) OR Amethopterin (Keyword) OR Mexate (Keyword) without additional filtering. All studies reporting on humans exposed to methotrexate and tested for Strongyloides were reviewed independently by two reviewers. Exclusion criteria were bone marrow transplantation, intrathecal route and methotrexate exposure completed $>1$ year prior to clinically apparent Strongyloides disease (CAD). Outcomes included $\mathrm{CAD}$, disseminated Strongyloides to the lungs (Strongyloides hyperinfection syndrome) or other organs (disseminated Strongyloidiasis) or death. High-dose (> 30mg/week) and low-dose methotrexate were analyzed separately. Descriptive statistics are reported.

Results: Fifty-six references were screened. After excluding papers available only in abstract form (which did not meet inclusion criteria) and duplicates, 53 articles were reviewed. Of these, 22 papers (including two small case series) met the inclusion criteria. Three additional case reports were identified from reference review. A total of 28 patients with clinically apparent Strongyloides diseases were identified. Fifty-seven percent of them had an underlying rheumatologic disease and the rest had hematologic disease. Hyperinfection syndrome was found in $46 \%$ of cases (similar in high and low-dose methotrexate categories). Disseminated Strongyloides was found in $21 \%$ of cases (12\% of low-dose and $36 \%$ of high-dose methotrexate categories). Death occurred in $30 \%$ of cases (19\% of low-dose and $45 \%$ of high-dose categories). All 11 patients on high-dose methotrexate received other immunosuppression. Similarly, among the 17 patients on low-dose methotrexate, 15 were on corticosteroids. The two patients on methotrexate monotherapy did not have either hyperinfection syndrome or disseminated Strongyloides, and responded well to treatment.

Conclusion: This systematic review showed that Strongyloides causes considerable morbidity and mortality in patients taking methotrexate. Additional immunosuppressives seem to increase the risk further. Prospective studies are needed to risk stratify patients and evaluate the benefits and cost effectiveness of screening and treating patients before initiation of low-dose methotrexate therapy.

\section{6}

Platelet Activation and Mitochondrial Release in Systemic Lupus Erythematosus

Maude Bouchard-Marmen (Université Laval, Quebec); Imène Melki (Université Laval, Québec); Anne-Sophie Julien (Clinical Research Platform, CHU de Québec-Université Laval Research Center, Québec); Eric Boilard (CHU de Québec-Université Laval Research Center, Québec); Paul Fortin (Department of Rheumatology, CHU de Québec-Université Laval, Québec)

Objectives: Platelets are now proposed as potential contributors in inflammation, possibly through the release of platelet microparticles (PMPs). On activation, platelets can release mitochondria, either contained in PMPs or as naked organelle, in the extracellular milieu. We aimed to determine whether there is a link between platelet activation, release of extracellular

Personal non-commercial use only. The Journal of Rheumatology Copyright $\subset$ 2018 . All rights reserved. 
mitochondria and clinical outcomes in systemic lupus erythematosus (SLE). Methods: We used high sensitivity flow cytometry to quantify PMPs and extracellular mitochondria on fresh blood samples from 76 SLE patients and 30 healthy controls. Surface expression of CD62P (P-selectin) and activated form of a $2 \mathrm{bb} 3$ were assessed to determine platelet activation. Platelet association with immunoglobulin $\mathrm{G}(\mathrm{IgG})$ was examined and quantified as mean fluorescence intensity (MFI). Mitochondrial DNA (mtDNA) was measured by quantitative PCR amplification. Spearman correlations between PMPs were calculated. ROC curves were used to assess predictive power of PMPs for SLE diagnosis. The effects of PMPs on clinical outcomes, damage (SDI $>=1$ vs 0 ), disease activity (SLEDAI $>=4$ vs $<4$ ), as well as presence of past thrombotic event, neurologic disorder and renal disorder, were studied using multivariate logistic regressions.

Results: We found that mtDNA and IgG+ platelets were potent predictors of SLE when compared to healthy controls with areas under the curve of 92\% (95\% CI 0.82-1.00) and 91\% (0.86-0.97), respectively. In SLE and controls, platelet mitochondria levels were negatively correlated with IgG+ platelets ( $r s=-0.53, \mathrm{p}<0.001$ ), while PMPs levels were positively correlated with platelet activation ( $\mathrm{rs}=0.39, \mathrm{p}<0.001)$. The majority $(60 \%)$ of microparticles found were of mitochondrial origin and harboured $\mathrm{IgG}+$. We obtained a negative association between damage and presence of platelet IgG+ (OR 0.21 [95\% CI 0.05-0.89]) and between disease activity and extracellular mitochondrial presence $(0.33$ [0.12-0.91]). No associations were found with the other clinical outcomes.

Conclusion: mtDNA and $\mathrm{IgG}+$ platelets may represent promising biomarkers in SLE. Whereas in normal conditions platelets might release their mitochondrial organelles inside microparticles, mitochondria may be released as naked organelles in pathological conditions such as SLE. Hence, the negative correlation between platelets mitochondria levels and $\mathrm{IgG}+$ platelet may be explained by the release of naked extracellular mitochondria with platelet activation in SLE.

\section{7}

Comparison of ACR and SLICC Classification Criteria in Childhood-Onset Systemic Lupus Erythematosus

Jessie Tao (Division of Rheumatology, SickKids Hospital; Faculty of Medicine, University of Toronto, Toronto); Linda Hiraki (Division of Rheumatology, SickKids Hospital; Division of Genetics, SickKids Research Institute; Faculty of Medicine, University of Toronto, Toronto); Deborah Levy (Division of Rheumatology, SickKids Hospital; Faculty of Medicine, University of Toronto, Toronto); Earl Silverman (Division of Rheumatology, SickKids Hospital; Divisions of Translational Medicine and Genetics, SickKids Research Institute; Faculty of Medicine, University of Toronto, Toronto)

Objectives: Systemic lupus erythematosus (SLE) remains a challenge to classify due to its heterogeneous nature. The American College of Rheumatology (ACR) and Systemic Lupus International Collaborating Clinics (SLICC) classification criteria were developed to differentiate SLE from other systemic autoimmune diseases. Although both are used in childhood-onset SLE (cSLE) research, neither has been validated for the pediatric population. Here we compare the sensitivities of ACR and SLICC criteria in cSLE and identify features that may increase the sensitivity of cSLE classification.

Methods: We conducted a retrospective study on all 722 patients diagnosed with cSLE at SickKids (Jan 1980-May 2017) based on expert pediatric rheumatologist opinion. Data from SickKids' Lupus Database were reviewed/validated against medical records prior to ACR and SLICC scoring. The sensitivities of ACR and SLICC scores were compared using McNemar's test. Subsequent analysis was performed to identify SLE features unique to each classification criteria and any remaining features that may be unique to cSLE.

Results: 694/722 (96.1\%) met SLICC criteria as compared to $668 / 722$ $(92.5 \%)$ who met ACR criteria $(\mathrm{p}<0.001) .47 / 54(87.0 \%)$ patients who did not meet ACR criteria fulfilled SLICC criteria as a result of: hypocomplementemia (29.1\%), alopecia (20.3\%), additional hematologic $(24.1 \%)$ and immunologic (13.9\%) criteria, Coombs anemia without hemolysis (7.6\%), and broadened definitions of CNS (2.5\%) and acute cutaneous (2.5\%) lupus. 21/28 (75.0\%) patients who did not meet SLICC criteria fulfilled ACR criteria as a result of: photosensitivity $(70.8 \%)$ and ACR lymphopenia criteria $(29.2 \%)$. If we modified SLICC criteria to include these features, sensitivity was $99.0 \%(\mathrm{p}<0.0001)$. Seven patients $(1.0 \%)$ were diagnosed with cSLE without meeting either set of criteria. 6/7 (85.7\%) of these patients had anti-Ro antibodies and none had features of primary Sjogren Syndrome (pSS). In adults, the inclusion of anti-Ro may decrease specificity for SLE due to anti-Ro association with pSS. This is not the case in cSLE due to the rarity of pediatric pSS.

Conclusion: SLICC criteria were significantly more sensitive than ACR criteria in the classification of cSLE within our cohort. If we modified SLICC criteria to include photosensitivity and ACR lymphopenia criteria, the sensitivity increased to $99.0 \%(\mathrm{p}<0.0001)$. If anti-Ro antibodies were added to the modified SLICC classification criteria, only one patient with a clinical diagnosis of cSLE would have been missed. Thus, we suggest that SLICC criteria are more sensitive than ACR criteria for cSLE classification and that further modification (addition of photosensitivity, ACR lymphopenia criteria, and/or anti-Ro antibodies) be considered in cSLE.

8

Cardiac Magnetic Resonance Imaging Role in Systemic Sclerosis-associated Pulmonary Hypertension: Analysis of 78 Patients

Karel Venne (Department of Medicine, Centre Hospitalier de l'Université de Montréal, Montreal); Sabrina Hoa (Rheumatology Division, Department of Medicine, Centre hospitalier de l'Université de Montréal (CHUM), Montreal); Martial Koenig (Rheumatology Division, Department of Medicine, Centre hospitalier de l'Université de Montréal (CHUM), Montreal); Jean-Richard Goulet (Rheumatology Division, Department of Medicine, Centre hospitalier de l'Université de Montréal (CHUM), Montréal); Eric Rich (Rheumatology Division, Department of Medicine, Centre hospitalier de l'Université de Montréal (CHUM), Montreal); Lada Miller (Rheumatology Division, Department of Medicine, Hôpital du Sacré-Cœur affiliated of Université de Montréal, Montreal); Anne Chin (Department of Radiology, Centre hospitalier de l'Université de Montréal (CHUM), Montreal); Carl Chartrand-Lefebvrel (Department of Radiology, Centre hospitalier de l'Université de Montréal (CHUM), Montreal); Pauline Gou (Rheumatology Division, Department of Medicine, Centre hospitalier de l'Université de Montréal (CHUM), Montreal); Jean-Luc Senécal (Rheumatology Division, Department of Medicine, Centre hospitalier de l'Université de Montréal (CHUM), Montreal); Tamara Grodzicky (Rheumatology Division, Department of Medicine, Centre hospitalier de l'Université de Montréal (CHUM), Montreal); Sandra Chartrand (Rheumatology Division, Department of Medicine, Hôpital Maisonneuve-Rosemont affiliated to Université de Montréal, Montréal)

Objectives: Pulmonary hypertension $(\mathrm{PH})$ in patients with systemic sclerosis (SSc) is one of the major causes of morbidity and mortality, despite current therapeutic interventions. Transthoracic echocardiography (TTE) as a screening tool has low sensitivity and is not well correlated to the severity of PH. Moreover, it is not specific as to the underlying cause of the disease. Few studies have used cardiac magnetic resonance imaging (MRI) for the investigation of $\mathrm{PH}$ in this population. In the hope of detecting this complication earlier, more data is needed to recommend cardiac MRI in the investigation of SSc-PH. By comparing the cardiac MRI results of SSc patients with and without $\mathrm{PH}$, we describe the differences in between the two groups and determine whether it could help in screening for PH in a large SSc population.

Methods: A retrospective analysis of our cohort of 432 SSc patients was performed. All patients routinely underwent screening for $\mathrm{PH}$, and diagnosis of $\mathrm{PH}$ was proven by right heart catheterization (RHC) in all suspected cases. Data from clinical, cardiopulmonary and serological investigations were analyzed. All living patients diagnosed with $\mathrm{PH}(\mathrm{n}=20)$ as well as a control group of 58 consecutive SSc patients without clinical suspicion of PH underwent a cardiac MRI. 
Results: A majority of patients had PH secondary to a left heart disease (LHD) (14 [58,3\%]) compared to $\mathrm{PH}$ secondary to primary arterial hypertension $(\mathrm{PAH})$ and from other causes $(5[20,8 \%]$ and $5[20,8 \%]$ respectively). Cardiac MRI showed statistically significant differences between $\mathrm{SSc}$ patients with and without $\mathrm{PH}$, respectively, for the measurement of the diameter of the main pulmonary artery (PA) (median (25th percentile - 75th percentile): 30.5 (23.0-41.0) vs $25.2(20.7-38.0) \mathrm{mm}, \mathrm{p}=0.0001)$, the right PA (22.7 (19.0-31.0) vs $18.8(12.9-27.2) \mathrm{mm}, \mathrm{p}=0.00002)$, the left PA (23.0 (16.0-30.0) vs $20.0(13.0-26.0) \mathrm{mm}, \mathrm{p}=0.003)$ and the ratio of the main PA to the ascending aorta $(0.97(0.80-1.15)$ vs $0.84(0.64-1.12), \mathrm{p}=$ $0.0007)$. There was a trend toward significance for the measurement of left and right ventricles parameters. No difference was found for the delayed gadolinium enhancement.

Conclusion: Cardiac MRI measurements of the diameter of the main PA, right PA, left PA as well as the ratio of the main PA to the ascending aorta were statistically significant. The primary investigation of $\mathrm{PH}$ could thus include cardiac MRI as a simple, reliable and noninvasive screening tool for an SSc population. Further research is needed to establish clear thresholds suggestive of a $\mathrm{PH}$ diagnosis.

9

Structural Damage in Patients with Very Early RA is Predicted with Clinical Measures of Baseline Disease Activity: DAS28 (CRP), SDAI, M-DAS28 and RAPID3 but not CDAI

Edward Keystone (Mount Sinai Hospital, University of Toronto, Toronto); Harris Ahmad (Bristol-Myers Squibb, Princeton); Yusuf Yazici (New York University School of Medicine, New York); Xiaoni Liu (Bristol-Myers Squibb, Princeton); Martin Bergman (Drexel University College of Medicine, Philadelphia); Meryem Maoui (Bristol-Myers Squibb, Montreal) Objectives: Clinicians rely on time-efficient, validated disease activity assessments to help accurately predict disease progression in patients with RA. The utility of the Routine Assessment of Patient Index Data 3 (RAPID3)1 in predicting structural damage progression is largely unknown, while that of DAS28 (CRP) 2 and modified (M-)DAS28 have been previously reported.3 This post hoc analysis using data from the AVERT (NCT01142726) study investigated the value of baseline clinical measures for predicting structural damage progression with MRI as a measure of progression.

Methods: AVERT was a 24-month, active-controlled trial with a 12-month, double-blind treatment period, during which MTX-naïve, ACPA-positive patients with early active RA were randomized (1:1:1) to abatacept $125 \mathrm{mg}$ weekly plus MTX or abatacept or MTX monotherapy, followed by a 12-month withdrawal period, during which all treatment was stopped.4 Logistic regression analysis, corrected for age, sex and corticosteroid use at baseline, was used to assess the correlation between baseline measures of disease activity (DAS28 [CRP], SDAI, CDAI, M-DAS28 and RAPID3) and the degree of structural joint damage, as assessed by MRI at 6 and 12 months. MRI erosion progression was defined as change from baseline greater than the smallest detectable change, which was calculated as $\mathrm{SD} /$ square root (2) x 1.96 (where SD is standard deviation of paired differences of change from baseline in total score between two readers).

Results: Logistic regression analysis was carried out for all randomized and treated patients who received abatacept + MTX $(n=119)$ or MTX monotherapy $(n=116)$. For these patients, DAS28 (CRP), M-DAS28 and RAPID3 at baseline were significant predictors of radiographic progression at Months 6 and 12 (Figure). Baseline SDAI was a significant predictor at Month 12 but not Month 6, and baseline CDAI was not a significant predictor at either time point (Figure). At Months 6 and 12, receiver operating characteristic curves showed that RAPID3 had the highest predictive value (area under the curve) for MRI progression (Table).

Conclusion: In this post hoc analysis of MTX-naïve, ACPA-positive patients with early RA, DAS28 (CRP), M-DAS28 and RAPID3 were significant predictors of structural damage progression at Months 6 and 12, as measured using MRI, with RAPID3 showing the highest predictive value for MRI progression.

\section{0}

Central and Peripheral Arterial Involvement in Kimura Disease

Mai Nguyen (University of Manitoba, Winnipeg); Ada Man (University of Manitoba, Winnipeg)

Background: Kimura disease (KD) is a rare inflammatory disorder characterized by soft tissue masses and lymphadenopathy with angiolymphatic proliferation and eosinophilic infiltration. Peripheral arterial involvement is rare and there have been no described cases of central arterial involvement in KD.

Case Presentation: A 26-year-old Filipino male with a 9-year history of biopsy-proven KD manifesting as subcutaneous masses, evanescent rash, peripheral eosinophilia, and elevated IgE presented with left sided weakness, facial droop and slurred speech while on low dose prednisone. Cyclosporine was discontinued 2 years prior. Neuroimaging revealed bilateral stenoses of the middle cerebral arteries (MCA) with a right caudate head and left posterior parietal lobe infarct. Imaging done a week prior for a similar self-resolving episode revealed less severe bilateral MCA stenoses for which aspirin was initiated. He was noted to have absent peripheral pulses. Subsequent computed tomography (CT) angiogram of the left arm revealed left subclavian narrowing and occlusion of the radial and ulnar arteries. C-reactive protein (CRP) was $37.7 \mathrm{mg} / \mathrm{L}$ and absolute eosinophil count was 1.69x10E9/L. Peak IgE level was 4789 IU/mL. ANA, dsDNA, ENAs, rheumatoid factor, antiphospholipid antibodies, lupus inhibitor, MPO antibody, PR3 antibody, and cryoglobulins were negative. Hepatitis B, C and human immunodeficiency virus were negative. Pulse methylprednisolone was started and he was discharged on aspirin, cyclosporine and a prednisone taper. Five months after discharge, he developed lower extremity claudication with radiographic narrowing and occlusions of the bilateral peroneal, anterior tibial, and posterior tibial arteries. New beading and stenosis of the left anterior cerebral (ACA) and left posterior cerebral arteries (PCA) were noted. Cyclosporine was discontinued and he received rituximab. Five months later, due to persistent rash, subcutaneous masses, and claudication symptoms, cyclosporine was restarted at an increased dose with improvement. Repeat imaging 2.5 years after his stroke revealed stable MCA stenoses and resolution of the ACA, PCA and left subclavian changes. Prednisone was tapered off 3 years after his stroke with resolution of symptoms, normalization of CRP and eosinophils, and reduction in $\operatorname{IgE}$ to $1070 \mathrm{IU} / \mathrm{mL}$.

Conclusion: Several cases of arterial involvement have been described in $\mathrm{KD}$, one with mesenteric arterial occlusion, and others with internal carotid artery occlusion, temporal artery thrombosis, and thromboangiitis obliterans. There have been no reported cases of intracranial arterial involvement to date. This is the first reported case of KD with simultaneous intracranial and peripheral arterial involvement treated with prednisone, cyclosporine, and rituximab.

11

Antinuclear Antibody (ANA) Testing and Antineutrophil Cytoplasmic Antibody (ANCA) Testing in a Tertiary Health Care Centre in Sherbrooke: An Assessment of the Adherence to the Guidelines and the Impacts on the Diagnosis and Health Care System

Maria Parfenova (University of Sherbrooke, Sherbrooke); Patrick Liang (Université de Sherbrooke, Sherbrooke)

Objectives: To describe antinuclear antibodies (ANA) and subserology ordering practices and to determine if their indications meet the recommendations for ANA testing at the Sherbrooke University Health Centre. To describe antineutrophil cytoplasmic antibodies (ANCA) testing practices and determine if they meet the current recommendations proposed for ANCA testing, at the same centre.

Methods: We identified the indications for the ANA and subserologies panel (Anti-SSA, anti-SSB, Anti-Jo1, Anti-Scl-70, Anti-Sm, Anti-U1 RNP) between 2012 and 2014 and compared to the guidelines for ANA testing and the Choosing Wisely recommendations. Moreover, the indications for ANCA tests were assessed and compared to the current guidelines for the appropriate testing of ANCA and the Choosing Wisely recommendations.

Personal non-commercial use only. The Journal of Rheumatology Copyright $(\subset) 2018$. All rights reserved. 
Variables included gender, age, ANA titer, subserologies panel, indication of ANA, ANCA $\geq 1: 20$, subtypes MPO and PR3, indications for ANCA, medical specialty, setting of the order and the final diagnosis.

Results: There were a total of 268 ANA tests that were included. In $35.8 \%$ of cases $(n=96)$, ANA was ordered as per recommendations versus $63.8 \%$ of cases $(n=171)$ without indications. There were 104 subserologies ordered and $55.8 \%$ were ordered at the same time as the ANA, against the Choosing Wisely recommendation of 2013. There were only $22 \%$ of ANA that were required for the final diagnosis. The 3 medical specialties that ordered ANA the most were rheumatology, gastroenterology and internal medicine (in descending order). The cost for ANAs ordered without appropriate indication is more than a thousand dollars in the time period studied. A total of 134 ANCA tests were included. There were $43 \%$ of ANCA that were ordered in line with the recommendations, $29 \%$ without recommendations and $27 \%$ for follow-ups. Moreover, 59\% of ANCA were not required for the final diagnosis. Clinical remission of subjects with ANCA was predicted in 100\% of cases, even before ordering the ANCA test for follow-up (negative predictive value). There were only $20 \%$ of ANCAs that influenced the subsequent management.

Conclusion: These results show that the rate of ANA and ANCA tests ordered in line with the recommendations remains low. Too many ANA subserologies are ordered at the same time as the ANAs. These orders have an important cost for the health care system that can be lowered by providing more education for professionals on avoiding unnecessary tests. Clinical assessment rather than ANCA testing should guide treatment changes especially when there is remission.

\section{2}

Antinuclear Antibody Specificities Present in Neutrophil Staining Patterns

May Choi (University of Calgary, Calgary); Carolina Auza (Inova Diagnostics, San Diego); Michael Mahler (Inova Diagnostics, San Diego); Chelsea Bentow (INOVA Diagnostics, San Diego); Erika Aron (INOVA Diagnostics, San Diego); Marvin Fritzler (University of Calgary, Calgary) Objectives: Anti-neutrophil cytoplasmic antibody (ANCA) detection using ethanol-fixed neutrophils is a well-established diagnostic test for ANCA-associated vasculitis (AAV). However, antinuclear antibodies (ANA) can also stain neutrophils and produce a perinuclear ANCA (P-ANCA) and other immunofluorescence patterns. Very little is known about the specific ANAs that may produce confounding ANCA staining. The purpose of this pilot study is to examine the frequency of ANCA positivity in known ANA positive sera.

Methods: Serum samples with well characterized ANA-reactivity were tested on ethanol-fixed neutrophils (NOVA Lite ${ }^{\circ}$ DAPI ANCA, Inova Diagnostics, San Diego, USA). These same slides were read using NOVA View (Inova Diagnostics). SLE-related autoantibodies as well as autoantibodies to special antigens (Ku, PCNA, RNA Pol III, Th/To, and Pm/Scl) were detected using an addressable laser bead immunoassay (ALBIA, Research Use Only).

Results: 24/43 (55.8\%) of ANA positive samples were positive for P-ANCA type staining on NOVA View and $18(41.9 \%)$ had monospecific autoantibodies. The frequency of P-ANCA type staining varied among the different ANA specificities, but was observed with monospecific dsDNA (1/1), Scl-70 (2/7), Jo-1 (1/1), and CENP (4/4) with neutrophils expressing a centromere pattern. For Scl-70, there was a titer-dependent effect as the two samples generating P-ANCA type staining had the highest anti-Scl-70 antibody titers (5080 and 5141 mean fluorescence intensity or MFI).

Conclusion: The prevalence of concomitant positive ANA and P-ANCA type staining was high (56\%). We observed differences among ANA fine-specificities with anti-CENP antibodies showing the most pronounced staining. However, anti-CENP antibodies had a distinct centromere pattern that allows for reliable exclusion on ANCA slides. Further studies with more well-characterized samples are needed to further analyze the impact of different ANA fine-specificities on P-ANCA and C-ANCA results.
13

Autoantibodies to Double-Stranded DNA with a Discordant ANA Result: False Positive or Unique Epitopes?

May Choi (University of Calgary, Calgary); Natalia Baeza (La Plata); Hector Arbillaga (Calgary); Alex Chin (Calgary Laboratory Services/University of Calgary, Calgary); Marvin Fritzler (University of Calgary, Calgary)

Background: Autoantibodies directed against double stranded DNA (dsDNA) are a serological hallmark of systemic lupus erythematosus (SLE). They are also a key component of the SLE classification criteria and have been historically associated with disease activity and lupus nephritis. Testing for dsDNA is indicated if the antinuclear antibody (ANA) is positive (classically a nuclear homogenous pattern) along with other signs and symptoms of SLE (i.e. high pre-test probability). Challenges in the reliable detection of anti-dsDNA include the polyclonal anti-dsDNA B cell response, the avidity of the autoantibodies and the spectrum of dsDNA targets. Certainly, different populations of anti-dsDNA have been associated with different disease and clinical phenotypes. As this case illustrates, not all anti-dsDNA antibodies are associated with homogeneous nuclear staining as detected by conventional indirect immunofluorescence (IIF) on HEp-2 substrates.

Case Presentation: A 69-year-old female patient with undifferentiated connective tissue disease (UCTD) presented with steroid-dependent symmetric polyarthritis, pleuritis, lymphopenia, and elevated inflammatory markers. Her ANA was negative on HEp-2 substrates from three different manufacturers (Euroimmun, Inova, ImmunoConcepts) although cytoplasmic speckled staining was noted. Remarkably, she had high titer anti-dsDNA confirmed by multiple assays (> 30,000 kIU/L by BioPlex (BioRad, Hercules, CA, USA), $667 \mathrm{IU} / \mathrm{mL}$ by chemiluminescence immunoassay (QUANTA Flash: Inova Diagnostics, San Diego, CA, USA), 1/320 on Crithidia luciliae (Inova Diagnostics), and 399.2 UI/mL on Quanta LITE). When $\mathrm{HEp}-2$ cells were treated with $0.1 \mathrm{~N} \mathrm{HCl}$ to remove nuclear proteins such as histones, the cytoplasmic staining was abolished but high titer (1/640) homogeneous nuclear staining was observed. After treatment of the extracted cells with DNase I, the ANA IIF once again became negative confirming that the reactivity on extracted cells was to DNA.

Conclusion: This patient with high titer anti-dsDNA but a negative nuclear IIF staining represents a serological enigma that has been observed in up to 1 in 150 sera with anti-dsDNA. The exact nature of the dsDNA reactivity in such cases is not clear but it strongly suggests a unique, hidden dsDNA epitope (cryptotope) that is exposed after histones and other nuclear proteins are removed from conventional HEp-2 substrates. Furthermore, since it is clear that unique dsDNA epitopes may be missed by the IIF ANA test, as in this case of UCTD, clinicians should be careful not to dismiss such results as false negative (in the case of the ANA IIF) or false positive (in the case of the anti-dsDNA results).

\section{4}

Clinical Features Associated with Anti-Ro52/TRIM21 in SLE

May Choi (University of Calgary, Calgary); Michael Mahler (Inova Diagnostics, San Diego); Michelle Jung (University of Calgary, Calgary); Claire Barber (University of Calgary, Calgary); Ann Clarke (University of Calgary, Calgary); Marvin Fritzler (University of Calgary, Calgary)

Objectives: Anti-Ro52/tripartite motif-containing 21 (TRIM21) antibodies, unlike some original thinking, are not part of the same macromolecular complex as anti-Ro60/SSA, and in some systemic autoimmune rheumatic diseases (SARDs), they are expressed quite separately. Ro52/TRIM21 functions as an E3 ubiquitin ligase as a member of the TRIM protein family involved in protein degradation pathways. In recent years, the clinical significance of this autoantibody is only beginning to be appreciated in SARDs. The purpose of this study was to develop a clearer understanding of anti-Ro52/TRIM21 in systemic lupus erythematosus (SLE) by clarifying clinical, demographic, and serological associations in a local cohort.

Methods: Patients fulfilling the American College of Rheumatology or Systemic Lupus International Collaborating Clinics (SLICC) Classification Criteria for SLE were enrolled in the Southern Alberta Registry for Lupus Erythematosus (STARLET) cohort (Calgary, Canada). Demographic,

Personal non-commercial use only. The Journal of Rheumatology Copyright (c) 2018. All rights reserved. 
clinical information, and sera were collected at time of enrollment. Autoantibodies directed to an array of SARD targets (Ro60/SSA, La/SSB, Sm, U1-RNP, dsDNA, ribosomal P (C22 peptide) as well as Ro52/TRIM21, were tested by an addressable laser bead immunoassay (ALBIA, Research Use Only). Pearson chi-square test and Mann-Whitney U tests were performed to determine any associations between the prevalence of anti-Ro52/TRIM21 and demographic, clinical features and other autoantibodies.

Results: 138 SLE patients were included; $89.1 \%$ female with median age of 31.3 years (interquartile range, IQR, 21.3-43.9) and disease duration of 12.0 years (3.8-20.8). The prevalence of anti-Ro52/TRIM21 was $21.0 \%$ (29/138) and often co-existed with anti-Ro/SSA $(79.3 \%, 23 / 29)$ and anti-La/SSB $(44.8 \%, 13 / 29)$. Monospecific anti-Ro52/TRIM21 was rare $(2.2 \%, 3 / 138)$. Patients with positive anti-Ro52/TRIM21 compared to those without the antibody, were more likely to have leukopenia (55.2 vs. 32.1, $\mathrm{p}=0.022$ ), pleuritis ( 44.8 vs. $22.9, \mathrm{p}=0.019)$, and negative anti-dsDNA (55.2 vs. $74.3, \mathrm{p}=0.045)$.

Conclusion: AntiRo52/TRIM21 antibodies were common in our SLE cohort $(21 \%)$ and were associated with anti-Ro60/SSA, anti-La/SSB, leukopenia, pleuritis, but were independent of anti-dsDNA. As anti-Ro52/TRIM21 in SLE is serologically and phenotypically quite distinct compared to systemic sclerosis or autoimmune inflammatory myopathies, different epitope specificities may be represented in each disease. There is evidence that activated Ro52/TRIM21 is perhaps protective against anti-dsDNA B cell responses, which may explain the lower frequency of anti-dsDNA in our anti-Ro52/TRIM21 positive patients. Results from larger cohorts and animals model knockout studies will provide further insight on its role as a pathogenic, diagnostics, and therapeutic biomarker of disease.

\section{5}

Economic Evaluation of Damage Accrual in a Nationwide Canadian SLE Cohort

May Choi (University of Calgary, Calgary); Yvan St. Pierre (McGill University, Montreal); Murray Urowitz (University of Toronto, Toronto); Dafna Gladman (University of Toronto, Toronto); Sasha Bernatsky (McGill University Health Centre, McGill University, Montreal); Evelyne Vinet (McGill University Health Centre, Montreal); Christian Pineau (Montreal General Hospital, Division of Rheumatology, Montreal); John Hanly (Dalhousie University and Nova Scotia Health Authority, Halifax); Christine Peschken (University of Manitoba, Winnipeg); Paul Fortin (Department of Rheumatology, CHU de Québec-Université Laval, Québec); Michelle Jung (University of Calgary, Calgary); Claire Barber (University of Calgary, Calgary); Susan Elliott (University of Waterloo, Waterloo); Jenna Dixon (University of Waterloo, Waterloo); Ann Clarke (University of Calgary, Calgary)

Objectives: We describe the costs associated with damage states in a Canadian-wide SLE cohort using multi-state modeling, which provides a dynamic representation of damage accrual in real time.

Methods: Patients fulfilling the revised ACR or Systemic Lupus International Collaborating Clinics (SLICC) Classification Criteria for SLE from 6 Canadian centres were enrolled. Participants completed validated health resource utilization (including hospitalizations, medications, physician visits, tests, and emergency room visits) and lost productivity questionnaires. Direct costs were calculated by multiplying health resources by their 2017 Canadian prices. Indirect costs included time loss and impaired productivity in labour force and non-labour force activities and were valued using age-sex specific wages from Statistics Canada. Annual costs associated with damage states (SLICC/ACR Damage Index [SDI]) were obtained from multiple regressions adjusting for age, race/ethnicity, and disease duration. To compute long-term estimates of direct/indirect costs, annual costs associated with each level of disease damage were multiplied by the expected duration in each state, forecasted using a multi-state Markov model (Bruce IN. Ann Rheum Dis 2015;74:1706-13). Future costs were discounted at a yearly rate of $3 \%$.

Results: 1361 patients participated, 90.4\% female, $71.0 \%$ Caucasian, mean age at diagnosis 33.1 years (SD 13.5), mean SLE duration at completion of economic questionnaire 16.8 years (SD 11.6), mean SLE Disease Activity Index (SLEDAI-2K) 2.71 (SD 3.21), and mean SDI 1.54 (SD 1.87). Annual direct $(\$ 18,620,95 \%$ CI: $\$ 15,850-21,390)$ and total costs $(\$ 46,523,95 \% \mathrm{CI}$ : $\$ 39,048-\$ 53,997)$ for those with the highest damage state (SDI of $\geq 5$ ) were 4.3- and 1.6-fold times higher than those with the lowest damage state (SDI of 0). Indirect costs did not differ when stratified by baseline SDI (SDI of $\geq$ 5 was $\$ 27,903,95 \%$ CI: $\$ 21,565-34,241$ and SDI 0 was $\$ 24,768,95 \%$ CI: $\$ 21,439-28,098)$ and exceeded direct costs by 3.9 -fold. Similar trends were observed for five- and ten-year cumulative costs. Total five- and ten-year cumulative costs for SDI of $\geq 5$ was $\$ 213,667$ (95\% CI: $\$ 179,094-248,240)$ and $\$ 387,108$ (95\% CI: $\$ 323,758-450,458)$ respectively. Conclusion: Patients with the highest baseline SDIs incurred cumulative direct costs that were 4.3-fold higher and total costs almost 2-fold higher than those with the lowest baseline SDIs. Indirect costs did not vary with SDI and patients with no or minimal damage still experienced considerably reduced productivity. Indirect costs exceeded direct, on average, by 3.9-fold, underscoring the importance of lost workforce productivity, and the need for actionable workplace and systems-level (i.e., government, policies, and society) interventions to improve the employment outcomes of those living with SLE. Best Abstract by a Rheumatology Resident. Supported by a CIORA grant.

\section{6}

Identification of a Rare Genetic Variant of OFD1 Gene in a Familial Form of Adult-onset Multiple Diaphyseal Insufficiency Fractures Associated with Facial and Teeth Hypoplasias

Marie-Eve Boisvert (CHU de Québec-Université Laval Research Centre, Quebec); Jacques Brown (Department of Rheumatology, CHU de Québec-Université Laval, Quebec); Rachel Laframboise (CHU de QuébecUniversité Laval, Quebec); Maxime Vallée (CHU de Quebec Research Centre, Quebec); Suzanne Morin (McGill University, Montreal); Edith Gagnon (CHU de Quebec Research Centre, Quebec); Arnaud Droit (CHU de Québec Research Centre, Quebec); Laetitia Michou (Department of Rheumatology, CHU de Québec-Université Laval, Quebec)

Objectives: The pathogenesis of atypical femur fractures (AFFs), a type of insufficiency fracture of the subtrochanteric or diaphyseal region of femur, is incompletely understood. They are thought to be associated with long-term use of bisphosphonates but also occur in patients who are naïve to pharmacological therapy. AFF have been reported in mineralization defects such as hypophosphatasia and rickets, or with Paget's disease of bone, all being associated with femur bowing, a risk factor for AFF. Here, we report a French-Canadian family in which eleven relatives have X-linked osteoporosis (without osteomalacia on bone biopsy) and/or dental and facial hypoplasia and/or adult-onset diaphyseal insufficiency fractures of long bones, with a radiological aspect akin to AFF.

Methods: In this family with X-linked insufficiency fractures, we first checked for mutations in our best candidate, the Plastin3 gene, previously reported in X-linked osteoporosis. Since no mutation was found in this gene, we performed, for cost-effectiveness reasons, a whole exome sequencing to identify the causal genetic variant. Basically, we performed whole exome sequencing and resequencing after a first bioinformatic filtering and intrafamilial segregation analysis with emphasis on $\mathrm{X}$ chromosome genetic variants.

Results: Surprisingly, a rare variant, p.A642D, of the OFD1 gene, was our only candidate on the $\mathrm{X}$ chromosome perfectly segregating with the phenotype within the family. In the literature, mutations of OFD1 gene were reported to be associated with the oral-facial-digital syndrome, which belongs to the group of ciliopathies. Ciliopathies are genetic disorders of the cellular cilia or the anchoring structure of the cilia or cilia dysfunction. Oral-facial-digital syndrome is characterized by malformations of the face, oral cavity and digits, and is transmitted in a X-linked pattern of inheritance with lethality in males. Although the affected relatives of this family were not diagnosed with oral facial digital syndrome before this study, their clinical phenotype was in accordance with a mild form of this heterogeneous

Personal non-commercial use only. The Journal of Rheumatology Copyright $(\subset) 2018$. All rights reserved. 
syndrome, which may also explain why we did not observe lethality in males in this family.

Conclusion: The case of this family is very interesting as it shows that stress fractures of long bones may occur in patients with oral facial digital syndromes. It may also contribute to a better understanding of the pathophysiology of diaphyseal insufficiency fractures and their association with oral health. Further studies are needed to understand the functional effects of this genetic variant on bone modeling and remodeling, and its possible contribution to other type of insufficiency fractures, such as AFF.

\section{7}

Ultrasound Finds Discordance between Disease Activity in the Hands and Feet of Patients with Rheumatoid Arthritis

Hanyan Zou (McMaster University, Hamilton); Karen Beattie (McMaster University, Hamilton); George Ioannidis (McMaster University, Hamilton); Maggie Larche (McMaster University, St Joseph's Healthcare Hamilton, Hamilton)

Objectives: Hands are frequently the main focus of routine examinations for patients with rheumatoid arthritis (RA) while feet are often neglected. Thus, patients with active disease in the feet but little or no activity in the hands may be inadequately treated. We compared clinical examination and ultrasonography (US) evidence of disease activity in the hands and feet in patients with RA.

Methods: Patients with RA (ACR criteria, disease duration 2-5 years) were recruited. Bilateral 2nd-5th metacarpophalangeal joints (MCPJs) and metatarsophalangeal joints (MTPJs) were clinically examined for swelling and tenderness. US of the 2nd-3rd MCPJs, 2nd-3rd proximal interphalangeal joints (PIPJs), and 2nd-5th MTPJs were graded for synovial thickening (ST) and power Doppler (PD) (0-3, $3=$ most severe), and presence of erosions by a rheumatologist. Total ST and PD scores were sums of the scores of each joint. ST grade $\geq 2$ in MTPJs and grade $\geq 1$ in MCPJs were interpreted as pathologic. The number of clinically symptomatic joints and pathologic joints on US were compared between hands and feet.

Results: This study included 22 patients $[\mathrm{n}=19$ female, mean $(\mathrm{SD})$ age $=$ 57.4 (7.6) years]. Of these, $9 \mathrm{had} \geq 1$ swollen MCPJ, $3 \mathrm{had} \geq 1$ swollen MTPJ, $3 \mathrm{had} \geq 1$ tender MCPJ and $11 \mathrm{had} \geq 1$ tender MTPJ. ST was present in the hands and feet of 14 and 21 patients, respectively. Total ST appeared higher in the feet [mean difference $(\mathrm{SD})=2.2(3.6)]$. $\mathrm{PD}$ was seen in the hands and feet of 11 and 6 patients, respectively. Total PD score appeared higher in the hands [mean difference $(\mathrm{SD})=1.5(2.89)$ ]. There was $\geq 1$ erosion in the hands and feet of 4 and 11 patients respectively. The number of patients with ST, PD and erosion in the MTPJs but not the hands was 7 , 2 and 8, respectively. In patients with no swollen MCPJs, there was ST in the MTPJs of 12 patients, PD in 4 patients, and erosion in 5 patients. In patients with no tender MCPJs, ST was found in the MTPJs of 18 patients, PD in 4 patients and erosion in 10 patients.

Conclusion: In this small cohort, swelling and PD appeared more prevalent in the hands. Tenderness and ST appeared more prevalent in the feet. Disease activity in the feet didn't appear to mimic activity in the hands. Thus, discordance between disease activity in the hands and feet emphasizes the need to assess the feet by clinical exam and US in patients with RA.

\section{8}

Knowledge Translation in Juvenile Idiopathic Arthritis Research in Canada: Comparing and Contrasting Parent and Adolescent Perceptions

Benjamin Rose-Davis (IWK Health Centre, Halifax); Julia Wright (Dalhousie University, Halifax); Michelle Batthish (McMaster Children's Hospital, Hamilton); Tania Cellucci (McMaster University, Hamilton); Ciaran Duffy (Children's Hospital of Eastern Ontario, Ottawa); Lori Tucker (BC Children's Hospital, Vancouver); Karen Duffy (Children's Hospital of Eastern Ontario, Ottawa); Adam Huber (IWK Health Centre, Halifax); Deborah Levy (Division of Rheumatology, SickKids Hospital; Faculty of Medicine, University of Toronto, Toronto); Bianca Lang (IWK Health Centre, Halifax); Dax Rumsey (University of Alberta, Edmonton); Janet
Curran (IWK Health Centre, Halifax); Elizabeth Stringer (IWK Health Centre, Halifax)

Objectives: The Research in Canadian Children with Childhood Arthritis Emphasizing Outcomes (ReACCh-Out) cohort has provided new knowledge about JIA outcomes in Canada. The aim of this study is to examine barriers and facilitators that influence the uptake of Canadian JIA research findings by parents of children with JIA (PJIA) and adolescents with JIA (AJIA)

Methods: PJIA and AJIA (> 12 yo) from four Canadian pediatric rheumatology centres were recruited for focus groups (FGs) that utilized a semistructured interview guide to gather information on their attitudes and experiences related to research on JIA occurring in Canada. Sessions were audio-recorded and transcribed for analysis. Themes were abstracted from transcripts using an open coding and general inductive approach.

Results: Two FGs occurred at each site (telephone interviews supplemented low turnout at 2 sites). 28 PJIA [22F; mean age 44.7(32-59)] and 19 AJIA [13F; mean age 14.2(13-17)] participated. Shared and contrasting themes emerged from the PJIA and AJIA groups. Both groups understood the purpose and value of research, but they lacked awareness around past or current JIA research initiatives. PJIA were keen to receive information from research, particularly at key time points (e.g. decision-making around treatments). Feeling overwhelmed by the volume of research information presented is a barrier. The majority of AJIA did not look for information from research on their own, and many do not perceive having a role in decisions around their own healthcare. AJIA were not interested in long-term outcomes of JIA; their priorities were to understand the impact of JIA on daily life (school, sports) and side effects of medications. Both PJIA and AJIA looked to their healthcare team for information, with many distrusting the internet as a source of information.

Conclusion: Understanding how to optimally deliver the most up-to-date information from research to PJIA, particularly when making decisions about medications requires further investigation. AJIA are unlikely to seek information on their own and overall their interests lie in the day to day management of their disease and impact on their life, as opposed to long-term outcomes. Supported by a CIORA grant.

\section{9}

Feasibility and Preliminary-efficacy of a Multi-faceted Physical Activity Counselling Program for Persons with Knee Osteoarthritis

Chance Park (Arthritis Research Canada, Coquitlam); EC Sayre (Arthritis Research Canada, Richmond); Linda Li (Rehab Sciences/Physical Therapy, University of British Columbia, Arthritis Research Canada, Richmond)

Objectives: Improving physical activity and reducing sedentary time are important for the management of knee osteoarthritis (OA). The study assessed the feasibility and preliminary-efficacy of a multi-faceted physical activity counselling program for persons with knee osteoarthritis (OA) for facilitating self-driven goal-setting, prior to running a full-scale randomized controlled trial.

Methods: Eligible participants with a diagnosis of or symptoms related to knee OA were enrolled in a one-group pre-post study. The intervention was a month-long, which included 1) an in-person education session; 2) a fitness tracker (Fitbit Flex 2); 3) FitViz, a Fitbit-compatible web application catered to persons with knee OA; 4) two biweekly (in-person and phone) counselling sessions with a physiotherapist. Outcome measures were taken before and after the intervention, and included: 1) time spent in moderate to vigorous physical activity (MVPA) in sessions of $>10$ mins at $3+$ metabolic equivalents (METs; primary outcome measure); 2) total daily steps; 3) time spent in sitting in sessions of $>20$ mins during the day. Preliminary-efficacy was assessed by using a matched pairs t-test on the outcome measures, and feasibility was assessed by the rates of participant enrollment, retention to the intervention and equipment loss.

Results: The eligibility screening process lasted from Oct 2016 - Feb 2017 (21 weeks), and 10 participants were successfully enrolled [9 women; mean age $63.1(\mathrm{SD}=15.7)$ years; mean BMI was $26.2(\mathrm{SD}=4.8)$ ]. All participants completed the study. The feasibility findings were a post-consent dropout rate of $0 \%$ and no equipment loss. Preliminary-efficacy findings were: 1 )

Personal non-commercial use only. The Journal of Rheumatology Copyright $\odot$ 2018. All rights reserved. 
MVPA; mean pre/post-intervention: 32.3/49.2, mean increase 16.9 [(95\% CI -3.6, 37.4); $\mathrm{p}=0.19] \mathrm{min} /$ day; 2) Step-count; mean pre/post-intervention: 5197.5/6220.4, mean increase: 1022.9 [(95\% CI -417.8, 2461.7); $\mathrm{p}=0.27]$ steps/day; 3) Sedentary time; mean pre/post-intervention: 596.4/983.4, mean increase: 387.0 [(95\% CI 276.6, 497.4); $\mathrm{p}=0.002] \mathrm{min} /$ day.

Conclusion: The study found a promising post-consent dropout rate of $0 \%$, and no financial damage due to equipment loss. However, participant enrolment rate was low, thus recruitment should be a focus during the full-scale randomized controlled trial. Preliminary-efficacy findings in MVPA and step-count showed an increase in outcome measures, although non-significant due to the limited sample size. There also was a paradoxical significant increase in sedentary time, which suggests a potential need for modification of the program to accentuate the importance of decreasing sedentary time in managing knee OA.

\section{0}

Evaluation of the Fracture Liaison Service within the Canadian Healthcare Setting: A Pilot Study

Matthew Wong-Pack (University of Toronto, Toronto); Ramy Khalil (McMaster University, Hamilton); George Ioannidis (McMaster University, Hamilton); Alexandra Papaioannou (Hamilton Health Sciences, Hamilton); Jonathan Adachi (McMaster University, St. Joseph's Healthcare, Hamilton); Arthur Lau (McMaster University, St. Joseph's Healthcare, Hamilton)

Objectives: The objective was to examine the fracture risk score of patients who sustained a fracture and were referred by the fracture liaison service to a single academic rheumatology practice. Fracture liaison services (FLS) support best practices for post-fracture osteoporosis care, aid in closing the care gap, and allow for the initiation of appropriate pharmacotherapy.

Methods: A retrospective cohort study was conducted for patients referred by the FLS Program within a tertiary care fracture clinic at Hamilton Health Sciences for assessment between January 2014 to July 2017. Patient demographics, risk factors, including fracture history were recorded. Fracture risk for each patient was determined using CAROC and FRAX.

Results: Over a three-year period, 320 patients were provided a referral by the FLS Program. Of those referred and assessed $(n=225)$, participants were 66.7 (11.6) years old, $78 \%$ female and a body mass index of 28.9 (6.1). 171 $(76 \%)$ patients presented with a major osteoporotic fracture (hip, clinical spine, forearm, or proximal humerus fracture) and 52 (23.1\%) with other fractures. $24(11.7 \%)$ patients assessed were on anti-resorptive therapy prior to fracture. Mean (SD) T-scores were: lumbar spine -1.0 (1.7) and femoral neck -1.6 (1.1). 148 (65.8\%) patients were on vitamin D supplementation with a mean (SD) dosage of 1525 (812) IU/day. 215 (95.6\%) of patients consumed calcium with an average of 713 (509) mg/day. 1 (0.4\%), 85 $(37.8 \%)$, and $138(61.3 \%)$ patients were found to be at low-risk, moderate-risk, and high-risk, respectively using CAROC. Patients were found to have a mean ten-year probability of sustaining a major osteoporotic fracture and hip fracture of $15.7(10.2) \%$ and $4.7(8.3) \%$, respectively using FRAX. Upon assessment, $136(60.4 \%)$ patients initiated or changed anti-resorptive therapy and $88(39.1 \%)$ were recommended vitamin D and calcium supplementation. Of those who cancelled $(n=38)$ or missed their appointment $(\mathrm{n}=38), 60(79 \%)$ patients sustained a major osteoporotic fracture and $16(21 \%)$ patients had other fractures.

Conclusion: Patients referred by the FLS Program received appropriate treatment to reduce fracture risk based on fracture risk status. On average, patients' calcium consumption was well below recommended daily requirement of $1200 \mathrm{mg} /$ day for those between 51-70 years of age. Current guidelines are to assess patients of age $>50$ with a fragility fracture. However, based on these findings, additional criteria / recommendations could be adapted for this program to refine the referral eligibility for patients screened and increase participation.

21

Predictors of Long-Term Retention of Methotrexate and Other DMARDs with Golimumab in Rheumatoid Arthritis and Psoriatic Arthritis: An Analysis from a Prospective, Observational Registry
Derek Haaland (Department of Medicine, McMaster University, Hamilton); Anna Jaroszynska (Oakville); Boulos Haraoui (Institut de Rhumatologie de Montréal, Montreal); Suneil Kapur (University of Ottawa, Ottawa); Jaqueline Stewart (Penticton); Wojciech Olszynski (University of Saskatchewan, Saskatoon); Keltie Anderson (University of Saskatchewan, Saskatoon); Raman Rai (McMaster University, Hamilton); Michael Starr (McGill University Health Centre, Montreal); Alexender Tsoukas (McGill, Montreal); Eliofotisti Psaradellis (JSS Medical Research, Montreal); Emmanouil Rampakakis (JSS Medical Research, Montreal); Cathy Tkaczyk (Janssen Inc, Toronto); Allen Lehman (Janssen Inc, Toronto); Francois Nantel (Janssen Inc, Toronto); Brendan Osborne (Janssen Inc, Toronto)

Objectives: Previous studies have suggested that concomitant methotrexate (MTX) therapy may increase the efficacy of biologic treatments. A scarcity of data exists on the benefits of combination therapy with golimumab (GLM) and MTX as well as other DMARDs (oDMARDs). The aim of this analysis was to compare the long-term retention of GLM monotherapy vs. combination therapy with MTX and/or oDMARDs and to explore independent predictors of retention in patients with rheumatoid arthritis (RA) and psoriatic arthritis (PsA) followed in Canadian routine practice.

Methods: BioTRAC is an ongoing, prospective registry of patients initiating treatment with infliximab or GLM for inflammatory arthritis or with ustekinumab for PsA. Eligible participants for this analysis included RA and PsA patients treated with GLM. Patients were excluded if they had follow-up < 24 months and were not discontinued. Treatment durability was assessed with the Kaplan Meier (KM) estimator of the survival function and Cox regression.

Results: 336 RA patients were included; no statistically significant differences existed in baseline characteristics. There were 195 (58.0\%) patients who discontinued with a KM-based mean (SE) time to discontinuation of 36.2 (1.7) months. Between group differences were observed with higher treatment durability for MTX+GLM+oDMARDs [39.8 (2.3)] months, followed by MTX+GLM [37.4 (3.4)], GLM+oDMARDs [27.2 (4.8)] and GLM monotherapy with $[25.2(3.0)](\mathrm{p}=0.025)$. Upon adjusting for potential confounders, higher durability was observed for the MTX+GLM+oDMARDs group vs. GLM monotherapy [hazard ratio -HR- (95\% CI): $0.59(0.36-0.96), p=0.032]$. Moreover, increased baseline DAS28 [HR (95\% CI): $1.17(1.03-1.32), \mathrm{p}=0.014]$ and previous use of MTX [HR (95\% CI): $1.73(1.00-3.00), p=0.052]$, were independently associated with premature treatment termination. 167 PsA patients were included; no statistically significant differences existed in baseline characteristics. There were 96 (57.5\%) patients who discontinued with a KM-based mean (SE) time to discontinuation of 34.7 (2.3) months. No between group differences were observed for treatment durability. However, upon adjusting for potential confounders, increased baseline MDGA [HR (95\% CI): 1.12 $(1.01-1.25), p=0.038]$, previous use of MTX [HR (95\% CI): 3.54 (1.09-11.45), $\mathrm{p}=0.035$ ] , and female gender [HR (95\% CI): $1.96(1.19-3.21), \mathrm{p}=0.008]$ were independently associated with premature treatment termination.

Conclusion: The results of this analysis have shown that combination therapy with GLM, MTX and other DMARDs is significantly associated with higher treatment durability compared to GLM monotherapy among RA patients. Although gender and MDGA were identified as significant independent predictors of long-term retention among PsA patients, treatment durability was not affected by concomitant MTX and DMARD use.

\section{2}

Prehypertension in Rheumatoid Arthritis: Underestimated Indicator of Cardiovascular Risk

Guadalupe Mendoza-Vázquez (Unidad de Investigación Biomédica 02, UMAE, HE Centro Médico Nacional de Occidente, Instituto Mexicano del Seguro Social (IMSS). Guadalajara, Jalisco, México.Programa de Doctorado en Ciencias Médicas, Facultad de Medicina, Universidad de Colima, México., Guadalajara); Alberto Rocha-Muñoz (Centro Universitario de Tonalá (CU Tonalá), Universidad de Guadalajara. Tonalá, Jalisco, México, Guadalajara); Jorge Gámez-Nava (Unidad de Investigación Biomédica 02, UMAE, HE Centro Médico Nacional de Occidente, Instituto Mexicano del 
Seguro Social (IMSS). Guadalajara, Jalisco, México, Guadalajara); Laura González-López (Departamento de Medicina Interna-Reumatología, Hospital General Regional 110, Instituto Mexicano del Seguro Social (IMSS). Guadalajara, Jalisco, México., Guadalajara); Mario Salazar-Páramo (Unidad de Investigación Biomédica 02, UMAE, HE Centro Médico Nacional de Occidente, Instituto Mexicano del Seguro Social (IMSS). Guadalajara, Jalisco, México., Guadalajara); Francisco Espinoza-Gómez (Programa de Doctorado en Ciencias Médicas, Facultad de Medicina, Universidad de Colima, México., Colima); Carlos Riebeling-Navarro (Unidad de Investigación en Epidemiología Clínica, Unidad Médica de Alta Especialidad, Centro Médico Nacional SXXI, IMSS., Ciudad de México); Arnulfo Nava-Zavala (Unidad de Investigación Biomédica 02, UMAE, HE Centro Médico Nacional de Occidente, Instituto Mexicano del Seguro Social (IMSS). Guadalajara, Jalisco, México., Guadalajara)

Objectives: Prehypertension (PHTN) includes systolic blood pressure in $\mathrm{mmHg}$, (SBP) of 120-139 or diastolic blood pressure (DBP) of 80-89. It is a risk factor for cardiovascular disease (CVD), further information is required for PHTA in RA. Therefore it is important to describe the frequency of PHTN, normotension (NT) and hypertension (HTN) in RA and to compare demographic, metabolic, and disease activity characteristics.

Methods: Cross-section analytical study. 250 women with RA (ACR1987) were included. They were classified in NT, PHTN and HTN pursuant to the JNC7 criteria. Demographic, CV risk and RA activity variables were included. ANOVA and Chi2 were used to analyze the results.

Results: The groups were NT $(n=65)$, PHTN $(n=101)$, HTN $(n=84)$. Comparisons for: age $54.2 \pm 10.9,57.6 \pm 10.8,59.5 \pm 9.9(\mathrm{p}=0.01)$; weight $64.3 \pm 12.3,66.9 \pm 10.9,69.0 \pm 13.1(\mathrm{p}=0.05)$; BMI $26.3 \pm 4.5,27.8 \pm$ 4.0, $28.8 \pm 4.9(\mathrm{p}=0.02)$; waist circumference (WC) $89.2 \pm 12.9,94.0 \pm$ $10.6,95.0 \pm 11.5(\mathrm{p}=0.01)$ were related to HTN but not to PHTN. The waist-to-height ratio (WHR) $0.57 \pm 0.09,0.60 \pm 0.07,0.62 \pm 0.08$ was associated with PHTN $(\mathrm{p}=0.02)$ and HTN $(\mathrm{p}=0.002)$. The percentage of fat mass ( $\geq 30 \%) 75 \%, 89 \%, 86 \%$ was associated with PHT ( $\mathrm{p}=0.05)$; WHR $(>0.55), 66 \%, 79 \%, 85 \%$ was associated with HTN $(\mathrm{p}=0.02)$; metabolic syndrome (MS) 29\%, 52\%, 67\% was associated with HTN (p < 0.001) and $\mathrm{WC} \geq 88 \mathrm{~cm}, 57 \%, 72 \%, 76 \%$ was associated with HTN ( $\mathrm{p}=0.03)$. The percentage of patients with disease activity (DAS28) 74\%, 55\%, 49\% were associated with NT ( $\mathrm{p}=0.006)$; the use of glucocorticoids $68 \%, 85 \%, 63 \%$ was associated with PHTN ( $\mathrm{p}=0.002)$; AINEs 92\%, 79\%, $88 \%$ was associated with NT $(\mathrm{p}=0.04)$

Conclusion: PHTN is associated with changes in body composition in RA. Cohort studies are required to clarify the relationship of PHTN and allow to propose strategies for PHTN management.

\section{3}

Clinical Characteristics Associated with Hypertriglyceridemic Waist Phenotype in Women with Rheumatoid Arthritis

Guadalupe Mendoza-Vázquez (Unidad de Investigación Biomédica 02, UMAE, HE Centro Médico Nacional de Occidente, Instituto Mexicano del Seguro Social (IMSS). Guadalajara, Jalisco, México.Programa de Doctorado en Ciencias Médicas, Facultad de Medicina, Universidad de Colima, México., Guadalajara); Alberto Rocha-Muñoz (Centro Universitario de Tonalá (CU Tonalá), Universidad de Guadalajara. Tonalá, Jalisco, México., Guadalajara); Rodolfo Guadiana-Lozano (Unidad de Investigación Biomédica 02, UMAE, HE Centro Médico Nacional de Occidente, Instituto Mexicano del Seguro Social (IMSS). Guadalajara, Jalisco, México., Guadalajara); Jorge Gámez-Nava (Unidad de Investigación Biomédica 02, UMAE, HE Centro Médico Nacional de Occidente, Instituto Mexicano del Seguro Social (IMSS). Guadalajara, Jalisco, México., Guadalajara); Laura González-López (Departamento de Medicina Interna-Reumatología, Hospital General Regional 110, Instituto Mexicano del Seguro Social (IMSS). Guadalajara, Jalisco, México., Guadalajara); Mario Salazar-Páramo (Unidad de Investigación Biomédica 02, UMAE, HE Centro Médico Nacional de Occidente, Instituto Mexicano del Seguro Social (IMSS). Guadalajara, Jalisco, México., Guadalajara); Francisco Espinoza-Gómez (Programa de Doctorado en Ciencias Médicas, Facultad de Medicina,
Universidad de Colima, México., Colima); Arnulfo Nava-Zavala (Unidad de Investigación Biomédica 02, UMAE, HE Centro Médico Nacional de Occidente, Instituto Mexicano del Seguro Social (IMSS). Guadalajara, Jalisco, México., Guadalajara)

Objectives: Rheumatoid arthritis (RA) is associated with risk factors for cardiovascular disease, which is its main cause of mortality. Among cardiovascular risk factors (CVR) dyslipidemias and systemic inflammation are included. The hypertriglyceridemic waist phenotype (HTWP) has been proposed as instrument for the identification of individuals with metabolic syndrome and therefore in the increase of CVR in several diseases. There is scarce information about HTWP in RA. Therefore, the goal of this assignment was to evaluate the frequency of HTWP and its association with the RCV variables in RA.

Methods: Cross-sectional study. We studied 125 women with RA who fulfilled the 1987 ACR criteria. Anthropometry, biompedance analysis were performed and the body mass index (BMI), DAS28 and HAQ-DI were determined. A lipid profile was performed, estimating the atherogenic index (AI) (Castelli; CT/HDL). HTWP was defined as: waist circumference $\geq 88 \mathrm{~cm}$ and triglycerides $\geq 150 \mathrm{mg} / \mathrm{dL}$. The patients were stratified in 2 groups: a) those with HTW b) those without HTW. Chi-square and Student's t test were performed for comparisons and a logistic regression model with odds ratio (OR) and confidence intervals (CI) at $95 \%$ for the association of variables studied with HTWP. A significant value with $p \leq 0.05$ was considered. This study was performed following the ethical principles of the Declaration of Helsinki.

Results: HTWP was found in 38 (30\%) patients. After comparison of subgroups with and without, patients with HTWP displayed higher values for all following variables: hypertension $(57.9 \%$ vs $37.9 \% \mathrm{p}=0.04)$, DM2 ( $23.7 \%$ vs $8.0 \%$ p $=0.02)$, BMI $(29.6 \pm 3.1$ vs $26.8 \pm 4.3, \mathrm{p}<0.001)$, fat mass $(39.2 \pm 4.8$ vs $34.7 \pm 6.7 \mathrm{p}<0.001)$ and $\mathrm{AI}(4.6 \pm 1.2$ vs $3.7 \pm 1.0$ $\mathrm{p}<0.001)$. There were no differences in DAS28 and HAD-DI. The logistic regression adjusted by age and disease evolution to evaluate different variables associated to CVR showed that HTWP conferred an $\mathrm{OR}=1.16$, $\mathrm{p}=0.001$

Conclusion: The presence of HTWP is associated with the increase of CVR in RA and its predictive capability of completion shall be studied.

\section{4}

Do Inflammatory Arthritis Inpatients Receiving Group Music Therapy Improve Pain Compared to Music Listening over the 6 Weeks using a Randomised Controlled Open-label Trial?: "One good thing about music, when it hits, you feel no pain"

Katherine Wright (GF Strong Rehabilitation Centre, Vancouver); Raheem Kherani (University of British Columbia, Richmond); Sophia Zhao (GF Strong Rehabilitation Centre, Vancouver); Charles Goldsmith (Simon Fraser University, Burnaby)

Objectives: Inflammatory arthritis (IA) includes a group of chronic autoimmune conditions that includes rheumatoid arthritis and other variants. It affects about $3 \%$ of the population. Patients often suffer with intolerable pain and associated disability. Pain management is rated by the patients as the highest priority in their overall disease treatment. The GF Strong Arthritis Rehabilitation inpatient program (GFS-ARS) has an interdisciplinary approach to pain management treatment. Listening to music is associated with reduced pain intensity levels and decreased opioid levels needed, increased functional mobility, reduced depression and decreased pain. There is evidence to support the use of active music therapy to aid in pain management. Music therapy in patients with IA is a novel area of study. More options for pain management are needed within this population, as studies within the last decade are indicating that use of NSAID's can cause an increase in cardiac, renal and gastrointestinal concerns.

Methods: Patients admitted to GFS-ARS were stratified into 4 strata: Rheumatoid Arthritis (RA), Surgery (S), RA no surgery (NS), other inflammatory Arthritis (OIA) surgery, and OIA, NS, each with a 1:1 allocation ratio using blocks of size 2 or 4 and were randomly assigned to Music Therapy (MT) or a Music Listening (ML) control group that listens to a relaxation

Personal non-commercial use only. The Journal of Rheumatology Copyright $(\subset) 2018$. All rights reserved. 
recording. Standardized tests were used to determine whether MT achieves different outcomes than ML with respect to pain (primary outcome) as well as self-efficacy, physical functioning and depression (secondary outcomes). Results: Below are the descriptive results of the 13 patients randomized (First patient randomized 2015-05-08; last one 2015-11-13). Table 1: Pain Descriptive Statistics Grp/n/Baseline (m, s)/Therapy End (m, s)/Change (m, s) ML 6,6.92,2.18,5.42,2.04,-1.50,1 .09 MT 7, 6.50,2.52, 4.57,2.28,-1.93,2.44 delta;95\% CI 0.42;-2.483,3.317, 0.85;-1.821,3.513, -0.44;-1.946,2.820 Grp $=$ Group, $\mathrm{n}=$ sample size, $\mathrm{m}=$ mean, $\mathrm{s}=$ standard deviation, delta $=$ mean difference, $95 \% \mathrm{CI}=95 \%$ confidence interval, Units are $\mathrm{cm}$ for Pain

Conclusion: Inpatients admitted to GFS-ARS were assessed for the effect of MT versus ML in IA. The baseline analysis indicate that randomization was successful and there were no meaningful differences at baseline between the MT and ML groups. This pilot study suggests the possible benefits of music therapy over music listening could benefit from a larger study to provide more convincing evidence of the merits of music therapy. The secondary outcome measures may provide added evidence for merits of music therapy.

25

Combining Tocilizumab with Methotrexate Improves Sustainability. A RWE Report from Quebec Database Rhumadata ${ }^{\circledR}$

Denis Choquette (Institut de Rhumatologie de Montréal, Montréal); Louis Bessette (Laval University, Quebec); Boulos Haraoui (Department of Medicine, Centre Hospitalier de l'Université de Montréal, Montréal); Frederic Massicotte (Institut de Rhumatologie de Montréal, Montréal); Jean-Pierre Pelletier (Institut de Rhumatologie de Montréal, Montréal); Jean-Pierre Raynauld (Institut de Rhumatologie de Montréal, Montréal); Marie-Anaiis Remillard (Institut de Rhumatologie de Montréal, Montréal); Diane Sauvageau (Institut de Rhumatologie de Montréal, Montreal); Angèle Turcotte (Centre d'ostéoporose et de rhumatologie de Québec, Québec); Edith Villeneuve (Institut de Rhumatologie de Montréal, Montréal); Louis Coupal (Institut de Rhumatologie de Montréal, Montréal)

Objectives: Biologic therapy targeting TNF have consistently demonstrated better efficacy and effectiveness when combined with a csDMARDS, most frequently methotrexate. Although pre-clinical protocols have shown short term efficacy of tocilizumab (TOCI) in monotherapy in very selected patients suffering from rheumatoid arthritis (RA), little is known on the long-term comparative sustainability (Monotherapy versus combo therapy). We evaluate here the comparative sustainability of tocilizumab in patients with rheumatoid arthritis treated with or without csDMARDS.

Methods: Data from RHUMADATA ${ }^{\circledR}$ patients with RA prescribed TOCI either as an initial or second biologic was analyzed. Patients were followed until treatment discontinuation, loss to follow-up or August 5th, 2017. The characteristics of selected patients were tabulated and the TOCI discontinuation rates of patients undergoing mono and combo therapy compared using Kaplan-Meier and parametric methods adjusting for potential confounder.

Results: A total of 119 patients with RA received TOCI in first or second intention. Of those, $42(35.3 \%)$ and $77(64.7 \%)$ received mono and combo therapy respectively. Most patients were women (76\%), mean age at diagnosis was 45.2( $\mathrm{SD}=12.6)$ years, and average age at treatment initiation was 55.2( $\mathrm{SD}=11.5)$ years. At treatment initiation, patients had an average disease duration of $9.6(\mathrm{SD}=9.6)$ years. HAQ and DAS28(4)-CRP at treatment initiation were respectively $1.22(\mathrm{SD}=0.64)$ and $4.65(\mathrm{SD}=1.17)$. Half and $12 \%$ of patients received hydroxychloroquine (HCQ) and sulfasalazine (SSZ) respectively. Age-adjusted Carlson's comorbidity index (ACCI) at baseline was $2.5(\mathrm{SD}=1.1)$, and $79 \%$ were RF-positive and $76 \%$ ACPA positive. Significant differences in retention rates between mono and combo TOCI therapy were observed (Figure). Mean retention time for mono and combo-therapy were respectively $2.80(\mathrm{SE}=0.33)$ and $5.57(\mathrm{SE}=0.41)$ years. Use of MTX remains significant ( $p$-value $=0.007)$ after adjusting for gender, age and disease duration at treatment initiation, HAQ, DAS28(4)-CRP, RF and ACPA, ACCI, and concomitant use of HCQ and SSZ.

Conclusion: Combining tocilizumab with csDMARDS significantly improves its sustainability.
26

Abatacept Retention Rates and Prognostic Factors of Retention in Patients with Rheumatoid Arthritis (RA) Treated in Canada: 2-Year Results from a Real-World Observational Study

Boulos Haraoui (Department of Medicine, Centre Hospitalier de l'Université de Montréal, Montréal); Denis Choquette (Institut de Rhumatologie de Montréal, Montréal); Louis Bessette (Department of Rheumatology, CHU de Québec-Université Laval, Québec); Majed Khraishi (Department of Medicine, Memorial University of Newfoundland, St. John's); Meryem Maoui (Bristol-Myers Squibb, Montreal); Julia Heitzmann (Excelya, Boulogne-Billancourt); Sean Connolly (Bristol-Myers Squibb, Montreal); Rieke Alten (Charite University, Berlin)

Objectives: To report 2-year retention rates in the Canadian cohort of the ACTION study (NCT02109666) and predictors of retention in the global cohort.

Methods: ACTION is a 2-year prospective, international, non-interventional study designed to provide long-term real-world data on abatacept retention in RA patients. The study was initiated in May 2008 and included patients with moderate-to-severe disease who initiated IV abatacept as a first- or subsequent-line biologic therapy. The 1-year interim analysis of ACTION was reported previously.1 Here we report the 2-year crude abatacept retention rate with $95 \% \mathrm{CI}$ (overall and by treatment line) in the Canadian cohort. Additional study outcomes from the global cohort include: 1) prognostic factors of retention and 2) EULAR response by treatment line and rheumatoid factor/anti-citrullinated protein antibody (RF/ACPA) seropositivity.

Results: In total, 228/229 Canadian patients were evaluable for analysis: 57 (25\%) biologic naïve and 171 (75\%) biologic failures. Duration of disease was longer in patients who failed previous biologics (17.5\% vs $28.7 \%$ for $6-10$ years and $37.4 \%$ vs $29.8 \%$ for $>10$ years duration). The overall 2 -year retention rate was $40 \%$ (95\% CI $33.5 \%-46.8 \%$ ), and was higher in biologic-naïve $(60.3 \%$ [ $45.6 \%-72.2 \%]$ vs -failure patients $(33.4 \%$ [26.2\% $-40.9 \%$ ]). Main reasons for abatacept discontinuation were lack of efficacy ( $78.6 \%$ in biologic-naïve vs $72.9 \%$ in -failure patients) and safety $(18.8 \%)$ in patients who failed previous biologics. In the global cohort $(\mathrm{N}=2350$; 673 biologic naive), the overall 2-year retention rate was $47.9 \%(95 \% \mathrm{CI}$ $45.7 \%, 50.0 \%)$, and it was also higher in biologic-naïve $(54.5 \%$ [50.4\%-58.3\%]) vs -failure patients $(45.2 \%$ [42.7\%-47.7\%]; $p<0.001$ Predictors of higher abatacept retention in the global cohort included: $\mathrm{RF} / \mathrm{ACPA}$ seropositivity $(\mathrm{p}=0.030$ biologic naïve; $\mathrm{p}=0.028$ biologic failure); diabetes ( $\mathrm{p}=0.044$ biologic naïve); geographic location, Canada, Spain and Italy vs Germany ( $\mathrm{p}<0.001$ biologic naïve); and abatacept combination therapy ( $p<0.001$ biologic failure). Only high Patient Global Assessment, ie $\geq 70$, ( $p=0.009$ biologic failure $)$ predicted lower retention. At 2 years, a greater proportion of biologic-naïve vs -failure patients had good/moderate EULAR response ( $\mathrm{p}=0.005)$; RF and/or ACPA seropositivity was associated with a significantly better EULAR response $(\mathrm{p}=$ 0.007)].

Conclusion: In a real-world Canadian cohort, higher abatacept retention rates were achieved by biologic-naïve vs -failure pts. In the global cohort, geographic location impacted 2-year abatacept retention and RF/ ACPA seropositivity were predictors of higher retention and better outcomes independent of treatment line. These results are consistent with the 1-year ACTION data.1 1. Nüßlein HG, et al. BMC Musculoskelet Disord 2015;16:176.

\section{7}

Can Leisure-time Physical Activity Level Predict Cardiovascular Risk in SLE Patients?

Alix St-Aubin (CHU de Québec - Université Laval, Quebec); Anne-Sophie Julien (Clinical Research Platform, CHU de Québec-Université Laval Research Center, Québec); Carolyn Neville (McGill University, Montreal); Ellie Aghdassi (Toronto Western Research Institute, Toronto); Stacey Morrison (Toronto Western Research Institute, Toronto); Jiandong Su (Toronto Western Hospital, Toronto); Janet Pope (Western University, 
Department of Medicine, Division of Rheumatology, London); Sara Hewitt (St. Joseph's Health Care, London); Christian Pineau (Montreal General Hospital, Division of Rheumatology, Montreal); Paula Harvey (Division of Cardiology, Women's College Hospital, Toronto); Michal Abrahamowicz (McGill, Montreal); Deborah Da Costa (McGill University Health Center, Montreal); Paul Fortin (Department of Rheumatology, CHU de Québec-Université Laval, Québec)

Objectives: Systemic lupus erythematosus (SLE) is a prototypic autoimmune disease that affects 1 women in 1000 in Canada. Cardiovascular disease contributes greatly to mortality in SLE as it contributes to $36.4 \%$ of the deaths. The purpose of this study was to see if the leisure-time physical activity (LTPA) level is a good indicator of cardiovascular risk in SLE population.

Methods: Patients $(n=287)$ were randomized either into the "now" group ( $n=144)$ which received a comprehensive behavioral intervention including a supervised exercise program in the first 12 months or the "later" group (n $=143$ ) which received usual care. At baseline and 12 months, patients were asked to complete questionnaires assessing LTPA level measured by the Aerobics Centre Longitudinal Study physical activity questionnaire (ACLS), the number of co-morbidities measured on the Self-Administered Comorbidity Questionnaire (SCQ) and coronary heart disease risk factor using the Framingham risk score. SLE damage and disease activity were also investigated. Finally, a flow-mediated dilatation (FMD) was performed. LTPA level were scored on the basis of hour of metabolic equivalent (MET) performed weekly: 1) sedentary group (no LTPA activity), 2) insufficiently active (<7.5 MET-h/week) and 3) active (> 7.5 MET-h/week). Logistic and linear regressions were used to assess the effect of LTPA level, study group and their interaction on dichotomous and continuous outcomes respectively. Models were also adjusted for age at baseline.

Results: A total of 276 patients were included in the analysis. At baseline, no associations with LTPA level were found regarding the Framingham score. There was even a tendency towards a negative association between the insufficiently active and sedentary groups and higher Framingham score $(p=0.0602)$. No associations were found for the FMD measures at baseline. For the comorbidities, associations were found within the later group, in which the active group had a lower number of comorbidities than the insufficiently active and the sedentary groups $(3 \pm 0$ vs. $5 \pm 1$ vs. $6 \pm 1, \mathrm{p}=$ $0.0230)$. At one year, no association were found for the FMD measure or the Framingham risk score and LTPA level.

Conclusion: Our data suggest that the LTPA level is not associated with the cardiovascular risk and the FMD measure at baseline but could potentially predict the number of comorbidities. Further research are needed to better understand the LTPA level in SLE patients and to tailor appropriate exercise-based interventions.

\section{8}

\section{Evaluation of the Indications for ANCA Testing and its Diagnostic Utility}

Noren Khamis (University of British Columbia, Vancouver); Touraj Khosravi-Hafshejani (University of British Columbia, Vancouver); Kamran Shojania (University of British Columbia, Vancouver); Michael Nimmo (University of British Columbia, Vancouver); Antonio Avina-Zubieta (University of British Columbia Faculty of Medicine; Arthritis Research Canada, Richmond); Natasha Dehghan (University of British Columbia, Division of Rheumatology, Vancouver)

Objectives: Anti-neutrophil cytoplasmic antibodies (ANCA) is a widely available screening test and there are no accepted guidelines to limit unnecessary testing. As part of a quality improvement initiative, this study assessed clinical indications and diagnostic outcomes of patients who were tested for ANCA.

Methods: A retrospective chart review of all inpatient ANCA tests ordered at a tertiary care centre in Vancouver, British Columbia from January 1, 2016 to December 31, 2016 was conducted by two reviewers using a structured form. Discrepancies were discussed and solved by consensus. ANCA test results, diagnostic outcomes, and indications for testing were determined.
Inpatient medical records from all positive ANCA test results [defined as elevated proteinase 3 (PR3) or myeloperoxidase (MPO)] were reviewed. In order to better understand the appropriateness of current ANCA testing practices, an additional random sample of all tests ordered (positive and negative) was also reviewed ( $\mathrm{n}=90,25.4 \%$ of all tests). Diagnosis of AAV was determined by biopsy results and notes from hospitalization.

Results: 354 ANCA tests were performed on 298 patients in the study year with $9(3.0 \%)$ patients receiving three or more tests. There were a total of 29 positive ANCA tests (8.2\% of all tests performed). Among patients with a positive ANCA, $19(65.5 \%)$ had a final diagnosis of AAV with $7(36.8 \%)$ patients already known to have AAV. Among all positive tests only 12 $(41.4 \%)$ tests resulted in a new diagnosis of AAV. Patients with a positive ANCA test who were not diagnosed with AAV had a final diagnosis of: inflammatory bowel disease [2 (20.0\%)], IgM nephropathy [2 (20.0\%)], IgA nephropathy [2 (20.0\%)], anti-glomerular basement membrane disease [1 $(10.0 \%)]$, viral bronchitis [1 (10.0\%)], MSSA bacteremia [1 (10.0\%)], or the diagnosis was not specified [1 $(10.0 \%)]$. From the random sample of all tests ordered, only $9(10.0 \%)$ of 90 tests were ordered because of suspicion for vasculitis. The majority of testing was for an unknown reason [47 (52.2\%) or for suspicion for a systemic autoimmune disease [24 (26.7\%)]. Patients with a final diagnosis of AAV had a statistically higher titer of PR3 compared with those who did not (115.2 vs 46.9, $\mathrm{p}<0.05$; normal range $0-20$ ).

Conclusion: The majority of patients who were tested for ANCA had a negative result and did not have a diagnosis of AAV. Furthermore, only a small percentage of tests were performed because of a suspicion of vasculitis. Developing appropriate testing guidelines may reduce costs and potential unnecessary investigations.

\section{9}

First Steps in Developing a Novel Decision Support Intervention for Pain Management Options in Juvenile Idiopathic Arthritis

Karine Toupin-April (Children's Hospital of Eastern Ontario Research Institute, Ottawa); Jennifer Stinson (The Hospital for Sick Kids, Toronto); Adam Huber (IWK Health Centre, Halifax); Ciaran Duffy (Children's Hospital of Eastern Ontario, Ottawa); Esi Morgan (Cincinnati Children's Hospital, Cincinnati); William Brinkman (Cincinnati Children's Hospital Medical Center, Cincinnati); Linda Li (Rehab Sciences/Physical Therapy, University of British Columbia, Arthritis Research Canada, Richmond); Laurie Proulx (Canadian Arthritis Patient Alliance, Ottawa); Lucie Brosseau (University of Ottawa, Ottawa); Isabelle Gaboury (Université de Sherbrooke, Sherbrooke); Janice Cohen (Children's Hospital of Eastern Ontario Research Institute, Ottawa); Paul Fortin (Department of Rheumatology, CHU de Québec-Université Laval, Québec); Peter Tugwell (University of Ottawa, Ottawa); Janique Gagnon (Children's Hospital of Eastern Ontario, Ottawa) Objectives: Juvenile idiopathic arthritis (JIA) is one of the most common causes of chronic musculoskeletal pain among youth, and can negatively impact all aspects of quality of life. However, pain is often under-recognized and pain management options are often not discussed thoroughly in clinical practice. To address the complexity of pain management options and challenges for youth to engage in in-depth discussions about these options, we propose to develop a decision support intervention for adolescents with JIA and their parents.

Methods: The intervention is being developed using a stepped approach. First, a systematic review was conducted to summarize the evidence for benefits and risks of pain management options in JIA. Second, a consensus meeting of rheumatology experts $(n=13)$, young adults with JIA $(n=2)$ and parents $(\mathrm{n}=1)$, as well as a follow-up Delphi survey were conducted to gain consensus on the elements to include in the decision support intervention. Third, focus groups with adolescents with JIA $(n=5)$ and their parents $(n=$ 3) were conducted to elucidate user profiles for this intervention. The next step consists in interviews of youth, parents and health providers to assess decision-making needs.

Results: A total of 22 randomized controlled trials (RCTs) were included. RCTs were found for massage, splints and orthoses, therapeutic exercises, psychosocial modalities, disease-modifying anti-rheumatic drugs, and

Personal non-commercial use only. The Journal of Rheumatology Copyright @ 2018 . All rights reserved. 
biologics. Both pharmacological and non-pharmacological pain management options were effective to varying degrees. Participants in the consensus meeting and the Delphi survey agreed that recent evidence for benefits, risks and inconvenience of pharmacological and non-pharmacological pain management options for JIA (and for pediatric chronic musculoskeletal pain and adult rheumatoid arthritis) should be presented in a user-friendly manner in the intervention. They also agreed to include an exercise to clarify adolescents' and parents' values and preferences. Participants involved in all phases of the project felt that the preferred format consisted of an electronic decision aid that could be used on a computer, tablet or smartphone periodically, and that could be discussed with health providers trained in decision coaching. Conclusion: The first phases of this research project have shown that a wide variety of treatment options can be effective and safe in reducing pain in JIA. Recent evidence for these pain management options, as well as a value clarification exercise, should be included in an electronic decision support intervention and discussed with trained health providers. Future steps include in-depth interviews to develop the intervention, as well as acceptability and usability testing.

30

Peer-Developed E-Learning Resource Can Address Rheumatology Knowledge Gaps in Junior Learners

Larissa Petriw (Queen's University, Kingston); Tabitha Kung (Queen's University, Kingston); Mala Joneja (Queen's University, Kingston)

Objectives: The symptoms related to Rheumatologic diseases are responsible for one third of visits to general practitioners, yet medical students and residents remain inadequately prepared to approach and manage Rheumatologic problems. There are few resources available to junior learners in Rheumatology; none combine clinical images, interactivity and case-based learning in an electronic format. Electronic learning (e-learning) provides a flexible medium for information presentation, multimedia and self-assessment in a mobile, searchable, user-friendly platform. Evidence suggests that peer-generated e-learning adds value, though most residents do not have extensive software development expertise. Here we present an interactive, peer-developed case-based Rheumatology e-book aimed to address these gaps.

Methods: Our e-book was written in the free program "iBooks Author" by Internal Medicine residents. The target audience was medical students and residents completing a rotation in Rheumatology. Key learning objectives were identified as the main sections of the e-book, including approaches to common rheumatologic presentations, case presentations of conditions commonly encountered over a rotation, and appendices (approaches to serology, medications and joint aspiration). Clinical images and self-assessments add interactivity. The e-book was evaluated by learners rotating through Rheumatology at Queen's University, with pre- and post-rotation surveys.

Results: The survey showed high satisfaction with the resource and improvement in learners' knowledge post-intervention. A total of 19 learners were surveyed; 14/19 reported using the e-book. All learners who used the resource agree the e-book is user-friendly (67\% strongly agree), well organized (56\% strongly agree), and has an appropriate level of material and cases (67\% strongly agree). All participants found the e-book useful for rotation preparation (56\% strongly agree, $44 \%$ agree) and would recommend it to a colleague (69\% agree, $31 \%$ strongly agree). All participants improved their comfort with Rheumatology topics on a Likert scale pre- and post-assessment. Pre-intervention global comfort level was 2.4/4 and post-intervention was $2.9 / 4$, with similar trends of improvement in the specific topics surveyed. The e-book itself was free and easy to create without significant additional training.

Conclusion: We present the first peer-developed e-learning resource of its kind in Rheumatology, aimed as an effective rotation resource for learners across the country. Although content writing remains labour-intensive, the iBook modality allows easy updating and minimal software knowledge by the writers. Conversion to a cross-platform format is possible for further distribution across a broader learner base. The e-book structure is adaptable to other medical education topics and is a user-friendly method of e-publication for busy residents interested in medical education.

\section{1}

Self-Perceived Risk of Cardiovascular Comorbidity Among Individuals Living with Rheumatoid Arthritis is Low: Results of a Systematic Literature Review

Olivia Ghosh-Swaby (Schulich School of Medicine and Dentistry, Western University, London); Bindee Kuriya (Sinai Health System, University of Toronto, Toronto)

Objectives: Individuals with rheumatoid arthritis (RA) are at an increased risk of developing cardiovascular disease (CVD). Patient perception of CVD in RA is not routinely evaluated. However, assessing awareness is an important first step to help tailor educational resources and treatment approaches to lower CVD risk. Our objective was to perform a systematic literature review evaluating awareness and perceived risk of CVD among individuals with RA.

Methods: Three electronic databases (Medline, EMBASE and Pubmed) were searched for English-language articles or abstracts between the years of 1990-2017. The main search terms and keywords pertained to RA, CVD, knowledge, perceptions and risk. Abstracts were screened for inclusion and selected independently by two reviewers.

Results: A total of 28 abstracts were screened and 12 underwent full text review. Three were interview-based studies (sample sizes of 14, 15 and111 patients) and 4 were questionnaire-based (sample sizes ranging between 113 and 208). Participants were predominantly female (77.5\%), mean age 55 years ( \pm 10 years), and almost half had post-secondary education $(42 \%)$. Forty percent of patients had a prior or current history of hypertension and/or high cholesterol. Those with the highest CVD risk (ascertained by the Framingham Risk Score) most frequently underestimated their risk as low, ranging between $56 \%$ to $96 \%$ of participants. The presence of any CVD risk factor (hypertension, high cholesterol, diabetes, obesity and smoking) was more common among patients who underestimated their CVD risk. Those with self-reported awareness about CVD or taking CVD medications (anti-hypertensive, lipid lowering agents) accurately perceived their risk as moderate or high compared to their validated risk score. The greatest gaps in knowledge were surrounding the effects of NSAIDs and exercise on CVD development. At the time of diagnosis, patients reported a preference to focus on control of their RA, rather than be concerned with CVD.

Conclusion: Individuals with RA report low perceived risk about CVD, despite a high frequency of traditional CVD risk factors. Efforts to understand the system-level, physician-level and patient-level barriers preventing optimal awareness of this comorbidity are needed. Only then, will interventions to improve CVD screening and management in RA be impactful and sustainable.

\section{2}

Process Evaluation of Employment and Arthritis: Making it Work, an Online Program to Help People with Inflammatory Arthritis Remain Employed

Kathy Tran (Simon Fraser University, Burnaby, Arthritis Research Canada, Richmond); Xi Xiang (Arthritis Research Canada, Richmond); Xiang Seah (Arthritis Research Canada, Vancouver); Brendan van As (Arthritis Research Canada, Richmond); Pam Rogers (Arthritis Research Canada, Richmond); Catherine Backman (Rehab Sciences/Occupational Therapy, University of British Columbia, Richmond); Monique Gignac (University of Toronto/ Institute for Work and Health, Toronto); Linda Li (Rehab Sciences/Physical Therapy, University of British Columbia, Arthritis Research Canada, Richmond); John Esdaile (University of British Columbia (Division of Rheumatology)/Arthritis Research Canada, Richmond); Diane Lacaille (University of British Columbia (Division of Rheumatology)/ Arthritis Research Canada, Richmond)

Objectives: Inflammatory arthritis (IA) commonly affects people's ability to work, yet few arthritis services exist addressing employment. We report on the process evaluation of the Making it Work program, an online 
self-management program to help people with IA deal with employment issues, performed in the context of a RCT testing its effectiveness at preventing work disability and improving at-work productivity.

Methods: Participants, recruited from rheumatology practices, a consumer organization (Arthritis Consumer Experts) and advertisements, were eligible if they had IA, were currently employed, aged 18-59, concerned about their ability to work. The program consists of five e-learning modules; five on-line group meetings using video conferencing led by a vocational rehabilitation counsellor (VRC); individual consults with an occupational therapist (OT) for an ergonomic work assessment and with a VRC.

Results: All program participants were included $(\mathrm{N}=278)$ [80\% female, mean (SD) age: 45(10) years, RA:52\%, AS:19\%, PsA:15\%, SLE:14\%]. Group meetings were successfully conducted online. Overall, participation was good. Median $(25 ; 75 \mathrm{Q})$ no. group meetings attended: $4(3 ; 5)$, with highest attendance in the first $(82 \%)$ and lowest in the fourth $(63 \%)$ meetings. Reasons for not attending included work obligations, health issues, family commitments, or other time constraints. $90 \%$ and $89 \%$ met with the OT and VRC. Completion of e-learning modules [Median(25;75Q) \% of total slides viewed] ranged from $49(0-100) \%$ for module 5 , to $100(10 ; 100) \%$ for modules $1 \& 2$. Not all content is relevant to all participants, depending on disease and job characteristics. All participants Jan-Dec 2016 completed a feedback survey post program $(n=69)$. Overall, participants were highly satisfied with the program. $94 \%$ would recommend it to others. Median [25Q;75Q] usefulness ratings $(0-10,0=$ not at all, $10=$ very useful $)$ were: 8 $[7 ; 10]$ for online modules; 9 [7.5;10] for group meetings; 8 [7;10] each for ergonomic \& VRC assessments. Median time to complete each module was $60 \mathrm{~min}$. Satisfaction with online group meetings was high: $93 \%$ were satisfied with the facilitation; $87 \%$ with group dynamic; $84 \%$ were comfortable with the online format. Median [25Q;75Q] ratings (1-10) for ability to follow group discussion: 10 [9;10]; getting to know other participants: 7 [7;10]; feeling listened to and understood: 9 [8;10]; feeling that the group was supportive: $9[7 ; 10]$.

Conclusion: Overall, online delivery of the Making it Work program was feasible and participants were highly satisfied. Our study demonstrates that self-management programs can be successfully delivered using an online format, which facilitates wider dissemination, greater convenience to patients, and lower costs.

\section{3}

A Review of Musculoskeletal Ultrasound Educational Programs in Rheumatology: Are these Based on Educational Theory?

Kamran Shaikh (Queen's University, Kingston); Mala Joneja (Queen's University, Kingston)

Objectives: Musculoskeletal ultrasound (MSUS) is increasingly used by rheumatologists to enhance their ability to diagnose and treatment rheumatic disease. Acquiring competency in MSUS is a procedural skill that may be enhanced by use of educational theories or frameworks. The goal of this paper is to review the literature describing educational curricula and teaching programs for MSUS, and establish whether any educational theories or frameworks were used to generate these programs.

Methods: We reviewed 6 databases (MEDLINE, EMBASE, Cochrane, CINAHL, ERIC, and Education Source Complete). We searched grey literature for guidelines for MSUS education based on the initial literature search results. We assessed full texts of the relevant articles to identify the educational programs, theories and frameworks utilised.

Results: 174 unique citations were identified with 67 being potentially relevant. There were several educational curricula to learn MSUS in the literature, of which only one explicitly used adult-learning theory. Numerous teaching modalities exist including training courses, electronic learning, self-directed learning, supervised/mentorship, specimen-based, multi-disciplinary and mixed approaches. None of the literature describing these modalities explicitly used educational theory as a basis for development of the teaching program.

Conclusion: There are several educational modalities with evidence for their use in teaching MSUS. Lack of standardized implementation of modalities and of outcome assessments preclude more detailed analysis as to the most effective MSUS educational techniques. There is a gap between theory and practice as few curricula are based on educational theories related to procedural skill acquisition. Consideration of educational theories may enhance effectiveness of educational programs to teach MSUS.

\section{4}

Musculoskeletal Point of Care Ultrasonography (POCUS) Training Among Canadian Post-Graduate Rheumatology Programs

Tenneille Tana (University of Ottawa, Ottawa); Noura Al-Osaimi (University of Ottawa, Ottawa); Johannes Roth (Children's Hospital of Eastern Ontario, Ottawa); Sibel Aydin (University of Ottawa, Ottawa Hospital Research Institute, Ottawa); Sue Humphrey-Murto (University of Ottawa, Ottawa)

Objectives: Over the years, there has been a growing interest in the use of point of care ultrasonography (POCUS). Despite increasing evidence demonstrating the clear benefits of POCUS in the clinical practice of rheumatology, uptake has been limited by Canadian Rheumatologists. Lack of training has been identified as a potential barrier. The purpose of this study, therefore, was to assess the current state of musculoskeletal POCUS training amongst the rheumatology post-graduate programs in Canada.

Methods: The survey was developed by three academic Rheumatologists and one trainee. Across Canada, all 15 Adult and 3 Pediatric Rheumatology post-graduate programs were surveyed via Survey Monkey.

Results: The completed response rates were 33\% (29/89) for PGY-4/5 rheumatology trainees and 39\% (7/18) for Program Directors (PD), where three and two respondents were from the Francophone programs in each category, respectively. Twenty-seven (93\%) residents and 6 (86\%) PD agreed or strongly agreed that POCUS is an important clinical tool in rheumatology. Eighty-three percent of residents and $62.5 \%$ of PD felt that POCUS should be a formal part of the rheumatology training. Thirteen $(45 \%)$ of the residents had access to a POCUS machine for use in a clinical setting; however, this access was limited to one clinic room in a limited number of ambulatory clinics, and no exposure was available within community rotations. Identified barriers included lack of expertise, standardized curriculums, POCUS machine, as well as the expense associated with external courses. Despite this, the majority (66\%) of residents plan to use POCUS in their future practice. To achieve their goals, residents considered the Canadian Rheumatology Ultrasound Society (CRUS) course, joint training amongst programs, and even initiating their own training program with experienced staff. Over $93 \%$ of resident responders and $100 \%$ of PD would be interested in a standardized musculoskeletal POCUS curriculum.

Conclusion: A National survey reveals musculoskeletal POCUS is considered important for clinical practice, yet barriers to training exist, including limited access to US machines (less than half had access), trained faculty, and a standardized curriculum. However, most residents in training still plan to use POCUS in their future practice. It is clear that both residents and PD felt POCUS should be a formal part of training and would be interested in a national standardized curriculum. These study conclusions are limited by the low response rate, and potential bias of respondents. Despite this, there appears to be considerable support for developing a standardized national POCUS curriculum in Canada.

35

Video-based Exercise Instruction Tool is Feasible, Acceptable, and May Increase Adherence to Home Exercise in JIA

Nikola Wilk (Montreal); Alexandra Sirois (McGill University, Montreal); Roya Taheri (MUHC Research Institute, Montreal); Sophie Laniel (Montreal Children's Hospital, Montreal); Michele Geoffrion (Montreal Children's Hospital, Montreal); Susan Bartlett (McGill University, Montreal); Claire LeBlanc (Montreal Children's Hospital, Montreal)

Objectives: Following JIA flares, regular rehabilitation exercises help increase joint range of motion $(\mathrm{ROM}) \&$ reduce pain. Typically JIA patients receive one $\mathrm{OT} / \mathrm{PT}$ exercise instruction session during a comprehensive clinic visit. We hypothesized online access to a video exercise program (VEP) specifically tailored to JIA patient needs would increase proper

Personal non-commercial use only. The Journal of Rheumatology Copyright @ 2018. All rights reserved. 
technique \& adherence to home exercises. Our pilot study (PARES I) compared acceptability \& use of English / French VEP with sketch diagrams on selected outcomes. Here we present results of exit interviews conducted with those patients \& parents.

Methods: We developed 50 VEP for 5 commonly affected joints in PARES I. Patients (1-17.9 yrs) undergoing standard OT/PT rehabilitation were randomized to videos posted on our hospital website or paper sketches for home use. Upon completion of PARES I, families were invited to participate in an optional exit interview conducted by two research assistants. Families who had been assigned sketch diagrams were asked to view the equivalent VEP before the interview. We explored perceptions of using VEP vs. sketches regarding proper technique, interest $\&$ adherence to exercise. Feedback \& preferences on video content \& quality was also queried. Interviews were audio recorded \& responses were summarized.

Results: 7 (4 sketch, 3 video) of 17 families (47\%) participated in exit interviews. 4 were parent-interviews $(3 \mathrm{~F}, 1 \mathrm{M})$; others were with adolescents. All patients were female (mean $8.6 \mathrm{yrs}$ ); $86 \%$ had 1 affected joint. Almost all $(86 \%)$ used home-based tools at least several times. Many (57\%) reported frequent difficulty remembering proper exercise-techniques; all believed having a tool to refer to at home was important. Compared with sketches, VEP was unanimously selected the preferred tool. While all acknowledged importance of adherence, given the option 'easy or difficult', most (71\%) reported it being at least somewhat difficult to do exercises daily. While some $(43 \%)$ believed VEP would increase adherence, three families acknowledged viewing multiple videos for different joints might increase nonadherence. Videos were judged the right length, with clear, easy to follow, age-appropriate instructions. Using children in videos of similar ages to patients was cited a strength. Suggestions included the addition of background music \& dialogue to motivate the completion of each exercise. Conclusion: Short video-based exercises were feasible \& acceptable to families of children with JIA, \& facilitated proper technique \& potentially adherence. Next steps involve refining \& expanding the exercise video bank $\&$ evaluating their use over time to improve JIA patient-outcomes.

\section{6}

\section{A Retrospective on the UCBeyond ${ }^{\circledR}$ Scholarship Program}

Michael Starr (McGill University Health Centre, Montreal); Liam Martin (University of Calgary, Calgary); Najma Aryan (UCB Canada Inc., Oakville) Objectives: Students with rheumatic diseases often have extra financial burdens when pursuing post-secondary education due to the high cost of their medical care. Recognizing this need UCB, through the one-of-a-kind UCBeyond ${ }^{\circledR}$ scholarship program, has offered financial support for students with systemic rheumatic diseases who are pursuing post-secondary education in Canada from 2011 to 2016. In total, 68 students have received UCBeyond ${ }^{\circledR}$ Scholarships. To show the importance and impact of this unique Scholarship, intended solely for Canadian students, we analyzed data from the top 147 applicants including demographics, diagnoses, and academic endeavors. Additionally, we conducted an in-depth interview with 3 award winners to gain further insight on how these scholarships have improved and impacted students' lives, education, and future plans.

Methods: Data was collected from UCBeyond ${ }^{\circledR}$ scholarship submissions of the top 30 applicants per year from 2011 to 2016 (omit 2015, data was lost), and from interviews with 3 past winners K.C., S.R., and S.D. All information was collected with the applicant's consent.

Results: Data were analyzed from 147 candidates. There were $123(84 \%)$ female and $24(16 \%)$ male applicants. Of the applicants studying Sciences (76), $43 \%$ (33) plan to pursue a career in healthcare, and $14 \%$ (11) plan to pursue a career in Rheumatology. In their submissions, applicants frequently emphasized the social value that the scholarship provides, in that it allows them to dedicate more time towards health management, education, and sports \& activities. We interviewed three prior UCBeyond ${ }^{\circledR}$ winners; K.C., S.R., and S.D. who shared their insights about their individual journeys as both patients and students. By evaluating these three cases, the impact of UCBeyond on each applicant's lives, futures, and education are highlighted. Conclusion: The UCBeyond Scholarship has touched the lives of a number of students. Post-secondary education is an expensive endeavor for any student. For a student with a systemic rheumatic disease, these expenses are much greater. The UCBeyond ${ }^{\circledR}$ scholarship is unique, and the effect of this investment in students living with inflammatory arthritic diseases is evident in our analysis of the top 147 candidates. The UCBeyond ${ }^{\circledR}$ Scholarship Program has successfully allocated approximately $\$ 765,000$ to Canadian students, thus helping these recipients pursue their academic dreams, and the impact of this has been highlighted by interviews with three of the past winners. Based on the feedback of previous recipients it is evident that this scholarship has made a substantial impact on the lives of students living with rheumatic diseases.

\section{7}

\section{Perspectives of Primary Care Providers Regarding Effective} Rheumatologic Consultation Notes

Neha Puri (University of Toronto, Toronto); Karishma Ramjee (University of Toronto, Toronto); Dana Jerome (Women's College Hospital, Toronto); Natasha Gakhal (Women's College Hospital, Toronto)

Objectives: Deficits in communication between primary care providers (PCPs) and specialists has been associated with a variety of adverse patient outcomes. Unsuccessful communication has been attributed to a lack of both teaching and standardization in regards to the consultation process. However, limited research exists on the characteristics of effective consultation notes (CNs). This gap in knowledge is important because multiple studies suggest that the quality of CNs can be improved by using standardized formats. Therefore, the goal of this study is to determine what PCPs referring patients to an academic outpatient rheumatology department believe to be important in CNs and, more specifically, their opinion of the current CNs.

Methods: PCPs who referred a new patient to the rheumatology department were invited to complete an anonymous survey. All PCPs to be included in the study were identified through the hospital's EMR and their contact information was obtained through the CPSO website. The participating PCPs were sent the following: 1 ) a cover letter explaining the purpose of the study 2) a one-page semi-structured survey 3) a copy of their patient's CN. Completed surveys were collected via fax. Completing the survey implied consent and participation was voluntary.

Results: A total of 15 responses were received (12\% response rate). $100 \%$ of PCPs agreed that their question/concern was addressed and that the management plan was made clear in the current $\mathrm{CN} .47 \%$ wanted additional information about medications and patient self-management. $100 \%$ agreed that information about the prognosis, long-term plan and steps for follow up is important to include. Furthermore, 93\% agreed that a rationale for diagnosis and information about management and medication recommendations is also important to include. $100 \%$ of the PCPs rated the overall quality of the consult as either good or excellent. Finally, PCPs recommended the following: separation of the plan and impression sections, inclusion of important diagnostic criteria, and creation of a "PCP action items list".

Conclusion: In general it appears that the current CNs are successfully communicating a clear management plan and addressing the question/ concern posed by the PCP. Furthermore, the PCPs appear to be satisfied with the overall quality of the current $\mathrm{CN}$. However, the following could be clarified: patient's prognosis, long-term plan, medications, diagnostic criteria, follow up and PCP action items. Future work includes using this information to modify the department's current $\mathrm{CN}$ template and conduct a further study to determine if the new CNs better meet the needs of the PCPs.

\section{8}

Hip Health Information Project - What Do Patients Want?

Wendy Watson (Vancouver Coastal Health, Vancouver); William Miller (University of British Columbia, Vancouver); Morag Crocker (Vancouver Coastal Health, Vancouver); Marie Westby (Vancouver Coastal Health Research Institute, Vancouver); Colleen O’Melinn (University of British Columbia, Vancouver)

Objectives: The number of total hip replacements (THR) in Canada is 
growing steadily, increasing direct and indirect healthcare costs. Preoperative education reduces hospital costs, optimizes surgical outcomes, accelerates rehabilitation and reduces pre-operative anxiety. Education that aims to improve functional ability prior to surgery by advising on exercise is known as prehabilitation (prehab). Several prehab interventions are described in the literature yet few include patients' input on the design of the education such that it addresses patients' preferences for content, timing and mode of delivery, specifically eHealth (e.g., online) technologies. This research explores the experiences and prehab learning preferences of adults undergoing THR.

Methods: Focus groups and interviews were used to explore patients' views. Eligible individuals were either waiting for THR (both before and after existing prehab education) or had THR within Vancouver Coastal Health. Participants were invited to take part in 90-min focus groups that were audiorecorded and transcribed verbatim. Data were collected between September 2016 and September 2017. Transcripts were analyzed using descriptive statistics and then examined by two independent researchers using an inductive thematic analysis approach. Data were analyzed to identify common themes with respect to prehab educational content and delivery.

Results: At the time of submission, nine focus groups $(n=48)$ and four interviews have been completed. Data from three focus groups of patients $(n=13)$ waiting for THR who had prehab education were analyzed. Four themes emerged regarding the experiences and learning preferences of adults undergoing THR: 1) "Inconsistency" - patients report variable knowledge and levels of anxiety about THR; 2) "Engagement" - patients wish to be engaged with both healthcare professionals and other patients through group education and peer support; 3) "More information" - patients desire more information about recovery from THR, including information on milestones, rehabilitation, daily activities and post-surgery resources; and 4) "Supplemental E-health" - patients are interested in online prehab as a way of obtaining early education and additional resources only if it supplements interactions with healthcare professionals and is easy to use and access.

Conclusion: To date, patients preparing for THR report varied levels of preparedness before surgery and express interest in education that incorporates meaningful interaction, either in-person or through an eHealth platform. Exploring the experiences and learning preferences for prehab in adults undergoing THR will facilitate the provision of accessible, effective and potentially cost-efficient education and the development of an eHealth prehab education program: Hip Health Information Project (HHIP).

\section{9}

Factors Affecting Consideration of a Career in Rheumatology by Internal Medicine Residents

Mary-Clair Yelovich (McMaster University, Hamilton); Karen Beattie (McMaster University, Hamilton); Mark Matsos (McMaster University, Hamilton)

Objectives: To date, several studies have examined factors contributing to internal medicine (IM) residents choosing to pursue a career in Rheumatology. Of note is that exposure to Rheumatology has been reported to be a key factor. The purpose of this study was to determine from Canadian IM residents at varying levels of training i) what factors have contributed to their considering a career in Rheumatology, ii) at what level of training increased exposure seemed to relate most to consideration of a career in Rheumatology, and iii) what kind of exposure to Rheumatology they thought might be beneficial.

Methods: A survey with 10 questions was developed by a rheumatologist. Links to online surveys (SurveyMonkey) were sent electronically to 12 IM residency programs across Canada, who were asked to distribute surveys to IM residents by email. Anonymous responses were received over a 3-month period. Responses were collated and summarized.

Results: There were 54 participants, 21 (39\%) PGY-1s, 31 (57\%) PGY-2s, and 2 (4\%) PGY-3s. Of these, 76\% selected "interest in the field" as being most important to them when choosing to pursue a particular subspecialty of IM. 64\% indicated that they had considered a career in Rheumatology (> $50 \%$ on VAS). $52 \%$ indicated that increased knowledge would affect their decision to pursue a career in Rheumatology ( $>50 \%$ on VAS). Of residents who had considered a career in Rheumatology, 64.6\% characterized their exposure to rheumatology as $>50 \%$ on VAS and $61.3 \%$ of them had completed their first clinical rotation in Rheumatology in PGY1. In contrast, of those who has not considered a career in Rheumatology $(<50 \%$ on VAS), $44 \%$ characterized their exposure to rheumatology as $>50 \%$ on VAS and $44 \%$ had completed their first clinical rotation in Rheumatology in PGY1, while $39 \%$ reported having had no clinical experience of Rheumatology during their residency. Clinical exposure was identified by the residents as important to increase their Rheumatology knowledge.

Conclusion: Interest in the field appears to be the most significant factor influencing residents in choosing Rheumatology. Earlier exposure, ideally in PGY-1, seems to relate to residents considering a career in Rheumatology. Clinical exposure was identified by the residents as important to increase their Rheumatology knowledge.

\section{0}

Improvements in Bone Marrow Edema, Erosions, and Synovitis of the Feet in Early Rheumatoid Arthritis

Mary-Clair Yelovich (McMaster University, Hamilton); Hanyan Zou (McMaster University, Hamilton); Myriam Allen (Université Laval, Hamilton); Saara Totterman (QMetrics Technologies, Rochester); Karen Beattie (McMaster University, Hamilton); Maggie Larche (McMaster University, St Joseph's Healthcare Hamilton, Hamilton)

Objectives: The evolution of bone marrow edema (BME), erosions, and synovitis in Rheumatoid Arthritis (RA) in the feet has been poorly studied. In the hands, it has been suggested that type of therapy may affect progression of erosions; anti-TNF therapy has been associated with limited bone repair and disease modifying anti-rheumatic drugs (DMARD) like Methotrexate with erosion progression. We aimed to quantify changes in bone marrow edema, erosions, and synovitis over two years in the feet of patients with early RA, with choice of therapy for each patient also noted. Methods: Patients newly diagnosed with RA (treatment naïve at baseline, ACR criteria) were recruited. Consenting participants were assessed at baseline, 1-year, and $\geq 2$ years after diagnosis by a rheumatologist for swollen and tender 2nd-5th metatarsophalangeal joints (MTPs) both feet. The most clinically symptomatic foot chosen at baseline was scanned using a $1.0 \mathrm{~T}$ peripheral MRI at each visit. The 2nd-5th MTPs were scored by a radiologist blinded to clinical results semi-quantitatively for synovitis (grade 0-3, total /12), the base and the head of MTP bones for BME (grade 0-3, total /24), and erosions (grade 0-10, total /80) according to OMERACT-RAMRIS. Therapy received after baseline was noted. Descriptive statistics characterized changes in each outcome over 2 years (follow-up minus baseline) and paired t-tests tested significance of changes. Results: This study included 19 patients $[\mathrm{n}=18$ females, mean (SD) age 54.2 (8.1) years]. The mean (SD) number of swollen and tender joints at baseline were 1.1 (1.6) and $4.6(2.8)$, respectively, and improved to $0.8(1.8)$ and $2.2(2.7)$ at 2-years. The mean (SD) change in synovitis over 2-years was $-1.1(2.4)(\mathrm{p}=0.06)$. BME significantly improved after one year (change $=-2.7(3.9), \mathrm{p}=0.014)$ and 2-years (change $=-2.9(4.7)(\mathrm{p}=0.021)$, with the greatest improvement in the first year. Significant improvement in erosions were seen after 1 -year (change $=-0.9,(1.6), p=0.035)$ which were generally sustained over 2 -years (change $=-0.9,(2.1), \mathrm{p}=0.061)$. At baseline, all patients were treated with single or combination DMARDs. At 1 -year, 4 patients were switched to a biologic therapy while the remaining 15 patients remained on DMARDs.

Conclusion: Improvements in BME, erosions, and synovitis were noted in early RA patients over the first two years of therapy, with most notable improvement noted in BME.

41

Incidence and Prevalence of Rheumatoid Arthritis in Saskatchewan Bindu Nair (University of Saskatchewan, Saskatoon); Regina Taylor-Gjevre (University of Saskatchewan, Saskatoon); Liying Wu (Saskatchewan Health Quality Council, Saskatoon); Shan Jin (Saskatchewan Health Quality

Personal non-commercial use only. The Journal of Rheumatology Copyright (C 2018. All rights reserved. 
Council, Saskatoon); Jacqueline Quail (Saskatchewan Health Quality Council, Saskatoon)

Objectives: Rheumatoid arthritis (RA) is a chronic inflammatory and destructive arthritis. Understanding the incidence and prevalence of RA within the province facilitates appropriate health care resource planning. The objective of this study is to estimate the incidence and prevalence of RA over time for the overall Saskatchewan population, for specific age range categories, and for gender.

Methods: Saskatchewan Provincial Administrative Health Databases (2001-2014) were utilized as data sources. Two RA case-definitions were employed: 1) > three physician billing diagnoses, at least one of which was submitted by a specialist (rheumatologist, general internist or orthopedic surgeon) within two years; 2) > one hospitalization diagnosis (ICD-9-CM code-714, and ICD-10-CA code-M05). Data from these definitions were combined to identify incidence and prevalence RA cases. Using this data, annual incidence and prevalence rates were calculated for the provincial population, specified age range categories and gender categories.

Results: The number of RA cases meeting the case definition increased from 3731 to 6223 over the study period. The incidence of RA disease demonstrated variation within the study period, ranging from $0.07 \%$ to $0.03 \%$. The prevalence of RA increased over time from $0.5 \%$ in $2001-2002$ to $0.7 \%$ in 2014-2015. Both incidence and prevalence rates rose with increasing age. While women had higher incidence and prevalence rates compared to men, there was no difference in overall trends of the age adjusted rates for both groups.

Conclusion: In Saskatchewan, the overall prevalence of RA is rising while there has been a recent peak in the incidence. A growing awareness of the need to identify RA early in the disease may be one of the factors contributing to these results.

\section{2}

Measurement Properties of Mood and Anxiety Assessment Tools in Systemic Lupus Erythematosus: A Systematic Review

Zana Alai (University of Manitoba, Winnipeg); Christine Peschken (University of Manitoba, Winnipeg); Carol Hitchon (University of Manitoba, Winnipeg); Annaliese Tisseverasinghe (University of Manitoba, Winnipeg) Objectives: High rates of mood and anxiety disorders are reported in patients with Systemic lupus erythematosus (SLE). These psychiatric complications are commonly assessed using questionnaire-based instruments, some of which incorporate somatic features that may be indistinguishable from the organic manifestation of SLE. Studies evaluating the validity of these patient-reported outcomes in SLE are scarce. Thus, we sought to appraise the evidence for use of various questionnaire-based tools to screen and monitor for depression and anxiety in people with SLE.

Methods: A systematic review using four major databases (Medline, Embase, Cochrane, PsychInfo), supplemented with grey literature, was conducted with help of an academic librarian to identify all studies evaluating one or more measurement properties of any mood and/or anxiety tool in adults (>16 years) with SLE. Two reviewers independently selected the studies and evaluated each study and instrument. The methodological quality of reports was assessed using COSMIN and the quality of each tool with respect to its measurement properties in SLE was summarized as per Terwee et al.

Results: From 2135 unique hits, 12 studies evaluating various versions of commonly-used depression (BDI, CES-D, HADS-D, PROMIS-D) and anxiety (HADS-A, PROMIS-A) instruments were included. Most studies excluded patients with prior neuropsychiatric illness. Sample sizes ranged from 20 to 204, and mean ages, from 34 to 49 years. The HADS, PROMIS, and BDI scales demonstrated good internal consistency, while the latter two also showed good test-retest reliability (one study each). Each instrument was illustrated to have some convergent validity (e.g. with SLENQ, MOS, or SF-36 subscales) in at least one study. The BDI, CES-D and HADS-D demonstrated good criterion validity for mood disorder (versus DSM5-based interview) in one study each. No study evaluated responsiveness. For testing internal consistency, all studies had poor quality, while for the other properties, about twothirds of studies had fair methodological quality. None were of high quality.
Conclusion: This is the first study to systematically review the evidence for use of various measurement tools for assessing anxiety and depression in SLE. We found limited evidence of reliability and validity for some of the instruments and no data on responsiveness. The generalizability of available data is limited by exclusion of patients with neuropsychiatric history, and paucity of confirmatory studies. Further assessment of measurement properties of these tools, especially responsiveness, in more inclusive SLE populations is needed to allow for better design and interpretation of studies of psychiatric comorbidity in this vulnerable population.

\section{3}

Correlation between Antibodies to the Phosphotidylserine/Prothrombin Complex (aPS/PT) and Anti-beta2glycoprotein-1-domain 1 (anti-beta2GP1-D1) and Vascular Thrombosis (VT) and/or Pregnancy Morbidity (PM)

Tania Pannu (University of Calgary, Calgary); Marvin Fritzler (University of Calgary, Calgary); Michelle Jung (University of Calgary, Calgary); Claire Barber (University of Calgary, Calgary); Yvan St. Pierre (McGill University, Montreal); Ann Clarke (University of Calgary, Calgary)

Objectives: aPS/PT is considered to be a risk factor for vascular thrombosis (VT) and/or pregnancy morbidity (PM). Anti-beta2GP1-D1 is potentially superior to anti-beta2GP1 in predicting VT/PM. We examined the correlation between anti-aPS/PT and anti-beta2GP1-D1 and the laboratory criteria for antiphospholipid antibody syndrome (APS): lupus anticoagulant (LAC), anticardiolipin (aCL), and anti-beta2GP1 and their association with VT/PM. Methods: Patients fulfilling the ACR or SLICC Criteria for SLE were enrolled in the University of Calgary Lupus Cohort. Serum from each patient was analyzed for aPS/PT (IgG/IgM) by ELISA (QUANTA Lite, Inova Diagnostics), anti-beta2GP1-D1 by chemiluminescence immunoassay (QUANTA Flash, Inova), LAC using tissue thromboplastin inhibition test and dRVVT, and aCL (IgG) and anti-beta2GP1 (IgG) by ELISA. VT/PM was based on patient self-report and confirmed by medical chart review. VT included arterial, venous, or small vessel thrombosis; PM included one fetal death $>10$ weeks, one premature birth $<34$ weeks or $>3$ spontaneous abortions $<10$ weeks. The Spearman correlation between aPS/PT and anti-beta2GP1-D1 and the conventional AP autoantibodies and between each autoantibody and VT/PM was calculated. The association between number of autoantibodies and VT/PM was assessed using univariate logistic regression. Results: 202 patients were included [91.7\% female; mean age at diagnosis 34.9 years, (SD 15.0); mean disease duration 15.1 years, (SD 11.8)). The \% of patients ever positive for aPS/PT, anti-beta2GP1-D1, LAC, aCL, and anti-beta2GP1 were $37.6,5.9,13.3,11.5$ and 12.1 , respectively. $32.2 \%$ had one VT/PM (VT 29.1\%, PM 4.0\%). The correlation (95\% CI) between IgG aPS/PT and LAC, aCL, anti-beta2GP1, and anti-beta2GP1-D1 was 0.54 $(0.44,0.64), 0.40(0.27,0.51), 0.43(0.30,0.54)$ and $0.29(0.14,0.42)$, respectively. The correlation between IgM aPS/PT and LAC, aCL, anti-beta2GP1 and anti-beta2GP1-D1 was $0.43(0.31,0.54), 0.16(0.02,0.29), 0.25(0.11$, $0.37)$ and $0.24(0.09,0.37)$. The correlation between anti-beta2GP1-D1 and LAC, aCL and anti-beta2GP1 was $0.28(0.14,0.42), 0.55(0.43,0.64)$ and $0.61(0.50,0.69)$. The correlation between $\mathrm{IgG} \mathrm{aPS} / \mathrm{PT}, \mathrm{IgM} \mathrm{aPS} / \mathrm{PT}$, anti-beta2GP1-D1, LAC, aCL, and anti-beta2GP1 and VT/PM was 0.17 $(0.03,0.30), 0.05(-0.09,0.19), 0.21(0.06,0.35), 0.22(0.08,0.35), 0.22$ $(0.08,0.35)$ and $0.24(0.10,0.37)$, respectively. The odds $(95 \% \mathrm{CI})$ of VT/PM increased incrementally by $46 \%$ for each additional autoantibody $(1.15,1.86)$

Conclusion: IgG aPS/PT and anti-beta2GP1-D1 are highly correlated with other AP antibodies, which along with the accepted laboratory criteria of APS, are all similarly correlated with VT/PM. IgG aPS/PT and anti-beta2GP1-D1 should be considered as criteria for APS

44

A Great Mimicker: Pitfalls for Rheumatologists in the Diagnosis of Whipple's Disease

Martha Decker (University of Alberta, Edmonton); Stan Houston (University of Alberta, Edmonton); Julinor Bacani (University of Alberta, Edmonton); 
Carrie Ye (Division of Rheumatology, University of Alberta, Edmonton) Introduction: Whipple's disease is a systemic infectious disease caused by Tropheryma whipplei involving arthralgias, malabsorption, diarrhea, and weight loss. Articular and constitutional symptoms are common and may precede other symptoms by several years.

Objectives: Review a case of Whipple's disease which presented as fever of unknown origin (FUO) mimicking malignancy and large vessel vasculitis (LVV), puzzling multiple specialists for several years.

Methods: After obtaining patient consent, we performed a chart review. Case: A 58 year-old woman with a 38-year history of refractory epilepsy, resulting in temporal lobectomy, was referred as an outpatient to our rheumatology clinic for 5 years of FUO and arthralgias after investigations for malignancy and infection were negative. A CT scan of the abdomen showed extensive low-attenuation mesenteric and retroperitoneal lymphadenopathy. Two separate excisional biopsies of mesenteric and inguinal lymph nodes showed no malignancy or infection. A $4.3 \mathrm{~cm}$ ascending aortic aneurysm was seen on ultrasound and CT scan (without cardiovascular risk factors) and a diagnosis of large LVV was considered.

The fevers were associated with increased seizures and repeated hospital visits; a trial of prednisone for presumed LVV was initiated while awaiting PET scan. The fevers resolved on oral prednisone $30 \mathrm{mg}$ daily, but recurred with tapering doses. Prednisone was discontinued after PET scan failed to show vasculitis. CT-angiogram was negative for aortic aneurysm and on reassessment of previous imaging, the finding of a possible aortic aneurysm was due to artifact from adjacent lymphadenopathy.

Repeat core biopsy of a mesenteric lymph node revealed non-necrotizing granulomatous inflammation and prominent histiocytes with periodic acid-Schiff (PAS)-positive intracellular material. Electron microscopy showed intra- and extra-cellular bacilli with a thick cell wall and triple-layered envelope, consistent with T. whipplei. Review of previous lymph node biopsies indicated the diagnosis was missed. PCR for T. whipplei was positive from both lymph node and CSF, confirming a diagnosis of Whipple's disease. Atypically, duodenal biopsy was negative for Whipple's disease. She was treated with ceftriaxone for two weeks followed by long-term combination doxycycline and hydroxychloroquine. The patient's epileptic control improved on therapy raising the suspicion that the seizure disorder was due to Whipple's disease.

Conclusion: Rheumatologists need to have a high index of suspicion for Whipple's disease in patients with FUO and joint symptoms. Potential pitfalls in diagnostic error include absence of typical abdominal symptoms, short-term response to prednisone therapy, potential mimickers of aneurysm on imaging and the need for pathologist-led specialized testing of tissue.

\section{5}

Risk of Inflammatory Bowel Disease in Patients with Psoriasis/Psoriatic Arthritis: A Population-Based Study

Bahar Moghaddam (University of British Columbia, Vancouver); Noren Khamis (University of British Columbia, Vancouver); Hamid Tavakoli (Arthritis Research Canada, Richmond); Antonio Avina-Zubieta (University of British Columbia Faculty of Medicine; Arthritis Research Canada, Richmond)

Objectives: Inflammatory bowel disease (IBD) and Psoriasis/Psoriatic Arthritis (PsO/PsA) are known to overlap in certain pathogenic pathways. Studies assessing the association of IBD and PsO/PsA are scarce. Our objective was to determine the risk of IBD (i.e. Crohn's Disease (CD) or Ulcerative Colitis (UC)) in patients with newly diagnosed $\mathrm{PsO} / \mathrm{PsA}$.

Methods: We used an administrative database from the province of British Columbia to conduct a retrospective matched cohort study (January1st, 1996 to December 31st, 2013) among patients meeting at least one of the following criteria: a) one diagnostic code for PsO/PsA by a rheumatologist or dermatologist; b) $\geq 2$ diagnostic codes for PsO/PsA more than 2 months apart in a two-year period by a non-rheumatologist or dermatologist physician; or c) $\geq 1$ hospitalization with diagnostic code for $\mathrm{PsO} / \mathrm{PsA}$. We ensured selection of incident cases by allotting a 5 -year run in period (i.e. no diagnosis of PsO/PsA prior to cohort entry time). We randomly selected age and gender matched controls from the general population for every identified case (non-PsO/PsA cohort). Our outcome of interest was a new diagnosis of IBD during follow up (CD or UC) using a validated algorithm. We performed Cox proportional hazard regression models adjusting for age, sex, socioeconomic status, Charlson's comorbidity index, hospitalizations and previous history of appendectomies.

Results: Among 83,924 newly diagnosed individuals with PsO/PsA and an equal number of controls, we identified 430 cases of IBD ( 228 cases of UC, 202 cases of $\mathrm{CD}$ ) in the $\mathrm{PsO} / \mathrm{PsA}$ cohort as compared to 247 cases ( 140 cases of $\mathrm{UC}, 107$ cases of $\mathrm{CD}$ ) in the non-PsO/PsA cohort. The corresponding incidence rate of IBD in the $\mathrm{PsO} / \mathrm{PsA}$ cohort was 67.3 per 100,000 personyear (95\% CI, 61.2-73.9), nearly two-fold higher than in the non-PsO/PsA (37.1 per 100,00 person-year (95\% CI, 32.3-42.7)). The age-, sex- and entry-matched hazard ratio (HR) for IBD in patients with PsO/PsA was 2.48 (95\% CI, 2.10-2.92). The fully adjusted model remained statistically significant $[\mathrm{HR}=2.42(95 \% \mathrm{CI}, 2.05-2.85)]$. The overall prevalence of IBD at the end of follow-up time was 7.62 person-years (PY)(standard deviation (SD) 4.58 PY) in the PsO/PsA cohort and 8.18 PY (SD 4.61 PY) in non-PsO/PsA cohort.

Conclusion: This general population-based study shows that patients with $\mathrm{PsO} / \mathrm{PsA}$ have a two-fold increased risk of IBD. This association suggest that having one inflammatory condition increases the risk of developing another inflammatory disease affecting other organs. The role of treatment or shared risk factors for IBD among PsO/PsA deserves further research.

\section{6}

Rituximab for Treatment of Refractory Lupus Enteritis: A Case-Report and Review of Literature

Bahar Moghaddam (University of British Columbia, Vancouver); Neda Amiri (Division of Rheumatology, University of British Columbia, Vancouver); Jennifer Reynolds (University of British Columbia, Department of Medicine, Division of Rheumatology, Vancouver)

Objective: Lupus enteritis is an uncommon and potentially life threatening manifestation of Systemic Lupus Erythematosus (SLE). While corticosteroids and non-biologic disease modifying anti-rheumatic drugs (DMARDs) are used for treatment, the role of biologics in management of refractory lupus enteritis is not well studied. Our objective is to report a case of DMARD refractory lupus enteritis with clinical response to rituximab.

Methods: A case report and literature review for treatment of lupus enteritis are presented.

Results: A 38 year-old, nulliparous, woman with a history of SLE was evaluated for recurrent abdominal pain, nausea and vomiting. She was diagnosed with SLE at the age of 24 with serology positive for ANA, anti-Smith, anti-RNA, anti-SSA and disease concordant anti-dsDNA levels. Clinical manifestations had included malar rash, arthritis, leukopenia, non-hemolytic anemia, and class II lupus nephritis. Medications at the time of evaluation included mycophenolate mofetil (MMF) and hydroxychloroquine. Over the last two years, she had had five hospital admissions for similar gastrointestinal symptoms. During her first admission, a diagnosis of lupus enteritis of the superior mesenteric artery (SMA) territory had been made based on thickened appearance of small bowel loops on CT scan of the abdomen. Colonoscopy was unremarkable with benign biopsies. During each admission, her symptoms abated with prednisone $1 \mathrm{mg} / \mathrm{kg}$ and outpatient escalation of DMARD therapy, with initial dose increase of azathioprine, then subsequent use of MMF

In the last six months prior to our evaluation however, her symptoms recurred with prednisone taper despite maximal MMF dosing. Repeat imaging of the abdomen showed dilated small bowel loops in the distal segment with terminal ileum involvement. Retrospective review of all previous abdominal imaging revealed that different portions of the bowel - extending beyond the SMA territory- had demonstrated thickening and "target sign" appearance with each presentation. She did not respond to IV pulse steroids and was admitted to the ICU. A subsequent CT scan showed ongoing bowel inflammation, worsening ascites, pleural effusions, a pulmonary embolism, and left renal vein thrombus. Given the patient's wish

Personal non-commercial use only. The Journal of Rheumatology Copyright @ 2018. All rights reserved. 
to preserve fertility, we chose to add rituximab rather than cyclophosphamide. The patient continues to do well with no relapse and a slow taper of prednisone.

Conclusion: To our knowledge, this is the third case report of rituximab used for treatment of DMARD refractory lupus enteritis. Though randomized controls of rituximab in SLE failed to meet primary endpoints, there may be potential for its use in lupus enteritis.

47

Drug-Related Retinal Toxicity in SLE

Elvis-Raymond Mukwikwi (University of Montreal, Montreal); Christian Pineau (Montreal General Hospital, Division of Rheumatology, Montreal); Evelyne Vinet (McGill University Health Centre, Montreal); Ann Clarke (University of Calgary, Calgary); Emil Nashi (McGill University Health Centre, Montreal); Fares Kalache (Montreal General Hospital, Division of Rheumatology, Montreal); Louis-Pierre Grenier (McGill University Health Centre, Montreal); Sasha Bernatsky (McGill University Health Centre, McGill University, Montreal)

Objectives: Hydroxychloroquine (HCQ) and chloroquine (CQ) are important in the treatment of systemic lupus erythematous (SLE) and other diseases. Retinal toxicity remains the most concerning, irreversible complication associated with these agents. Our aim was to study factors potentially associated with retinal toxicity within our SLE clinic cohort, using case-control analyses.

Methods: We reviewed data from the McGill University Health Center Lupus Clinic registry. Patients with American College of Rheumatology (ACR) SLE criteria are consecutively enrolled in this cohort. We identified patients who had retinal changes as assessed by the Systemic Lupus International Collaborating Clinics (SLICC)/ACR Damage Index. Their files were reviewed to confirm $\mathrm{HCQ} / \mathrm{CQ}$ retinopathy. For each case of retinopathy, we selected two SLE controls, matched on age of SLE diagnosis (within 5 years) and SLE duration. We compared the cases and controls in terms of race/ethnicity and clinical factors (duration of HCQ/CQ, average dose, renal damage) which may be predictors of retinal toxicity. We also determined if patients had attended annual ophthalmology assessments.

Results: Among the 326 patients who had been exposed to antimalarials, $14(4 \%)$ developed retinal toxicity. There were fewer Caucasians in the cases $(64.3 \%)$ versus controls $(85.7 \%)$ though the difference in proportions was not statistically significant. The average duration of $\mathrm{HCQ} / \mathrm{CQ}$ therapy was 18.7 years (95\% CI 11.9-25.5) in cases of retinal toxicity, and 17.5 years (95\% CI 13.7-21.3) in controls. The proportion of patients with an average dose exceeding current recommendations in those with retinal toxicity $(64.3 \%)$ was higher than controls (46.4\%) although the difference in proportions was not statistically significant. The percentage of individuals with renal damage among patients who developed toxicity (14.3\%) was similar to the control group (17.9\%). Regarding adherence to annual ophthalmologic assessments, similar proportions had missed at least one yearly assessment, after having been on antimalarial agents for 5 years or more $(50 \%$ of the cases and $65.4 \%$ of controls).

Conclusion: Four percent of patients developed anti-malarial retinal complications, over an average of 12.8 years of follow-up. We observed incomplete adherence to ophthalmology screening in many patients. The observation that cases tended to be non-Caucasians suggests race/ethnicity as a possible risk factor, but given the relatively limited study power the finding could be due to chance alone, and requires further study.

\section{8}

Ovariectomy Induced Bone Loss is Antagonized by Pulsed Electromagnetic Fields (PEMFs) and TNF-alpha and IL-6 Gene Knockouts in a Similar Mechanism

Siyi Zhu (Arthritis Research Canada, University of British Columbia; Rehabilitation Medicine Center/Provincial Rehabilitation Key Laboratory, West China Hospital, Sichuan University., Vancouver); Hongchen He (Rehabilitation Medicine Center/Provincial Rehabilitation Key Laboratory,
West China Hospital, Sichuan University, Chengdu); Chi Zhang (Rehabilitation Medicine Center/Provincial Rehabilitation Key Laboratory, West China Hospital, Sichuan University, Chengdu); Haiming Wang (Rehabilitation Medicine Center/Provincial Rehabilitation Key Laboratory, West China Hospital, Sichuan University, Chengdu); Chengfei Gao (Rehabilitation Medicine Center/Provincial Rehabilitation Key Laboratory, West China Hospital, Sichuan University, Chengdu); Qian Wang (Rehabilitation Medicine Center/Provincial Rehabilitation Key Laboratory, West China Hospital, Sichuan University, Chengdu); Quan Wei (Rehabilitation Medicine Center/Provincial Rehabilitation Key Laboratory, West China Hospital, Sichuan University, Chengdu); Xijie Yu (Laboratory of Endocrinology and Metabolism, Department of Endocrinology, National Key Laboratory of Biotherapy/Collaborative Innovation Center of Biotherapy and Cancer Center, West China Hospital, Sichuan University, Chengdu); Chengqi He (Rehabilitation Medicine Center/Provincial Rehabilitation Key Laboratory, West China Hospital, Sichuan University, Chengdu)

Objectives: Pulsed electromagnetic fields (PEMFs), as a safe and non-invasive method, could promote in vivo and vitro osteogenesis. However, the effect and underlying mechanisms of PEMFs on postmenopausal osteoporosis remain poorly understood. The present study is designed to investigate the effect of PEMFs on osteoporotic bone microarchitecture and abnormal bone metabolism together with its potential molecular mechanisms in ovariectomized (OVX) mice with different gene background.

Methods: Twenty 12-week Female wild-type (WT), TNF-alpha knockout (TNF-alpha-/-) or IL6 knockout (IL6-/-) mice, respectively, were sham-operated (SHAM) or subjected to OVX. After OVX, WT mice were equally assigned to the non-treatment and PEMFs groups. Mice in PEMFs group were subjected to daily 1-hour PEMFs exposure with $8 \mathrm{~Hz}, 3.8 \mathrm{mT}$ (peak value). Then all mice were euthanized after 4 weeks. Bone mass and skeletal microarchitecture were determined using micro-CT. Bone metabolism was assessed by histological analysis and qRT-PCR.

Results: OVX significantly increased the body weight only in WT mice without PEMFs treatment and decreased the uterine weight in all groups. The ratio of femoral trabecular bone volume to tissue volume (BV/TV), trabecular number ( $\mathrm{Tb} . \mathrm{N})$ and trabecular thickness $(\mathrm{Tb}$. Th) were significantly decreased in WT mice subjected to OVX, but increased in PEMFs group $(0.89,0.61,0.27$-fold respectively), TNF-alpha-/- mice $(1.54,1.29$, 0.30 -fold respectively) and IL6-/- mice (1.18, 0.75, 0.24-fold respectively). Furthermore, no difference in Micro-CT data analysis was found between PEMFs group and gene knockouts mice after OVX, although $38.5 \%$ and $45.1 \%$, respectively, increase in $\mathrm{BV} / \mathrm{TV}$ and $\mathrm{Tb} . \mathrm{N}$ were observed in TNF-alpha-/- mice when compared to the PEMFs group. The trends was further confirmed by bone histological analysis (H\&E staining). OVX failed to increase the number and size of TRAP-positive cells in PEMFs group and gene knockouts mice, although the phenomenon was more obvious in TNF-alpha-/- and IL6-/- mice. Real-time PCR revealed that PEMFs treatment and gene knockouts significantly up-regulated mRNA expressions of osteoblast related genes (Runx2 and Col1a1) and down-regulated WNT inhibitors mRNA (DKK1 and Sost) and osteoclast related mRNA (TRAP, MMP9, CTSK and TRAF6). The differences in mRNA level changes between PEMFs and gene knockouts were minor.

Conclusion: PEMFs alleviated ovariectomy induced bone loss by promoting skeletal anabolism and inhibiting skeletal catabolism possibly in a similar mechanism to TNF-alpha and IL-6 gene knockouts, which imply that TNF-alpha and IL-6 may become new potential targets for PEMFs in treating postmenopausal osteoporosis.

\section{9}

Different Regulatory Roles of TNF-alpha and IL-6 in Ovariectomy Induced Bone Loss

Siyi Zhu (Arthritis Research Canada, University of British Columbia; Rehabilitation Medicine Center/Provincial Rehabilitation Key Laboratory, West China Hospital, Sichuan University, Vancouver); Hongchen He 
(Rehabilitation Medicine Center/Provincial Rehabilitation Key Laboratory, West China Hospital, Sichuan University., Chengdu); Chengfei Gao (Rehabilitation Medicine Center/Provincial Rehabilitation Key Laboratory, West China Hospital, Sichuan University, Chengdu); Guojing Luo (Laboratory of Endocrinology and Metabolism, Department of Endocrinology, National Key Laboratory of Biotherapy/Collaborative Innovation Center of Biotherapy and Cancer Center, West China Hospital, Sichuan University, Chengdu); Ying Xie (Laboratory of Endocrinology and Metabolism, Department of Endocrinology, National Key Laboratory of Biotherapy/Collaborative Innovation Center of Biotherapy and Cancer Center, West China Hospital, Sichuan University, Chengdu); Haiming Wang (Rehabilitation Medicine Center/Provincial Rehabilitation Key Laboratory, West China Hospital, Sichuan University., Chengdu); Li Tian (Laboratory of Endocrinology and Metabolism, Department of Endocrinology, National Key Laboratory of Biotherapy/Collaborative Innovation Center of Biotherapy and Cancer Center, West China Hospital, Sichuan University, Chengdu); Xiang Chen (Laboratory of Endocrinology and Metabolism, Department of Endocrinology, National Key Laboratory of Biotherapy/ Collaborative Innovation Center of Biotherapy and Cancer Center, West China Hospital, Sichuan University, Chengdu); Xijie Yu (Laboratory of Endocrinology and Metabolism, Department of Endocrinology, National Key Laboratory of Biotherapy/Collaborative Innovation Center of Biotherapy and Cancer Center, West China Hospital, Sichuan University, Chengdu); Chengqi He (Rehabilitation Medicine Center/Provincial Rehabilitation Key Laboratory, West China Hospital, Sichuan University, Chengdu)

Objectives: An imbalance in bone formation relative to bone resorption and paralleled bone loss induced by ovariectomy (OVX) have been associated with elevated levels of pro-inflammatory cytokines. However, it is unclear what regulatory roles these cytokines act in mediating OVX-induced bone loss, and whether the adverse effects of these cytokines differ on bone metabolism. Here, we examined the effects of tumor necrosis factor-alpha (TNF-alpha) and interleukin-6 (IL6) gene knockout in preserving the bone loss induced by ovariectomy (OVX) and the mechanisms involved in bone metabolism.

Methods: Twenty 12-week Female wild-type (WT), TNF-alpha knockout (TNF-alpha-/-) or IL6 knockout (IL6-/-) mice, respectively, were sham-operated (SHAM) or subjected to OVX and euthanized after 4 weeks. Bone mass and skeletal microarchitecture were determined using micro-CT. Bone marrow stromal cells (BMSCs) from all three groups (WT, TNF-alpha-/- and IL6-/-) were induced to differentiate into osteoblasts or osteoclasts, and treated with 17-beta-Estradiol. Bone metabolism was assessed by histological analysis and qRT-PCR.

Results: OVX significantly increased the body weight only in WT mice and decreased the uterine weight in all three genotypes. The ratio of femoral trabecular bone volume to tissue volume, trabecular number and trabecular thickness were significantly decreased in WT mice subjected to OVX, but increased in TNF-alpha-/- mice (1.62, 1.34, 0.27-fold respectively) and IL6-/- mice (1.34, 0.80, 0.22-fold respectively). Furthermore, we observed a $29.6 \%$ increase in the trabecular number in TNF-alpha-/- mice when compared to the IL6-/- mice. Both, TNF-alpha-/- and IL6-/- BMSCs exhibited decreased numbers of TRAP-positive cells and an increase in ALP positive cells, with or without E2 treatment. While the knockout of TNF-alpha or IL6 significantly up-regulated mRNA expressions of osteoblast related genes (Runx2 and Col1a1) and down-regulated osteoclast related mRNA for TRAP, MMP9 and CTSK in vivo and in vitro, TNF-alpha knockout appeared to have roles beyond IL6 knockout in upregulating Colla1 mRNA expression and downregulating mRNA expressions of WNT-related genes (DKK1 and Sost) and TNF-related activation-induced genes (TRAF6).

Conclusion: While TNF-alpha and IL6 both had an important role in mediating OVX-induced bone loss, TNF-alpha had a stronger effect in inhibiting bone formation and enhancing TRAF6 mediated osteoclastogenesis than IL6, suggesting the role of different regulatory mechanisms governing TNF-alpha and IL6 action on bone metabolism.
50

Rheumatologic Immune-related Adverse Events Associated with Immune Checkpoint Inhibitor Therapy in Cancer Care: A Case Series from an Academic Center

Janet Roberts (Division of Rheumatology, University of Alberta, Edmonton); Anna Oswald (Division of Rheumatology, University of Alberta, Edmonton); Ina Cusnir (Division of Rheumatology, University of Alberta, Edmonton); Jason Soo (Division of Rheumatology, University of Alberta, Edmonton); Michael Smylie (Division of Medical Oncology, University of Alberta, Edmonton); John Walker (Division of Medical Oncology, University of Alberta, Edmonton); Michael Kolinsky (Division of Medical Oncology, University of Alberta, Edmonton); Quincy Chu (Division of Medical Oncology, University of Alberta, Edmonton); Naveen Basappa (Division of Medical Oncology, University of Alberta, Edmonton); Carrie Ye (Division of Rheumatology, University of Alberta, Edmonton)

Objectives: The last decade has seen an exponential increase in the use of immunotherapies in cancer treatment, with immune checkpoints proving to be a promising therapeutic target. Immune checkpoints are inhibitory pathways that modulate the immune response, and in health, serve to minimize damage to healthy tissue and prevent autoimmunity. In the setting of malignancy, tumor cells use immune checkpoints to their advantage, to evade the endogenous antitumor immune response. Hence, therapeutic targets blocking these pathways, release the brakes on pivotal immune system inhibitory mechanisms, with the goal of unleashing a robust antitumor response, but at the risk of concomitant induction of a host of immune related adverse events (IRAEs). While IRAEs are common, occurring in $70-90 \%$ of individuals, there are only small series of cases describing rheumatologic specific adverse events in the literature. We report here our experience at the University of Alberta, over an 18-month period, with patients who developed rheumatologic IRAEs secondary to immune checkpoint inhibitors (ICI).

Methods: All rheumatologists affiliated with the University of Alberta were contacted and 10 cases, diagnosed with rheumatic IRAEs in the context of ICI therapy, were identified. Following ethics approval a chart review was conducted and data including clinical presentation, treatment and outcomes collected.

Results: Presentations included; arthralgias (2), arthritis (4), vasculitis (1), sicca (1) and myositis (2). Two patients had pre-existing autoimmune disease. Malignancy types included: metastatic melanoma (4), metastatic squamous cell (1), non small-cell lung cancer (2), urothelial (1), metastatic renal cell (1) and hepatocellular carcinoma (1). Five patients received combination therapy with cytotoxic T-lymphocyte-associated antigen 4 (CTLA-4) and programmed cell death protein 1 (PD-1) inhibitors, and five received monotherapy with a PD-1 inhibitor. All patients were antibody negative. Treatments included systemic glucocorticoids, local steroid injections and disease-modifying anti-rheumatic drugs (hydroxychloroquine, methotrexate and sulfasalazine). Eight patients discontinued immunotherapy due to adverse events. Eight patients had sustained stability of their underlying malignancy, 2 had disease progression and there was one death.

Conclusion: Rheumatic IRAEs are an important complication of ICIs, and their incidence can only be expected to increase. Disease phenotypes likely differ from idiopathic rheumatic diseases, thus precluding specific rheumatic diagnoses at this time. Collaboration between centers is required to collect large cohorts of patients and identify trends in presentation, treatment and outcomes to guide the development of treatment recommendations.

\section{1}

Quality Improvement Project: Rheumatology Clinic Pre-visit Planning to Improve Patient Engagement

Janet Roberts (Division of Rheumatology, University of Alberta, Edmonton); Xing Sun (University of Alberta, Edmonton); Pamela Mathura (Alberta Health Services, Edmonton); Fabiola Nunez (University of Alberta, Edmonton); Lorelei Johnstone (Alberta Health Services, Edmonton); Sandra Rovensky (University of Alberta, Edmonton); Tobi Leder (Alberta Health Services, Edmonton); Elaine Yacyshyn (Division of Rheumatology, University of Alberta, Edmonton) 
Objectives: Many patients seen in an outpatient rheumatology clinic suffer from multi-system chronic diseases requiring complex care including the need for numerous medications, regular laboratory work and frequent visits with their primary care physician and other specialists. Pre-visit planning has been proposed as a key strategy for improving no-show rates and compliance in chronic diseases such as diabetes and hypertension. While various techniques have been used in pre-visit planning including pre-visit phone calls, questionnaires and checklists, none have been studied in the context of an outpatient rheumatology clinic, but may serve to improve patient preparedness, communication, clinic efficiency and ultimately outcomes in our specific patient population. Our primary objective was to identify key areas where gaps in communication may occur in all points of the care continuum, by involving all relevant stakeholders including the patient, clinic staff, nurses and rheumatologists, and to improve patient preparedness, engagement and clinic efficiency through the development of interventions to improve pre-visit planning.

Methods: A patient touch point map was created to determine the main process steps through the viewpoint of the patient. A patient engagement consultant assisted in developing a patient survey to consider the patient perspective on the efficacy of current methods used for pre-visit planning including the booking phone call, mailed appointment package and the www.albertarheumatology.com website. One hundred and twenty patients completed the survey with results used to develop interventions to improve pre-visit planning including: use of a standardized script during the booking call, updates to the appointment package using a patient-friendly format and posters placed in the clinic reception and nursing rooms to remind patients to come prepared for clinic appointments. These interventions were trialed with 10 patients and preparedness evaluated by the treating rheumatologist. A post-intervention telephone survey was carried out to collect patient feedback on the improvement interventions.

Results: Ten participants, 5 new and 5 follow-up patients completed the survey and reported feeling more prepared for clinic appointments. Appointment duration was reduced by an average of 7 minutes for new patients and 4 minutes for follow-ups. Clinic staff and physicians reported increased patient engagement and efficiency in the clinic.

Conclusion: Process changes demonstrated the importance of the patient's perspective in quality improvement. Improving patient engagement and preparedness through pre-visit planning lead to reduced clinic cycle time, improved clinic efficiency and increased patient and healthcare provider satisfaction.

52

Achieving Balance and Diversity in Patient Engagement in Research: Perspectives from Patients

Graham Macdonald (Arthritis Research Canada, Richmond); Jenny Leese (Arthritis Research Canada, Vancouver); Bao Tran (Arthritis Research Canada, Richmond); Alison Hoens (University of British Columbia, Vancouver); Sheila Kerr (Arthritis Research Canada, Richmond); Lianne Gulka (Arthritis Research Canada, Richmond); Wendy Lum (Arthritis Research Canada, Richmond); Linda Li (Rehab Sciences/Physical Therapy, University of British Columbia, Arthritis Research Canada, Richmond)

Objectives: The movement for patient engagement in research aspires to greater inclusion of and collaboration with patients at every point in the research cycle. Currently there is little empirical evidence concerning how patient engagement in research affects the lives of those involved. Our objective was to examine the experience of what it takes to be involved in research as a patient partner, focusing on how involvement affects daily life. Methods: This project was designed in a collaboration with patients with arthritis who had experience as research partners. Eligible participants were current or past members of a patient advisory board in an arthritis research centre. Participants were invited to take part in an hour-long interview. An iterative thematic analysis of the interviews was conducted using constant comparison method. Discussions with patient partners informed the development of themes.

Results: In 2015-2016, 22 participants were recruited, 21 (95\%) were female, ages ranged from 26 to 68 years, and time spent as a patient engaged in research varied from 1 month to 10 years. Sixteen $(73 \%)$ had IA, $9(41 \%)$ had OA, and $3(14 \%)$ had both. Fourteen $(64 \%)$ had at least one university degree. 12 participants $(55 \%)$ were employed fulltime, $9(41 \%)$ were retired, 2 were students and 2 were on disability benefits. Two themes were identified, the first concerned maintaining balance as a patient partner, in which many participants described in detail their experience as volunteers in a patient advisory board as being rewarding and purposeful, though sometimes difficult to balance with priorities such as family, work, and health. The second theme was drawn from the participants' concern at the difficulty of making patient engagement more diverse and reaching under-represented populations, noting issues such as gender imbalance, and poorer representation from marginalized communities, lower socioeconomic classes and people with limited educational opportunities.

Conclusion: Our findings show that for patients who have had the opportunity to be partners in research, it can be both rewarding and burdensome. Our participants also identified, with concern for the democratic ideal of inclusivity, that there are populations lacking the opportunity to be engaged in research and are not being reached or represented. This is of concern due to the important role that the social determinants of health play in determining quality of life for people affected by arthritis. Our findings suggest that increased efforts and resources and new strategies are needed to bring increased diversity to patient engagement in arthritis research.

\section{3}

Patients' Resource Preferences for a Knowledge Translation Toolkit for Hip and Knee Replacement Rehabilitation

Marie Westby (Vancouver Coastal Health Research Institute, Vancouver); Cheryl Koehn (Arthritis Consumer Expert, Vancouver); Alison Hoens (University of British Columbia, Vancouver)

Objectives: To identify patients' resource preferences and priorities for a knowledge translation toolkit (EQUIP-TJR) for implementing quality indicators (QIs) for TJR rehabilitation.

Methods: Online survey launched May 15, 2017 targeting adults on a surgical waitlist for hip or knee TJR for osteoarthritis OR who had a TJR in the past year. Recruitment strategies included posters in high volume surgical centres, surgeons' and physiotherapists' clinics, social media and e-mail blasts and e-newsletters in collaboration with our knowledge user partners. The survey consisted of three sections: 1) Rating toolkit resources on 5-point scale (definitely wouldn't use to definitely would use); 2) Overall rating of the EQUIP-TJR toolkit and its impact on rehabilitation care and outcomes using a 5-point scale (strongly disagree to strongly agree); and 3) Participant demographics. The survey took 15 minutes to complete and later in the recruitment process, participants were eligible to enter their names for a $\$ 100$ gift card prize draw. The University of BC Behavioural Research Ethics Board approved this study.

Results: At time of submission, 89 individuals had completed the online survey. A majority were from BC (58\%), female (75\%) and almost half were in the 55-64 years age range. There was a similar proportion of respondents undergoing hip (53\%) and knee (47\%) surgery and most $(67 \%)$ represented the post-operative phase. Of the proposed resources, the five identified as most likely to be used by patients (combined 'probably' and 'definitely would use') were: 1) Patient education booklet-98\%; 2) QI questionnaire$84 \%$; 3) QI checklist-80\%; 4) QI passport-72\%; and 5) Patient video vignette-71\%. An App with QI information and ability to track the quality of care in each stage of rehabilitation was rated least likely to be used. Respondents agreed/strongly agreed that the toolkit and its resources would help them to become more involved in their rehabilitation $(82 \%)$, track the quality of their rehabilitation care $(85 \%)$ and set goals and share expectations with their healthcare team $(83 \%)$. When asked whether knowing about the QIs and having the EQUIP-TJR toolkit would result in better care from their health care team and better outcomes after surgery, $65 \%$ and $75 \%$ agreed/strongly agreed, respectively.

Conclusion: Through an online survey, we learned that patients having hip and knee TJR prefer more traditional print-based educational resources to 
use the rehabilitation QIs. These responses will inform the development of the EQUIP-TJR toolkit to help patients engage in their rehabilitation care.

\section{4}

Efficacy of Janus Kinase (JAK) Inhibitors in Rheumatoid Arthritis: A Meta-analysis and Systematic Review

Tony Zhang (McMaster University, Hamilton); Nicholas Timmerman (University of Ottawa, Ottawa); Arthur Lau (McMaster University, St. Joseph's Healthcare, Hamilton)

Objectives: Janus kinase (JAK) inhibitors are a novel of class of disease modifying anti-rheumatic drugs for use in rheumatoid arthritis (RA). Tofacitinib was one of the first to have shown efficacy in the treatment of RA. Over last several years, newer evidence and more JAK inhibitors have become available. The purpose of this study is to systematically identify and evaluate the efficacy of JAK inhibitors in RA.

Methods: A literature search was performed using MEDLINE, EMBASE; Cochrane Central Register of Controlled Trials; and PubMed with original search on Nov 5, 2016 and updated in July 2017. Identified titles/abstracts were reviewed by TZ and NT. Only Randomized Controlled Trials were included. Our primary outcome was ACR 20 at week 12. Secondary outcomes were ACR 50, ACR 70, change in DAS28-CRP or DAS28-ESR, percentage of patients achieving DAS28-CRP or DAS28-ESR $<2.6$, change in HAQ-DI, change in patient global assessment of disease (GAD) activity at week 12 and 24. Data were extracted in duplicate and presented as relative risk ratios (RR) using the Review Manager 5.3.

Results: A total of 29 trials included for analysis. There were 13,662 RA patients with a mean age of 53 years old. Eight one percent were female. The mean disease of duration was 8 years. The baseline DAS28-CRP and DAS28-ESR was 5.6 and 6.3, respectively. Our primary endpoint ACR20 at week 12 was significantly higher in all JAK inhibitor groups comparing to placebos (tofacitnib RR 2.26, barictinib RR 1.71, peficitinib RR 1.58, decernotinib RR 2.52, upadacitnib RR 1.64, filgotinib RR 1.81, itacitinib RR 1.78). This was maintained at week 24. In terms of ACR 50 and ACR70 response, a greater amplitude of effect was noted in JAK inhibitors groups with RR in range of 2.27 to 6.68 with exception of peficitinib which did not reach statistical significance. Peficitinib and itacitinib both failed to show any superiority in a number of other outcomes, including percentage of patients reaching DAS28-ESR $<2.6$ and DAS28-CRP $<2.8$.

Conclusion: JAK inhibitors have been shown to be an effective class of medications in treatment of RA. Our analysis is limited by the heterogeneity of data. While we could not combine data across all JAK inhibitors, ACR20 response rate was shown to be significant for each individual JAK inhibitor. Greater efficacy was observed in ACR50 and ACR70. There seemed to be some intra-class differences with peficitnib and itacitinib lacking efficacy in certain outcome measures.

\section{5}

Gaps in Screening for Retinal Toxicity in Patients with Systemic Lupus Erythematosus and Rheumatoid Arthritis Exposed to Antimalarials: A Population Based-study

Mikameh Kazem (University of British Columbia, Vancouver); David Maberley (Vancouver Coastal Health, Vancouver); Hyon Choi (Massachusetts General Hospital/Harvard Medical School, Boston); Hamid Tavakoli (Arthritis Research Canada, Richmond); Kamran Shojania (University of British Columbia Faculty of Medicine; Arthritis Research Canada, Vancouver); John Esdaile (University of British Columbia (Division of Rheumatology)/Arthritis Research Canada, Richmond); Antonio Avina-Zubieta (University of British Columbia Faculty of Medicine; Arthritis Research Canada, Richmond)

Objectives: Background: Antimalarial (AM) drugs, such as hydroxychloroquine and chloroquine, are almost universally recommended for systemic lupus erythematosus (SLE) patients and remain a cornerstone of SLE care and to a lesser degree in rheumatoid arthritis (RA) care. Although AM are generally well-tolerated, a critical long-term adverse event is vision-threatening retinopathy. The Canadian guidelines recommend annual quantitative testing for bilateral visual field assessment (VFA) and funduscopic examination of the macula. Recently, the American Academy of Ophthalmology recommended adding spectral-domain optical coherence tomography (SDOCT) to ensure early identification of retinal toxicity. Objective: To evaluate adherence to the screening guidelines for retinal toxicity in all patients with RA and SLE in British Columbia (BC) who had been exposed to AM for 2-5 years prior to 2014 .

Methods: Using diagnostic codes in an administrative health database from the province of BC, Canada, we identified all prevalent cases of SLE and RA in 2014 that were exposed to AM based on prescription records. Outcome: we calculated screening rates for retinal toxicity, defined as at least one ophthalmology visit and a VFA or SDOCT procedure code within 2 years and 5 years prior to 2014

Results: We identified 7,427 prevalent cases who met the inclusion criteria. Further analysis showed that 6,426 (87\%), 5,842 (74\%), 5,387 (73\%) and $4,990(67 \%)$ had two, three, four and five consecutive years of exposure to AM prior to 2014, respectively. Overall, 50\% of patients had at least one annual visits to an ophthalmologist, when we added optometrist visits the rate increased up to $66 \%$. However, when we accounted for only ophthalmologists plus VFA or SDOCT rates within the 2-year and 5-year periods were $53 \%$ and $61 \%$, respectively. For both surveillance periods, patients seen by a rheumatologist had higher screening rates than those seen by non-rheumatologists (56\% versus $18 \%$, and $67 \%$ versus $32 \%$ for 2 -year and 5 -year periods, respectively).

Conclusion: Adherence to the screening guidelines for retinal toxicity was substantially low. However, patients seen by rheumatologists had higher rates of screening when compared to those seen by non-rheumatologists. This suggests an important gap in care and delay in implementation of screening guidelines for retinal toxicity due to AM exposure.

56

Muscle and Bone Strength in Children with Juvenile Idiopathic Arthritis: Results of a 6-month Home- and Group-based Exercise Intervention (LEAP Study)

Kristin Houghton (BC Children's Hospital, Vancouver); Heather MacDonald (University of British Columbia, Victoria); Heather McKay (University of British Columbia, Vancouver); Douglas Race (University of British Columbia, Vancouver); Adam Mitha (Queen's University, Kingston); Ciaran Duffy (Children's Hospital of Eastern Ontario, Ottawa); Lori Tucker (BC Children's Hospital, Vancouver)

Objectives: To 1) assess feasibility and safety of a 6-month home-based exercise program for children with juvenile idiopathic arthritis (JIA), 2) estimate the effect of participation in the exercise program on bone mass and strength, muscle force and power and clinical outcomes 3 ) determine if any changes in bone, muscle and clinical outcomes are maintained 6 months after completion of the exercise program.

Methods: We recruited 24 children with JIA to participate in a 6-month intervention that involved jumping exercises, resistance training and handgrip exercises and one group exercise session per month. We assessed lumbar spine bone mass (dual energy X-ray absorptiometry), distal tibia and radius bone microarchitecture and strength (high-resolution peripheral quantitative computed tomography), muscle function (jumping mechanography, dynamometry) and clinical outcomes (joint assessment, function, health-related quality of life) at baseline, 6- and 12-months. We assessed adherence using weekly activity logs. This study was part of a larger multisite (12 Canadian pediatric rheumatology centers), longitudinal observational cohort study of children with JIA (LEAP; Linking Exercise, Physical Activity and Pathophysiology in Childhood Arthritis; $N=707$ ).

Results: Thirteen children completed the 6-month intervention. Participants reported 9 minor adverse events, and post-exercise pain was rare $(0.4 \%)$. Grip strength increased by $4.3 \%$, on average, between baseline and 6 months, and was sustained at 12 months. Peak power (2-legged jump) did not change between baseline and 6 months; however, participants had a $7 \%$ increase between baseline and 12-months. Knee extensor total work increased by $15 \%$ between baseline and 6 months but was not sustained at 12 months.

Personal non-commercial use only. The Journal of Rheumatology Copyright $(\subset) 2018$. All rights reserved. 
Seven children $(54 \%)$ reported significant fatigue at baseline. Fatigue improved at 6 months (11.1 points) with improvement maintained at 12 months compared to baseline. Other muscle, bone or clinical outcomes did not change. Adherence to the exercise program was low (47\%) and decreased over time.

Conclusion: Children with JIA can safely and feasibly participate in a home-based exercise program designed to enhance muscle and bone strength. Gains in muscle strength and power did not translate to measurable improvement in bone outcomes in this small cohort. Fatigue improved, which may in turn facilitate physical activity participation. Low adherence and attrition (implementation factors) likely contributed to our inability to adequately assess the intervention effect on bone, muscle and clinical outcomes. Efforts are needed to address facilitators and barriers to participation in and adherence to exercise programs among children with JIA.

57

A New Model of Care for Pediatric Rheumatology in Ontario: Preliminary Results from Pilot Telemedicine Clinics Utilizing ACPACs Roberta Berard (Children's Hospital, LHSC, London); Sue Macqueen (The Arthritis Society, Kitchener); Michelle Diebold (Children's Hospital, London Health Sciences Centre, London); Ingrid Goh (The Hospital for Sick Children, Toronto); Anne MacLeod (The Arthritis Society, Thunder Bay); Christine O'Brien (Hospital for Sick Children, Toronto); Kristi Whitney (Hospital for Sick Children, Toronto); Brian Feldman (The Hospital for Sick Children, Toronto); Deborah Levy (Division of Rheumatology, SickKids Hospital; Faculty of Medicine, University of Toronto, Toronto)

Objectives: Twenty-five pediatric rheumatologists (PRs) service a population of 14 million Ontarians. To facilitate care to patients living in distance communities we proposed a new model of care using a local Advanced Clinician Practitioners in Arthritis Care (ACPAC) to engage with the PR via telemedicine. The first phase of this project reported our needs assessment of patients seen in London and Toronto. Of a total of 265 patient/caregiver respondents, $80 \%$ of patients travelled $>50 \mathrm{~km}$ with $13 \%$ travelling $>200 \mathrm{~km}$ with an associated cost $>\$ 50$ in $37 \%$ and $\geq 1$ missed day of work in $56 \%$ to attend PR visits. We present preliminary results from the second phase of this project: a survey of patient/parent satisfaction of the pilot ACPAC-PR telehealth clinics.

Methods: Twenty-five ACPACs interested in additional pediatric rheumatology training attended a two-day training program in September 2016. Of the 4 ACPACs participating in the pilot clinic phase, 3 had extensive pediatric experience and the fourth received on-site training. Patient/ caregiver perception of visit burden and satisfaction of the ACPAC-PR telehealth visit were surveyed. Quantitative data were summarized using descriptive statistics and qualitative data were analyzed using grounded theory.

Results: Three telehealth clinics with Windsor ACPAC-London PR (20 visits, 19 unique patients) and 4 with Thunder Bay ACPAC-Toronto PR (13 visits and 10 unique patients) have occurred to date. All 19 surveys were collected from the Windsor-London clinic and 3/8 distributed at the Thunder Bay-Toronto site. Nineteen $(86 \%)$ travelled $<25 \mathrm{~km}$ to the clinic, $16 / 22$ $(73 \%)$ spent $<\$ 25$ on travel and $27 \%$ did not spend any money. Six $(27 \%)$ missed 1/2 to 1 full day of work with no one missing more than 1 day. Over $90 \%$ of respondents strongly agreed/agreed that they were satisfied with the telemedicine process and interaction with the ACPAC and PRs. Nineteen $(86 \%)$ indicated that "the care I received today was equal to the care I receive in person" and that the wait time to see the PR was adequate (versus too short or too long) compared with an in person visit.

Conclusion: A model of care integrating ACPACs, telemedicine and PRs improves local access to pediatric rheumatology care while reducing the burden of travel and cost to families. Barriers to the full implementation of this model include the lack of funding for ACPACs to provide these outreach clinics as well as lack of (pediatric-trained) ACPACs in distant communities to sustainably provide this valuable service. Best Abstract on Paediatric Research by Young Faculty.
58

Systematic Review on Diagnostic Accuracy of MRI for Assessment of Sacroiliac Joints in Children and Adults

Vimarsha Swami (University of Toronto, Toronto); Dax Rumsey (University of Alberta, Edmonton); Jacob Jaremko (University of Alberta, Edmonton); Andrea Doria (University of Toronto, Toronto)

Objectives: Accurate and reproducible sacroiliac joint (SIJ) assessment on MRI in pediatric patients is challenging, often with high inter-observer variation. To facilitate consistent SIJ assessment, numerous scoring systems have been proposed. We sought to systematically review articles that evaluated diagnostic accuracy and reliability of existing MRI-based SIJ scoring systems for the evaluation of spondyloarthropathy in children and adults, and assess their applicability to pediatric patients.

Methods: Relevant studies were identified through a systematic literature search that included MEDLINE, EMBASE, and Cochrane Library Database. Studies reporting an element of diagnostic accuracy of an MRI-based SIJ scoring system were included: 1) reliability, 2) construct validity comparing MRI with other clinimetric measures, 3 ) criterion validity comparing MRI with a gold standard, and 4) ability of MRI to depict responsiveness to treatment. Canadian Task Force on Preventive Health guidelines were used to determine overall strength of recommendation (A-E) for scoring system utilization in clinical practice and research.

Results: From 38 eligible studies, five frequently utilized scoring systems were identified: the Spondyloarthritis Research Consortium of Canada (SPARCC) inflammation and structural scores, and the Leeds, Aarhus, and Berlin scoring systems. Together the SPARCC scores had the highest number of studies reporting reliability $(\mathrm{N}=13)$, construct validity $(\mathrm{N}=10)$, and responsiveness $(\mathrm{N}=13)$. Good inter-observer reliability with intra-class correlation coefficients (ICCs) $>0.8$ was reported in 9/13 (69\%) (SPARCC inflammation, structural), 5/6 (83\%), (Berlin), 1/1 (100\%) (Leeds), and 0 $(0 \%)$ (Aarhus) studies examining reliability. Seven $(70 \%)$ studies demonstrated correlation between SPARCC scores and ESR, CRP, and standardized clinical severity indices. Twelve (92\%) studies demonstrated decreases in SPARCC scores with treatment. No studies assessed criterion validity. No scoring systems dedicated to pediatric patients were found, with none taking into account age-related changes in bone marrow. Only 1 study on the SPARCC scoring systems included pediatric patients, and was not dedicated to solely pediatric patients

Conclusion: Currently no MRI scoring systems are available in the literature focusing on pediatric SIJ assessment. There is fair (grade B) evidence to recommend the use of the SPARCC method use, however adjustments are needed in this scale (e.g. grading for bone marrow changes over time) before routine use can be considered in pediatric patients.

\section{9}

Children with Enthesitis Have Worse Quality of Life, Function, and Pain, Irrespective of their Juvenile Arthritis Category

Dax Rumsey (University of Alberta, Edmonton); Jaime Guzman (University of British Columbia, Vancouver); Alan Rosenberg (University of Saskatchewan, Saskatoon); Adam Huber (IWK Health Centre, Halifax); Rosie Scuccimarri (McGill University, Montreal); Natalie Shiff (University of Florida, Gainesville); Alessandra Bruns (Université de Sherbrooke, Sherbrooke); Brian Feldman (The Hospital for Sick Children, Toronto); Dean Eurich (University of Alberta, Edmonton); ReACCh-Out Investigators (Edmonton)

Objectives: To estimate the impact of enthesitis on patient reported outcomes (PROs) in children with juvenile idiopathic arthritis (JIA), irrespective of their JIA category.

Methods: Children with JIA in the Research in Arthritis in Canadian Children emphasizing Outcomes (ReACCh-Out) cohort were studied. Entheseal tenderness by physician examination in 33 defined locations, Juvenile Arthritis Quality of life Questionnaire (JAQQ), Quality of My Life questionnaire (QoML), Childhood Health Assessment Questionnaire (CHAQ), and a pain severity visual analogue scale (VAS) were completed at enrolment, every six months for 2 years, and then yearly for up to 5 years.

Personal non-commercial use only. The Journal of Rheumatology Copyright $\subset$ 2018. All rights reserved. 
Analyses consisted of descriptive statistics, multivariate linear mixed models for longitudinal data, and ANCOVA.

Results: Among 1371 patients followed for a median of 35.3 months, 214 (16\%) had enthesitis, of whom 137 (64\%) were classified as having enthesitis-related arthritis (ERA). After adjusting for patient characteristics and JIA category, children with enthesitis reported higher JAQQ ( 0.41 points; $95 \%$ CI $0.22,0.59 ; 1=$ no difficulties, $7=$ difficulties all the time), higher CHAQ ( 0.13 points; $95 \%$ CI $0.06,0.20 ; 0=$ without any difficulty, $3=$ unable to do task), higher pain ( 0.93 points; $95 \%$ CI $0.63,1.24 ; 0=$ no pain, $10=$ maximum pain $)$ and lower QoML (-0.80 points; 95\% CI -1.09, -0.51 ; $0=$ the worst, $10=$ the best) scores than children without enthesitis, and these differences persisted during the five years after diagnosis.

Conclusion: Children with enthesitis, regardless of JIA category, report worse PROs than those without enthesitis. Physicians should assess for the presence of enthesitis in all children with JIA. Enthesitis should be considered as criterion for classification and included in the assessment of treatment response in JIA. Best Abstract on Paediatric Research by Young Faculty.

\section{0}

Quality of Referral Letters to Pediatric Rheumatology and its Impact on Access to Care

Alexandra Rydz (University of Alberta, Edmonton); Fangfang Fu (University of Alberta, Edmonton); Mark Drew (University of Alberta, Edmonton); Dax Rumsey (University of Alberta, Edmonton); Yan Yuan (University of Alberta, Edmonton); Mercedes Chan (University of Alberta, Edmonton)

Objectives: To describe the content of referral letters to pediatric rheumatology (PR) at a tertiary care pediatric center and to determine the impact of incomplete referral letters on time to PR assessment.

Methods: All new referral letters to PR at our centre over a 15-month period were prospectively evaluated by attending pediatric rheumatologists on a weekly basis during patient triage using a referrals' checklist. Letters were reviewed for eight components of a high quality referral: rheumatologic diagnosis of concern; patient symptoms; investigations; physical examination (musculoskeletal and general); co-morbidities; current and past management; and medications. Referrals for patients $>17$ years old were excluded. Basic patient demographics and referring physician specialty were also collected. Dates of triage decisions and resultant PR appointments were recorded, and times to PR appointments calculated. Where incomplete referrals required additional information from referring physicians, we documented dates this information was received and delays in triage times incurred. Final diagnoses made by rheumatologists were recorded retrospectively. Descriptive statistics were applied to the data.

Results: Referrals ( $\mathrm{n}=334,62 \%$ female, mean age 10.6 years) were received and analyzed from: family doctors $46.7 \%$, pediatric providers (including subspecialists) $40.1 \%$, and others (e.g., pediatric ENT) $13.2 \%$. The frequency of specific components included in referral letters were: patient symptoms $(316 / 334,94.6 \%)$; investigations $(224 / 334,67.1 \%)$; diagnosis of concern $(182 / 334,54.5 \%)$; musculoskeletal examination (159/334, 47.6\%); medications $(142 / 334,42.5 \%)$; current and past management $(133 / 334,39.8 \%)$; co-morbidities $(113 / 334,33.8 \%)$; and general examination $(86 / 334,25.7 \%)$. Further information was requested from 61/334 referrals (18.3\%) regarding one or more of: pertinent history $(57 / 61,93.4 \%)$; physical examination $(54 / 61,88.5 \%)$; diagnosis of concern (45/61, 73.8\%); and investigations $(27 / 61,44.3 \%)$. Where missing information was requested, the mean delay in time to triage was 4.1 weeks $(\mathrm{CI}$ : (1.9-6.2)). A final diagnosis was documented in 273 referrals, of which $50.9 \%$ had a confirmed rheumatic disease. The proportion of referrals resulting in a rheumatic diagnosis by provider was: $57.7 \%$ of those from pediatric providers; $46.7 \%$ from family doctors, and $45.2 \%$ from others. There was no significant difference between the likelihood of referrals from pediatric providers and family practice providers resulting in a true rheumatic diagnosis $(\mathrm{p}=0.1239)$.

Conclusion: Pertinent history and physical examination are the most commonly omitted components of referral letters to PR. Requesting missing information delayed triage. Only approximately half of PR referrals resulted in a PR diagnosis. These findings can inform medical education initiatives to facilitate access to timely PR care. Supported by a CIORA grant.

61

Patient Reported Outcomes using Smartphones in Rheumatology: A Scoping Review

Nathan Puhl (University of Alberta, Edmonton); Elaine Yacyshyn (Division of Rheumatology, University of Alberta, Edmonton)

Objectives: Patient reported outcomes (PRO) are quantifiable subjective symptoms recounted by patients representing important measures in rheumatologic disease. There has been increased interest in electronic assessment of PROs with widespread use of smartphones and the possibility of increased accuracy in capturing disease activity and improving disease management. This review aims to understand how patient reported outcomes are measured with smartphones; how these measurements correlate with objective clinical scores, and feasibility and development of such technology.

Methods: EMBASE and MEDLINE databases were searched using MeSH subject headings. Keywords related to: smartphones and rheumatic disease. Articles were assessed using a standard protocol for suitability, disease, outcomes, and app development.

Results: Combined bibliographic searches identified 262 articles after duplicates were removed. Titles and abstracts were then screened resulting in 43 articles. Of these, 13 were peer-reviewed papers while 30 were conference proceedings. 27 articles described PROs, with 10 of those correlated to objective disease activity scores and 8 describing at least part of the app development. The remaining 16 articles focused solely on app development and feasibility. Most articles evaluated technology use in rheumatoid arthritis (26), followed by juvenile idiopathic arthritis (JIA) (7). Other diseases included spondyloarthropathy, lupus, or inflammatory eye disease. Variable PROs were measured for inflammatory arthritis. In the 10 articles that compared PROs to disease activity measures, 5 demonstrated high correlation between the RAPID3, HAQ and patient reported tender or swollen joint counts with the DAS28, CDIA, SDAI, and physician global assessment. Passive activity was measured through step counters, trunk acceleration, and activity trackers in 7 articles, with 2 articles reporting significant correlation to DAS28. Articles regarding app development focused primarily on surveying patient stakeholders for opinions regarding app design, either with initial creation process or an iterative refinement process. 2 articles involved only rheumatologists in the development process, focusing on providers' willingness to use such technology in practise. Most of the articles reported favorable attitudes of patients and providers towards smartphone use.

Conclusion: This scoping review demonstrates the current literature regarding measuring PROs with smartphones is sparse, with the majority being conference proceedings. What data is available does suggest feasibility and utility incorporating PROs into practise for rheumatoid arthritis however there remains minimal standardization and validation regarding exact measures. As PROs are measured more frequently by accessible technology, validated studies need to be completed to evaluate effectiveness of these tools.

62

Clinical Audit of ANA Ordering Patterns in Rheumatology Referrals

Nadil Zeiadin (Queen's University, Kingston); Henry Averns (Rheumors, Kingston)

Objectives: Serum anti-nuclear antibody (ANA) tests have well-established clinical utility as diagnostic and prognostic markers, particularly in connective-tissue disorders such as SLE. However, interpreting results may be challenging, especially for primary care providers. Consequently, these tests are frequently ordered unnecessarily, which often results in further unnecessary testing, erroneous diagnosis or even inappropriate therapy. To decrease unnecessary testing, Choosing Wisely Canada has published recommended indications for ANA-testing. We sought to answer the following questions: 1. In what proportion of our rheumatology consultation requests

Personal non-commercial use only. The Journal of Rheumatology Copyright $(\subset) 2018$. All rights reserved. 
has an ANA been ordered? 2. Based on Choosing Wisely Canada Recommendations were these ANA tests reasonable? 3. Did ANAs directly lead to a consultation request?

Methods: We conducted a retrospective chart review in a general community rheumatology practice. We analyzed consecutive referrals for consultation received using a convenience sampling method. We determined from the information provided in the referral whether an ANA was indicated based on Choosing Wisely criteria, whether further investigations had been ordered and whether the consultation was initiated based primarily on a positive ANA result.

Results: We reviewed the e-charts of 100 consecutive new patients (new referrals analyzed from Oct 2016 to March 2017). 46\% of referrals had an ANA measured. Of these, $81 \%$ did not meet the indication criteria for ANA testing. Of the ANA's done without a clear indication, 59\% were negative, $33 \%$ of the ANA's fulfilling indications for testing were negative. Of all new referrals, $20 \%$ were requested primarily for a positive ANA. Of these, 13 referrals $(65 \%)$ had an ANA not fulfilling testing criteria.

Conclusion: We found ANA's are frequently ordered among patients referred for rheumatology consultation. Despite published guidelines for appropriate use of serum ANA, the vast majority of ANA testing is still done without a recognized indication. Furthermore, the unnecessary testing frequently results in referral to the rheumatology clinic as well as avoidable anxiety for patients. Consequently, more work is required in raising awareness of appropriate use of ANA testing to improve healthcare resource utilization. Our next step is to implement an educational initiative with primary care providers in our region regarding Choosing Wisely recommendations and appropriate use of the ANA test.

63

Intravenous Golimumab in Adult Patients with Active Psoriatic Arthritis: Efficacy and Safety Through 1 Year

Elaine Husni (Cleveland Clinic, Cleveland); Arthur Kavanaugh (University of California, San Diego, La Jolla); Francois Nantel (Janssen Inc, Toronto); Diane Harrison (Janssen Research \& Development, LLC, Spring House); Lilianne Kim (Janssen Research \& Development, LLC, Spring House); Kim Lo (Janssen Research \& Development, LLC, Spring House); Elizabeth Hsia (Janssen Research \& Development, LLC, Spring House)

Objectives: GO-VIBRANT evaluated safety and efficacy of intravenous (IV) golimumab (GLM) in adult patients (pts) with active psoriatic arthritis (PsA) over 1 year.

Methods: GO-VIBRANT is a Phase 3, multicenter, randomized, double-blind, placebo (PBO)-controlled trial. Biologic-naïve active PsA pts were randomized (1:1) to IV GLM $2 \mathrm{mg} / \mathrm{kg}$ at 0,4 , and every 8 weeks (q8w) or $\mathrm{PBO}$ at $0,4,12$, and 20 weeks (wks) with crossover to GLM $(\mathrm{PBO} \rightarrow \mathrm{GLM})$ at 24,28 , and $\mathrm{q} 8 \mathrm{w}$ through Wk52. Efficacy data from Wks24-52 and safety data through Wk60 are reported here.

Results: 480 pts were randomized (PBO: 239; GLM: 241). At Wk24, greater proportions of GLM vs PBO pts achieved ACR20 (77\% vs 24\%), ACR50 (54\% vs $6 \%$ ), ACR70 (33\% vs 3\%), and ACR90 (6\% vs 0\%). Mean improvement from baseline in HAQ-DI score was greater in GLM vs PBO groups $(-0.63$ vs -0.14$)$. At Wk24, greater proportions of GLM vs PBO pts achieved PASI75 (65\% vs 13\%), PASI90 (43\% vs $8 \%$ ), or PASI100 (26\% vs $6 \%$ ). Mean change from baseline in total modified vdH-S score in GLM vs $\mathrm{PBO}$ groups was -0.36 vs +1.95 . At Wk52, similar proportions of GLM vs PBO $\rightarrow$ GLM pts achieved ACR20 (76.8\% vs $77.0 \%)$, ACR50 $(58.1 \%$ vs $53.6 \%$ ), ACR70 (38.6\% vs $33.9 \%$ ), and ACR90 (13.7\% vs $11.3 \%$ ). Mean improvements from baseline in HAQ-DI score were maintained in GLM and $\mathrm{PBO} \rightarrow$ GLM groups $(-0.66$ and -0.56$)$. After crossover to GLM (Wk24), meaningful improvements in $\mathrm{PBO} \rightarrow \mathrm{GLM}$ group for ACR and HAQ endpoints were seen as early as Wk28. PASI responders at Wk52 for GLM vs $\mathrm{PBO} \rightarrow$ GLM pts were: PASI75 (71.9\% vs $60.6 \%)$, PASI90 (56.1\% vs $41.9 \%$ ), or PASI100 (28.6\% vs $18.7 \%$ ). Mean change from baseline in total modified vdH-S score was maintained for GLM $(-0.49)$. There was no increase in the mean change from baseline at $\mathrm{Wk} 52$ in $\mathrm{PBO} \rightarrow$ GLM group $(+0.76)$. Through Wk60, 460 pts received $\geq 1$ administration of GLM. Of these, $50.9 \%$ had $\geq 1 \mathrm{AE}$ and $5.2 \%$ had $\geq 1$ serious AE. The most common type of AE was infection (22.8\%). One death (acute hepatitis) and 2 malignancies (gastric cancer, colon cancer) were reported in GLM-treated pts. Two GLM-treated pts developed active TB. No opportunistic infection, anaphylaxis, or serum sickness reaction was reported. Four GLM-treated pts reported infusion reactions; none was serious.

Conclusion: In pts with active PsA, IV GLM maintained clinically meaningful improvements through 1 year. Through Wk60, the safety profile was consistent with other anti-TNFs, including subcutaneous GLM.

\section{4}

A Comparative Real-World Utilization Patterns of Innovator Infliximab and Biosimilar Infliximab in a Treatment Naïve and Switch Population from Germany: A Prescription Claims Analysis

Francois Nantel (Janssen Inc, Toronto); Emmanuel Ewara (Janssen Inc., Toronto); Vipul Jairath (Western University \& London Health Sciences Centre, London); Marilise Marrache (Janssen Inc., Toronto); Xenofon Baraliakos (Rheumazentrum Ruhrgebiet, Ruhr-University Bochum, Herne) Objectives: Retrospective prescription claims analysis comparing utilization patterns of innovator (IFX) and biosimilar (CT-P13) infliximab in treatment naïve population at 12 months and in patients continuing IFX vs switching to CT-P13 at 6 months.

Methods: QuintilesIMS ${ }^{\mathrm{TM}}$ longitudinal health insurance prescription data from Germany identified patients with an index claim of IFX or CT-P13 in all indications between Feb 2015-Oct 2016. Twelve-month post-drug initiation and 6-month post innovator-to-biosimilar switch retention analyses were conducted. All patients had sufficient claims history post-index and $>$ 2 claims of IFX or CT-P13 within the analysis period. Six-month analyses included: 1) matched analysis where each CT-P13 switch patient was matched to 5 IFX patients for IFX exposure prior to switch;2) unmatched analysis where CT-P13 switch patients were compared to the first 6 months of the IFX treatment naïve population. Log-binomial regression analyses were conducted to determine the relative risk (RR) of being retained on treatment at 6 or 12 months adjusted for age, sex, biologic treatment history and prescriber specialty.

Results: 6,491 patients had a claim of IFX or CT-P13. Of these, 1,160 and 1,324 patients had follow up time for inclusion in the 12-month naïve and 6-month post-switch analyses, respectively. Only IFX to CT-P13 switch utilization $(\mathrm{n}=101)$ was investigated. The risk adjusted probability of being retained on treatment after 12 months was $23 \%$ greater in the IFX group than in the CT-P13 group (RR IFX $=1.23,95 \%$ Confidence Interval $[\mathrm{CI}]$ $1.12-1.36, \mathrm{p}<0.0001)$. The probability of being retained was also greater in men, those who were bio-naive and those prescribed drugs by a rheumatologist. In the matched 6 months post-switch analysis the risk adjusted probability of being retained on treatment was $26 \%$ greater in the IFX maintenance group than in the CT-P13 switch group (RR IFX $=1.26,95 \%$ CI: $1.10-1.45$, $\mathrm{p}=0.0007)$. The probability of being retained was also greater in those prescribed drugs by a rheumatologist. Similar results were found in the unmatched analysis (RR IFX $=1.32,95 \%$ CI: 1.16-1.51, $<<0.0001$ ), where the probability of being retained was also greater in men and in those who were bio-naïve.

Conclusion: The findings from Germany demonstrate significant differences in the real-world utilization patterns of patients treated with IFX or CT-P13 in all labelled indications. Limitations of this study include a heterogenous population, disease severity and reasons for staying on treatment or switching could not be determined. Future analyses should capture clinical outcomes to better understand observed utilization patterns.

65

Is Earlier Golimumab Treatment Initiation in PsA and AS Patients Associated with Improved Outcomes?

Suneil Kapur (University of Ottawa, Ottawa); Proton Rahman (Memorial University, St. John's); Michelle Teo (Penticton); Jodie Reis (University of Saskatoon, Saskatoon); Rajwinder Dhillon (Niagara Falls); Pauline Boulos (Dundas); Raman Rai (McMaster University, Hamilton); Regan Arendse

Personal non-commercial use only. The Journal of Rheumatology Copyright (C) 2018. All rights reserved 
(University of Saskatchewan, Saskatoon); Julie Vaillancourt (JSS Medical Research, Montreal); Emmanouil Rampakakis (JSS Medical Research, Montreal); Allen Lehman (Janssen Inc, Toronto); Francois Nantel (Janssen Inc, Toronto); Brendan Osborne (Janssen Inc, Toronto)

Objectives: To assess the impact of disease duration on clinical and patient-reported outcomes in patients with psoriatic arthritis (PsA) or ankylosing spondylitis (AS) treated with subcutaneous golimumab (GLM) in Canadian routine practice.

Methods: BioTRAC is an ongoing, prospective registry of patients initiating treatment with infliximab or GLM for inflammatory arthritis or with ustekinumab for PsA. Eligible patients for this analysis included PsA and AS patients initiating GLM treatment since 2010. Disease duration at registry entry was categorized by tertiles as early (PsA: <2years; AS: < 1year), mid (PsA: 2-5years; AS: 1-2years) and long-term (PsA: $\geq 6$ years; AS: $\geq 3$ years) duration. Standard outcomes were assessed including low disease activity (LDA), remission (based on DAS28-ESR $<2.6$ and CDAI $\leq 2.8$ ), and minimal disease activity (MDA) achievement for PsA patients, and inactive disease (ASDAS < 1.3) for AS patients. The impact of disease duration on disease activity at 12 and 24-months was determined by multivariate logistic regression and general linear models adjusted for age, gender, prior biologic treatment, and baseline disease parameters.

Results: 253 PsA patients (54.4\% female) and 376 AS patients (58.6\% male) were included with a mean (SD) age of 52.7 (13.2) and 44.7 (13.3) years, respectively. Most patients were biologic naïve (PsA: 95.3\%; AS: 95.5\%). The mean (SD) duration of PsA and AS at baseline was 5.7 (7.7) and of 5.4 (9.8) years, respectively. In the PsA group, significantly greater improvements in MD global assessment (MDGA) and pain from baseline to month 24 were observed amongst patients treated at early term. Based on multivariate logistic regression analyses, patients treated at later stage disease were significantly less likely than patients treated early to achieve MDA (OR: 0.10; $\mathrm{p}=0.025$ ) and CDAI LDAS (OR: 0.06; $\mathrm{p}=0.002$ ) at 24-month. No association existed between PsA duration and LDA, remission and MDA achievement at 12-months. Among AS patients treated at early term, significantly greater improvements in BASFI from baseline to month 24 were observed $(-2.9,-1.4 ; \mathrm{p}=0.03)$. Based on multivariate logistic regression analyses, AS patients treated at mid- and long-term were significantly less likely than patients treated at early term to achieve inactive disease based on ASDAS at 12-months, with odds ratios of $0.21(\mathrm{p}=0.028)$ and $0.12(\mathrm{p}=$ $0.003)$, respectively. Disease duration was not associated with AS inactivity at 24-month.

Conclusion: The results of this analysis demonstrate that earlier treatment of PsA and AS with GLM in real-world is associated with improved outcomes, particularly in selected patient-reported outcomes at 12 and 24-months.

\section{6}

Validation of New Potential Targets for Remission and Low Disease Activity in Psoriatic Arthritis in Patients Treated with Golimumab Laura Coates (University of Oxford, Oxford); Proton Rahman (Memorial University, St. John's); Eliofotisti Psaradellis (JSS Medical Research, Montreal); Emmanouil Rampakakis (JSS Medical Research, Montreal); Brendan Osborne (Janssen Inc, Toronto); Allen Lehman (Janssen Inc, Toronto); Francois Nantel (Janssen Inc, Toronto)

Objectives: Treat to target recommendations in PsA state that the target of treatment should be remission or low disease activity (LDA). So far, the only validated target available was the minimal disease activity (MDA) criteria. Other potential targets have been developed including very low disease activity (VLDA) and the Disease Activity in PsA (DAPSA) score remission. Potential targets for LDA include DAPSA and clinical (cDAPSA) or the minimal disease activity (MDA) criteria for which modifications have been suggested to mandate individual components. Using an existing real-world cohort, the objectives were to calculate the proportion of patients achieving these criteria, their prognostic value and the overall patient impact of these disease states.

Methods: BioTRAC is an ongoing, prospective registry of inflammatory arthritis patients initiating treatment with infliximab, golimumab (GLM) or ustekinumab. PsA patients treated with GLM were included. Data collected at baseline, 6 and 12 months were used.

Results: A total of 188 patients (53\% female) were included with a mean disease duration of 5.5 years. VLDA was achieved in $2.1 \%, 17.2 \%$ and $15.6 \%$ of patients at baseline, 6 months and 12 months, respectively and those patients had a significant reduction in the number of TJC, SJC, enthesitis and PASI $(\mathrm{p}<0.002) .75 \%$ and $53.3 \%$ of patients in DAPSA and cDAPSA remission, respectively, also achieved VLDA $(\mathrm{p}<0.001)$. Patients who did not achieve cDAPSA nor DAPSA never achieved VLDA. Higher HAQ scores $(p<0.03)$ were observed in patients achieving remission with remaining dactylitis or active skin disease (BSA $\leq 10 \%$; cDAPSA only). The proportions of patients achieving MDA also achieving MDA Joints, MDA Skin, MDA Joints \& Skin and DAPSA LDA were $83.8 \%, 86.9 \%, 70.7 \%$ and $98.7 \%$, respectively. Patients achieving any MDA target had significantly lower SJC, TJC, PASI, dactylitis and enthesitis scores compared to non-achievers $(p<0.006)$. Patients achieving DAPSA or cDAPSA LDA showed significant reductions in SJC, TJC, PASI, dactylitis and enthesitis scores compared to non-achievers $(\mathrm{p}<0.05)$

Conclusion: VLDA is the most stringent new potential targets for remission in PsA. There was a high level of correlation between scores although residual activity in dactylitis and skin despite DAPSA remission has some impact on patients' function. MDA Joints, MDA Skin, MDA Joints \& Skin, DAPSA LDA and cDAPSA LDA represent new potential targets for LDA in PsA. Patients achieving either DAPSA endpoints, however, did not show a significant reduction in skin disease indicating that those two endpoints are more restricted to joint symptoms.

\section{7}

miR-21-5p as a Biomarker of Psoriatic Arthritis and Response to Treatment

Rohan Machhar (Toronto Western Hospital, Toronto); Justine Ye (Toronto Western Hospital, Toronto); Vinod Chandran (University of Toronto and University Health Network, Toronto); Dafna Gladman (University of Toronto, Toronto)

Objectives: Psoriatic arthritis (PsA) is an inflammatory arthritis occurring in patients with psoriasis. miRNA's are small non coding RNAs whose main function, at a post transcriptional level, is to modulate the expression of target genes via translation inhibition or mRNA degradation. Several studies have shown links between altered miRNA expression with the pathogenesis of several autoimmune disorders. A recent study identified miR-215p to be upregulated in early PsA and early rheumatoid arthritis (RA) and found down regulation post 12 weeks of methotrexate (MTX) treatment. Mir-21-5p was found upregulated in several inflammatory and autoimmune diseases such as systemic sclerosis, systemic lupus erythematous, RA, diabetes type 1 and psoriasis. The objective of this study was to determine the role of miR-21-5p as a biomarker for PsA and response to methotrexate.

Methods: Patients with PsA and psoriasis without arthritis (PsC) have been followed prospectively according to a standard protocol. Whole blood RNA samples collected in Tempus tubes from 40 patients with early PsA $(<2$ years disease duration and not receiving biologic therapy), 40 patients with PsC who have been confirmed by rheumatologist not to develop PsA ( $>10$ years disease duration, not receiving biologic therapy, and matched to PsA patients on age, sex, psoriasis duration, and age of psoriasis onset), and $42 \mathrm{HC}$ (matched to patients based on age, sex). RNA samples were also obtained from 30 PsA patients before and after 6 months of treatment with methotrexate. RNA was extracted using the Tempus Spin RNA Isolation Kit with a genomic DNA removal step. MiR-21-5p was validated using droplet digital PCR (ddPCR), a novel and sensitive technology for miRNA quantification. MiRNA-21-5p was compared among the three patient groups and in PsA patients before and after MTX.

Results: Significant up regulation of miR-21-5p was seen in both early PsA (122.7) and in PsC (58.5) in comparison to HC $(11.5, \mathrm{p}<0.001)$. There was more upregulation in early PsA compared with PsC ( $<<0.001)$, miR-21-5p was significantly down regulated 24 weeks post treatment in PsA patients

Personal non-commercial use only. The Journal of Rheumatology Copyright $(\subset) 2018$. All rights reserved. 
$(\mathrm{p}<0.008)$, the results correlated with the actively inflamed joints $(\mathrm{r}=0.897$, $\mathrm{p}=<0.0001)$.

Conclusion: Upregulation of miRNA $-21-5 p$ in patients with psoriatic disease compared to controls, and more so in PsA than PsC supports its role as biomarker for inflammation and for PsA. The down regulation post MTX treatment suggests a potential role as biomarker for response to treatment.

Mortality Rates and Causes in Psoriasis and Psoriatic Arthritis Patients Anastasiya Muntyanu (University of Toronto, Toronto); Ofir Elalouf (Toronto Western Hospital, Toronto); Justine Ye (Toronto Western Hospital, Toronto); Vinod Chandran (University of Toronto and University Health Network, Toronto); Dafna Gladman (University of Toronto, Toronto)

Objectives: Psoriatic arthritis (PsA) is chronic inflammatory musculoskeletal disease that affects about $30 \%$ of people with psoriasis, and can lead to joint damage and disability. There is evidence for increased risk of comorbidities and increased mortality risk. Our previous study demonstrated a standard mortality ratio of 1.62 . The goal of this study is to determine the changes in mortality rates and causes of death over the last four decades in patients with PsA followed prospectively.

Methods: Patients regularly followed at the University of Toronto PsA clinic between January 1, 1978-December 30, 2016 were included in the study. Patients are followed at 6-12 month intervals according to a standard protocol which includes clinical, laboratory and treatment information. Radiographs are performed at 2-year intervals. Information on deaths has been collected prospectively through interviews with relatives and family physicians, obituaries in the newspaper, as well as regular lost to follow up studies, most recently in 2017. Deaths are verified through regular linkage with the provincial cancer database and mortality database,

Results: Among 1456 patients followed over 14675.01 patient-years, there were 186 confirmed deaths (12.9\%) (89 females and 97 males), with a death incidence of 12.6 cases per 1000 patient-years. Major causes of death included malignant neoplasms, diseases of the circulatory system, diseases of the respiratory system, and unknown causes. The average age of onset of psoriasis was 27.6 years for the alive group, and 36.9 years for the deceased. Age at onset of PsA, was 37.1 years and duration 5.7 years for the alive group and 44.4 years with 10.4 years disease duration for the deceased. Duration of follow-up was 10 yrs for the alive group and 8 for the deceased. Deceased patients had more severe disease at presentation with higher number of clinically and radiologically damaged joints. $44 \%$ in the alive group were on biologics as compared to $16 \%$ in the deceased (although this may reflect the fact that biologics became available only in 2000). While the opposite trend was observed for DMARDS where $41 \%$ of the alive group were treated with DMARDs and $63 \%$ of the deceased group. The NSAIDs use for the two groups was comparable.

Conclusion: The rate of death was $1.26 \%$ per year. Patients who died were older at presentation, had longer disease duration, more severe disease. Further analyses are needed to understand the mortality risk and effect of therapies.

\section{9}

Efficacy and Safety of Ixekizumab in Patients with Active Psoriatic Arthritis and Previous Inadequate Response to TNF inhibitors: 52-week Results from a Phase 3 Study

Mark Genovese (Stanford University Medical Center, Palo Alto); Philip Helliwell (University of Leeds School of Medicine, Leeds); Bernard Combe (Hopital Lapeyronie, Montpellier); Joel Kremer (Albany Medical College, Albany); David Adams (Eli Lilly and Company, Indianapolis); Chin Lee (Eli Lilly and Company, Indianapolis); Lisa Kerr (Eli Lilly and Company, Indianapolis); Peter Nash (University of Queensland, Brisbane); Proton Rahman (Memorial University, St. John's)

Objectives: Ixekizumab (IXE), high affinity monoclonal antibody, selectively targets interleukin-17A. IXE was superior to placebo (PBO) in improving the signs and symptoms of PsA after 24-weeks of treatment (SPIRIT-P2; NCT02349295) active psoriatic-arthritis (PsA) patients who had an inadequate response to tumor-necrosis-factor inhibitors (TNFi).1 We report the Week-52 interim efficacy and safety findings of IXE treatment during the Extension Period (EP) of SPIRIT-P2 (Weeks24-156).

Methods: SPIRIT-P2 is a Phase-3, multicenter, double-blind study. All 363 patients had an inadequate response to 1-2 TNFi or were intolerant to TNFi. During Double-Blind Treatment-Period (DBTP; Weeks0-24), patients were randomly assigned 1:1:1 to subcutaneous administration of either $80 \mathrm{mg}$ IXE every 4 weeks $(\mathrm{Q} 4 \mathrm{~W} ; \mathrm{N}=122)$ or every 2 weeks $(\mathrm{Q} 2 \mathrm{~W} ; \mathrm{N}=123)$ following a $160 \mathrm{mg}$ starting dose at Week-0, or PBO $(\mathrm{N}=118)$. Of these, 310 patients completed DBTP and entered EP (Weeks 24-156). Patients randomized to IXE at Week- 0 continued the same dose regimen in the EP. PBO patients were re-randomized (1:1) to IXE Q4W or Q2W at Week-16 (inadequate responders) or 24. In this interim analysis, efficacy (up to Week-52) and safety (up to Week-156) were analyzed using EP population, defined as all patients who received at least 1 dose of study drug in EP. Missing values were considered non-response for categorical data and were imputed by modified baseline observation carried forward for continuous data.

Results: In DBTP, a significantly higher percentage of patients achieved ACR20 at Week-24 with IXE Q4W (53\%) or Q2W (48\%) than with PBO (20\%).1 EP included: 52 years mean age, $47 \%$ male, mean time since PsA onset 12 years, and mean tender and swollen joint counts at baseline were 23 and 12, respectively. For EP patients who were initially randomized to IXE Q4W or Q2W during DBTP, ACR20 responses at Week-52 were 68\% and 59\%, respectively. Patients treated with PBO (during DBTP) and re-randomized to IXE Q4W or Q2W during EP, ACR20 responses at Week-52 were $61 \%$ and $50 \%$, respectively. Majority of adverse events (AEs) were mild or moderate in severity. Serious AEs occurred in 15 patients, and one death occurred in EP population: a myocardial infarction in a PBO/IXE Q2W patient 502 days after starting IXE.

Conclusion: IXE demonstrated sustained improvement in the signs and symptoms of PsA across treatment groups during the EP. The safety profile of IXE observed in the EP population was consistent with the safety profile of the intent-to-treat population in the DBTP of SPIRIT-P2.

\section{0}

\section{The Skin Microbiome in Psoriatic Disease: A Systematic Review}

Meital Yerushalmi (University of Toronto, Toronto); Ofir Elalouf (Toronto Western Hospital, Toronto); Melanie Anderson (University Health Network, Toronto); Vinod Chandran (University of Toronto and University Health Network, Toronto)

Objectives: Psoriatic arthritis (PsA) is an inflammatory arthritis associated with psoriasis that affects $30 \%$ of psoriasis patients. Despite advances in our understanding of the role of genomics in these conditions, their pathogenesis is not fully understood. The growing interest in the role of the human microbiome in health and disease, afforded by advances in sequencing methods in recent years, prompted researchers to explore an important environmental factor in the pathogenesis of psoriatic disease: the skin microbiome. We aim to systematically review skin microbiome studies in psoriatic disease and define the microbiome characteristics of the psoriatic plaque.

Methods: Strategies to find studies investigating the bacterial skin microbiome of the psoriatic plaque using culture-independent sequencing technology were developed for Ovid databases Medline, Embase, Medline Epub Ahead of Print and In-Process \& Other Non-Indexed Citations, and Cochrane Central Register of Controlled Trials (CENTRAL). Appropriate subject headings and keywords were selected for each database, including terms for psoriatic disease, for bacteria or microbiota, and for skin. Results were limited to humans and English, and no limits for date or publication type were used. Two authors screened resulting citations independently of each other for inclusion and extracted data using a standardized data extraction form. Disagreements for inclusion were resolved by a third reviewer. Included studies were critically appraised through a review of methodology and study design.

Results: The searches resulted in 2985 citations, of which 8 studies (including 3 abstracts) met the inclusion criteria. 6 studies investigated the cutaneous microbiome of psoriasis only, and 2 studies compared its charac- 
teristics between psoriasis and PsA. Among psoriasis studies, reports of compositional shifts of plaque bacterial phyla vary between studies for most skin-dominant phyla. Likewise, no consensus was reached with respect to bacterial diversity of the plaque compared to unaffected or healthy skin. Heterogeneity in study design likely contributes to differences in findings. Sample collection technique included swabbing in 6 studies, biopsy in 1 study, and curette in 1 study. In addition, only 3 studies reported cutaneous microenvironment-based sample collection and data analysis, and variations existed in the exclusion of confounders such as antibiotics.

Conclusion: Owing to lack of consistency in methodology, no consensus can be drawn regarding the characteristics of the skin microbiome in psoriatic disease. Future studies employing standardized methodological techniques may improve our understanding of the role of the cutaneous microbiome in psoriatic disease.

71

The Value of Carotid Ultrasound in Cardiovascular Risk Stratification in Patients with Psoriatic Disease

Curtis Sobchak (University of Toronto, St. Catharines); Shadi Akhtari (Women's College Hospital, Toronto); Paula Harvey (Division of Cardiology, Women's College Hospital, Toronto); Dafna Gladman (University of Toronto, Toronto); Vinod Chandran (University of Toronto and University Health Network, Toronto); Richard Cook (University of Waterloo, Waterloo); Lihi Eder (Women's College Hospital, Toronto)

Objectives: Patients with psoriatic disease (PsD) are at a high risk of developing cardiovascular disease. The performance of clinical algorithms for cardiovascular risk stratification, such as the Framingham Risk Score (FRS), is sub-optimal in this patient population. The study aims were: 1) to assess whether subclinical atherosclerosis, as evaluated by carotid ultrasound, could predict future cardiovascular events (CVE) in patients with PsD and 2) to determine whether incorporation of imaging data could improve the properties of clinical cardiovascular algorithms.

Methods: In this cohort analysis, patients with psoriasis and psoriatic arthritis from the University of Toronto Psoriatic Disease Program underwent an ultrasound assessment of the carotid artery at baseline. The extent of atherosclerosis was assessed using mean carotid intima media thickness (cIMT in $\mu \mathrm{m}$ ) and total plaque area (TPA in $\mathrm{cm} 2$ ). Patients were followed according to a standardized protocol at 6-12 months intervals. Incident CVE that occurred following the ultrasound assessment were identified from the cohort database. A cardiologist reviewed the medical records related to each CVE and classified them to major CVE (myocardial infarction, stroke, revascularization or cardiovascular death) and minor CVE (angina, TIA, CHF exacerbation). The association between measures of carotid atherosclerosis and the risk of developing future CVE was evaluated using Cox proportional hazard models after adjusting for FRS.

Results: Overall, 607 patients with PsD were assessed and 34 patients had incident CVE which were confirmed by the cardiologist. 26 of the patients who developed CVE (76.4\%) were considered high risk by FRS, as compared to $146(25.4 \%)$ of those without events. In multivariable regression models, both TPA (Hazard Ratio (HR) 2.29, 95\% Confidence Interval (CI) 1.55, 3.37; p < 0.001), mean cIMT (HR 1.21, 95\% CI 1.07, 1.38; $\mathrm{p}=0.002$ ) and bilateral carotid plaques (HR 2.83, 95\% CI 1.03, 7.76, $\mathrm{p}=0.04)$ predicted incident CVE after controlling for FRS. The results were essentially similar after restricting the analysis to patients with major CVE and after excluding patients with a history of CVE at baseline.

Conclusion: Patients with PsD with subclinical carotid atherosclerosis are more likely to develop future incident CVE. Carotid ultrasound could potentially improve the identification of patients with psoriatic disease who are at high cardiovascular risk.

72

A Novel Role for the Psoriatic Arthritis Impact of Disease Questionnaire (PsAID)

Kishor Johnson (Schulich School of Medicine and Dentistry, London); Justine Ye (Toronto Western Hospital, Toronto); Vinod Chandran (University of Toronto and University Health Network, Toronto); Dafna Gladman (University of Toronto, Toronto)

Objectives: Psoriatic arthritis (PsA) is an inflammatory arthritis that occurs in about $30 \%$ of patients with Psoriasis (Ps). Recently, a new Patient Reported Outcome Measure (PROM), Psoriatic Arthritis Impact of Disease (PsAID) was specifically developed for PsA Patients. The two versions of the PsAID, PsAID-9 and PsAID-12, are scored on a Numeric Rating Scale (NRS) of 0-10. The Minimal Disease Activity (MDA) is a composite outcome measure for PsA patients, which uses the Health Assessment Questionnaire (HAQ) as one criterion. However, the HAQ does not correlate well with measures of disease activity as PsA disease duration increases, and its use in the assessment of disease activity has been questioned. Our objectives were to 1) validate the PsAID within our patient cohort, 2) determine if the PsAID can replace any of the other PROMs administered in the clinic, and 3) determine if the PsAID can replace the HAQ in the MDA.

Methods: Patients were recruited at the PsA Clinic at Toronto Western Hospital (TWH). All patients completed the PsAID and 10 other PROMs. Various measures of disease activity were recorded by a physician at each visit. Descriptive statistics (mean, median, SD, min, max) were calculated for all PROMs. PsAID cut-offs for use in the MDA were generated based on Remission (REM) and Low Disease Activity (LDA) disease states in the Clinical Disease Activity for Psoriatic Arthritis Index (cDAPSA).

Results: 115 patients completed the PsAID. There were 70 males, 45 females, with a mean PsA duration of $18.7( \pm 11.6)$ years. Mean scores of PsAID-9 and PsAID-12 were $3.4( \pm 2.4)$ and $3.2( \pm 2.3)$ respectively. The PsAID correlated moderately well with 9 of the PROMs administered in the clinic $(\mathrm{R} 2=0.51-0.78)$. Four PsAID cutoffs were generated for use in the MDA: REM PsAID-9, REM PsAID-12, LDA PsAID-9, and LDA PsAID-12. All four versions of the PsAID MDAs had a sensitivity greater than $85 \%$ with the HAQ MDA, and three versions of the PsAID MDA had a specificity greater than $85 \%$ with the HAQ MDA.

Conclusion: Our cohort had lower mean PsAID scores than previously reported series suggesting that our patients are monitored carefully. The only moderate correlations with other PROMs suggest that the PSAID cannot replace any of these PROMs. The high sensitivity and specificity of the PsAID MDA with the HAQ MDA suggest that the PsAID is an effective replacement for the HAQ in the MDA.

73

Lost-To-Follow-up Investigation in Psoriatic Arthritis

Alan Zhou (University of Toronto, Toronto); Anastasiya Muntyanu (University of Toronto, Toronto); Vivian Szeto (Toronto Western Hospital, Toronto); Justine Ye (Toronto Western Hospital, Toronto); Vinod Chandran (University of Toronto and University Health Network, Toronto); Dafna Gladman (University of Toronto, Toronto)

Objectives: Clinical databases are useful sources of information for cohort studies. However, loss-to-follow-up (LTF) can compromise internal validity of such studies. Our objectives were to: 1) Identify patients who were lost-to-follow-up (LTF) from the Psoriatic Arthritis (PsA) clinic; 2) Determine reasons for LTF; 3 ) Determine whether there were differences at first visit between patients seen regularly (ongoing) and those LTF.

Methods: Patients with PsA have been followed prospectively at the PsA clinic since 1978 with a standard protocol including demographics, clinical features, laboratory tests at 6-12 month intervals, and radiographs at 24 month intervals. LTF was defined as not seen in PsA clinic for at least 2 years as of June 2017. Descriptive statistics was used to compare LTF patients with ongoing patients. A phone questionnaire was administered to LTF patients to determine reasons for LTF, patient characteristics, and disease severity, and to invite them to return to clinic. Responses were compared among those who agreed to return and those who did not.

Results: Of 1436 patients registered at the PsA clinic who had more than one visit, 17 had appointments in summer of 2017, 177 had died, 75 had declined to be contacted from previous LTF studies, and 625 were ongoing. Of 542 patients identified as LTF in June 2017, no attempts were made to contact 141 who had been LTF since 1990. Of 401 patients who were called,

Personal non-commercial use only. The Journal of Rheumatology Copyright (c) 2018. All rights reserved. 
126 completed the questionnaire of whom 62 agreed to return to clinic, 58 were alive but declined or were unavailable to complete the questionnaire, 9 had died, and 208 could not be reached. Distance to clinic was the primary reason for becoming LTF, followed by being followed elsewhere, and doing well (no need for follow-up). 542 LTF patients were compared to 625 ongoing patients. LTF patients were younger, had longer disease duration at first visit, and more were unemployed. There were no significant differences in disease severity or co-morbidities between the two groups at first visit. Comparing returning LTF patients to non-returning LTF patients, there were no significant differences in disease severity or co-morbidities.

Conclusion: LTF over the past 39 years was approximately 30\%. Major reason for LTF was distance to clinic. LTF patients were not substantially different from ongoing patients at first visit, and those LTF who agreed to return were similar to those who did not. The influence of LTF on outcomes remains to be determined.

\section{4}

Changes in Lipid Levels and Incidence of Cardiovascular Events Following Tofacitinib Treatment in Patients with Psoriatic Arthritis: An Integrated Analysis across Phase 3 and Long-Term Extension Studies Dafna Gladman (University of Toronto, Toronto); Christina Charles-Schoeman (University of California, Los Angeles); Iain McInnes (University of Glasgow, Glasgow); Douglas Veale (St. Vincent's University Hospital and University College Dublin, Dublin); Bruce Thiers (Medical University of South Carolina, Charleston); Daniela Graham (Pfizer Inc, Groton); Cunshan Wang (Pfizer Inc, Groton); Thomas Jones (Pfizer Inc, Collegeville); Robert Wolk (Pfizer Inc, Groton); Ryan DeMasi (Pfizer Inc, Collegeville)

Objectives: Cardiovascular (CV) disease and cardiometabolic syndrome are common comorbidities/causes of mortality in psoriatic arthritis (PsA) patients. Tofacitinib is an oral Janus kinase inhibitor under investigation for PsA. We investigated changes in lipid levels and incidence of CV events in tofacitinib-treated PsA patients.

Methods: Data were analyzed for patients who received tofacitinib 5 or $10 \mathrm{mg}$ BID or placebo, integrated across two Phase (P)3 studies (OPAL Broaden [12 months; NCT01877668; including adalimumab control]; OPAL Beyond [6 months; NCT01882439]) and one ongoing LTE study (OPAL Balance [data cut-off May 2016; NCT01976364]). Lipid levels are reported from the placebo-controlled period (Months 0-3) of P3 studies. Blood pressure, hypertension events, and adjudicated major adverse CV events (MACE) are reported for all patients who received $\geq 1$ tofacitinib dose in P3 and LTE studies, pooled across doses. Incidence rates (IR; patients with events/100 patient-years) are reported.

Results: This analysis included 783 patients (776 patient-years of tofacitinib exposure); treatment duration was 1-927 days ( 230 months). After 3 months of treatment with tofacitinib 5 and $10 \mathrm{mg}$ BID, respectively, dose-dependent increases from baseline in low-density lipoprotein (LDL: $9.2 \%$ and $14.0 \%$ ) and high-density lipoprotein (HDL: $10.0 \%$ and 14.0\%) were observed; minimal changes were observed with placebo (LDL: $4.0 \%$; HDL: $-0.8 \%$ ). No changes in the LDL/HDL or total cholesterol/HDL ratio were shown with tofacitinib. Over 24 months in P3 and LTE studies, no clinically significant changes in systolic or diastolic blood pressure were observed. Hypertension events were reported in 38 patients (4.9\%; IR: 4.93 [95\% confidence interval: $3.49,6.77])$; 4 led to patient discontinuation, and 2 were serious adverse events. MACE were reported for 3 patients $(0.4 \%)$ receiving tofacitinib: sudden cardiac death (after 57 days of exposure), non-fatal myocardial infarction (197 days), and non-fatal ischemic stroke (80 days). The IR for $\operatorname{MACE}(0.38[0.08,1.11])$ was within the range reported in tofacitinib studies for psoriasis (IR 0.24 [0.15, 0.37]; 8,759 patient-years) and rheumatoid arthritis (IR 0.38 [0.30, 0.47]; 21,886 patient-years). No dose-dependent effects on blood pressure, hypertension, or MACE were apparent.

Conclusion: In tofacitinib-treated PsA patients, dose-dependent increases in lipid levels were observed at Month 3. In P3 and LTE studies, no clinically significant changes were seen in blood pressure over 24 months or incidence of hypertension over 30 months of observation. Incidence of MACE was within the range reported in tofacitinib studies in psoriasis and rheumatoid arthritis; however, the long latency of MACE requires longer-term observation.

\section{5}

Tofacitinib Treatment in Patients with Psoriatic Arthritis and Rates of Radiologic Progression According to Baseline CRP Levels: Results from a Phase 3 Clinical Study

Dafna Gladman (University of Toronto, Toronto); Oliver FitzGerald (St Vincent's University Hospital, Dublin); Arthur Kavanaugh (University of California, La Jolla); Daniela Graham (Pfizer Inc, Groton); Cunshan Wang (Pfizer Inc, Groton); Lara Fallon (Pfizer Canada Inc, Montreal)

Objectives: Tofacitinib is an oral Janus kinase inhibitor under investigation for the treatment of psoriatic arthritis (PsA). We evaluated radiographic progression over 12 months in patients with PsA treated with tofacitinib.

Methods: The Phase 3 OPAL Broaden (NCT01877668) study enrolled patients with an inadequate response to $\geq 1$ conventional synthetic disease-modifying antirheumatic drug, who were tumor necrosis factor inhibitor-naïve. Patients were randomized to receive oral tofacitinib $5 \mathrm{mg}$ twice daily (BID; $\mathrm{N}=107)$, tofacitinib $10 \mathrm{mg}$ BID $(\mathrm{N}=104)$, adalimumab $40 \mathrm{mg}$ subcutaneous once every 2 weeks (active control; $\mathrm{N}=106$ ), or placebo $(\mathrm{N}=105)$. Patients who initially received placebo advanced to tofacitinib at Month (M)3. Radiographs of the hands and feet at baseline and M12 were scored independently by two assessors using the van der Heijde-modified Total Sharp Score for PsA (mTSS; range 0-528); average scores were used. For radiographs missing at M12, mTSS was calculated by linear extrapolation from an earlier mTSS. Changes from baseline in mTSS, erosion, and joint space narrowing (JSN) were reported overall, and for patients grouped by baseline C-reactive protein $(\mathrm{CRP})>2.87 \mathrm{mg} / \mathrm{L}$ or $\leq 2.87$ $\mathrm{mg} / \mathrm{L}$. The proportions of patients with radiographic non-progression (defined as either an increase from baseline in mTSS $\leq 0.5$ or $\leq 0$ and less than the smallest detectable change [SDC; $\leq 0.66$ derived from this trial]) at M12 are reported for all patients.

Results: Across all treatment groups at baseline, mean mTSS score ranged from 10.4-17.6 (89.7-93.4\% had score > 0), mean erosion from 6.9-10.8 (89.7-93.4\% had score $>0$ ), and mean JSN from 8.6-13.7 (35.8-47.7\% had score $>0$ ). At M12, among patients treated with tofacitinib 5 and $10 \mathrm{mg}$ BID and adalimumab, respectively, minimal mean changes from baseline in mTSS score $(0.01,-0.01$, and -0.07$)$, erosion $(0.02,-0.00$, and -0.06$)$, and JSN $(-0.01,-0.01$, and -0.01$)$ were detected. Changes from baseline were similar in patients with CRP $>2.87 \mathrm{mg} / \mathrm{L}$ or $\leq 2.87 \mathrm{mg} / \mathrm{L}$. Radiographic non-progression at M12 using either threshold was observed in $>90 \%$ of patients in all three groups. At M12, 95.9\%, 94.9\%, and 97.9\% of patients receiving tofacitinib $5 \mathrm{mg}$ BID, tofacitinib $10 \mathrm{mg}$ BID, and adalimumab, respectively, were non-progressors based on radiographic changes $\leq \mathrm{SDC}$ in mTSS.

Conclusion: Patients with PsA receiving tofacitinib showed minimal mean changes in mTSS at M12, regardless of whether baseline CRP level was > $2.87 \mathrm{mg} / \mathrm{L}$ or $\leq 2.87 \mathrm{mg} / \mathrm{L}$. At M12, $>90 \%$ of patients who received tofacitinib or adalimumab met radiographic non-progression criteria.

\section{6}

Ixekizumab Provides Sustained Improvement in Signs and Symptoms in Patients with Active Psoriatic Arthritis: Two-Year Results from a Phase 3 Trial

Philip Helliwell (University of Leeds School of Medicine, Leeds); Eric Lespessailles (University of Orléans, Orléans); Catherine Shuler (Eli Lilly and Company, Indianapolis); Lotus Mallbris (Eli Lilly and Company, Indianapolis); Janelle Erickson (Eli Lilly and Company, Bismarck); Roy Fleischmann (University of Texas Southwestern Medical Center, Dallas); Dafna Gladman (University of Toronto, Toronto)

Objectives: To assess the efficacy and safety of ixekizumab (IXE), a high-affinity monoclonal antibody that selectively targets interleukin (IL)-17A. Over 2 years in SPIRIT-P1 Phase 3 study (NCT01695239) enrolling patients with active psoriatic arthritis (PsA). Previous results showed that IXE was superior to placebo (PBO) in achieving ACR20

Personal non-commercial use only. The Journal of Rheumatology Copyright $(\subset) 2018$. All rights reserved. 
response at Week 24 in biologic disease-modifying antirheumatic drugs (bDMARD)-naïve PsA patients.

Methods: During the SPIRIT-P1 double-blind treatment (DBTP; Weeks 0-24), 417 bDMARD-naïve patients with active PsA were randomized 1:1:1:1 to subcutaneous 80 -mg IXE (160-mg starting dose at Week 0 ) every 4 weeks (Q4W), every 2 weeks (Q2W), 40-mg adalimumab (ADA) Q2W (active reference-arm), or PBO. Of these, 381 patients entered the extension period (Weeks 24-52), followed by the long-term extension period (Weeks 52-156). Here, data are presented for combined extension periods (CEP; Weeks 24-108) in patients who completed the initial 24-week treatment and received $\geq 1$ dose of study drug during CEP. IXE-randomized patients continued on IXE throughout CEP and, if receiving PBO and ADA, were re-randomized (1:1) to IXEQ4W/Q2W at Week 16 (inadequate responders) or Week 24; ADA patients had an 8-week washout period before receiving IXE. Efficacy measures included ACR20/50/70 responses, HAQ-Disability Index (HAQ-DI) score, DAS28 based on C-reactive protein (DAS28-CRP), $75 \% / 90 \% / 100 \%$ improvement in the Psoriasis Area and Severity Index (PASI 75/90/100), Leeds-Enthesitis Index (LEI), and Leeds-Dactylitis Index-Basic (LDI-B). Categorical data were compared using a logistic regression model with missing values imputed by non-responder imputation, which treats inadequate responders as non-responders.

Results: IXE provided persistent improvement in ACR 20/50/70 response rates through 2 years of treatment (Total-IXEQ4W and Total-IXEQ2W: 61\% and $62 \% ; 46 \%$ and $53 \% ; 31 \%$ and $35 \%$ ). Similar improvements were seen for PASI 75/90/100 (Total-IXEQ4W and Total-IXEQ2W: 62\% and 68\%; $53 \%$ and $63 \% ; 43 \%$ and $56 \%$ ). The resolution of enthesitis and dactylitis in Total-IXEQ4W and Total-IXEQ2W groups was achieved in $46 \%$ and $46 \%$ [LEI (0)], and $74 \%$ and $73 \%$ [(LDI-B (0)] of patients, respectively. Frequency of treatment-emergent adverse events (AEs) were similar across treatment arms; majority were mild/moderate and serious AEs occurred in 46 patients. One death occurred (ADA/IXEQ4W patient suffered a cerebrovascular accident at Week 108).

Conclusion: In bDMARD-naïve patients, IXE demonstrated clinically significant improvement in the signs and symptoms of PsA across both treatment groups up to 2 years of treatment. The safety profile of IXE was similar to that in the DBTP of SPIRIT-P1, SPIRIT-P2, and other Phase 3 studies of IXE in patients with plaque psoriasis.

\section{7}

Myocarditis in Systemic Lupus Erythematosus: A Single Centre Experience

Jaya Tanwani (Toronto); Kostantinos Tselios (University of Toronto, Toronto); Dafna Gladman (University of Toronto, Toronto); Jiandong Su (Toronto Western Hospital, Toronto); Murray Urowitz (University of Toronto, Toronto)

Objectives: Myocarditis is reported in approximately 3\% of patients with systemic lupus erythematosus (SLE). Given its rarity, limited evidence exists regarding optimal treatment and prognosis. The present study aims to describe the characteristics of lupus myocarditis (LM) in a defined SLE cohort.

Methods: The Toronto Lupus Clinic database $(\mathrm{n}=1876)$ was retrospectively reviewed to identify patients with LM from 1970 to June 2017. LM definition was based upon a combination of clinical signs and symptoms, as well as electrocardiographic, imaging and biochemical criteria. Patients' charts were reviewed to confirm diagnosis. Data on clinical, diagnostic, therapeutic variables and outcomes were collected in a pre-specified data-retrieval form. Descriptive statistics were used.

Results: Thirty patients ( 25 females, 10 Blacks) with LM were identified for a prevalence of $1.6 \%$. Their mean age was $34.8 \pm 12.9$ years and median SLE duration was 3 years. Most of them (28/30) had active disease in other organ systems with a mean SLEDAI- $2 \mathrm{~K}=11.1 \pm 8.2$. Concomitant central nervous system involvement was observed in 8 patients (26.7\%), vasculitis in $4(13.3 \%)$, renal disease in $10(33.3 \%)$, musculoskeletal in $4(13.3 \%)$, serositis in 13 (43.3\%), mucocutaneous in $5(16.7 \%)$ and hematological in $3(10 \%)$. Active serology (increased anti-dsDNA and/or decreased C3/C4) was detected in 22/30 patients (73.3\%). From the cardiological perspective, concomitant pericarditis was diagnosed in $11 / 30$ (36.7\%), while 12 patients developed congestive heart failure (cardiogenic shock in two). ECG findings included isolated T-wave and ST-segment abnormalities in 75\% and $45.8 \%$ respectively. Moderate-to-severe systolic dysfunction (left ventricular ejection fraction $<50 \%$ ) was documented in $72 \%$ and wall motion abnormalities in $67 \%$. Cardiac biomarkers (mainly troponin) were elevated in $76.2 \%$. Glucocorticoids were administered to all patients (median prednisone dose $35 \mathrm{mg}$ /day), while immunosuppressives were used concomitantly in $21 / 30(70 \%)$, mainly consisting of azathioprine, mycophenolate mofetil and cyclophosphamide. Antimalarials were used in 18 patients (60\%). Supportive treatment included renin angiotensin system inhibitors (16/30), beta-blockers (17/30), calcium-channel blockers (7/30) and antiplatelets/anticoagulants (18/30). Complete (6/30) or partial (18/30, mild residual systolic dysfunction) resolution was documented in $80 \%$ of the patients. Two patients died in the acute phase due to cardiogenic shock and another four due to cardiomyopathy/congestive heart failure in the long term.

Conclusion: The prevalence of LM was $1.6 \%$ in our cohort. It was usually detected early during disease course and accompanied by generalized lupus activity, either clinical or serological. Prognosis was favourable with complete or partial resolution in $80 \%$ of the patients.

\section{8}

Monophasic Disease Course Pattern in Systemic Lupus Erythematosus Kostantinos Tselios (University of Toronto, Toronto); Dafna Gladman (University of Toronto, Toronto); Zahi Touma (Toronto Scleroderma Program, Division of Rheumatology, Toronto Western Hospital, University Health Network; Institute of Health Policy, Management and Evaluation, University of Toronto, Toronto); Jiandong Su (Toronto Western Hospital, Toronto); SLICC Investigators (Toronto Western Hospital, Toronto); Murray Urowitz (University of Toronto, Toronto)

Objectives: Disease course in systemic lupus erythematosus (SLE) follows three distinct patterns over time, namely relapsing-remitting, persistently active and prolonged quiescent. We recently showed that a small subgroup of patients might follow a "monophasic" course, characterized by an initial active clinical presentation, and followed by prolonged clinical remission. The aim of the present study was to assess the prevalence and characteristics of such patients.

Methods: The Toronto Lupus Clinic inception cohort (patients enrolled within 18 months from diagnosis, $\mathrm{n}=883$ ) was investigated. Prolonged clinical remission was defined as a clinical SLEDAI-2K $=0$ (serology excluded), achieved within five years since diagnosis and maintained for $\geq$ 10 years. No between visit interval $>18$ months was allowed. Descriptive statistics were used.

Results: From the 267 patients who met the inclusion criteria, 27 (24 females, 21 Caucasians) achieved prolonged clinical remission (prevalence $10.1 \%$ ) in 1.2 years since diagnosis (median). At diagnosis their mean age was $39 \pm 14.1$ years. Clinical manifestations included mucocutaneous involvement in $19(70.4 \%)$, arthritis in $16(59.3 \%)$, serositis in $5(18.5 \%)$, nephritis in 7 (25.9\%, class II in three, class III in one, class IV in three patients), central nervous system in $2(7.4 \%)$ and cytopenias in $15(55.6 \%)$. Serology was active in $13 / 27$ patients at enrolment (all with anti-dsDNA antibodies, 9 with concomitant low C3/C4). Initial SLEDAI-2K was $10.7 \pm$ 11.6. Ten years after achieving remission, $40.7 \%$ were still on antimalarials (from 59.3\%), 22.2\% on glucocorticoids (from 63\%) and $11.1 \%$ on immunosuppressives (from $33.3 \%$ ). Mean prednisone dose at 10 years was $5.3 \pm$ $3.2 \mathrm{mg} /$ day (from $16.8 \pm 9.5 \mathrm{mg} /$ day at enrolment), while SLICC/DI was 0.96 \pm 1.06 . Beyond 10 years, 7 patients $(25.9 \%)$ relapsed at $15.2 \pm 3.8$ years since diagnosis. Twenty of the 27 patients $(7.5 \%$ of the entire cohort) sustained remission for the entire length of follow-up (17.8 \pm 6.7 years) and thus had pure monophasic disease. Twelve patients (44.4\%) were not taking any medications after 10 years. Three patients died (11.1\%).

Conclusion: A monophasic disease course was observed in $7.5 \%$ of our inception SLE patients, while approximately half of them maintained remission without any medications after 10 years.

Personal non-commercial use only. The Journal of Rheumatology Copyright @ 2018. All rights reserved. 
79

What Does Evidence Based Medicine Tell Us on Treatments of Different Psoriatic Arthritis Subtypes? A Systematic Literature Review on Randomized Controlled Trials

Sibel Ureyen (University of Ottawa, Ottawa); Catherine Ivory (University of Ottawa, Department of Medicine, Division of Rheumatology, Ottawa); Umut Kalyoncu (Hacettepe University, Ankara); Jacob Karsh (University of Ottawa, Ottawa); Sibel Aydin (University of Ottawa, Ottawa Hospital Research Institute, Ottawa)

Objectives: The complexity of psoriatic arthritis (PsA) can be challenging when recruiting patients to clinical trials which might affect the homogeneity of the studies. The aim of this systematic literature review was to evaluate the inclusion criteria to be enrolled to randomized controlled trials (RCTs) in PsA to see whether different disease subtypes were targeted during the enrolment and/or whether different disease subgroups have been differentiated in the patient populations.

Methods: Medline, Embase and Cochrane databases were screened on October 4, 2016. The titles and the abstracts were independently screened by two investigators (SBU, CI). The selection criteria were patients with a diagnosis of PsA; RCTs on the efficacy of any treatment and age $\geq 18$ years. Studies other than RCTs were excluded. From the selected studies the inclusion criteria, the description of patient subgroups, the demographics of the study population, primary and secondary outcomes and efficacy data were retrieved.

Results: Within 2068 abstracts identified at screening, 76 articles and 52 abstracts were included in the final analysis. The main inclusion criteria were always determined by the number of joints, rather than the pattern of the disease. BASDAI, ASDAS, enthesitis and dactylitis were never a part of the inclusion criteria in any of the RCTs. Only 10 studies provided information about subtypes. Symmetrical polyarthritis was the majority of the patient population in most of the studies in a range between 52.2 to $66.9 \%$, followed by asymmetrical oligoarthritis in $9.8-42 \%$. The inclusion criteria being mostly based on tender and swollen joint counts resulted with the mean (SD) tender and swollen joints of 16.6-25.3 (10.4-18.3) and 9.6-25 (6.8-16.2), respectively. In terms of outcomes, all studies have used the responses in joints counts, mostly ACR 20/50/70 responses. Enthesitis or dactylitis were included in the primary outcomes in 5 RCTs and secondary in 20 RCTs. BASDAI was determined as the primary outcome only in one study and secondary in 3 studies. For the axial disease, 7 studies had given information about BASDAI changes or responses. The response in enthesitis and dactylitis was evaluated more frequently (21 studies). The efficacy on DIP disease or arthritis mutilans was never given.

Conclusion: There is good evidence in the literature to guide the clinicians on how to treat the PsA patients with polyarticular disease but there is a gap of knowledge on the treatment of the other disease subtypes such as oligoarticular disease.

\section{0}

A Closer Look at Enthesitis on Ultrasound: The Relationship between Individual Sonographic Findings and Physical Examination

Sibel Aydin (University of Ottawa, Ottawa Hospital Research Institute, Ottawa); Sibel Ureyen (University of Ottawa, Ottawa); Esen Gunal (Istanbul Medeniyet University, Istanbul); Conchi Gallego (Leeds Institute of Molecular Medicine, University of Leeds and NIHR Leeds Musculoskeletal Biomedical Research Unit, Leeds Teaching Hospitals, Leeds); Fatos Arslan (Istanbul Medeniyet University, Istanbul); Zoe Ash (Unit of Rheumatology, Hospital Universitario La Paz, Madrid); Dennis McGonagle (Leeds Institute of Molecular Medicine, University of Leeds and NIHR Leeds Musculoskeletal Biomedical Research Unit, Leeds Teaching Hospitals, Leeds); Helena Marzo-Ortega (Leeds Teaching Hospitals and University of Leeds, Leeds); Dafna Gladman (University of Toronto, Toronto); Lihi Eder (Women's College Hospital, Toronto)

Objectives: Enthesitis is a characteristic sign of spondyloarthritis. Physical examination is neither sensitive nor specific for assessing enthesitis. Ultrasonography (US) has the advantage of visualizing both soft tissue and bony changes in addition to detecting blood flow. Its comparison with physical examination is mostly reported with overall scores on multiple entheseal sites, rather than individual lesions/sites. In this study, we aimed to assess the correlation between various sonographic features and clinical enthesitis to understand the clinical significance of individual lesions on US. Methods: For this analysis, the raw data of 3 previous studies were used (1-3). Physical examination was determined as tenderness and/or swelling. The US scans were performed by 2 experienced investigators. Same elementary lesions were used to define enthesitis and presence/absence data was used in the analysis.

Results: Within 377 patients ( 341 with psoriatic arthritis, 36 with psoriasis), 2298 entheses were compared for US and physical examination. Patients with clinical Achilles enthesitis had more frequent abnormalities on US [hypoechogenicity (physical examination (+) vs (-): $40.7 \%$ vs $16.9 \%$; p < $0.001)$, thickening $(25.9 \%$ vs $12.9 \% ; \mathrm{p}=0.01)$, Doppler positivity $(16.7 \%$ vs $5.3 \% ; \mathrm{p}=0.002)$ and erosions $(14.8 \%$ vs $6.1 \% ; \mathrm{p}=0.02)]$. Similarly, hypoechogenicity $(43.3 \%$ vs $20.3 \%$; 0.03$)$, enthesophytes/calcifications $(46.7 \%$ vs $28 \% ; \mathrm{p}=0.03)$ and erosions $(13.3 \%$ vs $3.9 \% ; \mathrm{p}=0.016)$ at patellar tendon origin were more common in patients with clinical enthesitis. The clinical quadriceps enthesitis was related to hypoechogenicity on US $(69.6 \%$ vs $35.8 \% ; \mathrm{p}=0.001$ ) and patients with clinical enthesitis on the lateral elbow had more frequent Doppler signals $(20.8 \%$ vs $5.3 \% ; p=0.007)$. The rest of the entheseal sites were uncoupled from the US features, especially distal patellar tendon insertion and plantar fascia. In parallel with this analysis, the highest agreement between the clinical enthesitis and ultrasonographic features were seen for the Achilles enthesis and proximal patellar tendon origin for the same abnormalities as above, although the agreement was poor for all of them $(\mathrm{k}=0.08-0.19)$.

Conclusion: The link between physical examination and US for enthesitis is dependent on the anatomical site. Patellar tendon origin and Achilles enthesis are the 2 sites where physical examination is significantly linked to not only inflammatory changes but also US findings related to tissue damage, supporting the construct validity of US to visualize enthesitis. For other sites where physical exam is not linked to US, US may improve the assessment by eliminating the false positivities and negativities of the physical examination. 1. Polachek A, et al. ArthritisResTher.2017 2. Aydin SZ, et al.Dermatology.2012. 3. Arslan F, et al. Submitted to CRA2018.

81

Enthesitis: Are there Disease Specific Features on Ultrasound?

Fatos Arslan (Istanbul Medeniyet University, Istanbul); Esen Gunal (Istanbul Medeniyet University, Istanbul); Esra Kurum (University of California, California); Sibel Ureyen (University of Ottawa, Ottawa); Ayse Ozturk (Koc University, Istanbul); Sibel Aydin (University of Ottawa, Ottawa Hospital Research Institute, Ottawa)

Objectives: Ankylosing Spondylitis (AS) and Psoriatic Arthritis (PsA) share a variety of clinical and immunological features, such as enthesitis being frequently seen in both. Yet these are not the same diseases and distinct differences exist. The objective of the study was to investigate the similarities and differences of enthesitis for patients with AS and PsA using ultrasound (US).

Methods: Consecutive patients with AS and PsA were recruited and had an US scan blinded to the clinical features. Bilateral quadriceps tendon insertions, patellar tendon origins and insertions, Achilles tendon and plantar fascia insertions were scanned by US, categorizing the findings as "inflammation score' including the sum of hypoechogenicity, thickening, and entheseal Doppler scores, "damage score" by adding calcifications, erosions, and enthesophytes and "total US score" by summing both of these scores. These were compared in AS and PsA; then a linear regression model was adopted to test the significance of interactions for both the inflammation and damage scores adjusting for age, gender, BMI, clinical enthesitis, HLA-B27, antiTNF use and diagnosis.

Results: Seventy-nine patients with AS ( $36.7 \%$ female) and 85 patients with PsA (77\% female) were recruited. PsA patients had significantly higher inflammation scores than AS (11.3 (8) vs $9.2(6.9) ; \mathrm{p}=0.077)$, similar to

Personal non-commercial use only. The Journal of Rheumatology Copyright $\subset$ 2018 . All rights reserved. 
damage scores (10.4(5.5) vs 8.4(6), $\mathrm{p}=0.008)$ and total US scores (21.7 (12) vs $17.7(11.2), \mathrm{p}=0.013)$. Hypoechogenicity $(76.5 \%$ vs $58.2 \%, \mathrm{p}=0.019)$ and entheseal Doppler $(35.3 \%$ vs $15.2 \%, \mathrm{p}=0.004)$ were more frequent in PsA vs AS, whereas no significant differences were observed in the frequency of other elementary lesions. In multivariate analysis of the group on anti-TNF therapies, no significant differences between inflammation scores in PsA vs AS group were observed when adjusting for factors such as BMI, age, gender, clinical enthesitis and HLA-B27. In the group of patients without antiTNF, a difference in inflammation scores was observed between PsA and AS with females with PsA having 0.95 times less inflammation than females with AS. For damage scores, after adjusting for age, PsA patients have 4.22 times $\left(\mathrm{e}^{\wedge}(1.469-0.029)\right)$ more chronicity than their counterparts of similar age in the AS group which was observed independently of antiTNF therapy.

Conclusion: This study highlighted the differences of enthesitis on US for both AS and PsA. PsA patients have more damage on US at the entheseal insertions than AS, which may be linked to the abnormal response to trauma-biomechanical stress in PsA.

\section{2}

The Role of Obesity on Inflammation and Damage in Spondyloarthritis: A Systematic Literature Review on Body Mass Index and Imaging

Sibel Ureyen (University of Ottawa, Ottawa); Janet Dabague (ABC Medical Center I.A.P, Mexico); Lihi Eder (Women's College Hospital, Toronto); Dennis McGonagle (Leeds Institute of Molecular Medicine, University of Leeds and NIHR Leeds Musculoskeletal Biomedical Research Unit, Leeds Teaching Hospitals, Leeds); Sibel Aydin (University of Ottawa, Ottawa Hospital Research Institute, Ottawa)

Objectives: There is increasing interest to understand the inflammatory role of adipose tissue as well as the role of biomechanical stress on the pathogenesis of spondyloarthritis (SpA). The objective of this systematic literature review (SLR) was to evaluate the effect of obesity and/or body mass index (BMI) related to radiographic findings of SpA patients for both the axial and peripheral inflammation and damage.

Methods: Medline, Embase and Cochrane databases were screened on February 13,2017 . The titles and the abstracts were independently screened by two investigators (SBU, JD). Articles that have evaluated the link between BMI and the following imaging modalities, including plain radiography, ultrasound (US) and magnetic resonance imaging (MRI) were included. All included articles were investigated for the aim of study, demographic data, imaging modalities and the results of the studies relating to BMI during the full text review.

Results: Six hundred and thirteen articles were retrieved by the literature search, six of which met the inclusion criteria for the final analysis. Four of the articles were done in ankylosing spondylitis (AS), one in SpA and one in psoriatic arthritis (PsA), with a mean (SD) BMI of 23.3-30.9 (3.5-6.6) and mostly investigated the effect of BMI on axial disease. The major finding of this SLR was that higher BMI was closely related with existing new bone formation including syndesmophytes, enthesophytes and also higher modified Stoke Ankylosing Spondylitis Spinal Score on plain radiography. Fewer studies looked at the effect of BMI on the peripheral enthesis which found a moderately positive correlation between the Madrid Sonographic Enthesitis Index for enthesitis on US and BMI $(r=0.47 ; p<0.001)$. Gender was demonstrated to be a significant factor to influence this link in another study as BMI was correlated with US enthesophyte scores in males $(\mathrm{r}=$ $0.382, p<0.0001$ ), whereas there was no significant relationship between BMI and US enthesophyte scores in females (r: 0.141, p: NS). No studies met the inclusion criteria to be included in the SLR on MRI of the spine or the peripheral enthesis.

Conclusion: BMI is linked to both damage on the spine and peripheral enthesis and also entheseal inflammation as supported by the limited number of studies in the literature. Its effect on the spinal inflammation is not clear as the MRI studies are lacking.

83

Effect of Tofacitinib on Reducing Pain in Patients with Rheumatoid
Arthritis (RA), Psoriatic Arthritis (PsA), and Ankylosing Spondylitis (AS)

Alexis Ogdie (Division of Rheumatology, Department of Medicine, Perelman School of Medicine at the University of Pennsylvania, Philadelphia); Kurt de Vlam (UZ Leuven, Leuven); Iain McInnes (University of Glasgow, Glasgow); Philip Mease (Swedish Medical Center and University of Washington, Seattle); Philip Baer (Baer Weinberg MPC, Scarborough); Tatjana Lukic (Pfizer Inc, New York); Kenneth Kwok (Pfizer Inc, New York); Cunshan Wang (Pfizer Inc, Groton); Ming-Ann Hsu (Pfizer Inc, Groton); Anna Maniccia (Pfizer Inc, New York)

Objectives: Tofacitinib is an oral Janus kinase inhibitor for the treatment of RA, which has also been evaluated in other inflammatory rheumatic diseases (IRD) including PsA and AS. Pain impacts treatment adherence, assessment of disease improvement, and health-related quality of life in patients with IRD. We evaluated the effectiveness of tofacitinib in reducing pain in randomized controlled clinical trials in IRD patients.

Methods: Five patient populations treated with tofacitinib $5 \mathrm{mg}$ twice daily (BID), $10 \mathrm{mg}$ BID, or placebo were evaluated: [1] conventional synthetic disease-modifying antirheumatic drug (csDMARD)-inadequate response (IR) RA patients pooled (ORAL Scan, ORAL Sync, and ORAL Standard; $\mathrm{N}=2,066$ ), [2] tumor necrosis factor inhibitor (TNFi)-IR RA patients (ORAL Step; N = 399), [3] csDMARD-IR PsA patients (OPAL Broaden; N = 316), [4] TNFi-IR PsA patients (OPAL Beyond; $N$ = 394), and [5] AS patients (a Phase 2 study; $N=155$ ). Pain outcomes evaluated to Month (M)6 (Week [W]12 for AS) included Patient's Assessment of Arthritis Pain (PAAP) (RA and PsA populations), Short-Form Health Survey (SF)-36v2 Q7 (bodily pain in the past week), SF-36v2 Bodily Pain Domain (BP), EuroQol Five Dimensions Questionnaire Pain/Discomfort Domain (EQ-5D PD; all populations), and Bath Ankylosing Spondylitis Disease Activity Index (BASDAI) Q2 (level of AS neck, back, or hip pain/swelling) and Q3 (other pain/swelling) score (PsA and AS populations; PsA patients had spondylitis at screening and baseline BASDAI total score $>0$ ). Data were analyzed descriptively.

Results: In each RA or PsA csDMARD-IR and TNFi-IR population treated with tofacitinib, mean PAAP at baseline (5 mg BID, range 55.7-65.7 mm; $10 \mathrm{mg}$ BID, 54.4-60.1 mm) decreased as early as W2 (1st post-baseline assessment; $5 \mathrm{mg}$ BID, 45.8-49.8 mm; $10 \mathrm{mg}$ BID, 38.9-44.8 mm) and continued to decrease through M6 (5 mg BID, 31.6-34.4 mm, $10 \mathrm{mg}$ BID, 28.2-36.7 $\mathrm{mm}$ ); decreases were numerically greater vs placebo and magnitude of change in RA and PsA was similar. Improvements in SF-36v2 Q7, SF-36v2 BP, and EQ-5D PD were observed in RA and PsA csDMARD-IR and TNFi-IR populations, and in BASDAI Q2 and Q3 in the csDMARD-IR PsA and TNFi-IR PsA populations. In AS, improvements from baseline to W12 in mean SF-36v2 Q7, SF-36v2 BP, EQ-5D PD, and BASDAI Q2 were numerically greater with tofacitinib vs placebo.

Conclusion: Treatment with tofacitinib is associated with rapid and sustained reduction of pain in csDMARD-IR and TNFi-IR RA and PsA patients, and in patients with AS.

\section{4}

Addressing Rural and Remote Access Disparities for Patients with Inflammatory Arthritis through Videoconferencing and Innovative Inter-professional Care Models

Regina Taylor-Gjevre (University of Saskatchewan, Saskatoon); Bindu Nair (University of Saskatchewan, Saskatoon); Brenna Bath (University of Saskatchewan, Saskatoon); Udoka Okpalauwaekwe (University of Saskatchewan, Saskatoon); Meenu Sharma (University of Saskatchewan, Saskatoon); Erika Penz (University of Saskatchewan, Saskatoon); Catherine Trask (University of Saskatchewan, Saskatoon); Samuel Stewart (University of Saskatchewan, Saskatoon)

Objectives: To evaluate whether rheumatoid arthritis (RA) patients followed longitudinally using videoconferencing and inter-professional care support have comparable disease control to those followed in traditional in-person rheumatology clinics.

Methods: This was a randomized controlled trial for 85 RA patients

Personal non-commercial use only. The Journal of Rheumatology Copyright $(\subset) 2018$. All rights reserved. 
allocated to either traditional in-person rheumatology follow-up or video-conferenced follow-up with urban-based rheumatologists and rural in-person physical therapist examiners. Follow-up was every 3 months for 9 months. Outcome measures included disease activity metrics (DAS-28CRP, RA disease activity index (RADAI)), modified health assessment questionnaire (mHAQ), quality of life (EQ5D), and patient satisfaction (VSQ9).

Results: Of 85 participants, 54 were randomized to videoconferencing team model and 31 to traditional clinic (control group). Drop-out rates were high, with only $31(57 \%)$ from the videoconferencing and 23 (74\%) from the control group completing the study. Mean age for study participants was 56 years; $20 \%$ were male. Mean RA disease duration was 13.9 years. There were no significant between-group differences in DAS28-CRP, RADAI, mHAQ or EQ5D scores at baseline or over the study period. Satisfaction rates were high in both groups.

Conclusion: We found no evidence of a difference in effectiveness between inter-professional videoconferencing care and traditional rheumatology clinic for both provision of effective follow-up care and patient satisfaction for established RA patients. High drop-out rates reinforce the need for consultation with patients' needs and preferences in developing models-of-care. While use of videoconferencing/telehealth technologies may be a distinct advantage for some patients, there may be loss of travel-related auxiliary benefits for others. Supported by a CIORA grant

85

Let's Do Lunch! Connecting Students with the Real-Life Impact of Chronic Rheumatic Diseases

Regina Taylor-Gjevre (University of Saskatchewan, Saskatoon); Bindu Nair (University of Saskatchewan, Saskatoon); Udoka Okpalauwaekwe (University of Saskatchewan, Saskatoon); Anne Dooley (Saskatoon)

Objectives: People with chronic diseases comprise a substantial proportion of patients within many medical practices. Undergraduate medical students have limited opportunities for interacting with people with chronic rheumatic diseases. These conditions are generally successfully treated in outpatient settings which students tend to spend less time in. Early exposure is influential in generating future student interest and awareness.

Methods: At University of Saskatchewan, an extra-curricular opportunity was created for first year medical students to meet and share lunch with people diagnosed with chronic rheumatic disorders. Three lunches were scheduled. Twenty-four medical students and 22 patients attended. Feedback was obtained from both groups through questionnaires and separate focus groups. SPSS and NVivo were utilized for data analysis.

Results: Four themes were identified from the data. There was a high degree of satisfaction with sessions that were described as 'informative and transformative'. Students reported enhanced and detailed understanding around challenges of living with rheumatic diseases. Patient perceptions mirrored student responses indicating for example 'I made things more real to them'. Many student perceptions/ misperceptions around rheumatic diseases were reported to be clarified through dialogue with patients. Interest was expressed by several participating students in working with rheumatic disease patients in future. Patient participants also felt their contributions to conversations were influential in stimulating student interest in these chronic conditions.

Conclusion: Social connections over lunches with patients translating experiential knowledge was effective in stimulating medical student interest in these chronic diseases. Feedback suggested some success in enhancing understanding on real-life impact of rheumatic disease.

86

Geographic Variation in Incidence and Prevalence Rates for Rheumatoid Arthritis in Saskatchewan, Canada 2001-2014

Regina Taylor-Gjevre (University of Saskatchewan, Saskatoon); Bindu Nair (University of Saskatchewan, Saskatoon); Shan Jin (Saskatchewan Health Quality Council, Saskatoon); Jacqueline Quail (Saskatchewan Health Quality Council, Saskatoon)
Objectives: Rheumatoid arthritis is a destructive chronic inflammatory arthritis. Geographic or regional disease burden distribution in a largely rural province can inform development of health care delivery planning. Objective: To estimate and compare incidence/prevalence of RA in different geographic health regions and between urban/rural locations of residence within the province of Saskatchewan.

Methods: Saskatchewan Provincial Administrative Health Databases (2001-2014) were utilized as data sources. Two RA case-definitions were employed: 1) > three physician billing diagnoses, at least one of which was submitted by a specialist (rheumatologist, general internist or orthopaedic surgeon) within two years; 2) > one hospitalization diagnosis (ICD-9-CM code-714, and ICD-10-CA code-M05). Data from these definitions were combined to estimate annual RA incidence and prevalence. Annual incidence and prevalence rates across geographic regions and between rural and urban residences were examined.

Results: An increasing RA prevalence gradient was observed in a south to north direction within the province. In the 2014-15 Fiscal Year, the southern region of Sun Country had a $0.57 \%$ RA prevalence and the Northern Health Regions a prevalence of $1.15 \%$. Incidence rates fluctuated over time in all regions, but tended to be higher in Northern Health Regions. A higher RA prevalence trend was observed in rural residents over the study period.

Conclusion: Higher prevalence rates were observed for RA in Northern Health Regions than elsewhere in the province. Rural prevalence rates were higher than for urban residents. Health care delivery strategic planning will need to ensure appropriate access for RA patients throughout the province.

\section{7}

\section{A Multidisciplinary Rheumatoid Arthritis Group Class Results in} Improved Patients' Self-Efficacy

Rashmi Mandhane (Alberta Health Services, Edmonton); Caitlyn Giese (Alberta Health Services, Edmonton); Emma Vetsch (Alberta Health Services, Edmonton); Claudia Holody (University of Alberta, Edmonton); Kathy Cotton (Alberta Health Services, Edmonton)

Objectives: Self-efficacy is an individual's belief in their ability to perform a task. The objective of our study is to examine the effect of the Managing Your Rheumatoid Arthritis (RA) group management classes on RA patient self-efficacy. We hypothesized that RA patients demonstrate improved self-efficacy after attending the group management classes.

Methods: Between June 2015 and June 2017, 272 patients with RA were offered the Managing Your RA group management classes after assessment by the University of Alberta Hospital rheumatology occupational therapist (OT) and physical therapist (PT). Patients completed the Rheumatoid Arthritis Self Efficacy Scale (RASES) at four time points: initial assessment appointment $(n=225)$; beginning of group classes $(n=140)$, end of group classes $(\mathrm{n}=126)$ and at their two-month re-assessment appointment $(\mathrm{n}=$ 98). Paired-samples t-test analysis was conducted to examine differences from the beginning of the group classes to the end of the four-day group class.

Results: There was no difference in self-efficacy between those who attended the RA group $(n=107$, mean RASES $=110, S D=13)$ and those who did not attend the RA group $(n=118$, mean RASES $=107, \mathrm{SD}=14)$. There was no difference from the initial appointment RASES assessment (n $=107$; mean $\operatorname{RASES}=110, \mathrm{SD}=13$ ) and the start of the group class (mean RASES $=110, S D=11$ ). There was a significant improvement in the RASES from the first day of classes $(n=123$, mean RASES $=111, S D=11)$ and the last day of classes (mean RASES $=122, \mathrm{SD}=16 ; \mathrm{p}<0.0001$ ). There was also a significant improvement from the initial assessment $(\mathrm{n}=70$, mean $=$ $111, \mathrm{SD}=12$ ) and the follow-up reassessment (mean RASES $=116, \mathrm{SD}=$ 12). There was a significant decrease between the end of group $(n=75$, mean RASES $=122, \mathrm{SD}=17$ ) and the follow-up reassessment appointment (mean RASES $=118, \mathrm{SD}=13$ )

Conclusion: The Managing Your RA group management classes significantly increases self-efficacy of RA patients immediately after the classes. The increase in self-efficacy was sustained up to two-months after the classes were completed. Future studies will examine the change in RA patient 
self-efficacy over time in order to determine the optimal timing of a refresher intervention.

\section{8}

Time to Achieve Moderate/Low Disease Activity and Remission in RA Patients on Baricitinib Compared to Adalimumab, Methotrexate, and Placebo

Edward Keystone (Mount Sinai Hospital, University of Toronto, Toronto); Maxime Dougados (Paris Descartes University, Hôpital Cochin, Paris); Eric Ruderman (Northwestern Medical Group, Chicago); Baojin Zhu (Eli Lilly and Company, Indianapolis); Pedro Lopez-Romero (Eli Lilly and Company, Indianapolis); Hanne Lund (Eli Lilly and Company, Indianapolis); Anabela Cardoso (Eli Lilly and Company, Indianapolis); Douglas Schlichting (Eli Lilly \& Company, Indianapolis); Pindaro Martinez-Osuna (Eli Lilly and Company, Indianapolis); Robert Ortmann (Eli Lilly and Company, Indianapolis); Tore Kvien (Diakonhjemmet Hospital, Oslo); Susan Otawa (Eli Lilly and Company, Toronto)

Objectives: Baricitinib (BARI), an oral Janus kinase (JAK)1/2 inhibitor, has shown efficacy in DMARD-naive patients (pts) with RA and in pts with inadequate response to methotrexate (MTX-IR). In the EU, Japan and other countries, BARI is approved for treating moderate to severe RA in adults. This analysis evaluated time to achieve moderate disease activity (MDA), low disease activity (LDA), and remission in pts treated with BARI 4-mg vs placebo (PBO) or active comparators, MTX, or adalimumab (ADA 40-mg biweekly) from the RA-BEGIN and RA-BEAM trials.

Methods: In RA-BEGIN, DMARD-naive pts were randomized to BARI 4-mg once daily (QD), MTX or BARI 4-mg+MTX for 52 weeks (wks). In RA-BEAM, MTX-IR pts were randomized to PBO, BARI 4-mg QD or ADA for $52 \mathrm{wks}$ (PBO switched to BARI 4-mg at $24 \mathrm{wks}$ ). This analysis estimated time to achieve MDA (CDAI $\leq 22)$, LDA $(\mathrm{CDAI} \leq 10)$, and remission $(\mathrm{CDAI} \leq 2.8)$ in modified intent-to-treat pts and in the subset of pts with high baseline disease activity (HDA) defined as CDAI $>22$ from 2 trials. Cumulative incidence of MDA, LDA, and remission over 52 wks for RA-BEGIN and 24 wks for RA-BEAM was estimated. Hazard ratios between treatments were obtained using Cox proportional hazards regression after adjusting for region and baseline joint erosions (1-2 or $\geq 3$ erosions) without control for multiple comparisons.

Results: In DMARD-naive population, BARI 4-mg monotherapy pts were 1.6 times more likely to achieve MDA and LDA and twice more likely to achieve remission compared to MTX ( $<<0.001)$. Median time to MDA and LDA with BARI 4-mg treatment ( 2 and 12 wks, respectively) was shorter than with MTX (4 and $20 \mathrm{wks}$, respectively). BARI 4-mg+MTX performed similar to BARI 4-mg monotherapy. In MTX-IR population, BARI- 4-mg-treated pts were $1.7,2.3$, and 3.5 times more likely to achieve MDA, LDA, and remission than $\mathrm{PBO}(\mathrm{p}<0.001)$ and 1.0, 1.1, 1.4 times more likely to achieve MDA $(p=0.557)$, LDA $(p=0.295)$, and remission $(p=0.030)$ than ADA. Median time to MDA was 5 wks shorter with BARI 4-mg treatment ( 2 wks) than PBO (7 wks) but similar to ADA (2 wks). Median time to LDA with BARI 4-mg (12 wks) was 2 wks shorter than ADA (14 wks) whereas pts on PBO never reached the median time to LDA during the 24-wk study. Consistent results were obtained in pts with baseline HDA.

Conclusion: DMARD-naive and MTX-IR pts were more likely to achieve LDA and remission, and at a faster pace with BARI 4-mg.

\section{9}

Safety Profile of Baricitinib for the Treatment of Rheumatoid Arthritis up to 5.5 Years: An Updated Integrated Safety Analysis

Edward Keystone (Mount Sinai Hospital, University of Toronto, Toronto); Mark Genovese (Stanford University Medical Center, Palo Alto); Josef Smolen (Medical University of Vienna, Vienna); Tsutomu Takeuchi (Keio University, Tokyo); David Hyslop (Eli Lilly and Company, Indianapolis); William Macias (Eli Lilly \& Company, Indianapolis); Terence Rooney (Eli Lilly and Company, Indianapolis); Lei Chen (Eli Lilly and Company, Indianapolis); Christina Dickson (Eli Lilly and Company, Indianapolis); Jennifer Camp (Eli Lilly and Company, Indianapolis); Tracy Cardillo (Eli
Lilly \& Company, Indianapolis); Taeko Ishii (Eli Lilly and Company, Kobe); Kevin Winthrop (Oregon Health Sciences University, Portland); Susan Otawa (Eli Lilly and Company, Toronto)

Objectives: Baricitinib (BARI), an oral, selective inhibitor of Janus kinase (JAK) $1 / 2$, is approved in the EU, Japan and other countries for treating moderately to severely active RA in adults. We describe the drug's safety profile of the drug with updated data from an on-going long-term extension (LTE) study.

Methods: Long-term safety of once-daily BARI was evaluated in the "all-BARI-RA" dataset, which includes all patients (pts) with active RA exposed to any BARI dose from 8 randomized trials (4 Ph3, $3 \mathrm{Ph} 2,1 \mathrm{Ph} 1 \mathrm{~b})$ and 1 LTE study (data up to 01-Sept-2016). Previous all-BARI-RA analyses were provided for comparison (data up to 10-Aug-2015). Placebo (PBO) comparisons were evaluated for up to Wk 24 in the "PBO 4-mg" dataset from the $6 \mathrm{Ph} 2 / 3$ trials in which pts were randomized to BARI $4 \mathrm{mg}$, with censoring at rescue or treatment switch. Dose responses were evaluated based on the $4 \mathrm{Ph} 2 / 3$ trials in which pts were randomized to 2 or $4 \mathrm{mg}$ and includes data from the LTE (the "2-4mg-extended" dataset). Because of the latent period for malignancy, 2-4mg-extended was also analyzed without censoring for rescue or dose change (as-randomized analysis). Incidence rates (IRs) per 100 patient-years (PYs) were calculated.

Results: In the current analysis, 3492 pts received BARI for 6637 total PYs of exposure (an increase of over 2400 PYs from previous analysis); maximum exposure was $5.5 \mathrm{yrs}$. No differences were seen for BARI $4 \mathrm{mg}$ vs $\mathrm{PBO}$ in adverse events (AEs) leading to permanent study drug discontinuation, death, malignancy, serious infection, or major adverse cardiovascular event. Herpes zoster IR was significantly higher for BARI $4 \mathrm{mg}$ vs PBO (IR 4.3 vs $1.0 ; 4 \mathrm{mg}$ vs. $\mathrm{PBO}$, respectively). In $2-4 \mathrm{mg}$-extended, no significant differences were observed comparing BARI 2 vs $4 \mathrm{mg}$ for the above-mentioned events. Malignancy (excluding non-melanoma skin cancer) IRs were 0.5 and 1.3 for 2 and $4 \mathrm{mg}$, respectively, with as-treated analysis and 0.7 and 0.9 with as-randomized analysis. The following IRs were observed in the current all-BARI-RA: lymphoma (0.09), gastrointestinal perforation ( 0.05$)$, and tuberculosis $(0.15$, all in endemic areas). The IRs for these events were also similar to those previously reported. Fewer than $1 \%$ of pts discontinued due to abnormal laboratory results.

Conclusion: Patients with moderately to severely active RA, including patients exposed for up to 5.5 years, baricitinib maintained a safety profile that was similar to that previously reported 1 and acceptable in the context of demonstrated efficacy.

\section{0}

ACPA and RF as Predictors of Sustained Clinical Remission in Rheumatoid Arthritis Patients: Data from a Rheumatoid Arthritis Cohort

Janet Pope (Western University, Department of Medicine, Division of Rheumatology, London); Mohammad Movahedi (University Health Network, Toronto); Emmanouil Rampakakis (JSS Medical Research, Montreal); Angela Cesta (University Health Network, Toronto); John Sampalis (JSS Medical Research, Montreal); Claire Bombardier (Ontario Best Practices Research Institute, University of Toronto, Toronto General Research Institute, Toronto); OBRI Investigators (University Health Network, Toronto)

Objectives: Positive serology for anti-citrullinated protein antibody (ACPA) and rheumatoid factor $(\mathrm{RF})$ are included among the criteria for definitive RA diagnosis as per the 2010 ACR/EULAR classification criteria for rheumatoid arthritis (RA). Previous studies have shown that autoantibodies are positive predictors of response in rheumatoid arthritis (RA) patients treated with some biologics. The purpose of this study was to evaluate the interaction of RF and ACPA in predicting sustained clinical response in a large observational cohort of RA patients followed in routine clinical care. Methods: RA patients enrolled in the Ontario Best Practices Research Initiative (OBRI) registry, with active disease ( $\geq 1$ swollen joint), available autoantibody information, and at least 1 follow-up assessment were included in the analysis. Sustained clinical remission was defined as CDAI $\leq 2.8$ in

Personal non-commercial use only. The Journal of Rheumatology Copyright @ 2018. All rights reserved. 
at least 2 sequential visits separated by at least 3 and maximum of 12 months. Time to sustained remission was assessed with Kaplan-Meier survival analysis and multivariate cox regression.

Results: A total of 970 patients were included in the analysis, of whom 262 (27\%) were anti-CCPneg/RFneg, 60 (6.2\%) anti-CCPpos /RFneg, 117 (12.1\%) anti-CCPneg /RFpos, and 531 (54.7\%) anti-CCPpos /RFpos. At baseline, significant differences were observed between groups in age $(\mathrm{p}=$ $0.02)$, CDAI $(p=0.03)$, tender joint count $(\mathrm{p}=0.02)$, and HAQ $(\mathrm{p}=0.002)$, with anti-CCPpos /RFpos and anti-CCPpos /RFneg patients being youngest and having the lowest disease activity and disability. No differences were observed in terms of biologic use which occurred in $15.9 \%$ of patients.

Conclusion: These results suggest that anti-CCP but not RF positivity may be associated with improved response to anti-rheumatic medications in RA patients.

91

Reduction in Disease Activity in Patients with RA and an Inadequate Response to MTX: Baricitinib Compared to Adalimumab and Placebo Peter Nash (University of Queensland, Brisbane); Janet Pope (Western University, Department of Medicine, Division of Rheumatology, London); Anabela Cardoso (Eli Lilly and Company, Indianapolis); Marta Casillas (Eli Lilly and Company, Alcobendas); Douglas Schlichting (Eli Lilly \& Company, Indianapolis); Baojin Zhu (Eli Lilly and Company, Indianapolis); Scott Beattie (Eli Lilly \& Company, Indianapolis); Josef Smolen (Medical University of Vienna, Vienna)

Objectives: Baricitinib (BARI) is an oral Janus kinase (JAK)1/JAK2 selective inhibitor for the treatment of patients with moderately to severely active rheumatoid arthritis (RA). RA-BEAM was a phase 3 study in patients with RA and an inadequate response to methotrexate (MTX-IR), in which BARI demonstrated significant improvements in ACR20 response rates and DAS28-CRP compared to placebo (PBO) and adalimumab (ADA). This abstract examines the effects of BARI on disease activity scores and the improvement of disease activity compared to PBO and ADA utilizing CDAI, which does not include acute phase reactants and only uses clinical measures (adding physician and patient global assessments, to tender and swollen joint counts).

Methods: In RA-BEAM, 1305 patients were treated with $\mathrm{PBO}(\mathrm{N}=488)$, ADA $(\mathrm{N}=330)$ or BARI 4-mg $(\mathrm{N}=487)$, while continuing to receive background MTX. CDAI for the three treatment groups was determined at baseline (mean [SD] of 37.6 [12.8], 38.1 [12.0], 37.9 [13.0] for PBO, BARI, and ADA, respectively) and at each visit post baseline up to 24 weeks, and for BARI and ADA groups up to 52 weeks. In this analysis, CDAI and the improvement from baseline to Weeks 12 and 24 were compared between treatment groups using ANCOVA. The proportion of patients reaching a disease activity and improvement threshold at Weeks 12 and 24 were compared between treatment groups using logistic models. Analyses were not adjusted for multiplicity. Missing values were imputed using modified last observation carried forward.

Results: At baseline, across all treatment arms, 91\% of patients had high disease activity and $9 \%$ had moderate disease activity. Treatment with BARI resulted in significantly lower mean disease activity than PBO $(\mathrm{p}<0.001$ at Weeks 12 and 24) and ADA ( $\mathrm{p}=0.008$, Week 12; $\mathrm{p}=0.035$, Week 24). Fewer patients treated with BARI (16.4\%) remained in high disease activity at Week 24 compared to PBO $(47.6 \%, \mathrm{p}<0.001)$ and ADA $(22.9 \%, \mathrm{p}=$ $0.017)$. Patients treated with BARI had significantly greater improvement in the mean disease activity compared to PBO $(\mathrm{p}<0.001)$ and ADA $(\mathrm{p}=$ $0.023)$ at 24 weeks. A larger proportion of patients receiving BARI (86.2\%) were able to achieve at least a 12-point reduction in CDAI, the minimal clinically important difference in disease activity improvement, by Week 24 compared to patients receiving PBO $(52.4 \%, \mathrm{p}<0.001)$ or ADA $(77.5 \%$, $\mathrm{p}=0.001$ ).

Conclusion: In MTX-IR RA patients with moderate-to-severe CDAI-assessed disease activity, BARI significantly reduced the overall disease activity compared to PBO and ADA.
92

Additions to Methotrexate with Conventional and Biologic DMARDs in Rheumatoid Arthritis: Are there Difference in Subsequent Time to Treatment Failure?

Cristiano Moura (Department of Epidemiology, Biostatistics and Occupational Health, McGill University, Montreal); Vivian Bykerk (Hospital for Special Surgery, New York); Orit Schieir (Notre-Dame-de-Grace); Marie-France Valois (McGill University, Montreal); Susan Bartlett (McGill University, Montreal); Carol Hitchon (University of Manitoba, Winnipeg); Janet Pope (Western University, Department of Medicine, Division of Rheumatology, London); Gilles Boire (Université de Sherbrooke, Sherbrooke); Boulos Haraoui (Institut de Rhumatologie de Montréal, Montreal); Edward Keystone (Mount Sinai Hospital, University of Toronto, Toronto); Diane Tin (Southlake Regional Health Centre, Newmarket); Carter Thorne (Southlake Regional Health Centre, Newmarket); Sasha Bernatsky (McGill University Health Centre, McGill University, Montreal); CATCH Canadian Early Arthritis Cohort Investigators (Toronto)

Objectives: Our objective was to compare RA treatment strategies with conventional and biologic DMARDs after an initial MTX strategy was ineffective or associated with a severe adverse event.

Methods: We studied adults from a multicenter early arthritis cohort (enrolled from 2007- 2017 within one year of symptom onset). RA patients were eligible for our analyses if they initiated MTX (+/-other DMARDs) within 90 days of cohort entry and subsequently changed therapy (changed MTX route, lowered or stopped MTX or other DMARD, or added another DMARD or biologic). For this analyses, the time of medication change formed the time zero for a survival analyses of the second treatment approach. Patients were followed from time zero to assess discontinuations of, or additions to, their therapy. Multivariable survival models were used to compare outcomes. We generated hazard ratios (HRs) and 95\% confidence intervals (CI), comparing each of the treatment groups to oral methotrexate monotherapy.

Results: We included 911 RA patients initially exposed to MTX who had a first treatment failure. At time zero (time of initial failure), the most common second treatment strategies were MTX+ another DMARD (32.9\%) and non MTX DMARDs $(26.1 \%)$. The multivariable Cox regression analysis (adjusting for baseline characteristics: age, sex, co-morbidities, symptom duration, race, education, smoking, erosions, DAS-28, disease activity, corticosteroids, NSAIDs, and COXIBs) for the 911 RA patients suggested that those on biologics and those on triple therapy had a longer time to failure, compared to the group taking MTX oral monotherapy.

Conclusion: Our data suggest that, in those who fail initial MTX, RA patients given biologics or triple therapy remain on that treatment longer without further changes, versus those taking augmented MTX oral monotherapy. These data do not confirm clear differences in outcomes with respect to MTX (dual or triple) combinations, but width of confidence intervals precludes definitive conclusions in this regard.

\section{3}

Methotrexate Treatment Strategies in an Early Rheumatoid Arthritis Cohort

Cristiano Moura (Department of Epidemiology, Biostatistics and Occupational Health, McGill University, Montreal); Vivian Bykerk (Hospital for Special Surgery, New York); Orit Schieir (Notre-Dame-de-Grace); Marie-France Valois (McGill University, Montreal); Susan Bartlett (McGill University, Montreal); Carol Hitchon (University of Manitoba, Winnipeg); Janet Pope (Western University, Department of Medicine, Division of Rheumatology, London); Gilles Boire (Université de Sherbrooke, Sherbrooke); Boulos Haraoui (Institut de Rhumatologie de Montréal, Montreal); Edward Keystone (Mount Sinai Hospital, University of Toronto, Toronto); Diane Tin (Southlake Regional Health Centre, Newmarket); Carter Thorne (Southlake Regional Health Centre, Newmarket); Sasha Bernatsky (McGill University Health Centre, McGill University, Montreal); CATCH Canadian Early Arthritis Cohort Investigators (Toronto)

Objectives: Methotrexate (MTX) is recommended as part of initial therapy

Personal non-commercial use only. The Journal of Rheumatology Copyright (C) 2018. All rights reserved. 
in early RA, but practices range widely. The objective of this analysis was to describe MTX treatment in an early RA cohort, beginning with initial therapy and assessing time to treatment failure across various treatment strategies.

Methods: We studied adult patients from a prospective multicenter early arthritis cohort (enrolled 2007-2017 within one year of symptom onset) who fulfilled ACR/EULAR RA criteria. RA patients were eligible for our analyses if they initiated MTX (+/-other DMARDs) within 90 days of cohort entry. The first analyses determined time until 'failure' of that initial MTX-based therapy, from the time of first initiation, left-censored at cohort entry. Treatment failure definition included: change of route for MTX monotherapy, adding or stopping a DMARD/biologic, and changing dose/frequency of a DMARD or biologic, due to inefficacy or a serious adverse event.

Results: We studied 1,484 early RA patients, the majority initiating either MTX monotherapy (oral or subcutaneous) or MTX plus a second agent. At the time of entry into the early arthritis cohort, their mean (standard deviation, SD) age was 54 (15) years, their mean symptom duration was 5.6 months (2.8), their mean DAS28 scores were $5.3(1.4)$, and one third (38\%) were on oral steroids. Overall, 911/1464 $(61 \%)$ had a treatment failure, primarily due to inefficacy. The multivariate cox regression (adjusting for baseline characteristics: age, sex, co-morbidities, symptom duration, race, education, smoking, erosions, DAS-28, disease activity, corticosteroids, NSAIDs, and COXIBs.) for the first analyses showed that, compared to oral MTX monotherapy, all MTX strategies had longer time to failure.

Conclusion: Our data in early RA patients initially exposed to MTX suggest that compared to oral MTX, all other MTX strategies had longer time to failure. These data do not confirm clear differences in outcomes with respect to methotrexate DMARD combinations, as the width of confidence intervals precludes definitive conclusions in this regard.

\section{4}

\section{Changes in the Ontario Rheumatology Workforce}

Jessica Widdifield (Sunnybrook Research Institute, Toronto); Sasha Bernatsky (McGill University Health Centre, McGill University, Montreal); Vandana Ahluwalia (William Osler Health System, Ontario Rheumatology Association, Brampton); Claire Barber (University of Calgary, Calgary); Lihi Eder (Women's College Hospital, Toronto); Catherine Hofstetter (Toronto); Bindee Kuriya (Sinai Health System, University of Toronto, Toronto); Vicki Ling (Institute for Clinical Evaluative Sciences, Toronto); Anne Lyddiatt (Ingersol); Michael Paterson (Institute for Clinical Evaluative Sciences, Toronto); Janet Pope (Western University, Department of Medicine, Division of Rheumatology, London); Carter Thorne (Southlake Regional Health Centre, Newmarket)

Objectives: Our aim was to describe the Ontario rheumatologist workforce supply (number of providers and number per capita), diversity (provider characteristics) and activity (clinical workload) over the past decade.

Methods: We performed a repeated cross-sectional study of Ontario administrative health data from 2000 to 2014. Rheumatologists and their characteristics were identified using a validated physician registry. We used fee-for-service (FFS) billing claims to quantify activity levels expressed as full-time equivalents (FTE). Physicians below the 40th percentile of all Ontario rheumatologist FFS billings were classified as providing less clinical service ( $<1$ FTE). We also determined the number of clinical service days each year, and the number of rheumatologists working at least 209 days annually (an alternate FTE measure). Census data were used to express the number of rheumatologists per capita.

Results: There were 146 active rheumatologists (88 FTEs) in 2000, with a gradual increase to 187 rheumatologists (114 FTEs) in 2014. The overall provincial per capita supply was stable over time with 1 rheumatologist per 75,000 population $(0.6$ FTEs per 75,000$)$. The annual median (IQR) number of days providing clinical service decreased from 231 (193-259) days in 2000 to 207 (157-250) days in 2014. The proportion of rheumatologists with at least 10 daily patient encounters on at least 209 days/year showed a downward trend from $43(30 \%)$ rheumatologists in 2000 to $33(18 \%)$ rheumatologists in 2014. There was a shift in workforce demographics over time: two-thirds of rheumatologists were male in 2000 compared to half in 2014 , with only $34 \%$ males among physicians under age 45 years. Male rheumatologists had more patient encounters each year, and a higher proportion of males were identified as working at least 1 FTE (68\% vs. 53\% females). As of 2014, 22 (12\% of) rheumatologists were aged 65 years or older. The proportion of rheumatologists with hospital encounters/services has declined ( $87 \%$ in 2000 vs. $74 \%$ in 2014).

Conclusion: We observed changes in the Ontario rheumatology workforce over the past decade, with an increasing proportion of females and decreasing number of clinical service days. Although there has been an increase in the number of rheumatologists, the per capita supply has remained unchanged. Our findings have important implications for health human resource planning to meet the challenges facing the profession and needs of its patients. Supported by a CIORA grant.

\section{5}

Does Guideline-Based Care Improve Outcomes that Matter to Canadian Patients? Tighter Control, Less Suffering, and Greater Well-Being over the Past Decade in CATCH RA Patients

Susan Bartlett (McGill University, Montreal); Orit Schieir (Notre-Dame-de-Grace); Marie-France Valois (McGill University, Montreal); Carol Hitchon (University of Manitoba, Winnipeg); Janet Pope (Western University, Department of Medicine, Division of Rheumatology, London); Gilles Boire (Université de Sherbrooke, Sherbrooke); Boulos Haraoui (Department of Medicine, Centre Hospitalier de l'Université de Montréal, Montréal); Edward Keystone (Mount Sinai Hospital, University of Toronto, Toronto); Diane Tin (Southlake Regional Health Centre, Newmarket); Carter Thorne (Southlake Regional Health Centre, Newmarket); Vivian Bykerk (Hospital for Special Surgery, New York); CATCH Canadian Early Arthritis Cohort Investigators (Toronto)

Objectives: Best practice recommendations can increase quality of care and improve clinical outcomes, however the impact of recommendations on outcomes that matter most to patients have not been evaluated. We compared changes in patient reported outcomes (PROs) valued most by people with RA in the first year of follow up, as well as prior (2007-10) and subsequent (2011-16) to the 2010 release of Treat to Target and 2011 Canadian RA Treatment Recommendations.

Methods: Data included ERA adults enrolled in CATCH (Canadian early ArThritis CoHort) between 2007-16 who met 1987 or 2010 RA criteria and had active disease at enrollment. Standardized visits included clinical assessments, questionnaires, and laboratory tests every 3 months. Treatment was at the discretion of the rheumatologist and cohort investigators met annually to discuss ways to improve outcomes. We examined changes in DAS28; pain, fatigue, patient global (10 cm VAS); and HAQ at 6 and 12 months in the years prior to and after the release of guidelines.

Results: The sample included 1942 adults who were mostly female (72\%) with a mean (SD) age of 55 (15), 2 (2) comorbidities, and symptom duration of 6 (3) months. At enrollment, almost all (95\%) were in DAS28 moderate or high disease activity [MDA (42\%), HDA (53\%)], and were initially treated with csDMARDS (92\%) and MTX (75\%). Mean CDAI was 28.1, DAS28 was 5.2, patient global was 6.0, pain was 5.7, fatigue was 5.4, and HAQ was 1.1. As mean DAS28 scores decreased over the first year from 5.2 to 2.9, similar clinically meaningful improvements in patient global, pain, fatigue, and HAQ were also evident $(-3.0,-2.8,-2.3,-0.6$; p's $<.001)$. When comparing change in PROs in 2007-2010 vs 2011-2016, there were more rapid improvements in patient global and pain at 6 and 12 months (p's < .001) and similar improvements in HAQ and fatigue.

Conclusion: Results from this large country-wide study suggest that better disease control in the first year of RA translated to comparable improvements in pain, fatigue and disability-symptoms that patients identify as important-resulting in greater overall well-being. These data offer additional evidence supporting the importance of early identification and control of disease activity to improve long term outcomes and quality of life in people with RA.

Personal non-commercial use only. The Journal of Rheumatology Copyright @ 2018. All rights reserved. 
96

Time Trends over a Decade Show Earlier Intensified Medication Strategies and Improved Outcomes in Canadians with Early Inflammatory Arthritis

Orit Schieir (Notre-Dame-de-Grace); Marie-France Valois (McGill University, Montreal); Susan Bartlett (McGill University, Montreal); Kathleen Andersen (McGIll University, Beaconsfield); Carol Hitchon (University of Manitoba, Winnipeg); Janet Pope (Western University, Department of Medicine, Division of Rheumatology, London); Gilles Boire (Université de Sherbrooke, Sherbrooke); Boulos Haraoui (Institut de Rhumatologie de Montréal, Montreal); Diane Tin (Southlake Regional Health Centre, Newmarket); Carter Thorne (Southlake Regional Health Centre, Newmarket); Edward Keystone (Mount Sinai Hospital, University of Toronto, Toronto); Vivian Bykerk (Hospital for Special Surgery, New York); CATCH Canadian Early Arthritis Cohort Investigators (Toronto)

Objectives: Treatment recommendations aim to improve outcomes in rheumatoid arthritis (RA) through early identification and a treat-to-target approach. We examined recent trends over the past decade including patient characteristics, treatment strategies and disease activity in the first year of follow-up in a large Canadian early inflammatory arthritis (EIA) cohort.

Methods: Data were from individuals with EIA (RA or probably RA < 1 -year of symptom duration) enrolled in an ongoing prospective multi-center cohort study from 2007-2016 with complete DAS28 measures at 6 and 12-months. Protocolized visits included clinical assessments, questionnaires, and laboratory investigations every 3 months for the first year. Treatment was at the discretion of treating rheumatologist, and cohort investigators met annually to improve outcomes. We examined trends in patient characteristics, early treatment strategies with conventional(cs) and biologic(b) DMARDs and disease activity outcomes over 12-months (12M). Multivariable logistic regression was used to identify predictors of failure to achieve low disease activity (LDA) or remission (REM) by $12 \mathrm{M}$.

Results: Over 10 years, 2822 EIA patients were enrolled who were mostly female (70\%) and Caucasian (80\%). At study entry, 86\% met 1987 or 2010 ACR/ EULAR RA criteria; mean(sd) age was 54(15), symptom duration was 6(3) months. Most were treated with csDMARDs (92\%), often with MTX, as monotherapy or in combination with csDMARDs (77\%); $29 \%$ were prescribed oral steroids and $27 \%$ parenteral steroids. Baseline patient characteristics changed slightly over time with increases in age, male sex, education, income, and declines in current smoking (all p's $<0.01$ ). Baseline obesity rates, comorbidities and RA characteristics (serology, inflammatory markers, joint counts and disease activity indices) remained stable. Most $(87 \%)$ entered in moderate or high disease activity (MDA or HDA), and disease activity at 6 and 12M markedly improved over calendar time. DAS28 REM at $12 \mathrm{M}$ increased by over $30 \%$ (range $39 \%$ to $73 \%$, p < 0.0001 ), and $20 \%$ more achieved LDA or REM by $6 \mathrm{M}$ (range $48 \%$ to $68 \%, \mathrm{p}<0.0001$ ). Time trends in treatment showed earlier titration of MTX dosing to 20mg+, increases in subcut vs. oral MTX, earlier MTX combo therapies and more rapid escalation to bDMARDs (all p's $<0.05$ ). Older age, female sex, lower education, non-white, overweight/obese BMI, and more comorbidities were associated with inadequate response (persistent MDA or HDA) at 12 months (all p's < 0.05).

Conclusion: This study of Canadian EIA patients over time suggests that earlier, more intensified treatment promoted in practice recommendations has resulted in lower disease activity and a greater proportion of patients reaching the target of therapy.

97

Responsiveness and Minimally Clinically Important Differences of PROMIS Measures in Rheumatoid Arthritis

Susan Bartlett (McGill University, Montreal); Michelle Jones (Johns Hopkins School of Medicine, Baltimore); Clifton Bingham (Johns Hopkins, Baltimore)

Objectives: The ability to detect meaningful change in clinical status (responsiveness) is an important aspect of validity. Minimally clinically important difference (MCID) is a patient-centered construct reflecting the smallest difference of value to patients. PROMIS is a generic family of measures developed for use across chronic diseases. We examined the responsiveness and estimated MCIDs of selected PROMIS measures reflecting domains that people with RA have identified.

Methods: Data are from the first two visits of an observational trial in people with RA. Patients completed PROMIS Physical Function (PF), Pain Interference (PI), Participation Ability (PA), and Fatigue computer adapted tests and other patient-reported outcomes (PROs) using a tablet. At the second visit, patients also completed a 5-point item assessing change in RA status from the previous visit (much worse to much better) and the self-reported health rating. Descriptive statistics were calculated, and ANOVA was used to identify significant differences in scores on 9 PROMIS measures.

Results: The 196 RA patients who completed outcomes at clinical visits approximately 4 months apart were mostly female $(81 \%)$, white $(82 \%)$ with a mean age of 55 (13). All met ACR 1987 or 2010 criteria for RA. One-third reported the same health status at both visits (68 [35\%]); $13 \%$ were a little better; a similar number were much better; $27 \%$ were a little worse, and $7 \%$ were much worse. Correlations between PROMIS and other measures assessing similar domains ranged from 0.32 to 0.83 . Among patients reporting being "much worse", PROMIS scores worsened from 1.0 to 8.1 points (mean CDAI change 9.2)(Table). Among patients feeling much better, PROMIS scores improved from 1.6 to 6.9 points (mean CDAI change -6.6). Change in PROMIS scores were largest in relation to changing disease activity for symptoms highly relevant to RA (e.g., PI, fatigue, PF); notably patients with worsening in RA did not report higher anxiety or depression scores.

Conclusion: These initial data suggest PROMIS measures are responsive to clinical changes in RA status and contribute to the growing literature supporting the use of PROMIS measures to assess physical, social, emotional and health in people with RA.

\section{8}

Using PROs to Guide Patient-Centered Conversations and Care in Inflammatory Arthritis: The Patient Perspective

Clifton Bingham (Johns Hopkins, Baltimore); Katherine Smith (Johns Hopkins, Baltimore); Elaine DeLeon (Johns Hopkins School of Medicine, Baltimore); Michelle Jones (Johns Hopkins School of Medicine, Baltimore); Krista Gutierrez (Johns Hopkins School of Medicine, Baltimore); Allie Butanis (Johns Hopkins School of Medicine, Baltimore); Susan Bartlett (McGill University, Montreal)

Objectives: Although optimal care is patient-centered and grounded in shared decision-making (SDM) between patients and providers, rheumatologists often have little insight into the day-to-day experiences of their patients with inflammatory arthritis. We hypothesized that the use of validated patient-reported outcomes (PROs) querying RA symptoms and impact would provide insight into patient priorities, values, and preferences and facilitate SDM around treatment choices.

Methods: Participants in an observational study at an academic arthritis center completed PROMIS fatigue, pain, physical function, sleep, and participation on a tablet in the waiting room. Reports of results in numerical and graphical formats were available during the visit for review and discussion with the rheumatologist. Within 48 hours of the clinic visit, patients completed surveys about the relevancy and impact on the clinical visit. In-depth interviews were conducted with a subset.

Results: Survey data are from 68 patients who were mostly white $(85 \%)$, female (81\% with a mean age of 54 (13) and RA duration of 10 (9) years. Interviews with 15 participants provided additional support to themes identified in the survey. Almost all (94\%) reported the PRO question addressed important aspects of their health: "addresses a wide range of issues, which is great"; "It shows the impact my arthritis has on me, not just the pain"; "Helps give a better overall picture of what's going on". Most $(82 \%)$ reported the discussion of results during the visit improved communication and made it easier to raise issues: "I love seeing data and graphs it really helps me"; "It focuses...discussion with your physician so you can

Personal non-commercial use only. The Journal of Rheumatology Copyright $(\subset) 2018$. All rights reserved. 
address the most pressing, sort of prioritize, the issues you want to address"; "Doctor referred to my answers during discussion". A few (3\%) were unclear if PRO results had been reviewed, or whether care was impacted: "I think my doctor [already] treats me well".

Conclusion: Patients place high value on PRO information which gives insight into day-to-day life and unmet needs. Expanded PRO assessment with real time review of results provides an opportunity for more patient-centered RA care and SDM by guiding conversations and improving communication about disease-related symptoms and impacts that matter to patients. Funding PCORI IP2-PI0000737, SC14-1402-10818.

\section{9}

Using PROs to Guide Patient-Centered Conversations and Care in Inflammatory Arthritis: The Clinician Perspective

Susan Bartlett (McGill University, Montreal); Katherine Smith (Johns Hopkins, Baltimore); Elaine DeLeon (Johns Hopkins School of Medicine, Baltimore); Michelle Jones (Johns Hopkins School of Medicine, Baltimore); Krista Gutierrez (Johns Hopkins School of Medicine, Baltimore); Allie Butanis (Johns Hopkins School of Medicine, Baltimore); Clifton Bingham (Johns Hopkins, Baltimore)

Objectives: Although patient-reported outcomes (PROs) are routinely collected for research and quality purposes, they have not been routinely incorporated into the routine care of patients. Clinicians are best able to provide chronic disease care that is patient-centered when they have a better understanding of their patients' lived experiences. We hypothesized that having PRO results describing the range of symptoms and impacts of living with RA during clinic visits would provide insight into patient priorities, values, and preferences and facilitate SDM around treatment choices. Evidence for the impact of PROs on patient management from the clinician's perspective are presented.

Methods: Participants with RA in an observational study at an academic arthritis center completed PROMIS fatigue, pain, physical function, sleep, and participation on a tablet in the waiting room. Reports of results in numerical and graphical formats were available during the visit for review. Semi-structured interviews were used to query the experiences of rheumatologists and rheumatology fellows on the impact of PROs on RA patient management.

Results: Data are from interviews with 4 rheumatologists and 6 fellow trainees. Among rheumatologists, access to real-time PROMIS results during the visit was highly valued. All stated that completing questionnaires helped patient feel "heard" and discussing results made it clear that patients' experiences mattered. Several said reports prompted them to ask about symptoms they may have overlooked. There was concern that some symptoms (depression, anxiety) may have little to do with RA; identifying these without a clear pathway to resources was potentially problematic. All noted that how they used results differed depending on the needs of specific patients and the nature of the visit. Fellows expressed less certainty about their ability to control conversations, the value of additional PROs for RA care, or its impact on decision-making. Fellows also reported greater discomfort discussing results if they had not yet built a rapport with patients.

Conclusion: Expanded real-time assessments of RA symptom and impacts on day-to-day life as part of routine care appears to offer important new information to make RA care more patient-centered by informing discussions and facilitating shared decision-making around treatment. For trainees, the value of additional symptom information was balanced by concerns about greater time challenges, ways to integrate results into discussions and care plans, and availability of resources to address new problems that were identified. Results highlight opportunities to enhance broader communication skills training around potentially sensitive topics and quality of life in rheumatology training programs.

100

Validity of Screening Tools for Depression and Anxiety in Rheumatoid Arthritis

Carol Hitchon (University of Manitoba, Winnipeg); Lisa Zhang (University of Manitoba, Winnipeg); James Bolton (University of Manitoba, Winnipeg); Lesley Graff (University of Manitoba, Winnipeg); Lisa Lix (University of Manitoba, Winnipeg); Christine Peschken (University of Manitoba, Winnipeg); Jitender Sareen (University of Manitoba, Winnipeg); John Walker (University of Manitoba, Winnipeg); Charles Bernstein (University of Manitoba, Winnipeg); Ruth Ann Marrie (University of Manitoba, Winnipeg); CIHR Team in Defining the Burden and Managing the Effects of Psychiatric Comorbidity in Chronic Immunoinflammatory Disease (Winnipeg)

Objectives: Psychiatric comorbidity is common in rheumatoid arthritis (RA), contributes to adverse health outcomes but is often underrecognized. Improved detection is needed to facilitate management. Screening tools for depression and anxiety developed for the general population require validation in RA because symptoms common to both conditions may lead to criterion contamination. We sought to validate screening tools for depression and anxiety in RA patients.

Methods: RA patients recruited from outpatient rheumatology clinics completed several widely used screening instruments for depression and anxiety: 2 and 9-item versions of the Patient Health Questionnaire (PHQ2, PHQ9), Patient Reported Outcomes Measurement Information System ${ }^{\circledR}$ (PROMIS®) Depression Short Form-8a and Anxiety Short Form-8a, the Hospital Anxiety and Depression scale (HADs), the Overall Anxiety Severity and Impairment Scale (OASIS), and the General Anxiety Disorder-7 item scale (GAD-7) and for distress, the Kessler-6 scale. Psychiatric morbidity was formally assessed using the Structured Clinical Interview for DSM-IV-TR Axis I Disorders - Research version (SCID), a gold standard semi-structured interview to identify anxiety and mood diagnoses. Interviews were conducted by trained personnel blinded to the findings of the screening instruments. We report the percent of patients with depression and anxiety based on the SCID and screening tools and estimated the sensitivity (SENS), specificity (SPEC) positive predictive value (PPV), negative predictive value (NPV), and accuracy of each screening tool relative to the SCID, along with $95 \%$ confidence intervals $(95 \% \mathrm{CI})$. Test-retest reliability was assessed with the intra-class correlation coefficient (ICC).

Results: The 146 RA patients evaluated were predominantly female $(86 \%)$ and Caucasian (80\%), with a mean (SD) age of 58.9(11.7) years. The percent of patients with SCID diagnosed depression and anxiety were $11.0 \%$ and $18.5 \%$, respectively. The screening tools identified depression in $8.9 \%$ (HADS-D11) to $29.5 \%$ (PHQ9) of patients and anxiety in $13.0 \%$ (HADS-A11) to $42.5 \%$ (HADS-A8) of patients. The Kessler-6 identified distress in $88.4 \%$ of patients. For depression, the PHQ-2 performed the best (SENS(95\% CI) 0.88(0.62-0.98), SPEC 0.84 (0.76-0.90), PPV 0.40 (0.24-0.58), NPV $0.98(0.94,1.00)$ and accuracy $0.84(0.77-0.90)$. For anxiety, most measures had poor sensitivity but reasonable specificity. All measures had good test-retest reliability with ICC estimates ranging from $0.79(0.71,0.85)($ HADS-A) to $0.88(0.83,0.92)$ (PROMIS depression short form).

Conclusion: Clinical depression and anxiety affect a large proportion of RA patients. The short PHQ-2 (for depression) is feasible to incorporate into clinical practice. Despite the risk of overidentifying, use of screening tools would help identify RA patients needing further mental health assessment.

\section{1}

Gaining Access for Indigenous North Americans with Rheumatic Disease: A Pilot Study to Identify Barriers to Clinic Attendance

Amber Cogar (University of Manitoba, Winnipeg); Derek Kornelsen (University of Manitoba, Winnipeg); Christine Peschken (University of Manitoba, Winnipeg); David Robinson (University of Manitoba, Winnipeg); Carol Hitchon (University of Manitoba, Winnipeg); GAIN-RD Gaining Access for Indigenous North Americans with rheumatic disease (Winnipeg) Objectives: Rheumatic diseases have a high prevalence among Indigenous North Americans (INA), with associated higher rates of pain, disability, comorbidity, and mortality than other ethnic groups. This suggests a large care gap for INA with rheumatic disease. Unfortunately, this severe disease is accompanied by overwhelming evidence of underutilization of specialist

Personal non-commercial use only. The Journal of Rheumatology Copyright (C 2018. All rights reserved. 
care by INA arthritis patients (INAP), with fewer visits to specialists, frequent non-attendance at clinic visits, and frequent loss to follow-up. This leads to suboptimal rheumatology care delivery. As a first step to exploring barriers faced by INAP relating to clinic attendance we conducted semistructured interviews with health care providers who have experience in caring for INAP.

Methods: Health care providers (HCPs) and healthcare support workers (HCSWs) working in hospital-based internal medicine chronic disease subspecialty clinics in Winnipeg, Manitoba were invited to participate in semi-structured interviews of 30 - 60 minutes duration. Interviews focused on barriers to care for INA as perceived by participants or reported by INA patients to participants. Interviews were audiotaped and transcribed verbatim (AC). Interview transcripts were reviewed and coded for themes separately by two rheumatologists $(\mathrm{AC}, \mathrm{CH})$ using DeDoose software for qualitative analysis. Descriptive analysis of interviews is presented combined with informative quotations.

Results: A total of 8 interviews were conducted (3 HCP and $5 \mathrm{HCSW}$ ) before theme saturation was reached. Themes identified included: 1) difficulty notifying patients of appointments (often due to difficulty contacting patient resulting from frequent changes in contact information. This leads to patients not receiving notifications of appointment times);2) travel difficulties (particularly weather-related, as well as black-out periods from transportation services from northern communities); 3 ) personal issues limiting ability to attend appointments (e.g., not being able to find child care for a multi-day trip south to Winnipeg); 4) apprehension with travel to city; and 5) knowledge gaps (e.g., not appreciating the importance of attending for follow-up even when feeling subjectively well)

Conclusion: Understanding barriers to clinic attendance is a required first step to improving the delivery of rheumatology care to this vulnerable population. This preliminary analysis identified several actionable areas including improving systems for patient notification of appointments, and improving the patient experience potentially by increasing patient supports while attending clinics. Engaging patients in efforts to improve the delivery of rheumatology care is critical and future studies will obtain the patient perspective regarding barriers faced by INAP preventing clinic attendance.

\section{2}

Systematic Review of Randomized Controlled Trials Assessing Efficacy and Safety of Hydroxychloroquine in Systemic Lupus Erythematosus Kimberly Legault (McMaster University, Hamilton); Carlos Cuello-Garcia (McMaster University, Hamilton); Peter Tugwell (University of Ottawa, Ottawa)

Objectives: Effective treatment of systemic lupus erythematosus (SLE) has been hampered by the relative lack of therapies supported by good-quality evidence of benefit. Despite the longstanding use of hydroxychloroquine (HCQ) in SLE, systematic reviews of the evidence of benefit and summary estimates of treatment efficacy and harms are lacking. The objective of this Cochrane systematic review is to assess the benefit and harm of HCQ for the treatment of SLE.

Methods: All randomized controlled trials comparing HCQ at any dose with either placebo or no HCQ in adult patients with SLE were included. The major outcomes of interest were disease activity, steroid equivalent, quality of life, death, withdrawals due to adverse events, serious adverse events (SAEs), and serious infections. We searched CENTRAL, MEDLINE, EMBASE and Google Scholar. Two review authors independently screened titles, abstracts and full texts for inclusion, extracted data and assessed risk of bias for each study. Meta-analyses were carried out where meaningful. A 'Summary of findings' table was created with independent assessment of evidence quality for each outcome.

Results: The search strategy identified 3509 published studies, of which 144 were duplicate, 3297 were excluded after title and abstract screening, and 52 were excluded after full text screening, leaving 5 articles for inclusion (n $=164 \mathrm{HCQ}, \mathrm{n}=94$ placebo). Meta-analyses could not be performed on most measures due to heterogeneity of data. Across 5 studies, the HCQ groups showed improved disease activity with lower risk of flare compared to placebo groups (numerically or statistically). In 2 studies, steroid equivalent doses were numerically or statistically lower in the HCQ groups. One study described quality of life, with numerically higher scores in the HCQ group that did not reach statistical significance. There was one (HCQ) death reported. Across all studies, 7/164 in the HCQ groups withdrew and 5/94 in the placebo groups withdrew for adverse events. No studies specifically reported serious infections. The quality of evidence was generally low. Note: The results are preliminary and are awaiting peer review through the Cochrane Musculoskeletal Group.

Conclusion: This systematic review of the RCT evidence of the efficacy and safety of HCQ compared to placebo in SLE demonstrates low quality evidence of improvement in disease activity in patients taking HCQ compared to placebo, while showing no conclusive difference in quality of life, serious adverse events, steroid equivalents, or withdrawal due to adverse effects between groups, mortality and serious infections. Best Abstract on Research by Young Faculty.

\section{3}

Patterns of Aspirin Use in SLE Pregnancies within a Multinational Inception Cohort

Arielle Mendel (McGill University, Montreal); Sasha Bernatsky (McGill University Health Centre, McGill University, Montreal); SLICC Systemic Lupus Erythematosus International Collaborating Clinics (Toronto); Evelyne Vinet (McGill University Health Centre, Montreal)

Objectives: Because aspirin reduces the risk of preeclampsia in high-risk pregnancies by more than half, best practice guidelines recommend that aspirin be initiated in pregnant women with $\geq 1$ high-risk factors for preeclampsia, including SLE. Our objective was to assess the prevalence of aspirin use in SLE pregnancies within a multinational inception cohort, and compare aspirin use among those with and without additional preeclampsia risk factors

Methods: Premenopausal women aged 18-45 enrolled in the Systemic Lupus International Collaborating Clinics (SLICC) Registry (2000-2017) within 15 months of SLE onset were evaluated with yearly visits to update co-morbidities, pregnancy status, and medications. Study visits with a current pregnancy were assessed for aspirin use and preeclampsia risk factors. Aspirin use was compared over time and among those with and without traditional risk factors (i.e. hypertension, renal disease, diabetes, nulliparity, $\mathrm{BMI} \geq 35$, age $>40$ ), as well as disease-specific risk factors [i.e. antiphospholipid antibodies (+aPL), high disease activity i.e. SLEDAI $>12]$.

Results: We identified 297 women who had 479 pregnancies over the study period. Mean age during pregnancy was 31 (SD 4.9) years and 30\% were nulliparous. Half of the pregnancies $(51 \%)$ experienced $\geq 1$ traditional preeclampsia risk factors in addition to SLE, while a third (33\%) had +aPL. We observed aspirin use in $25 \%$ of pregnancies $(95 \%$ CI 22,29$)$ versus $22 \%$ $(95 \%$ CI 19,25) of visits before and after pregnancy among the same women. Aspirin use was similar among pregnancies with and without $\geq 1$ traditional risk factor for preeclampsia [25\% (95\% CI 20,31) versus $26 \%(95 \% \mathrm{CI}$ $21,32)$ ], while we observed a higher prevalence of aspirin use in those with $+\mathrm{aPL}$ [38\% (95\% CI 24,55)] versus those without [23\% (95\% CI 15,34)]. There was a significant difference in aspirin use based on maternal race/ethnicity, with $32 \%(95 \%$ CI 26,39) aspirin use in Caucasians versus $10 \%(95 \%$ CI 5,18$)$ for black women. Regional variability was observed in aspirin use (12-37\%). We could not establish a trend of increasing aspirin use over time.

Conclusion: In this cross-sectional analysis of SLE pregnancies, we observed that most women were not on aspirin and that half had additional preeclampsia risk factors. It is possible that aspirin was introduced at/or following the study visit when the pregnancy was documented, highlighting the importance of the treating rheumatologist in reviewing aspirin use and initiating it, if not already done, in pregnant SLE women. Our findings suggest black SLE women as a potentially vulnerable group during pregnancy, having the lowest prevalence of aspirin use. Best Abstract on Quality Care Initiatives in Rheumatology. 


\section{4}

Combined Hormonal Contraception use in SLE Women with and without Medical Contraindications

Arielle Mendel (McGill University, Montreal); Sasha Bernatsky (McGill University Health Centre, McGill University, Montreal); Christian Pineau (Montreal General Hospital, Division of Rheumatology, Montreal); Yvan St. Pierre (McGill University, Montreal); SLICC Investigators (Toronto Western Hospital, Toronto); Evelyne Vinet (McGill University Health Centre, Montreal)

Objectives: Combined hormonal contraceptives $(\mathrm{CHC})$ are contraindicated in specific medical conditions due to an increased risk of cardiovascular and thromboembolic events. Our objective was to assess the prevalence of $\mathrm{CHC}$ use in reproductive-aged women with systemic lupus erythematosus (SLE) with and without possible contraindications, and determine factors associated with possible inappropriate $\mathrm{CHC}$ use.

Methods: Premenopausal women aged 18-45 enrolled in the Systemic Lupus International Collaborating Clinics (SLICC) Registry (2000-2017) within 15 months of SLE onset were evaluated yearly to update drug use, including CHC. For each study visit, the presence of World Health Organization (WHO) Category 3 or 4 contraindications to CHC (e.g. smoker age $\geq 35$, hypertension, migraine with aura, antiphospholipid antibodies, stroke) was assessed. High disease activity (Systemic Lupus Erythematosus Disease Activity Index $>12$ or use of $>0.5 \mathrm{mg} / \mathrm{kg} /$ day of prednisone) was evaluated as a relative contraindication, as safety of estrogen in this setting is not established. Multivariate logistic regression examined the association between socio-demographic characteristics and $\mathrm{CHC}$ use with $\geq 1$ possible contraindications to estrogen.

Results: 1224 SLE women contributed 7756 study visits, of which 4477 (58\%) occurred in the presence of $\geq 1$ possible contraindications to $\mathrm{CHC}$. Women used CHC during 666 (9\%) visits, of which 310 (40\%) took place in the setting of $\geq 1$ possible contraindication. The most frequently observed WHO category- 3 or -4 contraindications among these visits were antiphospholipid antibodies (47\%), hypertension (37\%), and migraine with aura $(22 \%)$. Women with $\geq 1$ possible contraindication were less likely to be taking CHC (310/4477 visits, 7\% [95\% CI, 6-8]) compared to women with no contraindications (356/3279 visits, $11 \%$ [95\% CI 10-12]). Results of multivariate analysis were inconclusive.

Conclusion: In this international inception cohort of reproductive-aged SLE women, $\mathrm{CHC}$ use was low (9\% of visits) compared to general population estimates (greater than $35 \%$ ). CHC was used less frequently among women with contraindications, but a significant proportion (40\%) of CHC users possessed $\geq 1$ possible contraindications to $\mathrm{CHC}$. Further work is needed to examine factors behind contraceptive choices in SLE, and clarify the long-term outcomes associated with this exposure.

\section{5}

Can the Automated Neuropsychological Assessment Metrics (ANAM) Predict Cognitive Impairment Compared to a Comprehensive Neuropsychological Battery in Patients with Systemic Lupus Erythematosus (SLE)?

Zahi Touma (Toronto Scleroderma Program, Division of Rheumatology, Toronto Western Hospital, University Health Network; Institute of Health Policy, Management and Evaluation, University of Toronto, Toronto); Dorcas Beaton (University of Toronto/Institute for Work and Health, Toronto); Carmela Tartaglia (University Health Network, Toronto); Lesley Ruttan (University Health Network, Toronto); Sabrina Lombardi (University Health Network, Toronto); SLICC Investigators (Toronto Western Hospital, Toronto); Jiandong Su (Toronto Western Hospital, Toronto); Kenneth Colosimo (Toronto Western Hospital, Toronto); Michelle Vitti (Toronto Western Hospital, Toronto); Dennisse Bonilla (Toronto Western Research Institute, Toronto); Joan Wither (University of Toronto, Toronto); Marvin Fritzler (University of Calgary, Calgary); Robin Green (University Health Network, Toronto)

Objectives: The diagnosis of cognitive impairment (CI) is often delayed requiring use of a comprehensive battery $(\mathrm{CB})$ which imposes a time- and cost-burden. It would be beneficial to have a more expedient CI screening tool that could be applied in the ambulatory clinic setting. The Automated Neuropsychological Assessment Metrics (ANAM) is a computerized tool that can be used to screen for CI. We determined the ability of ANAM (v4) GNS Battery to predict CI in patients with SLE.

Methods: Consecutive patients $(n=98)$, aged 18-65 years, who attended a single center were administered the ANAM and CB on the same day. ANAM throughput scores were used to provide an estimate of 'cognitive efficiency'. Scores on the ANAM and CB were compared to a normative sample of age- and gender-matched healthy controls to obtain z-scores. The CB evaluates the major cognitive domains: manual motor speed and dexterity, simple attention and processing speed, visual-spatial construction, verbal fluency, learning and memory (visuospatial and memory), and executive functioning (untimed and timed). ANAM evaluates the major cognitive domains: attention and processing speed, memory, visual-spatial processing, executive functioning, abstract language function and fine motor processing. CI was operationalized on the CB and ANAM as a z-score of $\leq-1.5$ on $\geq 2$ domains or a $\mathrm{z}$-score $\leq-2.0$ on $\geq 1$ domains, or either. Performance of ANAM was compared against the CB using different CI definitions. Descriptive analysis was used to determine prevalence, sensitivity ( $\mathrm{Sn}$ ), specificity (Sp), Positive Predictive Value (PPV) and Negative Predictive Value (NPV).

Results: Of the 98 patients ( $90.8 \%$ female), the mean age at SLE diagnosis was $28.5 \pm 10.2$ and disease duration at enrolment was $15.5 \pm 10.0$ years. Prevalence of CI using CB ranged between $40.0-44.8 \%$ ( $\mathrm{z} \leq-1.5$ in $\geq 2$ domains and $z \leq-2.0$ in $\geq 1$ domains, respectively) and $55.2 \%$ for either. Prevalence of CI using the ANAM ranged between $30.8-39.3 \% \%$ ( $\mathrm{z} \leq-1.5 \mathrm{in}$ $\geq 2$ domains and $z \leq-2.0$ in $\geq 1$ domains, respectively) and $42.3 \%$ for either. ANAM sensitivity/specificity was 52/73\% and PPV/NPV was 70/55\% [based on $z \leq-1.5$ in $\geq 2$ domains or $z \leq-2.0$ in $\geq 1$ domains for ANAM and $C B]$.

Conclusion: ANAM is a promising tool for the assessment of CI in SLE. Future studies are required to determine if the sensitivity of the ANAM can be improved against the current CB.

\section{6}

Physician Resources and Activities in Canadian Academic Rheumatology Units 1998-2017

John Hanly (Dalhousie University and Nova Scotia Health Authority, Halifax); Canadian Council of Academic Rheumatologists (CCAR) (Dalhousie University and Nova Scotia Health Authority, Halifax)

Objectives: To evaluate changes in demographic features of adult and pediatric rheumatologists, time spent on clinical and academic activities and productivity of training programs in Canadian academic rheumatology centers between 1998-2017.

Methods: Members of the Canadian Council of Academic Rheumatologists performed standardized annual data collection. Information included physician and trainee demographics, recruitment, allocation of time to clinical care, teaching, research and administration, and pursuit of additional skills by trainees following core rheumatology training. Data collection was centralized, validated and stored in an electronic database.

Results: Over 19 years the number of academic rheumatologists increased by $72 \%$ from 172 (98 full-time, 74 part-time) to 295 ( 215 full-time, 80 part-time). The increase occurred for both adult $(106 / 152,70 \%)$ and pediatric $(17 / 20,85 \%)$ rheumatologists. The mean age increased from $47.8(31-76)$ to 51.1 (31-79) years and the male:female ratio changed from 2.6 to 0.8 . The mean proportion of time allocated to clinical care (54.2-57.8\%), teaching (15-17.3\%), research (18.8-22.1\%) and administration (6.4-7.6) was stable over time. The number of unfilled adult and pediatric faculty positions was 27 (12/16 centers) in 1998, reached a peak of 36 (14/16 centers) in 2008 and fell to 17 (10/16 centers) in 2017. These positions covered the spectrum of academic tracks and the most frequently cited barrier to recruitment was the lack of suitably qualified applicants. There were 38 Rheumatology trainees (adult and pediatric PGY4/5) in 1998 which reached a nadir of 25 in 2001 and rose steadily thereafter to 91 in 2017. The male:female ratio of trainees was lower than faculty in each year, changing from 1.11 in 1998 to 0.47 in 2017. In 1998, funding of training positions was shared by provincial

Personal non-commercial use only. The Journal of Rheumatology Copyright (c) 2018. All rights reserved. 
governments $(18 / 38,47 \%)$, Arthritis Society $(9 / 38,23 \%)$ and external sources $(11 / 38,30 \%)$ but this distribution changed to $86 \%, 1 \%$ and $13 \%$ respectively by 2017. Additional skills training following PGY5 was averaged in 2 year blocks and fell from $41.9 \%$ to $12.8 \%$ of trainees. Over time the number of individuals who pursued additional clinical training changed from 3.5 to 5.0 per year, clinical research from 8.5 to 5.5 and basic science research from 3.5 to 1.0 .

Conclusion: The national complement of academic rheumatologists has increased. Improved gender balance and increased trainees signal a vibrant interest in the specialty. The sustained commitment to clinical and academic responsibilities by faculty is reassuring, but reduction in post PGY5 training, especially in research methodology, is a concern.

\section{7}

Epidemiology and Survival of Systemic Sclerosis-Systemic Lupus Erythematosus Overlap Syndrome

Samar Alharbi (Toronto Scleroderma Program, Division of Rheumatology, Toronto Western Hospital, University Health Network; Division of Rheumatology, Mount Sinai Hospital; University of Toronto; Taibah University, Toronto); Zareen Ahmad (Toronto Scleroderma Program, Division of Rheumatology, Department of Medicine, Sinai Health Systems, University of Toronto, Toronto); Arthur Bookman (Toronto Scleroderma Program, Division of Rheumatology, Toronto Western Hospital, University Health Network, Toronto); Zahi Touma (Toronto Scleroderma Program, Division of Rheumatology, Toronto Western Hospital, University Health Network; Institute of Health Policy, Management and Evaluation, University of Toronto, Toronto); Jorge Sanchez-Guerrero (Toronto Scleroderma Program, Division of Rheumatology, Toronto Western Hospital, University Health Network; Division of Rheumatology, Mount Sinai Hospital; University of Toronto, Toronto); Nicholas Mitsakakis (Institute of Health Policy, Management and Evaluation, University of Toronto; Toronto Health Economics and Technology Assessment Collaborative, University of Toronto, Toronto); Sindhu Johnson (Toronto Scleroderma Program, Division of Rheumatology, Department of Medicine, Sinai Health Systems, University Health Network, Institute of Health Policy, Management and Evaluation, University of Toronto, Toronto)

Objectives: Systemic sclerosis (SSc) is an immune disorder characterized by vasculopathy and fibrosis that may overlap with another disease such as systemic lupus erythematous (SLE). Little is known about the epidemiology, clinical characteristics, and survival of SSc-SLE overlap (also called lupoderma). We evaluated the prevalence of SSc-SLE overlap syndrome, differences in SSc clinical characteristics and survival compared with SSc without SLE.

Methods: A cohort study was conducted including subjects who fulfilled the ACR-EULAR classification criteria for SSc and/or the ACR criteria for SLE. The primary outcome was the time from diagnosis to death from all causes. Survival was evaluated using Kaplan Meier curves and Cox Proportional Hazard models

Results: We identified 1252 subjects $($ SSc $n=1166$, SSc-SLE $n=86)$ with a SSc-SLE prevalence of $6.8 \%$. SSc-SLE were younger at diagnosis (37.9 years versus 47.9 years, $\mathrm{p}<0.001)$, more frequently had lupus anticoagulant $(6 \%$ versus $0.3 \%, \mathrm{p}<0.001)$, anticardiolipin antibody $(6 \%$ versus $0.9 \%, \mathrm{p}<$ $0.001)$, and pulmonary arterial hypertension $(\mathrm{PAH})(52 \%$ versus $31 \%, \mathrm{p}<$ $0.001)$. SSc-SLE less frequently had calcinosis $(13 \%$ versus $27 \%, \mathrm{p}=0.007)$, telangiectasia $(49 \%$ versus $75 \%, \mathrm{p}<0.001)$ and diffuse subtype $(12 \%$ versus $35 \%, \mathrm{p}<0.001)$. There were no significant differences in the occurrence of renal crisis (7\% versus $7 \%$ ), interstitial lung disease (40\% versus $34 \%$ ), and digital ulcers (38\% versus 32\%). SSc-SLE had better survival (median 26.1 versus 22.4 years), but this was not statistically significant (log rank $\mathrm{p}=$ $0.06)$. Female sex and diffuse subtype attenuated survival differences between groups (Hazard Ratio 0.70, 95\% CI 0.45, 1.11)

Conclusion: SSc-SLE are younger at diagnosis, more frequently have PAH, and less frequently have cutaneous manifestations of SSc. SSc-SLE patients should be monitored for pulmonary hypertension, interstitial lung disease, renal crisis and digital ulcers

\section{8}

Venous Thromboembolism in Systemic Sclerosis: Prevalence, Risk Factors and Impact on Survival

Sindhu Johnson (Toronto Scleroderma Program, Division of Rheumatology, Department of Medicine, Sinai Health Systems, University Health Network, Institute of Health Policy, Management and Evaluation, University of Toronto, Toronto); Nabil Hakami (Toronto Scleroderma Program, Mount Sinai Hospital, Toronto Western Hospital, Division of Rheumatology, Department of Medicine, University of Toronto, King Fahad Central Hospital, Ministry of Health, Toronto); Zareen Ahmad (Toronto Scleroderma Program, Division of Rheumatology, Department of Medicine, Sinai Health Systems, University of Toronto, Toronto); Duminda Wijeysundera (Department of Anesthesia and Pain Management, Toronto General Hospital, Department of Anesthesia and Institute of Health Policy, Management and Evaluation, University of Toronto, Toronto)

Objectives: Whether systemic sclerosis (SSc) confers increased risk of venous thromboembolism (VTE) is uncertain. We evaluated the incidence, risk factors and impact of VTE on SSc survival.

Methods: A cohort study of SSc subjects who fulfilled the ACR-EULAR classification criteria between 1970-2017 was conducted. Deep vein thrombosis was defined as thrombus on extremity ultrasound. Pulmonary embolism was defined as thrombus on thorax $\mathrm{CT}$ angiogram. Risk factors for VTE and time to all-cause mortality were evaluated.

Results: Of the 1181 subjects, 40 (3.4\%) experienced VTE events. The cumulative incidence of VTE was 2.7 (95\% CI 1.9, 3.7) per 1000 patient-years. PAH (OR 3.77 (96\% CI 1.83,8.17), peripheral arterial disease (OR 5.31 (95\% CI 1.99,12.92), ScL70 (OR 2.45 95\% CI 1.07.5.30), and anticardiolipin antibodies (OR 5.70 (95\% CI 1.16,21.2) were predictors of VTE. There were 440 deaths. There was no difference in survival between those with and without VTE (HR 1.16 (95\% CI 0.70,1.91). ILD (HR $1.54(95 \% \mathrm{Cl}$ $1.27,1.88$ ) and PAH (HR 1.35 (95\% CI 1.10,1 .65) were predictors of mortality. Conclusion: The risk of VTE in SSc is comparable to the general population. The presence of PAH, peripheral arterial disease, ScL70 and anticardiolipin antibodies are risk factors for VTE. VTE does not independently predict SSc survival.

\section{9}

Ethnic Variation in Systemic Sclerosis Morbidity and Mortality

Haifa Al-Sheikh (Toronto Scleroderma Program, Division of Rheumatology, Department of Medicine, Sinai Health Systems, University Health Network, University of Toronto, Toronto); Zareen Ahmad (Toronto Scleroderma Program, Division of Rheumatology, Department of Medicine, Sinai Health Systems, University of Toronto, Toronto); Sindhu Johnson (Toronto Scleroderma Program, Division of Rheumatology, Department of Medicine, Sinai Health Systems, University Health Network, Institute of Health Policy, Management and Evaluation, University of Toronto, Toronto)

Objectives: Systemic sclerosis (SSc) is an uncommon connective tissue disease characterized by pathological skin thickening and can involve multiple internal organs. Ethnic variations in SSc have been reported in clinical manifestations, severity of the disease as well as survival. Our aim was to compare the survival and disease manifestations across ethnicity among SSc patients.

Methods: The Toronto Scleroderma Program is the largest single-center, multiethnic, longitudinal SSc cohort in Canada. Patients are followed every 6 to 12 months using a standardized protocol. Patients who fulfilled the American College of Rheumatology-European League Against Rheumatism classification criteria for SSc and are 16 years of age or older were included in our retrospective cohort study. The study period was 1970-2017. Ethnicity was self-reported and was categorized as: Caucasian, African-American, Hispanic, Arab, East-Asian, First Nations or Persian. The primary outcome was the time from diagnosis to death from all causes. Secondary outcomes were differences in disease duration, SSc subtype, clinical manifestations, and serology. Survival probabilities and median survival times were determined using Kaplan-Meier survival curves. Cox proportional hazard models were used to estimate adjusted survival. 
Results: 1005 subjects were evaluated, the majority of whom were Caucasian $(\mathrm{n}=745(74 \%)$, African-American $\mathrm{n}=58(6 \%))$, South Asian $(n=69(7 \%))$, and East Asian $(n=80(8 \%))$. Compared to Caucasians, East Asians less frequently had calcinosis $(29 \%$ versus $9 \%, p=0.002)$, and esophageal dysmotility ( $88 \%$ versus $69 \%, \mathrm{p}=0.002$ ); African-Americans more frequently had interstitial lung disease $(31 \%$ versus $53 \%, p=0.007)$; and First Nation subjects more frequently had diffuse cutaneous disease (35\% versus $56 \%, \mathrm{p}=0.02)$ and diabetes $(5 \%$ versus $33 \%, \mathrm{p}=0.03)$. There were no differences across ethnicities in the prevalence of pulmonary hypertension, renal crisis, or digital ulcers. We found no difference in the short-term survival across ethnicities. However, in the long-term, there was trend for Hispanic subject to have better survival $(81.3 \%$ (95\% CI 63, 100), while First Nations $(58.3 \%$ (95\% CI 25, 100) and South Asian subjects $(52.6 \%(95 \%$ CI 32,87$)$ had worst survival at 15 years and 20 years, respectively. East Asians appear to have the longest median survival time 43.3 years. Conclusion: Ethnic variations in disease SSc disease manifestations are observed. However, in the setting of a universal health care system, this does not result in significant differences in survival.

\section{0}

Guillain Barre Syndrome as the First Presentation of Systemic Lupus Erythematosus

Erika Wall (University of Alberta, Edmonton); Steven Katz (University of Alberta, Edmonton)

Background: Systemic lupus erythematosus (SLE) has historically been referred to as "the disease of a thousand faces" for its varied presentations and diagnostic elusiveness. When the disease presents in an atypical manner, SLE may be missed even when sufficient evidence to support the diagnosis exists.

Case Presentation: A 32-year-old female admitted to the neurological intensive care unit was consulted to rheumatology for concern of systemic vasculitis. Four weeks prior to evaluation, the patient was seen in the emergency department for a ten-day history of generalized weakness, fatiguability, and paresthesias. She developed descending quadriparesis and areflexia and was given a diagnosis of Guillain Barre Syndrome (GBS). She was treated with plasma exchange (PLEX) and intravenous immune globulin (IVIG). After three weeks of hospitalization her muscle strength improved to 3-4/5 globally and she was transferred to a rehabilitation center. Within one day of transfer, the patient developed worsening weakness, chest tightness, and dysphagia. She required intubation for hypercapnic respiratory failure. Physical examination revealed complete absence of motor and reflex activity below the neck. There was generalized edema in the hands bilaterally but no joint effusions or rashes. There were no oral ulcerations. Complete blood count showed microcytic anemia, thrombocytosis, and leukocytosis. Serum creatinine was within normal limits. Urinalysis showed proteinuria and a significantly elevated protein to creatinine ratio. Immunologic testing showed positive ANA, anti-SSA, anti-RNP, anti-Sm, and p-ANCA with a negative MPO. ESR was elevated. Anti-centromere, anti-dsDNA, and APLA testing was negative. Complements were within normal limits. Nerve conduction studies showed globally diminished responses with sural sparing, positive fibrillation, and positive sharp waves consistent with GBS. Lumbar puncture showed mild albuminocytologic dissociation. Renal biopsy was consistent with lupus nephritis.

Discussion: The patient was given a diagnosis of neurologic SLE and was started on a three-day course of pulse solumedrol followed by high dose prednisone and cyclophosphamide as per Euro-Lupus protocol. Over the course of several weeks she significantly improved, was extubated, and began to regain functional capacity.

Conclusion: This case illustrates the challenge of arriving at a diagnosis of SLE in an atypical presentation. In addition, it highlights fascinating diagnostic differences in immune-mediated and vasculitic polyneuropathies. Finally, this case emphasizes that the value of explaining all inconsitencies and abnormalities in history and laboratory investigations is not limited to arriving at an academically correct diagnosis, but is the foundation of determining the most effective treatment and management plan for a patient.
111

Activation of Mammalian Target of Rapamycin in Renal Tissue of Patients with Scleroderma Renal Crisis: A Hypothesis-testing Study

Jessica Salituri (McGill University, Montreal); Marie Hudson (McGill University, Jewish General Hospital, Lady Davis Institute for Medical Research, Montreal); Patey Nathalie (University of Montreal, Montreal); Laeora Berkson (McGill University, Montreal); Murray Baron (McGill University, Jewish General Hospital, Montreal)

Objectives: Scleroderma renal crisis (SRC) is a rare but serious complication affecting $2-15 \%$ of patients with systemic sclerosis (SSc). Despite treatment with ACE inhibitors, outcomes for SRC patients are still poor, with one year mortality as high as $30 \%$. The cellular signaling mechanisms in SRC are not yet known. Mammalian target of rapamycin (mTOR) has been shown to be activated in vascular lesions of renal transplant patients with anti-phospholipid antibody syndrome (APLS). Given the similarities between the pathophysiology of SRC and APLS, we hypothesized that the mTOR pathway would also be activated in the renal vasculature of patients with SRC.

Methods: We retrospectively analyzed renal biopsies of four patients with scleroderma renal crisis in the Canadian Scleroderma Research Group cohort. Immunostaining was performed using anti-P-S6RP antibodies to evaluate the phosphorylation of mTOR, indicating pathway activation.

Results: All patients were female with a mean age of 57.25 ( $\mathrm{SD} \pm 18.3$ ). All had diffuse cutaneous SSc and satisfied the 2013 ACR Classification Criteria for SSc. Average disease duration at time of SRC presentation was 3.5 months ( $\mathrm{SD} \pm 5.7$ ). Two had anti-RNA polymerase III antibodies. All renal biopsies had histological features consistent with SRC. mTOR was activated in all four samples: three out of four samples showed phosphorylation in the renal vasculature and all showed phosphorylation in plasma cells and circulating inflammatory infiltrate.

Conclusion: Our preliminary results show mTOR activation in the vasculature of patients with SRC. Further work is needed to better characterize the difference between mTOR activation in SRC patients versus other renal pathologies. mTOR inhibitors are currently available and in development. If these findings are validated, they could inform further research into novel treatment targets for SRC. Best Abstract on Basic Science Research by a Trainee.

112

The Prevalence of Electrocardiographic Conduction Abnormalities in Systemic Sclerosis is Very Similar to that Found in Healthy Individuals: Data from the Canadian Scleroderma Research Group

Sophie Wojcik (McGill University Health Centre, Montreal); Christos Galatas (McGill University Health Centre, Montreal); Thao Huynh (Montreal University Health Centre, Montreal); Licia Iacoviello (IRCCS Istituto Neurologico Mediterraneo, Pozzilli); Simona Costanzo (IRCCS Istituto Neurologico Mediterraneo, Pozzilli); Giovanni de Gaetano (IRCCS Istituto Neurologico Mediterraneo, Pozzilli); Mianbo Wang (Lady Davis Institute for Medical Research, Montreal); Marie Hudson (McGill University, Jewish General Hospital, Lady Davis Institute for Medical Research, Montreal); Murray Baron (McGill University, Jewish General Hospital, Montreal)

Objectives: Electrocardiograms (ECG) are one of the modalities used to identify evidence of cardiac disease in Systemic Sclerosis (SSc). Commonly reported ECG abnormalities attributed to SSc include conduction abnormalities such as left anterior fascicular block (LAFB) and first-degree atrioventricular block (AVB). These abnormalities, however, are not infrequent in the general population. The objective of this study is to compare the prevalence of ECG conduction abnormalities in SSc and healthy controls.

Methods: ECGs were routinely performed on subjects in the Canadian Scleroderma Research Group cohort at their baseline visit, and compared to ECGs from a random sample of age and sex-matched controls from the Moli-Sani project database. This database consists of 24,325 healthy individuals $>35$ years of age living in the Molise region in Italy between March 2005 and April 2010. Two cardiologists read all of the ECGs using a

Personal non-commercial use only. The Journal of Rheumatology Copyright @ 2018 . All rights reserved. 
standardized approach. T-test, Chi-squared test and Fisher exact test were used to comparethe two groups. P-values $<0.05$ were considered significant. Results: SSc subjects $(\mathrm{n}=833 ; 86 \%$ females, mean age $56.3(+/-11.9)$ years, mean disease duration from first non-Raynaud's symptom $11.3(+/-9.3)$ years, $39.4 \%$ with diffuse cutaneous $\mathrm{SSc})$ and healthy controls $(\mathrm{n}=830$; matched to sex and age) were included. The prevalence of conduction abnormalities was similar in the SSc and control groups: left bundle branch block $1.7 \%$ and $1.6 \%(\mathrm{p}=0.85)$; right bundle branch block $3 \%$ and $2.8 \%(\mathrm{p}=$ $0.78)$; LAFB $3.1 \%$ and $3.9 \%(\mathrm{p}=0.41)$ and first-degree AVB $3.8 \%$ and $4 \%$ $(\mathrm{p}=0.89)$, respectively. Differences were found in the proportions of SSc subjects vs. controls with right atrial enlargement $(5 \%$ vs. $0.1 \%(\mathrm{p}<0.0001))$ and right axis deviation $(3.2 \%$ vs. $0.4 \%(\mathrm{p}<0.0001))$. Left atrial enlargement was also more common in SSc versus controls $(9.2 \%$ versus $1.6 \%$, respectively, $(\mathrm{p}<0.0001))$.

Conclusion: Although ECGs are routinely used to identify conduction abnormalities in SSc which are then attributed to the disease, our findings suggest that these are not specific to SSc, as they are identified in similar proportions of healthy individuals. The increased prevalence of signs of right heart stress are most likely secondary to pulmonary hypertension, rather than a direct effect of SSc on cardiac tissue. The only abnormality that we found more frequently in SSc, and which may indicate intrinsic cardiac disease, is left atrial enlargement. Best Abstract on Clinical or Epidemiology Research by a Trainee - Phil Rosen Award.

\section{3}

Treatment Algorithms for Systemic Sclerosis According to Experts Kyle Walker (University of Ottawa, Department of Medicine, Division of Rheumatology, Ottawa); Andreu Fernándes-Codina (Western University, Department of Medicine, Division of Rheumatology \& Internal Medicine Department, Hospital Universitari Vall d'Hebron, Universitat Autònoma de Barcelona, Barcelona); Janet Pope (Western University, Department of Medicine, Division of Rheumatology, London)

Objectives: Treatment options for Systemic Sclerosis (SSc) are limited (especially after first line treatment), and scarce data are available for choosing the order of treatment. The aim of this study was to update the SSc treatment algorithms obtained in 2012, based on SSc experts daily practice. Methods: An initial survey was designed based on the 2012 algorithms. The survey asked members of the Scleroderma Clinical Trials Consortium (SCTC, N = 160) whether they agreed with the 2012 algorithms or not, and which changes should be considered. Further consensus rounds were done to refine agreement ( 3 rounds in total); 62, 54 and 48 experts completed first, second and all surveys respectively.

Results: For scleroderma renal crisis (SRC), $82 \%$ of the experts agreed with the suggested algorithm (1st line angiotensin converting enzyme inhibitors [ACEI], 2nd and 3rd adding: calcium channel blockers [CCB] or angiotensin receptor blockers $[\mathrm{ARB}]$, and 4th alpha-blocker). For pulmonary arterial hypertension $(\mathrm{PAH})$, the agreement was $81 \%$. For mild $\mathrm{PAH}$, the majority suggested first phosphodiesterase 5 inhibitors (PDE5i), then endotelin receptor antagonists (ERA) plus PDE5i, then prostanoids; while for severe PAH 1st prostanoids, 2nd ERA plus PDE5i, 3rd ERA plus prostanoids. The Raynaud's phenomenon (RP) algorithms had $78 \%$ of agreement [mild (1st $\mathrm{CCB}$, 2nd adding PDE5i, 3rd ARB or switching to another CCB, 4th prostanoids), severe (1st CCB, 2nd adding PDE5i, 3rd ERA, 4th prostanoids)]. Sixty-eight percent of the experts agreed with the digital ulcer (DU) treatment scheme, suggesting 1st CCB, 2nd PDE5i. For interstitial lung disease (ILD) the agreement was $65 \%$, algorithms are shown in Figure 1. For skin involvement, agreement was $70 \%$. For patients with a modified Rodnan skin score (mRSS) of 10 1st methotrexate (MTX), 2nd mophetil mycophenolate (MMF); for mRSS 24 1st MTX, 2nd MMF; and for mRSS 32 1st MMF, 2nd MTX, 3rd IV cyclophosphamide (CYP), 4th autologous stem cell transplantation (ASCT). In inflammatory arthritis $79 \%$ agreed with 1st MTX, 2nd low dose glucocorticoids, 3rd hydroxychloroquine, 4th rituximab or tocilizumab. We have developed new cardiac and gastrointestinal algorithms.

Conclusion: Total agreement for SSc algorithms was considerable.
Combinations of PDE5i and ERA are being more frequently used than before. Prostanoids are a new 3rd line agent for active DU treatment. MMF is the preferred treatment for induction and maintenance in ILD. IV CYP and ASCT have been incorporated to treat advanced skin disease. Biologics may have a role in treating inflammatory arthritis. These algorithms may guide treatment in SSc.

\section{4}

\section{Trigeminal Neuralgia in Systemic Sclerosis}

Nancy Maltez (University of Ottawa, Department of Medicine, Division of Rheumatology, Ottawa); Marie Hudson (McGill University, Jewish General Hospital, Lady Davis Institute for Medical Research, Montreal); Yves Troyanov (Université de Montréal, Montréal); May Choi (University of Calgary, Calgary); Mianbo Wang (Lady Davis Institute for Medical Research, Montreal); Marvin Fritzler (University of Calgary, Calgary); Murray Baron (McGill University, Jewish General Hospital, Montreal); Douglas Smith (The Ottawa Hospital and University of Ottawa, Ottawa); Canadian Scleroderma Research Group (CSRG) (Montreal)

Objectives: Trigeminal neuralgia (TN) is characterized by pain and spasms affecting one or more divisions of the fifth cranial nerve. Of note, $\mathrm{TN}$ is one of the peripheral nervous system manifestations of systemic sclerosis (SSc), reported to be present in approximately $4 \%$ of patients. Proposed pathophysiologic mechanisms in this context include nerve entrapment and compression from mandibular bone resorption, a phenomenon seen in SSc due to pressure ischemia from overlying tight sclerotic skin compromising blood supply to bone. Previous studies of TN identified an association with overlap syndromes notably in patients with inflammatory myositis (IM), arthritis and interstitial lung disease (ILD). However, since there is a paucity of evidence concerning TN in SSc. we undertook a nested case-control study to identify associations between SSc and TN in a multi-centered SSc cohort. Methods: Data were retrieved from the Canadian Scleroderma Research Group (CSRG) registry, an open cohort of 1652 SSc subjects enrolled since 2004. Subjects with a physician-reported diagnosis of TN were identified at the baseline study visit (prevalent cases) and during follow-up (incident cases). Four SSc subjects without TN and matched to each case on study visit were identified as controls for either prevalent or incident cases. Sociodemographic, clinical and serological characteristics of cases and controls were compared. $P$ values $<0.05$ were considered statistically significant.

Results: $43(43 / 1652 ; 2.6 \%)$ prevalent and 36 incident $(36 / 6193$ total person-years follow-up; incidence rate 5.8 per 1000 person-years) TN cases were identified and matched to 144 and 172 controls, respectively. There were no significant differences in mean age, gender distribution and mean disease duration between cases and controls. Compared to controls, prevalent cases had more IM $(24.4 \%$ vs. $5.2 \%$, p $<0.001)$ and arthritis $(46.5 \%$ vs. $30.2 \%, \mathrm{p}=0.043)$. Similarly, incident cases also had more IM $(19.4 \%$ vs. $6.3 \%, \mathrm{p}=0.033)$ and arthritis $(50.0 \%$ vs. $16.2 \%, \mathrm{p}<0.001)$ compared to controls. There was a trend towards more ILD in prevalent ( $32.6 \%$ vs $23.8 \%$, $\mathrm{p}=0.241)$ and incident $(55.6 \%$ vs $40.6 \%, \mathrm{p}=0.105)$ cases compared to controls. U1RNP was numerically more frequent in cases versus controls (prevalent cases vs controls 9.4\% vs 5.4\%; incident cases vs controls $17.1 \%$ vs $5.8 \%) . \mathrm{Pm} / \mathrm{Scl}$ antibodies were infrequent and similar in prevalent $(2.9 \%$ vs $3.5 \%$ ) and incident (5.9\% vs $5.7 \%$ ) cases compared to controls.

Conclusion: This study provides novel evidence for a syndrome linking TN, IM, arthritis and possibly ILD. We propose that TN could be a consequence of active inflammation and represent a sign of disease activity in SSc.

\section{5}

Assessing the Educational Needs of Canadians with Systemic Sclerosis Teresa Semalulu (Northern Ontario School of Medicine, Thunder Bay); Karen Beattie (McMaster University, Hamilton); Maggie Larche (McMaster University, St Joseph's Healthcare Hamilton, Hamilton)

Objectives: Systemic sclerosis ( $\mathrm{SSc}$ ) is a connective tissue disease with clinical manifestations that range from minimal skin involvement to multiorgan dysfunction. The complex nature of the disease and the absence of disease modifying therapies makes it increasingly important to inform 
patients about their disease. An Educational Needs Assessment Tool (ENAT) was developed to assess the needs of people with rheumatic disease and validated in patients with SSc. We used the ENAT to solicit feedback from a sample of Canadians with SSc about their needs.

Methods: A cross-sectional convenience sample of Canadians with SSc was surveyed using an online adaptation of the ENAT questionnaire. Participants responded to questions/statements related to $7 \mathrm{SSc}$-associated topics using a 5-point Likert scale ( $1=$ not at all important; $5=$ extremely important $)$. The maximum ENAT score is 156 , which corresponds to a high need. Composite scores were derived for each of the seven areas of perceived educational need: managing pain, movement, feelings, information about scleroderma, treatments and supports. Descriptive statistics and frequencies were determined for patient characteristics. Total ENAT scores were determined for each participant. Unpaired t-tests and univariate linear regression analyses were used to identify differences or associations between patient characteristics and patient perceived needs.

Results: Fifty participants completed surveys [92\% female, mean (SD) age 52.2 (10.8) years, mean (SD) disease duration 10.9 (9.8) years, mean (SD) age at school completion 19.8 (3.6) years]. Eighty percent of patients wanted education related to scleroderma, $93 \%$ of whom stated that they wanted to know "lots of things" or "everything". The mean (SD) ENAT score for all participants was 111.9 (34.6). Highest needs were in domains related to knowledge about scleroderma, self-help and treatments. Participant desire for disease-related information was significantly associated with total ENAT $(b=26.00 ; p<0.001)$. ENAT scores were not different between sexes nor were they associated with age, duration of disease or age at school completion. Open-ended responses demonstrated additional needs for information related to $\mathrm{SSc}$ research.

Conclusion: SSc is a complex disease inciting relatively high educational needs among patients. Educating patients about disease manifestations, self and medical management, and research may be particularly beneficial. Patients with high educational needs about the disease also have high needs in other disease-related areas. Future studies should further explore the characteristics of patients who do not want disease-related education, the optimal medium for providing patient education to this heterogeneous group of patients and how perceived need may be linked to disease outcomes.

116

Improvement in Skin Score after 2 Years in Early Diffuse Cutaneous Systemic Sclerosis (dcSSc) Patients is Associated with Improvement in Multiple Other Domains of Disease Measurement

Boyang Zheng (McGill University Health Center, Montreal); Tatiana Nevskaya (Rheumatology Research, St. Joseph's Health Care, London); Janet Pope (Western University, Department of Medicine, Division of Rheumatology, London); Carl Baxter (MSD Ltd, Hoddesdon); Dena Ramey (Merck \& Co., Inc., Kenilworth); Murray Baron (McGill University, Jewish General Hospital, Montreal); Canadian Scleroderma Research Group (CSRG) (Montreal)

Objectives: Drug trials in dcSSc often use the change in modified Rodnan skin score (mRSS) as a primary outcome. The goal of this study was to determine if improvement in skin over time is associated with improvement in other domains in early dcSSc patients.

Methods: dcSSc patients with $\leq 5$ years disease duration followed over 2 years were identified from the Canadian Scleroderma Research Group registry. Changes in the degree of organ involvement were assessed using the Medsger Disease Severity Score (DSS) for each of the 9 systems as both continuous and dichotomous variables. $\mathrm{A} \geq 1$ point decrease was considered a clinical improvement. Pulmonary function tests, patient and physician reported global measures, Health Assessment Questionnaire (HAQ) and SF-36 physical component score (PCS) were also assessed. Bivariate, ANOVA and linear correlation (Pearson) analyses were performed.

Results: Of the 128 patients with 2 year follow up, $50 \%$ demonstrated skin improvement ( $\mathrm{mRSS}$ decrease of $\geq 5$ points and/or $\mathrm{a} \geq 25 \%$ reduction). Over two years, the average mRSS decreased from 22.6 to 18.1 for the entire cohort $(\mathrm{p}=0.0001)$. Physician global assessments of disease severity, activity and damage improved more in patients whose skin score improved ( $\mathrm{p}<0.003$ ). Improving mRSS was correlated with improving DSS for the following organ systems: lung, kidney, gastrointestinal, peripheral vascular, and the sum of all DSS scores without skin DSS. Compared to non-improvers, a greater proportion of skin-improvers had clinical lung improvement (39.4\% vs $17.2 \%, \mathrm{p}=0.006)$. Improvement in Forced Vital Capacity \% predicted (FVC\%) also correlated with skin improvement ( $\mathrm{r}=$ $0.33, \mathrm{p}=0.004)$. FVC $\%$ was stable in skin-improvers $(0.04 \%$ increase $)$ while declining by $6.5 \%$ in non-improvers, $p=0.026$. A higher proportion of skin-improvers also had improvement in joint/tendon involvement (50\% vs $21.2 \%, \mathrm{p}=0.017$ ) and improvement in any visceral organ involvement (renal, cardiac, pulmonary or gastrointestinal) $(60.3 \%$ vs $27.3 \%, \mathrm{p}=0.031)$. Skin-improvers had significantly improved HAQ scores compared to non-improvers $(-0.19$ point decrease vs. 0.18 point increase, $\mathrm{p}=0.001)$. Similarly, SF-36 PCS improved by 3.1 points in skin-improvers and worsened by 1.6 points in non-improvers, $\mathrm{p}=0.005$.

Conclusion: Over two years, improving skin scores in dcSSc patients were associated with an improvement in lung disease, joint/tendon involvement, global physician assessments, HAQ and SF-36 PCS. Skin-improvers had more overall visceral organ improvement. Our findings support the hypothesis that improvement in the severity of skin disease as a primary outcome in drug trials may be a surrogate for improvement in organ involvement and several other disease measurement domains.

\section{7}

Life Threatening Hughes-Stovin Syndrome without Manifestation of Behçet's Disease, Successfully Treated with Infliximab as Induction Therapy

Stuart Wiber (University of Saskatchewan, Regina); Robert McDougall (University of Saskatchewan, Regina); Zenon Belak (University of Saskatchewan, Regina); Patrick Duffy (University of Saskatchewan, Regina) Background: Hughes-Stovin Syndrome (HSS) was first characterized in 1959 and is a diagnosis for pulmonary artery aneurysm (PAA) with thrombosis. To date, there are reported to be 60 published cases of HSS, with a $58 \%$ mortality due to PAA rupture. The pathology is unknown. It is suspected to be a vasculitis akin to Behçet's Disease (BD), given that patients with HSS may or may not have features of BD, and additionally respond to similar treatment.

Case: A 22-year-old male with frank hemoptysis and hypoxemia was admitted to the intensive care unit for ventilation and intubation. He was found to have multiple bilateral pulmonary artery aneurysms on diagnostic imaging, the largest measuring $5.3 \mathrm{~cm}$, and a completely thrombosed and occluded superior vena cava, with thrombosis almost extending to the right atrium, as well as bilateral brachiocephalic vein thrombosis, and partial thrombosis of the right subclavian vein. He did not have any clinical findings or history consistent with BD. He was extensively worked up for infectious, hematological, autoimmune, anatomical and inflammatory etiologies for PAA and thrombosis. None of which yielded a definitive diagnosis. After multidisciplinary assessment, investigation and management by intensive care, rheumatology, infectious disease, hematology, respirology, thoracic surgery, and interventional radiology, the patient was diagnosed and treated for HSS.

Based on support from the literature and the context of this case, the patient was treated with a three-pronged approach to manage acute pulmonary artery rupture and stabilization of the remaining PAAs with coiling, initial immunosuppression with pulse steroids followed by induction with infliximab, and subsequent anticoagulation with warfarin after careful consideration given the risk of PAA rupture. Induction was achieved with infliximab $400 \mathrm{mg}$ iv q0, 2, and 6 weeks. Steroids were continued with an 8-month taper. The patient has remained in disease remission for 11 months, off steroids for 5 months, with azathioprine $100 \mathrm{mg}$ OD and infliximab $400 \mathrm{mg}$ q8 weeks maintenance.

Conclusion: Early recognition of HSS is essential with a multidisciplinary team to implement a three-pronged approach to managing PAA, immunosuppression, and consideration of anticoagulation. Given that this is a rare

Personal non-commercial use only. The Journal of Rheumatology Copyright (C 2018. All rights reserved. 
life-threatening syndrome, there are no large clinical trials to guide disease management. Induction is predominately achieved with cyclophosphamide. This case, however, illustrates that infliximab can successfully induce disease remission in life-threatening HSS. This bolsters the unfolding recognition that infliximab may be a reasonable and effective alternative to cyclophosphamide for first-line treatment of HSS

\section{8}

EGPA Presenting as a Non-vasculitic Cause of Eosinophilic Myocarditis Bohyung Min (University of Alberta, Calgary); Nathan Puhl (University of Alberta, Edmonton); Stephen Aaron (University of Alberta, Division of Rheumatology, Edmonton); Jan Tervaert (University of Alberta, Division of Rheumatology, Edmonton); Stephanie Keeling (University of Alberta, Division of Rheumatology, Edmonton)

Introduction: Eosinophilic myocarditis is a rare form of myocarditis with a diverse, and often difficult to determine etiology. Possible causes include hypersensitivity reactions, viral infections, hypereosinophilic syndrome, and eosinophilic granulomatosis with polyangiitis (EGPA). EGPA may start as a non-vasculitic disease with severe involvement of different organs such as the gut and/or heart.

Objective: Our aim was to report a case of (suspected) EGPA presenting as eosinophilic myocarditis on biopsy and discuss diagnostic and treatment challenges

Results: A 33 year old woman presented to hospital with a one-week history of chest pain and increasing dyspnea. Electrocardiogram and echocardiogram showed ST depression inferolaterally and left ventricular ejection fraction of $<10 \%$, with elevated troponin, brain natriuretic peptide, and peripheral eosinophilia of $11.2 \times 109 \mathrm{~L}$. Coronary angiography was normal. After admission in the hospital, she decompensated requiring venovenous extracorporeal membrane oxygenation and was transferred to our institution for consideration of cardiac transplantation. Left ventricular apex biopsy showed eosinophilic myocarditis, but no vasculitis. She was transitioned to a left ventricular assist device (LVAD) as her respiratory status had normalized. Past medical history included mild exercise-induced shortness of breath for which she was treated with Ventolin and Atrovent for the past six months. Furthermore, she had a history of rhino-sinusitis that was treated with over-the-counter decongestants.

At presentation, C-reactive protein was $63.6 \mathrm{mg} / \mathrm{L}$, total IgE was $796 \mathrm{kU} / \mathrm{L}$ whereas tests for ANCA were negative. No parasites and/or viruses were demonstrated, and the patient denied using drugs that could have been responsible for a hypersensitivity reaction. Bone marrow biopsy demonstrated hypereosinophilia, but was negative for malignancy, BCR/ABL, JAK2 kinase, and fusion protein mutations.

$\mathrm{CT}$ chest on presentation showed pulmonary edema (chest X-ray two weeks before presentation was normal). CT sinuses showed multiple sinus opacities and thickening of the mucosa.

Methylprednisolone $1 \mathrm{~g}$ IV daily for 3 days was started followed by oral prednisone $45 \mathrm{mg}$ BID in combination with high dose IVIG. Repeat echocardiogram demonstrated a moderate improvement. Unfortunately, she could not be weaned from the LVAD.

Conclusion: This case of eosinophilic myocarditis introduced multiple diagnostic and therapeutic challenges. Her presentation suggested eGPA with negative ANCA but without clear vasculitic manifestations. More intense immunosuppression using either Cyclophosphamide, Mepoluzimab, Omalizumab and/or Rituximab or not (with the goal to prepare the patient for immediate cardiac transplantation) will be discussed to determine optimal management of our patient.

\section{9}

\section{Paraneoplastic Granulomatosis with Polyangitis}

Stephen Morais (University of Massachusetts Medical School, Worcester); Emem Adolf-Ubokudom (University of Massachusetts Medical School, Worcester); Beata Skowronska-Cieslak (University of Massachusetts Medical School, Worcester)

Introduction: Granulomatosis with polyangitis (GPA) presenting with renal mass is a rare entity. The differential includes inflammatory pseudotumor and malignancy such as renal cell carcinoma (RCC) and lymphoma. Accurate diagnosis requires tissue evaluation to guide management.

Case Presentation: A 60 year-old male was referred to the emergency department by his urologist for weakness, fatigue, and 32 pound weight loss over a 3 month period. He was being evaluated for a recently identified 2 $\mathrm{cm}$ renal mass. On presentation he was afebrile, ill-appearing with tachycardia, leukocytosis, normocytic anemia, thrombocytosis, elevated serum creatinine, and dysmorphic RBCs with proteinuria on urine analysis. Further history revealed a recurrent sinusitis initially treated 3 months prior with Amoxicillin and methylprednisolone. He denied any paresthesias, wrist or foot drop, rash, bleeding, or arthritis. Suspicion for GPA prompted further evaluation notable for an erythrocyte sedimentation rate of $104 \mathrm{~mm} / \mathrm{hr}$, positive c-ANCA 1:640 titer with PR3 antibodies 162.1, normal complement levels, and a $2 \mathrm{~cm}$ hypervascular mass in his left kidney on abdominal imaging. He was started on steroid therapy and underwent diagnostic renal biopsies. Initial renal biopsy yielded pauci-immune acute necrotizing crescenteric glomerulonephritis with fibrinoid necrosis consistent with GPA. A second biopsy was requested of the renal mass and found RCC clear cell type. Staging confirmed malignancy limited to the lesion. Renal function, weakness, and sinusitis improved with steroid therapy. Shortly after he began outpatient Rituximab induction protocol for vasculitis and is scheduled for percutaneous ablation of his RCC.

Discussion: Renal involvement occurs in up to $80 \%$ of patients with GPA and $20 \%$ are present at diagnosis. The most common form is segmental necrotizing glomerulonephritis with proteinuria as seen in our patient. Rarely, patients present with a simultaneous renal mass in the form of inflammatory pseudotumor or RCC. Vandergheyst et al. reported 10 cases of inflammatory pseudotumor associated with GPA with variable improvement after immunosuppression, while Tastis et al. reported 5 cases of concomitant GPA and renal cell carcinoma noting the prevalence of RCC was significantly higher in GPA compared to a control group of rheumatoid arthritis. These two entities, pseudotumor and RCC, are not easily differentiated by radiography alone, which underscores the importance of tissue diagnosis in the evaluation of GPA with renal masses.

Conclusion: This case supports previous clinical observations in diagnosing GPA with simultaneous renal cell carcinoma. Clinicians should obtain tissue diagnosis in order to differentiate inflammatory pseudotumor from malignancy.

\section{0}

\section{Paraneoplastic Lymphocytic Cutaneous Vasculitis}

Stephen Morais (University of Massachusetts Medical School, Worcester); Alisha Lakhani (University of Massachusetts Medical School, Worcester); Katherine Upchurch (University of Massachusetts, Worcester)

Introduction: The differential diagnosis of secondary vasculitides is broad and challenging for physicians. Here we report a case of myelodysplastic syndrome (MDS) transformed to acute myelomonocytic leukemia (AML) presenting with lymphocytic cutaneous vasculitis in a patient with underlying autoimmune disease.

Case Presentation: A 60 year old Caucasian female with a history of CREST syndrome, Sjogren syndrome, treated hepatitis C, and MDS presented to the hospital for fever and lower extremity rash. Two weeks prior she was treated with Augmentin for sinusitis. She was ill-appearing, afebrile, with irregularly scattered erythematous tender subcentimeter lesions in her bilateral lower extremities. A cutaneous vasculitis was suspected. Differential diagnosis included infectious etiologies such as infective endocarditis, cryoglobulinemia, Sjogren syndrome associated vasculitis, medication-induced vasculitis, as well as hematologic malignancies in the setting of MDS. On admission, she had a normal WBC count, stable microcytic anemia and thrombocytopenia, with positive ANA and anti-centromere antibodies. Further auto-immune, inflammatory, and infectious blood work was negative, as was a transthoracic echocardiogram. A "punch" skin biopsy revealed perivascular lymphocytic and mononuclear cell infiltrate with focal fibrinoid necrosis of vessel walls consistent with lymphocytic cutaneous vasculitis. Her WBC count trended up to 32.9 with $74.5 \%$ monocytes

Personal non-commercial use only. The Journal of Rheumatology Copyright ( $) 2018$. All rights reserved. 
prompting a peripheral blood smear revealing predominantly mature cells. Flow cytometry of her blood revealed 30-35\% monocytic blasts suggestive of AML transformation. Bone marrow biopsy showed hyperplastic myeloid cells, $67 \%$ blasts with increased myeloid to erythroid ratio. CD34 + cells were elevated (20\%), confirming MDS transformation to AML. The patient underwent induction chemotherapy with ARA-C and Mitoxanthrone and at 1 month follow up had achieved remission of her leukemia with resolution of her vasculitic lesions.

Discussion: Though our patient had two existing diagnoses associated with cutaneous vasculitis (Sjogren's and hepatits C), the latter was treated and the former was a longstanding diagnosis with no systemic manifestations. Further, the lesions resolved with treatment of her AML providing evidence for a causal relationship. Autoimmune manifestations (AIM) are uncommon in MDS, occurring in 10-20\% of patients. Vasculitis accounts for $1.6 \%$ of these AIM, while malignancies are reported in $2.3-8 \%$ of patients with vasculitides. Fain et al reported 65 malignancies associated with vasculitides; $32.3 \%$ MDS, $29.2 \%$ lymphoma, and $36.9 \%$ solid tumors; AML is a rare manifestation.

Conclusion: The association between cutaneous vasculitis and lymphoid malignancies should always be considered in the differential diagnosis. Skin biopsy should be performed in patients with suspicious lesions and may prompt further hematologic studies.

121

\section{A Rare and Oft-Forgotten Cause of Symmetric Polyarthritis}

Stephen Morais (University of Massachusetts Medical School, Worcester); Emem Adolf-Ubokudom (University of Massachusetts Medical School, Worcester); Nancy Liu (University of Massachusetts Medical School, Worcester)

Introduction: Symmetric polyarthritis has a broad differential that can be challenging for providers. Clinicians need to maintain a high index of suspicion for great mimickers.

Case Presentation: A 30-year-old Brazilian female presented with a 4 month history of joint pain, swelling, and hours of stiffness effecting bilateral MCPS, PIPs, wrists, and MTPs associated with a diffuse rash, subcutaneous nodules, 26-pound weight loss, and hair thinning. Initial ER visit revealed polyarthritis and an ESR $38 \mathrm{~mm} / \mathrm{hr}$. She was treated effectively with 5 days of prednisone with recurrent skin rash and arthralgias after completion. Examination in rheumatology clinic revealed multiple papules on her face, skin thickening of upper chest and lower abdomen, and numerous lower extremity subcutaneous nodules. She had painful synovitis in MCPs, PIPs, wrists, and elbows with edema in her hands and feet. Neurological exam was normal. Laboratory revealed normal blood counts, electrolytes, renal and hepatic function. ESR $45 \mathrm{~mm} / \mathrm{hr}$ and serologic evaluation was significant only for rheumatoid factor $19 \mathrm{IU} / \mathrm{L}$; viral hepatitis serologies were non-reactive and Quantiferon gold indeterminate. Skin biopsy revealed diffuse perivascular and perineural granulomatous infiltrate, and Fite stain revealed numerous mycobacterium leprae, confirming the diagnosis of Hansen's disease. She was started on multi-drug treatment and experienced near complete resolution of articular and cutaneous symptoms at four-month follow-up.

Discussion: Lepromatous Leprosy, a chronic granulomatous infection, remains an important diagnostic consideration in patients presenting with symmetric polyarthritis; particularly those with skin and nerve manifestations with a negative anti-CCP. Despite the declining prevalence, endemic pockets in Brazil, Indonesia, and India exist with 213899 new cases reported worldwide in 2014. With increasing migration, physicians continue to see cases in developed nations, Massachusetts in this case. Four types of musculoskeletal manifestations are reported in nearly $75 \%$ of patients and may be the initial presenting symptom. These include a chronic polyarthritis, often symmetrical, clinically identical to RA. Bony erosions have also been reported, making distinction between RA and leprosy quite challenging. Several factors that may distinguish leprosy from RA include male sex, eyebrow loss, hypoaesthetic papules and nodules, early peripheral nerve damage and enlargement. Rheumatoid factor was positive in up to $26.6 \%$ of patients while anti-CCP is rarely positive.
Conclusion: Diagnosis of Hansen's Disease is often delayed and patients with articular symptoms are frequently treated for inflammatory arthritis. Increased awareness and high clinical suspicious can help avoid misdiagnosis, morbidity associated with untreated Leprosy and side effects of rheumatic medications.

\section{2}

\section{Leflunomide in Vasculitis: A Case Series}

Noura Mustapha (University of Toronto, Toronto); Simon Carette (University of Toronto, Toronto); Christian Pagnoux (University of Toronto, Toronto)

Objectives: Leflunomide has been used for the treatment of several autoimmune diseases, including rheumatoid arthritis and psoriatic arthritis. Few small case series and case reports also suggested some benefit in ANCA-associated vasculitides and other subtypes of vasculitis. Our primary objective is to analyze the patients who received Leflunomide for different vasculitides and report its clinical efficacy (sustained remission or relapse rate) and tolerability profile.

Methods: We conducted a retrospective analysis of the patients enrolled in the VCRC database who were followed at the Vasculitis Clinic of the Mount Sinai Hospital in Toronto and were treated with leflunomide as an induction or maintenance therapy for any type of vasculitis for at least 6 months, unless it was stopped due to side effects or inefficacy.

Results: A total of 32 patients were identified and analysed; mean duration of treatment was 3.4 years. $56 \%(n=18)$ of those patients were diagnosed with granulomatosis with polyangiits (GPA), $16 \%$ with Takayasu arteritis and $13 \%$ with eosinophilic granulomatosis with polyangiits (EGPA). The main reason for leflunomide initiation was disease relapse (34\%), suboptimal clinical response (38\%) or side effects (18\%) with previous treatments. Remission, defined as the absence of any detectable activity of the disease for at least 6 months, was achieved in $72 \%$ of the cases $(23 / 32$ patients), including $72 \%(n=13)$ of patients with GPA, $80 \%(n=4)$ of patients with Takayasu and all patients with MPA $(n=3)$. Leflunomide was ineffective in 5 patients (16\%): two patients had persistent sinus symptoms, one had a progression of renal involvement, one had new pulmonary nodules and one had a flare of skin vasculitis. Leflunomide was stopped due to side effects in 3 patients (alopecia, neuropathy and gastro-intestinal upset). One patient with GPA, who was treated for less than one year died of a cardiac arrest.

Conclusion: Leflunomide is a therapeutic option for vasculitides, including GPA or TAK. This medication has likely been underutilized. We intend to include more patients from the VCRC database, as well as from other Canadian centers.

\section{3}

Description of a Canadian Center's Cohort of 110 Patients with Eosinophilic Granulomatosis with Polyangiitis

Natalie Pulenzas (University of Toronto, Toronto); Simon Carette (University of Toronto, Toronto); Christian Pagnoux (University of Toronto, Toronto)

Objectives: Eosinophilic Granulomatosis with Polyangiitis (EGPA) is a rare vasculitis, with only a few published adult cohorts, none from Canada.

Methods: Data from patients with EGPA followed in the Mount Sinai Hospital's vasculitis clinic in Toronto were extracted from the chart, with their consent, and entered into the database developed by the Canadian vasculitis research network (CanVasc). Their main demographics, clinical manifestations at diagnosis, treatments and outcomes (vasculitis relapses, deaths) were analyzed.

Results: 110 patients were diagnosed with EGPA between 1967 and 2017; $51 \%$ were male; mean age at diagnosis was $47.7 \pm 16.1$ years. Mean delay between onset of asthma (confirmed in 105 [95.5\%]) and EGPA diagnosis was $106.3 \pm 150.8$ months, and between first symptoms of vasculitis and diagnosis was $36.0 \pm 61.0$ [range, $0-406$ ] months. The other most common symptoms at diagnosis were ENT manifestations (rhinitis 57.3\%, sinus polyps $41.8 \%$ ), lung infiltrates $(50.0 \%)$ and peripheral nerve involvement

Personal non-commercial use only. The Journal of Rheumatology Copyright $(\subset) 2018$. All rights reserved. 
(sensory neuropathy $51.8 \%$, motor neuropathy $39.1 \%$ ). Cardiac manifestations were recorded in $20(18.2 \%)$ and renal involvement in 23 patients (26.4\%) hematuria $20.9 \%$, proteinuria $11.8 \%$, none with end stage renal disease). Mean eosinophil count was $9.1 \pm 8.7 \times 109 / \mathrm{L}$. Fifty ( $45.4 \%)$ of the 106 patients with available ANCA by immunofluorescence at diagnosis were positive (p-ANCA in $82 \%$ ); $33(47.8 \%$ ) of the 69 patients with available ANCA by ELISA were positive (MPO-ANCA in $88 \%$ ). In addition to prednisone, cyclophosphamide was used for induction in $23(20.9 \%)$ patients. Biologics were given in only 7 patients $(6.4 \%)$ during the follow-up period. With a mean follow-up of $99.2 \pm 102.8$ months from diagnosis, vasculitis relapses occurred in 52 patients (47.3\%), after a mean of $70.5 \pm 77.9$ months from diagnosis, with 2 relapses occurring in 14 $(12.7 \%)$, and $\geq 3$ in $6(5.4 \%)$ patients. Two patients died, both due to malignancy. The only significant difference between ANCA+ vs. negative patients was the frequency of cardiac involvement, more common in the latter $(6.0 \%$ vs. $28.6 \% ; \mathrm{P}=0.002$ ). Relapse-free survival at 5 years was $78.2 \%$ [95\% CI: 67.8-85.5], comparable between the two groups. At last visit, 65 (59\%) patients were still on prednisone (mean dose, $6.5 \pm 11.2 \mathrm{mg} /$ day).

Conclusion: Main characteristics and outcomes of the patients in this first, large Canadian adult cohort appear similar to those reported in other countries. Cardiac manifestations were more common in ANCA-negative patients. Overall vasculitis-free survival was good, but patients were often steroid-dependent.

\section{4}

Conventional Immunosuppressants for the Treatment of Patients with Refractory or Relapsing Eosinophilic Granulomatosis with Polyangiitis Natalie Pulenzas (University of Toronto, Toronto); Simon Carette (University of Toronto, Toronto); Christian Pagnoux (University of Toronto, Toronto)

Objectives: Few series or placebo-controlled trials suggest that mepolizumab or rituximab may be effective for patients with relapsing or refractory eosinophilic granulomatosis with polyangiitis (EGPA). Surprisingly, comparable data on conventional non-biologic immunosuppressants, which have been prescribed for much longer, are lacking.

Methods: Data from EGPA patients followed at the Mount Sinai Hospital's vasculitis clinic (Toronto), entered into the Canadian vasculitis research network (CanVasc) database, were retrospectively reviewed. Eligible patients had to satisfy the same entry criteria as the main rituximab (Mohammad et al, ARD 2014) or mepolizumab studies (Wechsler et al, NEJM 2017), i.e. to have either a relapsing and/or refractory EGPA for at least 6 months. They had to then be started on azathioprine, methotrexate or leflunomide. Response rates were assessed at 6 and 12 months after treatment initiation. Remission was defined as a Birmingham Vasculitis Activity Score (BVAS) of 0 and prednisone dose $\leq 7.5 \mathrm{mg} / \mathrm{day}$ (definition A; mepolizumab trial) or with any dose of prednisone (definition B; rituximab series). Relapse was defined as the reappearance of symptoms leading to a BVAS increase and requiring new immunosuppressive therapy and/or increased prednisone dose.

Results: Among the 110 patients of the cohort, 25 met the eligibility criteria; $13(52 \%)$ had relapsing, 12 (48\%) had refractory EGPA. Seventeen (68\%) patients had received previous immunosuppressant(s), 8 (32\%) prednisone alone. Mean baseline prednisone dose was $14.6 \pm 12.4 \mathrm{mg} /$ day; the conventional immunosuppressant used was methotrexate in 14 patients, azathioprine in 10 , and leflunomide in 1 . Remission was achieved at some time during the first year in $12(48 \%)$ patients by definition $\mathrm{A}$, and $16(64 \%)$ using definition B (vs. 53\% in the mepolizumab trial [19\% in the placebo arm]; $49 \%$ in the rituximab series). At 6 and 12 months, 46.7\% (7/15; unknown status for 10 because no visit and/or missing prednisone dose) and $54.5 \%$ (6/11; unknown 14) of patients were in remission by definition A vs. $66.7 \%$ (10/15; unknown 10$)$ and $63.6 \%$ (7/11; unknown 14) by definition B, respectively. Four patients (16\%) had a vasculitis relapse (compared to $43 \%$ in the mepolizumab trial, which counted asthma exacerbations as flares; and $12 \%$ in the rituximab series). The immunosuppressant was stopped in 8 patients, due to ineffectiveness in 4 , liver toxicity in 2 , or other reasons.
Conclusion: Though retrospective biases must be acknowledged, the rates of remission observed in our study were substantial and should be kept in mind when interpreting results of studies on biologics in EGPA.

\section{5}

\section{A Rare Case of ANCA-associated Aortitis}

Saurash Reddy (University of Alberta, Edmonton); Richard Owen (University of Alberta, Edmonton); Elaine Yacyshyn (Division of Rheumatology, University of Alberta, Edmonton)

Background: ANCA vasculitides have traditionally been characterized as small-vessel diseases, with large-vessel vasculitis patients presenting with variable manifestations (i.e. Takayasu's arteritis and Giant cell arteritis [GCA]). ANCA-associated aortitis is a rare large-vessel vasculitis, with less than 25 described cases in the literature. It can present with potentially serious complications including aortic dissection, aortic rupture, and death. Case Presentation: A 64-year old previously healthy female was diagnosed with seronegative rheumatoid arthritis after having inflammation in her wrists and ankles in June 2013. A rheumatologist started her on prednisone after she declined DMARD therapy. Two months later, she presented to hospital with fatigue, weakness, and chest pain with elevated troponin. CT imaging revealed a $6.3 \mathrm{~cm}$ ascending aortic aneurysm with evidence of chronic Type B Dissection. After surgical repair, she was discharged on lowdose prednisone and oral methotrexate for management of her arthritis. Aortic biopsies showed no signs of vasculitis, and there was no other evidence of systemic vasculitis. Over three years of follow-up, she had persistently elevated inflammatory markers despite adequate arthritis control with the addition of hydroxychloroquine to the methotrexate. Serial CT scans showed post-surgical expansion of the aneurysm, requiring another endovascular procedure in 2015. A vasculitis workup was initiated to investigate the recurrent aneurysm, yielding a strongly positive MPO-ANCA titre with p-ANCA positivity in the setting of chronically intermittent proteinuria and hematuria on serial urinalyses. A diagnosis of MPO-ANCA-associated largevessel vasculitis was made due to suspected transient renal involvement and ANCA positivity. Given that her inflammatory markers, hematuria, and proteinuria had normalized at the time of diagnosis with no active arthritis or other vasculitis symptoms, a renal biopsy was not performed. She continued on combination therapy with methotrexate and hydroxychloroquine, without pursuing induction therapy with cyclophosphamide, due to patient preference for therapy.

Conclusion: This case highlights an unusual case of large vessel ANCA-related vasculitis. Previous cases describe large-vessel presentations (ie. aortitis, dissection) in conjunction with typical manifestations of ANCA small-vessel disease (hemoptysis, hematuria, visual symptoms). Evaluation of this type of presentation should include CT angiography to confirm aortic involvement, and both infectious and autoimmune workup for aortitis. Vessel biopsy should be pursued if possible along with evaluation for other manifestations of ANCA vasculitis (ie. lung, kidney, skin), and consideration of tissue biopsy. Treatment typically involves induction therapy with high-dose corticosteroid and cyclophosphamide, along with surgical intervention when indicated. Patients should be monitored with serial inflammatory markers and imaging for relapse.

\section{6}

\section{CNS Vasculitis in a Patient Treated with Long-term Tocilizumab}

Krista Rostom (University of Ottawa, Department of Medicine, Division of Rheumatology, Ottawa); Nataliya Milman (University of Ottawa, Ottawa)

Introduction: Tocilizumab (TCZ) is a monoclonal antibody against the interleukin 6 (IL-6) receptor used to treat rheumatoid arthritis and has recently shown results in giant cell arteritis. We describe a case of new onset central nervous system (CNS) vasculitis in a patient receiving long-term treatment with TCZ.

Case Presentation: A 49-year-old female presented with one month of headaches, disorientation, and odd behavior. Physical exam was normal except for disorientation and mild dysarthria. CT(A) of head and neck showed multiple bilateral lacunar infarcts and multifocal narrowing and 
beading of the vessels in the anterior and posterior circulation. Cerebral angiogram confirmed multifocal areas with beaded appearance and irregularities involving bilateral ACAs, left MCA, and bilateral PCAs, in keeping with vasculitis. ESR and CRP, ANA, ENA, anti-dsDNA, ANCA, antiphospholipid antibodies, HIV, syphilis, hepatitis B, hepatitis C, lyme serology, and blood cultures were negative. CSF had 32×106/L nucleated cells, 20x106/L RBCs, no oligoclonal bands, increased protein ( $0.63 \mathrm{~g} / \mathrm{L})$, and normal glucose $(3.3 \mathrm{mmol} / \mathrm{L})$. CSF cultures including HSV were negative. CT scans of the thorax, abdomen, and pelvis were normal.

Patient's past medical history was significant for Adult Onset Still's Disease (AOSD) diagnosed 15 years ago based on fevers, typical rash, and inflammatory arthritis. She had failed methotrexate, leflunomide, and anakinra, but her disease has been well controlled for the past 5 years on plaquenil $200 \mathrm{mg}$ bid, prednisone 5mg od, and tocilizumab 162mg SC q2weeks. In 2015, she had a stroke which was thought to be cardioembolic, though her initial echo was normal and she was subsequently lost to follow up.

Discussion: A clinical diagnosis of CNS vasculitis was made. Tocilizumab was stopped, and patient was given 3 daily pulses of $1 \mathrm{~g}$ of solumedrol IV with clinical improvement. Brain biopsy was requested, but the neurosurgery consultant felt that the risks of the biopsy were not warranted given classic findings on imaging, CSF, and clinical improvement with steroid therapy. Cyclophosphamide $600 \mathrm{mg} / \mathrm{m} 2$ IV monthly was added and the patient continues to improve five months into her disease course.

Conclusion: Our case could represent a paradoxical event of CNS vasculitis during treatment with TCZ. There are case reports of TCZ induced autoimmune diseases including leukocytoclastic vasculitis, sarcoidosis, and immune complex glomerulonephritis, but there are no previous reports of CNS vasculitis. Given the recent success using tocilizumab to treat giant cell arteritis, which rarely can affect the CNS, cases of possible paradoxical effects affecting the CNS are of interest.

127

Incomplete Kawasaki Disease with Coronary Aneurysms Presenting in an Adult Patient: A Case Report and Review of the Literature

Alice Mai (University of British Columbia, Vancouver); Mohan Stewart (University of British Columbia, Vancouver); Jason Kur (University of British Columbia, Vancouver)

Case Description: A 20-year-old previously healthy male presented with a 5-day history of fever, bilateral conjunctivitis, migratory arthritis, evanescent rash, headache, neck stiffness, pharyngitis, and intermittent diarrhea. Initial workup yielded a neutrophil-predominant leukocytosis, transaminitis, and elevated CRP but no other findings suggestive of an underlying infectious etiology. Within a few days of admission, he developed acute decompensated heart failure. An echocardiogram demonstrated a new dilated cardiomyopathy with an ejection fraction (EF) of 35\%. A subsequent cardiac workup included a CT coronary angiogram (CTCA), which unexpectedly demonstrated multiple aneurysms in all three major coronary vessels, a finding not seen on the previous echocardiogram. A diagnosis of incomplete Kawasaki Disease (KD) was made and treatment was initiated with IVIG and high dose ASA. The patient defervesced within a day of treatment. A repeat echocardiogram a week later demonstrated an improved $\mathrm{EF}$ of $65 \%$, and a repeat CTCA at that time showed stable coronary aneurysms. He was discharged on ASA with plans to monitor his aneurysms serially.

Discussion: KD is a medium-vessel vasculitis that predominantly occurs in children under the age of 5 . Adult-onset KD (AKD) is rare and often misdiagnosed. This has significant implications given that untreated KD increases the risk for coronary artery aneurysm (CAA). Monitoring the size and progression of CAAs is of great importance, as vascular abnormalities can lead to coronary artery thrombosis and resultant myocardial infarction (MI). The recommended method of screening and monitoring in pediatric patients is transthoracic echocardiography (TTE). However, TTE is limited in its ability to detect CAAs in the adult patient, as was demonstrated in our patient. In a review of 56 patients with $\mathrm{AKD}, 86 \%$ of incident coronary aneurysms were diagnosed by CTCA or catheter angiography and only $13 \%$ by TTE. CTCA has been reported to be as reliable as catheter angiography for visualization of the coronaries in $\mathrm{KD}$, but does impart a significant dose of radiation. Magnetic resonance angiography (MRA) has also been shown to be excellent at visualizing CAAs and does not involve radiation, but is not as sensitive as CTCA at visualizing distal CAAs.

Conclusion: Our case report demonstrates the importance of considering Kawasaki Disease in the adult patient, given the higher risk for cardiac sequelae with late diagnosis. Further research is needed to guide the optimal modality and frequency of CAA imaging in adult patients in whom TTE is insufficient.

\section{8}

Minocycline Associated Vasculitis: Case Report and Review of 35 Cases Yan Yeung (University of Western Ontario, London); Lillian Barra (University of Western Ontario, London)

Objectives: Minocycline is a synthetic tetracycline derivative with clinical uses including the treatment of acne vulgaris, rosacea, and rheumatoid arthritis. Minocycline has been associated with autoimmune phenomena including drug-induced lupus, autoimmune hepatitis, and vasculitis. We describe the first reported case of bowel infarction due to minocycline associated vasculitis in a 17 -year-old male patient. We then present the results of a literature review of all other published cases of minocycline associated vasculitis.

Methods: A literature search was performed on PubMed with the search term "minocycline vasculitis". English-language cases of minocycline associated vasculitis were included; we excluded cases where the primary diagnosis was minocycline induced lupus, autoimmune hepatitis, or other minocycline associated autoimmune phenomena. Additionally, the references of each relevant publication were then searched for additional cases of minocycline associated vasculitis.

Results: A 17 year-old male presented to the emergency room with fever, arthritis in his hands, and 3 days of increasing abdominal pain. He was on minocycline for 2 years for treatment of acne. He was brought to the operating room for presumed appendicitis, however, was found instead to have ischemic small bowel requiring resection. Anti-MPO antibodies were elevated, and small bowel pathology revealed Lymphoplasmacytic infiltrate of medium-sized mesenteric vessels. Minocycline was discontinued, his symptoms resolved, and anti-MPO antibodies normalized. No immunosuppression was required. 34 additional cases of minocycline associated vasculitis were identified. The median age was 22 years (range $15-55$ years) with 12 males and 23 females. The average duration of minocycline use before onset of vasculitis was 2 years (standard deviation $+/-1$ year). $74 \%$ of patients were pANCA positive, and of those patients, $42 \%$ were anti-MPO positive. The most common symptoms were fever (50\%), myalgia (43\%), fatigue $(37 \%)$, livedo reticularis (34\%), paresthesia (31\%), arthritis $(29 \%)$, and testicular pain (23\%). $60 \%$ of the cases met 1990 ACR classification criteria for polyarteritis nodosa. 29 biopsies were reported; they showed small and medium vessel involvement but there were no consistent findings otherwise. Treatment for all cases included minocycline discontinuation, however glucocorticoids and cyclophosphamide were used in 50\% and $11 \%$ of cases, respectively.

Conclusion: Minocycline can cause a systemic pANCA-associated vasculitis and should be suspected in patients with symptoms of a small-to-medium sized vasculitis who have been on minocycline for typically over a year. Discontinuation of minocycline can successfully treat minocycline associated vasculitis, however, additional immunosuppression with agents such as glucocorticoids and cyclophosphamide have also been used.

\section{9}

A Myositis Mimic: Positive Anti-SRP Antibody in a Patient with Facioscapulohumeral Muscular Dystrophy

Sarah Cribby (University of Calgary, Calgary); Sameer Chhibber (University of Calgary, Calgary); Paul MacMullan (University of Calgary, Calgary)

Introduction: We present a patient who was evaluated for possible inflammatory myositis, and found to have genetically confirmed facioscapulohumeral muscular dystrophy (FSHD).

Case Presentation: A 19-year-old gentleman was evaluated for progressive 
shoulder weakness and myalgias. He was found to have a positive anti-SRP (signal recognition peptide) antibody and referred to rheumatology for work up of a possible inflammatory myopathy. He described a 3-year history of progressive asymmetric muscle weakness, primarily involving his face and shoulder girdle. He had no dysarthria or dysphagia. His examination revealed weakness and wasting in the facial muscles (orbicularis oculi and orbicularis oris), subscapularis, biceps and pectoralis major bilaterally, more pronounced on the left. He had bilateral scapular winging. The remainder of his examination was normal. Based on the pattern of weakness, FSHD was suspected. Investigations revealed a mildly elevated CK (324 U/L) and a weakly positive anti-SRP antibody. EMG/NCS studies showed evidence of a proximal myopathy, but no evidence of inflammatory myositis. Genetic testing confirmed the diagnosis of facioscapulohumeral muscular dystrophy. Discussion: Hereditary muscular dystrophies can mimic inflammatory myopathies, especially when they present in adulthood, have rapidly progressive symptoms or limb girdle distribution of weakness. Facioscapulohumeral muscular dystrophy is the third most common adult muscular dystrophy. It has a characteristic regional and asymmetric pattern of muscle involvement, beginning with focal weakness in the shoulders, face and humeral muscles, with variability in the pattern and severity of subsequent muscle involvement.

Anti-SRP antibodies are seen 4-8\% patients with inflammatory myositis, and are associated with severe necrotizing myopathy, with rapidly progressive symptoms and poor response to therapy. A chronic form of anti-SRP myopathy with an earlier age of onset is also well described, and can be mistaken for FSHD. Anti-SRP antibodies have occasionally been detected in patients with other rheumatologic diseases, but have not been seen in patients with muscular dystrophy. Rarely, other myositis-specific antibodies have been reported in patients with muscular dystrophy, both in asymptomatic patients and patients with coexisting diagnoses of myositis or connective tissue disease.

Conclusion: This is the first report of the positive anti-SRP antibody in a patient with facioscapulohumeral muscular dystrophy. Limb girdle muscular dystrophies can mimic inflammatory myositis and are an important consideration in the evaluation of myopathy.

\section{0}

Survival and Post-Operative Outcomes following Lung Transplantation for Interstitial Lung Disease Secondary to Dermatomyositis and Polymyositis: A Case Report and Systematic Review of the Literature Sabrina Lue (Queen's University, Kingston); Taneisha McGhie (University of Toronto, Toronto); Bindee Kuriya (Sinai Health System, University of Toronto, Toronto)

Objectives: We present a case of interstitial lung disease (ILD) secondary to anti-melanoma differentiation-associated gene 5 (MDA5) antibody positive clinically amyopathic dermatomyositis (CADM) requiring lung transplantation that was complicated by rare events and resulted in death. Our objective was to conduct a systematic review to evaluate post-operative outcomes and survival of patients receiving lung transplantation for ILD secondary to dermatomyositis (DM) or polymyositis (PM).

Methods: Medline, Embase, Clinicaltrials.gov, and Cochrane were searched for published studies reporting lung transplant survival of adult patients with ILD secondary to DM or PM.

Results: A total of 2105 citations were screened, 95 articles were reviewed in full text, and 8 studies were included. Data were extracted from 3 retrospective studies, one case series, and 4 additional case reports. Post-transplant complications included acute and chronic rejection, cancer, infections, right heart failure, pulmonary reperfusion edema, cardiac tamponade, renal failure, and jejunal perforation. Survival outcomes were reported on 49 transplant patients, with 27 patients alive at the time of final follow-up, with ranges of a follow-up between 2 months to 3 years. Including case studies, the cumulative survival rates were $88 \%$ at 1 month, $71 \%$ at 6 months, $65 \%$ at 1 year, $55 \%$ at 2 years, and $44 \%$ at 3 years.

Conclusion: We describe a case of a patient with CADM who died due to complications following lung transplantation. This case is only one of four described cases of CADM treated with lung transplantation in the literature. There is a paucity of data on the clinical phenotypes and survival outcomes following lung transplantation for ILD secondary to DM and PM. Further information on subgroup outcomes could assist in selection and risk stratification of patients with DM or PM requiring lung transplantation.

\section{1}

Improving Methotrexate Knowledge in Chinese-speaking Patients with Inflammatory Arthritis: A Quality Improvement Initiative

Hasan Abdullah (University of Calgary, Calgary); Steven Katz (University of Alberta, Edmonton); Luojia Yang (Edmonton); Carrie Ye (Division of Rheumatology, University of Alberta, Edmonton)

Introduction: Methotrexate is the cornerstone of inflammatory arthritis therapy. However, suboptimal adherence to methotrexate therapy by patients is a significant barrier to achieving disease modifying effects. Studies have shown that medication adherence may be influenced by language barriers in patients who are non-native English speakers.

Objective: To study the effects of a Chinese methotrexate information sheet on Chinese speaking patients' understanding of methotrexate.

Methods: After obtaining ethics approval, 17 Chinese speaking patients with inflammatory arthritis taking methotrexate were recruited for this study from both community and academic rheumatology practices in Edmonton in the month of May 2016. A validated methotrexate knowledge questionnaire and the www.AlbertaRheumatology.com methotrexate information sheet were translated into Chinese characters. 2 Chinese methotrexate questionnaires were answered by each patient; a 'pre' questionnaire prior to, and a 'post' questionnaire after reading the Chinese methotrexate information sheet. Patients' questionnaires were scored out of 10 and the 'pre' and 'post' scores were compared. Information pertaining to education, self-assessed English proficiency and basic demographics were collected for each patient.

Results: On average, patients significantly improved their methotrexate knowledge score from 4.5/10 to 7.5/10 after reading the Chinese information sheet $(p=0.008)$. In particular, patients' knowledge of major side effects of methotrexate and folic acid's role in mitigating side effects improved significantly $(\mathrm{p}<0.05)$

Conclusion: Due to language barriers, patients whose first language is not English may have a poor baseline understanding of methotrexate but a simple quality improvement initiative of providing them a translated methotrexate information sheet in their native language can significantly improve their understanding of this drug. Given that lack of drug knowledge is a contributor to drug non-adherence, this is an important quality improvement measure that can be easily adopted to improve patient understanding and adherence.

\section{2}

Incidence of Infections in Early Arthritis

Meriem Kerbachi (McGill University, Montreal); Louis Bessette (Laval University, Quebec); Cristiano Moura (Department of Epidemiology, Biostatistics and Occupational Health, McGill University, Montreal); Sasha Bernatsky (McGill University Health Centre, McGill University, Montreal); Orit Schieir (Notre-Dame-de-Grace); Susan Bartlett (McGill University, Montreal); Carol Hitchon (University of Manitoba, Winnipeg); Janet Pope (Western University, Department of Medicine, Division of Rheumatology, London); Gilles Boire (Université de Sherbrooke, Sherbrooke); Boulos Haraoui (Department of Medicine, Centre Hospitalier de l'Université de Montréal, Montréal); Edward Keystone (Mount Sinai Hospital, University of Toronto, Toronto); Diane Tin (Southlake Regional Health Centre, Newmarket); Carter Thorne (Southlake Regional Health Centre, Newmarket); Vivian Bykerk (Hospital for Special Surgery, New York); CATCH Canadian Early Arthritis Cohort Investigators (Toronto)

Objectives: Few studies have focused on analyzing infection risk in recent-onset rheumatoid arthritis (RA).The main objective of this study was to estimate the incidence of infections in patients with early RA, focusing on a multi-centre Canadian cohort of RA patients enrolled within 12 months of symptom onset. 
Methods: All patients with early RA ( $<1$ year) enrolled in the cohort were studied. Infections have been reported in a standardized manner by patients at yearly assessments. In each year, a patient was allowed to contribute more than one infection event, provided the event was a new or recurrent (not chronic) infection. From January 2007 to November 2015, we tabulated the total number of infections and generated incidence rates of infections with 95\% confidence intervals $(\mathrm{CI})$.

Results: A total of 1728 RA patients met study criteria and were followed for an average of 3.2 years. At the time of recruitment, mean (standard deviation, $\mathrm{SD})$ age was 54.2 years (15.0), majority were Caucasian $(\mathrm{N}=$ $1,419,82.1 \%)$ and female $(\mathrm{N}=1,245,72.0 \%)$. Mean symptom duration at cohort entry was 5.8 months (3.0). The mean Disease Activity Score 28 was 5.1 (1.4), Simplified Disease Activity Index 28.6 (15.2) and Clinical Disease Activity Index 27.1 (14.4). One third $(571,33 \%)$ of these patients were on steroids at baseline and most $(\mathrm{N}=1,566,90.6 \%)$ were on DMARDs, with $1,262(73.0 \%)$ patients having been exposed to MTX. Biologics were used only by $41(2.37 \%)$ patients at the time of cohort entry. Over the study interval, we recorded 452 infections over a total of 5,525 person-years (81.8 events per 1000 patient-years) and over a quarter of these (125 events) involved hospitalization. Eighty four (18\%) of all infectious episodes were pneumonia (15.2 events per 1,000 patient years), with half of these pneumonias being associated with a hospitalization.

Conclusion: Our study provides novel data on infection in early RA patients in the real world. Reported infections in this Canadian cohort with early RA was not negligible. Half of reported pneumonia events in early RA involved hospitalization. A potential limitation was we did not attempt to confirm reported infections.

\section{3}

\section{Tumor Necrosis Factor Inhibitors for Sarcoidosis}

Stefanie Wade (University of Connecticut Internal Medicine Residency Program, West Hartford); Mollie Carruthers (University of British Columbia, Vancouver)

Objectives: Patients with sarcoidosis refractory to standard treatment are a therapeutic challenge and are often managed by a variety of specialists due to the heterogeneity of disease manifestations. We are now using tumor necrosis factor inhibitors (TNFi's) for severe or refractory disease. Current sarcoidosis literature lacks high quality analysis comparing the efficacy and safety of TNFi's for challenging cases of sarcoidosis. Our objective was to evaluate the efficacy of TNFi's in sarcoidosis resistant to standard treatment by meta-analysis.

Methods: A systematic English literature search using PubMed was performed. Search strategy involved screening for articles between 1996 and 2017 which reported use of TNFi's (etanercept (ETN), adalimumab (ADA), infliximab (INF), golimumab or certolizumab) for the treatment of biopsy proven sarcoidosis. We excluded case reports and sarcoidosis that developed while on a TNFi. Data regarding disease phenotype, past therapies, treatment outcomes, and adverse events were extracted by two reviewers and pooled in a meta-analysis.

Results: There were 470 abstracts identified. Selection was made of 102 original articles using TNF inhibitors in sarcoidosis. Fourteen studies were evaluated: five comparative trials and nine observational. The most robust studies were comparative trials in patients with pulmonary sarcoidosis. Variation in objective results (including pulmonary function tests) was seen. Adverse events leading to discontinuation were infrequent. Observational studies assessed patients with more wide ranging phenotypes including cutaneous, ocular, central nervous system (CNS), cardiac, pulmonary, liver, and musculoskeletal manifestations. Clear benefit with INF in neurosarcoidosis (when used with mycophenolate) and in multi-system sarcoidosis was seen. Both INF and ADA led to improved outcomes in uveitis. In one open label study, INF led to improvement in FDG PET in a majority of patients and improvement in FVC by $6.6 \%$. ADA improved or stabilized FVC in all patients with severe, symptomatic pulmonary sarcoid and led to improved 6MWT, Borg dyspnea score, and both patient and physician global assessment. An open label study with ETN for stage II and III pulmonary sarcoid was terminated early due to frequency of treatment failure. Case series support use in a wide range of extra-pulmonary organ systems, most notably neurosarcoidosis.

Conclusion: Current results from randomized and open label studies appear to support use of monoclonal antibody TNF inhibitors, for use in extra-pulmonary sarcoid disease, with particularly compelling evidence for use in uveitis and neurosarcoidosis.

\section{4}

Exposure to Mechanical Stress Associated with Poor Functional Outcomes in Patients with Axial Spondyloarthritis

Maysam Khalfan (Western University, London); Dafna Gladman (University of Toronto, Toronto); Robert Inman (University Health Network, Toronto); Lihi Eder (Women's College Hospital, Toronto); Sherry Rohekar (Western University, London)

Objectives: Heavy physical activity has previously been shown to result in poor functional outcomes in patients with ankylosing spondylitis (AS), a subtype of axial spondyloarthritis (AxSpa). This study assessed the impact of occupational mechanical stress on patient reported outcomes in a Canadian cohort of patients which includes all subtypes of axial spondyloarthritis, including non-radiographic spondyloarthritis (nr-AxSpa).

Methods: Patients enrolled in the SPondyloArthritis Consortium of Canada (SPARCC) database, a well-characterized longitudinal cohort of patients with $\mathrm{SpA}$, were assessed for occupational exposure to mechanical stress such as squatting, pushing, lifting, repetitive hand movements or use of vibrating tools. They were divided into two groups; a low stress group which included patients with no exposure or exposure to only one physical activity, and a high stress group which was exposed to two or more activities of physical stress. HAQ, BASDAI, BASFI, EQ5D, ASQOL, and SF36 mean scores were compared for these two groups, and the Mann-Whitney U test was used to test for significance.

Results: A total of 154 patients were included based on availability of information regarding their physical activity at work. The low stress group had 70 patients and the high stress group had 84 . Group characteristics revealed that the high stress group patients were younger, had a shorter disease duration, and had a higher percentage of patients with non-radiographic spondyloarthritis. The high stress group had statistically significant poorer scores in the HAQ (Low: 0.32 vs High: 0.46, p = 0.04), BASDAI (Low: 3.15 vs High: $3.79, \mathrm{p}=0.02$ ) and SF-PCS (Low: 43.6 vs High 39.9, p = 0.04) patient reported outcomes. They also scored worse on BASFI, ASQOL, EQ5D and SF-MCS, although not statistically significant. The significance in difference between the groups was lost when subjects were stratified according to smoking status.

Conclusion: Heavy physical activity at work was associated with worse functional outcomes in this Canadian cohort of patients with axial spondyloarthritis, however smoking plays a confounding role in this association. This information is helpful to guide further investigation of the utility of modifying mechanical stress exposure in order to improve outcomes in axial spondyloarthritis.

\section{5}

Comparison of the Bioavailability of a Single Dose of Certolizumab Pegol Injected either by a Pre-Filled Syringe or by an Auto-Injection Device

Béatrice Astruc (Biotrial, Rennes); Shikiko Watanabe (UCB Pharma, Anderlecht); Gerry Parker (UCB Pharma, Anderlecht); Jerry Syrotuik (UCB Pharma, Oakville); Réginald Hulhoven (UCB Pharma, Anderlecht)

Objectives: The pharmacokinetic (PK) properties of certolizumab pegol (CZP) have been shown to demonstrate a linear relationship between the dose administered (via intravenous and subcutaneous routes), the maximum plasma concentration (Cmax), and the area under the plasma concentration versus time curve (AUC). In multiple clinical studies, the half-life (t1/2) was approximately 14 days for all CZP dosage levels tested. CZP has been demonstrated to have good bioavailability (approximately $80 \%$ ) when given subcutaneously. This study compared the bioavailability of administering

Personal non-commercial use only. The Journal of Rheumatology Copyright (C) 2018. All rights reserved. 
CZP (2 x $200 \mathrm{mg}$ ) injections either by a pre-filled syringe (PFS) or by an auto-injection device (AI), in addition to investigating the safety and tolerability of both methods of administration.

Methods: This open-label, phase I study recruited healthy volunteers who were randomized into two parallel groups. On Day 1, study participants were injected with 2 x $200 \mathrm{mg}$ CZP either via a PFS or AI. CZP plasma concentrations were measured pre-dose, and post-dose at $12 \mathrm{~h}, 24 \mathrm{~h}$ and Days 2, 3 , $4,5,6,7,10,14,21,28,42,56$ and 84 . Safety and tolerability of CZP were assessed by recording adverse events (AEs) and serious AEs (SAEs), by each method of administration. Additionally, the tolerability of the injections, by PFS or AI, were assessed by a pain visual analog scale (VAS) and an injection site reaction questionnaire.

Results: 98 healthy volunteers were randomized to the PFS $(n=49)$ and AI $(n=49)$ groups. The mean plasma CZP concentration versus time profile of the AI and PFS were similar. PK parameters were similar between both modes of administration in terms of Cmax values (AI: $60.1 \mu \mathrm{g} / \mathrm{mL}$, PFS: $61.7 \mu \mathrm{g} / \mathrm{mL}$ ). The $90 \%$ confidence intervals $(\mathrm{CI})$ for the test $/$ reference geometric mean ratios in $\mathrm{Cmax}, \mathrm{AUC}(0-\mathrm{t}), \mathrm{AUC}$ and $\mathrm{t} 1 / 2$ were all contained in the bioequivalence range of $80-125 \%$, and no statistically significant difference was found for median tmax between the two modes of administration (point estimate $=0.0$ ). Safety assessments showed that the two injection methods were equally well-tolerated. Injections performed with the AI appeared to be less painful immediately after administration than injections given by the PFS, as well as being associated with less burning and redness.

Conclusion: CZP $400 \mathrm{mg}$ dose is bioequivalent whether administered by AI or PFS. Overall, CZP injections were safe and well-tolerated for both administration methods.

\section{6}

Incidence of Extra-Articular Manifestations in PsA and AS Patients Treated with Golimumab in Canadian Real-World

Michelle Teo (Penticton); Derek Haaland (Department of Medicine, McMaster University, Hamilton); John Kelsall (University of British Columbia, Vancouver); Isabelle Fortin (Centre de Rhumatologie de l'Est du Québec, Université du Québec à Rimouski, Rimouski); Pauline Boulos (Dundas); Raman Rai (McMaster University, Hamilton); Sanjay Dixit (Department of Medicine, McMaster University, Hamilton); Boulos Haraoui (Institut de Rhumatologie de Montréal, Montreal); Dalton Sholter (University of Alberta, Edmonton); Eliofotisti Psaradellis (JSS Medical Research, Montreal); Emmanouil Rampakakis (JSS Medical Research, Montreal); Brendan Osborne (Janssen Inc, Toronto); Francois Nantel (Janssen Inc, Toronto); Allen Lehman (Janssen Inc, Toronto)

Objectives: To describe the incidence of new onset extra-articular manifestations (EAMs) focusing on uveitis and inflammatory bowel disease (IBD) among SpA patients over 6 and 12 months of treatment with golimumab (GLM).

Methods: BioTRAC is an ongoing, prospective registry of patients initiating treatment with infliximab or GLM for RA, AS, or PsA, or with ustekinumab for PsA. Eligible participants for this analysis comprised GLM-treated AS $(n=395)$ and PsA $(n=257)$ patients. Presence of EAMs was defined as patients with uveitis or IBD. IBD data, however, was not collected for PsA patients. Results were based on patients with available data. Safety was ascertained by the incidence of adverse events (AEs) and serious adverse events (SAEs), reported per 100 patient-years (PY) of follow-up.

Results: Among AS patients, at baseline, the mean (SD) age was 45.3 (13.5) years and CRP was 14.1 (30.5) $\mathrm{mg} / \mathrm{L}$. Positive HLA B27 status was reported for $174(174 / 264 ; 65.9 \%)$ patients. History of uveitis was reported among $14.2 \%(\mathrm{n}=50 / 352)$ of AS patients. New-onset uveitis and IBD was reported for $2.3 \%$ (3.2 events/100 PY) and $1.1 \%$ (1.5 events/100 PY) of patients during follow-up, respectively. Results showed that CRP and HLA B27 status were not significantly associated with presence of EAMs at baseline. Overall, 419 AEs and 26 SAEs were reported by $199(50.4 \%)$ and $16(4.1 \%)$ patients by 12 months of treatment, respectively, for a total of $144.1 \mathrm{AEs} / 100$ PY and 8.9 SAEs/100 PY. A total of 5 AEs of uveitis were reported all of which were of mild severity, and either not $(n=4)$ or unlikely related $(n=$ 1) to the study drug. Among PsA patients, the mean (SD) age was 52.7 (13.2) years and CRP was $13.2(32.4) \mathrm{mg} / \mathrm{L}$ at baseline. A total of $9(9 / 80 ; 11.3 \%)$ patients had positive HLA B27 status. Patients with a history of uveitis was reported for $1.8 \%(n=4 / 224)$ of PsA patients. New-onset uveitis was reported for $0.4 \%$ (0.5 events/100 PY) of patients during follow-up. Overall, 254 AEs and 15 SAEs were reported by $123(47.9 \%)$ and $12(4.7 \%)$ patients by 12 months of treatment, respectively, for a total of $135.0 \mathrm{AEs} / 100 \mathrm{PY}$ and 8.0 SAEs/100 PY.

Conclusion: In patients with AS, development of uveitis and IBD was $2.3 \%$ and $1.1 \%$ during 12-month follow-up of GLM treatment, respectively. Among PsA patients, lower rates of new-onset uveitis were observed with $0.4 \%$. The current results should be interpreted with caution given the low number of cases of uveitis and IBD.

\section{7}

Central Reader Assessment of Imaging in Axial Spondyloarthritis: What is the Potential Impact on the Performance of the ASAS Classification Criteria?

Walter Maksymowych (University of Alberta, Edmonton); Raj Carmona (McMaster University, Hamilton); James Yeung (Private Practice, Vancouver); Jonathan Chan (University of British Columbia, Vancouver); Liam Martin (University of Calgary, Calgary); Ariel Masetto (Université de Sherbrooke, Sherbrooke); Sibel Aydin (University of Ottawa, Ottawa); Dianne Mosher (University of Calgary, Calgary); Stephanie Keeling (University of Alberta, Division of Rheumatology, Edmonton); Olga Ziouzina (University of Calgary, Calgary); Joel Paschke (Canadian Research Education (CaRE) Arthritis, Edmonton); Amanda Carapellucci (Canadian Research Education (CaRE) Arthritis, Edmonton); Robert Lambert (University of Alberta, Edmonton)

Objectives: The ASAS classification criteria provide the option to classify patients according to either imaging or clinical arms so that it may be possible to correctly classify patients even in the setting of unreliable assessment of imaging although the overall impact on the performance of these criteria is still unclear. We aimed to assess the impact of discrepancy between central and local imaging readers on the performance of the ASAS classification criteria in patients presenting with undiagnosed back pain to rheumatology practices.

Methods: The multicenter Screening for Axial Spondyloarthritis in Psoriasis, Iritis, and Colitis (SASPIC) Study is aimed at early detection of axial SpA. Consecutive patients $\leq 45$ years of age with $\geq 3$ months undiagnosed back pain with any one of psoriasis, acute anterior uveitis (AAU), or colitis undergo routine clinical evaluation by a rheumatologist for axial SpA. The rheumatologist determines the presence or absence of axial SpA after the clinical evaluation, again after the results of labs (B27, CRP) and radiography, and then after the results of local MRI evaluation. Imaging was also assessed centrally for features of axial SpA and detailed feedback on imaging findings provided to the local rheumatologist. The sensitivity and specificity of the ASAS classification criteria were calculated first according to the local rheumatologist diagnosis of axial $\mathrm{SpA}$ and secondly after incorporating imaging by central assessment.

Results: Central imaging assessment that included imaging was conducted in 180 patients $(50.6 \%$ male, mean age 34.8 years, mean back pain duration 7.0 years, B27+36.7\%) who were referred with AAU $(31.1 \%)$, psoriasis $(17.8 \%)$, Crohn's colitis $(36.7 \%)$, ulcerative colitis $(18.3 \%)$. Final diagnosis of axSpA was recorded in $82(45.6 \%)$ and 51 (28.3\%) following local and central assessment, respectively, with 38 (21.1\%) being re-categorized from axSpA to non-SpA and 7 (3.9\%) from non-SpA to axSpA following central assessment $(\mathrm{kappa}=0.48)$. Sensitivity/specificity of ASAS criteria was $79.3 \% / 78.6 \%$ per local diagnosis and $82.4 \% / 65.9 \%$ per central assessment For the 101 patients who had central assessment of MRI scans, final diagnosis of axSpA was recorded in 44 (43.6\%) and 24 (23.8\%) following local and central assessment, respectively, with $22(21.8 \%)$ being re-categorized from axSpA to non-SpA and 2 (2.0\%) from non-SpA to axSpA following central assessment (kappa $=0.49)$. Sensitivity/specificity of ASAS

Personal non-commercial use only. The Journal of Rheumatology Copyright (C) 2018. All rights reserved. 
criteria was $68.2 \% / 70.2 \%$ per local diagnosis and $87.5 \% / 66.2 \%$ following central assessment.

Conclusion: Incorporation of central imaging assessment leads to higher sensitivity but lower specificity of the ASAS criteria for axSpA.

\section{8}

Sustained Remission of Inflammation is Associated with Reduced Structural Damage on SI Joint MRI in Patients with Early Axial SpA: Evidence to Support the Concept of Treat-to-Target

Walter Maksymowych (University of Alberta, Edmonton); Pascal Claudepierre (Universite Paris Est Creteil, Creteil); Manouk de Hooge (Leiden University Medical Center, Leiden); Robert Lambert (University of Alberta, Edmonton); Robert Landewe (Amsterdam Rheumatology \& Immunology Center, Amsterdam); Anna Molto (Paris Descartes University, Hôpital Cochin, Paris); Désirée van der Heijde (Leiden University Medical Center, Leiden); Jack Bukowski (Pfizer, Collegeville); Heather Jones (Pfizer, Collegeville); Isabelle Logeart (Pfizer France, Paris); Lisa Marshall (Pfizer, Collegeville); Ronald Pedersen (Pfizer, Collegeville); Annette Szumski (inVentiv Health, Princeton); Bonnie Vlahos (Pfizer, Collegeville); Maxime Dougados (Paris Descartes University, Hôpital Cochin, Paris)

Objectives: Treat-to-target is acceptable in RA; however, it is unknown whether it will reduce/prevent disability, impairment of mobility, and structural damage in early axial $\mathrm{SpA}(\mathrm{axSpA})$ without radiographic sacroiliitis. We evaluated the impact of sustained clinical remission on MRI structural parameters, hypothesizing that patients with sustained inactive disease (ASDAS < 1.3) are more likely to achieve reduction in erosion (structural damage) and increase in backfill (a reparative process) on MRI of the SI joints (SIJ).

Methods: EMBARK (NCT01258738) and DESIR (NCT01648907) enrolled patients with early axSpA. EMBARK included 12 weeks of double-blind placebo-control, then open-label etanercept for 92 weeks. Patients in the DESIR observational cohort had no history of biologics and received no biologics for 2 years. T1 weighted MRI images of SIJ at baseline and 104 weeks were combined and anonymized; readers were unaware of film chronology and original patient cohort. Three experienced readers evaluated MRI images using the SpondyloArthritis Research Consortium of Canada SIJ Structural Score. Lesion change was considered present if $\geq 2$ of 3 readers measured change in same direction. ASDAS endpoints were assessed sequentially: sustained ( $\geq 2$ visits 6 months apart) inactive disease (ASDAS $<1.3)$ or moderate disease activity $(\geq 1.3$ to $<2.1)$; or no sustained response $(\geq 2.1)$. Net proportions of patients with decrease in erosion and increase in backfill were determined, unadjusted and adjusted for covariates that may affect development of lesions on MRI.

Results: From EMBARK and DESIR, 161 and 76 patients, respectively, were included. For patients in EMBARK with sustained ASDAS < 1.3, a greater percentage had decrease in erosion $(34 / 104,32.7 \%)$ than increase (5/104, 4.8\%); $<$ < 0.0001; without sustained response, $5 / 24$ (20.8\%) had decrease in erosion and $1 / 24(4.2 \%)$ had increase. This trend was also present in DESIR. Patients with sustained ASDAS < 1.3 in EMBARK: $22.1 \%$ had increase in backfill, $0 \%$ had decrease; $\mathrm{p}<0.0001$; in DESIR, $21.7 \%$ had increase, $0 \%$ had decrease; $\mathrm{p}<0.05$. For those without sustained response, difference between increase and decrease was smaller. Net percent of patients (adjusted) with sustained ASDAS $<1.3$ and erosion decrease: $22.6 \%$ and $9.3 \%$ for EMBARK and DESIR, respectively; without sustained response: $13.3 \%$ and $-10.1 \%$. Net percent of patients with sustained ASDAS $<1.3$ and backfill increase: $19.6 \%$ and $25.7 \%$ for EMBARK and DESIR, respectively; without sustained response: $8.7 \%$ and $6.0 \%$.

Conclusion: These data demonstrate a link between sustained ASDAS inactive disease and MRI structural endpoints. Clinical relevance of change in MRI SIJ erosion and backfill and their relationship to ankylosis development requires study.

139

Detection of Structural Lesions on T1 Weighted MRI versus Radiography of the Sacroiliac Joints in Early Axial SpA: 2-Year Data
Walter Maksymowych (University of Alberta, Edmonton); Pascal Claudepierre (Universite Paris Est Creteil, Creteil); Manouk de Hooge (Leiden University Medical Center, Leiden); Robert Lambert (University of Alberta, Edmonton); Robert Landewe (Amsterdam Rheumatology \& Immunology Center, Amsterdam); Anna Molto (Paris Descartes University, Hôpital Cochin, Paris); Désirée van der Heijde (Leiden University Medical Center, Leiden); Jack Bukowski (Pfizer, Collegeville); Heather Jones (Pfizer, Collegeville); Isabelle Logeart (Pfizer France, Paris); Lisa Marshall (Pfizer, Collegeville); Ronald Pedersen (Pfizer, Collegeville); Annette Szumski (inVentiv Health, Princeton); Bonnie Vlahos (Pfizer, Collegeville); Maxime Dougados (Paris Descartes University, Hôpital Cochin, Paris)

Objectives: Several methods exist for evaluating structural damage at the sacroiliac joint (SIJ): MRI, CT scan, radiography. A post-hoc analysis of SIJ images from EMBARK (NCT01258738) and DESIR (NCT01648907) evaluated: (1) association between presence/absence of erosion on MRI and presence/absence of erosion or sacroiliitis on radiographs at baseline and Week 104; (2) association between decrease/increase in erosion score on MRI and decrease/increase in erosion or sacroiliitis grade on radiographs between baseline and Week 104 .

Methods: All patients had early axSpA. EMBARK included 12 weeks double-blind placebo-control, then 92 weeks open-label etanercept. DESIR patients had no history of biologics and received no biologics for 104 weeks. MRI images from both studies were combined, anonymized, and read per patient; radiographs were combined, anonymized, and read separately from MRI. Readers were unaware of image chronology and original cohort. Three experienced readers individually evaluated T1 weighted MRI using SpondyloArthritis Research Consortium of Canada SIJ Structural Score; three different readers graded radiographic sacroiliitis using $\mathrm{mNY}$ grading system. Assessments were at level of each joint surface. Lesion presence/absence and decrease/increase was identified per patient if $\geq 2$ of 3 readers agreed. Statistical analyses included kappa coefficient of agreement and McNemar's test for discordance asymmetry.

Results: At baseline,156 (EMBARK) and 68 (DESIR) patients had MRI and radiographs available; erosion was present on both MRI and radiographs for 50/224 (22.3\%) patients and absent on both for 112/224 (50.0\%), kappa $=0.42$. Erosion was present only on MRI for $21.4 \%$ and only on radiographs for $6.3 \%, \mathrm{p}<0.0001$. Week 104 data $(\mathrm{N}=222)$ were similar to baseline. Results for erosion on MRI and sacroiliitis on radiographs were similar to those above; however, discordance was not significant. Decrease in erosion was present on both MRI and radiographs for 4/221 (1.8\%) patients and absent on both for 162/221 (73.3\%), kappa $=0.06$. Decrease in erosion was present only on MRI for $22.2 \%$ and only on radiographs for $2.7 \%$, p < 0.0001 . Results were similar for decrease in erosion on MRI and sacroiliitis grade on radiographs. Increase in erosion was present on both MRI and radiographs for $2 / 221(0.9 \%)$ patients and absent on both for $187 / 221$ $(84.6 \%)$, kappa $=0.03$. Increase in erosion was present only on MRI for $6.8 \%$ and only on radiographs for $7.7 \% ; \mathrm{p}=0.72$. Results were similar for increase in erosion on MRI and sacroiliitis grade on radiographs.

Conclusion: Findings of structural damage in the SIJ are observed differently on radiography and MRI in patients with early axSpA, and may appear to evolve differently.

\section{0}

Submetabolome Profiling with Differential Chemical Isotope Labeling Liquid Chromatography Mass Spectrometry and a Universal Metabolome Standard Reveals a Metabolite Profile with 99\% Accuracy for Rheumatoid Arthritis

Walter Maksymowych (University of Alberta, Edmonton); Derrick Blackmore (University of Alberta, Edmonton); Roman Eisner (City of Edmonton, Edmonton); Nan Wang (University of Alberta, Department of Chemistry, Edmonton); Liang Li (University of Alberta, Department of Chemistry, Edmonton); Zaeem Siddiqi (University of Alberta, Edmonton) Objectives: We aimed to employ metabolomic profiling that includes dansylation liquid chromatography mass spectrometry (LC-MS), resulting in 1000 -fold increase in detection sensitivity of amine/phenol-containing

Personal non-commercial use only. The Journal of Rheumatology Copyright @ 2018. All rights reserved. 
metabolites, and universal metabolome-standard (UMS) methodology in conjunction with differential chemical isotope labeling (CIL LC-MS), to target chemical group-based submetabolomes to identify a metabolite signature with high accuracy for RA.

Methods: 12C-dansylation and acid labeling of individual serological samples and 13C-dansylation and acid labeling of pooled samples from 47 age/gender matched healthy control subjects, 52 age/gender matched RA patients, and 46 patients with seropositive myasthenia gravis was undertaken. A total of 7,458 amine/phenol and 9954 organic acid metabolites were combined into a single data set for analysis. Metabolite concentrations were natural-log transformed. Model accuracy estimation was performed using 5 -fold cross-validation, and metabolites were selected using within-fold feature selection. Metabolites were ranked using Spearman correlation coefficient, and the top $\mathrm{n}$ were selected, with a varying $\mathrm{n}$. Training of the predictive model was done using a linear Support Vector Machine (SVM). After cross-validation, the final model formula was calculated on the entire data set using the same methodology as was evaluated using cross-validation. Cross-validation accuracy was further analyzed using randomly selected metabolites. Data processing and analysis was performed entirely in $\mathrm{R}$ (version 3.2.3). SVM was trained using the e1071 package (version 1.6-7) and cross-validation was done using the caret package (version 6.0-64).

Results: A total of 5711 metabolites were identified in all samples with orthogonal partial least squares discriminant analysis showing a clear separation of the 3 groups $(\mathrm{R} 2=0.98, \mathrm{Q} 2=0.80) .34$ serum metabolites were identified as potential RA biomarkers with correlation coefficients $\geq$ 0.80 . Cross-validation accuracy of top-ranked metabolites, according to Spearman's correlation, and using a varying number of metabolites shows that $99.1 \%$ accuracy for RA versus controls is achieved using only 4 metabolites.

Conclusion: CIL LC-MS metabolomic profiling and UMS methodology reveals that serum metabolomes of RA patients differ considerably from healthy and autoimmune disease.

\section{1}

Validation of Web-Based Calibration Modules for the Spondyloarthritis Research Consortium of Canada MRI Scores Using Game Psychology Walter Maksymowych (University of Alberta, Edmonton); Pamela Weiss (Children's Hospital Philadelphia, Philadelphia); Ulrich Weber (King Christian 10th Hospital for Rheumatic Diseases, Grasten); Sander Shafer (Gentofte Hospital, Copenhagen); Susanne Pedersen (University of Copenhagen, Copenhagen); Matthew Maksymowych (University of Alberta, Edmonton); Fardina Malik (Hospital for Special Surgery, New York); Georg Kroeber (Odense); Simon Krabbe (Copenhagen Center for Arthritis Research, Copenhagen); Mats-Peter Johansson (Gentofte Hospital, Copenhagen); Jacob Jaremko (University of Alberta, Edmonton); Eric Heffernan (St. Vincent's Hospital, Dublin); Riccardo Guglielmi (Ente Ospedaliero Cantonale, Lugano); Jonathan Cheah (Hospital for Special Surgery, New York); Hanne Boutrupe (University of Copenhagen, Copenhagen); Brian Trinh (Canadian Research Education (CaRE) Arthritis, Edmonton); Joel Paschke (Canadian Research Education (CaRE) Arthritis, Edmonton); Robert Lambert (University of Alberta, Edmonton)

Objectives: The application and appropriate use of imaging-based scoring instruments is usually an ad hoc process based on passive learning from published manuscripts and atlases while real-time interaction with instrument developers is often non-feasible. Moreover, most instruments lack knowledge transfer tools that would facilitate attainment of pre-specified performance targets for reader reliability. We aimed to 1 . To develop web-based calibration modules for the SPARCC MRI Scores based on consensus scores from these instrument developers, experiential game psychology, and real-time iterative feedback built into a scoring schematic directly on the MRI image. 2. To test the feasibility and attainment of pre-specified performance targets for reader reliability.

Methods: The scoring of MRI lesions of the sacroiliac joints uses the SPARCC methods on a web-based interface that includes a schematic of these joints adjacent to the MRI image. The SPARCC BME inflammation calibration module is comprised of 50 DICOM cases, each with scans from baseline and 12 weeks after the start of tumor necrosis factor inhibitor therapy. The SPARCC structural score is comprised of 40 cases, each with scans from baseline and after 2 years follow-up. Scans are scored blindedto-time-point. Continuous visual real-time feedback regarding concordance/discordance of scoring with expert readers is provided by a color-coding scheme. Reliability is assessed by real-time intra-class correlation coefficient (ICC). Accreditation for SPARCC BME score is achieved with status and change score ICC of $>0.8$ and $>0.7$, and for SPARCC structural status and change score an ICC of $>0.7$ and $>0.5 .14$ readers scored the SPARCC BME module and 11 the SPARCC structural module, 9 being inexperienced rheumatology fellows

Results: For the SPARCC BME score and the calibration-per-slice method, all 14 readers completed the scoring and attained the required level of proficiency. For the calibration-per-case method, 11 completed scoring and 7 attained proficiency for status scores and 5 for change scores. For the SPARCC structural scores and the calibration-per-slice method, all 11 readers completed scoring. Proficiency for status score was attained by 10 readers and proficiency for change was attained by $11,5,6,8$ readers for fat, erosion, backfill, ankylosis, respectively. All readers rated the modules as easy and intuitive with average time for reading each case for SPARCC BME and Structural being 9 min and 12 min, respectively.

Conclusion: Experiential web-based learning is an effective and feasible calibration tool to achieve proficiency targets in the scoring of MRI scans for SIJ lesions.

\section{2}

The Prevalence of Traditional Cardiovascular Risk Factors in Patients with Chronic Rheumatic Diseases and Adherence with Treatment Recommendations

Micaela Jacobson (University of Ottawa, Ottawa); Shadi Akhtari (Women's College Hospital, Toronto); Paula Harvey (Division of Cardiology, Women's College Hospital, Toronto); Dana Jerome (Women's College Hospital, Toronto); Jacqueline Hochman (Women's College Hospital, Toronto); Elizabeth Grigoriadis (Women's College Hospital, Toronto); Natasha Gakhal (Women's College Hospital, Toronto); Lihi Eder (Women's College Hospital, Toronto)

Objectives: The goal of the present study was to assess gaps in care regarding cardiovascular $(\mathrm{CV})$ risk factors of patients with chronic rheumatic diseases (CRD), including psoriatic arthritis (PsA), rheumatoid arthritis (RA), and ankylosing spondylitis (AS). Specifically, we examined the proportion of patients with undiagnosed, untreated, or undertreated hypertension (HTN), type II diabetes mellitus (DM), and dyslipidemia (DLP). We also examined body mass index (BMI) and current smoking status.

Methods: Participants were recruited from Women's College Hospital Rheumatology Clinic in Toronto, Canada. Inclusion criteria included minimum age 40 years, with no history of $\mathrm{CV}$ events (stroke, myocardial infarction, angina, or heart failure). Patients were interviewed for their smoking status and previous diagnosis and treatment with medications for DM, HTN, and DLP. Height, weight, and blood pressure were measured, plus serum lipid profile and HbAlc. Framingham Risk Score (FRS) was calculated to estimate 10-year probability of CV events. Based on FRS, patients were stratified into low (less than $10 \%$ ), moderate (10 to 19\%) and high risk ( $20 \%$ or higher) categories. The prevalence of under-diagnosis and under-treatment of DLP, DM, and HTN were then calculated based on local guidelines.

Results: A total of 70 RA patients, 50 PsA patients, and 14 AS patients were recruited. Ninety (67\%) patients were found to be overweight or obese (BMI of $25 \mathrm{~kg} / \mathrm{m} 2$ or higher), and 18 (13\%) were current smokers. Fifty-two (39\%) patients were found to have HTN $(\mathrm{BP}>140 / 90 \mathrm{mmHg})$. Three $(6 \%)$ were undiagnosed, $3(6 \%)$ were untreated, and $6(12 \%)$ were undertreated. Sixty $(45 \%)$ patients were found to have DLP. Eighteen $(30 \%)$ were undiagnosed, $22(37 \%)$ were untreated, and $10(17 \%)$ were undertreated. Fourteen $(10 \%)$ patients were found to have DM (HbAlc of $6.5 \%$ or higher). One was undiagnosed/untreated, and 4 (29\%) were undertreated. 
Conclusion: In the present study, a significant proportion of patients were found to have modifiable CV risk factors. Up to a third were undiagnosed, untreated, or undertreated, particularly for DLP. This high-risk group could benefit from improvement in the screening and management of $\mathrm{CV}$ risk factors to address the gaps in care identified in our study.

\section{3}

\section{Amyloid Arthropathy}

Mohammad Bardi (University of British Columbia, Vancouver); Drew Bowie (University of British Columbia, Victoria)

Background: Amyloidosis is a multisystem infiltrative disorder characterized by deposition of amyloid fibrils into tissue, altering their normal function. Clinical manifestations can vary widely depending on the fibril type, amount, and location of deposition. Deposition into articular and periarticular spaces can mimic rheumatic condition such as gout or rheumatoid arthritis.

Case Presentation: A 79 year old male was admitted for workup of acute-on-chronic kidney disease. During his admission, he experienced a fall and was incidentally found to have an inflamed left wrist. A CT was ordered to assess for fracture. Imaging demonstrated prominent subchondral cysts suggestive of underlying chronic crystal arthropathy. Rheumatology became involved due to a strong suspicion of gout. A joint aspiration was attempted but no significant effusion was present and a dual energy CT was unremarkable for uric acid deposition. Upon further review of the imaging, the possibility of amyloid arthropathy was raised given the extensive cystic appearance of his carpal bones without cortical disruption. Due to his progressive renal decline, and later finding of serum paraproteinemia a renal biopsy was performed that demonstrated features of light chain deposition consistent with AL amyloid. A subsequent bone marrow biopsy showed further AL amyloid deposits.

Conclusion: Amyloid arthropathy is an uncommon cause of erosive and destructive arthritis. That said, this case study demonstrates that it should be considered when clinical suspicion of crystal arthropathy and diagnostic testing are incongruent. AL Amyloid arthropathy, in particular, should be considered when investigations demonstrate paraproteinemia in the setting of arthritis. Additional evidence of amyloid arthropathy is found on CT imaging characterized as well-defined subchondral cysts with preserved cortical margins.

\section{4}

Best Practice Principles on Engaging Underserved Communities in Health Research: Review of the Literature

So Park (University of British Columbia, Vancouver); Susan Mills (University of British Columbia, Vancouver); Adeline Cui (University of British Columbia, Vancouver); Daisy Au (Multi-lingual Orientation Service Association for Immigrant Communities, Vancouver); Nancy Clark (University of Victoria, Victoria); Connie Davis (Centre for Collaboration, Motivation and Innovation, Vancouver); Guillermina Flores (University of British Columbia, Vancouver); Maylene Fong (Vancouver Coastal Health Authority, Vancouver); Clayon Hamilton (University of British Columbia, Vancouver); Shannon Holms (British Columbia Ministry of Health, Victoria); Cheryl Koehn (Arthritis Consumer Expert, Vancouver); Sharon Koehn (Simon Fraser University, Burnaby); Diane Lacaille (University of British Columbia (Division of Rheumatology)/ Arthritis Research Canada, Richmond)

Objectives: Underserved populations (UP) face more challenges in accessing care and in the quality of services they receive, but are far less represented in research than the general population. Thus, it is important for studies with clinical or healthcare policy implications to consider including or focusing on these populations. In preparation for our study on the barriers UP experience in self-managing arthritis and co-morbidities, we conducted a literature review on how to best engage members of UP as: 1) research partners through community-based organizations (CBOs); and 2) research participants.

Methods: Indexed databases (Medline/PubMed, CINAHL, Web of Science) and webpage searches (Google) were used to conduct the review. The first search was done on involving UP as research partners and participants. Inclusion criteria were: 1) English articles published after 2011;2) study taking place in Canada, USA, Australia, or Europe; and 3) results focused on UP engagement in research as partners or participants. The second search explored engaging partners and participants with addiction, mental illnesses, and arthritis in addition to the inclusion criteria above. UP included: 1) ethnic minorities; 2) indigenous; 3) older adult; 4) homeless; 5) low income; 6) refugees; and 7) immigrants. Relevant data and recommendations were extracted and synthesized.

Results: The research partner literature review resulted in 17 and 48 documents from unpublished and published literature, respectively. The research participant literature review identified a sample size with 20 unpublished and 56 published documents. Similar ideas on best practices were found in unpublished and published literature for both partner and participant reviews. Overarching principles for engaging UP partner and participants include: 1) clear and open communication; 2) identification and elimination of barriers; 3) time investment to build trust; 4) meaningful remuneration; 5) identification and mitigation of power differentials; and 6) time, resources, and flexibility for meaningful collaboration. How the above principles can be applied to the research process (ethics, engagement, recruitment, data collection and analysis, remuneration, and dissemination) will be discussed and concrete examples will be provided for illustration.

Conclusion: This study identified important principles for best practices that need to be implemented when conducting research with UP. We are currently implementing these principles in our study on barriers to self-management in persons with arthritis and co-mordbities. We are also using them as a foundation to develop a conceptual model for engaging UP in research that may help future researchers conducting studies with UP. Supported by a CIORA grant.

145

Access to Rituximab for Patients Comparing Variable Access in a Single Rheumatology Clinic

Raymond Chu (University of Alberta, Edmonton); Walter Maksymowych (University of Alberta, Edmonton); Steven Katz (University of Alberta, Edmonton); Elaine Yacyshyn (Division of Rheumatology, University of Alberta, Edmonton)

Objectives: The purpose of our study is to investigate the difference in time to access of rituximab therapy between patients with rheumatoid arthritis (RA) compared to patients with other autoimmune diseases.

Methods: A retrospective chart review was performed on 236 patients under the care of rheumatologists out of the University of Alberta, who had received rituximab from October 2012 to October 2017. Data extracted included the method of drug coverage, the date determining when a patient would require rituximab for treatment and the date of first infusion. A Cuzick's test of trend was performed on three groups. The first group includes patients with autoimmune disease (not RA) receiving coverage through the Short Term Exceptional Drug Therapy (STEDT) program, Alberta's publicly funded special access program for high cost drugs. The second group includes patients with autoimmune disease (not RA) who receive non-STEDT funding, encompassing compassionate care and insurance. The third group includes RA patients followed by the Rheumatoid Arthritis Pharmacovigilance Program and Outcomes Research in New Therapies (RAPPORT) clinic at the University of Alberta whom all receive non-STEDT funding. Analysis was conducted using STATA 13.

Results: Of the 236 patients, 154 patients were identified as having RA and followed by the RAPPORT clinic. Eighty-two patients were identified as having other autoimmune diseases (vasculitis, dermatomyositis, systemic lupus erythematosus, myositis, sarcoidosis, Sjogren's and ankylosing spondylitis). Of these 82 patients, 54 had their rituximab covered through Alberta's STEDT program and 28 patients received coverage from non-STEDT programs. The median time to access was 36 days (IQR: 25, 53) for RAPPORT patients, 30 days (IQR: 17, 44) for STEDT patients with other autoimmune diseases and 45.5 days (IQR: 33,63 ) for non-STEDT

Personal non-commercial use only. The Journal of Rheumatology Copyright $(\subset) 2018$. All rights reserved. 
patients with other autoimmune diseases. The Cuzick's test revealed a statistically significant trend between groups ( $\mathrm{p}$-value $=0.004$ ).

Conclusion: Our analysis concludes that public funding through the STEDT program, allows quicker access to rituximab compared to alternative sources of funding including compassionate care and insurance. RA patients who also receive non-STEDT coverage also receive rituximab more quickly, having the benefit of a dedicated clinic familiar with drug application processes. The result of this study indicates evidence of medication health economic disparity between access for patients with different conditions. This is concerning for patients who require medications quickly, for disease control, such as vasculitis patients, who appear to have less equity in access to rituximab. Further analysis is necessary to determine causes for this disparity and expedite access.

\section{6}

Prevalence and Metric of Depression and Anxiety in Lupus: A Systematic Review and Meta-analysis

Elvira Bangert (Queen's University, Kingston); Ahmed Moustafa (Western University, London); Sherief Marzouk (University of Toronto, Toronto); Jiandong Su (Toronto Western Hospital, Toronto); Lihi Eder (Women's College Hospital, Toronto); William Fung (University of Toronto, Toronto); Panos Lambiris (University of Toronto, Toronto); Zahi Touma (Toronto Scleroderma Program, Division of Rheumatology, Toronto Western Hospital, University Health Network; Institute of Health Policy, Management and Evaluation, University of Toronto, Toronto)

Objectives: To systematically review literature on 1) the prevalence of Depression and Anxiety in SLE patients, 2) the metrics of Depression and Anxiety and 3) the psychometric properties of different screening instruments. Methods: This review was conducted on the recommendation of PRISMA-R. Literature search (1954-2016) in Ovid Medline, Embase, Psyc INFO and CINAHL for articles on the prevalence of Depression and/or Anxiety in adult SLE patients using a specified neuropsychological instrument of cognitive function (CF) was conducted. Included studies were critically appraised and analyzed. The prevalence of Depression and Anxiety was studied for all instruments and Pooled Prevalence (PP) was determined in the commonly used instruments [Centre for Epidemiological Studies - Depression (CES-D), Beck Depression/Anxiety Inventory (BDI/BAI), Hospital Anxiety/ Depression Scales (HADS-D/A), Hamilton Rating Scales for Depression/Anxiety (HAM-D/A)].

Results: A total of 4394 references were identified, 278 were selected for detailed review and 121 were included in the final analysis. Depression: PP of Depression is $35.2 \%$ (95\% CI: 30.1\%-40.5\%) (Figure 1 (A)). Was obtained from 70 studies in 23399 SLE patients. Anxiety: PP of Anxiety is $24.2 \%$ (95\% CI: $17.9 \%-31.2 \%$ ) (Figure 1 (B)). Was obtained from 39 studies in 4495 SLE patients. CES-D: was utilized in 13 studies including 1856 SLE patients. Depression PP was $41.5 \%$ (95\% CI: 35.1\%-48.1\%). BDI/BAI: was utilized in 14 studies including 1355 SLE patients and 3 studies including 489 patients respectively. Depression PP was 39.9\% (95\% CI: 31.1\%-49.1) and Anxiety PP was $38.4 \%$ (95\% CI: 34.2\%-42.8). HADS-D/A: was utilized in 14 studies including 1238 SLE patients and 12 studies including 1099 patients respectively. Depression PP was $24.4 \%$ (95\% CI: $19.1 \%-30.1 \%$ ) and Anxiety PP was $38.3 \%$ (95\% CI: $29.1 \%-47.9 \%$ ). HAM-D/A: was utilized in 4 studies including 323 SLE patients and 5 studies including 269 patients respectively. Depression PP was $43 \%$ (95\% CI: $27.9 \%-58.7 \%$ ) and Anxiety PP was $37.3 \%$ (95\% CI: $31.7 \%-43.2 \%$ ). We found high variability in the prevalence of Depression and Anxiety, and high statistical heterogeneity (I2 $>75 \%$ ) which is attributed to the: 1 ) lack of standardization in the metrics and definitions of depression and anxiety in SLE, and 2) variability in demographics.

Conclusion: The prevalence of Depression and Anxiety are high $5.7 \%-78.6 \%$ and $1.1 \%-71.4 \%$ respectively, largely depending on the metrics. There is a lack of a standardized approach on how to measure and define Depression and Anxiety in SLE. CES-D, BDI, HAM-D yielded similar Depression prevalence, while HADS-D carried the lowest prevalence. All metrics of Anxiety yielded similar Anxiety prevalence.

\section{7}

Nailfold Videocapillaroscopy in Idiopathic Inflammatory Myopathies Elvira Bangert (Queen's University, Kingston); Marie Hudson (McGill University, Jewish General Hospital, Lady Davis Institute for Medical Research, Montreal); Evelyne Vinet (McGill University Health Centre, Montreal); Mianbo Wang (Lady Davis Institute for Medical Research, Montreal); Genevieve Gyger (McGill University, Jewish General Hospital, Montreal)

Objectives: In systemic sclerosis, a scleroderma pattern on nailfold videocapillaroscopy (NVC) (SSc) is well recognized. In idiopathic inflammatory myopathies (IIM), a scleroderma-like pattern, including giant-ramified capillaries, has been proposed. The aim of our study was to describe nailfold capillary abnormalities in IIM subsetted using newly proposed integrative criteria $[1,2]$.

Methods: We studied IIM subjects in the Canadian Inflammatory Myopathy Study (CIMS), a cohort of subjects with early onset disease followed prospectively. NVC images were acquired using the DS MEDICA Videocap (X200 magnification). The nailfolds of the second, third, fourth and fifth fingers of both hands were photographed and scored by an experienced rheumatologist. Microhemorrhages, giant capillaries, ectasias and ramified capillaries were scored using a standardized semi-quantitative scale $(0=$ no, $1=\leq 33 \%, 2=33-66 \%$, and $3=\geq 66 \%$ abnormalities per linear millimeter). Capillary density was scored both semi-quantitatively ( 0 if $\geq 7,1$ if $4-6$, or 2 if $\leq 3$ capillaries $/ \mathrm{mm}$ ) and quantitatively (mean number of capillaries $/ \mathrm{mm}$ ). Each NVC parameter, as well as disorganization and giant-ramified capillaries, were also scored as present or absent. Finally, the proportion of subjects with scleroderma-like, scleroderma (SSc), non-specific, and normal patterns were compared.

Results: 38 IIM subjects were included: 18 with DM (of which 9 had myositis specific antibodies and 9 were seronegative) and 20 with OM (of which 8 had anti-synthetase syndrome). Baseline characteristics of the subjects and NVC features and patterns are presented in Tables 1-3. Capillary density was lower than normal in both DM (mean 5.5/mm) and OM (mean $6.1 / \mathrm{mm}$ ). Giant-ramified capillaries were present in $22.2 \%$ of DM and $25 \%$ of OM subjects. SSc-like pattern was more common in DM (50\%) than OM $(25 \%)$, whereas active- or late-SSc pattern was more common in OM than DM (25\% vs $5.6 \%)$.

Conclusion: This is the first study of NVC using integrative criteria for IIM. We found differences in NVC features and patterns between DM and OM subsets. NVC may be an additional diagnostic tool in IIM. A larger study is ongoing to confirm these findings. 1. Benveniste et al. Curr Opin Neurol 2016;29(5):662-73. 2. Allenbach et al. Neuropathol Appl Neurobiol 2017;43(1):62-81.

\section{8}

Concomitant Septic Arthritis and Pseudogout after Total Knee Arthroplasty

Alexander Hofkirchner (The University of Western Ontario, London); Elvira Bangert (Queen's University, Kingston); Tanveer Towheed (Queen's University, Kingston)

Introduction: Although there have been few reports of pseudogout in patients after total knee arthroplasty (TKA), we know of only one reported case where both pseudogout and bacterial infection were found in a prosthetic knee. Here, we describe one such case of concomitant pseudogout and Parvimonas micra infection in a prosthetic knee.

Case Presentation: The patient, an 81-year-old woman with no history of crystalline arthropathy, presented with ill-defined pain in her left knee, which had undergone TKA three years prior. Synovial fluid aspirates were initially positive for calcium pyrophosphate crystals but negative for bacterial cultures, and many repeated arthrocenteses were required before arriving at this complicated diagnosis.

Conclusion: Clinicians must be aware that both pseudogout and septic arthritis may present in the same joint; however, early diagnosis and the prioritization of septic arthritis management are crucial in such cases to prevent further complications. 
149

Partnering in Research: Maximizing Benefits \& Minimizing Risks in Patient-Researcher Relationships

Jenny Leese (Arthritis Research Canada, Vancouver); Graham Macdonald (Arthritis Research Canada, Richmond); Bao Tran (Arthritis Research Canada, Richmond); Lianne Gulka (Arthritis Research Canada, Richmond); Alison Hoens (University of British Columbia, Vancouver); Sheila Kerr (Arthritis Research Canada, Richmond); Wendy Lum (Arthritis Research Canada, Richmond); Linda Li (Rehab Sciences/Physical Therapy, University of British Columbia, Arthritis Research Canada, Richmond)

Objectives: Patient engagement in research occurs when patients are meaningfully involved in governance, priority setting, conducting research, and/or knowledge translation activities. It has an ethical basis in the notion that people who are impacted by health research should be involved in research decision-making, if they choose. Little empirical evidence exists, however, to better understand ethical issues encountered in everyday practices of engagement, particularly from patients' perspectives. Our objective is to understand what is valued and at risk within relationships of patients and researchers engaging with each other in research, based on varied experiences of arthritis patients.

Methods: The project is jointly designed and conducted by researchers and patient partners. Participants were invited to take part in a one-hour in-depth interview, in-person or by phone. Eligible participants were current or past members of an arthritis patient advisory board in a research centre. A semistructured topic guide was used with prompts and probes to elicit detail about relationships with researchers. An iterative thematic analysis was conducted using constant comparison methods, and a relational ethics framework was applied to themes emerging from the data.

Results: 22 participants with arthritis (from 26 to 68 years old) were recruited. Twenty-one $(95 \%)$ were female, with time spent as a board member varying from 1 month to 10 years. Two themes emerged: 1) "Being Heard": Participants valued demonstrations of mutual respect within their relationships with researchers. This involved giving equal importance to the distinct contributions of patients and researchers in the research process; 2) "Being with Supportive People": Participants valued researchers who actively supported them to manage (actual and potential) negative physical and emotional impacts (e.g., fatigue, stress, uncertainty) of juggling engaging in research among other priorities in their daily lives.

Conclusion: Findings provide valuable insight into what is important, from patients' perspectives, in developing and maintaining good relationships between researchers and patients who are engaging with each other in research. They can serve as a guide for researchers and patients with chronic illness to identify and address ethical issues in how they relate to each other while engaging in research. They are a critical step to fostering everyday ethical practices of patient engagement in research that are anchored in patients' perspectives.

\section{0}

Effectiveness of a Fall-Prevention Intervention on Physical Performance and Anthropometric Characteristics in Older Adults with a High Rate of Arthritis

Dina Jones (West Virginia University, Morgantown); Sijin Wen (West Virginia University, Morgantown); Jennifer Eicher (West Virginia University, Morgantown); Corrie Mancinelli (West Virginia University, Morgantown)

Objectives: Tai Ji Quan: Moving for Better Balance (TJQMBB) is an evidence-based, community-delivered exercise program that improves balance and reduces falls. This translational study determined the effectiveness of TJQMBB in improving physical performance and anthropometric characteristics in a 16-week intervention in older adults in a new setting. Methods: Twenty faith-based organizations (FBOs) sponsored 16 TJQMBB classes at 15 FBOs in 7 rural counties. One-hour classes were held twice weekly for 16 weeks. Data were collected at baseline, 16 weeks, and 32 weeks via questionnaires (sociodemographics); anthropometric measurements (waist circumference, height, weight); and performance-based tests:
5- or 10-meter walk at usual pace (gait speed), timed up-and-go (TUG) (mobility), 4-square test (balance), and 5 times sit-to-stand (lower extremity strength). Participants were classified as high or low fall risk based on baseline TUG scores (low: $<12$ seconds, high: $\geq 12$ seconds). Mixed-effects models were used to longitudinally examine the change in each outcome from baseline to 16 weeks, baseline to 32 weeks, and across the 3 time periods, and to determine if improvement varied by baseline fall risk.

Results: Two-hundred thirty-seven people enrolled in the study. Participants ranged in age from 55 to 98 years (mean \pm SD: $71.5 \pm 9.2$ years) and were predominantly female (81\%). Arthritis was reported in $66 \%$ of participants. Data were available for 237 participants at baseline, 175 at 16 weeks, and 163 at 32 weeks. The mean baseline TUG score was $12.0 \pm 5.4$ seconds (range 5 to 43 seconds) with $32 \%$ of participants at high risk for falls. Waist circumference $(p=0.04)$ and scores on the TUG $(p<0.001)$, 4-square test ( $p<0.001)$, and chair stands $(\mathrm{p}<0.001)$ improved across time. Gait speed improved from baseline to 16 weeks $(\mathrm{p}=0.04)$, but not from baseline to 32 weeks or across time. There was no change in body mass index. Improvements in gait speed were greater in the low $(-0.1 \pm 0.4$ seconds $)$ versus high $(0.06 \pm 0.3$ seconds $)$ risk group $(\mathrm{p}=0.009)$, and greater in the high $(-2.6 \pm 5.8$ seconds $)$ versus low $(-0.6 \pm 1.7$ seconds $)$ risk group for the 4-square test $(\mathrm{p}<0.001)$.

Conclusion: The TJQMBB intervention was effective in improving waist circumference, mobility, balance, lower extremity strength, and walking speed in rural older adults in a new setting. Gains in walking speed were greater in the low fall-risk group while balance improved more in the high-risk group. The intervention was successful in improving balance in those with the greatest need.

\section{1}

\section{Methotrexate for Polymyalgia Rheumatica}

Leonardo Calderon (University of Ottawa, Ottawa); Samuel Whittle (Queen Elizabeth Hospital and University of Adelaide, Woodville); Catherine Hill (Queen Elizabeth Hospital, Woodville); Jinane Sayad (Ottawa); George Wells (University of Ottawa Heart Institute, Ottawa); Nataliya Milman (University of Ottawa, Ottawa)

Objectives: The mainstay treatment for polymyalgia rheumatica (PMR), an inflammatory disorder, is glucocorticoids. Therapy discontinuation can take several years, and long term glucocorticoid use exposes patients to side effects including but not limited to neuropsychiatric and metabolic disturbances, hypertension, and osteoporosis. Methotrexate, an inhibitor of tetrahydrofolate dehydrogenase, is used in low doses as a disease-modifying drug for rheumatoid arthritis and as remission maintenance agent in various rheumatologic conditions, including systemic lupus erythematosus, inflammatory myopathies, and systemic vasculitides. This review's objective is to elucidate the clinical benefits and harms of methotrexate for patients with PMR.

Methods: We conducted a Cochrane review of randomized control trials (RCT) within the databases Cochrane Central Register of Controlled Studies, MEDLINE and EMBASE. The seven major outcomes assessed at 52 weeks or closest reported time point were: proportion of patients who experienced at least one relapse, proportion of patients who achieved sustained discontinuation of glucocorticoids, proportion of patients in disease remission, physical function, short-term serious adverse effects (AE), withdrawals due to $\mathrm{AE}$, and glucocorticoid-related AE. Minor outcomes assessed included cumulative dose of glucocorticoids and mortality.

Results: Four RCTs were included totaling 194 participants. The proportion of patients who experienced at least one relapse was significantly lower in the methotrexate group compared to placebo group (RR 0.65[0.47-0.88], $\mathrm{p}<0.01$ ), and the proportion of participants in disease remission was significantly greater in the methotrexate than in the placebo group (RR 5.22 [1.28-21.29], $\mathrm{p}<0.05$ ); quality of evidence is low for these two outcomes. There were no significant differences in short-term serious AE, (Peto OR 0.24[0.04- 1.25], $\mathrm{p}=0.09$ ), withdrawals due to AE (Peto OR 0.52[0.14-2.02, $\mathrm{p}=0.35$ ), or glucocorticoid-related AE with the exception of cataracts (Peto OR 0.13[0.02-0.93], $\mathrm{p}=0.04)$ : for osteoporosis and fracture Peto OR

Personal non-commercial use only. The Journal of Rheumatology Copyright @ 2018. All rights reserved. 
$0.79[0.17-3.58], \mathrm{p}=0.76$; for hypertension RR 1.32[0.73-2.37], $\mathrm{p}=0.35$; for weight gain RR 1.32[0.83-2.11], $\mathrm{p}=0.24$ ); quality of evidence is very low for all AE-related outcomes. Physical activity was not measured within the studies included. The mean cumulative dose of glucocorticoids was significantly lower in the methotrexate group compared to placebo group, $2.57 \mathrm{~g} \pm 1.61$ versus $3.25 \mathrm{~g} \pm 1.40(\mathrm{MD}:-0.91,-1.25$ to $-0.57, \mathrm{P}<0.001$; moderate quality of evidence). There was no significant difference in mortality in the methotrexate compared to the placebo group (Peto OR $0.30[0.05-1.79], \mathrm{p}=0.19$; very low quality of evidence).

Conclusion: Results suggest methotrexate use may be warranted in individuals with PMR as demonstrated by consistent favourable trends in the major and minor outcomes investigated.

\section{2}

An International Delphi Exercise to Identify a Core Set of Items to Develop Classification Criteria for Scleroderma Renal Crisis

Emily-Ann Butler (Dalhousie University, Halifax); Mianbo Wang (Lady Davis Institute for Medical Research, Montreal); Christopher Denton (UCL, London); Marie Hudson (McGill University, Jewish General Hospital, Lady Davis Institute for Medical Research, Montreal)

Objectives: Scleroderma Renal Crisis (SRC) is characterized by malignant hypertension and acute kidney injury. Large gaps in our understanding of SRC persist. Indeed, the absence of a gold standard has hindered our ability to perform rigorous research on SRC. The Scleroderma Clinical Trials Consortium (SCTC) SRC Working Group was created to develop consensus and data-driven classification criteria for SRC. In the first phase of this project, we performed a scoping review of the literature to generate evidence-based items to define SRC (Hoa et al, Autoimmun Rev 2017). We undertook this second phase of the project to achieve consensus on a core set of items to define SRC.

Methods: A survey using the items identified by our scoping review was developed (REDCap platform, Vanderbilt University, Nashville, Tennessee). An international, multidisciplinary panel of experts from the SCTC, European Scleroderma Trials and Research Group (EUSTAR), the Canadian Scleroderma Research Group (CSRG), the Australian Scleroderma Interest Group (ASIG) was invited to participate. The Delphi exercise was performed in 3 rounds. In Round 1, participants were asked to identify omissions and clarify ambiguities. The survey was modified accordingly. In Round 2, participants were asked to rate the reliability and feasibility of the items using Likert-type scales ranging from 1-9 $(1=$ very unreliable/unfeasible, $5=$ uncertain, $9=$ very reliable/feasible), and to provide comments. In Round 3 , participants reviewed the results and comments of Round 2, and were asked to provide final ratings. Items rated as highly reliable and feasible (both median scores $\geq 7$ ) in Round 3 were selected as the provisional core set of items to define SRC.

Results: 216 experts were invited and 99 from 17 countries agreed to participate. Of the 31 items in the survey, consensus was achieved on items pertaining to hypertension, renal insufficiency, proteinuria and hemolysis. Consensus was not achieved on items pertaining to hematuria, thrombocytopenia, encephalopathy, retinopathy, hyperreninemia, cardiac dysfunction and histopathology.

Conclusion: This Delphi exercise allowed the generation of a provisional core set of items to define SRC. A nominal group discussion is planned for November 2017 to achieve final consensus on this core set. Future data-driven phases of the project are planned to validate this core set.

\section{3}

Adalimumab Induced Bullous Pyoderma Gangrenosum and Successful Treatment with Rituximab

Arina Garg (Louisiana State University, Shreveport); Samina Hayat (Louisiana State University, Shreveport)

Introduction: Pyoderma gangrenosum is a condition which causes tissue to become necrotic and is associated with various autoimmune and hematological diseases. There are two forms of pyoderma gangrenosum - typical ulcerative form which occurs in lower extremities, and atypical superficial form which occurs in other parts of the body. Etiology is secondary to immune dysfunction, particularly improper neutrophil functioning. Adalimumab is a fully human polyclonal TNF-alpha antibody which is used in the treatment of various autoimmune diseases, and has been used in the treatment of pyoderma gangrenosum when it is refractory to other medications. However, when adalimumab itself causes pyoderma gangrenosum, the treatment options can be limited.

Case Presentation: A 49 year old African American male with a history of hypertension, gout, obstructive sleep apnea and suspected rheumatoid arthritis presented to the ER with fever, generalized bullous rash and bilateral lower extremity ulceration for two weeks. Patient was being treated for suspected rheumatoid arthritis with prednisone and methotrexate for one year and was recently started on Adalimumab 40mg subcutaneously, in addition to methotrexate. One week after receiving the third dose of Adalimumab, he developed large bullae all over his body, with ulcerative lesions over the legs. On physical exam he was noted to have pustulobullous eruptions over abdomen, lower back, buttocks, and bilateral lower extremities, with large ulcerative lesions over distal aspect of bilateral lower extremities. Some bullous lesions burst draining suppurative grayish discharge. Labs were remarkable for WBC $23.7 \mathrm{~K} / \mathrm{uL}$, Hemoglobin $10.6 \mathrm{G} / \mathrm{dL}$, ESR $81 \mathrm{~mm} / \mathrm{hr}$, CRP $45.10 \mathrm{mg} / \mathrm{dL}, \mathrm{RF}<10$ and $\mathrm{CCP}<0.05$. Antibody to Adalimumab (ATA) levels were $15.7 \mathrm{U} / \mathrm{mL}$ (Ref $<1.7 \mathrm{U} / \mathrm{mL}$ ). PSTPIP1 genetic testing was negative. Skin biopsy from lower extremity showed lesions consistent with leukocytoclastic vasculitis and papillary dermal micro abscesses consistent with bullous variant of pyoderma gangrenosum.

Discussion: Patient was treated with local wound care and intravenous steroids. Rash initially responded to treatment, he was switched to tapering dose of prednisone and underwent surgical debridement and skin grafting on distal lower extremities. While tapering down steroids, his bullous rash recurred and he was started on Rituximab, after failing methotrexate. The rash healed completely after 2 cycles of Rituximab, and his steroids were tapered off.

Conclusion: Adalimumab is widely being used to treat various autoimmune diseases, however it may be associated with autoimmune phenomenon itself, such as bullous pyoderma gangrenosum. Rituximab can be successfully used to treat patients with pyoderma gangrenosum when it is caused by TNF-alpha antibody

\section{4}

A Case of Lupus Pernio Presenting with Upper Airway Obstruction Arina Garg (Louisiana State University, Shreveport); Samina Hayat (Louisiana State University, Shreveport)

Introduction: Lupus pernio, a cutaneous manifestation of sarcoidosis, is a chronic, raised indurated lesion of the skin, mainly distributed on the central areas of the face like alar rim, nasal tip and cheeks. The presence of lupus pernio can be associated with an increased risk for extra-cutaneous disease. In rare cases, it presents as granulomatous inflammation of upper respiratory tract and can result in airway obstruction.

Case Presentation: A 50 year old African American female with a known diagnosis of pulmonary sarcoidosis (not on any therapy) was referred by ENT with hoarseness of voice, dysphagia, shortness of breath and arthralgia. She had a previous CXR that showed hilar lymphadenopathy and had an ACE level $>95$. She had also undergone flexible laryngoscopy with video that showed scar web present between arytenoids extending anteriorly and occluding $>50 \%$ of glottic area. During rheumatology evaluation, she was noted to have a purplish, indurated rash on the nasal tip. The rest of her physical exam was unremarkable. On labs, CRP was $0.39 \mathrm{mg} / \mathrm{dL}$ and PR3-ANCA was negative. Repeat CXR showed residual mediastinal and hilar lymphadenopathy.

Discussion: Patient was diagnosed with lupus pernio with laryngeal involvement, as a manifestation of sarcoidosis, and started on high dose steroid therapy. She then underwent carbon dioxide laser ablation and balloon dilatation of supraglottic airway lesion. She was subsequently started on methotrexate therapy as her steroid was tapered down. With serial dilatations, steroid and methotrexate therapy, there was a marked decrease in the 
size of the glottic lesion on repeat laryngoscopy. The rash on the nasal tip and hoarseness also improved with therapy. She continues to be on methotrexate, while her steroids are being tapered off.

Conclusion: Lupus pernio, a cutaneous manifestation of sarcoidosis, when present may indicate involvement of upper respiratory tract from granulomatous inflammation leading to severe upper airway obstruction. Presence of lupus pernio rash should prompt evaluation for underlying sarcoidosis, and symptoms such as hoarseness and dysphagia should be further investigated. First line treatment is systemic corticosteroids with many requiring additional DMARD therapy. Concomitantly, endoscopic resection with carbon dioxide laser is recommended for high grade obstructing lesions.

155

\section{Restrospective Review of Idiopathic and Isolated Aortitis}

Dahye Hong (University of Alberta, Edmonton); Elaine Yacyshyn (Division of Rheumatology, University of Alberta, Edmonton)

Objectives: Aortitis is the inflammation of the aorta. Various etiologies include infections and inflammatory conditions such as systemic vasculitides and connective tissue diseases. However, aortitis can also be diagnosed in patients without a systemic disease, referred to as 'idiopathic aortitis'. A subset of this is 'isolated aortitis', where abnormalities are limited to the aorta and are absent in aortic branch vessels. Idiopathic aortitis is poorly defined, and no guidelines exist to direct the workup, treatment, and monitoring of these patients; thus there is great case-to-case variability. The objective of this study was to review the charts of aortitis patients to determine demographics, etiology of aortitis, use of systemic treatment, and long-term morbidity and mortality.

Methods: A retrospective review of medical records was conducted for patients who had surgical intervention for aortitis at Edmonton hospitals in 1998-2008. The patient list was generated from the pathology department, and the records were reviewed for patient demographics, clinical manifestations, etiology of aortitis, laboratory investigations and imaging, and surgical interventions. After initial hospital discharge, long-term outcomes were analyzed with outpatient medical records and subsequent inpatient hospital records, documenting any late complications, post-operative treatment, new lesions, and mortality.

Results: 15 patients were identified to have pathologically diagnosed aortitis that was surgically treated in Edmonton. Out of these, 5 had aortitis associated with a systemic condition, and 10 had idiopathic aortitis. 8 of the 10 with idiopathic aortitis had isolated aortitis. Only 40\% (2/5) of patients with systemic conditions were treated with systemic immunosuppression, while none of the idiopathic aortitis patients were treated systemically. On reviewing subsequent follow-up records, $60 \%$ (3/5) of patients with aortitis associated with systemic conditions had developed new vascular lesions on imaging, 20\% (1/5) had vascular complications, and 20\% (1/5) had death related to the aortitis or surgery. In the idiopathic aortitis group, $10 \%(1 / 10)$ had new lesions on subsequent imaging, 50\% (5/10) had complications, and none had death related to aortitis or surgery.

Conclusion: In patients diagnosed with aortitis in Edmonton, there was a significant portion with idiopathic aortitis. Both groups of idiopathic aortitis and aortitis associated with systemic diseases had inconsistent follow-up, and there was a number of patients who developed subsequent complications post-operatively. There appears to be a need for a systematic approach to aortitis. Further data from Calgary and Ottawa will be combined into a 3-centre study, which could potentially be used to develop an approach to aortitis.

\section{6}

\section{Hepatitis B Reactivation in Rheumatologic Patients}

Yassir Daghistani (University of British Columbia, Vancouver); Fergus To (University of British Columbia, Vancouver); Patrick Doyle (University of British Columbia, Vancouver); Hin Ko (University of British Columbia, Vancouver); Mel Krajden (University of British Columbia, Vancouver); Jason Kur (University of British Columbia, Vancouver); Alnoor Ramji (University of British Columbia, Vancouver); Kamran Shojania (University of British Columbia Faculty of Medicine; Arthritis Research Canada, Vancouver); Edward Tam (University of British Columbia, Vancouver); John Wade (University of British Columbia, Vancouver); Eric Yoshida (University of British Columbia, Vancouver); Graham Reid (University of British Columbia, Vancouver); Siegfried Erb (University of British Columbia, Vancouver); Mollie Carruthers (University of British Columbia, Vancouver) Objectives: The widespread usage of biologic disease modifying antirheumatic drugs (bDMARDs) in rheumatology has increased the risk of hepatitis B virus (HBV) re-activation. In two large, cross-sectional studies in rheumatoid arthritis and spondyloarthropathies internationally, the prevalence of chronic HBV infection was estimated at 3.0\% and 3.5\% respectively. $\mathrm{HBV}$ reactivation $(\mathrm{HBVr}$ ) after using immunosuppressive medicines has been observed in $6.8 \%$ of fulminant hepatitis cases in Japan. This study aims to quantify specific knowledge gaps that exist in rheumatologic practices with regards to the management of $\mathrm{HBVr}$ in rheumatologic patients, using a Canada-wide survey.

Methods: We initially conducted a short pilot survey of 15 rheumatologists and two rheumatology fellows at an inter-city rheumatology rounds to assess the discrepancy in practice with regards to dealing with HBV infection in patients started on biologic and non-biologic DMARDs. Subsequently, an expert panel of five hepatologists, two infectious disease specialists and four rheumatologists helped generate a final questionnaire. The final survey assessed knowledge in chronic hepatitis B monitoring and anti-viral prophylaxis with using biologic or non-biologic DMARDs or corticosteroids. A Canada-wide survey was sent to the members of the Canadian Rheumatology Association (CRA). The results were compared to the American Gastroenterology Association (AGA) guidelines.

Results: Seventeen responded to the initial pilot survey. Of the seven questions in the pilot survey, $47 \%$ of questions were either answered incorrectly or marked "I don't know". The final survey was sent to 521 members of the CRA and the response rate was $27.25 \%(\mathrm{n}=142)$. Interestingly, $8.8 \%$ of the responders thought not to refer their HBsAg positive patients to a gastroenterologist, although anti-viral prophylaxis and hepatology monitoring would be indicated. Over $50 \%$ of the respondents would order unnecessary tests such as anti-HBs or anti-HBc for monitoring a $\mathrm{HBsAg}$ positive patient on immunosuppressive therapy. $43 \%$ answered incorrectly to start anti-viral prophylaxis for HBsAg positive patients on DMARDs.

Conclusion: The results of our survey highlight particular knowledge gaps in monitoring and treating chronic hepatitis B in rheumatology patients. All patients with hepatitis B surface antigen positivity on immunosuppression including prednisone should be referred to gastroenterology. Similarly, patients with hepatitis B core antibody positivity on regimens considered to be at an increased risk of viral relapse, regardless of hepatitis surface antibody status, should be monitored regularly and started early on anti-viral prophylaxis.

\section{7}

Addressing Persistent Depressive Symptoms in Rheumatoid Arthritis Patients with Controlled Disease

Gilles Boire (Université de Sherbrooke, Sherbrooke); Patricia Dobkin (McGill University, Montréal); Isabelle Gaboury (Université de Sherbrooke, Sherbrooke); France Gervais (Rimouski); Marie-Claude Beaulieu (Université de Sherbrooke, Sherbrooke); Pierre Dagenais (Department of Medicine, Division of Rheumatology, Centre Hospitalier Universitaire de Sherbrooke, Université de Sherbrooke, Sherbrooke); Pasquale Roberge (Université de Sherbrooke, Sherbrooke); Sophie Roux (Université de Sherbrooke, Sherbrooke)

Objectives: Treat-to-target strategies aiming at remission have improved the outcomes of Rheumatoid Arthritis (RA) patients. The most frequent reason for not reaching remission remains the Patient Global Disease Activity (PGA) score; about a third of current patients are in Quasi-Remission (all components of ACR Boolean remission $\leq 1$ except PGA). PGA strongly correlates with Pain, Fatigue, Functional limitations and anxiety and depressive symptoms. Mindfulness-Based Stress Reduction (MBSR) may be effective to address the full array of RA patients' symptoms.

Personal non-commercial use only. The Journal of Rheumatology Copyright @ 2018. All rights reserved. 
Our objective is to evaluate the impact of MBSR on depressive symptoms, pain, fatigue, PGA and disease activity in RA patients with controlled clinical inflammation.

Methods: Support was obtained from CIORA for a pilot study. Approval was obtained from the CHUS Ethics committee. RA patients (from a pool of $\approx 2000$ followed at the CHUS) seen during regular follow up are evaluated for controlled disease ( $\leq 2 \mathrm{SJC}$ under stable treatment) and for depressive symptoms (Center for Evaluation Studies-Depression (CES-D) $\geq 16$ ). Participation to group MBSR (8 weekly sessions plus an additional day session) aiming at improving depressive symptoms is offered. Consenting patients will be randomized to a control (standard care) or MBSR group. Questionnaires (pain, fatigue, function, coping, anxiety, depression), Simple Disease Activity Index (SDAI), and serum and saliva samples will be collected at baseline and at 6 months. Qualitative analysis of hurdles and facilitators of patient participation is planned at the end of the MBSR session. At 6 months, patients in the control group will be offered the MBSR intervention. Follow-up post-MBSR is planned every 6 months up to 24 months Results: About $15 \%$ of RA patients with $\leq 2$ SJC and stable antirheumatic treatment report a CES-D score $\geq 16$. As expected, the CES-D score correlates with PGA, Pain and Fatigue levels. Recruitment is currently ongoing with a target of 30 patients (15 in each group). Results at 6 months of the first MBSR group and patient feedback on patient recruitment strategies will be available at the time of the annual meeting.

Conclusion: The need for an intervention to address persistent symptoms in RA patients with controlled inflammatory disease is real, although many patients appear reluctant to participate in a time-consuming intervention aimed primarily at improving depressive symptoms. Pending the results of the qualitative analysis, our experience suggests that putting more emphasis on Pain, Fatigue, Anxiety and Quality of life may be more appealing to patients. This may affect the recruitment strategy for the next MBSR groups. Supported by a CIORA grant.

\section{8}

Perceived Barriers and Facilitators of Vaccination Acceptance among Rheumatoid Arthritis Patients from the Perspective of Physician and Nurse Providers

Vincent Boucher (University of Quebec at Montreal, Montreal); Sandra Pelaez (McGill University, Montreal); Kim Lavoie (University of Quebec at Montreal, Montreal); Ines Colmegna (McGill University Hospital Centre, McGill University, Montreal)

Objectives: Despite national guidelines emphasizing the importance of immunization for infection prevention, vaccination rates remain suboptimal among rheumatoid arthritis (RA) patients. We aimed to explore health care professionals' perspectives regarding barriers and enablers of vaccine acceptance among RA patients, which will be targeted in a motivational communication intervention to increase vaccine uptake.

Methods: We conducted 3 focus group (FG) interviews with English-speaking rheumatologists, general practitioners, and nurses affiliated with an academic center. The purpose of the interviews was to ask questions about what they perceived to be the main barriers to and facilitators for vaccination acceptance among RA patients, in order to determine potential targets to develop an intervention. The interviews consisted of questions on vaccine barriers (e.g. "To what extent should discussions with patients about the importance of getting vaccinated for the flu/pneumonia be a priority?") and facilitators (e.g. "What do you believe are the main factors determining your RA patients' willingness to get the flu/pneumonia vaccines?"). Thematic analysis was conducted. All FG interviews were transcribed and uploaded into MAXQDA 12, which was used to code, categorize, compare, and contrast the data with the purpose of developing a conceptual understanding of identified themes related to barriers and facilitators of vaccine acceptance. Results: Eighteen health care professionals participated in the three FGs (nurses $n=5$, rheumatologists $n=7$, general practitioners $n=6$ ). Health care professionals identified barriers and facilitators to vaccination acceptance that were related to one of the following three categories: (1) the patient, (2) the provider, and (3) the healthcare system. The most salient perceived barriers to vaccination acceptance were patients' knowledge and beliefs (e.g. fears of side effects), followed by accessibility (e.g., difficulties accessing the healthcare centre and/or provider), and negative social media messages (e.g., Facebook). health care professionals' level of conviction of the importance of vaccination and conveying this to the patient, as well as taking the time to educate patients regarding vaccination, and listening to and supporting patients' decision-making, were seen as important facilitators of patients' acceptance of vaccination.

Conclusion: Health professionals recognize knowledge, beliefs, accessibility, and social media as main barriers to vaccine acceptance, and education and supporting patient's decision-making as key facilitators of vaccine acceptance. Enhancing patients' and providers' knowledge of the importance of vaccines for RA prevention, and promoting effective provider-patient communication, may help increase vaccine uptake among RA patients. Supported by a CIORA grant.

\section{9}

Systematic Review of Prognosis Studies in Childhood-onset Systemic Autoimmune Rheumatic Diseases in Adulthood

Kaien Gu (University of Manitoba, Winnipeg); Annaliese Tisseverasinghe (University of Manitoba, Winnipeg); Lily Lim (University of Manitoba, Winnipeg)

Objectives: Approximately 70,000 Canadian children live with childhood-onset (onset $<18$ years) systemic autoimmune rheumatic diseases (ChildSARDs), which comprise of childhood-onset systemic lupus erythematosus (SLE), Sjogren's syndrome, idiopathic inflammatory myositis (IIM), systemic sclerosis, and vasculitides. The survival of these patients has tremendously improved such that many now live into adulthood. However, little is known about ChildSARD outcomes or disease course in adulthood. Adult rheumatologists have minimal evidence to guide their management of these patients. We performed a systematic review to evaluate adulthood ( $\geq$ 18 years) outcomes of ChildSARD patients.

Methods: We developed a search strategy with an academic librarian to identify all original full-length English articles in MEDLINE and Embase (January 1990 - May 2017) reporting on any adulthood outcomes of ChildSARDs. Our strategy was iteratively refined and finalized after peer-review by librarians. Hand-searching supplemented the search. We excluded reviews, case reports/series, letters, editorials, and short reports. If a study included both pediatric and adult subjects, we assessed the study only if the median (or mean, assuming no skewed distribution) age at outcome ascertainment was $\geq 18$ years. Study designs, outcomes, and prognostic factors studied were recorded. Longitudinal study design refers to reporting of $\geq 3$ repeated occasions of outcome ascertainment. All studies were graded independently by two reviewers (after training for adequate agreement) using the Quality in Prognosis Studies (QUIPS) riskof-bias tool.

Results: Of 14,100 articles, 28 publications met our inclusion criteria. Only 2 studies (7\%) reported outcomes longitudinally. Only 10 (36\%) studies focused exclusively on adults. Publications are increasing: 4\% (1990-1999), 18\% (2000-2009), and 79\% (2010-2017). Most studies (82\%) were conducted in North America/ Europe. The most commonly studied disease was SLE (61\%). The most commonly reported outcomes were organ damage (29\%), cardiovascular outcomes (14\%), and mortality (14\%). The mean ages at outcome assessment ranged from 20-47 years for adult-only studies and 19-35 years for mixed studies. Moderate to high risk-of-bias was found in $100 \%$ for study participation, $90 \%$ for study attrition, $61 \%$ for prognostic factor measurement, $36 \%$ for outcome measurement, $89 \%$ for confounding, and $54 \%$ for statistical analysis.

Conclusion: There is a clear need for more information about adulthood outcomes of ChildSARDs. Longitudinal trajectory information is especially lacking. We recommend that future studies be undertaken in longitudinal cohorts and to focus on reporting longitudinal trajectory information. Adult outcomes should be separately reported from pediatric outcomes. Study populations should be clearly defined to allow for accurate MESH coding. 


\section{0}

Is Pressure Distribution in the Feet Associated with Imaging Evidence of Disease Activity in Patients with Early Rheumatoid Arthritis? Ashley Bergman (McMaster University, Hamilton); James Tanzil (McMaster University, Hamilton); Hanyan Zou (McMaster University, Hamilton); Monica Maly (McMaster University, Hamilton); George Ioannidis (McMaster University, Hamilton); Saara Totterman (QMetrics Technologies, Rochester); Karen Beattie (McMaster University, Hamilton); Maggie Larche (McMaster University, St Joseph's Healthcare Hamilton, Hamilton)

Objectives: Understanding the relationship between disease activity and pressure distribution in the feet of patients with early rheumatoid arthritis (RA) may improve our understanding of factors contributing to disease progression. We assessed the association between pressure distribution in the feet and imaging outcomes of disease activity in patients with early RA. We hypothesized that those with higher pressure in the forefoot (FF) relative to the hindfoot (HF) would have more evidence of active disease.

Methods: Patients with early RA (treatment naïve, ACR criteria) were enrolled. The most clinically symptomatic foot were scanned using Magnetic Resonance Imaging (MRI) and Ultrasonography (US). The 2nd-5th metatarsophalangeal joints (MTPJ) on MRI were graded according to OMERACT recommendations for synovitis (0-3), erosions (0-10), and bone marrow edema (BME) (0-3) by an MSK-trained radiologist. Using US, the same joints were graded for Power Doppler (PD) $(0-3,3=$ most severe). Total scores for each measure were obtained by summing the grades from each joint. Pressure distribution in the foot was obtained during barefoot standing using a capacitance mat (Matscan, Tekscan, Boston MA). Using TekScan software, peak pressure was identified for the HF and FF. A ratio of HF to FF peak pressure was calculated for each participant to reflect relative pressure distribution. Pearson correlation analyses determined associations between each MRI outcome and pressure distribution. Patients were also grouped according to total PD $(\leq 1$ and $\geq 2)$ score and a t-test examined potential differences in pressure distribution between those with and without active inflammation.

Results: Included in the study were 41 participants ( $n=33$ women), mean (SD) age $=51.9(10.3)$ years. The HF:FF ratio appeared lower in patients with a total $\mathrm{PD}$ score $\geq 2$ than $\mathrm{PD} \leq 1(\mathrm{n}=11$, mean $(\mathrm{SD})=1.4(0.5)$ versus $\mathrm{n}=30$, mean $(\mathrm{SD})=1.9(1.0))$, although the difference was not significant $(\mathrm{p}=0.18)$. This may have been affected by sample size and the relatively large standard deviations around the means. No notable associations were observed between pressure distribution and MRI measures of synovitis, erosions and BME $(\mathrm{p}>0.05)$.

Conclusion: Our data did not demonstrate that those with more active disease had preferential pressure distribution in the forefoot. However, potential differences in pressure distribution between patients with higher and lower PD scores warrant further investigation. The cross-sectional nature of our data do not allow us to determine if people with more active disease compensate by putting more pressure in their heels. Thus, the collection of longitudinal data is warranted.

\section{1}

Pressure Distribution in the Feet of Patients with Early Rheumatoid Arthritis: Is it Related to Clinical Symptoms or Patient-Reported Outcomes?

James Tanzil (McMaster University, Hamilton); Ashley Bergman (McMaster University, Hamilton); Hanyan Zou (McMaster University, Hamilton); Monica Maly (McMaster University, Hamilton); George Ioannidis (McMaster University, Hamilton); Maggie Larche (McMaster University, St Joseph's Healthcare Hamilton, Hamilton); Karen Beattie (McMaster University, Hamilton)

Objectives: Rheumatoid Arthritis (RA) damages the metatarsophalangeal joints (MTPJ) creating chronic pain. Little is known about the ways in which patients with RA distribute their weight across their feet in weight-bearing. Those who put more weight on their forefoot (FF) relative to their hindfoot (HF) may aggravate the disease and experience worse symptoms while others may attempt to compensate for symptoms by distributing more weight in their HF. To address this, we conducted a cross-sectional analysis to examine the associations between pressure distribution in the feet of patients with early RA and a) the Leeds Foot Impact Scale (LFIS) questionnaire and b) swollen and tender joint counts.

Methods: Adults (18-85 years old) with early RA (treatment naïve, ACR criteria) were enrolled. A rheumatologist assessed tenderness and swelling in each MTPJ (2-5) of both feet. Participants completed the LFIS questionnaire where higher scores represent more impairment and activity limitations. Participants stood barefoot on a capacitance mat (MatScan, Tekscan, Boston MA, USA). Pressure $(\mathrm{kPa})$ was recorded in 400 frames over 8 seconds. Pressure distribution throughout the foot was measured using TekScan software. Peak pressures in the HF and FF were identified by taking the average over 400 frames. The ratio of peak pressures in the HF relative to the FF (HF:FF) was calculated to reflect pressure distribution in the foot; a higher ratio reflects a preferential pressure distribution in the HF. Pearson correlation examined associations between clinical and self-reported outcomes and pressure distribution.

Results: Included in the study were 41 participants $(\mathrm{n}=33$ women), mean (SD) age $=51.9(10.3)$ yrs. Associations between HF:FF and the number of swollen joints $(r=-0.144, p=0.37)$ and tender joints $(r=0.258, p=0.10)$ were not significant. However, the association with tender joints approached significance suggesting that patients with more tender joints may place more pressure on their HF relative to their FF. LFIS outcomes were not associated with pressure distribution.

Conclusion: Associations between clinical and self-reported outcomes were not associated with pressure distribution in the feet in this cohort. Interpretation must consider the small sample size and the notion that some patients may have changed pressure distribution to compensate for symptoms while others may not. The potential association between tender joints and pressure distribution warrants further investigation. A longitudinal follow-up to investigate changes in weight distribution may help to resolve this.

\section{2}

Multifocal Central Nervous System Demyelination Secondary to Adalimumab in a Child with Enthesitis Related Arthritis: A Case Report Herman Tam (Department of Pediatrics and Child Health, University of Manitoba, Winnipeg); Mubeen Rafay (Department of Pediatrics and Child Health, University of Manitoba, Winnipeg); Carol Cooke (University of Manitoba, Winnipeg); Kerstin Gerhold (Department of Pediatrics and Child Health, University of Manitoba, Winnipeg)

Background: Tumor necrosis factor-alpha (TNF-alpha) inhibitors are therapeutic agents often used in the treatment of pediatric rheumatologic conditions. Central nervous system (CNS) demyelination is a rare but serious condition that has been seen in association with anti-TNF-alpha therapy but a causal relationship remains controversial. Adalimumab, a human monoclonal antibody against TNF-alpha, has been shown to be effective in children with Enthesitis-related arthritis (ERA). We performed a chart review followed by a literature review for search terms 'Adalimumab' and 'demyelination' and 'child or pediatric or infant' utilizing the databases: PubMed, EMBASE, and Scopus for all years available.

Case: A 15 year old boy with ERA refractory to Naproxen and Methotrexate was switched to Adalimumab subcutaneous injections every two weeks. Four months later, he presented with concerning neurological symptoms including vomiting, diplopia, nystagmus, headaches and confusion. An urgent MRI brain revealed multiple enhancing lesions involving the left parietal subcortical and cortical white matter, bilateral periventricular, and cerebellar regions, consistent with multifocal demyelination. His Adalimumab therapy was discontinued. He was investigated for infectious and post-infections immune mediated etiologies. Cerebral spinous fluid studies showed mildly elevated protein and negative oligoclonal bands. Extensive infectious work-up was negative. He was treated with intravenous pulse methylprednisolone for five consecutive days followed by a 21 day tapering of oral steroids. He had complete resolution of neurological symptoms shortly after treatment and remains asymptomatic to date. Follow-up brain MRI at 2 and

Personal non-commercial use only. The Journal of Rheumatology Copyright @ 2018. All rights reserved. 
10 months off Adalimumab showed improvements in multifocal areas of demyelination with decreased enhancement; but also a new small lesion in the right parietal white matter, which likely developed as a result of earlier Adalimumab therapy.

Discussion: The patient's ERA became active about two months after steroids treatment. He underwent intra-articular corticosteroid injections to his bilateral sacroiliac joints and was started on Indomethacin. Secukinumab, an anti-IL-17 agent, was added 8 months after discontinuation of Adalimumab due to failure of anti-inflammatory treatment.

Conclusion: We report the first demyelination disorder associated with Adalimumab in the pediatric population. Our literature search did not identify any pediatric case with CNS multifocal demyelination secondary to Adalimumab use across all indications. This case highlights a rare, but potentially serious side effect of Adalimumab that warrants careful monitoring. Long-term surveillance of Adalimumab safety profile may further elucidate the associations and mechanisms of such effect.

\section{3}

Update on Temporomandibular Joint Arthritis in Juvenile Idiopathic Arthritis

Ilya Kovalko (Queen's University, Kingston); Peter Stoustrup (University of Aarhus, Aarhus); Susanne Benseler (Section of Rheumatology, Department of Paediatrics, Alberta Children's Hospital/University of Calgary, Calgary); Marinka Twilt (Alberta Children's Hospital, Calgary)

Objectives: Juvenile idiopathic arthritis (JIA) is the most common form of arthritis in the pediatric population. Manifestation of arthritis in the temporomandibular joint (TMJ) presents unique challenges in diagnosis and treatment. This review critically analyzes recent literature, emphasizes new findings, and offers guidance on controversial clinical practices.

Methods: The literature review utilized EMBASE and MEDLINE databases from 2014 to August 2017. The search included English language papers on JIA involving the TMJ in the pediatric population, including historical and synonymous terms. Case studies were excluded.

Results: TMJ arthritis is potentially asymptomatic with long-term functional and morphological consequences. Diagnosis: Magnetic resonance imaging with gadolinium contrast (Gd-MRI) is the current gold-standard for diagnosis. A new TMJ-MRI scoring system has recently been published and includes eight items; bone marrow edema and enhancement; condylar flattening; effusions; erosions; synovial enhancement and thickening; and disk abnormalities. Clinical examination: Clinical orofacial examination for monitoring patients should include specific aspects of medical history, orofacial symptoms, muscle and TMJ function, orofacial function, and dentofacial growth. Each clinical examination should include; palpation of the TMJ and masticatory muscles; measurement of maximal mouth opening, mouth opening deviation, and condylar translation during opening; assessment facial morphology and symmetry. Treatment: Treatment of TMJ arthritis includes local and systemic drug therapy, treatment with orthodontic devices, and potentially maxilla-facial surgery. Intra-articular corticosteroid joint injections (IACI) were used frequently and showed favourable response with relief of symptoms and inflammatory changes. Recently concerning evidence has shown an increase in growth abnormalities in patients treated with IACI and caution is warranted. If TMJ arthritis is present at presentation Disease Modifying antirheumatic drugs (DMARDS) or biologics are indicated. Orthodontic functional appliances are an innovative and promising technique to treat the functional and morphological effects of this disease.

Conclusion: A novel Gd-MRI scoring system for diagnosing TMJ arthritis in JIA patients is available, enabling physicians to compare patients and treatment responses. Clinical examination should be performed in each patient at every visit and should include the functional and facial morphology assessment of the TMJ. Intra-articular injections should be used in certain patients with caution. A combination of systemic medications combined with orthodontic appliances is necessary to treat these patients, warranting a multidisciplinary approach

\section{4}

The Relationship of Serum Amyloid A, C-Reactive Protein, and Disease Activity in Periodic Fever Syndromes

Muhammed Dhalla (University of British Columbia and BC Children's Hospital, Vancouver); Jaime Guzman (University of British Columbia, Vancouver); Kristin Houghton (BC Children's Hospital, Vancouver); David Cabral (Division of Rheumatology, Department of Pediatrics, BC Children's Hospital Research Institute \& University of British Columbia, Vancouver); Kimberly Morishita (BC Children's Hospital, Vancouver); Andrea Human (BC Children's Hospital, Vancouver); Ross Petty (British Columbia Children's Hospital, Vancouver); Kelly Brown (Division of Rheumatology, Department of Pediatrics, BC Children's Hospital Research Institute \& University of British Columbia, Vancouver); Lori Tucker (BC Children's Hospital, Vancouver)

Objectives: Periodic fever syndromes (PFS) are characterized by episodes of intense inflammation, interspersed with periods of wellness. C-reactive protein (CRP) and serum amyloid A (SAA) are acute phase reactants that are used to assess disease activity, and sustained elevation of SAA may predict the development of amyloidosis. Our objective was to examine the relationship between SAA and CRP in children with PFS, and their relationship with disease activity.

Methods: Retrospective chart review of all children with PFS at the BC Children's Hospital pediatric rheumatology clinic, from 2010-2016, who had concurrent collection of SAA and CRP in a clinic visit; results from earliest paired collection were analyzed. Active disease was defined as any typical PFS episode having occurred within 3 months of clinic visit, and disease severity was determined by Physician Global Assessment (PGA). Reference range for SAA was $1.000-5.000 \mathrm{mg} / \mathrm{L}$; values $>16.000$ (the upper limit of measurement) were set to 16.000 . Reference range for CRP was $<5.0 \mathrm{mg} / \mathrm{L}$; values of $<5.0$ (the lowest measurement) were set to 5.0. SAA and CRP comparisons used Pearson correlation, and relationship with disease activity used a simple t-test.

Results: 30 patients $(14 \mathrm{~F}, 16 \mathrm{M})$ aged $1-15$ years (mean $7.3 \mathrm{y})$ were included, with diagnoses as follows: familial Mediterranean fever $(\mathrm{n}=5)$, TNF-receptor associated periodic syndrome (2), Hyper-IgD syndrome (1), cryopyrin-associated periodic syndrome (2), PFAPA (4), and unclassified PFS (16). SAA was significantly elevated in those with active versus inactive disease $($ mean active $=6.704 \mathrm{mg} / \mathrm{L}, \mathrm{SD} 6.65$; inactive $=1.161 \mathrm{mg} / \mathrm{L}, \mathrm{SD}$ $1.25 ; \mathrm{p}=0.05) ; \mathrm{CRP}$ trended towards elevation as well (mean active $=10.6$ $\mathrm{mg} / \mathrm{L}$, inactive $=5 \mathrm{mg} / \mathrm{L}, \mathrm{p}=0.265$ ). Higher levels of disease severity, measured by PGA, were associated with increasing levels of both SAA and CRP. There was a linear relationship between SAA and CRP $(r=0.52, p=$ 0.003 ), though there were several instances where one value was elevated while the other was normal.

Conclusion: In this single-center study of children with PFS, SAA and CRP measures correlate, are increased with active PFS, and are normal with inactive PFS. Interestingly there were several patients in whom either SAA or CRP, but not both, were elevated. Cut-off points for both assays limited the ability to more accurately define the relationship between the tests. Studying larger cohorts is needed to understand the clinical utility of these tests in determining disease states in PFS.

\section{5}

\section{Anti-Mup44 Autoantibodies in Inclusion Body Myositis}

Adam Amlani (Cumming School of Medicine, University of Calgary, Calgary); Mark Tarnopololsky (Pediatrics and Medicine, McMaster University Medical Center, Hamilton); Lauren Brady (Pediatrics and Medicine, McMaster University Medical Center, Hamilton); Marvin Fritzler (University of Calgary, Calgary)

Objectives: Sporadic Inclusion Body Myositis (sIBM) is an insidious onset, idiopathic inflammatory myopathy (IIM) characterized by slowly progressive muscle weakness that can evolve to significant disability. Unlike other IIM, there has not been a reliable biomarker to aid in the diagnosis, hence muscle biopsy and clinical findings remain the diagnostic standard. Autoantibodies to the $44 \mathrm{kDa}$ cytosolic 5'-nucleotidase 1A (cN1A: Mup44) 
have been reported as a potential biomarker for sIBM. The objective of our study was to determine the sensitivity and specificity of anti-Mup44 for sIBM in a cohort of neuromuscular patients.

Methods: Sera were collected from patients and controls and stored at $-80 \mathrm{C}$ until required for analysis. IgG antibodies to Mup44 (NT5C) were detected by an addressable laser bead immunoassay using a recombinant protein (Origene, Rockville, MD) with a cutoff of 400 median fluorescence units. Autoantibodies to other autoimmune inflammatory myopathy targets were detected by ALBIA (Inova Diagnostics, San Diego, CA) and anti-nuclear antibodies (ANA) detected by indirect immunofluorescence (IIF) on HEp-2 substrates (Inova Diagnostics). IIF staining patterns were classified according to the International Consensus on Autoantibody Patterns. Demographic and clinical data was obtained by chart review.

Results: 19/250 patients had sIBM. The remainder were healthy controls $(\mathrm{n}=28$ patients) or had other autoimmune conditions $(\mathrm{n}=97)$, autoimmune myopathies $(n=40)$, statin-related myopathies $(n=4)$, osteoarthritis $(n=$ 47 ), other neuromuscular or metabolic disorders ( $\mathrm{n}=13$ patients), or idiopathic ataxia $(\mathrm{n}=2) .11 / 19(57.9 \%)$ of the sIBM group, were positive for anti-Mup44 (sensitivity 0.58 ). By comparison, 1/19 (5\%) of the sIBM were positive for anti-HMGCR, but were negative for all other myositis-related antibodies (Jo-1, OJ, TIF1y, PL-12, SAE, EJ, MDA5, PL7, SRP, NXP2, MI-2). The frequency of anti-Mup44 in the non-sIBM control group was $12 \%$ (specificity 0.92 ). Of note, $3 / 28(10.7 \%)$ of the apparently healthy controls were positive for Mup44. Review of ANA IIF results for anti-Mup44 positive $(n=18)$ and anti-Mup44 negative sera $(n=31)$ indicated that there was no consistent pattern associated with anti-Mup44 positive sera.

Conclusion: The sensitivity and specificity of anti-Mup44 for IBM was 0.58 and 0.92 , respectively. Therefore, a normal anti-Mup44 test does not rule out SIBM (moderate sensitivity) whilst a positive test may be helpful with the canonical features of sIBM to rule in the disease (high specificity). Anti-Mup44 antibodies were not associated with a specific IIF staining pattern, hence screening using HEp-2 substrate is unlikely to be a useful predictor for the presence of these autoantibodies.

\section{6}

The French-Canadian Translation of the OA Go Away Tool

Lucie Brosseau (University of Ottawa, Ottawa); Karine Toupin-April (Children's Hospital of Eastern Ontario Research Institute, Ottawa); Gail Paterson (The Arthritis Society, Ottawa)

Objectives: The objectives of this study were to create a French-Canadian translation of the OA Go Away tool in order to examine the validity of the content as well as the reliability of test-retest.

Methods: A modified approach of the methodology from trans-cultural validation by Vallerand was adopted. A parallel translation of the OA Go Away tool was carried out initially by professionals and future professionals of rehabilitation. A primary committee of experts (P1) examined the translated version and created a preliminary experimental draft of the OA Go Away tool. This was then evaluated and modified by a second committee of experts (P2). Three patients with osteoarthritis of the knees evaluated the second experimental version and made minor consensual changes. Finally, a linguist examined this copy and an expert carried out a final translation. The principal co-researchers examined the problematic elements of this new version and proposed final modifications. The intra-class correlation coefficient (ICC) and the kappa coefficient have been used to examine the reliability of test-retest of the French-Canadian version of the OA Go Away tool. Results: The users (i.e. individuals with osteoarthritis of the knee $(n=21)$ ) judged all the final comments of the final version of the OA Go Away tool, with the exception of a few expressions for which they had made consensual modifications. The reliability of test-retest was acceptable (Kappa values between $0,236-0,941$ ) for the principal elements of the journal for the OA Go Away tool.

Conclusion: The five rigorous steps of the process permitted the production of a valid French-Canadian version of the OA Go Away tool. The FrenchCanadian version of the OA Go Away tool offers a moderate and reliable test-retest on average for all of its elements. This tool could be pertinent for French Canadian people with osteoarthritis of the hips or knees who engage in physical activity, as well as for healthcare professionals treating these individuals. This innovative tool, favouring the self-management of osteoarthritis, could be of interest to the international Francophone communities.

\section{7}

Comparing Effects of a Bisphosphonate Drug Holiday to Denosumab Treatment on Bone Mineral Density in Postmenopausal Women

Humna Amjad (University of Toronto, Brampton); Leon Li (McMaster University, Mississauga); Andrew Chow (Credit Valley Rheumatology, University of Toronto, Mississauga)

Objectives: There is limited research comparing bone mineral density outcomes during a bisphosphonate drug holiday to that of treatment on other antiresorptives, such as denosumab. The primary objective of this study is to compare bone mineral density outcomes of patients who directly transitioned to denosumab treatment from prior bisphosphonate use to those on a bisphosphonate drug holiday.

Methods: This is a retrospective chart review of patients followed in a community rheumatology clinic in Ontario, Canada. Patients who transitioned directly to denosumab treatment from previous bisphosphonate use and patients who were on a bisphosphonate drug holiday were identified. Baseline fracture risk and demographic information was collected. Information on the bone mineral densities and $\mathrm{T}$-scores for all patients was collected for two time intervals: (1) prior to discontinuing bisphosphonate treatment and (2) either during denosumab treatment or during the bisphosphonate drug holiday, both within a 6-18 month time period after bisphosphonate discontinuation. Bone mineral densities and T-scores were collected for the lumbar spine, femoral neck and total hip locations.

Results: A total of 346 post-menopausal women were eligible for the retrospective review. The denosumab group consisted of 220 patients (average age 77.3 years) and the drug holiday group included 126 patients (average age 70.5 years). There was an increase of 3.9 percentage points in lumbar spine bone mineral density from baseline with denosumab treatment $(95 \%$ $\mathrm{CI}, 2.6$ to 5.1), in comparison to a decrease of 0.3 percentage points for drug holiday patients ( $95 \% \mathrm{CI},-1.3$ to 0.6$)(\mathrm{P}<0.01)$. Denosumab also increased bone mineral density at the femoral neck by 5.3 percentage points $(95 \% \mathrm{CI}$, -3.8 to 14.3 ), in comparison to a decrease of 4.2 percentage points for drug holiday patients $(95 \% \mathrm{CI},-7.8$ to -0.7$)(\mathrm{P}=0.05)$. For total hip, bone mineral density decreased by 0.1 percentage points in patients on denosumab $(95 \%$ CI, -2.2 to 2.1) compared to a decrease of 2.0 percentage points for drug holiday patients $(95 \% \mathrm{CI},-4.1$ to 0.0$)(\mathrm{P}=0.19)$.

Conclusion: Our results demonstrate a significant increase in bone mineral density at the lumbar spine in patients on denosumab treatment in comparison to a drug holiday. There was no significant difference in bone mineral density outcomes for femoral neck and total hip locations between patients on denosumab treatment and those on a bisphosphonate drug holiday. There is need for further longitudinal research with a larger patient population to determine the generalizability of our results.

\section{8}

Treatment Strategies in Pediatric Primary Angiitis of the Central Nervous System: A Systematic Review

Anastasia Dropol (Alberta Children's Hospital, Calgary); Susanne Benseler (Section of Rheumatology, Department of Paediatrics, Alberta Children's Hospital/University of Calgary, Calgary); Brianna Ghali (University of Calgary, Calgary); Marinka Twilt (Alberta Children's Hospital, Calgary)

Objectives: Childhood primary angiitis of the central nervous system (cPACNS) is a devastating neurological disease that requires rapid diagnosis and immediate therapy to halt progression of inflammation. cPACNS affects both large and small arteries, and is divided into large vessel, angiography-positive disease (AP) that may progress over time (APP) or is self-limiting (APNP), and small vessel disease (AN) that is undetectable using conventional angiography, requiring brain biopsy for diagnosis. To date, no standardized treatment protocols exist for cPACNS, and evidence

Personal non-commercial use only. The Journal of Rheumatology Copyright @ 2018. All rights reserved. 
is limited to open-label cohort studies and case reports. The aim of this review was to summarize the literature and provide informed treatment recommendations.

Methods: A systematic review of cPACNS literature from January 2000 to July 2017 was conducted using OVID, MedLine, Pubmed, EmBase, Cochrane Database of Systematic Reviews, Cochrane Central Register of Controlled Trials, Clinicaltrials.gov, Vasculitis Foundation, European Vasculitis Society, CANVASC, Google Scholar and Web of Science. Potentially relevant articles were selected for full-text review using the STROBE checklist for cohort, case control, and cross-sectional studies if they met the following inclusion criteria: 1) reported treatment, 2) addressed pediatrics, 3 ) focused on the disease of interest, 4 ) included $\geq 5$ patients, 5) were original research and 6) were full-length articles. Reviews, expert opinions, editorials, case reports with $<5$ patients, articles lacking treatment information or non-English articles were excluded. A standardized assessment tool measured was used to gauge study quality, and treatment and outcomes were summarized. Based on summary analysis, treatment recommendations for cPACNS subtypes were provided.

Results: Of 2217 articles screened, 110 had fully text retrieval for inclusion criteria and 9 articles were included for detailed analysis. Only 7 studies were deemed high quality. No trials were available therefore no metaanalysis was possible. For APNP-cPACNS, the authors recommend long-term antithrombotic therapy and acute immunosuppression with intravenous (IV) methylprednisolone, followed by a 3-month oral prednisolone taper. APP-cPACNS and AN-cPACNS should be treated with induction therapy consisting of acute IV methylprenisolone followed by 6-12 months of prednisone taper, together with 6-months of IV cyclophosphamide therapy with trimethoprim/sulfamethoxazole. Maintenance therapy should consist of mycophenolate mofetil/mycophenolic acid. Long-term anticoagulation is also recommended in APP-cPACNS.

Conclusion: cPACNS is a life-threatening neurological disease that requires rapid initiation of treatment. This is the first systematic review of treatment literature in cPACNS and observational studies provide evidence for informed recommendations for treatment of cPACNS subtypes.

\section{9}

Celebrating Ability: Structured Art Workshops as a Therapeutic Coping Strategy for Patients with Juvenile Idiopathic Arthritis

Anastasia Dropol (Alberta Children's Hospital, Calgary); Nadia Luca (Section of Rheumatology, Department of Paediatrics, Alberta Children's Hospital/University of Calgary, Calgary); Susanne Benseler (Section of Rheumatology, Department of Paediatrics, Alberta Children's Hospital/University of Calgary, Calgary); Tommy Gerschman (Section of Rheumatology, Department of Paediatrics, Alberta Children's Hospital, Calgary); Nicole Johnson (Section of Rheumatology, Department of Paediatrics, Alberta Children's Hospital/University of Calgary, Calgary); Michael Lang (University of Calgary, Alberta Children's Hospital, Calgary); Jewel Loewen (Alberta Children's Hospital, Calgary); Maggie Mercer (Alberta Children's Hospital, Calgary); Alicia Ponzio (Calgary); Brian Rusted (University of Calgary, Calgary); Heinrike Schmeling (Section of Rheumatology, Department of Paediatrics, Alberta Children's Hospital/University of Calgary, Calgary); Rebeka Stevenson (Section of Rheumatology, Department of Paediatrics, Alberta Children's Hospital, Calgary); Leeanne Stringer (Calgary); Marinka Twilt (Alberta Children's Hospital, Calgary); Dwaraka Veeramreddy (Section of Rheumatology, Department of Paediatrics, Alberta Children's Hospital/University of Calgary, Calgary); Paivi Miettunen (Section of Rheumatology, Department of Paediatrics, Alberta Children's Hospital/University of Calgary, Calgary) Objectives: Juvenile Idiopathic Arthritis (JIA) affects nearly 20,000 Canadian children. Despite success with medications, managing "invisible" symptoms of pain, fatigue and psychosocial limitations remains challenging. Our objectives were to 1) create a Standardized Art Program to assess potential psychosocial benefits in JIA patients and 2) engage the local Hospital, University and general community through art workshops.

Methods: A prospective cohort of children with JIA > 6 years was referred to the Art and Arthritis Workshop from the local pediatric rheumatology clinic. Established artists led full day (7 hour) workshops focusing on children's abilities, using painting, sculpting and journaling techniques. The standardized structure included: a focused workshop theme, technique demonstration, and individual participant guidance. Community engagement involved volunteers from the local University's Faculty of Art, hospital staff, parents/siblings, and a journalist. Pre-workshop psychosocial health was assessed using the Pediatric Quality of Life Inventory Communication and Worry subsections (PedsQL 3.0 Arthritis Module; best score 100, range 0-100). Anonymous evaluations on workshop quality, location, time, and 'favourite part' were collected from participants.

Results: Nine workshops were held from January 2015-May 2017. Thirty-seven children (76\% female) attended at least one workshop (8-12 per session) for a total of 93 participants. Median age and time from diagnosis to first workshop was 11 years (interquartile range $=8-14.5$ ) and 52 months (interquartile range $=13-86.5$ ), respectively. Each subcategory of JIA was represented and to be inclusive, we also included children with other forms of inflammatory arthritis. The mean pre-project PedsQL subscore for Worry was $82 / 100$ (range 8-100) and Communication was $70 / 100$ (range $0-100$ ). A total of 16 parents, siblings, art graduates and hospital staff volunteered for at least one workshop, and the sessions were featured in newspapers and on Youtube. Post-workshop evaluations were provided by 64/93 (69\%) participants: $64(100 \%)$ participants enjoyed the workshops and 63 (98\%) approved the location, 58 (92\%) reported time was convenient, and 61 (95\%) indicated intention to attend a future workshop. As "favorite part", participants responded: 'seeing my painting come to life'; 'learning how artistic I can be'; 'good time for relaxation'; 'the wonderful freedom of [the artwork]' and 'meeting kids my age with arthritis'.

Conclusion: We implemented a structured Art Program for children with JIA that involved artists from the community and volunteers. Limitations in "communication" and "worry" on PedsQL were identified pre-workshop. Art creation may address this through facilitation of non-verbal communication and discovery of new strengths (art skills, peer engagement), while improving community engagement and disease awareness. Supported by a CIORA grant.

\section{0}

Kawasaki Disease in the Neonate: Case Report and Literature Review Fajer Altammar (IWK Health Centre, Halifax); Bianca Lang (IWK Health Centre, Halifax)

Background: Kawasaki Disease (KD) is an acute febrile vasculitic syndrome of early childhood and the leading cause of acquired heart disease in children in the developed world. It is extremely rare in neonates. We present a case of incomplete KD in a neonate and a review of the literature on neonatal KD.

Case Description: A previously healthy full term 15 day old Caucasian male with an unremarkable antenatal and perinatal history, presented on Day 2 of an illness characterized by fever, rash, irritability, and poor feeding. Examination revealed fever (39.6C), tachycardia (HR 180-210), tachypnea (RR 68), extreme irritability, and a generalized maculopapular rash, but was otherwise normal. His complete blood count, CRP and ESR were normal. Empiric intravenous antibiotics and acyclovir resulted in no improvement. On day 4 , he had ongoing fever and developed recurrent apnea, required supplemental oxygen, and was transferred to the pediatric intensive care unit. On day 5 , he developed bilateral non-purulent conjunctivitis, palmar erythema, bilateral non-pitting edema and erythema of his feet, and arthritis. His full septic work-up and viral studies were negative. On Day 6, after 5 consecutive days of fever and meeting 3 out of 5 criteria for KD, with no other obvious diagnosis, he received intravenous immunoglobulin and high dose aspirin for suspected incomplete KD. Over the next 48 hours, his extremity edema resolved, he no longer required supplemental oxygen, and fever did not recur. On day 9 of illness he had marked thrombocytosis and following discharge he developed distal extremity desquamation. Repeated echocardiograms excluded the presence of coronary artery aneurysms (CAA). 
Discussion: We believe this to be a rare case of incomplete KD in a neonate, in which timely IVIG administration led to resolution of the acute illness and may have prevented CAA. A comprehensive English-language medical literature review of KD presenting in the neonatal period, performed by searching the PubMed and Embase databases, revealed only fifteen case reports worldwide. Cases often presented with incomplete KD and unusual laboratory features including a normal CRP during the acute illness that was also seen in outpatient.

Conclusion: This case and our literature review should increase awareness that KD can rarely occur in the first month of life and may present with atypical features. Recognizing $\mathrm{KD}$ in a neonate enables appropriate treatment that can result in resolution of symptoms and may decrease the risk of cardiac complications.

\section{1}

Safety and Effectiveness of Ustekinumab for the Treatment of Psoriatic Arthritis over a 6 Month Period

Regan Arendse (University of Saskatchewan, Saskatoon); Anna Jaroszynska (Oakville); Derek Haaland (Department of Medicine, McMaster University, Hamilton); Pauline Boulos (Dundas); Isabelle Fortin (Centre de Rhumatologie de l'Est du Québec, Université du Québec à Rimouski, Rimouski); Raheem Kherani (University of British Columbia, Richmond); Ariel Masetto (Université de Sherbrooke, Sherbrooke); Jonathan Chan (University of British Columbia, Vancouver); Eliofotisti Psaradellis (JSS Medical Research, Montreal); Melissa Stutz (JSS Medical Research, Montreal); Brendan Osborne (Janssen Inc, Toronto); Francois Nantel (Janssen Inc, Toronto); Allen Lehman (Janssen Inc, Toronto)

Objectives: Ustekinumab (UST) is a fully human immunoglobin monoclonal antibody against interleukin-12 (IL-12) and interleukin-23 (IL-23) that has been proven safe and efficacious in randomized clinical trials for the treatment of PsA. The aim of the current analysis was to assess the safety and effectiveness of UST among PsA patients treated under Canadian routine clinical care

Methods: BioTRAC is an ongoing, prospective registry of patients initiating treatment with infliximab or golimumab for rheumatoid arthritis, ankylosing spondylitis, or PsA, or with UST for PsA. Eligible participants for this analysis included UST-treated PsA patients enrolled between 2014-2016. Clinical outcomes assessed at Baseline and the Month 6 visit were: SJC, TJC, pain (100mm visual analogue scale; VAS), PtGA and MDGA (100mm VAS), PASI, HAQ-DI, DAS-28, and joint counts for dactylitis and enthesitis. Safety was ascertained by the incidence of adverse events (AEs), reported per 100 patient years (PY) of follow-up. Descriptive statistics were produced for all variables, and between-visit changes in outcomes were assessed for statistical significance with the paired-samples t-test.

Results: A total of 63 UST-treated PsA patients were identified. Mean (SD) age and disease duration at Baseline was 52.8 (11.2) and 5.5 (8.1) years, respectively. A majority of patients were female $(63.5 \%)$, and $100.0 \%$ were biologic naïve. Changes from Baseline to Month 6 were statistically significant for all clinical outcomes ( $<<0.05)$, except for the PtGA $(58.2,49.1$; $\mathrm{p}=0.101)$ : SJC-28 (3.8, 1.6; $\mathrm{p}=0.014)$; TJC-28 (6.5, 3.4; $\mathrm{p}<0.001)$; Pain (55.1, 44.6; $\mathrm{p}=0.008) ; \operatorname{MDGA}(51.8,23.4 ; \mathrm{p}<0.001)$; PASI $(4.4,0.7 ; \mathrm{p}<$ $0.001)$; HAQ-DI (1.1, 0.8; $\mathrm{p}=0.019)$; DAS-28 $(4.0,3.5 ; \mathrm{p}=0.026)$. With respect to enthesitis and dactylitis, missing data did not permit assessment of improvement from baseline to Month 6 . At baseline, $50.9 \%$ of patients had some dactylitis and $34.9 \%$ had some enthesitis. Overall, 36 AEs were reported by 21 patients from Baseline to the Month 6 visit. The majority were mild in nature ( $n=26 / 36 ; 72.2 \%)$; "probable" and "very likely" relation to the study drug was determined for $11.1 \%(n=4 / 36)$ and $8.3 \%(n=3 / 36)$ of AEs, respectively. The most common AEs included General Disorders and Administration Site Conditions $(\mathrm{n}=7 / 21 ; 33.3 \%)$ and Infections and Infestations $(n=8 / 21 ; 38.1 \%)$. No serious AEs were reported.

Conclusion: The results of this analysis demonstrate that UST, administered to PsA patients in a routine clinical care setting, is a safe and effective treatment in improving clinical outcomes over 6 months of treatment.

\section{2}

Clinical Response to the First Biologic in Rheumatoid Arthritis Patients with Moderate Disease in a Real World Clinical Cohort

Xiuying Li (University Health Network, Toronto); Angela Cesta (University Health Network, Toronto); Mohammad Movahedi (University Health Network, Toronto); Claire Bombardier (Ontario Best Practices Research Institute, University of Toronto, Toronto General Research Institute, Toronto) Objectives: While most randomized trials assess the effectiveness of biologic DMARDs (bDMARDs) in rheumatoid arthritis (RA) patients with high disease activity, in the real world or routine care, patients with moderate disease activity are often treated with bDMARDs as well. This study aims to evaluate the effectiveness of the first biologic with or without conventional synthetic DMARD (csDMARDs) in patients with moderate disease activity. Methods: Biologic naïve patients enrolled in the Ontario Best Practices Initiative (OBRI), with moderate disease activity score (DAS28: > 3.2-5.1), or high disease activity score (DAS28: > 5.1) were included. Patients were required to remain on the biologic for 6 months and to have complete follow up data during this time period. Clinical response to their first biologic was measured by the change in DAS28 and by the proportion of patients who reached low disease activity (LDA) during the first 6 months of treatment. The change in DAS28 was assessed using linear regression modelling, adjusted for potential confounders (age, gender, disease duration, and physician global assessment). Multivariate logistic regression was used to compare the proportion of patients who reached LDA, adjusting for the same potential confounders.

Results: 443 patients were included. At initiation of their first bDMARD, 238 patients had a moderate DAS28 and 205 had a high DAS28. The two groups were similar with respect to age, gender, and disease duration. The mean (SD) DAS28 score was significantly lower for the moderate disease group compared to the high disease group (4.18 (0.54) vs 5.98 (0.63), p < $0.0001)$. There was a significant difference between the two groups in all DAS28 components, as well as the mean (SD) physician global (4.6 (2.0) vs $6.3(1.8), \mathrm{p}<0.0001)$. A significant change in DAS28 was found in both the moderate $[-0.89(95 \% \mathrm{CI}-1.12,-0.66)]$ and high $[-1.86$ (95\% CI -2.10 , -1.62)] disease groups, with greater improvement seen in the high disease group. A comparison of the change in DAS28 between the two groups was also significant $(0.97 \pm 0.16, p<0.0001)$. After 6 months of biologic treatment, a higher proportion of patients in the moderate group reached LDA, when compared to the high group (OR: $1.65 ; 95 \%$ CI: $1.03-2.65, p=$ $0.04)$.

Conclusion: Treatment with bDMARDs is effective in patients with moderate disease activity. While patients with high disease activity showed greater improvement after 6 months of biologic treatment, patients with moderate disease activity at initiation of a bDMARD were more likely to reach a LDA state.

\section{3}

Time to Discontinuation of Biologic Therapy by Mechanism of Action in Rheumatoid Arthritis: Results from the Ontario Best Practice Research Initiative (OBRI) Cohort

Mohammad Movahedi (University Health Network, Toronto); Sandra Couto (University Health Network, Toronto); Angela Cesta (University Health Network, Toronto); Claire Bombardier (Ontario Best Practices Research Institute, University of Toronto, Toronto General Research Institute, Toronto); OBRI Investigators (University Health Network, Toronto)

Objectives: Patients with rheumatoid arthritis (RA) may discontinue their biologic disease modifying antirheumatic drug (bDMARDs) due to non-response, loss of response or adverse events. However, time to discontinuation may be related to mechanism of action. We aimed to compare drug survival of tumor necrosis factor inhibitors (TNFi) versus non-TNFi in patients initiating bDMARD treatment in a Canadian (Ontario) observational cohort.

Methods: Patients enrolled in the Ontario Best Practice Research Initiative (OBRI) who started bDMARD therapy within 30 days before or any time after OBRI enrolment were included in the primary analysis. Patients were

Personal non-commercial use only. The Journal of Rheumatology Copyright @ 2018. All rights reserved. 
followed from bDMARD start until discontinuation/switching, death, lost to follow-up, or last visit, whichever came first. Time to discontinuation/switching of bDMARD due to (i) any reason, (ii) non-response, and (iii) adverse events (AEs), were assessed using Kaplan-Meier survival analysis for TNFi versus non-TNFi users. In the secondary analysis we investigated time to bDMARD discontinuation/switching in patients with no exposure to bDMARDs prior to OBRI enrolment (biologic naïve).

Results: Among the 962 patients included in the primary analysis, 174 (18.0\%) received non-TNFi and $788(82.0 \%)$ TNFi. Mean (SD) age and disease duration were $55.6(12.7)$ years and 8.8 (9.8) years, respectively, and the majority were females $(79.4 \%)$ and biologic naïve $(81.8 \%)$. TNFi included Etanercept, Adalimumab, Certolizumab, Golimumab, and Infliximab (Inflectra); and non-TNFi included Abatacept, Rituximab, and Tocilizumab. Over a mean (SD) follow-up of 2.0 (1.9) years, bDMARD discontinuation/switching was reported for $39.2 \%$ of patients, with not significant difference between TNFi and non-TNFi users (Logrank $\mathrm{p}=0.09$ ). There was also no significant difference due to non-response (Logrank $\mathrm{p}=$ 0.82 ) or adverse events (Logrank $\mathrm{p}=0.15$ ) between the two groups. At 2 years, more patients remained on TNFi $(64.0 \%)$ compared to non-TNFi $(55.0 \%)$. At 5 years, $50 \%$ and $43 \%$ of patients remained on TNFi and non-TNFi, respectively. A significant difference (Logrank $\mathrm{p}=0.01$ ) was found when the analysis was restricted to biologic naïve patients, with those receiving TNFi being more likely to remain on their medication.

Conclusion: Overall retention rate for biologics was comparable to findings in European registries. Similar to some studies, we found that patients stay on TNFi longer compared to non-TNFi, particularly in biologic naïve patients. However, there was no significant difference found between the two groups, for discontinuation or switching of bDMARDs due to non-response or adverse events. Further analyses are required to adjust for the effect of potential confounders (e.g. age, sex, disease activity, and other treatment regimens) on biologic discontinuation.

\section{4}

Incidence of Infusion Reactions to Intravenous Golimumab: Results from a Prospective, Real-World Community Registry

Rafat Faraawi (McMaster University, Kitchener-Waterloo); Andrew Chow (Department of Medicine, University of Toronto, Toronto); Majed Khraishi (Department of Medicine, Memorial University of Newfoundland, St. John's); Derek Haaland (Department of Medicine, McMaster University, Hamilton); Milton Baker (University of Victoria, Victoria); Cathy Tkaczyk (Janssen Inc, Toronto); Allen Lehman (Janssen Inc, Toronto); Francois Nantel (Janssen Inc, Toronto); Brendan Osborne (Janssen Inc, Toronto)

Objectives: Golimumab (GLM) is a monoclonal antibody targeting TNF-alpha, indicated for the treatment of adults with RA in combination with methotrexate (MTX). GLM-IV is recommended to be administered at a dose of $2 \mathrm{mg} / \mathrm{kg}$ given as a 30-minute intravenous (IV) infusion at weeks 0,4 and every 8 weeks thereafter. In two separate trials, GO-LIVE and GO-FURTHER, infusion reactions (IRs) were observed in a relatively small group of GLM-treated patients with $2.2 \%$ and $3.3 \%$ of patients having documented IRs, respectively. The GO-IV registry was initiated to evaluate the incidence and management of IRs with GLM-IV in a real-world Canadian practice setting.

Methods: GO-IV was a prospective, observational, non-interventional, multicentre study conducted at 11 Canadian sites from 2014-2016. GLM infusions were followed to document IRs and their management, pre-medication uses and adverse events (AEs). An IR was defined as any AE occurring during the infusion or within 1 hour post-infusion. Patients had to be $>18$ years of age, a confirmed diagnosis of RA, provide written consent for data collection, and be naïve to GLM (both subcutaneous and intravenous formulations).

Results: The study was terminated early due to lack of public listing for the drug. At that time, a total of 79 patients were enrolled and 62 of them were still ongoing. Reasons for premature discontinuation included AEs (7), lack of response (4), geographic issues (3), mis-diagnosis, switch to subcutaneous GLM or withdrawal of consent (one each). A total of 77 patients were included in the primary analysis and 78 in the safety analysis. Only 4 patients (5.1\%) documented an IR over 483 infusion visits (0.8\%), none of which classified as serious or leading to discontinuation. Three of those IRs occurred at the first infusion and one at infusion number three. Infusion-related AEs included palpitations, nausea, fatigue, infusion site pain, dizziness and headache (one each). The impact of pre-medication could not be established since only four infusions were pre-medicated with diphenhydramine and one with steroids. A total of 164 AEs was reported in 45 patients $(57.7 \%)$; 2 patients $(2.6 \%)$ reported a serious adverse event (acute myocardial infarction; multiple fractures, pneumothorax, concussion, traumatic haematoma and pneumothorax resulting from a fall). There was one incidence of a lipoma and no death. There were 30 infectious AEs reported in 24 patients.

Conclusion: The GO-IV registry shows that, in community-based infusion clinics, infusion reactions to GLM are uncommon and predominantly mild in nature.

\section{5}

Availability of Clinical Measures for Patients with Rheumatoid Arthritis in Integrated Delivery Networks who Receive a Biologic or Targeted Synthetic Disease-Modifying Antirheumatic Drug: A Real-World Analysis of an Electronic Health Records Database

Benjamin Chastek (Optum, Eden Prairie); Chieh-I Chen (Regeneron Pharmaceuticals, Inc, Tarrytown); Toshio Kimura (Regeneron Pharmaceuticals, Inc., Tarrytown); Jonathan Fay (Regeneron Pharmaceuticals Inc., Tarrytown); Stephanie Korrer (Optum, Eden Prairie); Stefano Fiore (Sanofi Genzyme, Bridgewater)

Objectives: This study examined the availability of clinical measures across IDNs among patients with RA who received a biologic or a targeted synthetic disease-modifying antirheumatic drug (tsDMARD).

Methods: In this retrospective analysis of the Optum One electronic health records (EHR) database, patients were 18 years or older, had rheumatoid arthritis (RA) diagnoses $\geq 7$ days apart between June 30, 2008 and July 31, 2015. For patients who switched from a tumor necrosis factor inhibitor (TNFi) to a different medication, the index date was the switch date. Among other patients with a prescription for a biologic or tsDMARD, the index date was the first prescription written. Analysis periods were "baseline" (1 year pre-index), or "follow-up" (1 year post-index). EHR reporting rates were determined for quantitative measures to guide treatment decisions: clinical measurements (height, weight, blood pressure, cholesterol, erythrocyte sedimentation rate [ESR], C-reactive protein [CRP], or tuberculosis [TB] test) and validated disease severity instruments (Routine Assessment of Patient Index Data [RAPID3], Disease Activity Score [DAS28], or Clinical Disease Activity Index [CDAI]). Mean ESR and CRP in follow-up were summarized.

Results: The 29,829 patients were $76.8 \%$ female, $80.1 \%$ age $\geq 45$ years, and 83.8\% Caucasian. Baseline TB reporting rate in EHRs was $18.8 \%$. EHR reporting rates in follow-up (median, 6 office visits) were: $49.0 \%$ ESR or CRP; and 6.5\% $\geq 1$ disease severity measure (5.8\% RAPID3, $0.2 \%$ DAS28, $0.6 \% \mathrm{CDAI}$ ). Data reporting in EHRs varied significantly across the 10 largest IDNs, including a range of $0.0 \%$ to $25.2 \%$ for disease severity measure reporting in follow-up. Mean \pm SD values for ESR and CRP in follow-up were $13.9 \pm 8.0 \mathrm{~mm} / \mathrm{hr}$ and $5.7 \pm 4.2 \mathrm{pcg} / \mathrm{mL}$, respectively; variations in ESR and CRP values were less pronounced than variations in EHR reporting rates.

Conclusion: In this analysis of EHR reporting of clinical data among RA patients in IDNs who switched from a TNFi or received a biologic or tsDMARD, approximately 1 in 5 had a TB test reported pre-index and half had ESR and/or CRP reported post-index. Validated measures of disease severity (RAPID3, DAS28, or CDAI) were reported infrequently, and EHR reporting was highly variable across IDNs. More consistent and more complete EHR reporting is recommended to assist rheumatologists in tracking whether treatment targets for RA are being met with biologic or tsDMARD therapy appropriately. 
176

Efficacy and Safety of Switching from Adalimumab to Sarilumab in an Open-label Extension of a Phase 3 Monotherapy Trial in Patients with Active Rheumatoid Arthritis

Gerd Burmester (Charité - University Medicine Berlin, Free University and Humboldt University Berlin, Berlin); Stefano Fiore (Sanofi Genzyme, Bridgewater); Chih-Chi Hu (Sanofi Genzyme, Bridgewater); Jonathan Fay (Regeneron Pharmaceuticals Inc., Tarrytown); Eun-Bong Lee (Seoul National University College of Medicine, Seoul); Mark Genovese (Stanford University Medical Center, Palo Alto)

Objectives: Sarilumab is a human mAb blocking the IL-6R-alpha. Efficacy and safety of sarilumab as monotherapy and combination therapy have been reported.1-3 In MONARCH (NCT02332590), adults intolerant of, inappropriate for, or inadequate responders to methotrexate received subcutaneous sarilumab (200 mg every 2 weeks [q2w]) or adalimumab (40 mg q2w) monotherapy for 24 weeks. 2 Sarilumab monotherapy demonstrated superiority to adalimumab monotherapy in reducing disease activity and improving physical function in patients with active RA.2 Safety profiles of both therapies were consistent with published data. Patients in MONARCH who completed the initial double-blind phase could continue in the open-label extension (OLE) in which all patients received sarilumab $200 \mathrm{mg}$ q2w monotherapy.

Methods: Disease activity, physical function, and safety were assessed regularly. Data were used as observed.

Results: A total of 321 patients completed MONARCH, 320 of whom entered the OLE; 155 switched from adalimumab to sarilumab (switch group), and 165 remained on sarilumab (continuation group). At OLE entry, mean DAS28-ESR was 4.46 and DAS28-ESR $\leq 3.2$ was $16.1 \%$ in the switch group vs 3.45 and $47.9 \%$, respectively, in the continuation group $(\mathrm{P}<0.0001$ for both endpoints). By Week 24 of the OLE, the proportion of patients in the switch and continuation groups who achieved DAS28-ESR $\leq 3.2$ was $49.7 \%$ vs $58.8 \%(\mathrm{P}=0.1033)$, DAS28-ESR $<2.6$ was $40.0 \%$ and $42.4 \%$ $(\mathrm{P}=0.6586)$, CDAI remission was $12.3 \%$ and $18.8 \%(\mathrm{P}=0.1054)$, and improvement in HAQ-DI of $\geq 0.3$ was $63.9 \%$ and $66.7 \%(P=0.6004)$, respectively. At Week 24 of the OLE, treatment-emergent adverse events (TEAEs; $63.9 \%$ vs $57.9 \%$ ), serious TEAEs $(9.0 \%$ vs $1.2 \%$ ), infections (34.2\% vs $23.6 \%)$, and serious infections ( $1.9 \%$ vs $0 \%)$ were observed in the switch and continuation groups, respectively, with 1 death in the switch group (malignancy) and no deaths in the continuation group. Discontinuations due to TEAEs occurred in $5.8 \%$ of patients in the switch group and $3.6 \%$ in the continuation group.

Conclusion: In the OLE of a randomized clinical trial, patients switching from adalimumab $40 \mathrm{mg}$ monotherapy to open-label sarilumab $200 \mathrm{mg} \mathrm{q} 2 \mathrm{w}$ monotherapy demonstrated improvements in physical function and in the signs and symptoms of RA, which became numerically similar to patients who were initially randomized to sarilumab $200 \mathrm{mg}$ q2w. Safety observations in the OLE were generally consistent with what was observed in the randomized portion of the study. References 1 . Genovese et al. Arthritis Rheumatol. 2015;67:1424-1437. 2. Burmester et al. Ann Rheum Dis. 2017;76:840-847. 3. Fleischmann et al. Arthritis Rheumatol. 2017;69:277-290.

\section{7}

The Impact of Disease Characteristics on Habitual Physical Activity and Sedentary Behaviour among Patients with Systemic Lupus Erythematosus Alexandra Legge (Dalhousie University, Halifax); Chris Blanchard (Dalhousie University, Halifax); John Hanly (Dalhousie University and Nova Scotia Health Authority, Halifax)

Objectives: Patients with systemic lupus erythematosus (SLE) are at increased risk for atherosclerotic cardiovascular disease (ASCVD). As sedentary behaviour and lack of physical activity are known ASCVD risk factors, we evaluated habitual physical activity levels among SLE patients using accelerometry. We also investigated the association between SLE disease characteristics and the amount of habitual physical activity and sedentary behaviour performed by SLE patients.

Methods: For this cross-sectional study, patients were recruited from an
SLE clinic at a single academic medical center. All participants met the ACR classification criteria for SLE. Validated instruments were used to measure disease activity (SLEDAI-2K), organ damage (SLICC/ACR Damage Index [SDI]), and functional status (HAQ). Laboratory data included ESR and serum CRP. Habitual physical activity was measured using triaxial accelerometers worn during waking hours for seven consecutive days. Minutes per day of sedentary time, light activity, and moderate-vigorous physical activity (MVPA) were recorded. SLE disease characteristics associated with time spent performing MVPA and time spent in sedentary behaviour were identified using multivariable linear regression, adjusting for demographic factors including age, sex, race, and education.

Results: There were 109 SLE patients (92\% female) with mean (SD) age 52.3 (14.5) years. High levels of sedentary behaviour were observed [mean (SD) sedentary time 10.1 (1.2) hours/day], accounting for $78.7 \%$ of total accelerometer wear time. Mean (SD) MVPA was low [32.6 (22.5) minutes/day]. Only 13/109 participants (11.9\%) met the Canadian Physical Activity Guidelines for MVPA ( $\geq 150$ minutes/week in 10 minute bouts). In univariable linear regression, SLE disease characteristics significantly associated with MVPA performance included the SDI (beta $=-0.187 ; \mathrm{p}=$ 0.005 ), HAQ (beta $=-0.207 ; \mathrm{p}=0.002$ ), SLEDAI-2K (beta $=-0.179 ; \mathrm{p}=$ 0.006 ), and ESR (beta $=-0.208 ; p=0.004$ ). In multivariable analysis, both the HAQ (beta $=-0.191 ; \mathrm{p}=0.003$ ) and SLEDAI-2K (beta $=-0.171 ; \mathrm{p}=$ 0.006 ) remained significantly associated with time spent performing MVPA. Specifically, higher HAQ and SLEDAI-2K scores were associated with lower MVPA performance. After adjustment for MVPA performance, none of the SLE disease characteristics we evaluated were significantly associated with time spent in sedentary behaviour.

Conclusion: SLE patients demonstrate suboptimal levels of habitual MVPA, as well as high sedentary behaviour. Our results highlight the potential negative impact of disease activity and functional disability on habitual physical activity in SLE. The findings identify a subgroup of SLE patients at increased risk for physical inactivity and will help inform the design of effective interventions to improve habitual physical activity levels in this population.

\section{8}

Habitual Physical Activity, Sedentary Behaviour and Cardiovascular Disease Risk Burden in Systemic Lupus Erythematous (SLE)

Alexandra Legge (Dalhousie University, Halifax); Chris Blanchard (Dalhousie University, Halifax); John Hanly (Dalhousie University and Nova Scotia Health Authority, Halifax)

Objectives: Patients with SLE are at increased risk for atherosclerotic cardiovascular disease (ASCVD). As sedentary behaviour and lack of physical activity (PA) are known ASCVD risk factors in the general population, we investigated the relationship between habitual PA levels and estimated 10-year ASCVD risk among patients with SLE. We also evaluated the association between habitual PA levels and individual ASCVD risk factors in SLE.

Methods: For this cross-sectional study, patients were recruited from an SLE clinic at a single academic medical center. All participants met the ACR classification criteria for SLE. Habitual PA was measured using triaxial accelerometers worn for seven consecutive days. Minutes per day of total sedentary time, prolonged sedentary bouts ( $\geq 30$ uninterrupted sedentary minutes), light activity, and moderate-vigorous physical activity (MVPA) were recorded. ASCVD risk factors included body mass index, blood pressure, fasting glucose, and lipid profile. Ten-year ASCVD risk was calculated using the 2013 American College of Cardiology (ACC) / American Heart Association (AHA) risk assessment tool. Associations between time spent in each PA category and calculated 10-year ASCVD risk were assessed using multivariable linear regression models. The relationships between PA at each intensity level and individual cardiovascular risk factors were also evaluated using multivariable linear regression, adjusting for age and gender. Results: There were 100 SLE patients (mean \pm SD age $52.4 \pm 14.4$ years; $92 \%$ female). On average, participants spent 10.0 hours/day being sedentary, 2.2 hours/day engaged in light activity, and 33.2 minutes/day engaged in

Personal non-commercial use only. The Journal of Rheumatology Copyright (c) 2018. All rights reserved. 
MVPA. Median (IQR) time spent in prolonged sedentary bouts was 35.4 (36.3) minutes/day. Only $11 \%$ of participants met the Canadian Physical Activity Guidelines for MVPA ( $\geq 150$ minutes/week in 10 minute bouts). Regression models demonstrated that time spent in MVPA was inversely associated with calculated 10-year ASCVD risk $(\mathrm{R} 2$ delta $=0.07 ; \mathrm{p}=0.008)$. Time spent in prolonged sedentary bouts was positively associated with 10 -year risk of ASCVD (R2delta $=0.06 ; p=0.015)$, independent of time spent in MVPA (R2 delta $=0.04 ; p=0.042)$. MVPA performance was also inversely associated with both systolic $(\mathrm{R} 2$ delta $=0.07 ; \mathrm{p}=0.005)$ and diastolic blood pressure ( $\mathrm{R} 2$ delta $=0.09 ; \mathrm{p}=0.003)$, after adjusting for age, gender, and antihypertensive use.

Conclusion: Among patients with SLE, those at higher risk for ASCVD perform less MVPA. Prolonged sedentary behaviour is associated with ASCVD risk in SLE, independent of MVPA performance. Our findings highlight the need for effective PA interventions to increase habitual MVPA levels and reduce prolonged sedentary time in this high-risk population.

\section{9}

Prospective Observational Study to Evaluate the Use of Musculoskeletal Ultrasonography to Improve Rheumatoid Arthritis Management: The Echo Study

Michael Stein (McGill Rheumatology, Royal Victoria Hospital, Montreal); Emmanouil Rampakakis (JSS Medical Research, Montreal); John Sampalis (JSS Medical Research, Montreal)

Objectives: Musculoskeletal Ultrasound (MSUS) has been shown to be superior to clinical examination in the detection of synovitis in patients with Rheumatoid Arthritis (RA), and can be used to improve diagnostic accuracy [1] and potentially monitor disease changes in order to make treatmen decisions aimed at optimizing patient care. Since the creation of the Canadian Rheumatology Ultrasonography Society (CRUS) in 2010, an increasing number of rheumatologists has been trained in the use of MSUS. The overall study objective is to compare the effectiveness of MSUS to Routine Care (RC) as a disease management tool in patients with moderate-to-severe RA for whom a change in treatment is indicated. The current analysis also assessed the predictive power of MSUS assessments. Methods: Descriptive interim analysis of the 'Echo' study, a prospective two-cohort, quasi-experimental study of patients diagnosed with active moderate-to-severe RA managed either with MSUS (within CRUS) or as per RC. In order to be eligible for the study patients must require a change in treatment as per the judgment of the treating physician. Patients are followed for 1 year with assessments at baseline, 3, 6, 9, and 12 months. Key outcome measures of interest include CDAI LDA/Remission, DAS-28 LDA/Remission, patient satisfaction and patient perception of participation in disease management.

Results: A total of 383 patients ( $71.5 \%$ female) with a mean (SD) age of 58.7 (11.7) years and disease duration of 7.0 (10.0) years were enrolled, without any significant differences between treatment groups. There were no differences between the two treatment groups with respect to the study outcomes. However, higher total US erosion score at baseline was associated with a lower rate of CDAI LDA at 12 months $(\mathrm{OR}=0.86 ; \mathrm{P}=0.047)$; higher total PD synovitis score at baseline was associated with a lower rate of CDAI LDA at 6 months ( $\mathrm{OR}=0.90 ; \mathrm{P}=0.010)$, and; higher total synovitis GREY scale at baseline was associated with lower rates of DAS28 LDA $(\mathrm{OR}=0.93$; $\mathrm{P}=0.026)$ and $\mathrm{DAS} 28$ remission $(\mathrm{OR}=0.94 ; \mathrm{P}=0.061)$ at 6 months.

Conclusion: MSUS assessments can be useful predictors of future disease remission in patients with RA. The impact of MSUS on disease management and clinical outcomes requires further study. Reference: 1. Colebatch AN et al. Ann Rheum Dis 2013, 72:804-814.

\section{0}

Teaching Musculoskeletal Ultrasound to Future Rheumatologists Shirley Lake (University of Toronto, Toronto); Pooneh Akhavan (Division of Rheumatology, Mount Sinai Hospital, Toronto); Maria Bagovich (University of Toronto, Toronto); Lihi Eder (Women's College Hospital, Toronto); Abe Chaiton (University of Toronto, Toronto)
Objectives: In Canada, Point-of-care musculoskeletal ultrasound (MSUS) has been inconsistently incorporated in rheumatology training programs and its value has not been established. The objective of our study is to describe a rheumatology residency MSUS course, the knowledge of MSUS skills obtained, and the views of the rheumatology residents regarding the MSUS course and MSUS in general.

Methods: Four half-day workshops were developed for PGY4 and 5 rheumatology residency program between October 2016 - June 2017. Each session, the residents spent 1 hour reviewing the anatomy of a specific joint area in the anatomy lab, a 45 minute didactic tutorial given by a rheumatologists with MSUS expertise, and then a 2 hour hands-on ultrasound practise with normal and volunteer patients with rheumatic diseases. Before the course, the residents completed a written multiple-choice test related to ultrasound image optimization and a survey to assess their previous MSUS experience and views regarding MSUS. The participants were asked to rate their agreement with statements on a scale of 1 (strongly disagree) to 5 (strongly agree). After the course, the same survey and written test were administered to evaluate the efficacy of the workshops. Descriptive statistics was calculated with median (range) for continuous variables and percentage for categorical variables

Results: 5 PGY5 and 3 PGY4 rheumatology residents participated in the pre-test survey, $75 \%$ female. $37.5 \%$ had done an MSUS course before Feedback from the workshop was positive, median rating 4.5/5 (range 3-5). Residents became more familiar with MSUS (14\% pre-course to $57 \%$ post-course) and their knowledge of MSUS improved. Median written test score was 4/10 (range 0-8) pre-course and 6/10 (range 3-8) post-course. Interestingly, residents felt that their physical exam technique was less adequate to diagnose pathology ( $62 \%$ pre-course and $43 \%$ post-course) and they were less confident in their placement of joint injections (75\% to 29\%). At the end of the course, residents cited how helpful the sessions were to teach anatomy and how they wanted more anatomy and ultrasound sessions. $86 \%$ of residents wanted to obtain more training in MSUS, 57\% felt they would use MSUS in future if they were more skilled, and $43 \%$ felt all rheumatologists should be familiar with MSUS.

Conclusion: Residents enjoyed the MSUS course, improved their knowledge, and wanted more MSUS training. More study is needed to understand the biggest impediments of continued MSUS usage, whether trainees pursued more training, and how many are using MSUS.

\section{1}

The Frequency and Cost of Repeat ANA Testing at Two University of Toronto-affiliated Teaching Hospitals

Shirley Lake (University of Toronto, Toronto); Natasha Gakhal (Women's College Hospital, Toronto); Amanda Steiman (Mount Sinai Hospital, Toronto); Ivan Blasutig (Children's Hospital of Eastern Ontario, Ottawa); Jessica Widdifield (Sunnybrook Research Institute, Toronto)

Objectives: Duplicate ordering of antinuclear antibody (ANA) testing can be unnecessary, potentially harmful, and costly. In 2014 , more than 275,000 ANA tests were performed in Ontario at a cost of $\$ 6,283,750$ annually ( $\$ 22.85$ per test). The number of tests ordered greatly exceeds the number of incident cases of systemic autoimmune rheumatic diseases. The goal of this study was to calculate the frequency and estimated costs of repeat ANA testing among rheumatologists at two University of Toronto-affiliated Teaching Hospitals

Methods: We evaluated ANA test-ordering practices at two University of Toronto-affiliated teaching hospitals (University Health Network and Mount Sinai Hospital) using the Lab Information System (LIS) over the period of January 2010 to February 2015. We identified all ANA tests performed (by indirect fluorescent antibody or ANA screening panel), as well as repeat testing for individual patients within 11 months of a previous test. We estimated the costs related to repeat testing, and assessed variation in repeat testing by rheumatologist. Unknown rheumatologist identifiers and rheumatologists with $<5$ tests were categorized together.

Results: We identified 14,966 ANA tests among 6,234 unique patients (25\% were male). In total, we identified 2,169 (14.5\%) repeat ANA tests within 
11 months of a prior test. Extrapolating the data, the total estimated cost of repeat testing was equivalent to $\$ 49,561.65$ over the five-year period (approximately $\$ 10,000$ per year in potentially unnecessary costs). Among 18 unique rheumatologists, repeat testing of patients ranged from $4 \%$ to $25 \%$. Conclusion: This study provides the first analysis of the frequency of repeated ANA testing by rheumatologists in Ontario. We observed an excess of $14.5 \%$ in repeat ANA testing and variation in repeat testing practices by rheumatologists. While we were unable to determine the clinical reason to support repeat testing, these findings provide insights and allow us to develop a rational and fiscally responsible way of ordering ANA tests.

\section{2}

In Support of Meaningful Assessment and Feedback: A Study of Reasoning Tasks Used During Clinical Case Review in the Rheumatology Clinic

Azin Ahrari (Western University, London); Mark Goldszmidt (Schulich School of Medicine, London)

Objectives: One of the challenges faculty face when assessing and offering feedback to residents is a lack of understanding of the meta-tasks shaping the very care we provide. While most are familiar with the clinical tasks (history taking, physical exam etc.), few are familiar with the metacognitive tasks (reasoning tasks - what we reason about when seeing a patient) that shape how we do the clinical tasks and make decisions. To date, reasoning tasks have been studied for admission case review in internal medicine. The purpose of this study was to build on this early work and explore reasoning tasks in the ambulatory rheumatology clinic in order to identify reasoning tasks addressed by residents and those introduced or elaborated upon by faculty. Results can then be used to better inform assessment and feedback in this new competency based era.

Methods: The study setting was an academic hospital-based rheumatology clinic. Data consisted of audio-recorded case review discussions between 8 faculty rheumatologists, 3 rheumatology fellows and 7 PGY1-3 internal medicine residents. In total, 26 case reviews were collected. Using the previously validated list of 3 overarching and 20 supportive reasoning tasks, we used a constant comparison approach to code and analyze these transcripts. Results: On average, 10 reasoning tasks were addressed/encounter. New consults focused more heavily on tasks related to exploring the most likely diagnosis/differential diagnosis, establishing management plans and identifying risk factors, whereas follow-up encounters focused on assessing rate of progression, response to treatment, estimating prognosis, assessing for complications and determining follow-up and monitoring. Faculty were most likely to introduce the omitted tasks of: weighing alternative treatment options and identifying complications associated with the diagnosis, investigations, or treatment. Furthermore, faculty were most likely to elaborate on the above tasks as well as: determining follow up, monitoring and consultation strategies; assessing rate of progression, and response to treatment and estimating prognosis

Conclusion: These findings can be used in three ways. First, the common patterns of omissions can be shared with residents as a way of better preparing them for their ambulatory encounters. Second, having a shared language around the metacognitive tasks shaping the rheumatology encounter can allow for more meaningful feedback with residents; helping them to see beyond the single case to broader patterns of their performance in rheumatology clinic. Finally, as we move forward with more competence based assessments, reasoning tasks can be used in the design of the assessment instruments themselves.

\section{3}

Case Based Learning Exercises versus Informal Teaching Session to Enhance Educational Experience on Rheumatology Rotation

Caroline Barry (Dalhousie University, Halifax)

Objectives: Medical students and post-graduate trainees may complete a rheumatology rotation at the Nova Scotia Rehabilitation Centre (NSRC) in Halifax, Nova Scotia. Educational opportunities include physical exam teaching, grand rounds and informal weekly lunchtime teaching to complement clinics. It is challenging to be competent in a specialty after a two or four week rotation. The goal of this project is to create case-based learning exercises to be completed by learners during their rotation to supplement knowledge and confidence in managing rheumatologic diseases. This is compared to current informal weekly lunchtime teaching with the rheumatologist on service.

Methods: Cased-based learning exercises were developed to be completed by learners during the 2017-2018 academic year. Cases highlight principles of assessment, diagnosis and management. Pilot topics include giant cell arteritis (GCA), polymyalgia rheumatica (PMR), monoarthritis and rheumatoid arthritis. Learners receive an electronic copy of cases with reading material at the start of the rotation to be completed using self-directed learning. A formal teaching session to review the cases occurs biweekly. This case-based session alternates on a cycle with the currently implemented informal weekly lunchtime teaching. A voluntary and anonymous feedback questionnaire is completed at the end of each two-week cycle.

Results: Four months of data are available for interpretation. From July 1st 2017 to November 10th 2017, 14 learners completed cases - 4 completed the GCA/PMR case, 6 the monoarthritis case and 4 the polyarthritis/ rheumatoid arthritis case. Comments from the qualitative review included preference for both types of teaching sessions: "Enjoyed being able to prepare ahead of time with cases," "Excellent resources and helpful guide for reading," "A combination of the two methods would help touch on areas of weakness or interest." From quantitative data, $86 \%$ of learners strongly agreed that resources and time were adequate in preparing for case-based exercises. $79 \%$ of learners felt both sessions were equal educational yield while $21 \%$ preferred case-based learning.

Conclusion: The feedback to date show learners enjoy a mixed educational experience between informal teaching sessions and case-based exercises. This project will continue over the 2017-2018 academic year with more cases development and data collected for consideration of permanently adopting case-based exercises as part of the rheumatology rotation experience. Feedback has allowed learners to suggest teaching methods they feel would be helpful on a rheumatology rotation which stimulates more medical education project opportunities.

\section{4}

"Aneurysm Osteoarthritis Syndrome: Blood Vessels, Bones and Groans" Caylib Durand (University of Calgary, Calgary); Susan Barr (University of Calgary, Calgary)

Background: This case report describes a 54F with Aneurysm Osteoarthritis Syndrome (AOS). She developed early onset osteoarthritis (OA) requiring a right total hip arthroplasty at age 39 and was found to have multiple aneurysms including the left iliac artery, right ophthalmic and cerebral arteries, and bilateral carotid arteries. Investigations for systemic rheumatic disorders were negative. The patient has 7 family members with AOS. Genetic testing revealed a mutation in the SMAD3 gene.

AOS is a newly identified syndromic connective tissue disorder, recently classified as Loeys-Dietz syndrome (LDS) type 3. It is a rare autosomal dominant disorder involving a mutation in the SMAD3 gene which codes for a protein in the TGF-beta signaling pathway. It is characterized by the early development of OA, arterial aneurysms, and mild craniofacial abnormalities. It is associated with high rates of death from arterial dissections and ruptures, and significant morbidity due to early OA.

Objectives: To raise awareness about the manifestations, risks and management of AOS. To perform a literature review and summary of the studies to date on AOS.

Methods and Results: A literature search for AOS was performed using Medline and EMBASE databases using keywords related to AOS. Only 31 studies were found, with most being descriptive in nature. One recent major study linked the SMAD3 gene mutation to AOS (Van de Laar et al Feb 2011). The limited literature on AOS suggests that further studies are needed to further characterize this rare disease and to identify different mutations that may be responsible for different clinical manifestations. This may provide a

Personal non-commercial use only. The Journal of Rheumatology Copyright $\subset$ C 2018. All rights reserved. 
better way to identify and classify patients that are at higher risk of death or complications.

Conclusion: AOS is a rare genetic condition that can result in significant mortality from ruptured aneurysms and poor quality of life due to early aggressive OA. Further studies of affected families are needed to further characterize the genetic mutations that contribute to this disease and to improve classification and risk stratification of patients to prevent mortality and improve quality of life. Education for early recognition and management of this disease is important in preventing disease complications. AOS may also serve as a model for understanding and targeting TBF-beta pathways in primary OA.

\section{5}

Measuring the ACPAC Program Trained Extended Role Practitioner (ERP) Workforce in Canada: A Profile of Practice Settings, Roles and Participation in Models of Arthritis Care in Canada

Lundon Katie (University of Toronto, Toronto); Amanda Pullan (University of Toronto, Toronto); Rachel Shupak (St. Michael's Hospital, Toronto)

Objectives: 1. To measure and map the ACPAC ERP workforce in Canada. 2. To present a snapshot of general practice characteristics relating to the ACPAC-trained ERP including the nature of their settings, roles and participation in the workforce as ERPs, and models of arthritis care in which they practise.

Methods: As part of quality assurance measures, graduates of the ACPAC program (www.acpacprogram.ca) were asked to contribute data pertaining to their current practice in the following categories: discipline, geographic location/setting (urban, community, remote/rural), participation in workforce as an ERP (\% FTE), nature and percent of practice (orthopaedic, rheumatology) as an ERP, age groups treated, and participation in different models of arthritis care. General practice locations of ACPAC program trained ERPs were geospatially plotted by province across Canada, as well as superimposed upon Ontario-derived LHIN (local health integrated network) based maps representing Rheumatologist distribution in Ontario.

Results: There have been 69 graduates of the ACPAC program with 66 in the current workforce (2 retired, one deceased); base disciplines include Physical Therapists $(n=49)$, Occupational Therapists $(n=13)$ and Registered Nurses $(n=7) .9$ remain working in traditional roles and 3 are in leadership roles leaving a residual of 54 in active ERP roles. The practice settings of these ERPs are as follows: urban (50\%); community (35\%); and remote/rural (15\%). The nature and percent of practice of these ERP roles are as follows: triage rheumatology $100 \%$ FTE $(20 \%)$ and fractional $<100 \%$ $(46 \%)$; triage orthopaedics $100 \%$ FTE $(6 \%)$ and fractional < $100 \%(15 \%)$; triage rheumatology and orthopaedics $100 \%$ FTE (9\%) and fractional < $100 \%(4 \%)$. The patient age-groups treated are adults/seniors (83\%), adults and paediatrics $(7 \%)$, and paediatrics $(10 \%)$. The ACPAC ERPs currently practise in community-based home care, community-based Rheumatologists' clinics, telehealth/ECHO, family health teams, hospital-based, visiting Rheumatologist and visiting ERP/fly in models of arthritis care.

Conclusion: It is important to understand the distribution and nature of practice settings of the highly trained advanced clinician practitioners in arthritis care, and recognize their potential to improve capacity in Rheumatology services delivered through different models of arthritis care. Aside from resource planning, this information is a practical step toward achieving improved connectivity between Rheumatologists and a network of ACPAC program trained ERPs across Canada which will ultimately benefit access to arthritis care for patients. Next steps include issuing a Pan-Canadian workforce survey which will explore attributes of all identified non-physician arthritis care specialists (Stand Up and Be Counted Too (2).

\section{6}

Development of the Patient Engagement in Research Scale

Clayon Hamilton (University of British Columbia, Vancouver); Alison Hoens (University of British Columbia, Vancouver); Tara Azimi (University of Alberta, Edmonton); Shanon McQuitty (Arthritis Research Canada,
Vancouver); Annette McKinnon (Arthritis Research Canada, Richmond); Kelly English (Arthritis Research Canada, Richmond); Linda Li (Rehab Sciences/Physical Therapy, University of British Columbia, Arthritis Research Canada, Richmond)

Objectives: The practice of engaging patients as partners when conducting arthritis research has grown in the last decade; however, no tool is available to measure success. This study aimed to identify items for a scale to measure meaningful patient engagement in research.

Methods: The Patient Engagement In Research (PEIR) Framework (1), developed using in-depth interviews of 18 patient partners with arthritis, was used to generate 120 items across eight domains. A 3-round Delphi process, involving online questionnaires and a teleconference discussion between rounds two and three, was then undertaken. Eligible Delphi panel members were adult ( $\geq 18$ years old) patients or their informal caregivers who engaged in research as patient partners in Canada within the last three years, and had internet access. Panelists rated in all 3 rounds the level of importance of each item on a 4-point adjectival scale ( 1 to 4 , higher $=$ more important). Three criteria were used to retain each item: a median rating of $\geq 3.25$, a rating of 3 or higher by $\geq 70 \%$ of panel members, and comments on its wording and importance. These criteria guided the decision of our research team, including four patient partners, on whether or not an item was retained, revised, or removed.

Results: We recruited 12 individuals (10 women, 11 Caucasians, and 1 Asian), from Alberta $(n=1)$, British Columbia $(n=9)$, and Ontario $(n=2)$, and they represented a variety of diseases, health-related conditions, and use of health care services such as rheumatoid arthritis, inflammatory bowel syndrome, multiple sclerosis, diabetes, stroke, neurodevelopmental disabilities, obesity, nutrition intervention implementation. Highest formal education ranged from high school diploma $(\mathrm{n}=1)$ to master's degree $(\mathrm{n}=$ 2). They were aged between $18-25$ and 76-85 years old. All panelists completed the questionnaires, except for one person in round two. A final set of 43 items was retained across the eight domains of the PEIR Framework: procedural requirements $(n=16)$, convenience $(n=4)$, contributions $(n=4)$, support $(n=5)$, team interaction $(n=3)$, research environment $(n=3)$, feel valued $(n=4)$, and benefits $(n=4)$. These items formed the initial Patient Engagement In Research Scale (PEIRS).

Conclusion: This project is the first to develop a tool for evaluating meaningful engagement of patients in research projects. Importantly, the Delphi process involved a synergy of patient partners as both participants and members of our research team to ensure the PEIRS is grounded in a patient perspective. The PEIRS is currently undergoing pretesting for content and face validation. (1)Hamilton et al. Health Expectations.2017;00:1-11.

\section{7}

A 38-year-old Man with Isolated Sternoclavicular Joint Swelling Konstantin Jilkine (Western University, London); Tristan Boyd (Rheumatology Division, St. Joseph's Health Care, London)

Case: A 38-year old man originally from the Philippines presented with a 4-month history of left sternoclavicular (SC) joint pain and swelling. $\mathrm{He}$ denied prior trauma, infectious symptoms, other joint involvement, or any features of seronegative spondyloarthropathy. Past medical history was unremarkable, with the exception of a $14 \times 8 \times 6 \mathrm{~cm}$ axillary abscess treated 10 years earlier with debridement and antibiotics; biopsy and cultures at that time were negative. MRI of the left SC joint demonstrated features of osteitis in the distal clavicle and manubrium, favoring trauma, however an inflammatory arthritis, such as SAPHO syndrome, could not be excluded triggering the referral to rheumatology. On examination, his vital signs were within normal limits. The left SC joint was tender to palpation with overlying, non-fluctuant soft tissue swelling with a slight violaceous discoloration. There were no other involved joints, cutaneous lesions, or lymphadenopathy. Cardiac, respiratory, abdominal examinations were normal. Blood work included: CRP 10.4, ESR 26, Hgb 154, WBC 9.6, Plt 466, and negative HLA-B27 antigen. Ultrasound-guided aspiration of the left SC joint was arranged, but no synovial fluid was obtained. Saline lavage revealed a non-inflammatory cell count and negative cultures. Given lack of evidence 
for an infectious etiology, an image-guided corticosteroid injection was arranged. His pain and swelling initially improved, however, his symptoms recurred four months later. CT thorax was arranged, which demonstrated destructive changes of the left SC joint with surrounding rim-enhanced fluid collections concerning for septic arthritis with multiloculated abscesses. Urgent repeat US-guided aspiration was arranged, which yielded $2 \mathrm{~mL}$ of turbid, purulent fluid. Fluid culture initially grew $1 \mathrm{cfu}$ of Staphylococcus epidermis and Propionibacterium acnes; AFB smear was negative. Four weeks later Mycobacterium tuberculosis (TB) was isolated. After conferring with Infectious Diseases, he was started on rifampin, isoniazid, pyrazinamide, and ethambutol. Orthopedic Surgery assessed the patient and felt irrigation and debridement was not indicated.

Discussion: Tuberculosis is a globally prevalent disease. SC joint involvement represents $<1 \%$ of osteoarticular TB, which itself accounts for $<2.5 \%$ of TB cases in Canada. Given its rarity, variable presentation, and non-specific findings, the diagnosis can be challenging.

Conclusion: This patient presented with monoarthritis, bland blood work, lack of systemic symptoms, and non-specific imaging. His initial fluid culture was negative. A high index of suspicion, healthy skepticism, and diligence in pursuing a complete workup is essential in identifying and treating such cases.

\section{8}

Managing Systemic Sclerosis: Assessing the Educational Needs of Rheumatologists

Zareen Ahmad (Toronto Scleroderma Program, Division of Rheumatology, Department of Medicine, Sinai Health Systems, University of Toronto, Toronto); Sindhu Johnson (Toronto Scleroderma Program, Division of Rheumatology, Department of Medicine, Sinai Health Systems, University Health Network, Institute of Health Policy, Management and Evaluation, University of Toronto, Toronto)

Objectives: Systemic sclerosis (SSc) is an uncommon, complex and heterogeneous condition, making it challenging to manage. Individual rheumatologists see relatively few cases and patient surveys identify numerous gaps in clinical care. There are no published data on the educational needs of rheumatologists caring for patients with SSc. We aimed to determine rheumatologists' self-rated knowledge and learning needs.

Methods: Survey questions were adapted from the EULAR Recommendations for the Treatment of SSc with reference to patient-identified care gaps. The survey was conducted on paper and on SurveyMonkey (a cloud-based online survey development software program). The target audience was Ontario rheumatologists, serving a population of 13.6 million. We sought to explore self-reported knowledge, experience, attitudes and perceived barriers in caring for SSc patients. Physician demographics and preferred educational methods were also collected. Gaps between perceived and desired knowledge were calculated to identify the greatest unmet learning needs.

Results: One hundred and eighteen responses were received with a response rate of $54 \%$. The greatest unmet learning needs were seen in the management of sexual dysfunction (average gap of 1.4 on a 5-point scale), pulmonary hypertension (1.1), interstitial lung disease (1.0) and gastrointestinal manifestations of the disease (1.0). The smallest learning gap concerned screening recommendations (0.7). 19\% of rheumatologists agreed with the statement "Scleroderma is an untreatable disease." Agreement with this statement was highest $(33 \%)$ among rheumatologists who treat relatively small numbers (1-10) of scleroderma patients.

Conclusion: We have identified several unmet learning needs regarding the management of SSc among rheumatologists. These can be used to inform future educational resources and programs for rheumatologists regarding $\mathrm{SSc}$ and to direct further research into their needs.

\section{9}

Psoriasis and the Risk of Foot and Ankle Tendinopathy or Enthesopathy in the Absence of Psoriatic Arthritis: A Population-Based Study

Ryan Lewinson (University of Calgary, Calgary); Isabelle Vallerand
(University of Calgary, Calgary); Laurie Parsons (University of Calgary, Calgary); Jeremy LaMothe (University of Calgary, Calgary); Alexandra Frolkis (University of Calgary, Calgary); Mark Lowerison (University of Calgary, Calgary); Gilaad Kaplan (University of Calgary, Calgary); Scott Patten (University of Calgary, Calgary); Cheryl Barnabe (University of Calgary, Calgary)

Objectives: Imaging studies in cutaneous psoriasis patients have demonstrated bone and tendon changes that are consistent with tendinopathy and enthesopathy, most commonly of the foot and ankle. However, in most cases, these lesions were asymptomatic and identified incidentally. It remains unknown whether patients with cutaneous psoriasis have an increased risk of developing clinically relevant tendinopathy or enthesopathy of the foot and ankle. Our objective was to determine if cutaneous psoriasis patients have an increased risk of clinically significant foot and ankle tendinopathy or enthesopathy compared to the general population.

Methods: Patients with cutaneous psoriasis and a general population cohort were identified in The Health Improvement Network (THIN), a general practice medical records database from the United Kingdom. All patients with psoriatic arthritis were excluded. Cox proportional-hazards models $($-alpha $=0.05)$ estimated the hazards ratio $(\mathrm{HR})$ for development of foot and ankle tendinopathy or enthesopathy among psoriasis patients, with adjustment for covariates such as age, sex, obesity status, smoking status, alcohol use, Charlson co-morbidity index, Townsend deprivation index, systemic psoriasis therapy, corticosteroid use, and fluoroquinolone use. Sensitivity analyses were performed to ensure psoriatic arthritis patients were excluded.

Results: We identified 78,630 cutaneous psoriasis patients and 5,983,338 persons from the general population. In an unadjusted model, cutaneous psoriasis patients had a $25 \%$ increased risk of developing foot and ankle tendinopathy or enthesopathy compared to the general population $(\mathrm{HR}=$ $1.25,95 \%$ CI $1.20-1.30, \mathrm{p}<0.0001)$. The HR remained unchanged and statistically significant after adjusting for covariates, and in numerous sensitivity analyses.

Conclusion: Patients with cutaneous psoriasis can have foot and ankle tendinopathy or enthesopathy without having psoriatic arthritis, presenting a diagnostic challenge to physicians. As the diagnosis of psoriatic arthritis can impact clinical management decisions, prompt referral to a rheumatologist should be initiated in these cases. Further research is needed to elucidate mechanisms contributing to the increased risk of foot and ankle tendinopathy or enthesopathy in patients with psoriasis.

\section{0}

Provincial Expenditures in Rheumatic Diseases: Trends and Disparities in Medical Care Costs

Mena Bishay (University of Alberta, Edmonton); Elaine Yacyshyn (Division of Rheumatology, University of Alberta, Edmonton)

Objectives: Autoimmune and inflammatory diseases encompass a broad spectrum of presentations amongst patient populations. This area is a continuously growing field accruing more costs with time. Few studies examining the economic impact of these diseases exist. We evaluated aggregate data for health expenditures on provincial and regional levels to study trends and disparities in costs.

Methods: Provincial health expenditure data from 2009-2016 was obtained from Alberta Health Services' Analytics, Data Integration, Measurement \& Reporting (DIMR) via their Tableau system. Aggregate data was obtained for the following disease groups: connective tissue disease and vasculitis (CTDV), rheumatoid arthritis (RA), spondyloarthropathy and other inflammatory arthropathies (SIA), and crystal arthropathies (CA). Matching data was also obtained for osteoarthritis (OA) for comparison. The following data were collected for each disease group: Total expenditures, total number of patients, number of male vs female patients, and average cost per person. Results: From 2009 to 2016, provincial costs have risen by $102 \%, 43 \%$, $60 \%$, and $87 \%$ respectively for CTDV, RA, SIA, and CA. The number of patients within each disease group has risen $68 \%, 25 \%, 32 \%$ and $54 \%$, respectively. When evaluating geographic distribution, total costs were

Personal non-commercial use only. The Journal of Rheumatology Copyright (c) 2018. All rights reserved. 
highest in the greater Calgary and greater Edmonton regions across all diseases, compiling 56-71\% of total provincial expenditures for these diseases. The greater Calgary region more often has higher average costs per patient compared to the greater Edmonton region, with differences up to $\$ 660$ per patient. In $2015-2016$, the average cost per patient for CTDV ranged from \$3810-\$4958/year for most locations, but for the remote west, rural south, and remote north regions average costs were $\$ 6256, \$ 7619$, and $\$ 9299 /$ year, respectively. Similar outliers were determined for multiple rural locations going back to 2009 , most often affecting the rural north, remote west, and remote north regions. Few outliers were seen in RA patients in the same timeframe, and none are seen in SIA, CA, or OA.

Conclusion: The number of patients with these autoimmune and inflammatory diseases has risen steadily since 2009. Costs for these diseases have also risen, however they have done so out of proportion to the patient population growth. Consistent differences exist in the cost per patient between the two largest centers in Alberta. Inequality in the cost of care for CTDV in remote/rural regions points to areas of inequity and patient access to care. The reason for these inequalities is not clear from this aggregate data, and requires further quality improvement studies and research.

\section{1}

Predictive Validity of Presenteeism Measures with Dual Answer Keys in Inflammatory Arthritis

Mira Donaldson (University of British Columbia, Vancouver); Alexandra Kobza (University of Ottawa, Ottawa); Dorcas Beaton (University of Toronto/Institute for Work and Health, Toronto); Hui Xie (Arthritis Research Canada/Faculty of Health Sciences at Simon Fraser University, Richmond); EC Sayre (Arthritis Research Canada, Richmond); Monique Gignac (University of Toronto/Institute for Work and Health, Toronto); Diane Lacaille (University of British Columbia (Division of Rheumatology)/ Arthritis Research Canada, Richmond)

Objectives: Employment studies in arthritis have emphasized the importance of decreased productivity at work, or presenteeism. However, consensus is still lacking on how to best measure this construct. Instruments used include the Work Limitations Questionnaire (WLQ), which measures the amount of time people are limited, and the Workplace Activity Limitations Scale (WALS), which measures the degree of difficulty. We modified the response keys of the WLQ and WALS, creating a dual answer key assessing both degree of difficulty and amount of time with difficulty. Previous work by our group has shown that scores obtained from combining responses to the dual answer keys exhibit good internal consistency, criterion and construct validity. The objective of this study was to evaluate the ability of the combined scores to predict future work cessation and number of work days missed due to arthritis.

Methods: A longitudinal study used baseline and 6-month data from the RCT of an employment intervention, the "Making It Work" Program. Inclusion criteria were: inflammatory arthritis, age 19-59, working at baseline, and concerns about arthritis affecting work. 327 participants were included (RA:173, PsA:48, SLE:42, AS:64). Combined scores were obtained by i) multiplying, and ii) adding, the scores of difficulty and time answer keys at the item level. We assessed the predictive ability of the combined scores, and the original scores, of the WALS and WLQ to predict work cessation due to arthritis within 6 months using binary logistic regression models, or number of work days missed due to arthritis using zero-inflated Poisson models.

Results: WLQ and WALS original and combined scores were significant predictors of future work cessation and number of work days missed. The WLQ combined scores predicted work cessation better than the original score (AIC:117.6 \& 118.2 for multiplicative and additive combinations, respectively vs. 125.4 for time) and work days missed (AIC:2179.8 \& 2177.8 vs. 2188.7). WALS original score (ie. degree of difficulty) predicted work cessation better than combined scores (AIC:95.8 vs. 109.3 \& 109.4). However, WALS combined scores predicted number of work days missed better (AIC:1696.9 \& 1673.4 vs. 1795.2).

Conclusion: Combined scores from dual answer keys applied to the WLQ and WALS significantly predicted future work cessation and number of work days missed. Using combined scores, rather than original answer keys, improved the ability of the WLQ to predict both work cessation and work days missed, but only improved the ability of the WALS to predict work days missed.

\section{2}

Measurement Properties of Presenteeism Measures with Dual Answer Keys in Inflammatory Arthritis

Mira Donaldson (University of British Columbia, Vancouver); Alexandra Kobza (University of Ottawa, Ottawa); Dorcas Beaton (University of Toronto/Institute for Work and Health, Toronto); Monique Gignac (University of Toronto/Institute for Work and Health, Toronto); Diane Lacaille (University of British Columbia (Division of Rheumatology)/ Arthritis Research Canada, Richmond)

Objectives: Employment studies in arthritis have emphasised the importance of decreased productivity at work, or presenteeism. Yet, how to best measure presenteeism remains challenging. The 'Work Limitations Questionnaire' (WLQ) and the 'Workplace Activity Limitations Scale' (WALS) are frequently used. The WLQ measures the amount of time people are limited, whereas the WALS measures the degree of limitation. We modified the response keys to the WALS and WLQ to measure both degree of difficulty and amount of time with difficulty. Our objective was to evaluate measurement properties, i.e. internal consistency and construct validity, of the WALS and WLQ with combined scores from dual answer keys.

Methods: A cross-sectional study used baseline data from the RCT of an employment intervention, the "Making It Work" Program. Inclusion criteria included: having inflammatory arthritis, currently employed, age 19-59, and having concerns about arthritis affecting ability to work. 364 participants were included (RA:195, PsA:54, SLE:46, AS:69; 77\% female, mean(SD) age: $45.9(9.8) \mathrm{yrs})$. Combined scores were obtained by i) multiplying and ii) adding, the scores of difficulty and time answer keys at the item-level. As no significant differences were observed between the two models, we report on the multiplicative model, which reflects consumers' preference. Internal consistency was analyzed using Cronbach's alphas; construct validity by measuring correlation (Spearman coefficients) between WALS or WLQ subscales and disease and work variables.

Results: Item-level analysis revealed a strong floor effect (WALS:16\%-56\%; WLQ:27\%-81\% for all items except one), likely reflecting the low limitation expected in a working sample. High (i.e. $\geq 0.7$ ) internal consistency (-alpha $0.70-0.82$ ) was found for WALS and all WLQ subscales except Physical Demands (0.67). As a priori hypothesized, moderate correlation was observed between the time (0.33-0.57), or combined (0.44-0.61), scores of WLQ subscales and work productivity impairment (WPAI) or work instability (WIS); with consistently higher correlations for combined than time scores. Moderate correlation was also observed between degree of difficulty (0.70-0.77), or combined (0.60-0.69), scores of WALS and WPAI, or WIS Notably, correlations were not higher for the WALS combined than degree of difficulty scores. Correlations with disease and job characteristics with time, difficulty, or combined scores of the WALS and WLQ met a priori hypothesized correlation levels.

Conclusion: Our previous research confirmed the value of measuring both degree of difficulty and amount of time, showing they measure different concepts. This research demonstrates that applying a dual answer key to two validated instruments shows good initial indicators of internal consistency and construct validity.

193

Incidence of Hip Fracture in Rheumatoid Arthritis Compared to General Population Controls, a Population-based Study

Allyson Jones (University of Alberta, Edmonton); Pierre Guy (University of British Columbia, Vancouver); Hui Xie (Arthritis Research Institute; Simon Fraser University, Vancouver); EC Sayre (Arthritis Research Canada, Richmond); Diane Lacaille (University of British Columbia (Division of Rheumatology)/ Arthritis Research Canada, Richmond) 
Objectives: Hip fractures have serious long-term effects, including a high 1-year mortality rate (usually 20-30\%) and poor functional recovery, with approximately $50 \%$ not attaining pre-fracture functional status. Although risk of hip fractures is known to be increased in rheumatoid arthritis (RA), few studies specifically look at the incidence of hip fractures in patients with RA compared to general population controls. We estimated the incidence of hip fractures in a population-based cohort including all incident RA cases in British Columbia, using administrative health data.

Methods: Using physician billing data and a previously published RA definition, we assembled an incident cohort of cases with RA onset between 1997 and 2009. Controls (with no diagnosis of RA or other inflammatory arthritis) selected randomly from the general population were matched 2:1 to RA patients on birth year, gender and index year. RA and controls with prior hip fractures, pathological fractures or Paget's disease were excluded. Hip fractures (ICD9-CM codes 820.0, 820.2; ICD10-CA codes S72.0, S72.1, S72.2) were identified using hospitalization data ( $\leq 25$ codes defining reason for admission or complications during hospitalization). Crude incident rates were calculated as number of new hip fractures per person-year (PY). Cox-regression models were used to compare age and sex adjusted risk of hip fracture in RA relative to controls.

Results: A total of 37616 RA patients and 75213 controls (mean age $57.3 / 57.2$ years, respectively; $66 \%$ females for each cohort) were followed until December 2014, yielding 360,521 and 732,249 PY, respectively. Incident hip fractures were observed in 1314 RA and 2083 controls. Mean(SD) age at time of fracture was 79.5(10.8) years in RA and 81.6(9.3) years in controls. Crude incidence rate was 3.6 and 2.8 per 1000 PY for RA and controls, respectively, yielding an incidence rate ratio (IRR) of 1.28 [95\% CI: $1.20 ; 1.37$ ]. Crude IRRs were slightly higher for men [All ages: $1.45(1.25 ; 1.69)$ vs. 1.24(1.14;1.34)] and decreased with age [ < 50: 2.11(1.40;3.18); 50-59: 2.78(2.10;3.68); 60-69: 1.48(1.24;1.77); 70-79: 1.14(1.02;1.27); 80-89: 1.11(0.97;1.27); > = 90: 1.08(0.68;1.70)]. Adjusting for age and sex, persons with RA had $28 \%$ greater risk of hip fracture than persons without (HR 1.28, 95\% CI 1.19, 1.37).

Conclusion: Risk of hip fractures in persons with RA is higher than age/sex matched controls particularly at younger ages. Given the impact of hip fractures, this has important implications for functional status and quality of life of people with RA. Further work on course of recovery, mortality and health services use post-fracture is needed. Supported by a CIORA grant.

\section{4}

Mortality Post Hip Fracture in Rheumatoid Arthritis Compared to General Population Controls, A Population-based Study

Allyson Jones (University of Alberta, Edmonton); Pierre Guy (University of British Columbia, Vancouver); Hui Xie (Arthritis Research Institute; Simon Fraser University, Vancouver); EC Sayre (Arthritis Research Canada, Richmond); Diane Lacaille (University of British Columbia (Division of Rheumatology)/ Arthritis Research Canada, Richmond)

Objectives: In prior work, we found a higher incidence of hip fractures in RA than age and sex matched general population controls (3.6 vs. 2.8 per 1000 PY). To assess burden of disease, we compared all-cause mortality post hip fracture in RA and general population controls.

Methods: Using physician billing data and a previously published RA definition, we assembled a population-based incident cohort of cases with RA onset between 1997 and 2009. Controls (with no inflammatory arthritis) were randomly selected from the general population, matched $2: 1$ to RA cases on birth year, gender and index year. People with prior hip fractures, pathological fractures or Paget's disease were excluded. Hip fractures were identified using hospitalization data. RA individuals and controls were followed from incident hip fracture to death, last health care use, or study end (Dec. 2014). Logistic regression was used to model in-hospital mortality (taking hospital transfers into account). Cox-proportional hazard models compared mortality risk in RA vs. controls with incident hip fractures over predefined time intervals post-fracture. Models were adjusted for age, sex, socio-economic status, rural/urban, hospital size, fracture type, and comorbidities measured in the year preceding hip fractures.
Results: The cohort included 37,616 RA patients and 75,213 controls, of whom 1,314 RA and 2,083 controls sustained an incident hip fracture and make up the study sample ( $78 \%$ and $80 \%$ females in RA and controls; mean(SD) age at time of fracture: 79.5(10.8) years for RA and 81.6(9.3) for controls). Deaths occurred in 715 RA patients and 1224 controls. Crude all-cause mortality rate post hip fracture for RA and controls was 457 and 610/1000 PY at 90 days, 229 and 280/1000 PY at 1 year, and 161 and 194/1000 PY at 5 years, respectively. After adjustment, mortality post hip fracture in RA did not differ significantly from persons without RA for in-hospital mortality [OR(95\% CI): $0.82(0.63 ; 1.07)]$; mortality was lower over the first 90 days [HR(95\% CI): 0.78(0.64, 0.96)], but did not differ significantly over later time intervals [HR( $95 \% \mathrm{CI}): 0.96(0.76 ; 1.22)$ over 91 days to 1 year, $0.89(0.78 ; 1.02)$ over $1-5$ years, and $0.85(0.68 ; 1.07) 5+$ years after hip fracture].

Conclusion: Despite a higher incidence of hip fractures in RA, mortality post hip fracture did not differ from controls, except for a lower risk of all-cause mortality 90-day post hip fracture, after adjusting for age, sex and other potentially confounding factors. Further research is needed to understand RA-specific characteristics influencing post-fracture mortality risk. Supported by a CIORA grant.

\section{5}

Prednisone Tapering Consensus Treatment Plans (CTP) for Pediatric Rheumatic Diseases: A Quality Improvement Initiative

Jayson Tan (Simon Fraser University, Vancouver); Jenny Tekano (BC Children's Hospital, Vancouver); Jayne Green (BC Children's Hospital, Vancouver); Kristin Houghton (BC Children's Hospital, Vancouver)

Objectives: Prednisone plays a central role in the treatment of pediatric rheumatic diseases but despite its widespread use, there are few guidelines and there is widespread variation in practice. We aimed to standardize prednisone prescribing practice in a shared care pediatric rheumatology model though 1) development of consensus treatment plans (CTP), 2) evaluation of adherence to the CTP and 3) evaluation of usefulness of CTP.

Methods: Eight prednisone CTPs (2 high dose regimens ( $1.5 \mathrm{mg} / \mathrm{kg}$ for 12 or 6 months' duration), 3 moderate dose regimens $(1 \mathrm{mg} / \mathrm{kg}$ for 6,3 , and 1 month duration) and 3 low dose regimens $(0.5 \mathrm{mg} / \mathrm{kg}$ for 3 or 1 month and $0.2 / \mathrm{kg}$ for 2 weeks) were developed after literature review and team consensus. An on-line prednisone calculator was developed, allowing for selection of a CTP or custom schedule. Weekly and monthly calendars were printed for children/parents and a single summary page created for healthcare providers (HCP) and the medical chart. We recruited 13 children who were prescribed prednisone. We assessed HCP and child/parent adherence to CTPs (medication logs, data collection forms) and HCP and child/parent perceived ease, usefulness, helpfulness and long-term understanding of prednisone dosing (questionnaire). Reasons for protocol deviations were recorded.

Results: 13 children completed the study. 8 children, 13 parents completed assessments. 5 physicians and 2 RNs completed 20 HCP assessments. Child/parent prednisone adherence was $69 \%$ at the initial visit and $80 \%$ at follow up visits. Compared to standard prednisone prescription guidance, the calendar was easy to follow (100\% child/parent, $75 \%$ of HCP), useful (95\% child/parent used the calendar on a daily or weekly basis, $85 \%$ HCP used the calendar at clinic visits), helpful tracking short term dose changes (100\% child/parent, $90 \% \mathrm{HCP})$ and helpful understanding the long-term prednisone management (100\% child/parent, $100 \% \mathrm{HCP}$ ). HCP report CTP has greatest use in initiating therapy. CTPs were modified by HCP in 54\% of children (themes - intercurrent illness, symptom control requiring change in tapering schedule). $\mathrm{RN}$ report a significant decrease in workload and the number of phone calls from families related to prednisone dose.

Conclusion: Our findings suggest prednisone CTPs help children / parents and HCP follow prednisone tapering schedules in a shared care clinic model. HCP commonly modified the CTPs based on individual disease related factors and refinement of CTPs will be informed by child / family feedback, $\mathrm{HCP}$ practice patterns, and new knowledge. CTPs may minimize medication errors, enhance compliance and reduce RN workload.

Personal non-commercial use only. The Journal of Rheumatology Copyright $(\subset)$ 2018. All rights reserved. 


\section{6}

Exploring How Individuals with Gout Experience an eHealth Supported Collaborative Care Model: An Explanatory Sequential Mixed Methods Study

Alyssa Howren (University of British Columbia Faculty of Pharmaceutical Sciences; Arthritis Research Canada, Vancouver); Nicole Tsao (University of British Columbia Faculty of Pharmaceutical Sciences; Arthritis Research Canada, Vancouver); Kamran Shojania (University of British Columbia Faculty of Medicine; Arthritis Research Canada, Vancouver); Hyon Choi (Massachusetts General Hospital/Harvard Medical School, Boston); Antonio Avina-Zubieta (University of British Columbia Faculty of Medicine; Arthritis Research Canada, Richmond); Alison Kydd (University of British Columbia Faculty of Medicine, Vancouver); Russell Friesen (Providence Health, Vancouver); Mary De Vera (University of British Columbia Faculty of Pharmaceutical Sciences; Arthritis Research Canada, Vancouver)

Objectives: We conducted mixed methods research to: (1) explain quantitative findings from the evaluation of the Virtual Gout Clinic (VGC), an eHealth supported collaborative care model involving rheumatology, pharmacy, and dietetics; and (2) explore how participants with gout experience this collaborative care model.

Methods: We applied an explanatory sequential mixed methods design nested within the longitudinal study of the VGC. In the quantitative phase, primary outcomes in the VGC, namely serum uric acid (SUA) and medication adherence as measured by the Compliance-Questionnaire Rheumatology 5-item (CQR5) were obtained at 12 months. The qualitative phase followed the principles of constructivist grounded theory and gathered data through one-on-one semi-structured interviews with participants after completion of the VGC. Participants were eligible for interviews if they met the following criteria: 1) diagnosed with gout by a rheumatologist; 2) enrolled in the VGC and received at least one pharmacist and one dietician consult; 3 ) have access to a phone; and 4) able to comprehend and speak English. The analysis was supported with NVivo 11 and followed the coding steps of initial coding and focused coding/categorizing, along with the constant comparative method. Interview participants were classified according to their quantitative outcomes (SUA, CQR5) from the VGC to facilitate interpretation and mixing with the qualitative data.

Results: Thirty-five individuals with gout participated in the VGC and $70 \%$ had achieved target SUA $(<360 \mu \mathrm{mol} / \mathrm{L})$ at 12 months. Of these, $12(10$ males) participated in qualitative interviews and 4 interview participants did not achieve target SUA and 3 were characterized as non-adherent CQR5. Integration of quantitative outcomes from the VGC with qualitative interviews indicate suboptimal health outcomes were explained by an identified theme on practical and perceptual barriers to gout management which comprised categories on lacking knowledge about the prognosis and treatment of gout and fearing side-effects of gout medications. Two constructed themes describe the participants' experience with the VGC: 1) 'describing experience with VGC' was characterized by learning with the VGC, receiving personalized support, and knowing someone cares; and 2) 'logistical considerations' of the VGC, included the need to optimize timing of the VGC and issues pertaining to coordination and accessibility with telehealth.

Conclusion: An eHealth-supported collaborative care model is a promising approach to optimize care delivery by providing both emotional and practical support to patients with gout. Persistent barriers suggest the need to provide ongoing support to patients beyond the initial diagnosis. Supported by a CIORA grant.

197

Is Shared Decision-Making Cost-Effective? An Evaluation of a Decision Aid for Triple Therapy in Rheumatoid Arthritis

Nick Bansback (University of British Columbia/Arthritis Research Canada, Vancouver); Tima Mohammadi (Centre for Health Evaluation and Outcome Sciences, Vancouver); Aslam Anis (University of British Columbia/ Arthritis Research Centre of Canada, Vancouver); James O'Dell (Department of Internal Medicine, University of Nebraska Medical Center, Omaha); Glen Hazlewood (University of Calgary, Calgary)
Objectives: Triple Therapy (a combination of 3 generic drugs) prior to a biologic, is the most cost-effective strategy for patients with rheumatoid arthritis. Many patients would prefer to use Triple Therapy, but utilization in Canada is low. We determined the potential value of an intervention that promotes informed shared decision-making between patients and clinicians around triple therapy use after failure of methotrexate.

Methods: We developed an economic model that compared a strategy where patient preferences for triple therapy use were integrated into treatment decisions, versus usual care. A previous study suggests that $59 \%$ of patients would choose triple therapy first if provided the comparative evidence of benefits, potential adverse events and dosing schedule. We assumed a cost of $\$ 50$ for a shared decision making intervention. Patient's trialling triple therapy first were assumed to switch to a sequence of biologics upon withdrawal. We extrapolated the influence of the initial treatment decision on subsequent disease progression, resource use such as hospitalizations, quality of life, mortality and Quality Adjusted Life Years (QALYs). Scenario analysis incorporated the process of shared decision-making into the benefits under different assumptions.

Results: Incorporating patient preferences was estimated to reduce average costs for a patient by $\$ 40,000$ over a lifetime through delaying the introduction of biologic therapy. Even under the most pessimistic assumptions regarding the potential for earlier biologic use delaying joint erosions, only 0.08 QALYs (28 days of full health) over a lifetime were estimated to be lost. The consequent incremental cost-effectiveness ratio for usual care vs the patient preference strategy is over $\$ 500,000 / \mathrm{Q} A L Y$. Incorporating process of shared decision-making offsets the negative QALYs, implying the patient preference strategy saves costs and increases QALYs.

Conclusion: This study demonstrates that systematically giving patients with rheumatoid arthritis who fail methotrexate an informed choice between triple therapy and a biologic as the initial treatment is highly cost-effective and even potentially cost-saving. This strategy allows patients who prefer biologic therapy to choose it, but saves costs by delaying the biologic initiation in patients who choose triple therapy first. This suggests that strategies for increasing shared decision-making through decision aids, and the infrastructure and incentives such as fee codes to implement this, could be cost-effective and should be explored to promote value based prescribing in rheumatology.

198

Economic Evaluation of Damage Accrual in the Systemic Lupus International Collaborating Clinics (SLICC) Inception Cohort

Megan Barber (University of Calgary, Calgary); Murray Urowitz (University of Toronto, Toronto); John Hanly (Dalhousie University and Nova Scotia Health Authority, Halifax); Ian Bruce (University of Manchester, Manchester); Li Su (Cambridge Institute of Public Health, Cambridge); Yvan St. Pierre (McGill University, Montreal); Vern Farewell (Cambridge Institute of Public Health, Cambridge); Ann Clarke (University of Calgary, Calgary); SLICC Systemic Lupus International Collaborating Clinics Investigators (SLICC, Pittsburgh)

Objectives: Little is known about the association of healthcare costs and damage accrual. We describe the costs associated with damage progression using multi-state modeling.

Methods: Patients fulfilling the revised ACR Criteria for SLE from 32 centres in 11 countries were enrolled in the SLICC Inception Cohort within 15 months of diagnosis. To supplement primary data collection, patients were sampled cross-sectionally at a single time between 4 and 17 years of disease duration on healthcare use and lost labour force/non-labour force productivity over the preceding year. Healthcare use was costed using 2017 Canadian prices (direct costs) and lost productivity using 2017 Statistics Canada age-sex specific wages (indirect costs). Annual costs associated with damage states (SLICC/ACR Damage Index [SDI]) were estimated from multiple regressions adjusting for age, sex, race/ethnicity, and disease duration. Five and 10-year cumulative costs were estimated by multiplying annual costs associated with each SDI state by the expected duration in each state, forecasted using a multi-state model and longitudinal 
SDI data from the SLICC Inception Cohort (Bruce IN. Ann Rheum Dis 2015;74:1706-13). Future costs were discounted at a yearly rate of $3 \%$. Results: 457 patients participated, $88.2 \%$ female, $44.6 \%$ Caucasian, mean age at diagnosis 33.6 years, mean disease duration at time of economic data 10.0 years, mean SLE Disease Activity Index (SLEDAI-2K) 2.97, and mean SDI 1.07. Annual costs were higher in those with a SDI $\geq 5$, with direct costs of \$20 014 (95\% CI \$14 270, \$25 757) versus \$ 4642 (95\% CI \$3187, $\$ 6098$ ) for a SDI $=0$. At SD1 $<=2$, hospitalizations and medications accounted for $54.3 \%$ of direct costs, whereas at SDI $\geq 3$, dialysis was responsible for $55.6 \%$. Cumulative 10 -year direct costs increased with increasing baseline SDI [SDI $\geq 5 \$ 175841$ (95\% CI $\$ 125374, \$ 226308)$ vs SDI $=0$ $\$ 45549$ (\$31 029, \$60 068)]. Indirect costs did not vary with baseline SDI. Conclusion: Patients with the highest baseline SDIs incurred cumulative 5 and 10-year direct costs approximately 4-fold higher than those with the lowest SDIs. However, indirect costs were influenced by factors other than SDI (potentially disease activity, quality of life, fatigue, plateauing of expectations regarding productivity later in the disease) and patients incurred considerable indirect costs even with no or minimal damage. This work demonstrates the substantial increase in direct costs in patients with higher damage, highlighting the cost savings potentially achieved by earlier introduction of therapies more effective at attenuating damage progression.

\section{9}

Improving Access to Rheumatologic Follow Up: A Quality Improvement Initiative

Chandra Farrer (Women's College Hospital, Toronto); Natasha Gakhal (Women's College Hospital, Toronto); Dana Jerome (Women's College Hospital, Toronto)

Objectives: Timely access to rheumatologic care in inflammatory arthritis is associated with improved patient outcomes (Ahluwalia et al 2014; Singh et al 2016). The use of extended role practitioners and optimization of booking have been shown to improve access to care (Newman et al 2004; Bush et al 2007). There are quality of care concerns regarding the discharge of inflammatory arthritis patients from specialist care but there are opportunities to explore discharge of non-inflammatory arthritis patients (Bukhari et al 2007; Badley et al 2012; Doerr et al 2013). Rheumatologists provide consultation to patients with osteoarthritis, fibromyalgia and non-articular pain, which can be managed by primary care or other health care providers (Badley et al 2012; Doerr et al 2013). Aim: The aim of the project is to improve access to rheumatology follow up as measured by decreasing the percent of patients unable to book a follow up appointment from $13 \%$ to $6 \%$ by May 31, 2017.

Methods: Using a mixed LEAN and time series study design, the change concepts of an extended role practitioner, discharge and booking templates were evaluated to determine the impact on access to follow up care in rheumatology.

Results: Significant improvement in the percent of patients unable to schedule a follow up was demonstrated. The change concepts of extended role practitioner and template optimization demonstrated improvement, while the discharge strategy did not demonstrate improvement. The balancing measures of new priority consult wait time, patient and staff satisfaction were maintained. Staff absences impacted access to rheumatologic follow up.

Conclusion: The optimization of extended role practitioner and booking templates facilitated access to rheumatology follow up care. Developing a strategy for staff absences could facilitate further improvement. Further study into discharge from rheumatology practice is warranted.

\section{0}

Pediatric Coronary Artery Ectasia - Rheumatic Disease Associations Tamara McMillan (University of British Columbia, Vancouver); Dion Pepelassis (University of Manitoba, Winnipeg)

Introduction: We present a case of coronary artery ectasia occurring in a patient with acute rheumatic fever (ARF). This is the second reported incidence in ARF and the first in a patient that did not have features of
Kawasaki disease (KD). Recognizing this association may prevent misdiagnosis and mismanagement of ARF as this clinical finding is much more commonly associated with $\mathrm{KD}$.

Case Presentation: A previously healthy nine year old Indigenous boy presented with history of fever for three days, new systolic and diastolic murmurs, cardiomegaly on CXR, prolonged PR interval on EKG, elevated inflammatory markers and elevated ASOT after a recent throat infection. He had no rash, subcutaneous nodules, abnormal movements nor arthritis/ arthralgias. He had no features of KD (changes to his lips or oral cavity, conjunctival injection, changes in his peripheral extremities, lymphadenopathy). His initial echocardiogram showed severe aortic valve insufficiency, moderate mitral valve insufficiency and right coronary artery ectasia. He was diagnosed with ARF based on his carditis, fever, elevated inflammatory markers and prolonged PR interval. Due to the dilated right coronary artery KD was considered but as he met no clinical criteria treatment was initiated for ARF. He was started on oral prednisone, high dose aspirin, enalapril, lansoprazole and penicillin at treatment doses. He recovered and was discharged on these medications with instructions to taper his prednisone and start prophylactic penicillin. At his four month follow up visit he had resolution of his right coronary artery ectasia. His echocardiogram otherwise continues to show severe aortic valve insufficiency, moderate mitral valve insufficiency and normal biventricular function.

Discussion: While ARF is considered to be quite rare in developed countries it has a relatively high prevalence rates in some communities, including Northern Manitoba and Ontario where rates are estimated to be 21.3 per 100 000. Incidence in the general Pediatric population of Canada is reported to be 2.9 per 1 million per year.

Conclusion: This case illustrates the importance of considering other diagnoses, including ARF in Pediatric patients presenting with coronary artery aneurysms and ectasia when other features of KD are not present. These two diseases have very different management both initially and long term, and they are both associated with significant morbidity and mortality long term if not managed correctly. Although ARF is thought to be nearly eradicated in developed countries the prevalence above highlights that this disease should still be considered in the appropriate clinical scenarios.

\section{1}

A Description of Diagnoses in a University Based Group Rheumatology Practice

Steven Katz (University of Alberta, Edmonton)

Objectives: This study aims to describe the frequency of rheumatic diagnoses in a large University based rheumatology practice. There are few studies which describe the range and frequency of diagnoses seen in a rheumatology practice, and none outside the Unites States or during the new era of biologic treatment. Gaining a better understanding of a rheumatology practice can have impact on recruitment into the specialty, training objectives and assessment, while also informing practitioners and payors about manpower needs within the field.

Methods: Twelve of fourteen Rheumatologists at the Kaye Edmonton Clinic in Edmonton, Alberta keep patient records on eClinician, an enterprise electronic medical record. ICD9 codes for all patient visits were extracted from eClinician between January 1 and December 31, 2016. The ICD9 codes were counted for frequency; those with a frequency of 10 or more were included; similar codes and/or diagnoses were grouped into common diagnostic categories. More than one ICD9 code may have been provided for a given clinic visit. Visit codes were further sorted by whether they represented a first visit or a follow up visit.

Results: In 2016, there were 10,593 total diagnoses assigned to 10,177 patients. 3006 diagnoses were assigned to 2779 new patients, and 7541 were assigned to 7398 follow up patients. A total of 384 unique ICD9 diagnostic codes were used, 274 for new visits and 276 for follow up. The 7 most common diagnoses for new patient visits were rheumatoid arthritis $(\mathrm{N}=435$, $14 \%$ of total new diagnoses), soft tissue pain ( $\mathrm{N}=272,9 \%)$, osteoarthritis $(\mathrm{N}=260,9 \%)$, fibromyalgia $(\mathrm{N}=190,6 \%)$, spondyloarthritis $(\mathrm{N}=102$, $3 \%)$, psoriatic arthritis $(\mathrm{N}=98,3 \%)$, and systemic lupus erythematosus

Personal non-commercial use only. The Journal of Rheumatology Copyright $\odot$ 2018. All rights reserved. 
(SLE) $(\mathrm{N}=96,3 \%)$. The 7 most common diagnoses for follow up patient visits were rheumatoid arthritis $(\mathrm{N}=2312,31 \%)$, SLE $(\mathrm{N}=580,8 \%)$, psoriatic arthritis $(\mathrm{N}=398,5 \%)$, small vessel vasculitis $(\mathrm{N}=371,5 \%)$, ankylosing spondylitis $(\mathrm{N}=294,4 \%)$, osteoarthritis $(\mathrm{N}=241,3 \%)$, and polymyalgia rheumatica $(\mathrm{N}=216,3 \%)$.

Conclusion: Inflammatory autoimmune diseases are commonly seen by rheumatologists at this University based centre, more for follow up visits than new patients. Rheumatoid arthritis is the most common diagnosis seen in both new and follow up visits. Further study at other university and community-based centres may help validate these results. These results may be useful to inform those guiding the rheumatology profession.

202

Attracting Internal Medicine Residents to Rheumatology: A 2018 Update

Steven Katz (University of Alberta, Edmonton)

Objectives: A 2009 Canadian study demonstrated Internal Medicine (IM) Residents were more likely to decide on a rheumatology subspecialty residency if they were given the opportunity for a rheumatology elective earlier in their medicine residency compared to other subspecialties. The study also demonstrated residents were more likely than other IM fields to complete their rheumatology training at the same school as IM. With a greater number of IM residents in Canada, the introduction of CaRMS to the subspecialty match, and more focus on job opportunities, this study was repeated to determine if these factors are still important to consider in attracting residents to rheumatology training.

Methods: Using data from the Canadian Post-MD Education Registry, the total number, percentage, and location of rheumatology trainees from English speaking Canadian IM programs were tracked between 2014-2016. Using a similar algorithm to the previous study, opportunities to complete a rheumatology rotation in each of year medicine training were calculated based on data posted by each program either on their home website or their CaRMS webpage, and correlated to the number of rheumatology trainees from each site. It was also determined how many first-year rheumatology trainees did their training at the same institution as their IM training over the same 3 academic years. Comparison was made to other medicine subspecialties (endocrinology, gastroenterology, infectious diseases, and nephrology) and the previous 2009 study which tracked residents between 2005-2007.

Results: 62/1002 (6\%) total residents entered a rheumatology training program in Canada between 2014-2016. This is greater than the 23/651 (3.5\%) who entered rheumatology training in the previous study. Compared to the previous study, a positive correlation between opportunities to complete a rheumatology rotation and rheumatology trainees was no longer identified. As before, there was still no correlation found for other IM fields. Seventy-three percent of rheumatology trainees completed their training at the same site as their core medicine residency, only slightly less than the 2009 study (78\%), and more than most other subspecialties except for infectious diseases $(78 \%)$.

Conclusion: Timing of rheumatology exposure may no longer be as important as a decade ago to attract residents to rheumatology residency training, although a majority of residents still complete their rheumatology and IM training at the same site. The most significant finding is the relative and absolute increase in rheumatology trainees overall compared to a decade ago. This may help inform future recruitment strategies and work force planning.

203

Improving Security and Accessibility of the Rheumatology Consult List: User Survey Results of Real World Testing of RheumDASH

Steven Katz (University of Alberta, Edmonton)

Objectives: Maintaining an up-to-date list of patients on the rheumatology in hospital consult team is an important aspect of patient care. Finding the right balance between maintaining patient security while ensuring team member accessibility is increasingly difficult as patient and electronic security standards escalate. RheumDASH was developed to address these issues. This study was performed to assess user satisfaction of this pilot project and determine areas for improvement.

Methods: RheumDASH was developed and launched in January 2017 as a secure, accessible, mobile web-based application with a paper based option that meets Alberta privacy standards to replace the previous consult list application (a Microsoft Word document on one poorly accessible computer) at the University Hospital in Edmonton. A survey of all users - staff rheumatologist, subspecialty residents and rotating residents - was undertaken in September 2017 to determine user "happiness" with RheumDASH compared to previous consult list experiences. The domains of user experience, security, accessibility, impact on patient care and handover, and overall impression were assessed. How users interacted with the application was also assessed. Free text positive and negative comments were also specifically elicited.

Results: $23 / 47$ (49\%) users completed the survey (13/18 staff/subspecialty fellows, 10/29 rotating residents). Compared to past experiences, $77.8 \%$ thought RheumDASH was equally or more user friendly, $91.3 \%$ thought it was equally easy or easier to access, $82.6 \%$ thought RheumDASH was more secure, while a majority felt RheumDASH positively impacted their ability to both provide patient care and improve patient handover. The overall impression of RheumDASH was: $8.7 \%$ Excellent, $65.2 \%$ Above Average, $21.7 \%$ Average, $4.4 \%$ below average and no scores of poor. $78.3 \%$ of users had personally updated the patient list, $94.4 \%$ of whom did it on their desktop and $27.8 \%$ on their mobile device. All users updated the list at some point at the start or end of the day, with $23.5 \%$ updating the list during patient rounds. $56.5 \%$ of users viewed the list both electronically and on paper, $21.7 \%$ electronically only while $21.7 \%$ viewed it on paper only. Specific positive comments were around the themes of accessibility, mobility, and security. Negative comments were primarily around poor formatting when printed and difficulty logging on to the application.

Conclusion: A beta version of an electronic secure accessible rheumatology consult list was well received. With the results demonstrated in this survey, a second version will be developed and implemented to account for the feedback provided.

\section{4}

Use of a Clinical Scoring System in Risk Stratifying Patients with Suspected Giant Cell Arteritis

Angela Hu (McMaster University, Hamilton); Karen Beattie (McMaster University, Hamilton); Sankalp Bhavsar (McMaster University, Hamilton); Kimberly Legault (McMaster University, Hamilton); Ryan Rebello (McMaster University, Hamilton); Nader Khalidi (McMaster University, St Joseph's Healthcare Hamilton, Hamilton)

Objectives: To examine the utility of a clinical scoring system for the risk stratification of patients with suspected giant cell arteritis (GCA) in accurately predicting diagnosis.

Methods: From a prospective database, we conducted retrospective chart reviews of patients with suspected GCA. Patients were assessed by a rheumatologist and categorized as low, moderate or high risk pre-test clinical impression. Given a normal scalp artery on MRI has been associated with negative temporal artery biopsy, the majority of patients deemed low/moderate risk underwent an MRI first and only received a temporal artery biopsy if results were positive or inconclusive. The Bhavsar-Khalidi (BK) score was developed based on clinical experience and a literature review of signs and symptoms with the highest likelihood ratios associated with GCA. Included in the BK score are the following criteria and their respective weightings: typical headache (3), atypical headache (1), scalp tenderness (2), jaw claudication (3), sudden visual loss (4), other visual symptoms (1), polymyalgia symptoms (1), constitutional symptoms (1), temporal artery tenderness (2), temporal artery decreased pulse (2), ESR > 40 or CRP $>10$ (1). A BK score $\leq 4=$ low, 5-7 = moderate, and $\geq 8=$ high A BK score was calculated for each patient and compared with a positive or negative diagnosis of GCA based on clinician final diagnosis. Agreement between BK score and physician pre-test impression was determined, as was 
sensitivity and specificity of physician pre-test impression compared to final GCA diagnosis.

Results: Included in the analyses were 78 patients. Using low and high risk patients, sensitivity of the BK score was $80 \%$, specificity was $72 \%$. Sensitivity of physician pre-test clinical impression was $42 \%$ and specificity was $91 \%$. Patients at moderate risk BK score were further analysed to examine which criteria were most predictive of a positive GCA diagnosis. Criteria with the highest associated odds ratios were: elevated CRP (OR 13.3) elevated ESR (OR 2.9) and sudden visual loss (OR 2.5). The kappa statistic between BK score and clinician pre-test impression was 0.82 (95\% $\mathrm{CI}=0.57-1.00)$.

Conclusion: The BK scoring tool may be useful for physicians to classify risk of GCA. While research supports the use of MRI for low-moderate risk patients, high risk patients should proceed to biopsy. Using certain markers (e.g. elevated CRP and ESR) to further stratify risk in moderate patients may help guide a clinician's decision to initiate steroid therapy. Future research will validate the BK score in a larger population and explore the tool's utility in primary care.

\section{5}

MDA5 Positive Clinical Amyopathic Dermatomyositis (CADM): A Single Centre Case Series of 10 Patients

Kun Huang (University of British Columbia, Vancouver); Kamran Shojania (University of British Columbia Faculty of Medicine; Arthritis Research Canada, Vancouver); James Yeung (Vancouver); Antonio Avina-Zubieta (University of British Columbia Faculty of Medicine; Arthritis Research Canada, Richmond)

Objectives: Positive MDA5 antibody has been reported in 50-73\% of CADM. About half of MDA5+ CADM patients die of rapid progressive interstitial lung disease (RP-ILD), defined as worsening dyspnea within 1 month that required high-flow oxygen therapy or ventilator support in previously stable patients. This study aims to review the clinical features and outcome of 10 cases of MDA5+CADM patients at a tertiary referral centre. Methods: Myositis antibody testing became available at Vancouver Coastal Health since 2014. We retrospectively reviewed clinical presentations, laboratories, imaging studies, treatment and outcome of patients with MDA5 antibody. The data is presented descriptively.

Results: Total 10 cases (3 male and 7 female) of MDA5+ CADM were identified (2014-2017). Mean age was $51.3 \pm 10.2$ years. Seven were Chinese, 2 Caucasians and 1 from Mauritius. All 10 patients had typical DM rash (heliotrope, Gottron's, mechanic's hands, periungual erythema). Cutaneous ulcers over the extensor surfaces of joints were seen in 4/10 and palmar papules in $2 / 10$, both of which were characteristic for MDA5+ CADM. Only 3/10 patients had CK elevation with little muscle weakness; all others had normal CK and no detectable weakness. ILD was present in all 10 patients. $6 / 10(60 \%)$ patients had RP-ILD and 4 died within 3 months of onset of worsening dyspnea, despite steroid, cyclophosphamide, Rituximab, cyclosporine and IVIG; 2 survived after receiving lung transplant. 4/10 (40\%) patients who were alive had stable ILD. 5/6 patients with RP-ILD (83.3\%) had coexisting Ro52 antibody whereas only $1 / 4$ patients with stable ILD (25\%) had Ro52. Of the 4 patients with stable ILD, one had worsening cutaneous ulceration and ILD that required IV cyclophosphamide; one responded to tacrolimus with improving respiratory symptoms; one had panniculitis refractory to IVIG, hydroxychloroquine, azathioprine and tofacitinib, and ultimately responded to IV cyclophosphamide; the last patient went with traditional Chinese medicine and lost follow up. None had underlying malignancy.

Conclusion: In this case series of 10 patients with MDA5+ CADM, 70\% were Chinese, a finding similar to previous reported higher incidence in Asians. Refractory panniculitis can be seen in MDA5+ CADM. Coexisting Ro52 may be predictive of worse prognosis. All patients had ILD with $60 \%$ RP-ILD and $40 \%$ mortality within 3 months. Lung transplantation was successful in 2 patients with RP-ILD. It is important to recognize the characteristic cutaneous and pulmonary features of this entity early on as mortality is high. Early intensive immunosuppression is instrumental for refractory cutaneous manifestation and/or progressive ILD.

\section{6}

Inflammatory Myositis Associated with Myasthenia Gravis with or without Thymic Pathology: Case Series and Literature Review

Kun Huang (University of British Columbia, Vancouver); Michelle Mezei (University of British Columbia, Vancouver); Kamran Shojania (University of British Columbia Faculty of Medicine; Arthritis Research Canada, Vancouver); Kristine Chapman (University of British Columbia, Vancouver); Neda Amiri (Division of Rheumatology, University of British Columbia, Vancouver); Natasha Dehghan (University of British Columbia, Division of Rheumatology, Vancouver)

Objectives: The association of myasthenia gravis (MG) and inflammatory myositis (IM) is rare and often only one of the diseases is diagnosed. Here, we report a case series of 6 patients with concurrent MG and IM who were followed at the Neuromuscular Disease Unit (NMDU) at a tertiary centre in Vancouver, BC. We also conducted literature review on clinical characteristics, diagnostic challenge and management of this condition.

Methods: In this study, we retrospectively examined patients seen at NMDU from 2004 to 2017 who had diagnosis of concurrent MG and IM. We reviewed medical records to assess their clinical presentations, laboratory findings, imaging studies and electrophysiological features. The data is presented descriptively.

Results: We identified 6 patients with MG-IM overlap. Three patients had simultaneous onset of MG and IM, 2 of whom presented with myasthenia crisis and fulminant myositis. In the other 3 patients, MG was the initial presentation and IM occurred 3-11 years after MG. Diagnosis of MG was confirmed with clinical features, electromyography and/or serology. All had symptoms of MG with predominant ocular or bulbar weakness. Among these 6 patients, 3 had underlying thymic pathology including two benign thymoma and one stage IV thymoma; all 3 patients had Acetylcholine Receptor (AChR) Antibody. Of the 3 patients with no thymic pathology by computed tomography (CT) or thymectomy, 1 had high positive AChR antibody and 2 were negative. Four patients had biopsies confirming the diagnosis of dermatomyositis or polymyositis. The other 2 patients declined biopsy; however, their MRI and EMG findings were consistent with IM. Only one patient had typical dermatomyositis rash. Among the 3 patients with underlying thymic pathology, thymoma were resected; all 3 were treated with high dose glucocorticoid, IVIG, and methotrexate with complete remission after 2 years. Of the 3 patients with no thymic pathology identified, one patient $(\mathrm{AChR}+)$ was in remission on mycophenolate and passed away from pancreatic cancer; two patients (AChR-) had refractory MG and IM, and both responded to rituximab.

Conclusion: In summary, this is one of the largest case series with MG-IM overlap with or without thymic pathology. It is very important to recognize such association and the different pattern of muscle involvement because therapies may be adjusted to treat both conditions. In patients with thymic pathology, conventional disease modifying agents, IVIG and glucocorticoid in addition to thymoma resection appear to be effective. In patients with refractory MG and myositis who were $\mathrm{AChR}$ negative, rituximab may be effective.

\section{7}

A Great Masquerader: Chronic Active Epstein Barr Virus for the Rheumatologist

Amieleena Chhabra (BC Children's Hospital, Vancouver); Raidan Alyazidi (BC Children's Hospital, University of British Columbia, Vancouver); Andrea Human (BC Children's Hospital, Vancouver)

Objective: To report an interesting presentation of chronic active Epstein Barr virus (CAEBV) as it pertains to rheumatologists.

Case Presentation: A previously healthy 9 year old girl presented to hospital with recurrent angioedema and mouth ulcers in the context of a 6 month history of perioral rash, intermittent fevers, night sweats, headaches, and fatigue. Examination revealed periorbital edema, tongue and lip swelling with diffuse oral mucosal ulceration. She was tachycardic (126 beats/minute), but had normal heart sounds, clear chest sounds, and palpable hepatomegaly. Neurological and musculoskeletal exams were normal.

Personal non-commercial use only. The Journal of Rheumatology Copyright (C 2018. All rights reserved. 
Ophthalmological exam showed bilateral anterior uveitis. Imaging included a chest radiograph showing trace pleural effusions and interstitial edema, echocardiogram showing coronary aneurysms, and abdominal ultrasound confirming hepatomegaly. Investigations revealed mild pancytopenia, transaminitis, elevated $\mathrm{IgG}$, and elevated von Willebrand factor (vWF) antigen, but normal CRP, ESR, complements and C1 esterase inhibitor. Antinuclear antibody 1:160, borderline positive anti-Ro, positive anti-smooth muscle antibody, negative anti-LKM, negative double stranded DNA, negative anti-nuclear cytoplasmic antibody profile, and negative other extractable nuclear antigens. Urinalysis was normal. The coronary aneurysms and elevated vWF antigen raised the possibility of systemic vasculitis, but the cytopenias, normal inflammatory markers, and unusual autoantibody profile were not in keeping with this diagnosis. An angiogram of the aorta, abdominal and renal vessels was normal. Infectious work up revealed high EBV PCR at $42,000 \mathrm{copies} / \mathrm{ml}$ in peripheral blood, suggesting an active EBV infection. However, the presence of positive EBV IgG and negative IgM, in the context of her unique constellation of features led to the consideration of CAEBV. Diagnosis was confirmed by a buccal mucosa biopsy showing superficial mucosal erosions with increased EBV positive cells on double staining (CD3 positive, CD20 negative). The EBV viral load was higher in peripheral NK and T cells, suggesting an NK/T cell subtype. She was initially treated with one dose of intravenous immunoglobulin at $2 \mathrm{~g} / \mathrm{kg}$ with marginal response, followed by 3 intravenous methylprednisolone pulses at $30 \mathrm{mg} / \mathrm{kg}$ with good response. She was started on aspirin for her aneurysms, azathioprine maintenance therapy, and steroids were tapered accordingly. Given the progressive, relapsing and life-threatening natural history of this disease, our patient is currently undergoing evaluation for hematopoietic stem cell transplant as potentially curative therapy.

Conclusion: This case highlights an important, albeit rare, differential diagnosis that rheumatologists should consider when evaluating patients with complex clinical presentations. CAEBV can manifest with a vast spectrum symptoms, many of which overlap with systemic autoimmune and inflammatory conditions.

\section{8}

Blastocystis Hominis Related Reactive Arthritis in a Recent Traveller Matthew Piche (Western University, London); Sherry Rohekar (Western University, London)

Case Presentation: 60 year old female referred for one month history of polyarthritis involving the 2nd, 3rd, 4th MCPs bilaterally, DIPs, PIPs and knees bilaterally. This occurred after receiving the Shingles vaccine along with feeling unwell with general malaise, nausea and a subjective fever followed by an erythematous and well-circumscribed, non pruritic rash between her shoulder blades extending to the buttocks. This resolved over 6-8 hours with taking antihistamines and did not return. Remaining unwell, two days later, she presented to community ER with bilateral swelling to MCPs, PIPs, DIPs, wrists, knees and feet. Advil and Tylenol was prescribed for symptom management. She had a history of loose stool for a number of years and used imodium on a PRN basis. Loose stool were somewhat more pronounced during the time of arthritis. Further questioning revealed recent trips to Morocco, Portugal, and South America with exploration of the local market cuisines of these countries. Labs and investigations included ANA 1:160, ENA negative, DsDNA 1, RF negative, ESR 66, CRP 146.3, cell count: WBC 7.5, HgB 114, Ptls 365, eosinophils 0.8, creatinine 58, ALT 44. Stool for ova and parasites was positive for blastocystis hominis.

Discussion: Blastocystis hominins is a protozoan with various life stages, is transmitted between animals and humans via ingestion of cysts and is not usually a pathogenic organism. This patient noted a marked improvement within two days of initiation of metronidazole characterized by decreased synovitis, pain, and improved range of motion. All case reports included patients who travelled to countries ranging from India and Nepal to Tanzania. The clinical picture was characterized by malaise and arthralgias with subsequent fulminant arthritis in immunocompetent patients. In accordance with the literature our patient improved with initiation of metronidazole within two days. This lends credence to the hypothesis that this clinical picture was precipitated by blastocystis hominis. Further interesting features of this case include the development of symptoms so soon after administration of the shingles vaccine and antibiotics to treat a UTI

Conclusion: This case illustrates what appears to be the first documented case of blastocystis hominis related reactive arthritis in the Canadian literature. The clinical presentation and improvement with metronidazole are in keeping with previous documented cases. This case is highlighted by unique features such as the concomitant administration of the shingles vaccine which may have acted in an immunomodulatory role which is unique to this case as compared to the known literature.

\section{9}

Efficacy of Commonly Prescribed Analgesics in the Management of Osteoarthritis: A Literature Review

Mohan Stewart (University of British Columbia, Vancouver); Jolanda Cibere (Arthritis Research Canada, Richmond); EC Sayre (Arthritis Research Canada, Richmond); Jacek Kopec (Arthritis Research Canada, Richmond) Objectives: Osteoarthritis (OA), the most common form of arthritis, can significantly impact patient quality of life through pain and difficulty with mobility. The most commonly used treatments for OA are non-surgical, and analgesia is a cornerstone of OA management. The aim of this review is to obtain current, literature-based estimates of the effect of common pharmacologic treatments on pain reduction in OA. In practice, patients experience pain relief likely as a result of drug effect in combination with an element of placebo effect. As such, we aim to include placebo effect in our estimates of OA pain reduction.

Methods: A MEDLINE search (2006-2016) was conducted for literature regarding acetaminophen, oral NSAIDs, topical NSAIDs, COX-2 inhibitors, and opioids in the treatment of OA pain. Randomized controlled trials that reported both baseline and endpoint pain, or baseline pain and mean change in pain, were included. Each treatment arm was counted separately in actively controlled trials. Effect on pain was estimated using relative change (RC) in pain, and expressed as percentage change. The RC metric enables assessment of pain reduction without subtracting placebo effect. An overall effect for each drug category was obtained as a weighted average of study-specific effects, with weights based on each study's sample size.

Results: 29 studies were included in this review. Relative change in pain was estimated in a total of 43 treatment arms (acetaminophen $n=6$, oral NSAIDs $n=9$, topical NSAIDs $n=8, \operatorname{COX}-2$ inhibitors $n=9$, and opioids $\mathrm{n}=11)$. Relative $(\%)$ changes in pain were found to be as follows: acetaminophen $=32.5$, oral NSAIDs $=34.3$, topical NSAIDs $=40.9$, COX-2 inhibitors $=36.9$, and opioids $=35.4$.

Conclusion: The effects of 5 major drug categories in the treatment of OA pain were reviewed and analyzed with data extracted from 29 studies published between 2006-2016. Acetaminophen was found to have an RC value close to that of oral NSAIDs. The effects of oral NSAIDs, COX-2 inhibitors, and opioids in reducing pain were similar to what has been demonstrated in previous literature. The greater RC value obtained for topical NSAIDs compared to oral NSAIDs may be attributed to differences in the drugs evaluated in oral NSAID vs. topical NSAID studies, differences in baseline pain between the two groups, and variation in the magnitude of placebo effect between oral and topical formulations.

\section{0}

Efficacy of Treatments for Pain Associated with Trapeziometacarpal (Thumb Base) Osteoarthritis: A Systematic Review (Part 1 Conservative Interventions)

Tokiko Hamasaki (Centre hospitalier de 1'Université de Montréal Research Center, Montreal); Sylvain Laprise (Physiotherapie MyoSynergie, Amos); Patrick Harris (Department of Surgery, Faculty of Medicine, Université de Montréal, Montreal); Nathalie Bureau (Centre hospitalier de l'Université de Montréal Research Center, Montreal); Nathaly Gaudreault (School of Rehabilitation, Faculty of Medicine and Health Sciences, Université de Sherbrooke, Sherbrooke); Lyne Lalonde (Faculty of Pharmacy, Université de Montréal, Montreal); Daniela Ziegler (Centre hospitalier de l'Université 
de Montréal Medical Library, Montreal); Manon Choinière (Centre hospitalier de l'Université de Montréal Research Center, Montréal)

Objectives: Trapeziometacarpal (thumb base) osteoarthritis (TMO) is a chronic, painful, debilitating disease, affecting 5-7\% of adults in North America. Even though the thumb is solicited in $50 \%$ of hand function, thus essential for daily activities, TMO management is not optimal because knowledge of evidence-based treatments is lacking. This systematic review (SR), therefore, aims to identify all of the existing interventions of different disciplines for TMO with documented efficacy in terms of pain, physical function, psychological well-being, quality of life, and/or treatment satisfaction. At this annual meeting, Part 1, the efficacy of the conservative interventions will be presented (Part 2, the efficacy of surgical interventions is still ongoing).

Methods: The protocol was developed based on the Cochrane Handbook for Systematic Reviews of Interventions, registered with PROSPERO, and published in BMJ Open. Bibliographic search was conducted by our academic librarian. The critical appraisal and data extraction of the included SRs, randomized controlled trials (RCT) and non-RCTs were separately performed by two authors. Efficacy estimate of a given outcome for a given intervention with respect to another one or a placebo (i.e., standardized mean difference for continuous variables and relative risk for dichotomous variables) was extracted from the SRs when available, otherwise computed from the RCTs. If no SR or RCT was available for the given outcome for the given intervention, non-RCT data were used to estimate its efficacy.

Results: Of 4986 identified references through bibliographic search, 21 SRs, 52 RCTs, and 47 non-RCTs met the selection criteria, including the following interventions: naproxen, transdermal steroids delivery, diclofenac gel, steroid/hyaluronate/saline injections, dextrose prolotherapy, acupuncture, leech therapy, stinging nettle, orthoses, laser therapy, manual therapy, exercises, combinations of orthosis-exercises/orthosis-exercises-joint protection/exercises-manual therapy, and surgical interventions. The weak to moderate levels of evidence support utilization of the following conservative interventions for TMO for pain and/or physical function: naproxen, leech therapy, dextrose prolotherapy, steroid/hyaluronate/saline injections, orthoses, manual therapy, and combinations of orthosis-exercises/ orthosis-exercises-joint protection/exercises-manual therapy. No studies explored efficacy on psychological well-being, and only a few, on quality of life, and/or treatment satisfaction.

Conclusion: Part 1 of this SR allowed to identify the conservative interventions for TMO whose efficacy was relatively well documented. However, there are only a few clinical trials available for each intervention, thus further studies are needed to provide stronger evidence. Ongoing Part 2 regarding the efficacy of different surgical interventions for TMO will complete this SR which will help healthcare providers to choose the best intervention for their TMO patients.

\section{1}

Cutaneous Leukocytoclastic Vasculitis Associated with an Intraarticular Injection of Hyaluronic Acid

Rachel Boyle (Dalhousie University, Halifax); Volodko Bakowsky (Dalhousie University, Halifax)

Case Presentation: A 58-year-old woman with osteoarthritis of the knee received an intra-articular knee injection of hyaluronic acid (HA). This was her first HA injection. One week after the injection, the patient developed a rash over her lower extremities and abdomen. $50 \mathrm{mg}$ of prednisone was administered for three days, followed by a tapering regime, and referral to rheumatology was made. The patient was reviewed in the rheumatology clinic two weeks later. The rash was persistent. She had no history of sinus issues, cough, hemoptysis, dyspnea, fever or arthralgia. There was no history of a previous similar rash, recent infections or any new medications. Her past medical history was remarkable for tachyarrhythmia-induced cardiomyopathy treated with ablation, obstructive sleep apnea and depression. Her current medications included prednisone $5 \mathrm{mg}$ daily, citalopram, lansoprazole, and acetaminophen with codeine. Her family history was significant for type I diabetes, lupus and rheumatoid arthritis. On examination, vital signs were stable and she was afebrile. Head and neck, chest and cardiovascular examinations were unremarkable. Knees demonstrated medial joint space tenderness without evidence of effusion. Cutaneous examination revealed palpable purpura predominantly over her lower extremities, with extension to the skin of her abdomen and forearms. Several areas of rash were confluent and beginning to ulcerate. Lab work up revealed normal $\mathrm{CBC}, \mathrm{Cr}$, LFTs, CRP, ESR and urinalysis. ANCA and ANA were negative. A chest $\mathrm{X}$-ray was reported normal. A diagnosis of isolated cutaneous leukocytoclastic vasculitis (LCV) was suspected. Due to the widespread distribution of her rash and ulceration, she was prescribed $40 \mathrm{mg}$ of prednisone daily with a slower tapering regimen. A skin punch biopsy of a fresh lesion was performed. Histologic examination confirmed LCV. The patient had difficulty tapering prednisone below $10 \mathrm{mg}$ initially due to recurrence of new skin lesions. After five months, however, she was able to taper off prednisone completely. She has not had recurrence of vasculitis over the past 3 years. Discussion: There is only one previous report of LCV related to an intraarticular injection of HA (1). Similarly, that patient also developed vasculitis on both legs, abdomen and arms within one week of the injection. There is also one report of vasculitis developing after cosmetic facial injections of HA (2).

Conclusion: To our knowledge this is the second report of LCV following an intra-articular HA injection and the first to describe the clinical course and resolution. (1) Huskisson, EC. Rheumatology 1999;38:602-07. (2) Alijotas $\square$ Reig, J. Dermatol Surg 2009;35:395-98.

\section{2}

Glucocorticoid-induced Osteoporosis Preventative Care in Rheumatology Patients: A Quality Assurance Study

Gregory Koller (University of Alberta, Edmonton); Steven Katz (University of Alberta, Edmonton); Carrie Ye (Division of Rheumatology, University of Alberta, Edmonton)

Objectives: Systemic glucocorticoid therapy is commonly used in the management of rheumatologic diseases. While their efficacy in treatment of inflammatory illness is indisputable, glucocorticoids also come with many side effects. A common, yet manageable, adverse effect is glucocorticoid induced osteoporosis (GIOP) with previous studies reporting fractures in 30 to $50 \%$ of patients receiving long-term glucocorticoid therapy. There are several publications that have shown GIOP care is not optimal in the general population (roughly $25 \%$ are started on pharmacotherapy and get baseline BMD scan); however, little is published on GIOP care amongst patients under the care of rheumatologists. This study looks at the proportion of rheumatology patients who received GIOP preventive care including calcium, vitamin D, pharmacotherapy, and BMD study within 6 months of starting a moderate dose of long-term prednisone.

Methods: A population based retrospective quality assurance study of adults seen at the University of Alberta Rheumatology Clinic was performed using Alberta's electronic outpatient medical record "eClinician." Records of adult patients prescribed prednisone from January 1st to December 31st, 2016 by a rheumatologist were initially included for review. The average prednisone dose within the first 3 monthsof prescription was calculated. Those whose average dose met the American College of Rheumatology (ACR) treatment guidelines for GIOP prevention $(\geq 7.5$ $\mathrm{mg}$ /day for $\geq 3$ months) were assessed for concurrent GIOP preventive care including supplementation of calcium and vitamin $\mathrm{D}$, prescription of pharmacotherapy, and requisition and completion of DXA scan within 6 months of starting prednisone. Exclusion criteria included: established osteoporosis; pre-existing pharmacotherapy; previous fragility fracture; DXA within 1 year prior to starting prednisone.

Results: A total of 745 discreet courses of prednisone were prescribed in 433 patients with 113 meeting the above inclusion criteria. Following the prednisone prescription, $83 \%$ (94/113) were supplemented with vitamin D and calcium, 50\% (56/113) were prescribed osteoporosis pharmacotherapy, and $46 \%(52 / 113)$ were given both. $25 \%$ (28/113) of patients had DXA imaging ordered by the rheumatologist within the first 6 months; of these, $86 \%(24 / 28)$ of patients completed the DXA. $16 \%(18 / 113)$ received

Personal non-commercial use only. The Journal of Rheumatology Copyright @ 2018. All rights reserved. 
calcium, vitamin D, pharmacotherapy, and completed BMD study. Multivariate analysis was performed looking at variables associated with utilization of GIOP measures.

Conclusion: Rheumatologists at our centre appear to have higher implementation rates of GIOP preventative care compared to studies of administrative databases of the general population. However, it remains suboptimal. We will explore variables associated with rates of GIOP care in order to identify populations in which to target further interventions.

213

Trends in Adherence to Glucocorticoid-Induced Osteoporosis Guidelines: Results from the CATCH Cohort

Stephanie Gottheil (Western University, London); Orit Schieir (Notre-Dame-de-Grace); Carter Thorne (Southlake Regional Health Centre, Newmarket); Gilles Boire (Université de Sherbrooke, Sherbrooke); Diane Tin (Southlake Regional Health Centre, Newmarket); Susan Bartlett (McGill University, Montreal); Boulos Haraoui (Department of Medicine, Centre Hospitalier de l'Université de Montréal, Montréal); Carol Hitchon (University of Manitoba, Winnipeg); Edward Keystone (Mount Sinai Hospital, University of Toronto, Toronto); Vivian Bykerk (Hospital for Special Surgery, New York); Janet Pope (Western University, Department of Medicine, Division of Rheumatology, London); CATCH Canadian Early Arthritis Cohort Investigators (Toronto)

Objectives: RA patients on chronic glucocorticoids are at high risk of osteoporosis and fracture. Guidelines on prevention of glucocorticoid-induced osteoporosis (GIOP) recommend using anti-osteoporotic medication (AOM), but adherence remains low. This study aimed to analyze AOM use in early RA, and to compare the application of ACR 2010 versus 2017 guidelines.

Methods: Data were obtained from the CATCH cohort, a multi-center prospective study of early RA patients. This analysis included participants $>50$, without baseline osteoporosis, with $>=6$ months followup. Adherence to 2010 guidelines was determined based on use of AOM in patients receiving $>=7.5 \mathrm{mg}$ prednisone daily for $>=3$ months. Adherence to 2017 guidelines was determined based on use of AOM in patients receiving $<=$ $2.5 \mathrm{mg}$ prednisone daily for $>=3$ months and FRAX $>=10$.

Results: Participants $(n=1468)$ had mean age $63(9), 933(64 \%)$ female, and mean DAS28-ESR 5.0 (1.4). $282(19 \%)$ patients met criteria for GIOP prophylaxis according to 2010 guidelines, and this number decreased over time. Only $54(19 \%)$ of eligible patients received AOM. Guideline adherence was higher when using 2017 criteria (27\%) vs. 2010 criteria (20\%).

Conclusion: In this study, approximately 1 in 5 patients met criteria for GIOP prevention according to 2010 ACR guidelines but only $19 \%$ received AOM. Chronic use of glucocorticoids decreased over time, suggesting either less frequent use or faster tapering. There was increased adherence when applying 2017 guidelines compared to 2010, suggesting that newer guidelines are more in keeping with current practice patterns.

\section{4}

Practice Variations in Treatment of Pediatric ANCA-associated Vasculitis (AAV) - A Pediatric Vasculitis Initiative (PedVas) Study Audrea Chen (University of British Columbia, Vancouver); Cherry Mammen (BC Children's Hospital, Vancouver); David Cabral (Division of Rheumatology, Department of Pediatrics, BC Children's Hospital Research Institute \& University of British Columbia, Vancouver); Kimberly Morishita (BC Children's Hospital, Vancouver)

Objectives: Routine practices of physicians for corticosteroid (CS) treatment of children with ANCA-associated vasculitis (AAV) are not previously well described. The optimal CS dosing and weaning schedule for remission induction is not known. Our objective is to provide a description of CS use in AAV patients with renal disease in an international cohort.

Methods: Patient data was obtained from ARCHiVE, an international pediatric vasculitis registry (40 contributing sites). Patients diagnosed with AAV $<18$ years old with pauci-immune glomerulonephritis on renal biopsy, hematuria, proteinuria, or a decrease in renal function requiring dialysis were included. CS treatment course and kidney function were examined at time of diagnosis (TOD), 4-6 months after presentation (post-induction (PI)) and at 12 months. GFR, estimated using bedside Schwartz equation, was used to stratify renal function into normal GFR (>90 ml/min/1.73m2), mildly reduced (MiR) (60-90 ml/min/1.73m2), moderately reduced (MoR) (30-60 $\mathrm{ml} / \mathrm{min} / 1.73 \mathrm{~m} 2)$, severely reduced $(\mathrm{SR})(15-30 \mathrm{ml} / \mathrm{min} / 1.73 \mathrm{~m} 2)$ and renal failure (RF) $(<15 \mathrm{ml} / \mathrm{min} / 1.73 \mathrm{~m} 2)$.

Results: 112 patients met inclusion criteria. 74\% had granulomatosis with polyangiitis (GPA), and 16\% microscopic polyangiitis (MPA). At TOD, 50\% had normal or MiR-GFR. 13\% had RF and $19 \%$ of patients required dialysis at TOD. For induction treatment, $37 \%$ received $>1.5 \mathrm{mg} / \mathrm{kg} / \mathrm{day}$ of CS (Hi-CS), $51 \%$ received $0.5-1.5 \mathrm{mg} / \mathrm{kg} /$ day of CS (Mod-CS) and $3 \%$ did not receive CS. In addition to oral CS at TOD, $44 \%$ had $\leq 3$ methylprednisolone pulses (IVMP), and 25\% received more than 3 IVMPs. Clinical features at TOD included: alveolar hemorrhage (37\% Hi-CS, 33\% Mod-CS), hypertension (27\% Hi-CS, 23\% Mod-CS), nephrotic syndrome (20\% Hi-CS, 5\% Mod-CS), GFR reduction (46\% Hi-CS, 60\% Mod-CS) and renal failure (20\% Hi-CS, $19 \%$ Mod-CS). At PI, $68 \%$ of patients were weaned to $0.1-0.5 \mathrm{mg} / \mathrm{kg} /$ day of prednisone, and $12 \%$ were on $<0.1 \mathrm{mg} / \mathrm{kg} / \mathrm{day}$ of CS At 12 months, $43 \%$ of patients were taking $<0.1 \mathrm{mg} / \mathrm{kg} / \mathrm{day}$ of CS and $36 \%$ were receiving $0.1-0.5 \mathrm{mg} / \mathrm{kg} /$ day.

Conclusion: The spectrum of renal impairment at TOD in patients with AAV and renal disease ranges from those with normal or MiR-GFR (50\%) to patients requiring dialysis (19\%). There is significant variability in CS treatment at TOD and CS weaning over the first 12 months of disease. Future analysis will examine whether such variations correlate with outcomes such as remission and damage.

\section{5}

The Link between Psoriasis, Psoriatic Arthritis and the Onset of Diabetes

Vivian Szeto (Toronto Western Hospital, Toronto); Justine Ye (Toronto Western Hospital, Toronto); Alan Zhou (University of Toronto, Toronto); Vinod Chandran (University of Toronto and University Health Network, Toronto); Dafna Gladman (University of Toronto, Toronto); Cheryl Rosen (University Health Network, Toronto Western Hospital, Toronto)

Objectives: Psoriasis currently affects more than 125 million people in the world. Approximately, $30 \%$ of patients with psoriasis will develop psoriatic arthritis (PsA) and are also at a higher risk of developing diabetes. The aim of this project was to determine whether patients with psoriasis and diabetes are at a higher risk for developing PsA than those who do not have diabetes. Methods: In this study, 2 cohorts were analyzed: 1) PsA patients followed in clinic since 1978 (PsA cohort) and 2) patients with psoriasis without PsA followed since 2006 (PsC cohort). The date of onset of psoriasis and PsA and the PASI scores were retrieved from the database and chart reviews. Patients documented to have diabetes were contacted to confirm date of onset of diabetes, type of diabetes, and treatment. The data was then statistically analyzed using Student's t-test and Chi-square to test the differences between the 2 cohorts.

Results: The PsC cohort included 642 patients of whom 62 patients developed PsA (converters) while the remaining 581 patients have not developed PsA (non-converters). $11 \%$ of the converters developed diabetes, while only $5 \%$ of the non-converters developed diabetes. Among the converters, $29 \%$ developed diabetes before PsA while $71 \%$ of the patients developed diabetes after their diagnosis of PsA. Whereas among the non-converters $33 \%$ of the patients developed diabetes before their psoriasis diagnosis and $67 \%$ developed their diabetes after their psoriasis diagnosis. The PsA cohort comprised of 1437 patients of whom 81 were diagnosed with diabetes. Of these 81 patients, $32 \%$ were diagnosed with diabetes before their PsA onset while 68\% were diagnosed with diabetes after the onset of PsA. The highest average PASI score in those diagnosed with diabetes before their PsA was 7.8 while those who were diagnosed with diabetes after their PsA onset had an average PASI score of 9.4. The standard prevalence ratio of diabetes based on Ontario's (Canada) population data (1996-2013) was calculated to be 1.35 and 1.12 for the PsA and PsC cohort, respectively.

Conclusion: These results show that patients with psoriasis and diabetes are 
not at a higher risk of developing PsA. Patients with psoriatic disease are more likely to develop diabetes after the diagnosis of PsA than before. Based on the standard prevalence ratio, patients already diagnosed with PsA have a slightly higher likelihood of developing diabetes.

\section{6}

Effect of Biologic DMARDs on Weight in Patients with Inflammatory Arthritis

Britney Jones (University of Alberta, Edmonton); Imran Hassan (University of Alberta, Edmonton); Walter Maksymowych (University of Alberta, Edmonton); Elaine Yacyshyn (Division of Rheumatology, University of Alberta, Edmonton)

Objectives: Inflammatory arthritis (IA) is a chronic disorder, including patients with Rheumatoid and Psoriatic arthritis. Currently, the estimated prevalence of IA in Canada is approximately $1 \%$. Obesity rates are higher in patients with IA than the general population and extremes of weight have been associated with higher disease activity. As there is an elevated rate of cardiovascular disease in this population, obesity is an important modifiable risk factor. Conflicting evidence exists around weight change associated with biologic Disease Modifying Antirheumatic Drugs (boDMARDs). This study sought to examine if weight or body mass index (BMI) is correlated with treatment with a boDMARD in patients with inflammatory arthritis. Secondary objectives examined disease activity as a function of weight change.

Methods: This was a retrospective analysis of 2373 patients enrolled in the Rheumatoid Arthritis Pharmacovigilance Program and Outcomes Research in Therapeutics (RAPPORT) Database. This database is a cohort of Rheumatoid and Psoriatic arthritis patients in Northern Alberta that captures demographic information including weight at baseline, 1 month, 3 months, 6 months, and subsequently yearly. Weight in pounds and body mass index were tracked over time in months using a random effect model with subject specific random effects. We considered DAS 28 into the model as a covariate.

Results: There was a small but statistically significant increase in weight (0.02 lbs, p $=<0.001)$ and BMI $(0.006 \mathrm{lbs}, \mathrm{p}=<0.001)$ over time. There was also a negative association between DAS 28 and increased weight $(-0.56, \mathrm{p}<0.001)$ as well as BMI $(-0.08, \mathrm{p}<0.001)$ suggesting an increase in weight was associated with lower disease activity.

Conclusion: Our data shows a small increase in weight associated with boDMARDs that is of uncertain clinical significance. Patients with IA on boDMARDs tend to gain weight over time. This may be reflective of improved disease control. The negative association between increased weight and decreased DAS 28 supports this assertion. Extremes of weight and BMI have previously been shown to be risk factors for poor long-term outcomes in patients with IA and it is important to keep in mind when counseling patients about lifestyle modifications. Future analysis could compare the rate of weight change in patients with IA to that of the general population as weight gain is associated with aging in general.

\section{7}

Need for Educational Activities on the Use of Methotrexate for Rheumatic Diseases Targeting Non-Rheumatologist Physicians: Results of a Survey in Guinea

Yan Liu (McGill University, Montreal); Aly Kamissoko (Ignace Deen Hospital, Conakry); Girish Mody (University of KwaZulu-Natal, Durban); Ines Colmegna (McGill University Hospital Centre, McGill University, Montreal); Carol Hitchon (University of Manitoba, Winnipeg)

Objectives: Methotrexate (MTX) is standard of care and first-line therapy for rheumatoid arthritis (RA) patients. Due to limited rheumatology manpower, the care of RA patients in developing countries of Africa is mainly provided by non-rheumatologists. Understanding how physicians use MTX is critical to assess the need and define the focus of future educational interventions.

Methods: In partnership with the sole rheumatologist in the country, we surveyed non-rheumatologist physicians from a university affiliated hospital in Conakry, the capital of Guinea, regarding their experience and comfort with prescribing methotrexate for rheumatic disease. This hospital is one of the two national hospitals and has 113 physicians. We report descriptive statistics. Values are $\mathrm{n}(\%)$ or median (range) unless otherwise indicated. Results: 41 physicians (35\% of university affiliated hospital physicians; 1 community based physician) completed the questionnaire. The majority were internists (75\%), family physicians (11\%) and orthopedic surgeons (14\%) with a median (range) number of years in practice of 5 (1-12). Only three physicians surveyed (7\%; 2 internists, 1 family physician) reported having previously used MTX for rheumatic diseases, with a range of 3-5 new prescriptions per year. MTX was prescribed mainly for RA. When all respondents were asked about their level of comfort to initiate MTX or to do the required follow-up of a RA patient on MTX with a scale of 0 (very uncomfortable) to 10 (very comfortable), 37 providers reported median values (range) of 2 (0-10) for both questions. When given a hypothetical situation of a RA patient whose disease status remained uncontrolled with MTX at a weekly dose of $10 \mathrm{mg}, 5 / 33$ physicians would increase the dose of MTX up to a range of $15-20 \mathrm{mg} /$ week, $3 / 33$ would add prednisone while keeping the patient on the same dose of MTX, and most (25/33) would refer to another physician due to lack of comfort in the management. The physicians reported their preferred modalities to increase confidence in using MTX for RA patients were taking a course (32/41), and having hands-on experience with an experienced physician (24/41). The highest priorities identified to improve the treatment of RA patients were training (17/41) and access to quality drug/laboratory testing (12/41).

Conclusion: Among non-rheumatologists, self-reported lack of confidence in prescribing MTX could delay treatment of patients with rheumatic conditions. Enhancing training for non-rheumatologists and advocating for policies to promote an equitable access to MTX and laboratory services are key to optimize RA treatment in countries with limited rheumatology manpower.

\section{8}

Prospective Validation of the Scleroderma Skin Patient-Reported Outcome (SSPRO) in Diffuse Cutaneous Systemic Sclerosis (dcSSc)

Ada Man (University of Manitoba, Winnipeg); Nancy Dgetluck (Corbus Pharmaceuticals, Inc., Norwood); Barbara White (Corbus Pharmaceuticals, Inc., Norwood)

Objectives: Skin thickening is a distressing feature of systemic sclerosis (SSc). The severity and extensiveness of skin thickening, however, does not directly correlate to its effect on the SSc patient's health-related quality of life (HRQOL). Rather, the location of the skin thickening and its effects on physical discomfort, function, or appearance, may be important. Yet, no skin-specific patient-reported outcome (PRO) in SSc has been routinely used, and SSc skin health currently is assessed only with a physician measure, the modified Rodnan skin score (mRSS). In this study, we prospectively examined the validity of a recently developed skin-specific PRO, Scleroderma Skin PRO (SSPRO), in a clinical trial of an anti-fibrotic therapy in dcSSc patients.

Methods: SSPRO has 18 items representing 4 HRQOL scales as related to skin involvement: physical symptoms, physical function, emotional, and social effects. Anabasum is a preferential cannabinoid receptor type 2 agonist that was tested for safety and efficacy in dcSSc in a double-blind randomized placebo-controlled Phase 2 trial (JBT101-SSc-001). Efficacy outcomes included the SSPRO, Patient Global Assessment (PtGA), HAQ-DI, Physician Global Assessment (MDGA), mRSS, and FVC \%. Construct validity was examined by correlating baseline SSPRO and other outcome scores (Pearson's Correlation Coefficient). Internal consistency was estimated (Cronbach's -alpha). Effect size (ES, ratio of mean change in outcome score from baseline to 12 weeks, to the standard deviation of the total score at baseline) was calculated to assess responsiveness to change. Results: SSPRO was administered to $41 \mathrm{dcSSc}$ participants. Internal consistency was high (0.87-0.92). SSPRO correlated strongly with PtGA (0.63), HAQ-DI (0.52), and MDGA (0.51), and weakly with mRSS (0.45) showing convergent validity (all $\mathrm{p}<0.05$ ). As expected, SSPRO did not correlate

Personal non-commercial use only. The Journal of Rheumatology Copyright $\odot$ 2018. All rights reserved. 
with FVC \%, showing divergent validity. The SSPRO total mean score demonstrated improvement in anabasum-treated $(\mathrm{N}=26)$ compared to placebo-treated subjects $(\mathrm{N}=15)$ at 12 weeks, $\mathrm{LS}$ means difference $(\mathrm{SE})=$ $-16.9(6.0), P=0.004$. The ES $(n=41)$ was moderately high at 0.51 , also demonstrating SSPRO's responsiveness to change. This ES was higher than mRSS (0.38), PtGA (0.35), and HAQ-DI (0.25), and similar to MDGA (0.57).

Conclusion: SSPRO is the first PRO developed specifically to address the SSc patient's assessment of their skin health. Its performance characteristics in this clinical trial with dcSSc patients demonstrate validity in all aspects examined, including responsiveness to change. Therefore, SSPRO will be a useful outcome measure in the assessment of therapeutic efficacy, providing complementary, patient focused information, to the widely used mRSS

\section{9}

The Effectiveness and Safety of Certolizumab Pegol in Rheumatoid Arthritis Patients in a Canadian Practice Setting: Two Year Results from the Non-Interventional F-alphasT CAN Study

Louis Bessette (Department of Rheumatology, CHU de Québec-Université Laval, Québec); Boulos Haraoui (Department of Medicine, Centre Hospitalier de l'Université de Montréal, Montréal); Andrew Chow (Department of Medicine, University of Toronto, Toronto); Isabelle Fortin (Centre de Rhumatologie de 1'Est du Québec, Université du Québec à Rimouski, Rimouski); Sanjay Dixit (Department of Medicine, McMaster University, Hamilton); Majed Khraishi (Department of Medicine, Memorial University of Newfoundland, St. John's); Derek Haaland (Department of Medicine, McMaster University, Hamilton); Sami Elmoufti (UCB Pharma, Oakville); Jerry Syrotuik (UCB Pharma, Oakville); Saeed Shaikh (Department of Medicine, McMaster University, Hamilton)

Objectives: The objective of F-alphasT CAN was to assess the real-life effectiveness and safety of certolizumab pegol (CZP) in rheumatoid arthritis (RA) patients in a Canadian practice setting.

Methods: F-alphasT CAN was a two-year prospective, observational, noninterventional, non-comparative, post-marketing study conducted between December 22, 2010-December 16, 2016. F-alphasT CAN evaluated CZP use in Canadian adults with moderate-to-severe, active RA. The primary efficacy variable was to assess clinical effectiveness of CZP in achieving 28-joint count Disease Activity Score (DAS28) $<2.6$ after 2 years. DAS28 was derived using CRP when available; when unavailable at all visits, ESR was used providing it was assessed consistently throughout the study. Incidence of CZP-related treatment-emergent adverse events (TEAEs) were monitored during the study. The full analysis set (FAS), defined as all patients with a baseline DAS28 value $\geq 2.6$, who took $\geq 1$ dose of CZP and provided a valid post-baseline DAS28 measurement, was used for all clinical effectiveness measurements. The safety set (SS), which included all patients having taken $\geq 1$ dose of CZP, was used for all safety measurements.

Results: 546 patients were enrolled into the study (SS $=546$; FAS $=451$ ); $229(41.9 \%)$ completed to Week 104. The mean age (SS) was 56 years, and $78 \%$ were female. Median disease duration at baseline was 4.6 years (range $0-50$ years). At baseline, disease characteristics (mean [SD]; FAS) were: DAS28 5.2 (1.1) $(\mathrm{n}=451)$, swollen joint count 9.2 (5.2), tender joint count 11.7 (7.0), Patient's Assessment of Arthritis Pain by Visual Analogue Scale (PtAAP [VAS]) 59.0 (25.3), Clinical Disease Activity Index (CDAI) 32.9 (12.8), and Health Assessment Questionnaire-Disability Index (HAQ-DI) 1.4 (0.7). At Week 104, DAS28 $<2.6$ and DAS28 $\leq 3.2$ were achieved by 90/451 (20.0\%) and 128/451 patients (28.4\%), respectively. Mean (SD) score at Week 104, in PtAAP was 28.1 (25.7), HAQ-DI 0.84 (0.73), and CDAI 9.43 (10.04). CZP-related TEAEs, including some additionally reported through a patient support program, were consistent with the known safety profile of CZP; (TEAEs: 162/546 [29.7\%]; serious TEAEs 20/546 [3.7\%], of which $1.8 \%$ [10/546] were serious infections, most commonly pneumonia $(4 / 546[0.7 \%])$. No cases of tuberculosis were reported. Five deaths were reported during the study ( 4 unrelated to CZP [two respiratory tract malignancies, one intracranial aneurysm, and one due to cardiovascular complications], and 1 unknown cause).
Conclusion: The F-alphasT CAN study demonstrated that 2-year CZP therapy was an effective RA treatment in routine clinical practice, and identified no new CZP-related safety signals.

\section{0}

Improvement in Work Productivity and Fatigue of Certolizumab Pegol in Rheumatoid Arthritis Patients in a Canadian Practice Setting: Two Year Results from the Non-Interventional F-alphasT CAN Study

Louis Bessette (Department of Rheumatology, CHU de Québec-Université Laval, Québec); Boulos Haraoui (Department of Medicine, Centre Hospitalier de l'Université de Montréal, Montréal); Andrew Chow (Department of Medicine, University of Toronto, Toronto); Isabelle Fortin (Centre de Rhumatologie de l'Est du Québec, Université du Québec à Rimouski, Rimouski); Sanjay Dixit (Department of Medicine, McMaster University, Hamilton); Majed Khraishi (Department of Medicine, Memorial University of Newfoundland, St. John's); Derek Haaland (Department of Medicine, McMaster University, Hamilton); Sami Elmoufti (UCB Pharma, Oakville); Jerry Syrotuik (UCB Pharma, Oakville); Saeed Shaikh (Department of Medicine, McMaster University, Hamilton)

Objectives: To assess the real-life effect of certolizumab pegol (CZP) on workplace and household productivity, and fatigue, in RA patients in a Canadian practice setting.

Methods: F-alphasT CAN was a two-year prospective, observational, non-interventional, non-comparative, post-marketing study, conducted between December 22, 2010-December 6, 2016. F-alphasT CAN evaluated CZP use in Canadian adults with moderate-to-severe RA. The primary objective assessed the proportion of patients achieving Disease Activity Score (DAS28) $<2.6$ after 2 years. The full analysis set (FAS), defined as patients with a baseline DAS28 $\geq 2.6$, who took $\geq 1$ dose of CZP and provided a valid post-baseline DAS28 measurement, was used for all productivity and fatigue measurements. The validated arthritis-specific Work Productivity Survey (WPS) and Fatigue Assessment Scale (both 0-10 scales) were used to assess RA impact on household and workplace productivity, and fatigue.

Results: 546 patients were enrolled into the study (FAS = 451); 229 completed to Week 104, and 90/451 (20.0\%) achieved the primary endpoint of DAS2 < 2.6. At baseline, $41.7 \%$ of patients who completed the WPS questionnaire (187/448) were employed. Of the 261 unemployed patients, $31 \%(81 / 261)$ were unable to work due to arthritis, $15.3 \%$ (40/261) were homemakers, and 43.3\% (113/261) were retired. Mean (SD) patient-reported assessment of fatigue at baseline was $6.6(2.2)(n=445)$. At Week 104, $42.4 \%$ of patients who completed the questionnaire (86/203) were employed. Of the 116 unemployed, $26.7 \%$ (31/116) were unable to work due to arthritis, $13.8 \%$ (16/116) were homemakers, and $44.8 \%$ (52/116) were retired. Employment status was missing for one patient at Week 104. At Week 104 employed patients reported reduced absenteeism (mean [SD] workdays: 0.5 [2.3] versus 3.3 [7.2] at baseline), and reduced presenteeism (work productivity reduced by at least half; 0.7 [3.4] versus 7.2 [10.3] at baseline) over the previous month due to arthritis. Patients reported fewer days of household work missed/month compared to baseline (mean [SD] 4.0 [9.1] versus 10.3 [10.5]) and the mean [SD] level of arthritis interference with household productivity was reduced (mean 2.8 [3.0] versus 6.1 [3.0] at baseline; scale 0-10). By Week 104, fewer days of family, social or leisure activities were missed due to arthritis (mean 1.7 [5.2] versus 4.6 [7.7] at baseline). Patient assessed fatigue was reduced by 2.1 (2.8) from baseline. Conclusion: In this study, over a 2-year follow-up period, CZP treatment improved productivity in the workplace and the household, and reduced fatigue.

\section{1}

Alopecia Areata Secondary to the use of Leflunomide in Patients with Rheumatoid Arthritis: A Case Report and Literature Review Ina Cusnir (Division of Rheumatology, University of Alberta, Edmonton); Jill Hall (University of Alberta, Edmonton); Carrie Ye (University of Alberta, Edmonton) 
Background: Leflunomide is a disease modifying anti-rheumatic drug (DMARD) that has been licensed for the treatment of rheumatoid arthritis (RA) since 1998. Commonly reported adverse effects include diarrhea, nausea, hypertension, headache, transient hair loss, and hepatotoxicity. Objective: To describe a patient with RA treated with leflunomide who developed alopecia areata (AA) and to discuss similar published cases. A chart review of this case and a literature search on this topic were performed. Case Presentation: A 39 year old female, diagnosed with seronegative RA in 1999, was well controlled on methotrexate $25 \mathrm{mg}$ sc weekly and hydroxychloroquine $300 \mathrm{mg}$ po daily until May 2016 when she developed increased disease activity, thus leflunomide $20 \mathrm{mg}$ po daily was added. She responded well and experienced only minor adverse effects (loose stools). Leflunomide was decreased to $20 \mathrm{mg}$ every other day in August 2016, however this led to increased arthralgias and thus she increased it back to $20 \mathrm{mg}$ daily but decreased methotrexate to $15 \mathrm{mg}$ sc weekly. No other medication changed was made, but several months later, patchy alopecia was discovered on the left side of her scalp. It continued to worsen until the bald patch developed to approximately 10 centimeters in length. Leflunomide was discontinued in March 2017 (with a return to full dose methotrexate) and within a couple months hair regrowth commenced, although with a darker pigment than before. Laboratory tests, including liver enzymes, remained normal throughout.

Discussion: We believe the AA was due to the leflunomide for several reasons. First, notwithstanding the association between methotrexate and AA, the patient had no prior history of hair loss prior to initiation of leflunomide despite long-term use of methotrexate; second, it resolved following its discontinuation. Further, 3 similar cases have been reported in the literature, within which only 1 other patient was taking leflunomide concomitantly with methotrexate ( 2 were using TNF inhibitors). We determined this reaction to be "probable" on the Naranjo adverse drug reaction probability scale with a score of 7 out of 13 .

Conclusion: Leflunomide-induced alopecia areata appears to be very uncommon, but if identified to be culprit and discontinued, can be reversible.

\section{2}

Smoking and Opioid Use is Associated with Symptom Severity in Rheumatoid Arthritis

Angela Karellis (JSS Medical Research, Montreal); Emmanouil Rampakakis (JSS Medical Research, Montreal); John Sampalis (JSS Medical Research, Montreal); Martin Cohen (McGill University Health Centre, Montreal); Michael Starr (McGill University Health Centre, Montreal); Peter Ste-Marie (McGill University Health Centre, Montreal); Yoram Shir (McGill University Health Centre, Montreal); Mark Ware (McGill University Health Centre, Montreal); Mary-Ann Fitzcharles (McGill University Health Centre, Montreal)

Objectives: Cigarette smoking, both current and past, is a risk for incident rheumatoid arthritis (RA), even for those with low exposure rates of 1-10 pack years. As smoking is associated with opioid use in patients with chronic pain, the aim of the current analysis was to examine disease status for RA patients and the relationship between current cigarette smoking and opioid use.

Methods: As part of a study to evaluate cigarette and marijuana smoking in rheumatic disease patients, 1000 consecutively attending rheumatology patients completed an anonymous self-administered questionnaire including: pain severity on visual analog scale (VAS), patient global assessment (PtGA) and cigarette or marijuana smoking status. Concomitant physician recorded information included: diagnosis, sociodemographics, co-morbidities, treatments for RA and physician global assessment (PGA). Patients were categorized according to current smoking status and opioid use. Patient characteristics were compared between groups with one-way ANOVA.

Results: 248 patients were diagnosed with RA [mean (SD) age $=62.4(14.3)$ years and $77.4 \%$ female] stratified by smoking status and opioid use: 9 patients were current smokers and opioid users, 186 patients non-smokers and non-opioid users, and 53 patients current smokers or opioid users. Unemployment/ disability was statistically different between groups (current smokers \& opioid users vs. non-smokers \& non-opioid users vs. current smokers or opioid users: $11.1 \%$ vs. $3.3 \%$ vs. $13.5 \% ; p=0.015)$. Current smokers and opioid users reported significantly worse disease, including higher mean (SD) PGA [4.4 (1.6) vs. 2.3 (2.2) vs. 3.8 (2.4); p < 0.001], PtGA [4.5 (2.5) vs. $2.9(2.6)$ vs. $3.9(2.8) ; \mathrm{p}=0.021]$ and pain VAS [6.6 (2.0) vs. 3.7 (2.7) vs. 4.9 (3.1); $\mathrm{p}=0.001]$. In regard to medication use, current smokers and opioid users took significantly more medications for disease management [mean (SD): 3.6 (1.3) vs. 1.8 (1.0) vs. $2.3(1.1) ; \mathrm{p}<0.001$ ], specifically NSAIDs $(77.8 \%$ vs. $39.8 \%$ vs. $54.7 \% ; \mathrm{p}=0.019)$ and anti-epileptics $(22.2 \%$ vs. $3.2 \%$ vs. $7.5 \%$; $\mathrm{p}=0.020)$ with a trend towards more antidepressant use $(22.2 \%$ vs. $4.8 \%$ vs. $5.7 \%$; $=0.088)$.

Conclusion: Current smoking and opioid use is significantly associated with increased disease severity and other medication use, indicating that RA patients who smoke experience greater symptom severity and may use chemical coping methods to alleviate symptoms.

\section{3}

Pharmacokinetics (PK) \& Pharmacodynamics (PD) Results of a Double-blind Randomized Parallel Arm Study Comparing 3 Anti CD20 Monoclonal Antibodies in Moderate to Severe Seropositive Rheumatoid Arthritis Inadequately Responding to Methotrexate Patients

Vikram Haridas (Sushruta Multispeciality Hospital, Hubballi); Rahul Katta (Katta Hospital and S.R. Kalla Memorial General Hospital, Jaipur); Ajit Nalawade (Inamdar Multispecialty Hospital, Pune); Sandeep Kharkar (CARE Hospital, Nagpur); Vyacheslav Zhdan (M.V.Sklifosovskyi Poltava Regional Clinical Hospital, Department of Rheumatology. Higher State Educational Institution of Ukraine Ukrainian Medical Stomatological Academy, Poltava); Olena Garmish (SI NSC M.D. Strazhesko Institute of Cardiology of NAMSU, Kiev); Luis Lopez-Lazaro (Dr Reddy's Labs SA, Basel); Sonica Batra (Dr Reddy's Laboratories Ltd., Bachupally); Suresh Kankanwadi (Dr Reddy's Laboratories (EU) Ltd, Cambridge)

Objectives: DRL_RI is a proposed biosimilar to rituximab (Rituxan ${ }^{\circledR}(\mathrm{RP})$ in USA; Mabthera ${ }^{\circledR}$ (RMP) in the European Union).Objectives were to compare the pharmacokinetics (PK) and pharmacodynamics (PD), efficacy, safety and immunogenicity of DRL_RI, RP and RMP in patients with rheumatoid arthritis (RA).

Methods: Randomized, double-blind, parallel group, comparative study of DRL_RI, RP, and RMP in combination with stabilized doses of non-biological DMARDs in 18-65 years old patients with moderate to severe active RA, biologics naïve and with inadequate response to methotrexate (at least 6 months treatment, the last 3 at stable dose). Patients were randomised to receive $2 \times 1000 \mathrm{mg}$ rituximab doses 2 weeks apart as DRL_RI, RP or RMP by intravenous infusion. Samples for PK and PD were collected over 24 weeks. PK was assessed using noncompartmental methods. PK bioequivalence was defined as $91 \%$ confidence interval for the geometric mean ratio (GMR) between two compared products (T/R) within 80 to $125 \%$ for the parameters AUC0-14days, after first infusion; AUC0-t, after second infusion and AUC $0-\infty$ over entire course. The primary PK population was antidrug antibody (ADA) negative patients. A sensitivity analysis was done including all patients regardless of ADA results. All pairwise comparisons were evaluated. Peripheral blood B-cell (flow cytometry determined CD19+ cells) depletion kinetics was compared using time to depletion to below $20 \%$ lower limit of normal (LLN) and to below the limit of detection (LOD). Efficacy, safety, tolerability and immunogenicity are separately presented.

Results: 276 patients (approximately equal numbers per arm) were included. PK comparisons showed that DRL_RI fulfilled the Protocol bioequivalence definition with both RP (T/R GMR and 91\% CI 100.80\% $(94.62 \%$, $107.38 \%)$ for AUC0-14days; $100.37 \%(92.30 \%, 109.14 \%)$ for AUC0- $\infty$, and $101.55 \%(92.60 \%, 111.36 \%)$ for AUC0-t and RMP (T/R GMR and $91 \%$ CI $95.45 \%(89.60 \%, 101.68 \%)$ for AUC0-14days; $93.58 \%(85.98 \%, 101.85 \%)$ for AUC0- $\infty$, and $94.83 \%(86.52 \%, 103.93 \%)$ for AUC0-t). Bioequivalence between the RP and the RMP was also proven. Sensitivity analysis results were consistent. PD: B-cell depletion was rapid. Median time to depletion below $20 \%$ of LLN was $3.0 \mathrm{~h}$ in all groups. No significant differences between groups were found. For depletion to below LOD only the first

Personal non-commercial use only. The Journal of Rheumatology Copyright @ 2018. All rights reserved. 
quartile was reached being $675.0 \mathrm{~h}$ for DRL_RI and RP; 2042h for RMP without significant differences.

Conclusion: Pharmacokinetic bioequivalence was proven between DRL_RI and reference products as well as between the reference products. There were no statistically significant differences between the study drugs in time to B-cell depletion using two different cutoffs.

\section{4}

Double-blind Randomized Parallel Arm Study of 3 Anti CD20 Monoclonal Antibodies in Patients with Moderate to Severe, Seropositive Rheumatoid Arthritis Inadequately Responding to Methotrexate Based Therapy. Efficacy Safety \& Immunogenicity Results Vikram Haridas (Sushruta Multispeciality Hospital, Hubballi); Rahul Katta (Katta Hospital and S.R. Kalla Memorial General Hospital, Jaipur); Ajit Nalawade (Inamdar Multispecialty Hospital, Pune); Sandeep Kharkar (CARE Hospital, Nagpur); Vyacheslav Zhdan (M.V.Sklifosovskyi Poltava Regional Clinical Hospital, Department of Rheumatology. Higher State Educational Institution of Ukraine, Ukrainian Medical Stomatological Academy, Poltava); Olena Garmish (SI NSC M.D. Strazhesko Institute of Cardiology of NAMSU, Kiev); Luis Lopez-Lazaro (Dr Reddy's Labs SA, Basel); Sonica Batra (Dr Reddy's Laboratories Ltd., Bachupally); Suresh Kankanwadi (Dr Reddy's Laboratories (EU) Ltd, Cambridge)

Objectives: DRL_RI is a proposed biosimilar to rituximab (Rituxan ${ }^{\circledR}(\mathrm{RP})$ in USA; Mabthera ${ }^{\circledR}$ (RMP) in the European Union).Objectives were comparing pharmacokinetics (primary), pharmacodynamics, efficacy, safety and immunogenicity of these products over 24 weeks.

Methods: Randomized, double-blind, parallel group study of rituximab in combination with stable doses of non-biological DMARDs in patients with moderate to severe RA who were aged 18-65 years, biologics-naïve and inadequate responders to methotrexate (at least 6 months treatment, last 3 at stable dose). Patients received 2x1000 mg intravenous rituximab doses 2 weeks apart as DRL_RI, RP or RMP. Efficacy criteria: standard ACR20/50/70 endpoints; DAS28-CRP at 4 weeks intervals. Safety criteria: adverse events and clinical laboratory. Immunogenicity was assessed as detection rate, titer and neutralising character of anti-drug antibodies (ADA). Pharmacokinetics and pharmacodynamics are separately reported. ACR20/50/70 rates with their 95\% confidence intervals (CI) were determined by logistic regression adjusted by region and gender. Immunogenicity rates were compared using two-sided Clopper Pearson 95\% confidence intervals. DAS28-CRP was compared using a generalised estimated equation model adjusting by baseline score, region and gender.

Results: 276 patients (approximately equal numbers per arm) were included. Patient characteristics were comparable at baseline. At week 24, ACR20 was comparable between DRL_RI and both RP [DRL_RI-RP difference $(95 \%$ CI) $2.8 \%(-11.18,16.81)]$, and RMP (DRL_RI-RP difference (95\% CI) $3.6 \%$ $(-10.54,17.73))$. ACR50 was also comparable (DRL_RI-RP difference $(95 \%$ CI) $4.4 \%(-10.73,19.52)$; DRL_RI-RP difference (95\% CI) $0.9 \%$ (-14.45, $16.18)$ ) and for ACR70 [DRL-RP difference $(95 \%$ CI) $2.3 \%(-8.97,13.49)]$, DRL_RI and RMP [DRL-RP difference $(95 \%$ CI) $1.9 \%(-9.47,13.24)]$. Reference products were comparable to each other. ADAs predose were found in $3(3.3 \%), 2(2.2 \%)$ and no subjects in the DRL_RI, RP and RMP arms. At week 24, ADA were found in $4(4.6 \%), 12(14.0 \%)$ and $7(8.0 \%)$ subjects in DRL_RI, RP and RMP arms, respectively. The respective median (range) titers were 4 (1-8); 1 (1-16) and 9 (1-256). At week 24, neutralising ADA were found in one patient per arm versus none predose. Adjusted differences (95\% confidence interval) in DAS28-CRP between treatments were: DRL_RI-RP -0.06 $(-0.33,0.21)$; DRL_RI-RMP $0.16(-0.13,0.44)$ and RP-RMP 0.21 (-0.06, 0.49 ), suggesting that all treatments were comparable. Safety findings (AEs, laboratory abnormalities) were comparable for all 3 treatments.

Conclusion: DRL_RI showed comparable efficacy with the RP and the RMP as evaluated by the ACR20/50/70 rates, DAS28-CRP scores and comparable immunogenicity and safety.

225

Impact of Residential Area on the Profile of Rheumatoid Arthritis
Patients Initiating their First Biologic DMARD: Results from the Ontario Best Practices Research Initiative (OBRI)

Raman Joshi (Brampton Civic Hospital, William Osler Health System, Brampton); Mohammad Movahedi (University Health Network, Toronto); Emmanouil Rampakakis (JSS Medical Research, Montreal); Angela Cesta (University Health Network, Toronto); John Sampalis (JSS Medical Research, Montreal); Claire Bombardier (Ontario Best Practices Research Institute, University of Toronto, Toronto General Research Institute, Toronto); OBRI Investigators (University Health Network, Toronto)

Objectives: Access to care and management of Rheumatoid Arthritis (RA) patients may differ based on residential area which, in turn, can affect the real-world effectiveness of anti-rheumatic medications. The aim of the current analysis is to describe differences in the profile of patients initiating their first biologic disease modifying antirheumatic drug (bDMARD) based on their residence in urban vs. rural areas.

Methods: RA patients enrolled in the OBR initiating their first bDMARD within 30 days prior to or following enrolment were included in the analysis. Parameters compared included patient sociodemographics (age, gender, race, education level, marital status, smoking status, annual household income, health insurance coverage), disease duration, disease severity parameters (Disease Activity Score (DAS), Simple Disease Activity Index, Clinical Disease Activity Index (CDAI), Swollen and Tender Joints (SJC28, TJC28), Medical Doctor Global Assessment (MDGA), Patient Global Assessment (PGA), Health Assessment Questionnaire - Disability Index (HAQ-DI), presence of erosion), bDMARD type, and concomitant anti-rheumatic medications including conventional synthetic disease modifying antirheumatic drug (csDMARDs), non-steroidal anti-inflammatory drugs (NSAIDs), and steroids.

Results: A total of 629 RA patients were included of whom $522(83 \%)$ resided in urban areas and $107(17 \%)$ in rural areas. Other than marital status (urban vs. rural: $64.6 \%$ vs. $82.2 \%$ married; p < 0.001 ) no significant differences in sociodemographics were observed between groups. However, patients from urban areas were less likely to have an erosion $(55.7 \%$ vs. $62.8 \% ; \mathrm{p}=0.2)$, had numerically lower TJC28 (7.2 vs. 7.9; $\mathrm{p}=0.43)$, and numerically lower SJC28 (6.6 vs. 7.1; $\mathrm{p}=0.42$ ) at bDMARD initiation. Type of bDMARD (anti-TNF vs. other mechanism of action) was comparable between groups ( $87.9 \%$ on anti-TNF) as was concomitant treatment with csDMARDs ( $85.7 \%$ on csDMARDs), NSAIDs (19.7\% on NSAIDs). The concomitant use of steroids was significantly lower in patients from urban areas $(21.6 \%$ vs $30.1 \%$; $\mathrm{p}=0.04)$.

Conclusion: Important differences may exist between profiles of RA patients residing in rural versus urban areas in initiating of their first bDMARD. The implications on treatment outcomes should be assessed.

\section{6}

Viral Exposures as a Risk Factor for Rheumatoid Arthritis: Summarizing the Evidence

Fatima Kudaeva (Western University, London); Mark Speechley (Western University, London); Neil Klar (Western University, London); Janet Pope (Western University, Department of Medicine, Division of Rheumatology, London)

Objectives: Different viral exposures have been implicated in the etiology of rheumatoid arthritis (RA) via several mechanisms of immune activation (such as molecular mimicry). The purpose of this systematic review was to summarize the evidence relating to the association between putative viral exposures and the development of RA.

Methods: A systematic literature search was conducted in September 2017 using MEDLINE-OVID, EMBASE-OVID, PUBMED and Cochrane library databases from inception. Only studies published in English were included. For the meta-analysis, only studies reporting frequencies of RA with viral serology, or case-control studies were included.

Results: After the primary screen of 5763 citations, 45 studies were selected for review. Studies had poor quality. All results are given with Odds or Hazard Ratios and 95\% Confidence Intervals and compared to controls unless if otherwise stated. The detection of Epstein-Barr virus (EBV) was

Personal non-commercial use only. The Journal of Rheumatology Copyright (C) 2018. All rights reserved. 
comparable between RA and controls $(\mathrm{N}=24$ studies, $\mathrm{OR} 1.05(0.68,1.61)$, $p=0.82$ ). The odds of parvovirus B19 (PBV19) infection were not increased in RA patients $(\mathrm{N}=4$ studies, OR $1.51(0.66,3.49), \mathrm{p}=0.33)$. Patients with hepatitis $\mathrm{C}(\mathrm{HCV})$ are at increased risk of RA $(\mathrm{N}=1$ study, HR $2.03(1.27$, $3.22), \mathrm{p}<0.01)$, with no increased risk with hepatitis $\mathrm{B}(\mathrm{HBV})(\mathrm{N}=1$ study, HR $1.09(0.74,1.63), p>0.05)$. Seronegative 'RA' developed in $36-80 \%$ of patients with polyarthritis after Chikungunya fever (CHIKV) $(\mathrm{N}=2$ studies). The odds of RA did not significantly differ between patients with or without preceding infections $(\mathrm{N}=1$ study, OR $0.96(0.82,1.13)$ for sinusitis, 0.99 $(0.86,1.14)$ for tonsillitis, and $1.03(0.73,1.45)$ for pneumonia, $\mathrm{p}>0.05$ for all comparisons).

Conclusion: Studies about the risk of RA after viral exposures suffer from inconsistent methodological quality. The findings of RA after CHIKV are interesting but may actually be a more self-limited inflammatory arthritis (as follow up was short in most studies). There seems not to be a risk of sustained RA after PBV19 infection. It is difficult to interpret the HCV data as viral hepatitis can cause a rheumatoid factor (RF) production for other reasons (e.g. cryoglobulinemia). There is not enough evidence to support an association between some viruses and RA development, but they probably lead to RA in genetically susceptible individuals. The effect of epidemic respiratory viruses such as Influenza on the risk of RA is poorly understood. Influenza infection may increase RF production but no association with RA onset has been found.

\section{7}

Retention Rates of TNF Inhibitors and Abatacept Used as a First Biologic DMARD in the Treatment of Rheumatoid Arthritis: 8 Years of Experience from the Rhumadata ${ }^{\circledR}$ Registry

Denis Choquette (Institut de Rhumatologie de Montréal, Montréal); Louis Bessette (Laval University, Quebec); Evo Alemao (Bristol-Myers Squibb, Princeton); Boulos Haraoui (Department of Medicine, Centre Hospitalier de l'Université de Montréal, Montréal); Frederic Massicotte (Institut de Rhumatologie de Montréal, Montréal); Mondher Mtibaa (Bristol-Myers Squibb Canada, Saint-Laurent); Eleonora Muratti (Bristol-Myers Squibb, Montreal); Jean-Pierre Pelletier (Institut de Rhumatologie de Montréal, Montréal); Roelien Postema (Bristol-Myers Squibb, Uxbridge); Jean-Pierre Raynauld (Institut de Rhumatologie de Montréal, Montréal); Marie-Anaïs Remillard (Institut de Rhumatologie de Montréal, Montréal); Diane Sauvageau (Institut de Rhumatologie de Montréal, Montreal); Angèle Turcotte (Centre d'ostéoporose et de rhumatologie de Québec, Québec); Edith Villeneuve (Institut de Rhumatologie de Montréal, Montréal); Louis Coupal (Institut de Rhumatologie de Montréal, Montréal)

Objectives: Sustainability of treatment is important to consider when selecting a therapy for chronic conditions such as RA. Sustainability is a useful clinical marker for both long-term efficacy and safety. A recently published randomized controlled trial has demonstrated similar efficacy and safety profiles between abatacept (ABA) and adalimumab over 2 years (1, 2). Our objective is to assess the long-term sustainability of $\mathrm{ABA}$ and anti-TNFs following treatment failure with a conventional synthetic DMARD (csDMARD) in comparable cohorts of patients (pts) with RA.

Methods: Data from pts with RA seen at two tertiary centers and prescribed either ABA or a TNF inhibitor (adalimumab, certolizumab, etanercept, golimumab or infliximab) as their first biologic (b)DMARD after 1 January 2006 were extracted from the RHUMADATA ${ }^{\circledR}$ registry. The choice of therapy was a joint decision between the pt and the treating physician. Pts were followed until either they discontinued treatment, were lost to follow-up or the cut-off date of 9 January 2017. Pt baseline characteristics were compared using descriptive statistics and the cumulative incidence of biologic agent discontinuation using Kaplan-Meier methods. Overall differences in the discontinuation rates of biologic agents were tested using the log-rank test.

Results: Overall, 641 pts met study criteria; 82 pts received ABA and 559 $\mathrm{TNF}$ inhibitors (adalimumab $=136$, certolizumab $=52$, etanercept $=226$, golimumab $=88$ and infliximab $=57$ ) as first-line treatment following inadequate response to csDMARDs. No clinically significant differences in baseline characteristics were noted between treatment groups. Most pts were diagnosed after January 2000 (72.5\%) and were women (77.5\%). Average age at diagnosis was $47.1(\mathrm{SD}=13.4)$ years, with a mean disease duration of 7.2 (7.8) years, and a mean CDAI of 43.1 (32.5) at baseline. No significant differences in retention rates were observed in the ABA and anti-TNF groups. On average, pts treated with anti-TNFs and ABA maintained their treatment for 1.59 (1.91) and 1.90 (2.08) years, respectively. Lack of efficacy $(47.6 \%)$ and adverse effects $(22.0 \%)$ were the most commonly cited reasons for treatment discontinuation.

Conclusion: Abatacept and TNF inhibitors demonstrate similar sustainability at 8-year, supporting studies 1,2 that demonstrate that abatacept used after csDMARDs inadequate response is as safe and effective as a TNF targeting agents in the long term. References: 1 . Schiff M, et al. Ann Rheum Dis 2014;73:86-94. 2. Schiff M, et al. Ann Rheum Dis 2008;67:1096-1103.

\section{8}

Abatacept Shows Better Sustainability than TNF Inhibitors when Used Following Initial Biologic DMARD Failure in the Treatment of Rheumatoid Arthritis: 8 Years of Real-World Observations from the Rhumadata ${ }^{\circledR}$ Clinical Database and Registry

Denis Choquette (Institut de Rhumatologie de Montréal, Montréal); Louis Bessette (Laval University, Quebec); Evo Alemao (Bristol-Myers Squibb, Princeton); Boulos Haraoui (Department of Medicine, Centre Hospitalier de l'Université de Montréal, Montréal); Frederic Massicotte (Institut de Rhumatologie de Montréal, Montréal); Mondher Mtibaa (Bristol-Myers Squibb Canada, Saint-Laurent); Eleonora Muratti (Bristol-Myers Squibb, Montreal); Jean-Pierre Pelletier (Institut de Rhumatologie de Montréal, Montréal); Roelien Postema (Bristol-Myers Squibb, Uxbridge); JeanPierre Raynauld (Institut de Rhumatologie de Montréal, Montréal); Marie-Anaïs Remillard (Institut de Rhumatologie de Montréal, Montréal); Diane Sauvageau (Institut de Rhumatologie de Montréal, Montreal); Angèle Turcotte (Centre d'ostéoporose et de rhumatologie de Québec, Québec); Edith Villeneuve (Institut de Rhumatologie de Montréal, Montréal); Louis Coupal (Institut de Rhumatologie de Montréal, Montréal)

Objectives: In the absence of biomarkers predicting response to a specific therapy, the choice of second biologic is based mostly on habit and availability of an alternative agent. Traditionally, a second anti-TNF was the preferred option, but recent registry data point to better responses and retention if a drug with a different mode of action is prescribed. Our aim is to assess the long-term retention of abatacept (ABA) and TNFi following first biologic (b)DMARD inadequate response in RHUMADATA ${ }^{\circledR}$ registry patients (pts) with RA.

Methods: Data from RHUMADATA ${ }^{\circledR}$ pts with RA prescribed either ABA or TNFi as the second bDMARD after 1 January 2006 were analysed. Pts were followed until treatment discontinuation or 9 January 2017 cut-off. Pt characteristics were compared using descriptive statistics, bDMARD discontinuation rates using Kaplan-Meier methods, and proportional hazard models were used to identify predictors of treatment discontinuation.

Results: Data for 92 and 194 pts prescribed ABA or a TNFi, respectively, as second-line treatment were extracted. No clinically significant differences in baseline characteristics were noted between treatment groups. Most pts were women $(76.2 \%)$, average age (SD) was 45.1 (13.3) years at diagnosis and disease duration 10.8 (9.0) years. Most pts were stopping an anti-TNF agent: $97 \%$ of those who were switched to ABA and $83 \%$ of those who were prescribed a second anti-TNF. Overall, $77.6 \%$ of pts stopped their first bDMARD after $>6$ months of treatment (secondary failure). Significant differences in retention between ABA and TNFi groups (log-rank $\mathrm{p}=0.0002$ ) were observed (Table, Figure). Results remained unchanged for pts treated with TNFi only in first line, and primary/secondary failure of the first bDMARD did not affect sustainability of the second agent. Lack of efficacy (57.7\%) and AEs (16.5\%) were the most commonly cited reasons for treatment discontinuation

Conclusion: Abatacept has better sustainability over a second line TNFi in RA patients having failed one prior bDMARD.

Personal non-commercial use only. The Journal of Rheumatology Copyright @ 2018. All rights reserved. 
Dose Reduction of Baricitinib in Patients with Rheumatoid Arthritis Achieving Sustained Disease Control: Results of a Prospective Study Tsutomu Takeuchi (Keio University, Tokyo); Mark Genovese (Stanford University Medical Center, Palo Alto); Boulos Haraoui (Institut de Rhumatologie de Montréal, Montreal); Zhanguo Li (Peking University People's Hospital, Beijing); Li Xie (Eli Lilly and Company, Indianapolis); Rena Klar (Quintiles IMS Holdings Inc, Durham); Ana Pinto Correia (Eli Lilly and Company, Indianapolis); Susan Otawa (Eli Lilly and Company, Toronto); Pedro Lopez-Romero (Eli Lilly and Company, Indianapolis); Inmaculada de la Torre (Eli Lilly and Company, Madrid); Terence Rooney (Eli Lilly and Company, Indianapolis); Josef Smolen (Medical University of Vienna, Vienna)

Objectives: In patients (pts) with active rheumatoid arthritis (RA) and inadequate response (IR) to DMARDs, phase 3 studies demonstrated efficacy of baricitinib (2-mg and 4-mg). Larger, more rapid treatment effects were observed with baricitinib 4-mg than 2-mg. Baricitinib is currently approved in the EU, Japan, and other countries for the treatment of moderately to severely active RA in adults. The objective of this study was to investigate the effects of baricitinib dose step-down in pts who achieved sustained disease control with baricitinib 4-mg.

Methods: Pts with RA who completed a baricitinib phase 3 study could enter a long-term extension (LTE) study. In the LTE, pts who received baricitinib 4-mg for $\geq 15$ months, and who achieved sustained low disease activity ([LDA] - CDAI score $\leq 10$ for pts from RA-BUILD, RA-BEAM, RA-BEACON) or remission (CDAI $\leq 2.8$ for pts from RA-BEGIN) at 2 consecutive visits $\geq 3$ months apart, were re-randomized in a blinded manner to either continue baricitinib 4-mg or step down to 2-mg. Pts could be rescued (to baricitinib 4-mg) if CDAI score was $>10$, or $>2.8$ for pts from RA-BEGIN. Efficacy and safety were assessed through 48 weeks following re-randomization.

Results: The majority of pts in both groups maintained the state of LDA or remission over the 48-week period. However, dose reduction to 2-mg resulted in significant increases in disease activity at 12,24, and 48 weeks. Dose reduction also resulted in a more rapid time to relapse (defined as loss of step-down eligibility criteria), with significantly more pts relapsing over 48 weeks compared to the 4-mg group $(\mathrm{p}=0.001)$. Higher proportion of the patients reported LDA (79.6\% vs $66.9 \%)$ and remission $(40.4 \%$ vs $32.7 \%)$ with baricitinib 4-mg compared to 2-mg at Week 48, respectively. Rescue rates were $9.6 \%$ for baricitinib 4-mg, and $18.3 \%$ for baricitinib 2-mg. Most rescued pts could regain LDA or remission with the 4-mg dose. Dose reduction was associated with a numerically lower rate of non-serious infections; rates of serious adverse events and adverse events leading to discontinuation were similar across groups.

Conclusion: These data indicate that disease control was better maintained on the 4-mg dose than 2-mg. Nonetheless, most stepped-down pts could maintain LDA or remission, or recapture control with return to the 4-mg dose if needed. Stepping down to a dose of 2-mg daily may be a reasonable consideration for some pts after having achieved sustained LDA or remission on the 4-mg dose.

\section{0}

Real-World Effectiveness and Safety of Subcutaneous Abatacept in Biologic-Naive vs. Biologic-Experienced RA Patients: The Abatacept Best Care Study

Boulos Haraoui (Department of Medicine, Centre Hospitalier de l'Université de Montréal, Montréal); Janet Pope (Western University, Department of Medicine, Division of Rheumatology, London); Isabelle Fortin (Centre de Rhumatologie de l'Est du Québec, Université du Québec à Rimouski, Rimouski); Emmanouil Rampakakis (JSS Medical Research, Montreal); John Sampalis (JSS Medical Research, Montreal); Francoise Romeyer (Bristol-Myers Squibb Canada, Saint-Laurent); Joseph Atallah (BristolMyers Squibb Canada, Saint-Laurent); Louis Bessette (Department of Rheumatology, CHU de Québec-Université Laval, Québec)

Objectives: Efficacy and safety of subcutaneous (SC) abatacept in managing rheumatoid arthritis (RA) has been demonstrated in numerous controlled clinical trials. However, real-world data of SC abatacept in routine care are scarce. The aim of this interim analysis is to compare the effectiveness and safety of SC abatacept as first or second biologic agent in Canadian patients enrolled thus far in Abatacept Best Care (ABC).

Methods: $\mathrm{ABC}$ is a prospective, multicenter, randomized study aimed at comparing a T2T approach vs. standard of care in real-life management of patients with RA starting SC abatacept as first or second line biologic agent, and describing adherence of physicians to recommended T2T treatment guidelines while collecting data on real-life use of SC abatacept. General linear models were used to assess the impact of prior biologic use on treatment effectiveness

Results: 276 patients (74.3\% females) were included with a mean (SD) age of 59.7 (11.7) years and duration since RA diagnosis of 7.5 (8.9) years; of these $214(77.5 \%)$ and $189(68.5 \%)$ had available follow-up data at 6 and 12 months, respectively. Prior biologic use was reported for $39.5 \%(60.5 \%$ were biologic-naïve). Overall, baseline disease parameters were comparable with the exception of time since RA diagnosis (9.0 vs. 6.4 years; $\mathrm{P}<0.001$ ) which was significantly higher in biologic experienced patients and TJC28 (10.5 vs. 9.1; $\mathrm{P}=0.062)$ which was numerically higher. After 6 months treatment, both patient groups experienced significant improvements in all disease parameters which were maintained or further enhanced by 12 months. However, upon adjusting for baseline disease activity, improvements in CDAI $(\mathrm{P}=0.020)$, physician global $(\mathrm{P}=0.011)$, TJC28 $(\mathrm{P}=0.031)$, SJC28 $(P=0.009)$, and HAQ $(P=0.037)$ at 6 months were significantly higher in biologic-naive patients (Table 1). Similar results were obtained at 12 months. A total of $533 \mathrm{AEs}$ were reported for $162(58.7 \%$ ) patients, $67.0 \%$ of biologic-experienced patients and $53.3 \%$ of biologic-naïve patients (256.1 vs. 211.2 events/100 PY). Most common AEs were upper respiratory infections (biologic-experienced vs. biologic-naive: $4.6 \%$ vs. $9.0 \% ; 5.2$ vs. 14.6 events/100 PY) and bronchitis (7.3\% vs. 7.8\%; 8.4 vs. 10.2 events/100 PY). Serious AEs and serious infections were reported for $26(9.4 \%)$ patients $(10.1 \%$ vs. $9.0 \% ; 16.8$ vs. 13.2 events per $100 / \mathrm{PY})$ and $5(1.8 \%)$ patients ( $0.9 \%$ vs. $2.4 \%$; 0 vs. 1.5 events/100 PY), respectively.

Conclusion: Results of the current analysis suggest that SC abatacept has similar safety in biologic-naïve and -experienced patients with more improvement in outcome measures in the former.

\section{1}

\section{Role for S100A12 in Mediating Vascular Inflammation}

Dara Mairiang (Division of Rheumatology, Department of Pediatrics, BC Children's Hospital Research Institute \& University of British Columbia, Vancouver); Giulia Armaroli (Department of Pediatric Rheumatology and Immunology, University Children's Hospital, Münster); Jordan Chiu (Division of Rheumatology, Department of Pediatrics, BC Children's Hospital Research Institute \& University of British Columbia, Vancouver); Stephanie Hughes (Division of Rheumatology, Department of Pediatrics, BC Children's Hospital Research Institute \& University of British Columbia, Vancouver); Christoph Kessel (Department of Pediatric Rheumatology and Immunology, University Children's Hospital, Münster); David Cabral (Division of Rheumatology, Department of Pediatrics, BC Children's Hospital Research Institute \& University of British Columbia, Vancouver); Kelly Brown (Division of Rheumatology, Department of Pediatrics, BC Children's Hospital Research Institute \& University of British Columbia, Vancouver)

Objectives: Chronic Primary Vasculitis of childhood describes a group of serious multisystem, diseases characterized by inflammation of blood vessels to major body organs. Improved understanding of disease and the identification of biomarkers of disease activity is critical for patient care, particularly for informing the safe stopping of treatment when disease activity is low. Previously, serum levels of neutrophil-derived, pro-inflammatory molecule S100A12 has been demonstrated to correlate with vasculitis activity in pediatric vasculitis patients. Our aim is to investigate if S100A12 is a potential driver of vascular endothelial cell activation in vitro.

Methods: Human umbilical vein endothelial cells (HUVEC) that are repre-

Personal non-commercial use only. The Journal of Rheumatology Copyright $\subset$ 2018 . All rights reserved. 
sentative of small to medium sized vessels and human coronary artery endothelial cells (HCAEC) representative of large vessels were employed. Cells lines were stimulated directly with S100A12 (5 - $20 \mathrm{mg} / \mathrm{ml})$. Alternatively, cell lines were stimulated with supernatants from S100A12-activated peripheral blood mononuclear cells (PBMC) from healthy adults in the absence or presence of blocking antibodies $(1 \mathrm{mg} / \mathrm{ml})$ for common pro-inflammatory cytokines (TNF-alpha, IL6 and IL1-beta). Stimulation with TNF-alpha $(10 \mathrm{ng} / \mathrm{ml})$ or LPS $(0.5-100 \mathrm{ng} / \mathrm{ml})$ were used as positive controls for endothelial cell activation that was measured by mRNA expression (PCR) and protein expression (flow cytometry and ELISA) of the pro-inflammatory cytokine IL- 8 and the endothelial cell adhesion molecules ICAM-1, and VCAM-1.

Results: S100A12 does not directly activate HUVEC or HCAEC as occurred in response to LPS or TNF-alpha. In contrast, HCAEC, but not HUVEC, were responsive to supernatants derived from S100A12-activated PBMC as evidenced by induced expression of the genes encoding IL-8, ICAM-1, and VCAM-1. This induction appears highly dependent on IL-1-beta as we observed significant reduction in IL-8 expression in the presence of an anti-IL1-beta antibody.

Conclusion: S100A12 is a biologic marker that is sensitive to changes in disease activity in childhood chronic primary vasculitis. While S100A12 does not directly activate large- or medium-sized endothelial cells in vitro, we provide evidence that it may be an indirect activator of large vessel endothelial cells via stimulation of IL-1-beta from peripheral blood mononuclear cells.

\section{2}

Resolving the Synovial Fluid Proteome and Peptidome for Disease-specific Mediators of Inflammatory Arthritis

Shalini Mahendran (Mount Sinai Hospital, Toronto); Eleftherios Diamandis (Department of Laboratory Medicine and Pathobiology, University of Toronto, Toronto); Vinod Chandran (University of Toronto and University Health Network, Toronto)

Objectives: Sensitive screening tools of inflammatory arthritides are greatly lacking. Biochemical tests for the diagnosis of rheumatoid arthritis (RA) are unable to identify one third of patients who do not present with serum biomarkers. Similarly, psoriatic arthritis (PsA) has no clinically validated biomarkers for the early detection of joint disease in psoriasis patients. Soluble biomarkers, including proteins and peptides, can provide an immediate reflection of pathological changes due to disease. In arthritis, putative markers can be discovered in the synovial fluid (SF). Using mass spectrometry (MS), we aim to provide a detailed analysis of the proteome and peptidome of RA and PsA SF.

Methods: 10 SFs from PsA, RA and control patients were investigated under label-free MS-based methods. Proteomic fractions underwent reduction, alkylation, and trypsin digestion while peptidomic fractions were desalted using solid-phase extraction. All samples were subjected to liquid-chromatography tandem MS followed by data extraction using MaxQuant 1.5.5.1. Results: Quantification of the SF proteome revealed 100 differentially expressed proteins in RA SF and 118 differentially expressed proteins in PsA SF relative to the control group. Similarly, our peptidomic results demonstrated increased proteolytic activity in the inflammatory arthritides with the identification of 41 proteins originating from 177 peptides in RA SF and 32 proteins originating 170 peptides in PsA SF relative to the control. Ficolin2 (FCN2) and heterogeneous nuclear ribonucleoproteins C1/C2 (HNRNPC) were uniquely identified in PSA SF and both have previously been observed to stimulate production of autoantibodies in related inflammatory diseases. These results warrant further investigation into autoantibody production in PsA SF and serum. The identification and upregulation of proteins and peptide fragments with antimicrobial activity including FCN2, ficolin-3 (FCN3), bactericidal permeability-increasing protein (BPI), neutrophil defensin 3 (DEFA3), histone H2A type 1-J (HIST1H2A) and histidine-rich glycoprotein (HRG) suggest an important role of the microbiome in the onset of inflammatory arthritides. This is especially of interest in the context of PsA where there is increasing research into the role of the gut microbiota in the development of the disease.
Conclusion: The use of high resolution MS facilitates the identification of uniquely and differentially expressed proteins and peptides which may be critical to understanding the progression of inflammatory arthritides. Ultimately, this will allow for the development of therapeutic interventions and minimally-invasive biomarker panels for diagnostic and predictive purposes.

\section{3}

Methotrexate Enhances the Immunopotency of Human Mesenchymal Stromal Cells

Maximilien Lora (Research Institute MUHC, Montreal); Natalia Shimabukuro (Research Institute MUHC, Montreal); Anastasia Cheng (Research Institute MUHC, Montreal); Marie Hudson (McGill University, Jewish General Hospital, Lady Davis Institute for Medical Research, Montreal); Ines Colmegna (McGill University Hospital Centre, McGill University, Montreal)

Objectives: Mesenchymal stromal cells (MSC) comprise a heterogeneous population of multipotent cells with the capacity to modulate responses against allo- and autoantigens. Increased donor age and chronic inflammatory diseases reduce MSC immunopotency (IP). Methotrexate (MTX), the mainstay of treatment in rheumatoid arthritis (RA), enhances the function of regulatory T-cells. We tested the in vitro effect of methotrexate on human adipose derived MSC, specifically its ability to boost the IP of dysfunctional MSC.

Methods: Adipose derived human MSC were characterized according to the International Society for Cellular Therapy criteria. MTX uptake by MSC (FITC-MTX) was assessed by flow cytometry (FC). MSC viability was determined by 7-Amino-actinomycin D (7-AAD) staining (FC) and MSC levels by 2',7' -dichlorofluorescin diacetate (DCFDA) (FC). The effect of MTX on MSC immunopotency was evaluated in allogeneic MSC-mediated: T-cell suppression assays (IP assays FC). Passage 4 MSC were incubated for $48 \mathrm{~h}$ with (MSC+) or without (MSC-) MTX $(10 \mu \mathrm{M})$. Following extensive washes, conditioned media (CM from MSC collected 96h post MTX treatment) and cells (MSC collected 7 days post MTX treatment) were obtained from $\mathrm{MSC}+$ and MSC- conditions. IP assays were performed to compare the effect of (a) CM-MSC+ versus CM-MSC-, and (b) MSC+ versus MSC-, on suppressing the proliferation of $\mathrm{CD} 3 / \mathrm{CD} 28$ activated T-cells.

Results: Reduced folate carrier (RFC) is expressed on MSC and intracellular MTX was detected at Day 2 of MTX treatment and persisted until Day 4 post-MTX. MTX treatment was associated with an increase in ROS levels (DCFDA-GMFI 1.65 fold higher in MSC+ than MSC- samples, $\mathrm{p}<0.05 ; \mathrm{n}$ =6) and reduced MSC viability (7AAD-MSC+: $83.3 \pm 2.9 \%$ vs 7AAD-MSC-: $90.1 \pm 3.6 \%, \mathrm{p}<0.05 ; \mathrm{n}=6$ ). CM-MSC+ suppressed T-cells significantly better than CM-MSC- $(66.80 \pm 5.97 \%$ vs $39.33 \pm 9.35 \%$, $\mathrm{p}<0.05 ; \mathrm{n}=12)$. Similarly, MSC+ had better IP than MSC- $(20.11 \pm 3.92 \%$ vs $24.42 \pm 4.83 \%, \mathrm{p}<0.05 ; \mathrm{n}=11)$.

Conclusion: MTX treatment enhances the immunopotency of dysfunctional MSC. The modulation of the MSC secretome by MTX mediates part of this effect as MTX improves MSC-mediated:T-cell suppression both in cell-cell contact dependent and independent (supernatant transfer) conditions.

\section{4}

Plasma Cytokines Assessed at Diagnosis are Associated with Non-Response to Methotrexate in Rheumatoid Arthritis Patients

Martin Pelletier (CHU de Québec-Université Laval Research Center, Québec); Paul Fortin (Department of Rheumatology, CHU de Québec-Université Laval, Québec); Marie-Pier Longchamps (CHU de Québec-Université Laval Research Center, Québec); Geneviève Parent (CHU de Québec-Université Laval Research Center, Québec); Hadrien Benk-Fortin (CHU de Québec-Université Laval Research Center, Québec); Anne-Sophie Julien (Clinical Research Platform, CHU de Québec-Université Laval Research Center, Québec); Nathalie Amiable (CHU de Québec-Université Laval Research Center, Quebec); Emmanuelle Rollet-Labelle (CHU de Québec-Université Laval Research Center, Quebec); Laetitia Michou (Department of Rheumatology, CHU de

Personal non-commercial use only. The Journal of Rheumatology Copyright (c) 2018. All rights reserved. 
Québec-Université Laval, Quebec); Louis Bessette (Department of Rheumatology, CHU de Québec-Université Laval, Québec); Philippe Tessier (CHU de Québec-Université Laval Research Center, Québec)

Objectives: Methotrexate (MTX) is the first line treatment for patients with rheumatoid arthritis (RA). For over $30 \%$ of patients, MTX fails to diminish DAS28 score in a timely and satisfactory manner. The challenge is to find a way to identify these non-responders at the time of diagnosis. The aims of this study were to determine whether plasma cytokines could be used as biomarkers of early RA and of therapeutic response to MTX.

Methods: Thirty RA patients receiving MTX (mean age $58 \pm 12$ y.o. with symptom duration of $6 \pm 3$ mo.) from the Group for Early Arthritis Research (GEAR) / CHU de Québec SARD Biobank Data Repository (SBDR). Patients were evaluated clinically at baseline and 6 mo., and classified as responders to MTX or non-responders according to the DAS28CRP EULAR Classification (good response vs moderate/none). Plasma cytokines were measured at baseline by multiplex assay or ELISA and compared to age- and sex-matched healthy controls. Bivariate logistic regression was used to identify RA patients and non-responders based on cytokine concentration or detection, with Firth bias correction when needed. Optimal cut points were found using distance criteria and their predictive performance was assessed.

Results: Plasma concentrations of IL-1RA, CCL11/Eotaxin, CXCL10/IP-10, CCL2/MCP-1，CCL4/MIP-1-beta，CCL5/RANTES, CXCL12a/SDF-1-alpha and calprotectin were significantly different in RA patients compared to controls. IL-21, IL-22, IL-1-alpha, CXCL1/GRO-alpha were detected differently in RA plasma. IL-1RA and CCL4/MIP-1-beta were good predictors of RA with sensitivity and specificity of approximately $95 \%$ when dichotomized with cut points of 280 and 124 , respectively. Thirty-five percent of patients were non-responders to MTX. IL-1-alpha, IL-18, IL-17A and IL-27 were less frequently detected in non-responders. Conclusion: Several cytokines differentially expressed in plasma are associated with early RA. Absence of detection of IL-1-alpha, IL-17A and IL-27 and low concentration of IL-18 was associated with non-response to MTX. We propose that these cytokines could be used as predictors of MTX response in RA.

\section{5}

Plasma Cytokines as Biomarkers to Classify Patients with Systemic Autoimmune Rheumatic Diseases

Lindsay Ouellet (Université Laval, Quebec); Marie-Pier Longchamps (CHU de Québec-Université Laval Research Center, Québec); Anne-Sophie Julien (Clinical Research Platform, CHU de Québec-Université Laval Research Center, Québec); Nathalie Amiable (CHU de Québec-Université Laval Research Center, Quebec); Emmanuelle Rollet-Labelle (CHU de Québec-Université Laval Research Center, Quebec); Alena Ikic (Department of Rheumatology, CHU de Québec-Université Laval, Quebec); Alexandra Albert (Department of Rheumatology, CHU de Québec-Université Laval, Quebec); Laetitia Michou (Department of Rheumatology, CHU de Québec-Université Laval, Quebec); Louis Bessette (Department of Rheumatology, CHU de Québec-Université Laval, Québec); Philippe Tessier (CHU de Québec-Université Laval Research Center, Québec); Martin Pelletier (CHU de Québec-Université Laval Research Center, Québec); Paul Fortin (Department of Rheumatology, CHU de Québec-Université Laval, Québec)

Objectives: Systemic autoimmune rheumatic diseases (SARDs) are characterized by abnormal immune responses and chronic inflammation. Diagnosis is based on clinical symptoms, assessment of general markers of systemic inflammation and presence of autoantibodies. We aimed to establish whether cytokines can help to discriminate between rheumatoid arthritis (RA), systemic lupus erythematosus (SLE) and systemic scleroderma (SSc).

Methods: One hundred and eighty one patients ( $76 \%$ female) from the CHU de Québec-Université Laval SARD Biobank Repository Database (SBRD) were included in the study. Mean age and disease duration were $53 \pm 15$ y.o. and $7 \pm 9$ years, respectively. The first biospecimen collected from each patient was used: RA patients (62) were incident cases (disease duration of
$0,05 \pm 0.22$ years) while SLE (84) and SSc (35) patients were prevalent cases (disease duration of 9,95 \pm 10.31 years and 9,28 \pm 9.18 years, respectively). Thirty-four cytokines involved in inflammation, immunoregulation, chemotaxis and growth were quantified in plasma by multiplex assays using Luminex technology. The concentration of frequent cytokines was treated as continuous variable, while those detected in less than $25 \%$ of patients were analyzed as present or not. Bivariate multinomial regression model was used to predict diagnosis of patients based on cytokine profile. Results are presented as odds ratio with $95 \%$ confidence interval.

Results: Plasma concentration of IL-1RA [OR 1,01 (95\% CI 1.01-1.01) for a 10-unit increase] and CCL4/MIP-1-beta [1.02 (1.01-1.02)] were higher in RA patients compared to SLE, while it was lower for CCL2/MCP-1 [0.98 (0.97-0.99)] and CCL5/RANTES [0.99 (0.98-0.99)]. IL-1-beta [0.12 (0.05-0.25)] and IL-7 [0.26 (0.12-0.57)] were detected significantly less often in RA than SLE patients, while IL-21 [4,58 (1,93-10,89)] and IL-1-alpha [4.15 (2.04-8.44)] were detected significantly more often. CCL5/RANTES was the only discriminator between SSc and SLE $[0.98$ (0.97-0.99)]. Concentrations of all other cytokines were similar between SSc and SLE.

Conclusion: The expression of 8 out of 34 plasma cytokines was significantly different in plasma of RA, SLE and SSc patients. A diagnosis of RA was predicted by higher levels of IL-1RA, CCL4/MIP-1-beta, IL-21 and IL-1-alpha, and by lower levels of CCL2/MCP-1, CCL5/RANTES, IL-1-beta and IL-7. CCL5/RANTES levels also discriminated between SLE and SSc. We propose that quantification of specific plasma cytokines could be used as biomarkers to assist in the diagnosis of RA, SLE and SSc. Acknowledgement: This project was partly funded by Dr Fortin's Canada Research Chair on SARDs. Unrestricted funds to support the CHU de Québec-Université Laval SBRD were granted by Pfizer, Amgen, BMS, Abbvie, Roche, Sanofi-Genzyme and Merck \& Co.

\section{6}

Microparticles of Platelet, Red Blood Cell and Endothelial Cell Origin are Differently Decorated with Autotaxin and more Abundant in Systemic Lupus Erythematosus

Anne-Sophie Julien (Clinical Research Platform, CHU de Québec-Université Laval Research Center, Québec); Stephan Hasse (CHU de Quebec-Université Laval Research Center, Quebec); Anne-Claire Duchez (CHU de Quebec-Université Laval Research Center, Quebec); Chenqi Zhao (CHU de Québec-Université Laval Research Center, Quebec); Paul Fortin (Department of Rheumatology, CHU de Québec-Université Laval, Québec); Sylvain Bourgoin (CHU de Québec-Université Laval Research Center, Quebec)

Objectives: Autotaxin, the enzyme that produces lysophosphatidic acid, and cell-derived microparticules (MP) are present in biological fluids including blood, cerebrospinal and synovial fluid samples. MP in the blood originate mainly from platelets, red blood cells, and vascular endothelial cells. MP are potential markers of rheumatic diseases and may be associated with disease activity or comorbidity such as premature atherosclerosis. In this study we evaluated in systemic lupus erythematosus (SLE) the levels of MP derived from platelets, red blood cells, and endothelial cells that were decorated with autotaxin and their association with clinical variables such as SLE disease activity and damage

Methods: Blood samples were obtained from 76 SLE patients and 50 age- and sex-matched controls. Demographic and clinical variables were collected. Plasma autotaxin was assessed using ELISA. Combinations of fluorochrome-conjugated antibodies against human platelet antigen (anti-CD41), red blood cell antigen (anti-CD235a), endothelial cell antigen (anti-CD31), or autotaxin were used to quantify MP (EVs/ $\mu$ l) by high sensitivity flow cytometry. Distribution of MP between groups was compared using Wilcoxon tests. Multivariate logistic regression analyses were used to assess the association of MP with disease activity (SLE Disease Activity Index $>=4$ ) and damage (SLICC Damage Index $>=1$ ).

Results: Plasma ATX levels tend to be higher in SLE compared to controls (medians 267 vs $230 \mathrm{ng} / \mathrm{mL}, \mathrm{p}=0.07$ ). Plasma CD41+, CD235a+ and 
CD41-CD31+ MP were higher in SLE compared to controls, with Cohen's $\mathrm{d}$ effect size of $0.43,0.87$ and 0.36 , respectively. CD41+ and CD41-CD31+ MP decorated with autotaxin in control and SLE plasmas were similar (16.4\% vs $13.4 \%$ and $86.3 \%$ vs $87.8 \%$, respectively). The ratio of CD235a+ MP harboring autotaxin was decreased in SLE (7.2\%) compared to controls (23.8\%). The levels of plasma ATX and MP subtypes were not associated with activity nor damage. However, use of prednisone significantly reduced plasma ATX levels (medians $221 \mathrm{vs} 280, \mathrm{p}=0.03$ ). Treatment with antihypertensive drugs decreased the levels of CD235a+ MP decorated with autotaxin (medians $914 \mathrm{vs} 9147, \mathrm{p}=0.001)$ and the use of antimalarial drugs enhanced plasma levels of CD41+ MP (medians 7499 vs 2436, $p=0.02$ ).

Conclusion: Blood MP levels are increased in SLE. The use of prednisone but not of other drugs is associated with a reduction of plasma ATX level. Antihypertensive drugs and antimalarial drugs may affect specific groups of MP in the blood. Our observations indicate that various MPs are decorated with ATX that may impact on the development of cardiovascular diseases such as atherosclerosis.

\section{7}

Demyelinating Disorders in RA Offspring Exposed to TNF Inhibitors Evelyne Vinet (McGill University Health Centre, Montreal); Susan Scott (McGill University Health Centre, Montreal); Cristiano Moura (Department of Epidemiology, Biostatistics and Occupational Health, McGill University, Montreal); Christian Pineau (Montreal General Hospital, Division of Rheumatology, Montreal); Jeffrey Curtis (University of Alabama at Birmingham, Birmingham); Sasha Bernatsky (McGill University Health Centre, McGill University, Montreal)

Objectives: Adult RA patients exposed to TNFi have an increased risk of demyelinating disorders compared to unexposed RA subjects. Most TNFi are monoclonal immunoglobulins and are actively transported across the placenta, reaching higher fetal than maternal blood levels. As the fetal blood-brain barrier allows passage of circulating immunoglobulins into the central nervous system, offspring exposed to TNFi might be more susceptible to adverse neurological events. Thus, we evaluated demyelinating disorders in RA offspring exposed to TNFi during pregnancy compared to unexposed RA offspring, as well as to children from the general population. Methods: The "PregnAncies in RA mothers and Outcomes in offspring in the United States (PAROUS)" cohort includes all women with $\geq 1$ hospitalization for delivery after RA diagnosis, identified through MarketScan database (2011-2014), and a randomly selected control group of women, matched $\geq 4: 1$ for age, year of delivery, and state of residence. Only women continuously enrolled within MarketScan for $\geq 12$ months prior to delivery and with available child linkage were included in PAROUS. We defined TNFi exposure based on $\geq 1$ filled prescription during pregnancy and/or the preconception period. We ascertained demyelinating disorders in the offspring based on $\geq 1$ hospitalization and/or outpatient visit with a diagnostic code related to multiple sclerosis, transverse myelitis, and/or optic neuritis. The event of interest was ascertained from birth until end of MarketScan enrolment, end of study period, and/or death, whichever came first.

Results: We identified 2455 RA offspring and 11018 matched controls. Among RA offspring, 399 (16.2\%) were exposed to TNFi in the preconception and/or gestational period, while 2056 (83.7\%) were unexposed both during the pregnancy and preconception periods. In utero exposures to corticosteroids and non-biologic DMARDs were more common in RA pregnancies than in unaffected pregnancies. We did not identify any case of demyelinating disorders in RA offspring exposed to TNFi and RA offspring unexposed to TNFi. In the matched group of children born to unaffected mothers and unexposed to TNFi, one case of demyelinating disorder was identified $(0.009 \%$; $95 \%$ CI $0.000,0.005)$.

Conclusion: In the largest cohort of RA offspring exposed to TNFi ever assembled, we did not identify any case of demyelinating disorders. However, as demyelinating disorders are a rare event during childhood (observed in less than $0.01 \%$ of our general population control group), we cannot conclude that RA offspring exposed to TNFi do not have an increased risk due to limited study power.

\section{8}

Serious Infections in SLE Offspring Exposed to Biologic DMARDs

Evelyne Vinet (McGill University Health Centre, Montreal); Susan Scott (McGill University Health Centre, Montreal); Christian Pineau (Montreal General Hospital, Division of Rheumatology, Montreal); Sasha Bernatsky (McGill University Health Centre, McGill University, Montreal)

Objectives: Belimumab and rituximab are monoclonal immunoglobulins, which are actively transported across the placenta during pregnancy, resulting in fetal exposure. We evaluated serious infections in SLE offspring exposed to belimumab and rituximab in the preconception and/or gestational period compared to unexposed SLE offspring, as well as to children from the generalpopulation.

Methods: The "SLE Pregnancies in the US Registry (SPUR)" cohort includes all women with $\geq 1$ hospitalization for delivery after SLE diagnosis, identified through MarketScan database (2011-2014), and a randomly selected control group of women, matched $\geq 4: 1$ for age, year of delivery, and state of residence. Only women continuously enrolled within MarketScan for $\geq 12$ months prior to delivery and with available child linkage were included in SPUR. We defined belimumab and rituximab exposure based on $\geq 1$ filled prescription during pregnancy and/or the preconception period. We ascertained serious infections in the offspring based on $\geq 1$ hospitalization with infection as a primary diagnosis, $\leq 12$ months of life.

Results: We identified 910 SLE offspring and 9066 matched controls. Mean maternal age was identical among SLE offspring and children born to unaffected mothers [both 33 years, standard deviation (SD) 4]. Only $3(0.3 \%)$ and $1(0.1 \%)$ SLE offspring were exposed respectively to belimumab and rituximab during the preconception and/or gestational period. No case of serious infection occurred in SLE offspring exposed to either belimumab and/or rituximab. The percent of serious infections in SLE offspring unexposed to biologic DMARDs was identical $(2.5 \%, 95 \%$ CI $1.9,3.8)$ to non-SLE offspring $(2.5 \%, 95 \%$ CI $2.2,2.8)$.).

Conclusion: In the largest cohort of SLE offspring ever assembled, we were unable to demonstrate a marked excess risk for serious infections in those exposed to belimumab and/or rituximab during pregnancy versus unexposed children, as the use of these biologic DMARDs was infrequent during pregnancy, limiting our study power.

\section{9}

\section{Indications for Cesarean Delivery in SLE Pregnancies}

Evelyne Vinet (McGill University Health Centre, Montreal); Paul Fortin (Université Laval, CHU de Québec, Quebec); Stéphanie Roberge (Université de Laval, Québec); Emmanuel Bujold (Centre Hospitalier de l'Université Laval (CHUL), Quebec); Nils Chaillet (Centre Hospitalier de l'Université Laval (CHUL), Quebec)

Objectives: Pregnant SLE women are at increased risk of cesarean delivery compared to pregnant women from the general population. Yet, to date, no one has evaluated the indications for cesarean delivery in SLE pregnancies. Using the large population-based "QUAlity of care, obstetrics RISk MAnagement, and mode of delivery (QUARISMA)" cohort, we assessed indications for cesarean delivery in SLE pregnancies versus unaffected pregnancies.

Methods: The QUARISMA cohort includes 184,952 pregnancies and was established as part of a cluster-randomized, controlled trial, which aimed to evaluate the effect of a 1.5-year intervention, involving audits of indications for cesarean delivery and feedback to healthcare professionals, at 32 hospitals in Quebec (2008-2011). The intervention had a small effect on the rate of cesarean delivery (Chaillet, NEJM, 2015). As part of the trial, in-hospital data, including maternal SLE status and information on indications for cesarean delivery, were abstracted by trained professionals from the medical records. For the present study, we individually matched 4:1 SLE pregnancies to unexposed pregnancies on maternal age, parity, body mass index (BMI), hospital of delivery, QUARISMA intervention group, and intervention period. We identified cesarean deliveries in SLE and unexposed pregnancies, and ascertained their indications. We performed multivariate

Personal non-commercial use only. The Journal of Rheumatology Copyright $\subset$ 2018 . All rights reserved. 
analyses to estimate the risk of cesarean delivery for any indications, as well as the risk of cesarean delivery for fetal distress (e.g. cardiac distress, prematurity, intra-uterine growth restriction, stillbirth), maternal disease, and/or preeclampsia in SLE pregnancies versus controls, adjusting for BMI and multiple births.

Results: We identified 122 SLE pregnancies and 488 unexposed pregnancies. Cesarean deliveries occurred in 35/122 (29\%) of SLE pregnancies versus 132/488 (27\%) of unexposed pregnancies. Among the 35 SLE cesarean deliveries, indications included (but were not limited to) cardiac distress in $34 \%$, prematurity in $29 \%$, maternal disease in $20 \%$, and preeclampsia in $11 \%$. Among the 132 unexposed cesarean deliveries, $28 \%$ were due to cardiac distress, $10 \%$ to prematurity, $4 \%$ to maternal disease, and $6 \%$ to preeclampsia. In multivariate analyses, there was a trend for an increased risk of cesarean delivery for any indications in SLE versus unexposed pregnancies (OR 1.6; 95\% CI 0.9,2.9). Moreover, SLE pregnancies had a substantially increased risk of cesarean delivery for fetal distress, preeclampsia, and/or maternal disease versus unexposed pregnancies (OR 2.6; 95\% CI 1.2,5.3).

Conclusion: Cesarean deliveries in SLE pregnancies are more often due to fetal distress, preeclampsia, and/or maternal disease than cesarean deliveries in pregnancies from the general population.

\section{0}

Time to Pregnancy in Women with Systemic Lupus Erythematosus Meriem El-bakali (Université de Montréal, Montreal); Sasha Bernatsky (McGill University Health Centre, McGill University, Montreal); Christian Pineau (Montreal General Hospital, Division of Rheumatology, Montreal); Evelyne Vinet (McGill University Health Centre, Montreal)

Objectives: Women diagnosed with systemic lupus erythematosus (SLE) during the reproductive period have fewer children than unaffected women. Multiple disease-related factors might influence family size in SLE women, including impaired fertility. However, until now, no one has assessed if women with SLE have a prolonged time to pregnancy (TTP). Therefore, we aimed to measure TTP in women with SLE of reproductive age and compare the occurrence of delayed TTP (i.e. $\geq 12$ months) to general population figures.

Methods: Female subjects with SLE from the Montreal General Hospital Lupus Clinic, aged 18-45 years, were enrolled between 2012 and 2017. SLE diagnosis was defined in accordance with the ACR classification criteria for SLE. At baseline and annual follow-up visits, the investigators administered a detailed reproductive questionnaire, wherein the TTP was measured. TTP was assessed retrospectively for all pregnancies occurring prior to the baseline visit and prospectively for all pregnancies occurring over the follow-up period. Disease damage index [i.e. SLICC damage index (SDI) score] and the mean disease activity (i.e. SLEDAI score) over a maximum of 5 years were recorded, previous cyclophosphamide (i.e. ever/never) and other relevant drug exposures. Descriptive statistics were calculated.

Results: A total of 333 women with SLE completed the questionnaire. Among these women, 135 never had a pregnancy and 198 conceived at least once. In women having $\geq 1$ pregnancies, we identified 400 pregnancies for which the TTP was assessed. The TTP was measured retrospectively in 347 pregnancies, of which 226 and 121 respectively occurred prior and after SLE diagnosis. We observed a TTP $\geq 12$ months in $4.9 \%$ (95\% CI 2.6, 8.8) of pregnancies occurring prior to diagnosis and in $9.1 \%(95 \% \mathrm{CI} 4.9,16.0)$ of pregnancies after diagnosis. 43 pregnancies occurred over the study period and $11.6 \%(95 \%$ CI $4.4,25.9)$ had a TTP $\geq 12$ months. None of the women with prolonged TTP had prior cyclophosphamide exposure nor were exposed to steroids while attempting to conceive. However, they tended to be slightly older (median 33 vs 31 years) and more likely to have a SDI $\geq 1$ [60\% (95\% CI 17,92$)$ vs $28 \%(95 \%$ CI 14,47$)$ ] compared to women with TTP $<12$ months.

Conclusion: Our findings suggest that the occurrence of delayed TTP after SLE diagnosis is similar to that of the general population, which has been consistently reported at approximately $10 \%$. However, larger observational studies, including notably an unexposed group of women without SLE, are needed to confirm our results.

\section{1}

Cardiotoxicity and Myotoxicity of Antimalarials: A Case-based and Systematic Review of the Literature

Shane Cameron (University of Manitoba, Winnipeg); Annaliese Tisseverasinghe (University of Manitoba, Winnipeg); Christine Peschken (University of Manitoba, Winnipeg)

Objectives: Antimalarials are strongly recommended as long-term therapy for systemic lupus erythematosus patients. Antimalarials are known to be associated with retinopathy; cardiac and neuromuscular toxicity has also been reported. With increased long-term exposure, the frequency of reported retinopathy has increased, but there is little understanding of risks for cardiac and neuromuscular toxicity. We therefore conducted a case based and systematic review of the literature.

Methods: MEDLINE, EMBASE and Cochrane Library databases were searched for case studies involving antimalarial cardiac and neuromuscular toxicity, or randomized controlled trials (RCTs) and observational studies involving adverse events associated with antimalarials. The search was not restricted by condition requiring antimalarial prescription. Quality assessments were performed with the Cochrane Risk of Bias tool for RCTs, and the Newcastle-Ottawa Scale for observational studies. Two individuals reviewed citations for inclusions/exclusion at each stage (title, abstract and full-text review).

Results: After duplicate removal, 5953 references were identified. After fulltext review, 28 RCTs and 15 observational cohort/case control studies were included in the systematic review, and 114 publications including 145 individual cases comprised the case study review. Mean duration of antimalarial exposure was 10-14 years for the cases of reported cardiomyotoxicity, but only 4.7 years for neuromyotoxicity; $<20 \%$ of patients with these toxicities had retinopathy. This suggests different risks for these 3 toxicities. Antimalarial exposure in the RCTs ranged from 6-132 months, with no reported cardio-or neuromyotoxicity. As no RCT specifically monitored for antimalarial cardio-or neuromyotoxicity, (although 2 studies performed serial electrocardiographs) quality was low with a high risk of bias. Of the 15 non-randomized studies, two retrospective studies identified myopathy as reasons for drug discontinuation in 2/156 and 6/719 patients. In 2 cross-sectional studies, one found bundle branch blocks in $3 / 85$ patients on antimalarials, another identified antimalarial myopathy in $15 / 119$ patients by muscle biopsy. The overall quality of the evidence was very low due to high risk of bias.

Conclusion: The evidence supports previous views that risk of cardio- and neuromyotoxicity due to antimalarials is relatively uncommon, and usually associated with long-term use. However, the quality of the evidence is very low, resulting in very weak conclusions. We were unable to find convincing evidence of specific risk factors that would help guide clinicians, nor was there enough evidence to provide an estimate of risk. Large scale longitudinal prospective studies with rigorous evaluation for these adverse events are needed, and any RCTs including these antimalarials should systematically assess patients for cardio-and neuromyotoxicity.

242

Trajectories of Quality of Life in an Inception Cohort of Lupus Patients and their Determinants

William Fung (University of Toronto, Toronto); Lillian Lim (Dept. Rheumatology, The Hospital for Sick Children, Toronto); Jiandong Su (Toronto Western Hospital, Toronto); Zahi Touma (Toronto Scleroderma Program, Division of Rheumatology, Toronto Western Hospital, University Health Network; Institute of Health Policy, Management and Evaluation, University of Toronto, Toronto)

Objectives: Systemic lupus erythematosus (SLE) is a chronic disease with varying disease activity levels and morbidities over time; this can affect patients' health related quality of life $(\mathrm{QoL})$. In clinical practice, we observe substantial heterogeneity in how patients' QoL evolve over time. This study aims to: 1) Determine if there are latent classes of QoL trajectories (T) among SLE patients as measured by the Medical Outcome Survey Short Form 36 (SF-36) and 2) Identify predictors of latent class membership. 
Methods: This is a single centre retrospective longitudinal inception SLE cohort. Annual SF-36 has been collected prospectively per clinical protocol. Only patients with $\geq 3$ SF-36 questionnaires, with the 1st within 2 years of diagnosis, were studied. The primary outcomes were the physical component (PCS) and mental component scores (MCS) of the SF36. Group based trajectory modelling was performed using Proc Traj (SAS). One model each was fitted for the PCS and MCS. The best model was determined by a combination of clinical plausibility and statistical criteria - number of patients in each group and fitting Bayesian Information Criterion (BIC). Models with the lowest BIC and groups containing $>5 \%$ of the total sample size were selected as the final models. Predictive effects of baseline variables on class membership were tested by logistic regressions.

Results: 171 patients with follow up to 10 years were analyzed. Mean age at SLE diagnosis was $35.7+13.4$ years, and $88 \%$ were female. For PCS, 2 classes of T were identified: T1 persistently very low PCS (54.6\%) and T2 low PCS with slight improvement (45.4\%). For MCS, 3 classes of T were identified: T1 persistently very low MCS (28.4\%), T2 low MCS improving over time (32.4\%) and T3 average MCS improving over time (39.2\%). After adjusting for other factors, PCS T2 was significantly associated with younger age at SLE diagnosis (OR 0.95 [95\% CI 0.92-0.97, p = 0.0001]) and trending towards significance for higher baseline disease activity (OR 1.04 [95\% CI 0.998-1.09, $\mathrm{p}=0.06])$. Regressions did not show significant results for MCS. Conclusion: The physical (PCS) and mental health components (MCS) of SF36 follows distinct classes of trajectory in SLE. Some patients have persistently very low PCS and MCS while others show mild improvement from below average to near or above average scores. Younger age at lupus diagnosis was associated with better PCS trajectory (T2).

\section{3}

Adherence to Antimalarials and Risk of Type 2 Diabetes Mellitus in Patients with Systemic Lupus Erythematosus: A Population-Based Cohort Study

Hans Haag (University of British Columbia, Vancouver); EC Sayre (Arthritis Research Canada, Richmond); Antonio Avina-Zubieta (University of British Columbia Faculty of Medicine; Arthritis Research Canada, Richmond); Mary De Vera (University of British Columbia Faculty of Pharmaceutical Sciences; Arthritis Research Canada, Vancouver)

Objectives: Aside from their effect on disease activity in systemic lupus erythematosus (SLE) antimalarials have been shown additional benefits such as reducing the risk of diabetes. However, a recent systematic review reported sub-optimal adherence to antimalarials with rates as low as $25 \%$. As medication adherence mediates patient outcomes, our objective was to evaluate the association between adherence to antimalarials and risk of type 2 diabetes mellitus (T2DM) among SLE patients.

Methods: Using a population-based database that includes all residents of British Columbia, Canada, we conducted a retrospective, longitudinal cohort study of patients with incident SLE and incident antimalarial use. We established drug courses for antimalarials - defining each new course when a 90-day permissible gap had been exceeded between refills and calculating corresponding proportion days covered (PDC). Our primary exposure was adherence to antimalarials according to three categories: 1 ) adherent (PDC $\geq 0.90)$; 2) non-adherent $(0<\mathrm{PDC}<0.90)$; and 3$)$ discontinuer $(\mathrm{PDC}=0$, no drug). T2DM outcomes were defined using International Classification of Disease 9th and 10th Revision Codes, and Canadian Drug Identity Codes for anti-diabetic medication (first date of either encounter). We used multivariable Cox's proportional hazards models with time-dependent variables to evaluate the association between adherence to antimalarials and risk of T2DM.

Results: The study cohort included 1,498 patients with incident SLE, with mean age of $44.4 \pm 14.8$ years and $1,360(90.8 \%)$ women. Mean number of antimalarial prescriptions/courses over follow-up was $23.2 \pm 37.7 / 2.1$ \pm 1.8 , with mean course duration of $553.9 \pm 820.8$ days. Over median 4.62 years of follow-up, we recorded 140 incident cases of T2DM. After adjusting for age, gender, comorbidities, and concomitant medications, the hazard ratio (HR) for those who were adherent to antimalarials was 0.61 (95\% confidence interval $[\mathrm{CI}], 0.40-0.93)$ as compared to discontinuers, suggesting a protective effect of adherence to antimalarials. In contrast, the HR for those who were non-adherent was 0.78 (95\% CI, $0.50-1.22)$ as compared to discontinuers. Sensitivity analyses involving permutations of permissible gaps (i.e. 120, 180 days) and PDC cut-off (i.e. 0.80) did not materially change our results.

Conclusion: These population-based data show a protective effect of adherence to antimalarials on risk of T2DM in SLE patients. Given the effectiveness of antimalarials in treating SLE as well as additional benefits, findings emphasize the need to raise awareness, among health professionals and patients with SLE, of the importance of adherence to these therapies.

244

Gallbladder Involvement in Lupus: A Case Report and Review of the Literature

Jesse Heyland (University of Calgary, Calgary); Dianne Mosher (University of Calgary, Calgary)

Case Presentation: A 22 year old female initially presented with symptoms of a transient ischemic attack and was diagnosed with systemic lupus erythematosus and secondary antiphospholipid antibody syndrome. During the first few days of her hospitalization, she developed abdominal pain localized to the right upper quadrant accompanied by nausea and vomiting. Serial abdominal ultrasounds showed progressive and evolving gallbladder wall thickness, and a mildly positive sonographic Murphy's sign. Her GGT (gamma glutamyl transferase) peaked at 362. General surgery was consulted and a HIDA (hepatobiliary iminodiacetic acid) scan was performed which was normal. She was diagnosed as having acute acalculous cholecystitis in the setting of systemic lupus erythematosus. She was treated conservatively with antibiotics and immunosuppression with a good clinical response. She was asymptomatic at time of discharge.

Conclusion: Systemic lupus erythematosus can rarely result a gallbladder involvement. A literature review was performed to assess our understanding of the pathophysiology, natural history and treatment outcomes of this condition. Cholecystitis in the setting of lupus has several proposed mechanisms: biliary dyskinesia, vasculitis, and vasculopathy in the setting of antiphospholipid antibodies. Untreated, it can commonly result in gangrene and perforation. Finally, more recent studies have shown that they can be managed conservatively with glucocorticoids.

\section{5}

Assessment of the Psychometric Properties of Patient-Reported Outcomes of Anxiety and Depression in SLE

Andrew Kwan (Queen's University, Toronto); Sherief Marzouk (University of Toronto, Toronto); Helia Ghanean (University of Toronto, Toronto); Kishwar Ali (UHN, Toronto); SLICC Investigators (Toronto Western Hospital, Toronto); Jiandong Su (Toronto Western Hospital, Toronto); Zahi Touma (Toronto Scleroderma Program, Division of Rheumatology, Toronto Western Hospital, University Health Network; Institute of Health Policy, Management and Evaluation, University of Toronto, Toronto)

Objectives: Mood disorders (MD) and anxiety disorders (AD) are amongst the most common manifestations of neuro-psychiatric SLE. Currently, the screening and diagnosis for MD and $\mathrm{AD}$ in ambulatory settings are delayed and often missed due to the lack of standardized valid questionnaires for assessing MD and AD in patients with SLE. This study aims to: 1) determine the prevalence of MD and AD in SLE patients using the Center for Epidemiological Studies-Depression Scale [CES-D], Hospital Anxiety and Depression Scale [HADS], and Beck Anxiety Inventory [BAI] questionnaires. 2) Study the criterion validity and interpretability of CES-D, HADS and BAI, and 3) evaluate their diagnostic accuracy when compared to the assessment of an independent psychiatric assessment using the Mini-International Neuropsychiatric Interview (MINI) - our gold standard. Methods: A cross-sectional study of consecutive SLE patients $(n=203)$, aged 18-65 and attending the Toronto Lupus Clinic between June 2017-September 2017, was performed. A subset of 118 participants were screened for MD and AD using the CES-D, HADS, and BAI, and underwent

Personal non-commercial use only. The Journal of Rheumatology Copyright @ 2018. All rights reserved. 
the MINI at their routine clinic visit. Conventional cut-off scores were used to indicate the prevalence of MD and AD: CES-D $\geq 16$; HADS-D $\geq 8$; HADS-A $\geq 8$; BAI $\geq 22$. Sensitivity, specificity, PPV, and NPV were evaluated against the MINI. Receiver operator characteristic (ROC) curves and the Youden Index were utilized to determine the optimal cut-off scores for CES-D, HADS and BAI.

Results: The prevalence of MD ranged from 27.6\% (HADS-D) to $45.8 \%$ (CES-D) and the prevalence of anxiety ranged from $29.1 \%$ (BAI) to $44.8 \%$ (HADS-A). ROC curves showed that the CES-D (AUC 0.85, 95\% CI: $0.75-0.95$ ) slightly outperformed HADS-D (AUC $0.83,95 \%$ CI: 0.73-0.93), compared to MD ascertained by MINI. The sensitivity, specificity, PPV, and NPV for CES-D at the optimal cut-off was $78 \%, 81 \%, 42 \%$, and $95 \%$, respectively. The diagnostic accuracy of HADS-A also outperformed BAI with AUC's of 0.67 (95\% CI: 0.52-0.83) and 0.65 (95\% CI: 0.50-0.80), respectively, for AD. Analysis by Youden index exhibited optimal cut-offs for CES-D (26), BAI (21), HADS-A (11), and HADS-D (8) that improved their sensitivity and specificity as screening metrics for MD and AD in SLE patients.

Conclusion: This study assessed the criterion validity and interpretability of patient-reported outcome tools: BAI, HADS, and CES-D for MD and AD screening in SLE patients. Compared to the gold standard, CES-D outperformed HADS-D, and HADS-A outperformed BAI. These results suggest that SLE-specific cut-offs may improve diagnostic accuracy of current screening metrics in lupus.

\section{6}

IgA Vasculitis and Lupus Nephritis: A Rare Combo

Mohammad Bardi (University of British Columbia, Vancouver); Natasha Dehghan (University of British Columbia, Division of Rheumatology, Vancouver)

Background: IgA vasculitis (formerly known as Henoch-Schönlein purpura) is generally seen in children between the ages of 3 to 15 with $10 \%$ of cases seen in adults. The incidence in adults is 5 per 100,000. Here we report the first adult case of IgA vasculitis presenting with lupus nephritis in a previously healthy adult.

Case Presentation: A 46-year-old woman with a prior history of resected thymoma eight months prior who presents to the emergency department with a one-week history of bilateral ankle and wrist pain, along with edema and purpuric rash over the lower extremities. She was treated with indomethacin and referred to rheumatology. On follow-up, laboratory data was remarkable for a mild pancytopenia with lymphopenia, proteinuria, hemoglobinuria and a high anti-nuclear antibody (ANA) titer. She also had positive antibodies to dsDNA, Smith, Histone, and RNP. There was a concern for a connective tissue disorder, but a skin biopsy showed cutaneous small-vessel vasculitis with positive staining for $\operatorname{IgA}$ and $\mathrm{C} 3$ on immunofluorescence. This supported a diagnosis of IgA vasculitis. Further investigations for small vessel vasculitis were negative. Given that she had slowly progressive proteinuria, she went on to have a renal biopsy that showed mesangial hypercellularity with IgG deposits more than IgA along with $\mathrm{C} 1 \mathrm{q}$ deposits that were consistent with class IIIa focal proliferative lupus nephritis. Immunosuppression with prednisone and mycophenolate-mofetil was initiated that lead to progressive normalization of her renal function with the resolution of proteinuria and normalization of her pancytopenia.

Conclusion: To our knowledge, this is the first case of IgA vasculitis presenting concurrently with lupus nephritis. This case highlights the importance of confirming a diagnosis with tissue whenever possible and to maintain a high suspicion for overlapping conditions even when they have not been previously encountered.

\section{7}

Pancreatitis as the Initial Manifestation of Systemic Lupus Erythematosus: A Case Report and Review of the Literature

Tooba Ali (Queens University, Kingston); Mark Matsos (McMaster University, Hamilton); Kimberly Legault (McMaster University, Hamilton) Lupus affects multiple organs; pancreatitis due to lupus has been reported in both the adult and pediatric population. Pancreatitis as the first manifes- tation of lupus is exceedingly rare. We report the case of a 21-year-old previously healthy female of Filipino descent who presented with severe pancreatitis without a clear etiology. The accompanying constellation of fever, alopecia, ulcers, photosensitivity and positive dsDNA, antiSM, SSA and SSB antibodies strongly suggested systemic lupus erythematosus. She improved with treatment with steroids, hydroxychloroquine, and azathioprine. We performed a review of the literature for pancreatitis as the first presentation of SLE. Fourteen cases have been reported in the English literature since 1980 of lupus presenting with pancreatitis. All were females ranging from age 13 to 54 . Fever, photosensitivity, and arthralgia were common. Hematological and renal involvement were the common organs involved. Three patients in the case series died from severe pancreatitis and associated lupus flare.

\section{8}

\section{Would-be Mimickers of Vasculitis}

Tooba Ali (Queens University, Kingston); Marie Clements-Baker (Queen's University, Kingston)

Case Presentation: A 54-year-old gentleman has a history of p-ANCA associated inflammatory pachymeningitis that required posterior fossa craniotomy and resulting hearing loss and $\mathrm{CN}$ deficits. His headaches and vertigo improved with high dose steroids which are slowly tapered off. $\mathrm{He}$ remained well on follow up. A year later he develops acute aortic insufficiency and cardiomyopathy requiring urgent aortic valve replacement and pacemaker insertion. Pathology of his aortic valve suggests lymphohistiocytic valvulitis. He remains in remission on low dose steroids and cardiac function slowly returns to normal. Another year later he develops new peripheral arthritis, worsening non-ischemic cardiomyopathy and peripheral neuropathy acutely over few weeks. The patient endorses new drug use including methyl amphetamine which may account for the new cardiomyopathy. However repeat p-ANCA levels are also significantly higher and EMG confirms new vasculitic neuropathy in peripheral nerves. A nerve biopsy is suggestive of inflammatory neuropathy. High dose steroids are begun for immunosuppression and drug use is discontinued while the patient is in hospital. Neuropathy and cardiomyopathy improve, however, it is difficult to ascertain if either the steroids or discontinuation of drug use contributed to the improvement.

Conclusion: This case illustrates the importance of a thorough history taking including illicit substance use as drug use can be a mimicker of vasculitis.

\section{9}

Prevalence, Characteristics, and Geographic Distribution of Systemic Sclerosis in Manitoba

Ali Almaleki (University of Manitoba, Winnipeg); Leigh-Anne Shafer (University of Manitoba, Winnipeg); Ada Man (University of Manitoba, Winnipeg)

Objectives: To estimate the prevalence, describe the clinical characteristics, and determine the geographic distribution of the systemic sclerosis ( $\mathrm{SSc}$ ) population in Manitoba.

Methods: All rheumatologists in Manitoba were surveyed for the SSc patients they currently follow. At the academic hospital, a database with SSc diagnoses extracted from physician billing codes collected from 1990 to 2016 was used. In the community clinics, electronic medical record systems operational from 2013-2017 were queried for SSc billing codes. All available charts were reviewed to confirm SSc diagnoses for patients at the academic hospital and 6/9 community clinics. An SSc case was defined as: 1) a living resident of Manitoba as of January 1,2017,2) $\geq 17$ years old, and 3) have a rheumatologist-diagnosis of SSc. Overlap disease with SSc was accepted if SSc was the predominant condition. Prevalence was calculated based on the 2016 census data. To account for the uncertainty of diagnosis of patients with unreviewed charts, we estimated prevalence estimates with sensitivity analyses assuming that 0.7 to 0.9 of such patients would have SSc. Data collected included age, date of diagnosis, gender, SSc subtype, and SSc manifestations. Postal codes were used to determine geographic distribution of the SSc population. 
Results: A total of 318 to 336 SSc patients were identified in Manitoba, corresponding to a prevalence estimate of 249 to 263 per million. We found higher prevalence (432 to 546 per million) in 2 sets of adjacent census divisions in northwest and eastern Manitoba. Of patients with available data $(\mathrm{N}=253), 14 \%$ were male, $86 \%$ were female, with mean age (SD) of 60 years (12.6). Mean age (SD) at diagnosis was 49 years (14.4). $74 \%$ had limited cutaneous SSc, 17\% had diffuse cutaneous SSc, 7\% had overlap disease, and $2 \%$ had sine scleroderma. Of patients with available data to calculate scores for the 2013 ACR/EULAR SSc classification criteria $(\mathrm{N}=$ 118), we found that more patients from the academic hospital met the criteria than those from community clinics. There was a greater proportion of patients with diffuse disease, and with younger age of diagnosis seen at the academic hospital.

Conclusion: The prevalence of SSc in Manitoba was estimated to be between 249 to 263 per million, which is in line with estimates from other parts of Canada and United States. Further cases may be found by surveying other physicians who may care for SSc without rheumatologists. Geographic areas with increased prevalence will be further investigated and confirmed.

250

Long-term Outcome of Patients with Systemic Sclerosis: Predictors of Organ Damage using Trajectory Models in a Large Canadian Scleroderma Inception Cohort

Leah Sinai (Schulich School of Medicine, London); Tatiana Nevskaya (Rheumatology Research, St. Joseph's Health Care, London); Mianbo Wang (Lady Davis Institute for Medical Research, Montreal); Murray Baron (McGill University, Jewish General Hospital, Montreal); Janet Pope (Western University, Department of Medicine, Division of Rheumatology, London)

Objectives: To study the course of internal organ damage over time in SSc patients using an inception cohort longitudinal design to identify damage trajectories, and test if damage trajectories have prognostic advantages over the traditional SSc classification into diffuse and limited subsets.

Methods: SSc patients from the CSRG database who had disease duration of $<5$ years from the onset of first non-Raynaud's symptoms with follow up data available were included. Internal organ involvement was assessed by Medsger's severity scale but considered as irreversible/damage only if the same (or higher) severity grade was present at the next annual visit. Outcome was the trajectory of organ damage over time as measured by the visceral organ damage score in lung, cardiac and renal systems. Latent class mixed models were fitted with the package "lcmm" in R 3.2.0. A lower Bayesian Information Criteria value indicated a preferred model. After selection of the classes, the number of patients and trajectory shapes of each class were determined. Multivariate logistic analysis was performed.

Results: Three main damage trajectories were identified in $305 \mathrm{SSc}$ patients. All SSc patients with rapid accumulation of damage early in disease course $(\mathrm{n}=11)$ had active disease at baseline (EScSG-AI $\geq 3)(\mathrm{p}=0.001)$. Compared to patients with the most favorable trajectory $(n=257)$, they initially had (all < 0.01$)$ : a higher mRSS $(22.3 \pm 11.6$ vs $11.8 \pm 10.8)$, both in upper and lower body areas, hyperpigmentation, all had mRSS $>7$ and more frequently mRSS $>25$ with a rapid skin progression rate and a shorter duration from RP onset to diagnosis. Rapid progressors showed more severe musculoskeletal involvement at first visit (tendon friction rubs finger flexion contractures, myositis), peripheral vascular disease, severe lung (lower $\operatorname{DLCO}(57 \pm 24 \%$ vs $76 \pm 24 \%, \mathrm{p}=0.01)$, more frequent $\mathrm{FVC}<70 \%(\mathrm{p}=$ $0.001)$ ), severe GI disease, severe cardiac and kidney involvement. Total Medsger's severity score was higher among rapid progressors $(13.3 \pm 3.2$ vs $8.0 \pm 3.9, \mathrm{p}=0.0001)$. RNA-polymerase III antibodies were more common while anti-centromere antibodies were absent in all rapid progressors $(p=0.015)$. The best predictors in the final model of rapid damage trajectory were FVC $<70 \%$, younger age of onset [45-54 years], renal involvement and tendon friction rubs (all $\mathrm{p}<0.01$ ). Neither skin score, nor SSc sybtype were predictive of rapid accumulation of organ damage.

Conclusion: Early SSc patients can be classified into three main damage trajectories based on the pace of visceral damage accumulation over disease course irrespective of SSc subtype.

\section{1}

Changes in Skin Scores Predict Internal Organ Involvement in Early Diffuse Systemic Sclerosis

Tatiana Nevskaya (Rheumatology Research, St. Joseph's Health Care, London); Murray Baron (McGill University, Jewish General Hospital, Montreal); Carl Baxter (MSD Ltd, Hoddesdon); Dena Ramey (Merck \& Co., Inc.,, Kenilworth); Canadian Scleroderma Research Group (CSRG) (Montreal); Janet Pope (Western University, Department of Medicine, Division of Rheumatology, London)

Objectives: To estimate whether severity and changes of skin thickening over 12-months in incident diffuse cutaneous systemic sclerosis ( $\mathrm{dcSSc})$ are related to progression of visceral disease.

Methods: Diffuse cutaneous SSc patients from the CSRG database who had disease duration from the onset of first non-Raynaud's symptom $\leq 5$ years with no evidence of end-stage internal organ damage and/or significant comorbidity at initial visit with 1 year follow-up (FU) data were included. Internal organ involvement was based on Medsger severity scores and CSRG definitions. Patient- and physician-assessed measures of health status were assessed. Adjusted univariate and multivariable regression analyses were used to study the association between skin thickening and subsequent organ damage.

Results: Higher baseline mRSS was significantly associated with worse disease severity (Medsger's total severity score without skin component, physician and patient global assessments), functional disability (HAQ-DI) and worse quality of life (SF-36 PCS) at baseline and 1-year FU. Baseline mRSS was not predictive of progression of organ involvement over one year. However, improvement in mRSS was found in $42 \%$ of patients and was associated with a lower activity index $(\mathrm{p}=0.0001)$. Over 1 year, those who improved on mRSS had less FVC decline (FVC changes $2.62 \pm 8.96 \%$ vs $-2.51 \pm 9.36 \%, \mathrm{p}=0.011)$ and better HAQ $(-0.16 \pm 0.39$ vs $0.08 \pm 0.53, \mathrm{p}=$ 0.006), compared with non-improvers. Cardiac involvement (new or worsening) was less in mRSS improvers $(10 \%$ vs $26 \%, p=0.018 ; 12 \%$ vs $31 \%, \mathrm{p}=0.008)$, new and progression in any internal organ $(18 \%$ vs $34 \%$, $\mathrm{p}=0.021 ; 31 \%$ vs $54 \%, \mathrm{p}=0.004)$, and multiple internal organ progression $(4 \%$ vs $17 \%, p=0.017)$ at 1 year and less severity score $(p=0.007)$. Skin score worsening occurred in $16 \%$ and was associated with further progression of joint/tendon involvement and renal involvement at 1-year FU visit $(p=0.032)$. Those with mRSS improvement showed a correlation between the degree of improvement (mRSS values) and improvement in disease severity by both physician assessment $(\mathrm{p}=0.002)$ and total Medsger's severity score without skin component $(p=0.005)$. After adjustment for confounders, mRSS improvement showed a protective effect on any new/progression of any internal organ involvement $(\mathrm{p}=0.020 / \mathrm{p}=$ $0.006)$ and HAQ $(\mathrm{p}=0.001)$.

Conclusion: High initial mRSS was linked to severity of joint contractures, worse function and quality of life, but was not predictive of visceral disease progression over a year. mRSS improvement was indicative of less frequent progression of internal organ involvement, less severe disease and improved HAQ.

252

Biopsy-positive versus Negative Giant Cell Arteritis: Clinic Presentation and Predictive Factors

Faiza Khokhar (McMaster University, Hamilton); Vanessa Ocampo (University of Toronto, Toronto); Karen Beattie (McMaster University, Hamilton); Angela Hu (McMaster University, Hamilton); Sankalp Bhavsar (McMaster University, Hamilton); Nader Khalidi (McMaster University, St Joseph's Healthcare Hamilton, Hamilton)

Objectives: Giant Cell Arteritis (GCA) is a granulomatous arteritis that usually affects the aorta and/or its major branches. It has a predilection for branches of the carotid and vertebral arteries, especially the cranial arteries, including the temporal artery. Temporal artery biopsy (TAB) is the gold

Personal non-commercial use only. The Journal of Rheumatology Copyright $\odot$ 2018. All rights reserved. 
standard to diagnose GCA; however, a negative biopsy does not exclude GCA. Whether patients with a positive TAB present differently than those without remains unknown. Thus, we characterized patient symptoms in those with and without a positive TAB and examined potential group differences. Methods: Retrospective chart reviews of patients with possible GCA involving the temporal arteries who underwent a TAB between 2000 and 2016 were performed. Extracted data included sex, age, biopsy result, TAB length, duration of glucocorticoid use and cumulative glucocorticoid dosage prior to biopsy. Laboratory markers (ESR, CRP) and symptoms potentially related to GCA were also extracted. Descriptive statistics and frequencies were determined for all outcomes in all individuals. Proportions of individuals in each biopsy group that reported symptoms were also determined. Group differences were not compared due to the small sample size. Results: Included in the analyses were 38 patients; 24 female (63\%), mean (SD) age 72.3 (9.7) years. Biopsy-proven GCA was confirmed in 14 patients (37\%). The mean age, TAB length and number of days on glucocorticoids prior to biopsy were virtually equivalent between groups with and without a positive TAB. The mean ESR and CRP levels for the negative biopsy group were $47.3(21.8) \mathrm{mg} / \mathrm{L}$ and $59.0(103.4) \mathrm{mg} / \mathrm{L}$, respectively. For the positive biopsy group, corresponding values were $54.5 \mathrm{mg} / \mathrm{L} \mathrm{(29.7)} \mathrm{and} 66.5 \mathrm{mg} / \mathrm{L}$ (33.4). Despite small numbers, there were apparent differences between those with versus without a positive TAB in the proportions of cases of scalp tenderness ( $71 \%$ vs $46 \%$ ), jaw claudication ( $43 \%$ vs $8 \%$ ), polymyalgia rheumatica (50\% vs $17 \%)$, and vision loss (21\% vs $17 \%)$. The proportions of headache complaints, temporal artery abnormalities on examination and mean temporal artery length appeared similar between groups.

Conclusion: In examining patients with possible GCA, there appeared to be a higher proportion of individuals with a positive TAB with scalp tenderness, jaw claudication, polymyalgia rheumatica and visual symptoms than with a negative TAB. Caution must be used in interpreting these results given the small sample size, yet they warrant further investigation. A larger sample size may help decipher differences in biopsy positivity rates based on TAB length, ESR and CRP.

\section{3}

Gangrene and Amputation Resulting from an Atypical Case of ANCA-associated Vasculitis

Arundip Asaduzzaman (North York General Hospital, Toronto)

Background: The ANCA-associated vasculitides (AAV) classically affect small blood vessels of the skin, joints, peripheral nerves, glomeruli, and respiratory tract. Limb ischemia or necrosis are thought to be very rare manifestations, being previously described only in a small number of case reports, and usually occurring in conjunction with more typical multisystem disease.

Case Presentation: A 51 year-old man with a history of hypertension presented to a community rheumatology clinic with psoriasis, peripheral inflammatory polyarthritis, anterior uveitis, and systemic symptoms. His investigations were remarkable for positive RF of $30 \mathrm{IU} / \mathrm{mL}$ and chronic CRP elevation greater than $100 \mathrm{mg} / \mathrm{L}$. His presentation was initially attributed to psoriatic arthritis, and he was treated sequentially with methotrexate, sulfasalazine, and a short course of prednisone. Due to inadequate response, adalimumab was started. Three days after receiving the first dose, he developed acute pneumonitis requiring mechanical ventilation. $\mathrm{He}$ then manifested a progressive foot drop, diffuse splinter hemorrhages, severe gangrene of the toes, increase in RF titre to $184 \mathrm{IU} / \mathrm{mL}$, and strongly positive c-ANCA (anti-PR3 $95 \mathrm{U} / \mathrm{mL}$ ). Although he had normal renal function, he had an active urinary sediment, and renal biopsy demonstrated a crescentic pauci-immune glomerulonephritis. He met the ACR/EULAR provisional 2017 classification criteria for granulomatosis with polyangiitis on the basis of his clinical and serologic features. The disease was controlled with high dose corticosteroids, PLEX, and oral cyclophosphamide. Unfortunately, there was irreversible tissue loss necessitating mid-foot amputation.

Discussion: This case illustrates a rare presentation of ANCA-associated vasculitis, with significant morbidity resulting from limb gangrene. Possible mechanisms for limb ischemia in AAV include active arteritis of medium- sized vessels, in situ thrombosis as a result of a pro-inflammatory state, or a combination of these factors. This case is also unusual in its temporal progression, which led to diagnostic uncertainty. The differential diagnosis evolved to include psoriatic arthritis, rheumatoid arthritis with vasculitis, cryoglobulinemic vasculitis, infective endocarditis, and ultimately granulomatosis with polyangiitis. This highlights the importance of appropriately revising a diagnosis in the face of discordant clinical and serologic features.

\section{4}

\section{Venous Thrombosis in Behcet's Syndrome}

Tahir Kanji (Queen's University, Kingston); Marie Clements-Baker (Queen's University, Kingston)

Objective: To describe a case report and literature review for venous thrombosis in Behcet's Syndrome.

Case Report: Mr. AG is a 23 year old gentleman with a past medical history of HLA-B27 positive peripheral arthritis, asthma, depression, episode of orchitis in 2009 and iron-deficiency anemia with a normal colonoscopy. He presented to his primary care physician's office with syncope and was found to have a large IVC thrombus with subsegmental PE and large right DVT. $\mathrm{He}$ was started on dalteparin and transitioned to rivaroxaban. He then presented to hospital several weeks later with worsening fatigue, dyspnea, increasing abdominal girth and was found to have progressive thromboses despite rivaroxaban along with Budd-Chiari syndrome. He underwent catheter directed thrombolysis but this was not successful. Worsening right leg pain with swelling and radiologic evolution of his right leg DVT prompted initiation of high dose heparin, IVIG and anti-thrombin 3 infusions. His review of systems was positive for 2 year history of recurrent oral ulcers and posterior truncal acne but negative for genital ulcers, ocular symptoms or pathergy-like reactions. His exam showed stable vitals, unremarkable oral, cardiorespiratory, abdominal and neurologic exams. His musculoskeletal exam showed right knee swelling, warmth and tenderness. His investigations revealed inconclusive immunofluorescence for ANCA but positive ELISA for MPO and PRE at titres of 74 and 44. His hypercoagubility, infectious and malignancy work-up was negative. He was initiated on cyclophosphamide pulse intravenously and transitioned to azathioprine maintenance therapy.

Discussion: Behcet's syndrome is a vasculitis syndrome of variable vessel size which affects veins more than arteries. Lower extremity vein thrombosis is the most frequent manifestation of vascular involvement. However, Budd-Chiari syndrome is a rare and serious complication of Behcet's syndrome. Histologically, a predominantly neutrophilic vasculitis around the vaso vasorum is typical of Behcet's syndrome. Other than that, vascular walls may show fibrous thickening, accompanied by non-specific inflammatory infiltrate. The current data indicates that the pathogenesis of thrombosis is not due to a coagulation abnormality. Two retrospective studies have suggested that anticoagulant treatment is ineffective in preventing venous thrombosis and rather immunosuppressant therapy should be considered the mainstay of therapy.

Conclusion: Venous thrombosis is a common manifestation of Behcet's Syndrome. Immunosuppression rather than anticoagulation should be mainstay of therapy.

\section{5}

Echocardiographic Screening in Childhood Onset Systemic Lupus Erythematosus

Georgina Tiller (BC Children's Hospital, Vancouver); Lori Tucker (BC Children's Hospital, Vancouver); Kimberly Morishita (BC Children's Hospital, Vancouver); Jaime Guzman (University of British Columbia, Vancouver); Kristin Houghton (BC Children's Hospital, Vancouver); Andrea Human (BC Children's Hospital, Vancouver); Angelyne Rivera (BC Children's Hospital, Vancouver); Ross Petty (British Columbia Children's Hospital, Vancouver); Shreya Moodley (BC Children's Hospital, Vancouver); David Cabral (Division of Rheumatology, Department of Pediatrics, BC Children's Hospital Research Institute \& University of British Columbia, Vancouver) 
Objectives: Cardiac manifestations are commonly seen in childhood onset systemic lupus erythematosus (SLE), and can be asymptomatic. Our clinical practice is to perform routine screening following SLE diagnosis and then two yearly, or as indicated if abnormalities are identified. The aim of this study is to determine the frequency of abnormal ecocardiogram (ECHO) findings identified by either routine screening or clinical indication in a cohort of children with SLE, and determine the association with SLE disease activity.

Methods: Patients diagnosed with SLE from 2005 -2015 were identified from our records (cutaneous or drug induced lupus were excluded) $(\mathrm{N}=115$; 20 charts reviewed to date). From retrospective chart review we collected all ECHO results, clinical and demographic data both at diagnosis and at time of ECHO testing including SLEDAI score and ESR as disease activity measures.

Results: Of the 20 patients included in this analysis, mean age at diagnosis was 13.7 y. $90 \%$ (18/20) had at least one ECHO. 10/18 (56\%) had an ECHO within three months of diagnosis. The mean time to first ECHO was $4.8 \mathrm{~m}$ (range 0-31); the median SLEDAI score at initial ECHO was 10 (IQR 3-13) and ESR 32 (IQR 11-62). Initial ECHO was requested routinely in $78 \%$ $(14 / 18)$ and for clinical indications (chest-pain, murmur, dyspnea, hypertension) in $22 \%(4 / 18)$. Of the $22 \%(4 / 18)$ with abnormalities (3 pericardial effusion, 1 LVH), median SLEDAI was 19.5 (IQR 9-25.5) and ESR 72 (IQR $7-136)$. Of 78\% (13/18) with no abnormalities, median SLEDAI was 6 (IQR 2-10) and ESR 30 (IQR 12.5-54.5). Abnormalities were found in 7\% (1/14) of ECHOs requested routinely and 75\% (3/4) requested for clinical indication. $72 \%(13 / 18)$ had at least two echocardiograms with mean time interval to second ECHO 1.9y (range 0.1-3.5). The median SLEDAI at second ECHO two was 4 (IQR 0-11), ESR 16 (IQR 8.5-35). Median SLEDAI score of the 2 patients with abnormal second ECHO was 13.5 and ESR 132; both had abnormalities in initial ECHO; one was performed for clinical indication.

Conclusion: Screening with echocardiography occurred in most pediatric patients diagnosed with SLE. Although one quarter of patients had an abnormal finding on initial echocardiogram, more findings were detected when there was a clinical indication. SLEDAI score and ESR did not appear to be associated with an increased risk of ECHO abnormalities. The 'Choosing Wisely Canada' campaign encourages smart and effective healthcare choices; this review may inform the optimal practice of echocardiographic screening in pediatric lupus.

\section{6}

\section{Ethnicity in Children with Auto-Inflammatory Disease in British Columbia}

Lori Tucker (BC Children's Hospital, Vancouver); Jenny Tekano (BC Children's Hospital, Vancouver); Kimberly Morishita (BC Children's Hospital, Vancouver); David Cabral (Division of Rheumatology, Department of Pediatrics, BC Children's Hospital Research Institute \& University of British Columbia, Vancouver); Kristin Houghton (BC Children's Hospital, Vancouver); Jaime Guzman (University of British Columbia, Vancouver); Andrea Human (BC Children's Hospital, Vancouver); Kelly Brown (Division of Rheumatology, Department of Pediatrics, BC Children's Hospital Research Institute \& University of British Columbia, Vancouver) Objectives: Published cohorts of patients with auto-inflammatory diseases (AID) show distinct patterns of occurrence; however, ethnicity in AID in a multi-ethnic country has not been well described. Our objective is to examine the ethnic backgrounds of a cohort of children from British Columbia (BC) with auto-inflammatory disease.

Methods: Children diagnosed with an AID and followed in the provincial Auto-Inflammatory Disease Clinic at the BC Children's Hospital, Vancouver, BC were enrolled into a longitudinal database (Jan 2016-present). Patients with a complete baseline visit were included in this analysis, and data extracted are: diagnosis (physician-assigned in the AID clinic), demographics, and parent self-reported ethnicity. Parents self-recorded all known ethnicity of all 4 grandparents, using modified Statistics Canada ethnicity listing which included additional ethnic groups known to have high rates of AID. Current Statistics Canada population estimates of ethnicity in BC (2006 census) were used to determine over- or under- representation of ethnicity.

Results: 58 children with AID were included, with a mean age at diagnosis of 5.5 y (range 1.4-16.6 y). Patient diagnoses included: Periodic fever, aphthous stomatitis, pharyngitis, and adenopathy (PFAPA; $n=19$ ), Familial Mediterranean fever (FMF; $\mathrm{n}=8$ ); TNF Receptor associated periodic syndrome (TRAPS; $n=4)$; Behcet's syndrome $(n=4)$; unclassified periodic fever syndrome $(n=20)$; and one each having Blau syndrome, Mevalonate kinase deficiency, and Cryopyrin-associated periodic syndrome. The majority of patients (84\%) were mixed ethnicity, with English/Irish/Scottish and European predominating, reflecting the general BC population. Seven children (12\%) with AID have First Nations background, with 4 having PFAPA and 3 Behcet's syndrome (5\% FN ethnicity in the BC population). Thirteen children (22\%) have Asian origin, with diagnoses of FMF, TRAPS, and unclassified AID. No children with PFAPA had Chinese, Filipino, South or South-East Asian background, although collectively these ethnicities comprise approximately $10 \%$ of the $\mathrm{BC}$ population.

Conclusion: Children with First Nations ethnicity were over-represented in this AID cohort, which is a new observation and may relate to the genetic origins of the West Coast FN peoples. Children with East and South Asian ethnicity seemed to be under-represented compared to the general $\mathrm{BC}$ population. Canadian patients with AID may have different ethnic background from previously reported European cohort studies, and further study including patients from other provinces would provide a more complete picture of AID in Canada.

\section{7}

Providing Patient and Family Education and Support for Children with Rheumatic Diseases and their Families: Cassie and Friends Annual Family Day in BC

Lori Tucker (BC Children's Hospital, Vancouver); Jennifer Wilson (Vancouver); Kristin Houghton (BC Children's Hospital, Vancouver); Kimberly Morishita (BC Children's Hospital, Vancouver); Jaime Guzman (University of British Columbia, Vancouver); Kelly Brown (Division of Rheumatology, Department of Pediatrics, BC Children's Hospital Research Institute \& University of British Columbia, Vancouver); Debbie Setton (Vancouver); Jayne Green (BC Children's Hospital, Vancouver); Jenny Tekano (BC Children's Hospital, Vancouver); Iris Davidson (BC Children's Hospital, Vancouver); Heather Best (BC Children's Hospital, Vancouver); Danielle Eccles (BC Children's Hospital, Vancouver); David Cabral (Division of Rheumatology, Department of Pediatrics, BC Children's Hospital Research Institute \& University of British Columbia, Vancouver) Objectives: To describe the growth and impact of an annual Family Day event on children with rheumatic diseases and their families in BC.

Methods: Family Day, an annual event starting in 2009, is organized and funded by Cassie and Friends, a registered BC charity dedicated to transforming the lives of children with rheumatic diseases and their families. It is a full day, multi-track conference held outside the hospital environment, organized collaboratively by the pediatric rheumatology team members at BC Children's Hospital and the Cassie and Friends volunteer conference committee. The program includes lectures, round-table discussions, and patient panels for parents, with both age-related recreational and educational activities for children and youth (i.e. peer to peer support). Content and format of the program is informed annually by feedback from previous Family Days and advice from pediatric rheumatology team members. Formal feedback from parent attendees is provided by a post-event survey.

Results: Registration has increased annually from 141 attendees in 2015 to 372 in 2017 with a wait list. In 2017, attendees were: 81 children and youth with rheumatic diseases, 68 siblings, and 150 parents and care-givers. A total of 72 speakers and volunteers participated; 28 medical professionals as speakers and volunteers, and 10 young adults with rheumatic diseases as volunteers. Families came from 29 communities throughout BC. 59\% of attendees completed the post-event survey. The 2017 event was rated as very good or excellent by $98 \%$ of respondents, with most stating they would

Personal non-commercial use only. The Journal of Rheumatology Copyright @ 2018. All rights reserved. 
recommend Family Day to other families, and planned to attend the event next year. Parents enjoyed opportunities to meet other parents and health professionals in small interactive groups, and asked for more actionable strategies to help them with their children. The post-meeting questionnaire identified what families perceived as their greatest challenges in dealing with their child's rheumatic disease: day-to-day family life, pain, promoting well-being, school, medications and treatments, and fear.

Conclusion: A family-centered interactive educational and recreational day offers the opportunity for peer-to-peer connections for both patients and parents, as well as education and support. In addition, Cassie and Friends Family Day is promoting the formation of a strong patient and family community, inclusive of health professionals, that addresses the needs of children with rheumatic diseases and their families.

\section{8}

Perspectives on Uptake of and Adherence to a 6-month Home- and Group-based Exercise Intervention for Children with Juvenile Idiopathic Arthritis (LEAP Study)

Douglas Race (University of British Columbia, Vancouver); Joanie Sims-Gould (University of British Columbia, Vancouver); Lori Tucker (BC Children's Hospital, Vancouver); Heather MacDonald (University of British Columbia, Victoria); Lindsay Nettlefold (University of British Columbia, Vancouver); Ciaran Duffy (Children's Hospital of Eastern Ontario, Ottawa); Jennifer Stinson (The Hospital for Sick Kids, Toronto); Kristin Houghton (BC Children's Hospital, Vancouver); Michele Gibbon (Children's Hospital of Eastern Ontario, Ottawa); Elizabeth Stringer (IWK Health Centre, Halifax); Shirley Tse (Hospital for Sick Children, Toronto); Debbie Feldman (Université de Montréal, Montreal); Heather McKay (University of British Columbia, Vancouver)

Objectives: To identify barriers and facilitators to the uptake of and adherence to a 6-month home-based exercise program for children with juvenile idiopathic arthritis (JIA).

Methods: A convenience sample of children with JIA (ILAR criteria) $(\mathrm{n}=$ $18)$ and their parents $(n=23)$ were recruited from a group of 23 child-caregiver dyads participating in an exercise intervention study; the Linking Exercise, Activity and Pathophysiology Exercise Intervention (LEAP-EI). Child-caregiver dyads completed in-depth, semi-semi-structured, one-to-one interviews with a trained interview moderator prior to starting the exercise program and at the end of the 6-month program

Results: Interviews with 17 children ( 8 girls, 9 boys) and 17 parents / guardians (13 mothers, 1 father, 1 grandmother, and 2 mother/father combined) were conducted prior to the exercise intervention and interviews with 14 children ( 8 girls, 6 boys) and 14 parents/guardians (13 mothers and 1 mother/father combined) were conducted after the exercise intervention. Thematic analysis of pre-exercise program interview transcripts revealed three reasons child-caregiver dyads initiated the exercise program: 1) potential health benefits, 2) selflessness and 3) parental support. Analysis of post-exercise intervention transcripts identified four main themes within a priori themes of barriers and facilitators to program adherence: 1) parental support, 2) enjoyment, 3) time pressures (subthemes: time requirement of exercise, scheduling, forgetting) and 4) physical ailments.

Conclusion: Major barriers to and facilitators to exercise for children with JIA fell into three categories: personal, social and programmatic factors. These barriers were not unlike those that emerged for unaffected children and youth. There is a desperate need to develop effective strategies to engage children in physical activity and to overcome barriers that prevent them from doing so. Parental support is a key facilitator of exercise adherence and future initiatives may potentially engage children and their families in developing solutions to enhance their participation in and commitment to physical activity.

\section{9}

Children with Auto-inflammatory Diseases in British Columbia, Canada: Years of Delay Before Diagnosis

Lori Tucker (BC Children's Hospital, Vancouver); Jenny Tekano (BC Children's Hospital, Vancouver); David Cabral (Division of Rheumatology, Department of Pediatrics, BC Children's Hospital Research Institute \& University of British Columbia, Vancouver); Jaime Guzman (University of British Columbia, Vancouver); Kristin Houghton (BC Children's Hospital, Vancouver); Kimberly Morishita (BC Children's Hospital, Vancouver); Kelly Brown (Division of Rheumatology, Department of Pediatrics, BC Children's Hospital Research Institute \& University of British Columbia Vancouver)

Objectives: Auto-inflammatory diseases (AInD) often first present in childhood. The protean manifestations of fever, rash and other inflammatory symptoms can be confused with common childhood illnesses. Disease identification and correct diagnosis may be delayed due to low recognition of AInD among community health care providers.

Methods: Consecutive patients seen in the provincial tertiary care pediatric AInD clinic at the BC Children's Hospital consented to participate in a longitudinal patient registry called CAN-Fever. Baseline disease data was collected from parents and hospital charts of subjects enrolled January 2016-August 2017. Subjects with complete CAN-Fever enrollment data were included, with data extracted on demographics, diagnosis, and time from symptom onset (parent report) to diagnosis.

Results: 66 patients (M:F 35:31, age at diagnosis mean 6.6 y, range 1-16 y) were enrolled into CAN-Fever with complete baseline data. Unclassified periodic fever syndrome $(n=20)$, Periodic fever, aphthous stomatitis, pharyngitis and adenopathy syndrome (PFAPA) (15), and Chronic recurrent multifocal osteomyelitis (CRMO) (15) were the most comment diagnoses; less frequent diagnoses were: Familial Mediterranean Fever (FMF) (8), TNF receptor associated periodic syndrome (TRAPS) (3), Behcet's syndrome (3), Cryopyrin-associated periodic syndrome (CAPS) (1), and Mevalonate Kinase Deficiency (1). Children with CRMO were older at diagnosis (mean 10.4 y) compared with children with periodic fever syndromes (mean $5.6 \mathrm{y}$; $\mathrm{p}<0.0001$ ). Median duration from symptom onset to diagnosis was $2.8 \mathrm{y}$ (IQR 2.0, $3.6 \mathrm{y}$; range 0.4-10.6) for children with any periodic fever syndrome, compared to 2 y (IQR 0.8,3.1 y; range 0-7.9) for CRMO. Five patients were diagnosed more than 8 years after symptom onset ( 2 with PFAPA, 3 unclassified). There was no significant differences in time from symptom onset to diagnosis between AInD diagnoses, with FMF having shortest duration (median $1.83 \mathrm{y}$ ) and PFAPA, Behcet's, CAPS, and unclassified syndromes all having median 3 years or longer before diagnosis was made. Median duration from AInD diagnosis to study enrollment was $1.7 \mathrm{y}$ (IQR 0.4,5.1 y; range 0-16.5)

Conclusion: Most children with $\mathrm{AInD}$ in $\mathrm{BC}$ did not receive a diagnosis for years, delaying opportunity for effective treatment. Poor recognition of AInD in the general medical community and previous lack of a dedicated expert clinical centre may be contributing factors. 
Author Index

\section{A}

Aaron S, 118

Abdullah H, 131

Abrahamowicz M, 27

Adachi J, 20

Adams D, 69

Adolf-Ubokudom E, 119, 121

Aghdassi E, 27

Ahluwalia V, 4, 94

Ahmad H, 9

Ahmad Z, 107, 108, 109, 188

Ahrari A, 182

Akhavan P, 180

Akhtari S, 71, 152

Al-Osaimi N, 34

Al-Sheikh H, 109

Alai Z, 42

Albert A, POD13, 235

Alemao E, 227, 228

Alharbi S, 107

Ali K, 245

Ali T, 247, 248

Allen M, POD09, 40

Almaleki A, 249

Altammar F, 170

Alten R, 26

Alyazidi R, 207

Amiable N, POD13, 234, 235

Amiri N, 46, 206

Amjad H, 167

Amlani A, 165

Andersen K, 96

Anderson K, 21

Anderson M, 70

Anis A, 197

Arbillaga $\mathrm{H}, 13$

Arendse R, 65, 171

Armaroli G, 231

Aron E, 12

Arslan F, 80, 81

Aryan N, 36

Asaduzzaman A, 253

Ash Z, 80

Astruc B, 135

Atallah J, 230

Au D, 144

Auza C, 12

Averns H, 62

Avina-Zubieta A, 28, 45, 55, 196 , 205, 243

Aydin S, POD10, 34, 79, 80, 81, 82, 137

Azimi T, 186

B

Bacani J, 44

Backman C, 32

Baer P, 83

Baeza N, 13

Bagovich M, 180

Baker M, 174

Bakowsky V, 211

Bangert E, 146, 147, 148
Bansback N, POD07, 197

Baraliakos X, 64

Barber C, POD06, 14, 15, 43, 94

Barber M, 198

Bardi M, 143, 246

Barker T, POD03

Barnabe C, POD06, 1,189

Baron M, 111, 112, 114, 116, 250, 251

Barr S, 184

Barra L, 128

Barry C, 183

Bartlett S, 35, 92, 93, 95, 96, 97 . 98, 99, 132, 213

Basappa N, 50

Bath B, 84

Batra S, 223, 224

Batthish M, 18

Baxter C, 116, 251

Beaton D, 105, 191,192

Beattie K, POD09, 17, 39, 40, $115,160,161,204,252$

Beattie S, 91

Beaulieu M, 157

Belak Z, 117

Bell M, 4

Benk-Fortin H, 234

Benseler S, 163, 168, 169

Bentow $\mathrm{C}, 12$

Berard R, 57

Bergman A, 160, 161

Bergman M, 9

Berkson L, 111

Bernatsky S, 15, 47, 92, 93, 94, 103, 104, 132, 237, 238, 240

Bernstein C, 100

Bessette L, POD13, 25, 26, 132, 219, 220, 227, 228, 230, 235, 234

Best H, 257

Bhavsar S, 204, 252

Biln N, POD01

Bingham C, 97, 98, 99

Bishay M, 190

Blackmore D, 140

Blanchard C, 177, 178

Blasutig I, 181

Boilard E, 6

Boire G, POD01, 92, 93, 95, 96, $132,157,213$

Boisvert M, 16

Bolton J, 100

Bombardier C, 4, 90, 172, 173, 225

Bonilla D, 105

Bookman A, 107

Bouchard-Marmen M, 6

Boucher V, 158

Boulos P, 65, 136, 171

Bourgoin S, 236

Boutrupe H, 141

Bowie D, 143

Boyd T, 187

Boyle R, 211

Brady L, 165
Brinkman W, 29

Brooks S, 4

Brosseau L, 29, 166

Brown J, 16

Brown K, 164, 231, 256, 257, 259

Bruce I, 198

Bruns A, 59

Bujold E, 239

Bukowski J, 138, 139

Bulloch A, 1

Bureau N, 210

Burmester G, 176

Butanis A, 98, 99

Butler E, 152

Bykerk V, 92, 93, 95, 96, 132, 213

\section{C}

Cabral D, 164, 214, 231, 255, 256, 257, 259

Calderon L, 151

Cameron S, 241

Camp J, 89

Canadian Council of Academic Rheumatologists (CCAR), 106

Canadian Early Arthritis Cohort Investigators, 92, 93, 95, 96, 132, 213

Canadian Scleroderma Research Group (CSRG), 114, 116, 251

Carapellucci A, POD10, 137

Cardillo T, 89

Cardoso A, 88, 91

Carette S, 122, 123, 124

Carl C, 8

Carmona R, POD10, 137

Carrier N, POD01

Carruthers M, 133, 156

Casillas M, 91

Cellucci T, 18

Cesta A, 4, 90, 172, 173, 225

Chaillet N, 239

Chaiton A, 180

Chambers D, POD03

Chan J, POD10, 137, 171

Chan M, 60

Chandran V, 67, 68, 70, 71, 72, $73,215,232$

Chapman K, 206

Charles-Schoeman C, 74

Chartrand S, 8

Chartrand-Lefebvrel C, 8

Chastek B, 175

Cheah J, 141

Chen A, 214

Chen C, 175

Chen L, 89

Chen X, 49

Cheng A, 233

Chhabra A, 207

Chhibber S, 129

Chin A, 8, 13

Chiu J, 231

Choi H, 55, 196
Choi M, 12, 13, 14, 15, 114

Choinière $\mathrm{M}, 210$

Choquette D, 25, 26, 227, 228

Choudhury S, POD03

Chow A, 167, 174, 219, 220

Chu Q, 50

Chu R, 145

Cibere J, 209

CIHR Team in Defining the Burden and Managing the Effects of Psychiatric

Comorbidity in Chronic Immunoinflammatory Disease, 100

Clark N, 144

Clarke A, 14, 15, 43, 47, 198

Claudepierre P, 138, 139

Clements-Baker M, 248, 254

Coates L, 66

Cogar A, 101

Cohen J, 29

Cohen M, 222

Colmegna I, POD02, 5, 158, 217, 233

Colosimo K, 105

Combe B, 69

Connolly S, 26

Cook R, 71

Cooke C, 162

Costanzo S, 112

Cotton K, 87

Coupal L, 25, 227, 228

Couto S, 173

Cribby S, 129

Crocker M, 38

Cuello-Garcia C, 102

Cui A, 144

Curran J, 18

Curtis J, 237

Cusnir I, 50, 221

D

Da Costa D, 27

Dabague J, 82

Dagenais P, 157

Daghistani Y, 156

Davidson I, 257

Davis C, 144

de Gaetano G, 112

de Hooge M, 138, 139

de la Torre I, 229

de la Torre M, POD02

De Vera M, POD14, 196, 243

de Vlam K, 83

Decker M, 44

Dehghan N, 28, 206, 246

DeLeon E, 98, 99

DeMasi R, 74

Denton C, 152

Dgetluck N, 218

Dhalla M, 164

Dhillon R, 65

Diamandis E, 232

Dickson C, 89

Diebold M, 57

Personal non-commercial use only. The Journal of Rheumatology Copyright (c) 2018. All rights reserved. 
Dixit S, 136, 219, 220

Dixon J, 15

Dobkin P, 157

Donaldson M, 191, 192

Dooley A, 85

Doria A, 58

Dougados M, 88, 138, 139

Doyle P, 156

Drew M, 60

Droit A, 16

Dropol A, 168, 169

Duchez A, 236

Duffy C, 18, 29, 56, 258

Duffy K, 18

Duffy P, 117

Durand C, 184

E

Eccles D, 257

Eder L, 71, 80, 82, 94, 134, 142, 146,180

Eicher J, 150

Eisner R, 140

El-bakali M, 240

Elalouf $\mathrm{O}, 68,70$

Elliott $S, 15$

Elmoufti S, 219, 220

English K, 186

Ennis D, 2

Erb S, 156

Erickson J, 76

Esdaile J, 32, 55

Espinoza-Gómez F, 22, 23

Eurich D, 59

Ewara E, 64

F

Fallon L, 75

Faraawi R, 174

Farewell V, 198

Farrer C, 199

Fay J, 175, 176

Feldman B, 3, 57, 59

Feldman D, 258

Fernandes A, POD01

Fernándes-Codina A, 113

Fiore S, 175, 176

Fitzcharles M, 222

FitzGerald O, 75

Fleischmann R, 76

Flores $\mathrm{G}, 144$

Fong M, 144

Fortin I, 136, 171, 219, 220, 230

Fortin P, POD13, 6, 15, 27, 29, 234, 235, 236, 239

Friesen R, 196

Fritzler M, 12, 13, 14, 43, 105, 114, 165

Frolkis A, 1, 189

Fu F, 60

Fung W, 146, 242

G

Gaboury I, POD08, 29, 157

Gagnon E, 16
Gagnon J, 29

Gaining Access for Indigenous

North Americans with

rheumatic disease, 101

Gakhal N, POD04, 37, 142, 181, 199

Galatas C, 112

Gallego C, 80

Gámez-Nava J, 22, 23

Gao C, 48, 49

Garg A, 153, 154

Garmish O, 223, 224

Gaudreault N, 210

Genovese M, 69, 89, 176, 229

Geoffrion M, 35

Gerhold K, 162

Gerschman T, 169

Gervais F, 157

Ghali B, 168

Ghanean H, 245

Ghosh-Swaby O, 31

Gibbon M, 258

Giese C, 87

Gignac M, 32, 191, 192

Gladman D, 15, 67, 68, 71, 72, $73,74,75,76,77,78,80,134$, 215

Goh I, 57

Goldsmith C, 24

Goldszmidt M, 182

González-López L, 22, 23

Gottheil S, 213

Gou P, 8

Goulet J, 8

Graff L, 100

Graham D, 74, 75

Green J, 195, 257

Green R, 105

Grenier L, 47

Grigoriadis E, 142

Grodzicky T, 8

Gu K, 159

Guadiana-Lozano R, 23

Guglielmi R, 141

Gui Y, POD01

Gulka L, 52, 149

Gunal E, 80, 81

Gutierrez K, 98, 99

Guy P, 193, 194

Guzman J, 59, 164, 255, 256, 257,259

Gyger G, 147

H

Haag H, 243

Haaland D, 21, 136, 171, 174, 219,220

Hakami N, 108

Hall J, 221

Hamasaki T, 210

Hamilton C, 144, 186

Hanley G, POD14

Hanly J, 15, 106, 177, 178, 198

Haraoui B, 21, 25, 26, 92, 93, 95 , $96,132,136,213,219,220$,
$227,228,229,230$

Haridas V, 223, 224

Harris P, 210

Harrison D, 63

Harrison M, POD07

Harvey P, 27, 71, 142

Hassan I, 216

Hasse S, 236

Hayat S, 153, 154

Hazel E, POD02

Hazlewood G, 197

He C, 48,49

$\mathrm{He} \mathrm{H}, 48,49$

Heffernan E, 141

Heitzmann J, 26

Helliwell P, 69, 76

Hewitt S, 27

Heyland J, 244

Hill C, 151

Hiraki L, 7, POD12

Hitchon C, 5, 42, 92, 93, 95, 96 , $100,101,132,213,217$

Hladunewich M, POD12

Hoa S, 8

Hochman J, 142

Hoens A, 52, 53, 149, 186

Hofkirchner A, 148

Hofstetter C, 94

Holms S, 144

Holody C, 87

Hong D, 155

Houghton K, 56, 164, 195, 255, $256,257,258,259$

Houston S, 44

Howren A, 196

Hsia E, 63

Hsu M, 83

Hu A, 204, 252

$\mathrm{Hu}$ C, 176

Huang K, 205, 206

Huber A, 18, 29, 59

Hudson M, POD02, POD07, 111, $112,114,147,152,233$

Hughes S, 231

Hulhoven R, 135

Human A, 164, 207, 255, 256

Humphrey-Murto S, 34

Husni E, 63

Huynh T, 112

Hyslop D, 89

\section{I}

Iacoviello L, 112

Ikic A, 235

Inman R, 134

Ioannidis G, POD09, 17, 20, 160, 161

Ishii T, 89

Ivory $\mathrm{C}, 79$

J

Jacobson M, 142

Jairath V, 64

Jamal S, 2

Jaremko J, 58, 141
Jaroszynska A, 21, 171

Jerome D, 37, 142, 199

Jilkine K, 187

Jin S, 41, 86

Johansson M, 141

Johnson K, 72

Johnson N, 169

Johnson S, 107, 108, 109, 188

Johnstone L, 51

Joneja M, 30, 33

Jones A, 193, 194

Jones B, 216

Jones D, 150

Jones H, 138, 139

Jones M, 97, 98, 99

Jones T, 74

Joshi R, 225

Julien A, POD13, 6, 27, 234, 235,236

Jung M, 14, 15, 43

K

Kalache F, 47

Kalyoncu U, 79

Kamissoko A, 217

Kanji T, 254

Kankanwadi S, 223, 224

Kaplan G, 1, 189

Kapur S, 21, 65

Karellis A, 222

Kargard M, POD06

Karsh J, 79

Katie L, 185

Katta R, 223, 224

Katz S, 110, 131, 145, 201, 202, 203, 212

Kaul P, POD05

Kavanaugh A, 63, 75

Kazem M, 55

Keeling S, POD05, POD10, 118, 137

Kelsall J, 136

Kerbachi M, 132

Kerr L, 69

Kerr S, 52, 149

Kessel C, 231

Keystone E, 9, 88, 89, 92, 93, 95 , $96,132,213$

Khalfan M, 134

Khalidi N, 204, 252

Khalil R, 20

Khamis N, 28, 45

Kharkar S, 223, 224

Kherani R, 24, 171

Khokhar F, 252

Khosravi-Hafshejani T, 28

Khraishi M, 26, 174, 219, 220

Kim L, 63

Kimura T, 175

Kiss A, POD04

Klar N, 226

Klar R, 229

Ko H, 156

Kobza A, 191, 192

Koehn C, POD07, 53, 144

Personal non-commercial use only. The Journal of Rheumatology Copyright @ 2018. All rights reserved. 
Koehn S, 144

Koenig M, 8

Kolinsky M, 50

Koller G, 212

Kopec J, 209

Kornelsen D, 101

Korrer S, 175

Kovalko I, 163

Krabbe S, 141

Krajden M, 156

Kremer J, 69

Kroeber G, 141

Kudaeva F, 226

Kung T, 30

Kur J, 127, 156

Kuriya B, 31, 94, 130

Kurum E, 81

Kvien T, 88

Kwan A, 245

Kwok K, 83

Kydd A, 196

L

Lacaille D, 32, 144, 191, 192 . 193, 194

Laframboise R, 16

Lake S, POD04, 180, 181

Lakhani A, 120

Lalonde L, 210

Lambert R, POD10, 137, 138, 139,141

Lambiris P, 146

LaMothe J, 189

Landewe R, 138, 139

Lang B, 18, 170

Lang M, 169

Laniel S, 35

Laprise S, 210

Larche M, POD09, 17, 40, 115, 160,161

Laskin C, POD12

Lau A, 20, 54

Lavoie K, 158

Laxer R, 3

LeBlanc C, 35

Leder T, 51

Lee C, 69

Leese J, 52, 149

Legault K, 102, 204, 247

Legge A, 177, 178

Lehman A, 21, 65, 66, 136, 171, 174

Lespessailles E, 76

Levy D, POD12, 7, 18, 57

Lewinson R, 1, 189

Li L, 19, 29, 32, 52, 140, 149, 167,186

Li X, 172

Li Z, 229

Liang P, POD01, 11

Lim L, 3, 242

Lim L, 159

Ling V, 94

Liu N, 121

Liu X, 9
Liu Y, 217

Lix L, 100

Lo K, 63

Loewen H, 5

Loewen J, 169

Logeart I, 138, 139

Lombardi S, 105

Longchamps M, POD13, 234, 235

Lopez-Lazaro L, 223, 224

Lopez-Romero P, 88, 229

Lora M, 233

Lowerison M, 1, 189

Luca N, 169

Lue $\mathrm{S}, 130$

Lukic T, 83

Lum W, 52, 149

Lund $\mathrm{H}, 88$

Luo G, 49

Lyddiatt A, 94

Lynd L, POD14

M

Maberley D, 55

Macdonald G, 52, 149

MacDonald H, 56, 258

Machhar R, 67

Macias W, 89

MacLeod A, 57

MacMullan P, 129

Macqueen S, 57

Mahendran S, 232

Mahler M, 12, 14

Mai A, 127

Mairiang D, 231

Maksymowych M, 141

Maksymowych W, POD01, POD10, 137, 138, 139, 140, $141,145,216$

Malik F, 141

Mallbris L, 76

Maltez N, 114

Maly M, 160, 161

Mammen C, 214

Man A, 10, 218, 249

Mancinelli C, 150

Mandhane R, 87

Maniccia A, 83

Maoui M, 9, 026

Marotta A, POD01

Marra C, POD14

Marrache M, 64

Marrie R, 100

Marshall L, 138, 139

Martin L, POD10, 36, 137

Martinez-Osuna P, 88

Marzo-Ortega H, 80

Marzouk S, 146, 245

Masetto A, POD01, POD10, 137, 171

Massicotte F, 25, 227, 228

Mathura P, 51

Matsos M, 39, 247

McDougall R, 117

McGhie T, 130

McGonagle D, 80, 82
McInnes I, 74, 83

McKay H, 56, 258

McKinnon A, 186

McMillan T, 200

McQuitty S, 186

Mease P, 83

Melaku Z, 5

Melki I, 6

Melkie A, 5

Meltzer M, 5

Mendel A, 103, 104

Mendoza-Vázquez G, 22, 23

Mengistu Y, 5

Mercer M, 169

Mezei M, 206

Michienzi S, POD01

Michou L, POD13, 16, 234, 235

Miettunen P, 169

Miller L, 8

Miller W, 38

Mills S, 144

Milman N, 126, 151

Min B, 118

Mitha A, 56

Mitsakakis N, 107

Mody G, 217

Moghaddam B, 45, 46

Mohammadi T, 197

Molto A, 138, 139

Moodley S, 255

Morais S, 119, 120, 121

Morgan E, 29

Morin S, 16

Morishita K, 164, 214, 255, 256, 257, 259

Morrison S, 27

Mosher D, POD10, 137, 244

Moura C, 92, 93, 132, 237

Moustafa A, 146

Movahedi M, 90, 172, 173, 225

Mtibaa M, 227, 228

Mukwikwi E, 47

Muntyanu A, 68, 73

Muratti E, 227, 228

Mustapha N, 122

$\mathbf{N}$

Nagaraj S, POD06

Nair B, 41, 84, 85, 86

Nalawade A, 223, 224

Nantel F, 21, 63, 64, 65, 66, 136, 171,174

Nash P, 69, 91

Nashi E, 47

Nathalie P, 111

Nava-Zavala A, 22, 23

Nettlefold L, 258

Neville C, 27

Nevskaya T, 116, 250, 251

Nézondet-Chetaille A, POD13

Nguyen $\mathrm{M}, 10$

Nimmo M, 28

Nunez F, 51

O

OBRI Investigators, 90, 173, 225
O'Brien C, 57

O’Dell J, 197

O'Melinn C, 38

Ocampo V, 252

Ogdie A, 83

Okpalauwaekwe U, 84, 85

Olszynski W, 21

Ortmann R, 88

Osborne B, 21, 65, 66, 136, 171, 174

Oswald A, 50

Otawa S, 88, 89, 229

Ouellet L, 235

Owen R, 125

Ozturk A, 81

$\mathbf{P}$

Pagé N, POD13

Pagnoux C, 122, 123, 124

Pannu T, 43

Panopalis P, POD02

Panwar J, 3

Papaioannou A, 20

Parent G, 234

Parfenova M, 11

Park C, 19

Park S, 144

Parker G, 135

Parsons L, 189

Paschke J, POD10, 137, 141

Paterson G, POD08, 166

Paterson M, 94

Patten S, 1, 189

Pedersen R, 138, 139

Pedersen S, 141

Pelaez S, 158

Pelletier J, 25, 227, 228

Pelletier M, POD13, 234, 235

Penz E, 84

Pepelassis D, 200

Peschken C, 15, 42, 100, 101, 241

Pesson K, POD03

Petriw L, 30

Petty R, 164, 255

Piche M, 208

Pineau C, 15, 27, 47, 104, 237, 238, 240

Pinto Correia A, 229

Ponzio A, 169

Pope J, POD11, 27, 90, 91, 92 , $93,94,95,96,113,116,132$, 213, 226, 230, 250, 251

Postema R, 227, 228

Proulx L, 29

Psaradellis E, 21, 66, 136, 171

Puhl N, 61, 118

Pulenzas N, 123, 124

Pullan A, 185

Puri N, 37

Q

Qendro T, POD02

Quail J, 41, 86

$\mathbf{R}$

Race D, 56, 258

Personal non-commercial use only. The Journal of Rheumatology Copyright (c) 2018. All rights reserved. 
Rafay M, 162

Rahman P, 65, 66, 69

Rai R, 21, 65, 136

Ramey D, 116, 251

Ramjee K, 37

Ramji A, 156

Rampakakis E, 21, 65, 66, 90, 136, 179, 222, 225, 230

Raynauld J, 25, 227, 228

ReACCh-Out Investigators, 59

Rebello R, 204

Reddy S, 125

Reid G, 156

Reis J, 65

Remillard M, 25, 227, 228

Reynolds J, 46

Rich E, 8

Richards C, 5

Riebeling-Navarro C, 22

Rivera A, 255

Roberge P, 157

Roberge S, 239

Roberts J, 50, 51

Robinson D, 101

Rocha-Muñoz A, 22, 23

Rogers P, 32

Rohekar S, 134, 208

Rollet-Labelle E, 234, 235

Romeyer F, 230

Rooney T, 89, 229

Rose-Davis B, 18

Rosen C, 215

Rosenberg A, 59

Rostom K, 126

Roth J, 34

Roux S, 157

Rovensky S, 51

Ruderman E, 88

Rumsey D, 18, 58, 59, 60

Rusted B, 169

Ruttan L, 105

Rydz A, 60

\section{$\mathbf{S}$}

Sadatsafavi M, POD14

Salazar-Páramo M, 22, 23

Salituri J, 111

Sampalis J, 90, 179, 222, 225, 230

Sanchez-Guerrero J, 107

Sareen J, 100

Sauvageau D, 25, 227, 228

Savill J, POD01

Savu A, POD05

Sayad J, 151

Sayre E, 19, 191, 193, 194, 209, 243

Schieir O, 92, 93, 95, 96, 132, 213

Schlichting D, 88, 91

Schmeling H, 169

Scott S, 237, 238

Scuccimarri R, 5, 59

Seah X, 32

Semalulu T, 115
Senécal J, 8

Setton D, 257

Shafer L, 249

Shafer S, 141

Shaikh K, 33

Shaikh S, 219, 220

Sharma M, 84

Shiff N, 59

Shimabukuro N, 233

Shir Y, 222

Shojania K, 28, 55, 156, 196 , 205, 206

Sholter D, 136

Shuler C, 76

Shupak R, 185

Siddiqi Z, 140

Silverman E, POD12, 7

Sims-Gould J, 258

Sinai L, 250

Sirois A, 35

Skowronska-Cieslak B, 119

SLICC Investigators, 78, 103 , 104, 105, 198, 245

Smith D, 114

Smith K, 98, 99

Smolen J, 89, 91, 229

Smylie M, 50

Sobchak C, 71

Soo J, 50

Speechley M, 226

Spitzer K, POD12

Spooner L, POD07

St-Aubin A, 27

St. Pierre Y, 15, 43, 104, 198

Starr M, 21, 36, 222

Ste-Marie P, 222

Stefanelli V, POD03

Steiman A, POD12, 181

Stein M, 179

Stevenson R, 169

Stewart J, 21

Stewart M, 127, 209

Stewart S, 84

Stimec J, 3

Stinson J, 29, 258

Stoustrup P, 163

Stringer E, 18, 258

Stringer L, 169

Stutz M, 171

Su J, 27, 77, 78, 105, 146, 242, 245

Su L, 198

Sun X, 51

Swami V, 58

Sweezie R, 4

Syrotuik J, 135, 219, 220

Szeto V, 73, 215

Szumski A, 138, 139

T

Taheri R, 35

Takeuchi T, 89, 229

Tam E, 156

Tam H, 162
Tan J, 195

Tana T, 34

Tanwani J, 77

Tanzil J, 160, 161

Tao J, 7

Tarnopololsky M, 165

Tartaglia C, 105

Tavakoli H, 45, 55

Taylor-Gjevre R, 41, 84, 85, 86

Tekano J, 195, 256, 257, 259

Teo M, 65, 136

Tervaert J, 118

Tessier P, POD13, 234, 235

Thiers B, 74

Thorne C, 92, 93, 94, 95, 96, 132,213

Tian L, 49

Tiller G, 255

Timmerman N, 54

Tin D, 92, 93, 95, 96, 132, 213

Tisseverasinghe A, 42, 159, 241

Tkaczyk C, 21, 174

To F, 2, 156

Torres M, POD03

Totterman S, POD09, 40, 160

Touma Z, 78, 105, 107, 146, 242, 245

Toupin-April K, POD08, 29, 166

Towheed T, 148

Tran B, 52, 149

Tran K, 32

Trask C, 84

Trinh B, 141

Troyanov Y, 114

Tsao N, POD14, 196

Tse S, 3, 258

Tselios K, 77, 78

Tsoukas A, 21

Tucker L, 18, 56, 164, 255, 256, $257,258,259$

Tugwell P, POD08, 29, 102

Turcotte A, 25, 227, 228

Turk M, POD11

Twilt M, 163, 168, 169

$\mathbf{U}$

Upchurch K, 120

Ureyen S, 79, 80, 81, 82

Urowitz M, 15, 77, 78, 198

V

Vaillancourt J, 65

Vallée M, 16

Vallerand I, 1, 189

Valois M, 92, 93, 95, 96

van As B, 32

van der Heijde D, 138, 139

Veale D, 74

Veeramreddy D, 169

Venne K, 8

Vetsch E, 87

Villeneuve E, 25, 227, 228

Vinet E, 15, 47, 103, 104, 147 237, 238, 239, 240

Vitti M, 105
Vlahos B, 138, 139

\section{W}

Wade J, 156

Wade S, 133

Walker J, 100

Walker J, 50

Walker K, 113

Wall E, 110

Wang C, 74, 75, 83

Wang $\mathrm{H}, 48,49$

Wang L, POD12

Wang M, 112, 114, 147, 152, 250

Wang N, 140

Wang Q, 48

Ware M, 222

Watanabe S, 135

Watson W, 38

Weber U, 141

Wei Q, 48

Weiss P, 141

Wells G, 151

Wen S, 150

Westby M, 38, 53

White B, 218

White T, POD06

Whitney K, 57

Whittle S, 151

Wiber S, 117

Widdifield J, 94, 181

Wijeysundera D, 108

Wilk N, 35

Wilson J, 257

Winthrop K, 89

Wither J, 105

Wojcik S, 112

Wolk R, 74

Wong B, POD04

Wong-Pack M, 20

Wright J, 18

Wright K, 24

Wu L, 41

$\mathbf{X}$

Xiang X, 32

Xie H, 191, 193, 194

Xie L, 229

Xie Y, 49

Y

Yacyshyn E, 51, 61, 125, 145, $155,190,216$

Yang L, 131

Yazici Y, 9

Ye C, 44, 50, 131, 212, 221

Ye J, 67, 68, 72, 73, 215

Yeh V, POD03

Yelovich M, 39, 40

Yerushalmi M, 70

Yeung J, POD10, 137, 205

Yeung Y, 128

Yoshida E, 156

Yu X, 48, 49

Yuan Y, 60 
Zeiadin N, 62

Zhang C, 48

Zhang L, 100

Zhang T, 54
Zhao C, 236

Zhao S, 24

Zhdan V, 223, 224

Zheng B, 116

Zhou A, 73, 215
Zhu B, 88, 91

Zhu S, 48, 49

Ziegler D, 210

Ziouzina O, POD10, 137

Zou H, POD09, 17, 40, 160, 161 